\title{
THE SEARCH FOR SUPERSYMMETRY: PROBING PHYSICS BEYOND THE STANDARD MODEL
}

\author{
Howard E. HABER \\ Department of Physics, University of California, Santa Cruz, CA 95064, U.S.A. \\ and \\ Stanford Linear Accelerator Center, Stanford, CA 94305, U.S.A. \\ and \\ G.L. KANE \\ Randall Laboratory of Physics, University of Michigan, Ann Arbor, MI 48109, U.S.A.
}

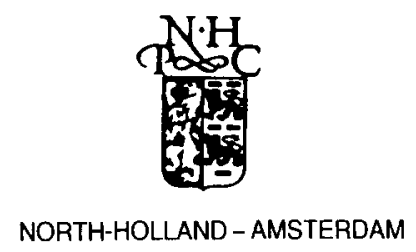




\title{
THE SEARCH FOR SUPERSYMMETRY: PROBING PHYSICS BEYOND THE STANDARD MODEL
}

\author{
H.E. HABER* \\ Department of Physics, University of California, Santa Cruz, CA 95064**, U.S.A. \\ and \\ Stanford Linear Accelerator Center, Stanford, CA 94305, U.S.A. \\ and \\ G.L. KANE** \\ Randall Laboratory of Physics, University of Michigan, Ann Arbor, MI 48109, U.S.A. \\ Received 11 May 1984
}

\section{Contents:}

1. Supersymmetry-modelling physics beyond the standard model

1.1. Introduction

1.2. Theoretical motivation for expecting physics beyond the standard model

1.3. Motivation for supersymmetry

1.4. Supersymmetry as a model of physics beyond the standard model

1.5. Photino (and other supersymmetric particle) interactions

1.6. Comments

1.7. Remarks on references

2. Notation and nomenclature

3. Survey of the supersymmetric particle spectrum

3.1. Scalar-leptons

3.2. Scalar-neutrinos

3.3. Scalar-quarks

\begin{tabular}{|c|c|}
\hline & 3.4. Gluinos \\
\hline 77 & 3.5. Charginos \\
\hline \multirow[t]{2}{*}{77} & 3.6. Neutralinos \\
\hline & 3.7. Goldstinos \\
\hline 79 & 4. $W$ and $Z$ decay at the $\bar{p} p$ colliders \\
\hline 82 & $\begin{array}{l}\text { 4.1. The decay of } \mathrm{W}, \mathrm{Z} \text { into supersymmetric scalar-lep- } \\
\text { tons }\end{array}$ \\
\hline 83 & 4.2. The decay of $\mathrm{W}, \mathrm{Z}$ into supersymmetric fermions \\
\hline 87 & $\begin{array}{l}\text { 4.3. Comments on } \mathrm{W} \text { and } \mathrm{Z} \text { decay into supersymmetric } \\
\text { particles }\end{array}$ \\
\hline 88 & 5. Supersymmetry in $\mathrm{e}^{+} \mathrm{e}^{-}$(and related) physics \\
\hline 89 & 5.1. Continuum $\mathrm{e}^{+} \mathrm{e}^{-}$production of supersymmetry \\
\hline 90 & 5.2. Quarkonium decay into supersymmetric particles \\
\hline 91 & 5.3. Supersymmetry at the $Z^{0}$ \\
\hline 91 & 5.4. Supersymmetry in two-photon physics and miscel- \\
\hline 96 & laneous processes \\
\hline 102 & 6. Supersymmetry at hadronic colliders \\
\hline
\end{tabular}

* Supported in part by the National Science Foundation, grant PHY-8115541 and in part by the Department of Energy, contract DE-AC0376SF00515.

** Supported in part by the Department of Energy, contract DE-AC02-76ER01112

*** Permanent address.

Single orders for this issue

PHYSICS REPORTS (Review Section of Physics Letters) 117, Nos. 2-4 (1985) 75-263.

Copies of this issue may be obtained at the price given below. All orders should be sent directly to the Publisher. Orders must be accompanied by check.

Single issue price Dff. 114.00, postage included. 
7. Supersymmetry in ep physics

7.1. Supersymmetric components of the nucleon

7.2. Associated production of supersymmetric particles at ep machines

8. Indirect constraints on supersymmetric parameters

8.1. $g-2$ of the electron and muon

8.2. Flavbr changing neutral currents

8.3. Parity violation in the strong interactions

8.4. The $\rho$-parameter

8.5. $C P$-Violation

8.6. Rare decays of $\mathrm{K}$ and $\pi$

8.7. Cosmological constraints

8.8. Proton decay and baryon number violation

8.9. Anomalies

9. Theoretical expectations for masses

10. Alternative models and assumptions

10.1. Enlarging the effective low energy gauge group

10.2. Enlarging the particle content of the low energy theory

10.3. Theories which violate $R$-parity

10.4. A phenomenologically important Goldstino (or gravitino)

10.5. Grand unification and supersymmetry

11. Future prospects and conclusions
Appendices

A. Spinor notation and conventions 196

B. How to construct Lagrangians for an SU(2) $\times U(1)$ mode] of broken supersymmetry

1. Recipe for constructing Lagrangians

2. A model of supersymmetric $\operatorname{SU}(2) \times U(1)$ in which the gauge symmetry breaks to $\mathrm{U}(1)$

C. The Lagrangian and Feynman rules

1. Mixing of scalar-leptons (or quarks) 208

2. Mixing of charged gauginos and higgsinos 209

3. Mixing of neutral gauginos and higgsinos 214

4. The Feynman rules 219

D. Feynman rules for Majorana particles 233

E. Sample calculations 239

1. $\mathrm{e}^{+} \mathrm{e}^{-} \rightarrow \bar{\gamma} \bar{\gamma} \quad 239$

2. ' $\mathrm{S}_{0} \rightarrow \tilde{\gamma} \bar{\gamma}$

3. $\mathrm{e}^{+} \mathrm{e}^{-} \rightarrow \gamma \tilde{\gamma} \tilde{\gamma},{ }^{3} \mathrm{~S}_{1} \rightarrow \gamma \bar{\gamma} \tilde{\gamma}$

4. $\mathrm{e}^{-} \mathrm{e}^{-} \rightarrow \tilde{\mathrm{e}}^{-} \overline{\mathrm{e}}^{-}$

5. ${ }^{1} \mathrm{~S}_{0} \rightarrow \tilde{\gamma} \tilde{\gamma}$ revisited 246

6. $\mathrm{e}^{+} \mathrm{e}^{-} \rightarrow \bar{\nu} \bar{\nu}$

7. Cancellation of quadratic divergences 250

Note added in proof 251

References 251

\begin{abstract}
:
In this paper we survey methods by which supersymmetry (or other new physics) could be observed in experiments at present and future accelerators. We review some of the motivation for supposing supersymmetry might be a symmetry of nature even though there is presently no evidence for it. We try to systematize the necessary new notation, and discuss in some detail how to calculate results, with considerable emphasis on pedagogical completeness. We summarize present limits on the existence of supersymmetric partners of ordinary particles, and show how to get improved quantitative limits if supersymmetric particles are not detected, so that eventually it is possible to be sure they are either detected or do not exist on the mass scale accessible to experiments.
\end{abstract}

\title{
1. Supersymmetry - modelling physics beyond the standard model
}

\subsection{Introduction}

At present (circa 1983) there is a very successful model which describes either quantitatively or (in the absence of precise computations) qualitatively nearly all available data pertaining to the strong, weak and electromagnetic interaction phenomena. This is an amazing state of affairs when one considers that just sixteen years ago, there was no theory of the weak or strong interactions. Apart from quantum electrodynamics (QED), particle physics phenomena were at best described by piecemeal phenomenological models with no fundamental theory in sight. Today, the Glashow-Weinberg-Salam theory [1.1] accurately describes weak and electromagnetic (now referred to as electroweak) phenomena, and quantum chromodynamics (QCD) [1.2] is accepted as the theory of the strong interactions. Thus, all of low energy particle physics phenomena (including the recent discovery of the $\mathrm{W}^{ \pm}$and $Z^{0}$ bosons [1.3]) fits well with what has come to be called the Standard Model - a gauge theory based on $S U(3) \times S U(2) \times U(1)$. Only one piece in the puzzle is missing - the spin zero elementary Higgs boson needed by the Standard Model for spontaneous symmetry breaking (which is responsible for masses for the $\mathrm{W}^{ \pm}, \mathrm{Z}^{0}$ and fermions). Although one could argue that it is only a matter of time until the Higgs boson will be discovered (depending on its mass which is not fixed by the theory), it is widely 
thought that deeper problems exist, connected with the Higgs boson, which suggest that it is necessary to look beyond the Standard Model to understand the Higgs sector of the theory.

If there is new physics beyond the Standard Model, several questions naturally arise, of which we will consider two. First, at what energy scale will the new physics enter and, second, how will the new physics be identified? There are strong theoretical arguments, which we shall summarize below, that the new physics will enter in the range $100 \mathrm{GeV}-1 \mathrm{TeV}$. This is a very exciting possibility since the lower end of this energy range is already being probed for the first time (by the CERN $\bar{p} p$ collider). Other facilities now under construction aim to study this energy range in detail. These facilities include the $Z^{0}$ factories SLC and LEP, an $\mathrm{e}^{-} \mathrm{p}$ facility HERA and the TEVATRON $\bar{p} p$ collider at FERMILAB. In about a decade we expect pp collisions at superhigh energies (up to about $40 \mathrm{TeV}$ in the center-of-mass) at the U.S. Superconducting Super Collider (SSC) and perhaps at a European supercollider. For completeness, we summarize some of the properties of the various accelerators in table 1.

The second question raised above (how will the new physics be identified?) is harder to answer and is the subject of this report. Although we deal explicitly with the new phenomena expected from supersymmetry, and we believe there are good physics arguments for thinking that supersymmetry might be a valid symmetry in nature, it is still true that no one knows what form the new physics will take. It is worth emphasizing that even if nature is not supersymmetric, there is much to be learned about ways the new physics might appear from carefully examining any specific theory.

By far the most intensely studied class of theories as a possible candidate for new physics beyond the Standard Model has been supersymmetry. In addition to having certain properties needed in order to solve various theoretical puzzles, supersymmetric theories contain a large number of experimental consequences.

The purpose of this report is to review and summarize the experimental consequences of supersymmetry. This includes tightening the constraints on the parameters of supersymmetric models (in the absence of new phenomena) as well as identifying ways by which supersymmetry (and in general, signals of new physics beyond the Standard Model) can be discovered. We have attempted to make this review particularly useful for experimentalists and for theorists who are not yet experts in supersymmetry. Some of the necessary theoretical machinery is pedagogically explained and emphasis is placed on describing the most likely ways to discover supersymmetry if it exists. In addition, we hope that this review can be used as a springboard which can lead to new ideas or more detailed work on identifying

Table 1

Summary of present and future colliders currently expected to exist

\begin{tabular}{lllll}
\hline Machine & Max $\sqrt{s}(\mathrm{GeV})$ & $\mathscr{L}\left(\mathrm{cm}^{-2} \mathrm{~s}^{-1}\right)$ & Turn on & Type \\
\hline PETRA & $30-45$ & $\sim 10^{31}$ & Now-1984 & $\mathrm{e}^{+} \mathrm{e}^{-}$ \\
PEP & $\sim 30$ & $\sim 10^{31}$ & on & $\mathrm{e}^{+} \mathrm{e}^{-}$ \\
TEVATRON & & & & \\
collider & 2000 & $10^{30}-10^{31}$ & 1986 & $\overline{\mathrm{p}}$ \\
Spp̄S collider & $\sim 640$ & $10^{29}$ & 1984 & $\overline{\mathrm{p} p}$ \\
& $\sim 640$ & $10^{30}$ & 1986 & $\overline{\mathrm{p} p}$ \\
SLC & $\sim 100$ & $\sim 6 \times 10^{30}$ & $1986-87$ & $\mathrm{e}^{+} \mathrm{e}^{-}$ \\
LEP & $100-170$ & $\sim 2 \times 10^{31}$ & $1988-89$ & $\mathrm{e}^{+} \mathrm{e}^{-}$ \\
HERA & $\sim 310$ & $\sim 5 \times 10^{31}$ & 1990 & $\mathrm{e}^{ \pm} \mathrm{p}$ \\
Tristan & $60-70$ & $\sim 10^{31}$ & $1987-88$ & $\mathrm{e}^{+} \mathrm{e}^{-}$ \\
& 170 & $\sim 5 \times 10^{31}$ & $\sim 1989$ & $\mathrm{e}^{ \pm} \mathrm{p}$ \\
SSC & $\sim 40000$ & $\sim 10^{33}$ & $1995 ?$ & $\mathrm{pp}$ \\
\hline
\end{tabular}


supersymmetry in future experiments. Basically, we can summarize our goal as being either to ensure that evidence for supersymmetry is found if it exists, or to become confident that it is absent at all energies accessible to experiment if it does not exist.

An aspect of searching for supersymmetric particles that is important and somewhat subtle is that they may not be detectable by normal procedures. Indeed, it has been demonstrated that gluinos, and also the partners of neutral and of charged electroweak gauge bosons, could have existed in data from the ISR or from PETRA but not have been observed. This could have happened mainly because large amounts of energy and momentum can be carried off by supersymmetric partners. We will see that there are a number of ways for supersymmetric partners to be discovered in experiments in progress, as well as future ones.

Our plan for the rest of this report is as follows. We will review next some theoretical arguments that suggest the appearance of new physics below about $1 \mathrm{TeV}$, and the reasons for pursuing a detailed study of the consequences of supersymmetry. Next, general features common to all supersymmetric models are briefly mentioned. Supersymmetry leads to a doubling of the degrees of freedom so that both naming conventions and symbols can easily get out of hand. Thus, in chapter 2 we emphasize the notation which we shall use in the rest of this report. Chapter 3 begins our tour through the various new supersymmetric particles. In each case we discuss the signatures of their decays, the most likely machines at which to produce the new particles, existing experimental limits and their loopholes, and future possibilities. Chapters 4-7 then examine specific physics areas (as dictated by the type of machine) at which new supersymmetric particles may be produced and detected. We discuss in turn: $\mathrm{W}^{ \pm}$ and $\mathrm{Z}^{0}$ production and decay at the $\mathrm{p} \overline{\mathrm{p}}$ collider, $\mathrm{e}^{+} \mathrm{e}^{-}$physics, hadron machines in general, and ep collider physics. In chapter 8 , we summarize indirect constraints that are imposed on supersymmetric theories. These can come from, for example, the absence of flavor changing neutral currents, the absence of parity violation in the strong interaction, and cosmological constraints. In chapter 9, we discuss the question of theoretical expectations for masses of new particles, briefly summarizing the range of expectations from various theoretical approaches to supersymmetry. To be complete, chapter 10 mentions some alternatives beyond the minimal supersymmetric extension of the Standard Model. Some conclusions and a summary table on event topologies which would signal supersymmetry are given in chapter 11. Pedagogical discussions of various technicalities of supersymmetry are provided in a series of appendices. Appendix A describes our spinor notation and the relevant conventions. Recipes for converting two-component to four-component notation are given. Appendix B describes how to build an interaction Lagrangian for an $\mathrm{SU}(2) \times \mathrm{U}(1)$ model of broken supersymmetry. Appendix $\mathrm{C}$ provides the complete interaction Lagrangian needed in this report along with the Feynman rules. This requires a discussion of possible mixings (in both the supersymmetric fermion and scalar sectors) which lead to physical states that are nontrivial combinations of the interaction eigenstates. Appendix D discusses how to deal with (four-component) Majorana fermions which necessarily appear in supersymmetric theories. Finally, appendix E provides some sample computations illustrating the results of the previous appendices.

\subsection{Theoretical motivation for expecting physics beyond the standard model}

While our report is (obviously) concentrated on the more phenomenological aspects of supersymmetric theories, we think it is important to discuss why we thought it worth all the effort, why we believe it is worthwhile for experimenters to spend the time needed to look for superpartners, and why 
we think it is worthwhile for theorists to do further work to help pinpoint signatures and production of superpartners.

The discussion can be divided as follows. First, there are aesthetic or philosophical reasons to believe that the Standard Model is incomplete (described below in this section). This belief is widely shared among theorists, although there are large differences of emphasis and interpretation. Second, accepting the conclusion that some new physics is needed - why should supersymmetry be taken seriously as the solution? What are the physics reasons why so many theorists find supersymmetry so attractive? We give this discussion in the spirit that we are trying to explain our (widely shared) views, rather than convince.

There are three kinds of reasons why the Standard Model is incomplete. First, it contains many arbitrary assumptions and parameters. Why are left-handed fermions in SU(2) doublets and righthanded ones in SU(2) singlets? Why three colors? Why is electric charage quantized $\left(q_{\mathrm{d}}=q_{\mathrm{e}} / 3\right)$ ? How many generations are there? Why do the Cabibbo-Kobayashi-Maskawa (CKM) [1.4] angles and the weak mixing angle have the values they do? Etc. There is no guarantee that any of these questions can be answered, but most physicists hope they can.

Second, the Standard Model, or QED, is not asymptotically free, so ultimately, at some energy scale, its interactions must become strong. Even though this may be an astronomically high scale, it suggests that in principle the Standard Model is the low energy effective theory of a more fundamental one.

The above two arguments do not suggest in any way, known at present, that supersymmetry is a particularly good approach to going beyond the Standard Model, although it could be relevant to them. However, the following argument does. If one calculates radiative corrections to the mass of the Higgs boson of the Standard Model, e.g. from a fermion loop in the propagator, one has a loop integral of the form

$$
\int \mathrm{d}^{4} P\left[1 /\left(P-m_{f}\right)\left(P+\not K-m_{f}\right)\right]
$$

for a Higgs of momentum $K$. This diverges quadratically for large $P$, independently of $K$, so it gives a correction $\delta m^{2} \sim \Lambda^{2}$ where $\Lambda$ is the scale beyond which the low energy theory no longer applies. Could the Higgs boson mass in fact be superheavy [1.5]? For some Higgs mass of the order of a few $\mathrm{TeV}$, the Higgs self-coupling gets too strong, and we should not be observing the apparently successful perturbation theory at low energies. Since corrections larger than this mass scale would seem equally unphysical, we expect the new physics to give an effective cutoff scale below a few $\mathrm{TeV}$. In fact, the Higgs vacuum expectation value, which determines $m_{\mathrm{w}}$ and, in principal, the fermion masses, is about $250 \mathrm{GeV}$, and it is this number that needs a fundamental explanation. These arguments are subtle since the above quadratic divergence is independent of the momentum of the Higgs, and thus can be subtracted off. However, if the full theory contains any scale $\Lambda^{\prime}$ much above a few $\mathrm{TeV}$, similar corrections from coupling to objects at the heavy scale will give corrections $\delta m^{2} \sim \Lambda^{\prime 2}$, so the Higgs mass and vacuum expectation value will again get too large unless there is a mechanism to prevent it.

So far, three kinds of attempts have emerged to try to deal with the above problem. One approach is to have quarks, leptons, and gauge bosons be composite objects [1.6]. While this eliminates the problem at today's level, it simply displaces it without improving our understanding, and we will not consider it further at present. A second approach is to eliminate fundamental scalars from the theory by making them composites of new fermions - the Technicolor approach [1.7]. This is a good idea, but at present it has two major problems; (1) it seems to be technically very difficult to construct a workable model [1.8], 
and (2) nearly all models predict the existence of charged technipions (which behave like charged Higgs bosons) with mass below about $25 \mathrm{GeV}$. So far these have not been observed at $\mathrm{e}^{+} \mathrm{e}^{-}$colliders [1.9]. They would be excluded if a heavy quark were observed decaying semileptonically, for example $t \rightarrow b \ell \nu$. [The decay $\mathrm{t} \rightarrow \mathrm{bH}^{+}$is only semiweak and would dominate if allowed [1.10]. If it dominated, one only gets e, $\mu$ via a chain $\mathrm{H} \rightarrow \tau \nu, \tau \rightarrow \ell \nu \bar{\nu}$, so the e, $\mu$ are too soft to mimic the hard lepton in $\mathrm{t} \rightarrow \mathrm{b} \ell \nu$. Thus observation of $\mathrm{t} \rightarrow \mathrm{b} \ell \nu$ would imply that if any $\mathrm{H}^{+}$exists then $m\left(\mathrm{H}^{+}\right)>m_{\mathrm{t}}-m_{\mathrm{b}}$.]

The third approach is to use a higher symmetry to eliminate the quadratic divergence in the Higgs mass, which can be technically arranged in supersymmetric theories, as we will indicate in the next section. To summarize, the presence of fundamental scalars in the theory is difficult to understand unless something new is added. In a supersymmetric theory the scalars can be a natural part of the theory, and it is possible technically to have both light and heavy scalars in a natural way.

It is useful to restate the above arguments in a more theoretical (and more technical) manner. Let $\mu_{2}$ be the scale at which $S U(2) \times U(1)$ breaking takes place. We assume that the Standard Model is the low energy approximation of some more fundamental theory which becomes relevant at a scale $\mu_{1}$. Examples of such a scale are the grand unification [1.11] scale $M_{\mathrm{GUT}} \sim 10^{14}-10^{17} \mathrm{GeV}$ and the Planck mass $M_{\mathrm{p}} \sim 10^{19} \mathrm{GeV}$ where gravitational effects become significant on a microscopic level. We may imagine calculating the mass of the Standard Model Higgs using the fundamental theory. The output of this theoretical computation would be a scale dependent mass parameter evaluated at the fundamental scale $\mu_{1}$. The relevant quantity for the low energy theory is the running mass evaluated at the scale $\mu_{2}$. These two quantities are related by an equation which has the schematic form [1.12]:

$$
m_{\mathrm{H}}^{2}\left(\mu_{2}\right)=m_{\mathrm{H}}^{2}\left(\mu_{1}\right)+C g^{2} \int_{\mu_{2}^{2}}^{\mu_{1}^{2}} \mathrm{~d} k^{2}+R g^{2}+\mathrm{O}\left(g^{4}\right)
$$

where $g$ is a coupling constant, $C$ is dimensionless and $R$ grows almost logarithmically with $\mu_{1}$ as $\mu_{1} \rightarrow \infty$. The term proportional to $C$ diverges quadratically when $\mu_{1} \rightarrow \infty$. The advantage of eq. (1.2) is that it involves finite quantities only and does not require knowledge of the theory beyond the scale $\mu_{1}$. Equation (1.2) illustrates the theoretical problems described above. First, in order that $m_{\mathrm{H}}^{2}\left(\mu_{2}\right) \ll \mu_{1}^{2}$, one has to fine-tune the parameter $m_{\mathrm{H}}^{2}\left(\mu_{1}\right)$ extremely accurately to cancel the second term in eq. (1.2) which is of order $\mu_{1}^{2}$. This is called the fine-tuning problem. It has been also referred to as the naturalness problem [1.7,1.12-1.15]; clearly the 'natural' value for $m_{\mathrm{H}}^{2}\left(\mu_{2}\right)$ is a number of order $\mu_{1}^{2}$. A related problem is the hierarchy problem $[1.12,1.16]$, which asks the question: Why is $\mu_{2} \ll \mu_{1}$ ?

It is interesting to see how various theoretical approaches attempt to solve these problems. In Technicolor approaches, there are no light elementary Higgs particles; the light Higgs-like objects are composites of more fundamental fermions. Thus, in eq. (1.2) one interprets $\mu_{1}$ to be the scale at which the composite nature of the Higgs boson is revealed. We take $\mu_{2}$ to be of order the W mass, $m_{\mathrm{w}}$. Naturalness is satisfied if we require $g^{2} \mu_{1}^{2} \sim m_{\mathrm{w}}^{2}$, or $\mu_{1} \sim m_{\mathrm{w}} / g \sim \mathrm{O}(1 \mathrm{TeV})$, which implies that new physics must enter by the $1 \mathrm{TeV}$ scale. In supersymmetry, one finds that the theory has no quadratic divergences at all. In terms of eq. (1.2), supersymmetry guarantees that $C \equiv 0$. This occurs via cancellations in certain Feynman diagrams which are insured by requiring that each known particle has a supersymmetric partner of the same mass and coupling. Because supersymmetry is broken in nature, the degeneracies which led to $C=0$ are no longer exact. Still, as long as effects of supersymmetry become relevant by a scale $\mu_{1} \sim 1 \mathrm{TeV}$ (e.g., new supersymmetric particles with masses below $\mu_{1}$ ), 
naturalness is once again preserved. If $C=0$ (or more precisely, $C=0$ for $\mu_{1}>m_{\mathrm{w}} / g$ ), eq. (1.2) implies that corrections to the relation $m_{\mathrm{H}}\left(\mu_{2}^{2}\right) \approx m_{\mathrm{H}}\left(\mu_{1}^{2}\right)$ are at most of order $\mu_{2}$.

We emphasize that the solution to the naturalness (fine-tuning) problem necessarily predicts that new physical phenomena must exist at a scale of $m_{\mathrm{w}} / g \sim O(1 \mathrm{TeV})$ or below. In the case of supersymmetry, this new physics consists of a spectrum of new supersymmetric particles (partners of the ordinary particles) which have masses no greater than about $1 \mathrm{TeV}$ and in some cases may be substantially lighter.

At this point, the hierarchy problem is not solved since we do not understand why $m_{\mathrm{H}}^{2}\left(\mu_{1}\right) \ll \mu_{1}^{2}$. However, it is plausible that the theory may guarantee that $m_{\mathrm{H}}^{2}\left(\mu_{1}\right) \equiv 0$. In such a scenario, one can have an explanation of why $\mu_{2} \ll \mu_{1}$. There are examples of supersymmetric models with this property although it is by no means guaranteed.

\subsection{Motivation for supersymmetry}

One reason why supersymmetry is of considerable interest is that it can in principle deal with the problem of the quadratic Higgs mass divergence, described above. In supersymmetric theories there is always a loop of superpartners accompanying the loop of normal particles; the extra minus sign that goes with any fermion loop, plus the supersymmetric relations between masses and couplings, guarantee that the coefficient of the divergence is zero. We give an explicit example as one of the calculations of appendix E.

The requirement that the unrenormalized theory has no quadratic divergences to all orders in perturbation theory implies that the theory is supersymmetric, and all supersymmetric theories satisfy this requirement $[1.17,1.18]$. Thus in unbroken supersymmetric theories it would be possible to understand why corrections to scalar boson masses are not large, and how a complete theory could contain several widely separated scales.

The main reason for expecting that supersymmetric particles will be detected with masses below a $\mathrm{TeV}$, given the fact that the supersymmetry is broken (since the superpartners are not observed with masses of the normal particles), is that the above argument will fail if the masses of superpartners are too large. Since the supersymmetry is, by hypothesis, relevant to fixing the weak scale of $250 \mathrm{GeV}$, one expects (crudely speaking) that the superpartners should differ in mass from the normal particles by no more than about $250 \mathrm{GeV}$.

This argument is confirmed in models. While there is no compelling supersymmetric model, all the ones studied produce some detectable superpatrners that are light, often with masses well below $m_{\mathrm{w}}$. More technically, the models can be tuned to have a larger scale of supersymmetry breaking and still account for $m_{\mathrm{w}}$ at tree level, with no partners below $m_{\mathrm{w}}$, but then they are unstable and have to be retuned as soon as radiative corrections are included. Models where such radiative corrections are less than the masses themselves have detectable, light superpartners. Note we have not commented in this discussion on where the fundamental scale of supersymmetry breaking is. It can be at a very high scale. What matters is that the mechanism that feeds down to account for the weak scale will also give masses to superpartners around that scale.

There are several other reasons why theorists find supersymmetry attractive. They may well be good reasons, but they do not imply (contrary to the situation with the above argument) that the supersymmetric partners should be observed on any particular scale. Consequently, we will list them here, but not discuss them in detail. (1) Nature has shown that it likes gauge theories, and supersymmetry is the next logical gauge theory to try [1.19]. Further, the spin degree of freedom is not fundamentally understood or integrated into the gauge theory structure at present, while it would be with supersym- 
metry. (2) Supersymmetric theories are mathematically better behaved, perhaps even finite [1.20]. Some theorists feel that eventually only supersymmetric quantum field theories will be considered meaningful. But the supersymmetry of these theories need not be observable in the low energy effective theory of quarks, leptons, and gauge bosons, although it could be. (3) Locally supersymmetric theories relate the generators of supersymmetric transformations to the generators of space-time transformations, so they couple the theory of gravity to the supersymmetric theory [1.21]. Many theorists hope that by this connection gravity can eventually be unified with the strong and electroweak forces.

Since the connection to gravity is obscure but potentially so exciting, we will try to indicate intuitively how it comes about. What happens is that the generators of supersymmetry transformations have the property that applying them twice to any boson or fermion field gives a space-time transformation, which means they must be related to the general theory of coordinate transformations, i.e. gravity. To see how this happens in a simple model [1.22], called the massless Wess-Zumino model [1.23], imagine a complex scalar $\phi$ and a Majorana fermion $\psi$ connected by supersymmetry. One can have a Lagrangian including interactions,

$$
\mathscr{L}=\partial_{\mu} \phi^{*} \partial^{\mu} \phi+\frac{1}{2} \mathrm{i} \bar{\psi} \gamma \cdot \partial \psi-g^{2}|\phi|^{4}-g(\phi \bar{\psi} \psi+\text { h.c. }) .
$$

Just as with the usual $V$ or $A$ currents, one can form here a local supercurrent connecting $\psi, \phi$,

$$
S_{\mu}=\partial_{\sigma} \phi^{*} \gamma^{\sigma} \gamma_{\mu} \psi+\mathrm{i} g \gamma_{\mu} \phi^{* 2} \psi
$$

This current is conserved for massless $\phi, \psi$, so we can define a conserved charge (which is also a spinor) as usual,

$$
Q=\int \mathrm{d}^{3} x S_{0}
$$

Then, using standard commutation relations for $\phi, \psi$ we find that applying $Q$ twice, in the sense of the anticommutator (since it is a fermion), gives

$$
\{Q, \bar{Q}\}=2 \gamma^{\mu} P_{\mu}
$$

where $P_{\mu}=\mathrm{i} \partial_{\mu}$. On the other hand, $Q$ is the spin $1 / 2$ operator that transforms states, $Q|\phi\rangle=|\psi\rangle$, $Q|\psi\rangle=|\phi\rangle$. So we see that supersymmetric transformations are intimately tied up with space-time ones. The anticommutator above explicitly arises because of the derivative operations needed in forming the supercurrent. [The models which explicitly relate a low energy supersymmetry to supergravity do so in a more technical way, requiring arguments beyond those discussed above [1.24].]

These last three arguments provide good reasons for studying supersymmetry mathematically, and for hoping that experimental evidence for supersymmetry will appear, but they are neutral on whether the experimental evidence needs to appear at accelerator energies.

\subsection{Supersymmetry as a model of physics beyond the standard model}

In the rest of this chapter, we shall briefly describe the basic characteristics of supersymmetric theories relevant for the discussion of its phenomenological implications. In particular, we shall 
elucidate the assumptions which dominate most of the discussion in this report. (For a brief look at alternative ideas, see chapter 10.) Then we mention the main experimental signatures which are the hallmarks of supersymmetry. We end the chapter with some comments on the general question of how to seek out new phenomena beyond the Standard Model in future experiments.

Supersymmetry is a symmetry that relates fermionic and bosonic degrees of freedom. It therefore predicts new supersymmetric particles which are partners of all the known particles and differ in spin by half a unit. In addition, the total number of bosonic and fermionic degrees of freedom must be equal. As an example, corresponding to a given quark there are two scalar-quarks. The counting works as follows: We can count degrees of freedom conveniently by regarding separately the left-handed and right-handed quark chiralities $q_{\mathrm{L}}$ and $q_{\mathrm{R}}$. Correspondingly, there will be two complex scalar-quarks $\tilde{q}_{\mathrm{L}}$ and $\tilde{q}_{\mathrm{R}}$. Note that $q_{\mathrm{L}}$ and $\tilde{q}_{\mathrm{L}}$ each have two degrees of freedom so that the counting matches. (Another way to view the counting is that the Dirac spinor $q$ consists of a two-component quark and its antiquark which corresponds in number to two scalar-quarks and their scalar-antiquarks.) We may therefore enumerate the minimal set of new supersymmetric particles that are needed; this is given in table 4 displayed in chapter 2.

The following properties are noteworthy. The supersymmetric particles carry with them quantum numbers (with the exception of spin) identical to those of their ordinary particle partners. For example, like the gluon, the gluino is a color octet, flavor singlet, $C=-1$ object. The scalar-electron, like the electron, carries a conserved electron number. One should also note that there are two Higgs doublets listed in table 4 along with their supersymmetric partners. The reason here is a technical one involving the generation of fermion masses. In the ordinary electroweak theory, one can use one Higgs doublet to write down all possible Higgs-fermion Yukawa interaction terms. When the Higgs acquires a vacuum expectation value, the fermions acquire mass. In supersymmetry, not all Higgs-fermion interaction terms which occurred in the non-supersymmetric theory are allowed (some terms would violate supersymmetry). As a result, one finds that with one Higgs doublet, only quarks of a given charge can acquire mass. One needs at least two Higgs doublets to give mass to both up-type and down-type quarks (and leptons).

One might wonder why the supersymmetric particle spectrum cannot be more economical. We know that spin zero Higgs bosons are required in the theory; why not have them as the scalar partners of the leptons (and quarks)? This was tried many years ago by Fayet [1.25], but was not successful. There are a number of reasons why such a scenario seems not to work. First, it would have to be the scalar partner of the (left-handed) neutrino which acquired a vacuum expectation value which broke $\mathrm{SU}(2) \times \mathrm{U}(1)$ down to U(1).* But the (scalar) neutrino carries a lepton number, so that we would also spontaneously break a lepton number. This is certainly undesirable, although it is not as restricted phenomenologically as one might expect [1.26]. Second, if Higgs bosons were the partners of quarks they would carry color quantum numbers and could lead to low energy baryon number violation. There are other reasons that are more technical and involve details of model building. It appears likely that separate Higgs bosons and their fermionic supersymmetric partners are required in supersymmetric models.

Finally, note the effect of supersymmetry breaking on the spectrum (shown in table 4 in chapter 2). First, the mass degeneracy of a particle and its supersymmetric partner is lifted. A sensible theory would have to explain why the supersymmetric particle masses are all lifted above their partners (enough to avoid experimental detection to date). Second, mass matrices for the supersymmetric particles can mix particles which carry the same values of conserved quantum numbers. Thus, the supersymmetric

\footnotetext{
* If the scalar partner of a charged lepton (or quark) gained a vacuum expectation value then electromagnetism (or color) would be broken.
} 
particles listed in table 4 are first listed as 'interaction eigenstates' only. The correct mass eigenstates would have to be determined by experiment (or by a theoretical model). For example, in addition to scalar-quarks of different generations mixing through a super-Cabibbo-Kobayashi-Maskawa matrix [1.27], we can also have $\tilde{q}_{\mathrm{L}}$ and $\tilde{q}_{\mathrm{R}}$ mixing. Another example of this phenomena is the mixing of the photino, zino and two neutral higgsinos. Discussion of such mixing can be found in appendix $\mathrm{C}$ and sections 3.5 and 3.6. The symbols and names for mass eigenstates are listed in the third column of table 4.

The supersymmetric spectrum in table 4 contains neutral massive Majorana fermions such as the photino. As a result there can be fermion number violation as exemplified by such reactions as $\mathrm{e}^{-}+\mathrm{e}^{-} \rightarrow \tilde{\mathrm{e}}^{-}+\tilde{\mathrm{e}}^{-}$which occurs via the $\mathrm{t}$-channel exchange of a Majorana photino. This would not however be seen in the laboratory as such because the scalar-electrons would quickly decay $\left(\tilde{\mathrm{e}}^{-} \rightarrow \mathrm{e}^{-}+\right.$ $\tilde{\gamma})$ so that no fermion number violation would seem to occur. If the photino and gluino were massless, then a generalization of fermion number conservation could be defined. In the context of supersymmetry, the relevant symmetry is a continuous global symmetry called an R-symmetry $[1.25,1.28]$. We will argue in section 3.4 that the gluino cannot be massless in which case this continuous R-symmetry must be broken. It turns out that despite this breaking, a discrete R-symmetry (almost) always remains.* This leads to the multiplicatively conserved quantum number which is called R-parity [1.29]. All ordinary particles are assigned an R-parity of +1 , whereas the supersymmetric partners have an $\mathrm{R}$-parity of -1 . Formally, one can define the R-parity of any particle of spin $j$, baryon number $B$ and lepton number $L$ to be $R=(-1)^{2 j+3 B+L}$. This has a number of important consequences. First, supersymmetric particles must be produced in pairs. That is, because in laboratory experiments the initial state contains no supersymmetric particles, the final state can only contain an even number of such particles. Second, there must be a lightest supersymmetry particle. Due to R-parity conservation, such a particle could not decay into only non-supersymmetric particles. In the case of spontaneously broken global supersymmetry, the lightest supersymmetric particle is the Goldstino [1.30] (since it is massless). However, in models involving supergravity, the Goldstino is absorbed into a gravitino [1.31] which in most recent models is not the lightest supersymmetric particle (see chapter 9). Therefore, with the exception of section 3.7 where Goldstino phenomenology is discussed, we will assume that the Goldstino is irrelevant for the search for supersymmetry. Having said that, the supersymmetric particle most likely to be the lightest (and hence stable) is the photino, although the scalar-neutrino is also a good possibility [1.51] (see section 3.2). Complications due to mixing with other neutral Majorana fermions could alter this assumption as discussed in section 3.6, so the lightest state could be a mixture of photino, zino, and higgsino. However, for the most part, we will assume for simplicity that the photino is light (it could even be massless!) and stable. If the scalar-neutrino is the lightest supersymmetric partner, then photinos would decay (e.g., $\tilde{\gamma} \rightarrow \nu \tilde{\nu})$. This possibility has implications for a number of experiments, and will be discussed where appropriate. Throughout the review we will speak as if the photino (or appropriate mass eigenstate) were the lightest supersymmetric partner and stable. If scalar-neutrinos or Goldstinos are lighter minor modifications will be needed in some of the discussions.

The third implication of the R-parity quantum number is that the production cross section for any light supersymmetric particle like the photino is likely to be of weak-interaction size. To see this, consider $\mathrm{e}^{+} \mathrm{e}^{-} \rightarrow \tilde{\gamma} \tilde{\gamma}$. This occurs via t-channel exchange of a scalar-electron. Because the scalar-electron is heavy (certainly $\tilde{M}_{\mathrm{e}} \geq 22 \mathrm{GeV}[1.32]$ ), the virtual ẽ exchange suppresses the $\tilde{\gamma}$ production cross section. Likewise, the $\tilde{\gamma}$ interaction cross section is of weak-interaction size because it can only interact

\footnotetext{
* The discrete group that remains is $\mathrm{Z}_{2}$ or addition modulo 2 .
} 
with matter via the exchange of a heavy scalar-electron or scalar-quark. Consequently, experimentally, the $\tilde{\gamma}$ will behave rather like a neutrino. This observation, which would also be true for the lightest stable supersymmetric particle, is the basis for most of the experimental signatures of supersymmetry. [Note one possible exception to the above rule which occurs if gluinos ( $\tilde{\mathrm{g}})$ are light. Because of the existence of a gõ $\tilde{g}$ vertex, one can pair produce gluinos without an intermediate heavy supersymmetric particle exchange. Thus $\tilde{g}$ cross sections can be very large, which will be discussed in detail in section 3.4.]

We have implicitly assumed above that with the exception of the photino and the gluino, all supersymmetric particles will be heavy. This is for the most part true - as an experimental fact. We provide in table 2 the current experimental limits on supersymmetric particle masses [1.9, 1.32-1.34]. Note that there are still a few partners which could be very light, in particular the scalar-neutrinos.

The entire discussion above has assumed that R-parity is an exact conservation law. If it were broken, then the lightest supersymmetric particle would be unstable. Almost certainly, such a situation would also require the violation of lepton number (e.g., $\tilde{\gamma} \rightarrow \gamma+\nu$ ). We will briefly consider this alternative in section 10.3. Otherwise, we will always assume that R-parity is exact.

Based on the discussions above, it is clear that there will be one basic experimental signature which could distinguish supersymmetry from Standard Model physics. Namely, once supersymmetric particles are produced, they will eventually decay into photinos which, like neutrinos, will simply escape the apparatus (except for neutrino and photino detectors). But neutrinos are almost always produced in association with a charged lepton, while the photino is often produced with no hard charged leptons present. Consequently, we have the possibility of finding events where substantial amounts of energy and momentum are lost but no lepton is seen. This is the hallmark of supersymmetry and is the basis of almost all experimental searches for supersymmetric phenomenon. Of course, occasionally, one can think of Standard Model background which have similar signals. A dramatic example is an event which should occasionally occur at (pp and) $p \bar{p}$ colliders where $p \bar{p} \rightarrow Z^{0}+X, Z^{0} \rightarrow \nu \bar{\nu}$. Therefore, a detailed consideration of various supersymmetric signals and backgrounds needs to be done on a case-by-case basis, and occupies most of this report.

Table 2

Present and foreseeable future experimental status of superpartners (notation is defined in chapter 2)

\begin{tabular}{|c|c|c|c|c|}
\hline Superpartners & Present limits & Soon ... & $\cdots$ & . . . . . about 1989 \\
\hline $\bar{\ell}^{ \pm}$ & $\begin{array}{l}\bar{M}_{c} \geq 22 \mathrm{GeV}[1.32] \\
\tilde{M}_{\mu}>18 \mathrm{GeV}[1.33] \\
\tilde{M}_{\tau} \geq 16 \mathrm{GeV}[1.9,1.33]\end{array}$ & $\begin{array}{l}\sim 30 \mathrm{GeV} \\
-20 \mathrm{GeV}\end{array}$ & $\begin{array}{l}{\left[\mathrm{e}^{+} \mathrm{e}^{-} \rightarrow \tilde{\gamma} \tilde{\gamma} \gamma\right]} \\
{\left[\mathrm{e}^{+} \mathrm{e}^{-} \rightarrow \tilde{\ell} \overline{\bar{\ell}}\right]}\end{array}$ & $\begin{array}{l}\sim 65 \mathrm{GeV} \\
\sim 45 \mathrm{GeV}\end{array}$ \\
\hline$\tilde{\nu}$ & $M\left(\bar{\nu}_{\tau}\right)+M\left(\tilde{\nu}_{\ell}\right) \geq m_{\tau}[1.35]$ & & {$\left[\mathrm{e}^{+} \mathrm{e}^{-} \rightarrow \tilde{\nu}_{\ell} \overline{\bar{\nu}_{\ell}}\right.$} & $\left.\mathrm{W} \rightarrow \bar{\ell} \bar{\nu}_{\ell}\right]$ \\
\hline$\tilde{q}$ & $\begin{array}{l}\tilde{M} \geq 18 \mathrm{GeV} \\
\text { (if } \tilde{\mathrm{q}} \rightarrow \mathrm{q} \tilde{\gamma} \text { ) }\end{array}$ & $\sim 40 \mathrm{GeV}$ & $\begin{array}{l}{\left[\mathrm{e}^{+} \mathrm{e}^{-} \rightarrow \overline{\mathrm{q}} \overline{\bar{q}}\right]} \\
{[\overline{\mathrm{p}} \mathrm{p} \rightarrow \tilde{\mathrm{q}} \overline{\mathrm{q}} \mathrm{X}]}\end{array}$ & $\begin{array}{l}\sim 40 \mathrm{GeV} \\
-70 \mathrm{GeV}\end{array}$ \\
\hline$\overline{\mathbf{g}}$ & $\tilde{M}>2-5 \mathrm{GeV}[1.34]$ & $\sim 40 \mathrm{GeV}$ & $\begin{array}{c}{[\overline{\mathrm{p} p} \rightarrow \overline{\mathrm{g}} \overline{\mathrm{g} X}]} \\
150 \mathrm{GeV} \text { if } \overline{\mathrm{g}} \rightarrow \mathrm{g}\end{array}$ & $\tilde{y}$ is large) \\
\hline$\tilde{\chi}^{0}(\tilde{\gamma} \ldots)$ & None & $\bar{M}_{x_{1}}+\tilde{M}_{x_{2}} \leqslant$ & {$\left[\mathrm{e}^{+} \mathrm{e}^{-} \rightarrow \bar{\chi}_{2} \tilde{X}_{1}\right]$} & $\tilde{M}_{x_{1}}+\tilde{M}_{x_{2}} \leqslant 95 \mathrm{GeV}$ \\
\hline$\tilde{\chi}^{ \pm}(\tilde{W} \ldots)$ & $\begin{array}{l}\tilde{M} \geq 20 \mathrm{GeV}[1.52] \\
\text { (for some } \mathrm{BR} \text { ) }\end{array}$ & $\begin{array}{l}\sim 20 \mathrm{GeV} \\
\tilde{M}_{\chi^{+}}+\tilde{M}_{\chi^{0}} \\
\leq 50 \mathrm{GeV}\end{array}$ & $\begin{array}{l}{\left[\mathrm{e}^{+} \mathrm{e}^{-} \rightarrow \bar{\chi}_{\mathrm{i}}^{+} \tilde{\chi}_{\mathrm{i}}^{-}\right]} \\
{\left[\mathrm{W}^{ \pm} \rightarrow \bar{\chi}^{+} \tilde{\chi}^{0}\right]}\end{array}$ & $\begin{aligned} \sim 40 \mathrm{GeV} \\
\tilde{M}_{x^{+}}+\tilde{M}_{x^{0}}<70 \mathrm{GeV}\end{aligned}$ \\
\hline
\end{tabular}




\subsection{Photino (and other supersymmetric particle) interactions}

Since a good deal of the discussion will deal with signatures for supersymmetry, we consider here explicitly the details of how photinos interact. We will rely heavily on this section throughout the review.

The detection of supersymmetric partners is always based on either (a) detecting the interaction of a light superpartner at the end of a decay chain (usually thought of as a photino) or, (b) missing $p_{\mathrm{T}}$ because such a particle escapes a detector, or both. The missing $p_{\mathrm{T}}$ can be distinguished from that of a neutrino because no charged lepton accompanies the photino.

If the particle involved is a higgsino or a Goldstino or a scalar-neutrino there are some differences (see below) but the main points are the same, so we will emphasize the photino case. For photinos and Goldstinos the interactions with ordinary matter were calculated by Fayet [1.36].

The incoming photino interacts by exciting or exchanging a scalar-quark as in fig. 1. The quarks and gluons are constituents inside the hadron. The $\tilde{\gamma}$-hadron cross section is then, from the standard parton model formalism [1.37],

$$
\sigma_{\mathrm{Int}}^{\tilde{\gamma}}=\sum_{q} \int \mathrm{d} x q(x) \hat{\sigma}(\hat{s})
$$

where $\hat{\sigma}$ is the constituent ( $\tilde{\gamma} \mathrm{q} \rightarrow \tilde{\mathrm{q} X}$ ) cross section, $s$ is the $\tilde{\gamma}$-hadron c.m. energy squared, and $\hat{s}=x s$. We shall neglect the contribution from $\tilde{\gamma} \mathrm{g} \rightarrow \mathrm{q} \tilde{\mathrm{q}}$. A more complete calculation will not appreciably alter the conclusions below. The $\tilde{\mathrm{q}}$ can decay to $\tilde{\mathrm{gq}}$ if $\tilde{M}_{\mathrm{g}}^{2}<x s$, which may be satisfied for the relevant $x, s$; otherwise $\mathrm{q} \tilde{\gamma}$ is available. If the $\tilde{\mathrm{g}} \mathrm{q}$ mode is allowed it will dominate because the coupling is stronger. For beam dump analysis the mass of $\tilde{\mathrm{g}}$ is, by the hypothesis being tested, sufficiently small that the $\tilde{\mathrm{g} q}$ mode dominates.

The constituent cross section is of the form

$$
\hat{\sigma} \sim\left(\alpha \alpha_{\mathrm{s}} / \tilde{M}_{\mathrm{q}}^{4}\right) \hat{s}
$$

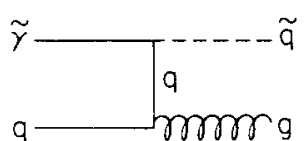

(a)

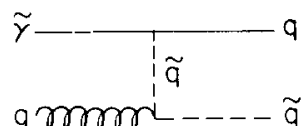

(c)

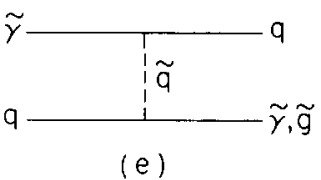

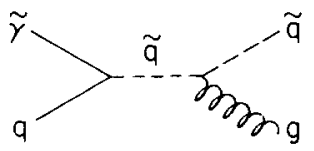

(b)

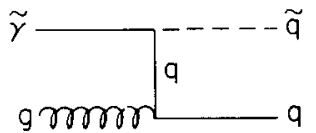

(d)

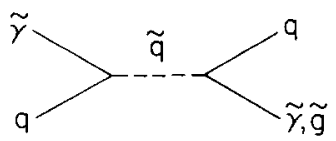

(f)

Fig. 1. Photino interactions with matter. 
assuming $\hat{s} \ll \tilde{M}_{\mathrm{q}}^{2}$ (if $\hat{s} \sim \tilde{M}_{\mathrm{q}}^{2}$ considerable revision is needed). Putting $\alpha_{\mathrm{s}}=0.15$, and using (1.8) in (1.7), the full interaction cross section is (including all numerical factors) [1.36, 1.38]

$$
\sigma_{\mathrm{lnt}}^{\bar{\gamma}} \approx 2 \times 10^{-37} \tilde{E}_{\gamma}\left(m_{\mathrm{w}} / \tilde{M}_{\mathrm{q}}\right)^{4} \tilde{F}\left(\tilde{M}_{\mathrm{g}}^{2}, s\right) \quad \mathrm{cm}^{2} .
$$

$\tilde{F}$ is the analogue of the standard structure function $F_{2}$,

$$
\tilde{F}=\sum_{q} \int_{\tilde{M}_{\mathbf{g}}^{2} / s}^{1} \mathrm{~d} x q(x)\left(1-\tilde{M}_{\mathbf{g}}^{2} / x s\right)^{2}\left(1+\tilde{M}_{\mathbf{g}}^{2} / 8 x s\right) e_{\mathbf{q}}^{2} .
$$

For $\tilde{M}_{\mathrm{g}}^{2} \rightarrow 0, \tilde{F}=F_{2} \simeq 0.15$. The phase space corrections for $\tilde{M}_{\mathrm{g}}^{2} \neq 0$ can be significant for $\tilde{M}_{\mathrm{g}} \geqslant 1 \mathrm{GeV}$. The result for $\sigma \tilde{\gamma}_{\mathrm{Int}}$ has been written in the usual form of a neutrino cross section; $\tilde{E}_{\gamma}$ is the photino lab

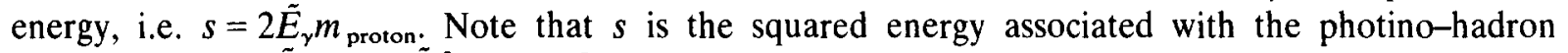
system. If $m_{\mathrm{w}} \simeq \tilde{M}_{\mathrm{q}}$ and $\tilde{M}_{\mathrm{g}}^{2} \ll x s, \sigma_{\mathrm{Int}}^{\tilde{\gamma}}$ is a few times larger than a neutrino charged current cross section. Thus photinos will interact in beam dump detectors, but will not interact in collider detectors. The interaction in a beam dump detector gives, as a signature, an event with no hard charged lepton and very little missing energy or $p_{\mathbf{T}}$, so it is a neutral current candidate, but one with different characteristics from neutrino events. In particular, its distributions are those for scalar currents, and there is less missing energy, $p_{\mathrm{T}}$. The signature in a collider is the $p_{\mathrm{T}}$ carried away (with no associated hard $\ell^{ \pm}$).

With the above remarks in mind one can understand the main signatures discussed below. The above discussion is given assuming photinos are light or massless. Appropriate caveats if they are heavy are given in various discussions below. Note also that the result for the interaction cross section goes as $\tilde{M}_{\mathbf{q}}^{-4} ;$ if scalar-quarks were very heavy, photino interactions would get very weak.

\subsection{Comments}

We close this introductory chapter with a few philosophical remarks and some comments.

(a) We have argued that the likelihood of new physics beyond the Standard Model in the $100 \mathrm{GeV}$-few $\mathrm{TeV}$ region is very high. We have also argued that supersymmetry is an excellent candidate for describing this new physics. However, it is entirely possible that although new physics will be uncovered in this energy regime, it will not at all be related to supersymmetry. (Perhaps supersymmetry is relevant only at the Planck scale!) If this were the case, is all the discussion on the search for new phenomena described in this report irrelevant? We think not. In searching for new phenomena, it is important to consider how new physics can emerge from Standard Model backgrounds. In some sense, supersymmetry is serving as a toy model for new physical phenomena which we anticipate in the next energy regime. In this regard, supersymmetry has one decided advantage. Because it is a weak coupling theory, one may perform detailed calculations of a whole assortment of possible new physics. In each case, detailed study of Standard Model backgrounds then reveals where new physics can be uncovered. As a simple example, consider the fact that, until recently, experimenters at PEP and PETRA were throwing away events with over $50 \%$ of the visible energy missing. The reason was that this cut easily got rid of $2-\gamma$ background and beam-gas events. However, it also cuts away potential new physics, including possible supersymmetry signals. The value of a detailed study which can be provided in the context of supersymmetry is that new experimental signatures can be found which have the 
potential for uncovering new physics. Thus, supersymmetry is playing the role of a specific model of how new physics beyond the Standard Model can be observed experimentally. Even if supersymmetry is not correct in practice, it will have served a useful purpose in emphasizing some ways to discover new physics.

(b) We want to emphasize that our treatment has very little model dependence for two reasons. First, most of the model dependence in discussions of supersymmetric models arises because the origin of the supersymmetry breaking is not understood. This shows up in a model dependence of the masses of superpartners. But we discuss all searches and limits as functions of masses, so we are not affected by the model dependence of masses. When mass matrices cause mixing of weak eigenstates some model dependence does enter, and we will discuss that in context. Second, we only consider $N=1$ supersymmetry, that is, the theory with one generator of supersymmetric transformations. If the true theory had more generators it would be richer in phenomena, but all we say would remain valid. That is because the effective low energy theory must be an $N=1$ supersymmetry to be compatible with the SU(2) $)_{\mathrm{L}} \times$ U(1) electroweak theory [1.14]. For $N \geq 2$ one has weak $S U(2)$ multiplets consisting of both left- and right-handed fermions which can be connected by a supersymmetric transformation, which does not occur in the Standard Model. We lose no generality by considering the phenomenology of $N=1$ supersymmetry.

(c) We assume, of course, the validity of the Standard Model. Then we can compute all properties of superpartners which depend on their color, weak, or electromagnetic charges, such as production cross sections, branching ratios, etc.

(d) We will discuss in a limited way how to distinguish one supersymmetric signal from another, or from other possible new physics, but our emphasis will be on how to detect any new signal. When something is discovered it will be a great pleasure to think about what it is.

(e) In general a new part of physics could be detected by finding a new particle or a new interaction. We have concentrated on finding particles for several practical reasons. At accelerators new particles are much easier to detect than new interactions, and they provide a qualitatively new phenomenon rather than a quantitative deviation. We consider effects of interactions in chapter 8 , and describe them in some detail; by their nature they allow many interpretations even if found, while a new particle that behaves like a gluino or a neutralino points strongly toward a supersymmetric interpretation. Also, many indirect searches have been pushed quite far, while there are large open windows for finding new particles.

(f) We do not explicitly review models or model building, although we occasionally mention results of models in context, and in chapter 9 we make a brief survey. Recent reviews of models appear in refs. $[1.19,1.24]$.

(g) Finally, we note that it is not a problem for supersymmetric ideas that superpartners have not yet been found. As we will discuss in chapter 9 , most of them are expected to have masses of order $m_{\mathbf{w}}$, and could not have been detected yet. Those that could have been observed, such as the gluino, are allowed to be heavy enough to have been unobservable so far. But as we will see, the machines available in the next decade will have a high probability of detecting supersymmetric partners if supersymmetry is relevant to understanding the weak scale.

\subsection{Remarks on references}

In this report we have endeavored to review all literature pertaining to the search for supersymmetry which we have seen (through the end of 1983). The references are arranged so as to be self-contained 
within each chapter. In addition, we have found various review articles on supersymmetry [1.39-1.49] extremely useful in learning the techniques of supersymmetry and applying them to the task at hand. In a review of this magnitude, it is inevitable that some relevant references will have been overlooked. We sincerely apologize at this point to any authors who we may have unintentionally slighted. Finally, we note that a significant literature on supersymmetry has been accumulating in 1984 since the completion of this work. Due to the lack of time and space we must leave the compilation of these references to the next review.

\section{Notation and nomenclature}

Since all the supersymmetric theories which we discuss require a partner for each normal particle, there is considerable new notation required. People have used various notations already. After some consideration, involving usefulness, aesthetics, and the need for communicating over the phone, we have settled on the notation given in tables 3 and 4 .

For each standard particle the superpartner is shown. The main subtlety is the need for column 3 of table 4; the weak eigenstates will mix, giving mass eigenstates that are linear combinations of weak eigenstates, and that require separate names because they have different properties.

In the tables we have not made explicit the chiralities of the fermions and their partners. To be fully explicit, one has as weak eigenstates the usual left-handed electron $e_{L}$ (in an $S U(2)$ doublet) and right-handed electron $e_{R}\left(\right.$ an $S U(2)$ singlet), the left-handed up quark $u_{L}, u_{R}, d_{L}, d_{R}$, etc. Each of these has a scalar partner $\tilde{e}_{L}, \tilde{e}_{R}, \tilde{u}_{L}, \tilde{u}_{R}$, etc. When necessary we will make the $L$ and $R$ explicit.

To discuss production and decay of supersymmetric particles, one requires a full interaction Lagrangian, which is given in appendix C. However, apart from an occasional factor of two and a few subtle and uncommon interactions, one can construct for pedagogical purposes the necessary vertices by simply taking all Standard Model three-particle vertices and replacing ordinary particles by their superpartners in pairs. Thus the vertex ee $\gamma$ gives

$$
\mathrm{e}^{+} \mathrm{e}^{-} \gamma \rightarrow \tilde{\mathrm{e}}^{+} \mathrm{e}^{-} \tilde{\gamma}+\tilde{\mathrm{e}}^{-} \mathrm{e}^{+} \tilde{\gamma}+\tilde{\mathrm{e}}^{+} \tilde{\mathrm{e}}^{-} \gamma
$$

Table 3

Rules for names

(1) Supersymmetric partners have a (). Masses of supersymmetric particle are denoted by $\tilde{M}$ (whereas a lower case $m$ is used for ordinary particle masses).

(2) Weak-interaction eigenstates are denoted by mnemonic letters such as $\bar{W}, \tilde{Z}, \tilde{B}, \tilde{\mathrm{q}}, \tilde{\ell}, \tilde{\mathrm{g}}$, etc. They are named by an appropriate use of 'ino' for superpartners of bosons - wino, zino, bino, photino, gluino (collectively called gaugino), higgsino, etc.-and by the prefix 'scalar' for superpartners of fermions (scalar-quark, scalar-electron, scalar-neutrino, etc.).

(3) Mass eigenstates can be different from weak eigenstates when several states can mix. In practice this matters especially for spin $1 / 2$ partners of gauge bosons and Higgs bosons (i.e. the gauginos and higgsinos), and for some scalar partners. In general the charged spin 1/2 mass eigenstates are called charginos and denoted $\tilde{\chi}_{i}^{ \pm}$, and the neutral spin $1 / 2$ mass eigenstates are called neutralinos and denoted $\tilde{\chi}_{j}^{0}$. Mass eigenstates corresponding to the scalars $\tilde{q}_{L}$ and $\tilde{q}_{R}$ are denoted $\tilde{q}_{1}$ and $\tilde{q}_{2}$.

(4) When the mass eigenstates have specific couplings they may be denoted by lower case letters to indicate the coupling. For the charginos, if the coupling is W-like we call $\tilde{\mathrm{w}}^{ \pm}$a wino, if the coupling is Higgs-like we call $\tilde{h}^{ \pm}$a higgsino,and if the coupling is intermediate we use 'wiggsino'. Similarly, for the neutralinos, if $\bar{\chi}_{\mathrm{i}}^{0}$ couples like a photon (or Z, Higgs) it will be denoted $\bar{\gamma}$ (or $\bar{z}, \tilde{h}^{0}$ ) and called photino (or zino, higgsino). If the $\bar{\chi}_{\mathrm{i}}^{0}$ coupling is intermediate it will be called a 'ziggsino'.

(5) The wiggsino and ziggsino states which consist of exactly one half gaugino component and one half higgsino component are special since in the supersymmetric limit they are mass eigenstates. We will use special notation for these states which are explicitly defined in eqs. (B25) and (B35) of appendix $\mathrm{B}$. The wiggsino states are denoted by $\bar{\omega}_{1}$ and $\bar{\omega}_{2}$; the ziggsino states are denoted by $\bar{\zeta}_{1}$ and $\bar{\zeta}_{2}$. 
Table 4

\begin{tabular}{|c|c|c|c|c|c|c|}
\hline \multirow{3}{*}{$\begin{array}{l}\text { Normal } \\
\text { particles }\end{array}$} & \multirow[b]{3}{*}{ Symbol } & \multicolumn{3}{|c|}{ Supersymmetric partners } & \multirow{2}{*}{\multicolumn{2}{|c|}{$\begin{array}{l}\text { Mass eigenstates with } \\
\text { specific couplings }\end{array}$}} \\
\hline & & \multirow{2}{*}{$\begin{array}{l}\text { Weak interaction } \\
\text { eigenstates }\end{array}$} & \multicolumn{2}{|c|}{ Mass eigenstates } & & \\
\hline & & & Symbol & Name & Symbol & Name \\
\hline $\begin{array}{r}\mathrm{q}=\mathrm{u}, \mathrm{d}, \mathrm{s} \\
\mathrm{c}, \mathrm{b}, \mathrm{t}\end{array}$ & $\tilde{\mathrm{q}}_{\mathrm{L}}, \overline{\mathrm{q}}_{\mathrm{R}}$ & scalar-quark & $\tilde{q}_{1}, \tilde{q}_{2}$ & scalar-quark & & \\
\hline$\ell=\mathrm{e}, \mu, \tau$ & $\tilde{\ell}_{\mathrm{L}}, \bar{\ell}_{\mathrm{R}}$ & scalar-lepton & $\bar{\ell}_{1}, \bar{\ell}_{2}$ & scalar-lepton & & \\
\hline$\nu=\nu_{\mathrm{e}}, \nu_{\mu}, \nu_{\tau}$ & $\tilde{\nu}$ & scalar-neutrino & $\dot{\nu}$ & scalar-neutrino & & \\
\hline $\mathrm{g}$ & $\tilde{\mathrm{g}}$ & gluino & $\tilde{\mathrm{g}}$ & gluino & & \\
\hline$W^{ \pm}$ & $\tilde{W}^{ \pm}$ & wino & & & $\tilde{\mathrm{w}}^{ \pm}$ & wino \\
\hline $\mathrm{H}_{1}^{+}$ & $\tilde{\mathrm{H}}_{1}^{+}$ & higgsino & $\bar{x}_{1,2}^{ \pm}$ & charginos & $\tilde{\mathrm{h}}^{ \pm}$ & higgsino \\
\hline $\mathrm{H}_{2}^{-}$ & $\tilde{\mathrm{H}}_{2}^{-}$ & higgsino & & & $\tilde{\omega}_{1}, \tilde{\omega}_{2}$ & wiggsino \\
\hline$\gamma$ & $\bar{\gamma}$ & photino & & & $\bar{\gamma}$ & photino \\
\hline$Z^{0}$ & $\tilde{\mathbf{Z}}^{0}$ & zino & & & $\tilde{\mathrm{z}}$ & zino \\
\hline $\mathrm{H}_{1}^{0}$ & $\overline{\mathrm{H}}_{1}^{0}$ & higgsino & $\tilde{\chi}_{\mathrm{i}}^{0}$ & neutralinos & $\tilde{\mathrm{h}}_{1}, \tilde{\mathrm{h}}_{2}$ & higgsino \\
\hline $\mathrm{H}_{2}^{0}$ & $\tilde{\mathrm{H}}_{2}^{0}$ & higgsino & & & $\tilde{\zeta}_{1}, \tilde{\zeta}_{2}$ & ziggsino \\
\hline$\left(W^{3}\right)$ & $\left(\tilde{\mathbf{W}}^{3}\right)$ & (wino) & & & $\left(\tilde{w}^{3}\right)$ & (wino) \\
\hline$(\mathrm{B})$ & $(\tilde{\mathbf{B}})$ & (bino) & & & $(\tilde{b})$ & (bino) \\
\hline
\end{tabular}

This table gives the nomenclature we use for supersymmetric partners. For the partners of bosons, mixing generally occurs among the weak eigenstates, so one needs different symbols and names for weak and for mass eigenstates. Couplings of weak eigenstates are determined by the theory, but couplings of mass eigenstates depend on the amount of mixing; for mass eigenstates the names and symbols are either generic $\left(\tilde{\chi}_{i}\right)$ or reflect the couplings. As always, $Z^{0}$ and $\gamma$ are linear combinations of $\mathrm{W}^{3}, \mathrm{~B}\left[\gamma=\cos \theta_{\mathrm{w}} B+\right.$ $\left.\sin \theta_{\mathrm{w}} W^{3}, Z=-\sin \theta_{\mathrm{w}} B+\cos \theta_{\mathrm{w}} W^{3}\right]$, with the same combination of $\tilde{\mathrm{W}}^{3}, \overline{\mathrm{B}}$ giving $\tilde{Z}^{0}, \tilde{\gamma}$. If supersymmetry is a spontaneously broken global symmetry there is also a Goldstone fermion called the Goldstino and denoted $\tilde{G}$. In supergravity theories, the Goldstino is absorbed into a massive gravitino $\tilde{\mathrm{g}}_{3 / 2}$ which is the spin $3 / 2$ partner of the graviton.

all with strength $-e\left(e^{2} / 4 \pi=\alpha=1 / 137\right)$. Similarly,

$$
\mathrm{q} \overline{\mathrm{q}} \gamma \rightarrow \overline{\tilde{\mathrm{q}}} \mathrm{q} \tilde{\gamma}+\tilde{\mathrm{q}} \overline{\mathrm{q}} \tilde{\gamma}+\tilde{\mathrm{q}} \overline{\tilde{q}} \gamma
$$

all with strength $e e_{\mathrm{q}}\left(e_{\mathrm{q}}\right.$ is the quark charge), and

$$
\mathrm{q} \overline{\mathrm{q}} \mathrm{g} \rightarrow \overline{\tilde{q}} \mathrm{q} \tilde{g}+\tilde{\mathrm{q}} \overline{\mathrm{q}} \tilde{\mathrm{g}}+\overline{\tilde{q}} \tilde{\mathrm{q}} \mathrm{g}, \quad \mathrm{ggg} \rightarrow \tilde{\mathrm{g}} \tilde{g} g,
$$

all with strength $g_{\mathrm{s}}\left(g_{\mathrm{s}}^{2} / 4 \pi=\alpha_{\mathrm{s}} \approx 0.15\right)$. For particles that mix, one proceeds as above for the weakinteraction eigenstates, and then replaces chargino and neutralino weak eigenstates by the appropriate combination of mass eigenstates. This procedure allows one to understand what processes can occur, with about what strength. The techniques needed for precise calculations are given in the appendices.

\section{Survey of the supersymmetric particle spectrum}

\subsection{Scalar-leptons}

There are two scalar-leptons, denoted by $\tilde{\ell}_{\mathbf{L}}$ and $\tilde{\ell}_{\mathbf{R}}$, for each generation of leptons, $\ell$. That is, there 
is one $\left(\tilde{\ell}_{\mathrm{L}}\right)$ associated with the left-handed lepton $\ell_{\mathrm{L}}$ (which is in a weak SU(2) doublet), and a different one $\left(\tilde{\ell}_{\mathrm{R}}\right)$ associated with the right-handed lepton $\ell_{\mathrm{R}}$ (which is a weak SU(2) singlet). In general, $\tilde{\ell}_{\mathrm{L}}$ and $\tilde{\ell}_{\mathbf{R}}$ are expected to mix; the mass eigenstates will be (model dependent) linear combinations of these states as discussed in appendix C.1. For simplicity, we will usually work with the $\tilde{\ell}_{\mathrm{L}}$ and $\tilde{\ell}_{\mathrm{R}}$ states. When it is unimportant, we will drop the $\mathrm{L}, \mathrm{R}$ subscript and simply use the symbol $\tilde{\ell}$. It is straightforward to produce scalar-leptons in $\mathrm{e}^{+} \mathrm{e}^{-}$colliders and we shall concentrate on their signatures at these machines, briefly mentioning other possible methods of production. Little is changed here from the original analysis of Farrar and Fayet [3.1], apart from consideration of what happens if photinos are not massless, and of some other production channels. First we will study the scalar-lepton decay signatures.

\section{(A) The decay of the scalar-lepton}

If photinos are light, the scalar-lepton decays rapidly, with essentially $100 \%$ branching ratio into $\tilde{\ell}^{ \pm} \rightarrow \ell^{ \pm} \tilde{\gamma}$ :

$$
\Gamma\left(\tilde{\ell}^{ \pm} \rightarrow \ell^{ \pm} \tilde{\gamma}\right)=\alpha\left(\tilde{M}_{\ell}^{2}-m_{\ell}^{2}-\tilde{M}_{\gamma}^{2}\right) p_{\ell} / \tilde{M}_{\ell}^{2},
$$

where $p_{\ell} \equiv \lambda^{1 / 2}\left(\tilde{M}_{\ell}^{2}, m_{\ell}^{2}, \tilde{M}_{y}^{2}\right) / 2 \tilde{M}_{\ell}$ is the lepton momentum in the rest frame of the scalar-lepton. The photino interacts too weakly to be detected in a normal collider detector, so it escapes (see section 1.5). Hence, in the $\tilde{\ell}^{ \pm}$rest frame, if we neglect the mass of the lepton, at least half the energy of the event escapes.

If the photino is heavier than the scalar-lepton then the scalar-lepton decay depends on what is the lightest supersymmetric particle. For example, it could be stable, although there are limits on the existence of new stable charged particles [3.2] (as well as astrophysical constraints [3.3]). If the scalar-neutrinos were lighter, the $\beta$-decay type process $\tilde{\ell} \rightarrow \ell \nu \tilde{\nu}$ occurs at a weak interaction rate or faster (depending on the mass of the wino which mediates the decay). Since $\Gamma \sim \tilde{M}_{\ell}^{5}$ and $\tilde{M}_{\ell} \geq 18 \mathrm{GeV}$, this decay is probably too rapid to detect at a collider. For the most part, however, we will assume that $\ell^{ \pm} \tilde{\gamma}$ is the dominant decay mode.

\section{(B) The production of scalar-leptons and present limits}

Because charged scalar-leptons interact electromagnetically, $\mathrm{e}^{+} \mathrm{e}^{-}$physics is the ideal environment to look for them. The charged scalar-leptons are straightforward to produce in pairs via $\mathrm{e}^{+} \mathrm{e}^{-} \rightarrow \tilde{\ell}^{+} \tilde{\ell}^{-}$. They are produced by direct channel $\gamma$ and $\mathrm{Z}^{0}$ as for any charged scalar; for $\mathrm{e}^{+} \mathrm{e}^{-} \rightarrow \tilde{\mathrm{e}}^{+} \tilde{\mathrm{e}}^{-}$we must also include t-channel photino and zino exchange. One photon exchange predicts $\frac{1}{4} \beta^{3}$ units of $R$ each for $\tilde{\ell}_{\mathrm{L}}^{ \pm}, \tilde{\ell}_{\mathrm{R}}^{ \pm}$and a $\sin ^{2} \theta$ production distribution. Because of the p-wave threshold factor of $\beta^{3}$, a rise in $R$ can only be observed far above threshold. However, because of the loss of half the energy of the event into unobserved photinos, it is possible to identify the occurrence of scalar-lepton production. Specifically, $\mathrm{e}^{+} \mathrm{e}^{-} \rightarrow \tilde{\ell}^{+} \tilde{\ell}^{-}$appears as the production of acolinear $\ell^{+} \ell^{-}$pairs with lots of missing energy. There is some background from $\tau^{+} \tau^{-}$events but this is calculable background which can be subtracted, and it behaves differently kinematically. Background from $\gamma \gamma$ events can be easily cut away. Note that the central production $\left(\sin ^{2} \theta\right.$ distribution) of scalar-leptons alleviates the $\gamma \gamma$ contamination as well as beam-gas events which would also appear with large amounts of missing energy. The discussion above shows that, assuming a light photino, the mass limits one can set on the scalar-lepton mass should be close to the electron beam energy. (The limits would be less stringent for heavy photinos or alternate decay modes.)

All present detectors have results on limits on scalar lepton masses based on the nonobservation of $\mathrm{e}^{+} \mathrm{e}^{-} \rightarrow \tilde{\ell}^{+} \tilde{\ell}^{-}$[3.4]. Combining them, one can exclude $\tilde{\mathrm{e}}$ and $\tilde{\mu}$ up to about $18 \mathrm{GeV}$ and $\tilde{\tau}$ to $16.5 \mathrm{GeV}$, as 
long as a (nearly) massless photino is assumed. Better limits on ẽ masses can be obtained by other techniques to be discussed below.

At larger center-of-mass energies $\sqrt{s}, \mathrm{Z}^{0}$ exchange will become important. The differential cross section still exhibits a $\sin ^{2} \theta$ distribution. By integrating, one obtains the total cross section for scalar lepton production $(\tilde{\ell} \neq \tilde{\mathrm{e}})[3.5]$ :

$$
\begin{aligned}
\sigma\left(\mathrm{e}^{+} \mathrm{e}^{-} \rightarrow \tilde{\ell}_{\mathrm{i}}^{+} \tilde{\ell}_{\mathrm{i}}^{-}\right)= & \left(\pi \alpha^{2} / 3 s\right) \beta^{3}\left[e_{\mathrm{i}}^{2}+8 r e_{\mathrm{i}}\left(T_{3 \mathrm{i}}-e_{\mathrm{i}} \sin ^{2} \theta_{\mathrm{w}}\right)\left(1-4 \sin ^{2} \theta_{\mathrm{w}}\right)\right. \\
& \left.+16 r^{2}\left(T_{3 \mathrm{i}}-e_{\mathrm{i}} \sin ^{2} \theta_{\mathrm{w}}\right)^{2}\left(1+\left(1-4 \sin ^{2} \theta_{\mathrm{w}}\right)^{2}\right)\right],
\end{aligned}
$$

where $e_{\mathrm{i}}$ and $T_{3 \mathrm{i}}$ are the electric charge and third component of the weak isospin, given by $e=-1$ and $T_{3 \mathrm{i}}=-1 / 2$ for charged scalar-leptons, and the parameter $r$ is given by

$$
r=s /\left[16\left(s-m_{\mathrm{z}}^{2}\right) \cos ^{2} \theta_{\mathrm{w}} \sin ^{2} \theta_{\mathrm{w}}\right]
$$

(appropriate width corrections apply for $s \simeq m_{z}^{2}$ ).

For $\mathrm{e}^{+} \mathrm{e}^{-} \rightarrow \tilde{\mathrm{e}}^{+} \tilde{\mathrm{e}}^{-}$, we cannot neglect the $\mathrm{t}$-channel photino exchange. If we neglect $\mathrm{Z}^{0}$ and zino exchange graphs $\left(\sqrt{s} \ll m_{z}\right)$, the result in the limit of zero photino mass is [3.1]

$$
\frac{\mathrm{d} \sigma}{\mathrm{d} \cos \theta}\left(\mathrm{e}^{+} \mathrm{e}^{-} \rightarrow \tilde{\ell}_{\mathrm{L}}^{+} \tilde{\ell}_{\mathrm{L}}^{-}\right)=\frac{\pi \alpha^{2} \beta^{3} \sin ^{2} \theta}{8 s}\left[1+\left(1-\frac{4 K}{1-2 \beta \cos \theta+\beta^{2}}\right)^{2}\right],
$$

where $K=1$ for $\tilde{\ell}=\tilde{\mathrm{e}}$ and $K=0$ otherwise. An identical formula is obtained for $\mathrm{e}^{+} \mathrm{e}^{-} \rightarrow \tilde{\ell}_{\mathbf{R}}^{+} \tilde{\ell}_{\mathbf{R}}^{-}$. Note that the t-channel photino exchange changes the angular distribution substantially leading to an increased cross section near $\theta=0$. For larger $\sqrt{s}$, the $Z^{0}$ and zino contributions will become important and an exact form for the cross section including interferences will be required. The effects of nonzero photino mass can lead to some interesting changes; in particular an enhancement of the rate near threshold. Additional information can also be gained by using polarized beams. These topics have been discussed in refs. [3.102, 3.103].

One can be sensitive to scalar-electron masses larger than the beam energy by using two different techniques, both of which assume light photinos. The first method, suggested by Gaillard, Hall and Hinchliffe [3.6], involves associated production of the scalar-electron and photino via $\mathrm{e}^{+} \mathrm{e}^{-} \rightarrow \mathrm{e}^{+} \tilde{\mathrm{e}}^{-} \tilde{\gamma}$ as shown in fig. 2. (Calculations of this process can also be found in refs. [3.104, 3.105].) Assuming that $\tilde{\mathrm{e}} \rightarrow \mathrm{e}+\tilde{\gamma}$, one effectively is searching for $\mathrm{e}^{+} \mathrm{e}^{-} \rightarrow \mathrm{e}^{+} \mathrm{e}^{-} \tilde{\gamma} \tilde{\gamma}$. The signal one looks for consists of an acoplanar $\mathrm{e}^{+} \mathrm{e}^{-}$pair and substantial missing energy in the event. The advantage of this process is that (for light photinos), one is sensitive to scalar-electron masses larger than the beam energy (see fig. 3). Expressions for the differential cross sections were computed using the Weizsacker-Williams equivalent photon approximation and are given in ref. [3.6]. Here, we note that the total cross section (normalized

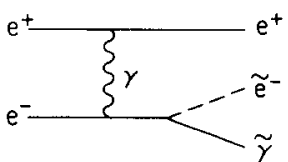

(o)

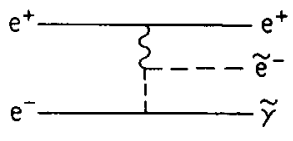

(b)

Fig. 2. Graphs for $\mathrm{e}^{+} \mathrm{e}^{-} \rightarrow \mathrm{e}^{+} \tilde{\mathrm{e}}^{-} \overline{\boldsymbol{\gamma}}$. 


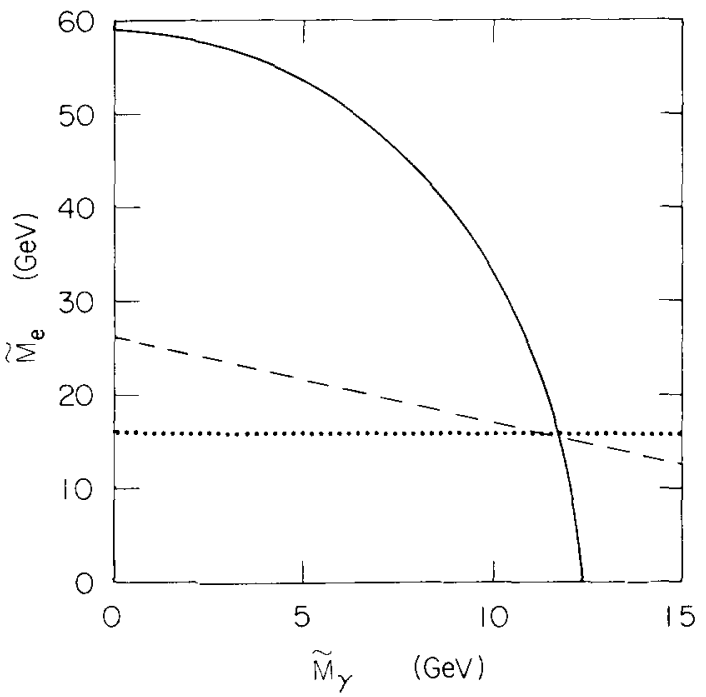

Fig. 3. Domains of scalar-electron masses vs. photino masses that are accessible to searches in $\mathrm{e}^{+} \mathrm{e}^{-}$collisions at the level of $10^{-38} \mathrm{~cm}^{2}$. The solid line corresponds to $\mathrm{e}^{+} \mathrm{e}^{-} \rightarrow \bar{\gamma} \dot{\gamma} \gamma[3.11,3.12,3.106]$, the dashed line corresponds to $\mathrm{e}^{+} \mathrm{e}^{-} \rightarrow \mathrm{e}^{ \pm} \mathrm{e}^{ \pm} \bar{\gamma}[3.6,3.104,3.105]$, and the dotted line corresponds to $\mathrm{e}^{+} \mathrm{e}^{-} \rightarrow \overline{\mathrm{e}}^{+} \overline{\mathrm{e}}^{-}[3.1]$. This figure has been taken from ref. [3.12].

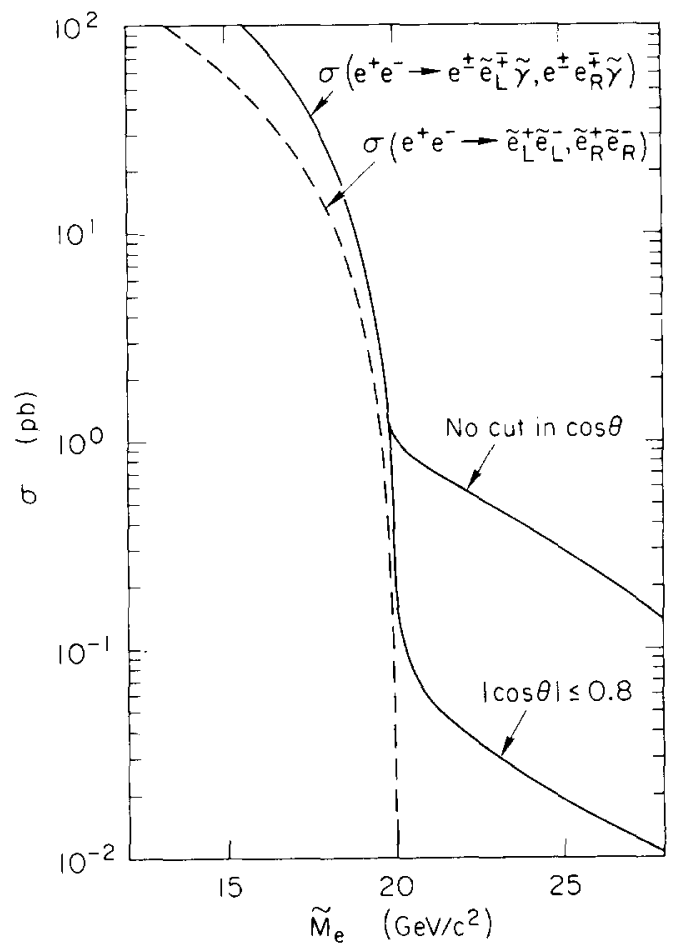

Fig. 4. The integrated cross section for $\mathrm{e}^{+} \mathrm{e}^{-} \rightarrow \mathrm{e}^{+} \tilde{\mathrm{e}}^{-}$(dashed line) and for $\mathrm{e}^{+} \mathrm{e}^{-} \rightarrow \mathrm{e}^{ \pm} \mathrm{e}^{-} \bar{\gamma}$ (solid line) vs. scalar-electron mass at $\sqrt{ }^{-}=40 \mathrm{GeV}$. We assume that $\tilde{M}_{\mathrm{e}}=\bar{M}_{\mathrm{eR}} \equiv \bar{M}_{\mathrm{c}}$. The effect of a cut on the center of mass scattering angle $(\theta=$ angle between the beam and observed final state $\mathrm{e}^{ \pm}$) is shown. This figure has been taken from ref. [3.105].

to $\left.\sigma\left(\mathrm{e}^{+} \mathrm{e}^{-} \rightarrow \gamma^{*} \rightarrow \mu^{+} \mu^{-}\right)\right)$is

$$
R\left(\mathrm{e}^{+} \mathrm{e}^{-} \rightarrow \mathrm{e}^{+} \tilde{\mathrm{e}}_{\mathrm{L}}^{-} \bar{\gamma}\right)=\frac{\alpha}{12 \pi} \log \left(\frac{E}{m_{\mathrm{e}}}\right)\left[\frac{2}{x}+18-54 x+34 x^{2}+3\left(3-3 x-4 x^{2}\right) \log x-9 x \log ^{2} x\right],
$$

where $x \equiv \tilde{M}_{\mathrm{e}}^{2} / 4 E^{2}$ for an electron beam energy of $E$ (and similarly for the production of $\tilde{e}_{\mathrm{R}}$ ). A complete calculation has been performed in ref. [3.105]; the results (compared to the cross section for $\mathrm{e}^{+} \mathrm{e}^{-} \rightarrow \tilde{\mathrm{e}}^{+} \tilde{\mathrm{e}}^{-}$) are shown in fig. 4. Although the cross section drops dramatically for $\tilde{M}_{\mathrm{c}}>\sqrt{ } \bar{s} / 2$, the characteristics of this process (acoplanar $\mathrm{e}^{+} \mathrm{e}^{-}$pair along with missing $p_{\mathrm{T}}$ ) should be sufficient to make it useful in setting mass limits for the scalar-electron. The only obvious 'background' which cannot be easily eliminated with cuts is the production of some new heavy lepton [3.7]. Eventually, this background could be distinguished by the fact that it will contribute a unit of $R$ to the total $\mathrm{e}^{+} \mathrm{e}^{-}$cross section as compared with the $1 / 4$ unit of $R$ (along with a slow threshold turn-on) for each new scalar, and by the presence of other decay modes for the heavy lepton (such as $\nu_{\ell} \pi$ ).

This method has already been used by two groups to set new limits on ẽ masses. They find [3.8] that $\tilde{M}_{\mathrm{e}}>22 \mathrm{GeV}$ at $95 \% \mathrm{CL}$. (This limit assumes that the two scalar-electron masses, e.g. $\tilde{M}_{\tilde{e}_{\mathrm{L}}}$ and $\tilde{M}_{\tilde{e}_{\mathrm{R}}}$, are unequal, with one mass much larger than the other. Then, the limit applies to the lighter mass. If $M_{\tilde{e}_{\mathrm{L}}}=M_{\tilde{e}_{\mathrm{R}}}$, then the experimental limit one obtains is around $1 \mathrm{GeV}$ larger.)

Note that in the previous discussion, it was assumed that the scalar-electron was produced on-shell. 
This need not be the case and one might consider computing $\mathrm{e}^{+} \mathrm{e}^{-} \rightarrow \mathrm{e}^{+} \mathrm{e}^{-} \tilde{\gamma} \tilde{\gamma}$ allowing the scalar-electron to be off-shell. This was done by Chen, Wald and Weinstein [3.9] who claimed it was possible to observe this process at PEP even if $\tilde{M}_{\mathrm{e}}$ were as large as $50 \mathrm{GeV}$ although no formulas were provided. Their results have been disputed by Ellis and Hagelin [3.10] who have estimated (assuming $M_{\mathrm{e}} \gg \sqrt{ } s$ ):

$$
\sigma\left(\mathrm{e}^{+} \mathrm{e}^{-} \rightarrow \mathrm{e}^{+} \mathrm{e}^{-} \tilde{\gamma} \tilde{\gamma}\right)=0.5 \times 10^{-39}\left(50 \mathrm{GeV} / \tilde{M}_{\tilde{\mathrm{e}}}\right)^{4} \mathrm{~cm}^{2}
$$

Taking an upper limit of $10^{-38} \mathrm{~cm}^{2}$ as observable (there are experiments proposed at PEP which claim to be sensitive to such levels), one finds sensitivity to $\tilde{M}_{\tilde{\mathrm{e}}} \leqslant 23 \mathrm{GeV}$ which is approximately as well as one was able to do using the previous method at the current PEP energy of $\sqrt{s}=29 \mathrm{GeV}$.

The second method for obtaining more severe mass limits for the scalar-leptons was suggested by Grifols et al. [3.95], Fayet [3.11] and by Ellis and Hagelin [3.12]. They proposed a search for $\mathrm{e}^{+} \mathrm{e}^{-} \rightarrow \tilde{\gamma} \tilde{\gamma} \gamma$ which is a radiative correction to $\mathrm{e}^{+} \mathrm{e}^{-}$annihilation into photino pairs. The calculations of refs. [3.95, $3.11,3.12$ ] involve a number of approximations. A more exact calculation of this process has recently been performed independently by three groups [3.106, 3.114, 3.115]. The advantage of the extra photon is that it serves to tag the event. Otherwise, because the photinos are assumed to escape, the event would be invisible. (This method is just a variation on the neutrino counting experiments [3.13], whereby one counts . neutrinos by measuring $\mathrm{e}^{+} \mathrm{e}^{-} \rightarrow \nu \bar{\nu} \gamma$.) In the case of photinos, the cross section is sensitive to the scalar-electron mass because it appears in the propagator (see fig. 5). Hence, measurement of this process could be sensitive (if the photino were light) to scalar-electron masses which are as large as $60 \mathrm{GeV}$ as indicated in fig. 3. Because the photino could be heavy, limits for all the processes discussed above should be presented as an excluded region on a graph of $\tilde{M}_{\ell}$ vs. $\tilde{M}_{\gamma}$. Of course, observation of $\mathrm{e}^{+} \mathrm{e}^{-} \rightarrow \gamma+\mathrm{X}$ where X is invisible does not necessarily imply supersymmetry. However, observation of such a signal beyond that required for three families of neutrinos would be extraordinarily important and would require some new physics, whose nature would have to be determined separately.

Preliminary experimental results on $\mathrm{e}^{+} \mathrm{e}^{-} \rightarrow \gamma+\mathrm{X}$ already exist [3.14]. Interpreting those results as the nonobservation of $\mathrm{e}^{+} \mathrm{e}^{-} \rightarrow \tilde{\gamma} \tilde{\gamma} \gamma$, the MAC collaboration finds that $\tilde{M}_{\mathrm{e}}>29 \mathrm{GeV}$ at $90 \% \mathrm{CL}$. (This result assumes that the two scalar-electron masses are unequal, with one mass much larger than the other. If $\tilde{M}_{\tilde{e}_{L}}=\tilde{M}_{\tilde{e}_{R}}$, then the limit is around $5 \mathrm{GeV}$ larger.) Improved experiments are now under way $[3.14,3.15]$ which will result in the best limits available on scalar-electron masses.

So far the discussion has concentrated on present day $\mathrm{e}^{+} \mathrm{e}^{-}$machines. In the near future, we will be able to use $Z^{0}$ factories (SLC and LEP) to search for new particles. It is straightforward to calculate the two-body decays of $Z^{0}$ into scalar-lepton pairs. Assume for simplicity that $\tilde{e}_{L}$ and $\tilde{e}_{R}$ are degenerate in mass. Then the decay rate is $[3.16]$ :

$$
\Gamma\left(\mathrm{Z}^{0} \rightarrow \tilde{\mathrm{e}}_{\mathrm{L}}^{+} \tilde{\mathrm{e}}_{\mathrm{L}}^{-}+\tilde{\mathrm{e}}_{\mathrm{R}}^{+} \tilde{\mathrm{e}}_{\mathrm{R}}^{-}\right) / \Gamma\left(\mathrm{Z}^{0} \rightarrow \mathrm{e}^{+} \mathrm{e}^{-}\right)=\frac{1}{2}\left(1-4 \tilde{M}_{\mathrm{e}}^{2} / m_{\mathrm{z}}^{2}\right)^{3 / 2} .
$$

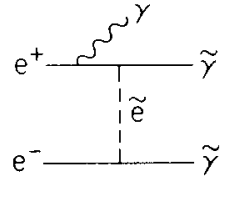

(a)

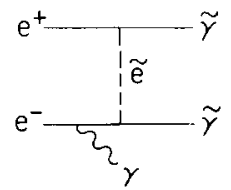

(b)

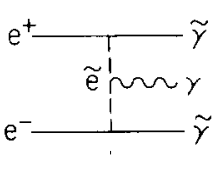

(c)

Fig. 5. Graphs for $\mathrm{e}^{+} \mathrm{e}^{-} \rightarrow \bar{\gamma} \bar{\gamma} \gamma$. 
In addition, it is of interest to consider rates for $\mathrm{Z}^{0} \rightarrow \mathrm{e}^{ \pm} \mathrm{e}^{\mp} \tilde{\gamma}$ and $\mathrm{Z}^{0} \rightarrow \mathrm{e}^{+} \mathrm{e}^{-} \tilde{\gamma} \tilde{\gamma}$ which are analogous to the processes already considered in this section. The process $\mathrm{Z}^{0} \rightarrow \tilde{\mathrm{e}}^{ \pm} \mathrm{e}^{\mp} \tilde{\gamma}$ has been computed by Johnson and Rudaz [3.107]. They find that for $\tilde{M}_{\mathrm{e}}>m_{\mathrm{z}} / 2$, the rate is severely suppressed so that effectively one will not be sensitive to scalar-electron masses larger than $m_{\mathrm{z}} / 2$ in $\mathrm{Z}^{0}$ decay. For further discussion, see section 5.3. We expect similar conclusions to be drawn from $\mathrm{Z}^{0} \rightarrow \mathrm{e}^{+} \mathrm{e}^{-} \tilde{\gamma} \tilde{\gamma}$. $\mathrm{Z}^{0} \rightarrow \tilde{\gamma} \tilde{\gamma}$, which must occur at one loop, is expected to occur with a very small branching ratio* if $\tilde{\gamma}$ is a pure photino state, so that using neutrino-counting techniques here (i.e., $\mathrm{e}^{+} \mathrm{e}^{-} \rightarrow \mathrm{Z}^{0} \gamma, \mathrm{Z}^{0} \rightarrow \tilde{\gamma} \tilde{\gamma}$ ) would be pointless. However, if significant higgsino components were mixed into the state we usually call the photino (see section 3.6) then its coupling to the $\mathrm{Z}^{0}$ could be very large, resulting in an observable signal in $\mathrm{e}^{+} \mathrm{e}^{-} \rightarrow \gamma \tilde{\gamma} \tilde{\gamma}$ on the $\mathrm{Z}^{0}$.

Hadron colliders are not a likely place to discover scalar-leptons. The relevant production mechanisms are (real or virtual) $Z^{0} \rightarrow \tilde{\ell}^{+} \tilde{\ell}^{-}$(see section 4.1) or $\bar{q} q \rightarrow \tilde{\ell}^{+} \tilde{\ell}^{-}$. The signal consists of an $\ell^{+} \ell^{-}$pair (with no definite mass for the pair) and missing $p_{\mathrm{T}}$ due to the escaping photinos. However, production cross sections are small and appropriate cuts are required to separate the signal from a larger Drell-Yan $\ell^{+} \ell^{-}$background.

One needs to keep in mind that all limits on the $\tilde{\ell}^{ \pm}$mass depend on how it decays. Presumably it is not stable. If it can decay $\tilde{\ell}^{ \pm} \rightarrow \ell^{ \pm} \tilde{\gamma}$, then all the above discussion applies and the limit depends on $\tilde{M}_{\gamma}$. Even if $\tilde{\ell}^{ \pm} \rightarrow \ell^{ \pm} \tilde{\gamma}$ is not allowed, the limit from $\mathrm{e}^{+} \mathrm{e}^{-} \rightarrow \tilde{\gamma} \tilde{\gamma} \gamma$ still applies and depends on $\tilde{M}_{\gamma}$. If $\tilde{\ell}^{ \pm} \rightarrow \ell^{ \pm} \nu \tilde{\nu}$ (say, because $\tilde{\nu}$ is the lightest supersymmetric particle), the limit on the $\tilde{\ell}^{ \pm}$mass depends on $\tilde{M}_{\nu}$.

\subsection{Scalar-neutrinos}

In the Standard Model, neutrinos are (usually) taken as massless. The right-handed neutrino is assumed either not to exist or to be very heavy. When we examine the scalar partner of the neutrino, it follows that we need only consider $\tilde{\nu}_{\mathrm{L}}$ (which we shall henceforth denote as $\tilde{\nu}$ ). We assume that a $\tilde{\nu}_{\mathrm{R}}$, if it exists, is irrelevant for present day (low energy) physics.

The properties of the scalar-neutrino $\tilde{v}$ are harder to specify than those of the corresponding scalar-leptons. This is due to the fact that the possible decay modes of $\tilde{\nu}$ are very sensitive to various (at present unknown) mass parameters of other supersymmetric particles. Thus, first we must provide a careful discussion of possible scalar-neutrino decay channels, and then consider the experimental signatures of scalar-neutrinos produced at various accelerators.

\section{(A) The decay of the scalar-neutrino}

We will concentrate here on $\tilde{\nu}_{\mathrm{e}}$; other flavors will be discussed at the end of this subsection. The first possibility is that the scalar-neutrino is stable. This will occur if the scalar-neutrino is the lightest supersymmetric particle. ${ }^{* *}$ At present, this possibility cannot be ruled out; it even seems consistent with cosmological constraints on stable particles [3.20,3.21]. Such scalar-neutrinos would behave more or less as ordinary neutrinos; the weakness of their interactions would depend on the size of the corresponding scalar-lepton mass.

The second case occurs when there exists a chargino or a neutralino lighter than the scalar-neutrino allowing for the decays $\tilde{\nu} \rightarrow \tilde{z} \nu$ and/or $\tilde{\nu}_{\mathrm{e}} \rightarrow \tilde{\mathbf{w}}^{+} \mathrm{e}^{-}[3.22]$. For example,

\footnotetext{
"In the supersymmetric limit, $\mathrm{BR}\left(\mathrm{Z}^{0} \rightarrow \tilde{\gamma} \tilde{\gamma}\right) \equiv 0$ since it is related by supersymmetry to the process $\mathrm{Z}^{0} \rightarrow \gamma \gamma$ which cannot occur due to Yang's theorem [3.17].

${ }^{* *}$ As stated in section 1.4, we assume the existence of a conserved R-parity which guarantees that the lightest supersymmetric particle is stable. However, certain unconventional models where the scalar-neutrino gets a vacuum expectation value (thus breaking R-parity and lepton number) $[3.18,3.19]$ would allow even a light scalar-neutrino to decay via $\bar{\nu} \rightarrow \nu \gamma$. The possible decay $\bar{v} \rightarrow \nu \tilde{\mathrm{G}}(\tilde{\mathrm{G}}=\mathrm{Goldstino})$ will also be ignored here; see section 3.7 .
} 


$$
\Gamma\left(\tilde{\nu}_{\mathrm{e}} \rightarrow \tilde{\mathrm{w}}^{+} \mathrm{e}^{-}\right)=\left(\alpha M_{\nu} \sin ^{2} \phi_{+} / 4 \sin ^{2} \theta_{\mathrm{w}}\right)\left(1-\tilde{M}_{\mathrm{w}}^{2} / \tilde{M}_{\nu}^{2}\right)^{2}
$$

where $\phi_{+}$is the mixing angle which indicates the amount of wino component in the chargino, $\tilde{\mathrm{w}}$. If such is the case, the scalar-neutrino could be detected through the decays of the resulting chargino. Detecting charginos will be discussed in great detail in section 3.5.

The only other possible two-body decay occurs through a one-loop graph: $\tilde{\nu} \rightarrow \nu+\tilde{\chi}^{0}$ where $\tilde{\chi}^{0}$ is a neutralino which does not have a tree coupling $\tilde{\nu} \nu \tilde{\chi}^{0}$. We consider the situation when $\tilde{\chi}^{0}$ is the photino $\tilde{\gamma}$. (Although $\tilde{\chi}^{0}$ could be a higgsino $\tilde{h}^{0}$ if it were light enough, one must recall that higgsinos couple fermions to their scalar partners with strength proportional to the fermion mass. Hence, barring exceptional circumstances, this possibility may be neglected.)

The decay $\tilde{\nu} \rightarrow \nu \tilde{\gamma}$ was computed by Barnett, Haber and Lackner [3.23]. They assumed an SU(2) $\times$ $\mathrm{U}(1)$ model of softly broken supersymmetry where the only supersymmetry breaking consisted of heavy masses for the scalar-quarks and scalar-leptons [3.24]. In such a model, the photino is massless, the charginos $\left(\tilde{\omega}_{1}\right.$ and $\left.\tilde{\omega}_{2}\right)$ and charged Higgs scalars are degenerate in mass with the W, and there are two neutralinos $\left(\tilde{\zeta}_{1}\right.$ and $\left.\tilde{\zeta}_{2}\right)$ degenerate in mass with the $Z^{0}$. The diagrams contributing to $\tilde{\nu} \rightarrow \nu \tilde{\gamma}$ are shown in fig. 6 . In a more complicated model, the details would change but one expects that the basic results remain the same. Their result was that to one-loop, the effective $\tilde{\nu} \nu \tilde{\gamma}$ vertex is given by

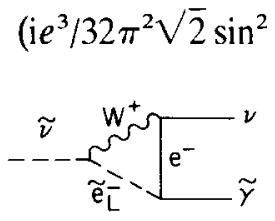

(a)

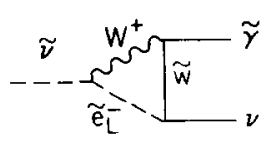

(c)

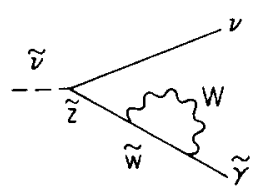

(e)

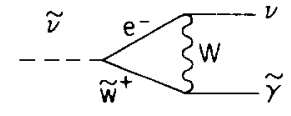

(b)

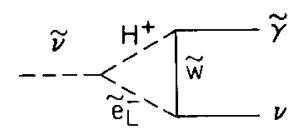

(d)

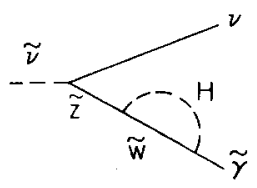

(f)

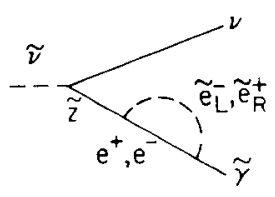

(g)

Fig. 6. One-loop diagrams for $\tilde{\nu} \rightarrow \tilde{\gamma} \nu$. These are the contributing graphs in a supersymmetric $\mathrm{SU}(2) \times \mathrm{U}(1)$ model with the supersymmetry broken only by explicit mass terms for the scalar-quarks and scalar-leptons. Note that in (e), the loop consists of either $\mathrm{W}^{-} \tilde{\omega}_{2}^{+}$or $\mathrm{W}^{+} \tilde{\omega}_{\mathrm{i}}$. Similarly in (f) with $\mathrm{H}$ replacing $\mathrm{W}$. In (g) the loop consists of either $\tilde{\mathrm{e}}_{\mathrm{L}}^{-} \mathrm{e}^{+}$or $\overline{\mathrm{e}}_{\mathrm{R}}^{+} \mathrm{e}^{-}$as well as all relevant members from other generations of leptons, quarks and their scalar partners.

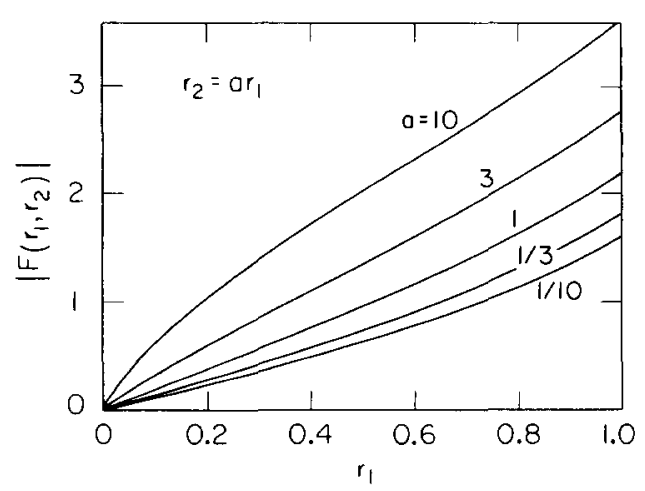

Fig. 7. A graph of the function $F\left(r_{1}, r_{2}\right)$ defined by eq. (3.9) for various values of $r_{1}$ and $r_{2}=a r_{1}$. The function is actually negative definite, so we plot its absolute value. This figure has been taken from ref. [3.24]. 
where $r_{1} \equiv \tilde{M}_{\nu}^{2} / m_{\mathrm{w}}^{2}$ and $r_{2}=\tilde{M}_{\mathrm{e}}^{2} / m_{\mathrm{w}}^{2}\left(\tilde{M}_{\nu}\right.$ and $\tilde{M}_{\mathrm{c}}$ are the scalar-neutrino and scalar-electron masses respectively). A graph of $F\left(r_{1}, r_{2}\right)$ for various values of its arguments is given in fig. 7 . An explicit formula and useful properties of the function $F\left(r_{1}, r_{2}\right)$ are given in ref. [3.24]. It is now easy to compute the resulting decay rate

$$
\Gamma\left(\tilde{\nu}_{\mathrm{s}} \rightarrow \nu+\tilde{\gamma}\right)=\left(\tilde{M}_{\nu} \alpha^{3} / 128 \pi^{2} \sin ^{4} \theta_{\mathrm{w}}\right)\left|F\left(r_{1}, r_{2}\right)\right|^{2}
$$

which leads to a lifetime (in seconds)

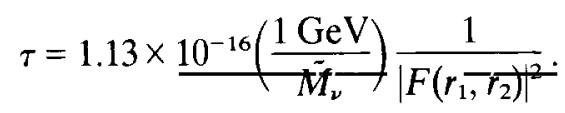

In many models one finds that the $\nu \tilde{\gamma}$ decay mode is the dominant one. In this case, the signature of scalar-neutrino production is the same as if the scalar-neutrino were stable; the photino interacts weakly in matter and escapes along with the neutrino.

Excepting decays into $\tilde{\mathrm{z}}^{0} \nu$ and $\tilde{\mathrm{w}}^{+} \mathrm{e}^{-}$, the only way for a decaying scalar-neutrino to leave observable tracks is for it to decay into multi-body (greater than two) final states. Here, we discuss three- and/or four-body decay modes depicted in fig. 8 . We need not discuss these two cases separately because any kinematically allowed three-body decay will contain a supersymmetric particle in the final state which will immediately decay itself. As an example, if $\tilde{\nu}_{\mathrm{e}} \rightarrow \nu_{\mathrm{e}} \mathrm{e}^{+} \tilde{\mathrm{e}}_{\mathrm{L}}^{-}$is possible, one finds immediately that $\tilde{\mathrm{e}}_{\mathrm{L}}^{-} \rightarrow \mathrm{e}^{-} \tilde{\gamma}$ with a width given by eq. (3.1). By computing the four-body phase space including finite widths, one automatically includes the three-body decay configuration.

Results are given in figs. 9 and 10 taken from ref. [3.24]. They may be summarized as follows. Multi-body decays of the scalar-neutrino are negligible except in the following circumstances. First if the scalar-neutrino and scalar-quark masses are nearly degenerate and much larger than the gluino mass then the dominant four-body decays are $\tilde{\nu} \rightarrow \mathrm{e}^{-} \tilde{\mathrm{g}}(\mathrm{u} \overline{\mathrm{d}}, \mathrm{c} \overline{\mathrm{s}}, \ldots)$ and $\tilde{\nu} \rightarrow \nu \tilde{\mathrm{g}} \mathrm{q} \overline{\mathrm{q}}$ with branching ratios competitive with the $\nu \tilde{\gamma}$ decay mode. If the scalar-neutrino and scalar-lepton masses are nearly degenerate (but the scalar-quark masses are much heavier), then the dominant four-body modes are $\tilde{\nu}_{\mathrm{e}} \rightarrow \nu_{\mathrm{e}} \mathrm{e}^{-} \ell^{+} \tilde{\gamma}(\ell=\mathrm{e}, \mu, \tau)$ although their branching ratios of $\sim 10^{-3}$ make them quite rare. Finally, if the

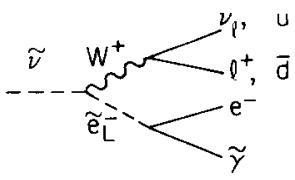

(a)

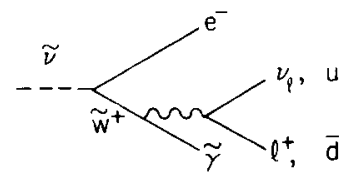

(b)

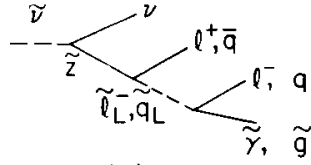

(c)

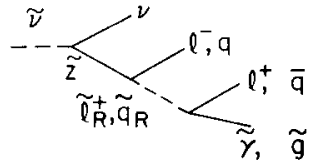

(d)

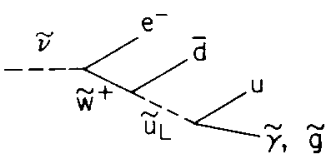

(e)

Fig. 8. Four-body decays of the scalar-neutrino. See caption to fig. 6 . Note that we use the symbols $u$ and $d$ for all up-type and down-type quarks, etc. For convenience, the Cabibbo angle is neglected. 


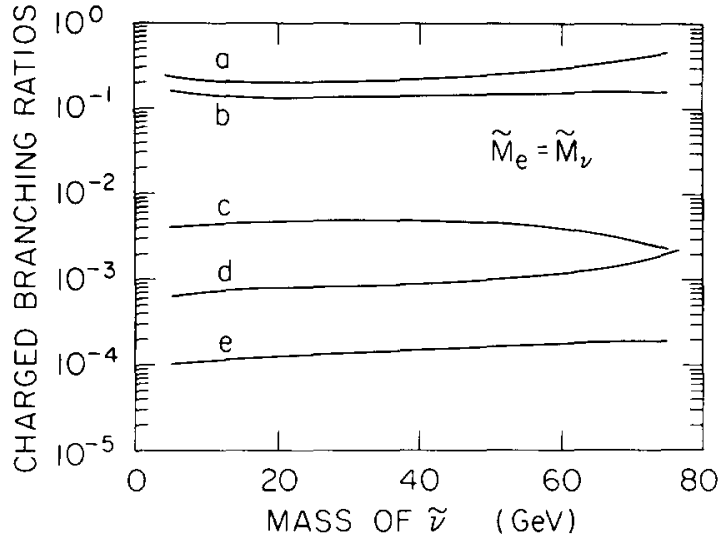

Fig. 9. Branching ratio for four-body modes of the scalar-neutrino. We label the various modes as follows: (a) $\mathrm{e}^{-} \mathrm{ud} \bar{g}+\mathrm{e}^{-} \operatorname{cs} \tilde{g}$; (b) $\Sigma_{\mathrm{i}} \nu \overline{\mathrm{q}}_{\mathrm{i}} \mathrm{q} \overline{\mathrm{g}}$; (c) $\mathrm{e}^{-} \mathrm{u} \overline{\mathrm{d}} \tilde{\gamma}+\mathrm{e}^{-} \mathrm{c} \bar{\gamma} \tilde{\gamma}$; (d) $\nu \mathrm{e}^{+} \mathrm{e}^{-} \tilde{\gamma}$; (e) $\nu \mu^{+} \mu^{-} \tilde{\gamma}$ or $\nu \tau^{+} \tau^{-} \tilde{\gamma}$. The rates for $\nu \mu^{+} \mathrm{e}^{-} \tilde{\gamma}, \nu \tau^{+} \mathrm{e}^{-} \tilde{\gamma}$, and $\Sigma_{\mathrm{i}} \nu \overline{\mathrm{q}}_{\mathrm{j}} \mathrm{q}_{\mathrm{i}} \tilde{\gamma}$ occur approximately at the level of (d). We use a value of $\alpha_{\mathrm{s}}=0.2$. We have taken the charged scalar-lepton and scalar-quark masses to be equal. This figure has been taken from ref. [3.24].

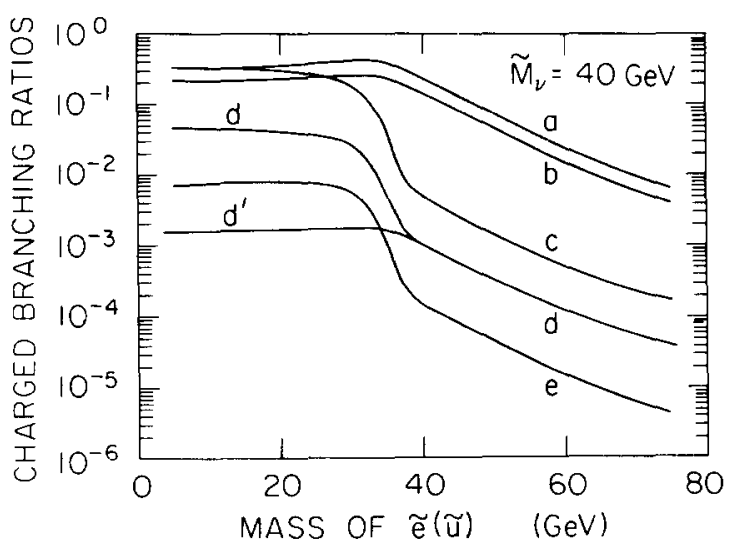

Fig. 10. Branching ratio of charged decay modes of $\bar{\nu}$ as a function of $\tilde{M}_{e}$ (which is assumed to be equal to $\tilde{M}_{u}$ ). We have chosen $\tilde{M}_{\nu}=$ $40 \mathrm{GeV}$. Note that for $\tilde{M}_{\mathrm{e}}<\tilde{M}_{\nu}$, the dominant decays are actually three-body decays containing a physical ẽ (or ũ) in the final state. The labelling here is the same as in fig. 9 except that we have to distinguish between $\mathrm{d}$ (which corresponds to $\nu_{\ell} \ell^{+} \mathrm{e}^{-} \tilde{\gamma}$ final states) and $\mathrm{d}^{\prime}$ (which corresponds to $\nu \bar{q} q \tilde{\gamma})$. This figure has been taken from ref. [3.24].

appropriate scalar-quarks and/or scalar-leptons are lighter than the scalar-neutrino, then the scalarneutrino decays nearly $100 \%$ of the time into four-bodies via an on-shell scalar-quark and/or scalarlepton. Hence, if the scalar-neutrino is produced with a large momentum, its four-body hadronic decays will tend to appear as jet-like events with some missing energy. The consequences of these decay modes will be seen in the context of the various ways of producing scalar-neutrinos experimentally.

So far, we have concentrated on the decay of the $\tilde{\nu}_{\mathrm{e}}$. It is important to consider whether any of our conclusions would change for the decay of other flavors of scalar-neutrinos $\left(\tilde{\nu}_{\mu}, \tilde{\nu}_{r}\right)$. The answers depend on various masses and mixing angles in the supersymmetric model which is used. For example, a diagram analogous to fig. 8e would allow $\tilde{\nu}_{\mu} \rightarrow \mu^{-} \mathrm{e}^{+} \tilde{\nu}_{\mathrm{e}}$ if the mass difference between $\tilde{\nu}_{\mu}$ and $\tilde{\nu}_{\mathrm{e}}$ were large enough. Furthermore, mixing between $\tilde{\nu}_{\mu}$ and $\tilde{\nu}_{\mathrm{e}}$ (analogous to Cabibbo mixing in the quark sector) could lead to unusual decay signatures. However, there are strong phenomenological constraints on the mass differences between successive generations of scalar-quarks and scalar-leptons (see chapter 8 ). We shall summarize arguments there which indicate that it is likely that the three generations of scalar-neutrinos are nearly degenerate in mass. If such is the case, one can simply repeat the discussions of this section regarding the decay of the $\tilde{\nu}_{\mu}$ and $\tilde{\nu}_{\tau}$. Furthermore, mixing effects would be negligible because in the limit that the scalar-neutrinos are exactly degenerate in mass, electron, muon and tau numbers are separately conserved global quantum numbers. But if it should turn out that scalarneutrinos of different families are not degenerate, some further consideration should be given to $\tilde{\nu}_{\mu}, \tilde{\nu}_{\tau}$ decays.

\section{(B) Production of scalar-neutrinos}

We summarize the various signatures of scalar-neutrinos in $\mathrm{e}^{+} \mathrm{e}^{-}, \overline{\mathrm{p}} \mathrm{p}$, and $\mathrm{e}^{-} \mathrm{p}$ physics. The most likely place to discover scalar-neutrinos is in $\mathrm{W}^{+}$and/or $\mathrm{Z}^{0}$ decay. If the decays are kinematically allowed, then

$$
\Gamma\left(\mathrm{Z}^{0} \rightarrow \tilde{\nu} \tilde{\bar{\nu}}\right) / \Gamma\left(\mathrm{Z}^{0} \rightarrow \nu \bar{\nu}\right)=\frac{1}{2}\left(1-4 \tilde{M}_{\nu}^{2} / m_{z}^{2}\right)^{3 / 2},
$$




$$
\Gamma\left(\mathrm{W}^{+} \rightarrow \tilde{\mathrm{e}}_{\mathrm{L}}^{+} \tilde{\nu}_{\mathrm{e}}\right) / \Gamma\left(\mathrm{W}^{+} \rightarrow \mathrm{e}^{+} \nu_{\mathrm{e}}\right)=\frac{1}{2}\left\{\left[\left(m_{\mathrm{w}}^{2}-\tilde{M}_{\nu}^{2}-\tilde{M}_{\mathrm{e}}^{2}\right)^{2}-4 \tilde{M}_{\nu}^{2} \tilde{M}_{\mathrm{e}}^{2}\right] / m_{\mathrm{w}}^{4}\right\}^{3 / 2}
$$

In the absence of new particles, we expect $\mathrm{BR}\left(\mathrm{W}^{+} \rightarrow \mathrm{e}^{+} \nu_{\mathrm{e}}\right) \approx 8 \%$ and $\mathrm{BR}\left(\mathrm{Z}^{0} \rightarrow \nu \bar{\nu}\right) \approx 6 \%$ which implies nonnegligible production of scalar-neutrinos in $\mathrm{W}^{+}$and $\mathrm{Z}^{0}$ decay.

In W decay, there can be two kinds of events. If the dominant scalar-neutrino decay mode is into $\nu \tilde{\gamma}$ (or if it is stable), then the scalar-neutrino and its decay products are not observed. The emitted scalar-electron decays immediately (its lifetime is too short to leave a visible track) into $\mathrm{e}^{-} \tilde{\gamma}$ and the $\tilde{\gamma}$ is not observed [3.25-3.27]. Such events will appear as $\mathrm{W}^{ \pm} \rightarrow \mathrm{e}^{ \pm} \mathrm{X}$ where the emitted electron is isolated and is sometimes hard. The characteristics of such events are quite different from $\mathrm{W}^{ \pm} \rightarrow \mathrm{e}^{ \pm} \nu$ decays in a number of ways. First, the average $p_{\mathrm{T}}$ of electrons coming from $\mathrm{W} \rightarrow \tilde{\mathrm{e}} \tilde{\nu} \rightarrow \mathrm{e} \tilde{\gamma} \tilde{\nu}$ is roughly half that of electrons coming from

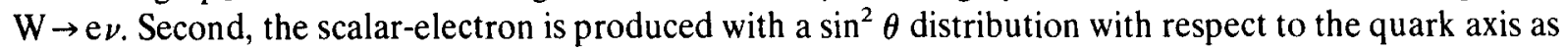
compared with a $(1 \pm \cos \theta)^{2}$ distribution for the $\mathrm{e}^{\mp}$ coming from $\mathrm{W} \rightarrow \mathrm{e} \nu$ events. Background events from $\tau$-decays, possible new heavy leptons, semi-leptonic decays of heavy quarks, and $\mathrm{W}$ production due to sea-sea annihilation must be carefully considered. This we discuss further in chapter 5 . If the scalar-neutrino decays into charged particles, a scalar-neutrino event will most likely consist of an isolated electron and an accompanying hadronic jet. Contamination from heavy quark production where one quark decays semi-leptonically is far more severe here.

Scalar-neutrinos could be produced in $\mathrm{Z}^{0}$ decay. Although it is in principle possible to observe this at the CERN $\bar{p}$ p collider, the absence of very high luminosity makes it unlikely as a copious source of scalar-neutrinos. Large $\mathrm{Z}^{0}$ cross sections at SLC and LEP and an adequate scalar-neutrino branching ratio imply one of three possible outcomes: the scalar-neutrino will be discovered there, limits on its mass close to $m_{z} / 2$ will be obtained, or the $\tilde{\nu}$ decays are mainly invisible. Whereas an invisible scalar-neutrino decay is an advantage in $\mathrm{W}$ decay, it is highly undesirable in $\mathrm{Z}^{0}$ decay. Note that if $\mathrm{Z}^{0} \rightarrow \tilde{\nu} \tilde{\bar{\nu}}$ and both scalar-neutrinos decay invisibly, one way to detect it is to employ neutrino counting techniques [3.13]. (Another is through the effect on $\Gamma\left(\mathrm{Z}^{0}\right)$ which we discuss in chapter 4.) Namely, run the energy of the $Z^{0}$ factory slightly above the $Z^{0}$ mass and look for $\mathrm{e}^{+} \mathrm{e}^{-} \rightarrow \gamma+$ nothing, corresponding to $\mathrm{e}^{+} \mathrm{e}^{-} \rightarrow \gamma \mathrm{Z}^{0}, \mathrm{Z}^{0} \rightarrow \nu \bar{\nu}, \tilde{\nu} \bar{\nu}, \ldots$ On the other hand, if the scalar-neutrino has substantial charged decay modes, chances of observing scalar-neutrinos are excellent. The most spectacular type of event occurs when $Z^{0} \rightarrow \tilde{\nu} \bar{\nu}$, one scalar-neutrino decays invisibly into neutrals and the other one decays into a jet of charged hadrons. These 'one-sided events' are very striking; sample events simulated by Monte Carlo in ref. [3.27] are shown in fig. 11. Finally, if scalar-neutrinos decay entirely into charged particles, then high multiplicity events will occur with only a small amount of energy lost to two outgoing photinos; these may be difficult to untangle from conventional events.

One additional advantage to the high luminosity $\mathrm{Z}^{0}$ factories is that one can be sensitive to rare decay modes of the scalar-neutrino. As we mentioned in the previous subsection, the decay rate $\tilde{\nu}_{\mathrm{e}} \rightarrow \nu_{\mathrm{e}} \mathrm{e}^{-} \ell^{+} \tilde{\gamma}(\ell=\mathrm{e}, \mu, \tau)$ is likely to be rare (see figs. 9 and 10$)$. Still, in a large sample of $\mathrm{Z}^{0}$, an occasional one-sided event where the charged particles consisted of, say, $\mathrm{e}^{-} \mu^{+}$or $\mathrm{e}^{-} \tau^{+}$would be extremely striking. Not many such events are needed to attract attention.

The events described above can also occur in continuum $\mathrm{e}^{+} \mathrm{e}^{-}$physics. Here, $\mathrm{e}^{+} \mathrm{e}^{-} \rightarrow \tilde{\nu} \overline{\tilde{\nu}}$ can occur by both s-channel $\mathrm{Z}^{0}$ exchange and $\mathrm{t}$-channel wino exchange (see appendix E.6). The signatures discussed above are also relevant here. In addition, if the wino is light enough, a third mechanism shown in fig. 12 is possible. One possible advantage of $\mathrm{e}^{+} \mathrm{e}^{-} \rightarrow \mathrm{e}^{+} \tilde{\mathrm{w}}^{+} \tilde{\nu}_{\mathrm{e}}$ is that this signature can be observed even if the scalar-neutrino decays entirely into neutrals. More details may be found in chapter 4.

If we replace the $\mathrm{e}^{+}$in fig. 12 with a proton, we have a mechanism for producing scalar-neutrinos in ep physics. However, the rates for this mechanism [3.104] are too small to be observed. A far better way 


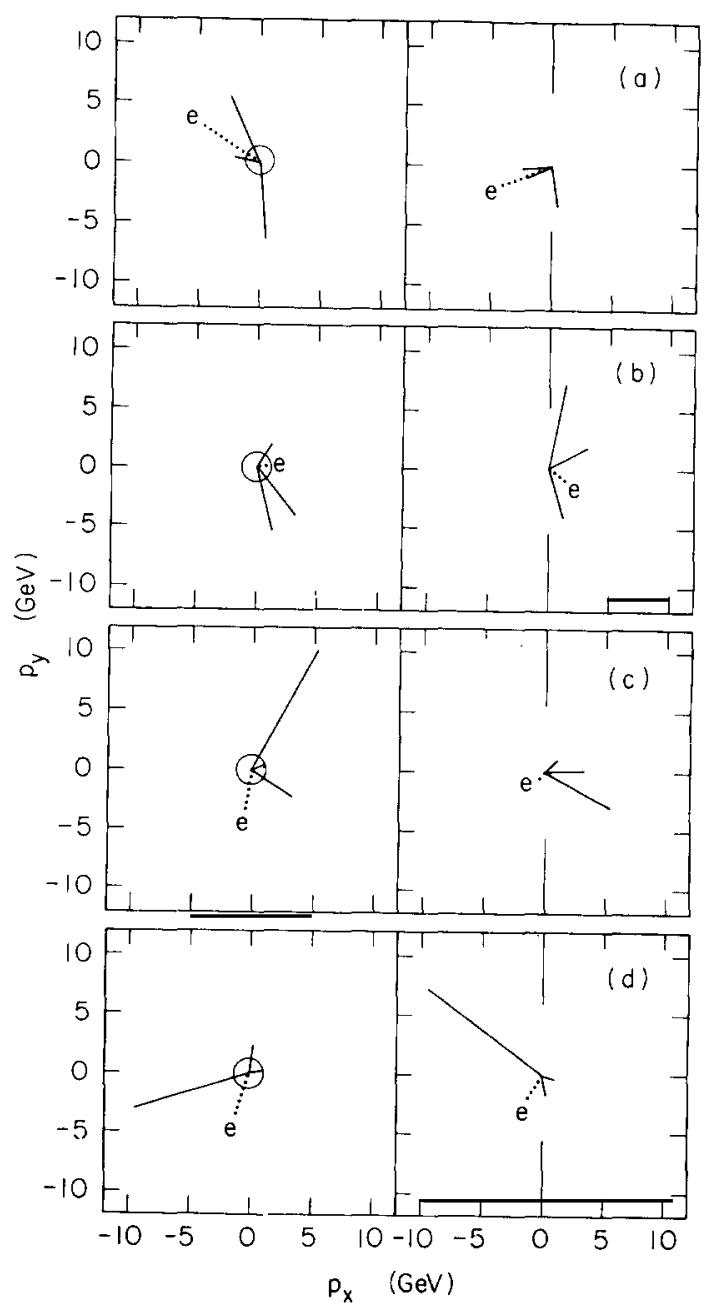

Fig. 11. Simulated events of $\mathrm{e}^{+} \mathrm{e}^{-} \rightarrow \tilde{\nu} \overline{\bar{\nu}}$. One of the scalar neutrinos decays into eud̄ $\bar{g}$ and the other one decays invisibly. In these "typical events' $\sqrt{s}=42 \mathrm{GeV}$, and $\bar{M}_{\nu}=18 \mathrm{GeV}$. Each event is shown in two views: first with the beam pipe perpendicular to the plane of the projection and second with the beam pipe going from top to bottom of the plane of projection. The beam pipe has been marked in both views. The dotted lines correspond to the electron whereas the solid lines represent the gluino and the two quarks. The resultant hadron jets will usually be relatively narrow. This figure has been taken from ref. [3.27].

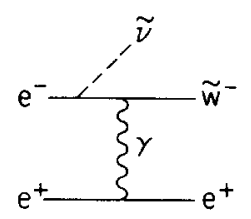

(a)

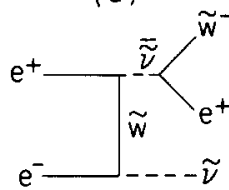

(d)

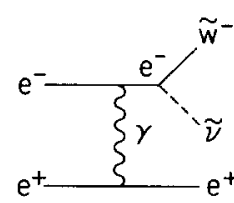

(b)

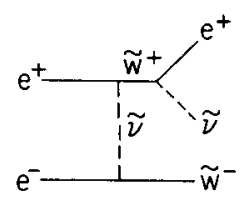

(c)

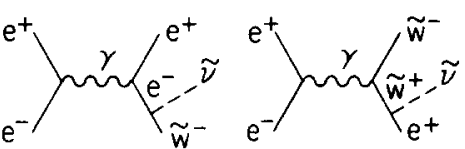

Fig. 12. Graphs for $\mathrm{e}^{+} \mathrm{e}^{-} \rightarrow \mathrm{e}^{+} \tilde{\nu} \tilde{\mathrm{w}}^{-}$. Note that if we replace the $\mathrm{e}^{+}$with a proton in (a) and (b) we obtain graphs for ep $\rightarrow \tilde{\nu} \tilde{w}^{-} p$.

to produce $\tilde{\nu}$ at ep colliders is via ep $\rightarrow \tilde{\nu} \tilde{\mathrm{q}}+\mathrm{X}$ (which is the supersymmetric version of the standard neutral current process ep $\rightarrow \nu+\mathrm{X}$ ). If the $\tilde{\nu}$ decays into a four-body final state this event would be distinguished by the appearance of hadronic jets associated with the lepton vertex. Details can be found in chapter 7.

Finally, one can in principle produce scalar-neutrinos in association with scalar-quarks in deep inelastic neutrino production. The main problem here is one of accessible energy scales. Because the 
neutrino beams must be incident on fixed targets, the total center of mass energy for the interaction will be too low to compete with the other methods described here for producing scalar-neutrinos.

(C) Existing experimental limits and future possibilities

At present, the limits on scalar-neutrinos are virtually nonexistent. The best one can do so far is to observe [3.28] that there would be modifications to $\tau$ decay properties if the decay $\tau \rightarrow \tilde{\nu}_{\ell} \tilde{\nu}_{\tau} \ell$ could occur, as in fig. 13. Depending on the wino mass, this gives limits which qualitatively imply $\tilde{M}\left(\tilde{\nu}_{\tau}\right)+$ $\tilde{M}\left(\tilde{\nu}_{\ell}\right) \geq m_{\tau}$ for $\ell=\mathrm{e}, \mu$. Similar remarks obviously apply for $\mu \rightarrow \tilde{\nu}_{\mu} \tilde{\nu}_{\ell} \mathrm{e}$.

As for a future increase in experimental limits or discovery, the best hope is the combined study of scalar-neutrino production from both $\mathrm{W}$ and $\mathrm{Z}^{0}$ decay. In chapter 11 , we provide our best guess at accessible mass limits during the coming decade.

\subsection{Scalar-quarks}

Scalar-quarks come in two varieties: $\tilde{\mathrm{q}}_{\mathrm{L}}$ and $\tilde{\mathrm{q}}_{\mathrm{R}}$. The discussion is analogous to the one regarding scalar-leptons; we shall use conventions similar to those stated at the beginning of section 3.1. Since scalar-quarks carry fractional electric charge, and less color than gluinos, they are produced with smaller cross sections than scalar-leptons at $\mathrm{e}^{+} \mathrm{e}^{-}$colliders, and smaller cross sections than gluinos at hadron machines. Further, because they have quark jets as decay products their signatures may not be the most favorable for detection. Only if they happen to be lighter than other supersymmetric partners would they be the first to be observed. Nevertheless, they do have significant production cross sections and acceptable signatures.

\section{(A) Decays}

Scalar-quarks always have a coupling to $\tilde{\mathrm{g}}, \tilde{\gamma}$ so if the decays

$$
\begin{aligned}
& \tilde{\mathrm{q}} \rightarrow \mathrm{q} \tilde{\gamma}, \\
& \tilde{\mathrm{q}} \rightarrow \mathrm{q} \tilde{\mathrm{g}},
\end{aligned}
$$

are kinematically allowed they will occur and dominate. The $\tilde{\mathrm{g}}$ mode is larger by $C \alpha_{\mathrm{s}} / \alpha e_{\mathrm{q}}^{2}$, where $C=4 / 3$ is a color factor and $e_{\mathrm{q}}$ is the quark charge in units of $e$, if $\tilde{M}_{\mathrm{g}}=\tilde{M}_{\gamma}$. Equation (3.15) gives a complicated signature since usually $\tilde{\mathrm{g}} \rightarrow \mathrm{q} \overline{\mathrm{q}} \tilde{\gamma}$ but eq. (3.14) can give a very good signature of a quark jet and missing $p_{\mathbf{T}}$.

The rate for $\tilde{q} \rightarrow q \tilde{\gamma}$ is

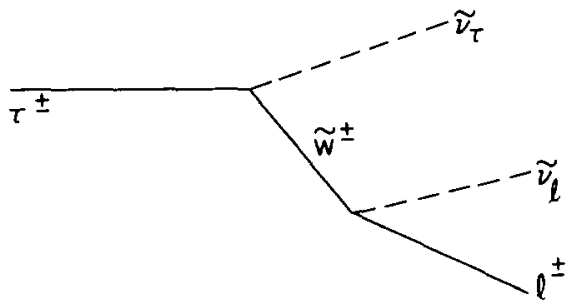

Fig. 13. Graph for $\tau^{ \pm} \rightarrow \bar{\nu}_{\tau} \tilde{\nu}_{\ell} \ell^{ \pm}$. 


$$
\Gamma(\tilde{\mathrm{q}} \rightarrow \mathrm{q} \tilde{\gamma})=\alpha e_{\mathrm{q}}^{2}\left(\tilde{M}_{\mathrm{q}}^{2}-\tilde{M}_{\gamma}^{2}-m_{\mathrm{q}}^{2}\right) p_{\mathrm{q}} / \tilde{M}_{\mathrm{q}}^{2}
$$

where $p_{\mathrm{q}}=\lambda^{1 / 2}\left(\tilde{M}_{\mathrm{q}}^{2}, \tilde{M}_{\gamma}^{2}, m_{\mathrm{q}}^{2}\right) / 2 \tilde{M}_{\mathrm{q}}$ is the quark center-of-mass momentum; for $\tilde{\mathrm{q}} \rightarrow \mathrm{q} \tilde{\mathrm{g}}$ multiply by $\frac{4}{3} \alpha_{\mathrm{s}} / \alpha e_{\mathrm{q}}^{2}$ and replace $\tilde{M}_{\gamma}$ with $\tilde{M}_{\mathrm{g}}$.

\section{(B) Production and signatures}

Scalar-quarks can be produced at $\mathrm{e}^{+} \mathrm{e}^{-}$or hadron colliders, or in heavy particle $\left(\mathrm{Z}^{0}, \mathrm{~W}^{ \pm}, \mathrm{t}, \ldots\right)$ decays.

(a) In $\mathrm{e}^{+} \mathrm{e}^{-}$collisions the spin-zero scalar-quarks of flavor $\mathrm{q}$ are produced with a cross section*

$$
\mathrm{d} \sigma / \mathrm{d} \Omega=\left(3 \alpha^{2} / 8 s\right) e_{\mathrm{q}}^{2} \beta^{3} \sin ^{2} \theta,
$$

where $e_{\mathrm{q}}$ is the electric charge of quark $\mathrm{q}$ in units of $e, \beta=\left(1-4 \tilde{M}_{\mathrm{q}}^{2} / s\right)^{1 / 2}$, and $\theta$ is the center-of-mass production angle. (A color factor of 3 has been included.) If the scalar-quarks were stable they would appear as a jet pair. Since they decay with short lifetimes, just as c-quarks or b-quarks do, they must be searched for in terms of their decay products. The only signature which is likely to be useful is $\tilde{q} \rightarrow q \tilde{\gamma}$, giving a two-body decay which is isotropic in the $\tilde{\mathrm{q}}$ rest frame. The $\tilde{\gamma}$ escapes a collider detector, so one gets missing energy and $\boldsymbol{p}_{\mathbf{T}}$ with acolinear and acoplanar jets - that is, one final jet will generally not lie in the plane of the beams and the other final jets.

For massless photinos half the energy is carried off on the average; for massive photinos the energy carried off is even larger. Thus considerable care needs to be taken with experimental cuts to avoid reducing the signal too much. Because $\tilde{M}_{\gamma}$ is not known, experimental limits for a given $\tilde{M}_{\mathrm{q}}$ will be different as the assumed value of $\tilde{M}_{\gamma}$ is changed if no signal is detected. Compared to scalar-leptons, the observable scalar-quark cross section is suppressed both by the fractional electric charge and by the fact that one is experimentally sensitive (for the most part) to the (presumably) less prevalent $\mathrm{q} \tilde{\gamma}$ decay mode. (If the gluino is sufficiently light, the q $\mathrm{g}$ mode will dominate because of the dependence on $\alpha_{\mathrm{s}}$ ) Hence, it is more difficult to detect scalar-quarks than scalar-leptons of the same mass.

One interesting possibility arises from the study of indirect constraints on supersymmetric models (to be discussed in chapter 8). In order that flavor changing neutral currents be adequately suppressed, one finds that scalar-quark masses of different flavors cannot be too different. (The exact bound applies to scalar-quarks of a given charge; see eq. (8.6).) It then may happen that more than one (maybe all) flavors of scalar-quarks may be closer in mass than the $\mathrm{e}^{+} \mathrm{e}^{-}$beam resolution. If this happens, one could pass the scalar-quark threshold and suddenly find multiple flavors of scalar-quarks being produced (thus enhancing the overall rate).

(b) In hadron collisions there are several ways to produce scalar-quarks. The basic process can produce $\tilde{\mathrm{q}} \overrightarrow{\tilde{q}}, \tilde{\mathrm{q}} \tilde{\mathrm{q}}, \tilde{\mathrm{q}} \tilde{\gamma}$, with elementary processes as shown in fig. 14 . Which process dominates depends on masses.

The signatures are different, and which is best depends on the sizes of cross sections, on detector capabilities, and on Standard Model backgrounds. For example,

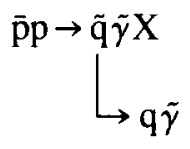

${ }^{*}$ Equation (3.17) neglects the s-channel $\mathrm{Z}$ exchange graph. This may be accounted for by using eq. (3.2) with appropriate values for $e_{\mathrm{i}}$ and $T_{3 \mathrm{i}}$. 

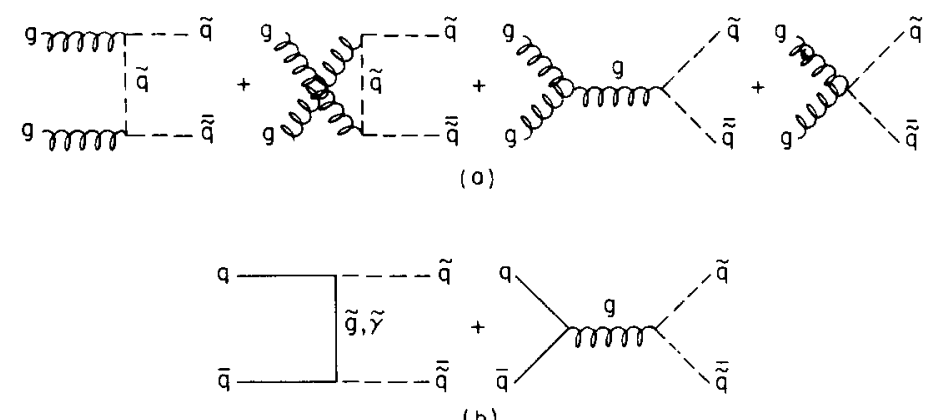

(b)

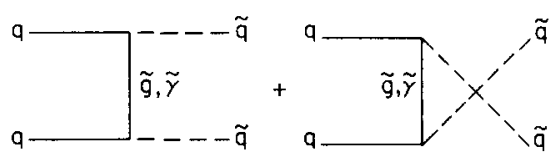

(c)

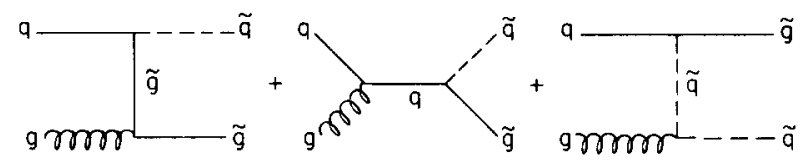

(d)

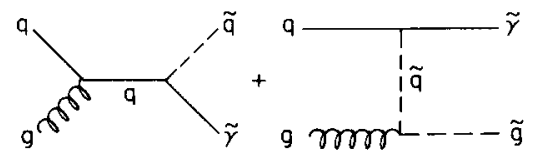

(e)

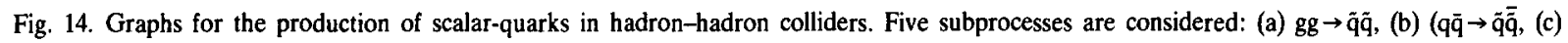
$q q \rightarrow \tilde{q} \bar{q}$, (d) $q g \rightarrow \tilde{q} \tilde{g}$ and $(e) q g \rightarrow \tilde{q} \tilde{\gamma}$.

gives a rather good potential signature with a single quark jet detected and large missing $\boldsymbol{p}_{\mathrm{T}}$, but it almost certainly has a smaller cross section (depending on masses) than $\tilde{\mathrm{q}} \overline{\tilde{q}}$. Further, there is some QCD background, and additional background from production of $Z^{0}+\mathrm{g}$ with $Z^{0} \rightarrow \nu \bar{\nu}$ (an $18 \%$ branching ratio in the Standard Model). While the $\tilde{\mathrm{q}} \overline{\tilde{q}}$ cross section decreases for heavy $\tilde{\mathrm{q}}$, if both scalar-quarks decayed via $\tilde{\mathrm{q}} \rightarrow \mathrm{q} \tilde{\gamma}$ the signature may be quite good, with hard acoplanar quark jets allowing identification of individual events that would not occur in the Standard Model.

Some studies of signals and backgrounds have been done [3.5,3.29-3.35], and there is room for extensive further study of the variations with $\sqrt{s}, \tilde{M}_{\mathrm{q}}, \tilde{M}_{\gamma}, \tilde{M}_{\mathrm{g}}$. It is particularly important to identify the regions where one can hope to identify $\tilde{\mathrm{q}} \overline{\tilde{q}}$ or $\tilde{\mathrm{q}} \tilde{\gamma}$ on an event-by-event basis, since then a relatively small number of events is needed and large masses can be produced.

(c) There are several ways scalar-quarks can appear in decays. They will couple to the $Z^{0}$, so $Z^{0} \rightarrow \tilde{q} \overline{\tilde{q}}$ occurs for each flavor if $\tilde{M}_{\mathrm{q}}<m_{z} / 2$. Counting both $\tilde{\mathrm{q}}_{\mathrm{L}}$ and $\tilde{\mathrm{q}}_{\mathrm{R}}$ we obtain a formula equivalent to eq. (3.7). Thus the effect of the superpartners on the $Z^{0}$ would be an increase of the width by a factor $3 / 2$ if all scalar-quarks were degenerate with the associated quarks. This is reduced by the phase-space suppression for heavy scalar-quarks. For example, assuming $M_{\mathbf{q}}>20 \mathrm{GeV}$, we would have 


$$
\Gamma\left(\mathrm{Z}^{0} \rightarrow \overline{\tilde{\mathrm{q}}}_{\mathrm{L}} \tilde{\mathrm{q}}_{\mathrm{L}}+\overline{\tilde{\mathrm{q}}}_{\mathrm{R}} \tilde{\mathrm{q}}_{\mathrm{R}}\right)<0.38 \Gamma\left(\mathrm{Z}^{0} \rightarrow \overline{\mathrm{q}} \mathrm{q}\right) .
$$

The analogous decays occur for the $W^{ \pm}$; one can have $W^{+} \rightarrow \tilde{t} \overline{\tilde{b}}$, $\tilde{\mathrm{u}} \overline{\tilde{d}}$, etc. In cases where $\tilde{\mathrm{q}} \rightarrow \mathrm{q} \tilde{\gamma}$, these events are hard to identify, but not out of the question, especially for $W^{ \pm}$tagged by associated production with a gluon.

(d) Scalar quarks can be produced at ep colliders. The most interesting reactions to study are the supersymmetric analogs of the charged current process $(\mathrm{ep} \rightarrow \nu \mathrm{X})$ and the neutral current process $(\mathrm{ep} \rightarrow \mathrm{eX})$. The corresponding supersymmetric processes are ep $\rightarrow \tilde{\nu} \tilde{\mathrm{q}} \mathrm{X}$ and ep $\rightarrow \tilde{\mathrm{e}} \tilde{\mathrm{q} X}$ respectively [3.5, $3.58,3.108$ ] as shown in fig. 15. In general, these events will be difficult to extract from the larger ordinary charged and neutral current processes. There is one exception which occurs when the scalar-neutrino decays appreciably into four-body final states (see figs. 9 and 10). If such is the case, then the signature for ep $\rightarrow \tilde{\nu} \tilde{\mathrm{q} X}$ will consist of a multiparticle decay at the lepton vertex associated with the production of a hadronic jet at the hadron vertex, with some transverse energy of the event eventually lost due to escaping photinos. This could be the most dramatic signal of scalar-quarks. Further discussions can be found in chapter 7 .

\section{(C) Present limits}

Limits on the existence of scalar-quarks come from two sources. First, qualitatively, scalar-quarks

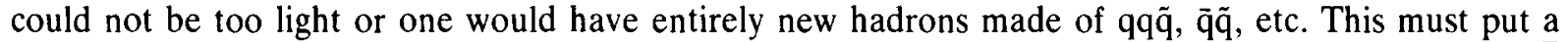
limit of order one $\mathrm{GeV}$ on scalar-quark masses. If scalar-quarks were rather light, one would have $\tilde{\mathrm{q}} \overline{\tilde{q}}$ bound states analogous to $\psi, Y$, etc. [3.36,3.5]. Since these are not found, the scalar-quarks must be too heavy. The bound states produced in $\mathrm{e}^{+} \mathrm{e}^{-}$collisions would be $\mathrm{p}$-wave, with smaller leptonic widths, so the constraints are not as strong as for quarks. The situation has been analyzed by Nappi [3.36], who finds that $e_{\mathrm{q}}=2 / 3$ scalar-quarks can be excluded if they are lighter than about $3 \mathrm{GeV}$, but limits are not found for $e_{\mathrm{q}}=-1 / 3$ scalar-quarks.

More quantitatively, limits can be placed from the absence of a signal in $\mathrm{e}^{+} \mathrm{e}^{-}$collisions. We are only aware of one such result at present, from the JADE collaboration, giving for $95 \%$ CL limits [3.37]

$$
3.1 \mathrm{GeV}<\tilde{M}_{\mathrm{q}}<17.8 \mathrm{GeV}, \quad e_{\mathrm{q}}=2 / 3, \quad 7.4 \mathrm{GeV}<\tilde{M}_{\mathrm{q}}<16.0 \mathrm{GeV}, \quad e_{\mathrm{q}}=-1 / 3,
$$

based on assuming $\tilde{\mathrm{q}} \rightarrow \mathrm{q} \tilde{\gamma}$ is the dominant decay with light $\tilde{\gamma}$ and examining the resulting acolinearity. JADE also gives some limits based on the possibility that scalar-quarks are stable and form neutral or charged stable hadrons, presumably by binding with light quarks, giving heavy fermions. From $\mathrm{d} E / \mathrm{d} x$ or the absence of visible energy a large range of kinematically accessible masses can be excluded.

It is important to note that these limits depend on the decay $\tilde{\mathrm{q}} \rightarrow \mathrm{q} \tilde{\gamma}$ dominating and photinos being very light. If gluinos are less massive than scalar-quarks, so $\tilde{\mathrm{q}} \rightarrow \mathrm{q} \tilde{\mathrm{g}}$ dominates, then with $\tilde{\mathrm{g}} \rightarrow q \bar{q} \tilde{\gamma}$ one has $\tilde{\mathrm{q}} \rightarrow \mathrm{qq} \overline{\mathrm{q}} \tilde{\gamma}$, giving a very difficult signature. However, even in this case the highly spherical event will allow limits to be set or a signal detected; but no published analysis is relevant.

Finally, one can get some limits from the absence of $q \rightarrow \tilde{q} \tilde{\gamma}$. Since $b$ decay is dominated by $b \rightarrow c \bar{f} f^{\prime}$, the decay $b \rightarrow \tilde{b} \tilde{\gamma}$ is not allowed - otherwise it would dominate being electromagnetic in strength. Thus

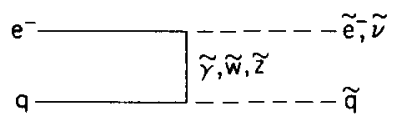

Fig. 15. Graph of parton subprocess responsible for ep $\rightarrow \tilde{e} \tilde{q} X$ and $e p \rightarrow \tilde{\nu} \tilde{q} X$. 
one can conclude (very crudely) $\tilde{M}_{\mathrm{b}}+\tilde{M}_{\gamma} \geqslant m_{\mathrm{b}}$, with similar remarks for charm, etc. If a t-quark is observed via its semi-leptonic decay one can analogously conclude immediately that $\tilde{M}_{\mathrm{t}}+\dot{M}_{\gamma} \geq m_{\mathrm{t}}$. Similar remarks can be made with $\tilde{\gamma}$ replaced by $\tilde{\mathrm{g}}$.

We conclude that on the basis of a direct analysis of data charge-2/3 scalar-quarks seem to be excluded with masses below about $17.8 \mathrm{GeV}$, while for charge- $1 / 3$ scalar-quarks there seems to be a window from about $1 \mathrm{GeV}$ to $7.4 \mathrm{GeV}$. We conjecture that there is a way to close this window, and that in fact it is closed, but a careful analysis has not yet been done. If in fact there were a $\tilde{\mathrm{q}}_{-1 / 3}$ with $\tilde{M}<7 \mathrm{GeV}$, it would imply a significant violation of isospin conservation in strong interactions. One would compute the violation along the same lines as the parity-violating isospin violation studied by Duncan [3.38]; since one has $\Delta \tilde{M} \sim 10 \mathrm{GeV}$ and $\tilde{M} \sim 7 \mathrm{GeV}$ in loops, the operators generated would be large, and are presumably excluded by strong isospin conservation. This analysis needs to be carried out in detail. Assuming we have understood this situation correctly, it appears that at the present time that scalar-quarks of either charge should be required to be heavier than $17 \mathrm{GeV}$ if the decay $\tilde{q} \rightarrow q \tilde{g}$ does not occur. On the other hand, if gluinos are lighter than scalar-quarks the decay sequence $\tilde{\mathrm{q}} \rightarrow q \tilde{\mathrm{g}}$, $\mathrm{g} \rightarrow \mathrm{q} \overline{\mathrm{q}} \tilde{\gamma}$ will dominate, and none of the collider tests will apply. Then from the analysis of Nappi, plus the requirement of strong isospin invariance, we can only deduce $\tilde{M}_{\mathrm{q}} \geq 3 \mathrm{GeV}$.

\section{(D) Future possibilities}

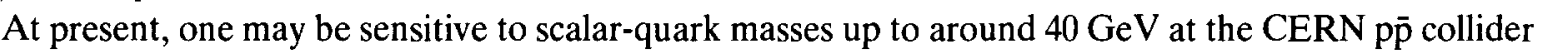
under favorable assumptions. When data is available from the FNAL Tevatron a significant window will be available. We are not aware of any detailed studies on the detectable mass range for FNAL energies and luminosities and the available detectors. Presumably $\tilde{q}$ masses of order $75 \mathrm{GeV}$ will be accessible, with larger masses in certain favorable situations. Data could be available at $\mathrm{e}^{+} \mathrm{e}^{-}$colliders at about the same time scale as at FNAL which could search up to about $40 \mathrm{GeV}$ for $\tilde{M}_{\mathrm{q}}$. Once the FNAL studies are available they will not be improved on until a multi-TeV, high luminosity, super collider is available. Cross sections for production of $\tilde{q}$ at FNAL in various processes are given by Dawson et al. [3.34]. To see what $\tilde{q}$ masses could be detected it is necessary to study the signal and the competing QCD background with the detector characteristics in mind. If gluinos are lighter than scalar-quarks, then the discovery of scalar quarks at hadron colliders is likely to be difficult; hence no useful limit on their masses can be obtained there.

Under favorable conditions it might be possible to discover the scalar-quark in an ep collider such as HERA. This would be feasible by looking for ep $\rightarrow \tilde{\nu} \tilde{q}+X$ only if the scalar-neutrino has an appreciable decay branching ratio into four-body final states. Unfortunately, once again, we cannot get a useful limit on the scalar quark mass if no such events are seen, because if $\tilde{\nu} \rightarrow$ unobserved neutrals is the dominant decay, the above signature is lost.

\subsection{Gluinos}

Gluinos $(\tilde{\mathrm{g}})$ are the spin $1 / 2$ supersymmetric partners of the gluons $(\mathrm{g})$. In the supersymmetric limit, the gluino is a massless two-component fermion (the supersymmetry insures that the number of degrees of freedom of the gluino is equal to two, which is the number of polarization states of a massless gluon). Supersymmetry breaking will in general allow the gluino to acquire a mass. However, the counting of degrees of freedom remains unchanged, so that in this case the gluino must be a massive Majorana 
fermion.* The dominant interactions of gluinos are governed by the $\tilde{g} \tilde{g} g$ vertex which is the supersymmetric version of the three-gluon vertex; in both cases the strength of the vertex is given by the strong QCD coupling constant $g_{s}$. Because the gluino is a self-conjugate Majorana fermion it has a definite charge conjugation $(\mathrm{C})$ of -1 . $^{* *}$ In this section we will take the gluino mass to be arbitrary, and discuss constraints on this mass from presently available experimental data as well as ways to detect gluinos at present and future machines, and what limits can be assigned if they are not found.

\section{(A) The decay of the gluino}

Gluinos are expected to be copiously produced in hadronic machines (see section B which follows). The ease of their detection is very sensitive to their possible decay modes. There is the usual subtlety here in that gluinos (which are color octets) would live in new supersymmetric hadrons [3.39-3.45]. If the gluino is sufficiently heavy (say $\tilde{M}_{\mathrm{g}} \geq 1-3 \mathrm{GeV}$ ), the lifetime of such hadrons would be approximately equal to the lifetime calculated for a free gluino. In this sense, gluinos would behave in much the same manner as a new heavy quark would. For light gluinos, the discussion is much more subtle. We shall begin by assuming that the gluino is heavy enough so that it is appropriate to treat the gluino as a free particle. A discussion of the case of the light or massless gluino follows at the end of this section.

The dominant decay of the gluino is likely to be a three-body decay into q $\tilde{q} \tilde{\gamma}$ as depicted in fig. 16. We present the differential decay rate assuming the photino has mass $\tilde{M}_{\gamma}$. Let us define $x=\tilde{E}_{\gamma} / \tilde{M}_{\mathrm{g}}$ and $y=\tilde{M}_{\gamma} / \tilde{M}_{\mathrm{g}}$. Physically, $x$ is a measure of the missing energy of the gluino decay, since we assume that the photino escapes detection. Then we find [3.46] (in the approximation of massless quarks and assuming that the scalar-quark masses are large, i.e., $\tilde{M}_{\mathrm{L}}, \tilde{M}_{\mathrm{R}} \gg \tilde{M}_{\mathrm{g}}$ )

$$
\frac{\mathrm{d} \Gamma}{\mathrm{d} x}=\frac{\alpha \alpha_{\mathrm{s}} e_{\mathrm{q}}^{2} \tilde{M}_{\mathrm{g}}^{5}}{2 \pi}\left(\frac{1}{\tilde{M}_{\mathrm{L}}^{4}}+\frac{1}{\tilde{M}_{\mathrm{R}}^{4}}\right)\left(x^{2}-y^{2}\right)^{1 / 2}\left[x\left(1-\frac{4}{3} x\right)+y(1-2 x)-\left(\frac{2}{3}-x\right) y^{2}+y^{3}\right],
$$

where $e_{\mathrm{q}}$ is the quark charge in units of $e$. The range for $x$ is $y \leq x \leq\left(1+y^{2}\right) / 2$. Integrating over photino energies leads to a total decay rate

$$
\begin{aligned}
& \Gamma(\tilde{\mathrm{g}} \rightarrow \tilde{\gamma} q \overline{\mathrm{q}})=\frac{\alpha \alpha_{\mathrm{s}} e_{\mathrm{q}}^{2} \tilde{M}_{\mathrm{g}}^{5}}{96 \pi}\left(\frac{1}{\tilde{M}_{\mathrm{L}}^{4}}+\frac{1}{\tilde{M}_{\mathrm{R}}^{4}}\right) \\
& \times\left[\left(1-y^{2}\right)\left(1+2 y-7 y^{2}+20 y^{3}-7 y^{4}+2 y^{5}+y^{6}\right)+24 y^{3}\left(1-y+y^{2}\right) \log y\right] .
\end{aligned}
$$
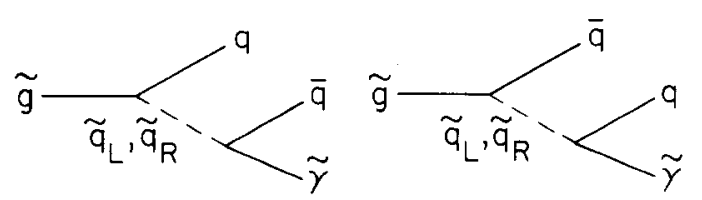

Fig. 16. Graphs for $\tilde{\mathbf{g}} \rightarrow q \bar{q} \bar{\gamma}$. The quarks q may run over all possible flavors such that the decay is kinematically allowed.

\footnotetext{
* In principle, one can artificially introduce an additional color octet of ordinary (matter) fermions which could then combine with the Majorana gluinos and produce massive Dirac gluinos [3.39]. Such models appear to be quite artificial and we shall assume throughout this paper that gluinos (as well as photinos) are Majorana fermions.

${ }^{* *}$ This follows from the fact that the gluon is odd under $C$. Such a concept is useful only in the presence of $C$-conserving interactions.
} 


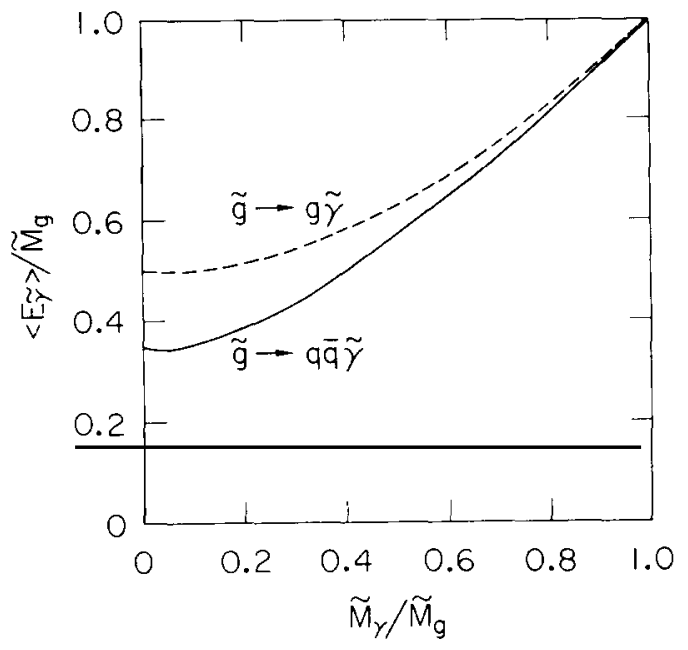

Fig. 17. Photino energy lost in gluino decay. We plot the average energy $\left\langle E_{\hat{\gamma}}\right\rangle$, in the gluino rest frame, carried off by the photino in the decay $\overline{\mathrm{g}} \rightarrow \mathrm{q} \overline{\mathrm{q}} \bar{\gamma}$ (solid line) and $\overline{\mathrm{g}} \rightarrow \mathrm{g} \bar{\gamma}$ (dashed line).

The average photino energy $\left\langle\tilde{E}_{\gamma}\right\rangle$ is easily computed from eq. (3.21). In fig. 17, we plot $\left\langle\tilde{E}_{\gamma}\right\rangle$ against $y \equiv \tilde{M}_{\gamma} / \tilde{M}_{\mathrm{g}}$.

Since $\tilde{\mathrm{g}} \rightarrow \mathrm{q} \overline{\mathrm{q}} \tilde{\gamma}$ is likely to be the dominant $\tilde{\mathrm{g}}$ decay mode, the free gluino lifetime is given by the inverse of $\Gamma(\tilde{\mathrm{g}} \rightarrow \mathrm{q} \overline{\mathrm{q}} \tilde{\gamma})$. It is a typical 'weak' decay since $\Gamma \sim \alpha \alpha_{\mathrm{s}} \tilde{M}_{\mathrm{g}}^{5} / \tilde{M}_{\mathrm{q}}^{4}$, assuming $\tilde{M}_{\mathrm{g}}$ is fairly heavy. However, as emphasized below, it is expected that light bound gluinos do not decay as free particles but as off-mass-shell bound ones which have a larger effective mass and thus a shorter lifetime [3.44].

A possible competing decay is the two-body mode $\tilde{\mathrm{g}} \rightarrow \mathrm{g} \tilde{\gamma}$ [3.46]. Since this latter process involves three $C=-1$ particles, it is a $C$-violating process. However, $C$-violation is expected quite naturally in supersymmetric theories. Weak interaction effects in general lead to mass splitting between the $\tilde{q}_{L}$ and

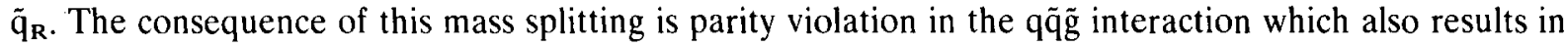
$C$-violation (since $C P$ is approximately conserved). The decay $\tilde{\mathrm{g}} \rightarrow \mathrm{g} \tilde{\gamma}$ occurs at one-loop where the loop consists of a quark and scalar-quark. The decay rate is found to be

$$
\Gamma(\tilde{\mathrm{g}} \rightarrow \mathrm{g} \tilde{\gamma})=\frac{\alpha_{\mathrm{s}}^{2} \alpha e_{\mathrm{g}}}{8 \pi^{2}} \frac{\left(\tilde{M}_{\mathrm{g}}^{2}-\tilde{M}_{\gamma}^{2}\right)^{3}}{\tilde{M}_{\mathrm{g}}^{\mathrm{s}}} \sum_{j}\left(I_{\mathrm{L} j}-I_{\mathrm{R}_{j}}\right)^{2},
$$

where we sum over the flavors $j$ occurring in the loop and $I$ is a complicated function of quark, scalar-quark and gluino masses. Exact formulas are given in ref. [3.46]. Here we note one useful limit: Let $R \equiv \tilde{M}_{\mathrm{g}}^{2} / \tilde{M}_{\mathrm{k}}^{2}$ and $r=m_{\mathrm{q}}^{2} / \tilde{M}_{\mathrm{k}}^{2}$ where $\tilde{M}_{\mathrm{k}}$ is the scalar-quark mass and $\mathrm{k}=\mathrm{L}$ or $\mathrm{R}$. Then, for small gluino mass $(R \ll 1)$,

$$
I_{\mathrm{k}}=\frac{-R}{4(\longmapsto-r)^{2}}\left[1+r+\frac{2 r \log r}{1-r}\right]+\mathrm{O}\left(R^{2}\right) .
$$

If in addition, the quark mass is negligible $(r \ll 1)$, we find that $I_{\mathrm{k}} \approx-R / 4$.

To compare $\tilde{\mathrm{g}} \rightarrow \mathrm{g} \tilde{\gamma}$ to $\tilde{\mathrm{g}} \rightarrow \tilde{\gamma} \mathrm{q} \overline{\mathrm{q}}$, let us count only one flavor of massless quarks and take the photino to be massless. Then, using eqs. (3.23) and (3.24) we find 


$$
\Gamma(\tilde{\mathrm{g}} \rightarrow \mathrm{g} \tilde{\gamma}) / \Gamma(\tilde{\mathrm{g}} \rightarrow \mathrm{q} \tilde{\mathrm{q}} \tilde{\gamma})=3 \alpha_{\mathrm{s}}\left(\tilde{M}_{\mathrm{R}}^{2}-\tilde{M}_{\mathrm{L}}^{2}\right)^{2} / 4 \pi\left(\tilde{M}_{\mathrm{L}}^{4}+\tilde{M}_{\mathrm{R}}^{4}\right) .
$$

The factor on the right-hand side depends on unknown scalar-quark masses but could be nonnegligible. In some models the $\mathrm{L}$ and $\mathrm{R}$ eigenstates differ in mass by a factor of two or more, giving $\left(\tilde{M}_{\mathrm{R}}^{2}-\right.$ $\left.\tilde{M}_{\mathrm{L}}^{2}\right)^{2} /\left(\tilde{M}_{\mathrm{L}}^{4}+\tilde{M}_{\mathrm{R}}^{4}\right)>\frac{1}{2}$. Furthermore, if the photino mass is significant (i.e. $y=\tilde{M}_{\gamma} / \tilde{M}_{\mathrm{g}}$ close to 1 ), it follows from eqs. (3.21) and (3.23) that the three-body rate vanishes as $(1-y)^{2}$ relative to the two-body rate. Having a significant two-body decay rate can have important phenomenological implications as we shall discuss in the next section.

Finally, we briefly mention more exotic decays of the gluino. If the gluino and photino were degenerate (or the photino was heavier) and if in addition the scalar-neutrino were lighter, then four-body decays of the gluino such as $\tilde{\mathrm{g}} \rightarrow \mathrm{ude} \mathrm{e}^{-} \tilde{\nu}$ and $\tilde{\mathrm{g}} \rightarrow \mathrm{q} \overline{\mathrm{q}} \nu \tilde{\nu}$ would be permitted [3.46]. These rates could be important only for an unusual choice of parameters, in which case the gluino would be quite long lived.

Given the gluino lifetime as calculated above, we need to determine the lifetime of the supersymmetric hadron in which the gluino lives. Clearly, we need only consider the (one or two) lightest supersymmetric hadrons since the heavier ones will decay strongly into lighter ones. Gluinos could be constituents of either new fermions qq̃ $\tilde{g}$ (meiktinos, in the terminology of ref. [3.42]) and g̃g (glueballinos) or new bosons $\tilde{g} \tilde{g}$ (gluinonia). For heavy gluinos, the total lifetime of the states containing one $\tilde{g}$ will be roughly given by the lifetime of the free gluino. However the photino energy spectrum of the decaying supersymmetric hadron will be modified near the kinematic endpoint $x=\tilde{E}_{\gamma} / \bar{M}_{\mathrm{g}}=\frac{1}{2}\left(1+y^{2}\right)$. This occurs for two reasons: First, higher order QCD corrections lead to logarithmic singularities at the endpoint which must be summed to all orders. Second, bound state corrections are important in this regime. One can apply an analysis similar to the one used in ref. [3.47] for semi-leptonic decays of B and D mesons. Typically, the result is that the photino spectrum roughly agrees with that from a freely decaying gluino as long as $x$ is not near its kinematic endpoint. As $x$ approaches its maximum, the spectrum rapidly cuts off to zero (as opposed to approaching a nonzero constant as indicated in eq. (3.21)).

For light gluinos the analysis is less certain. Franco [3.44] attempted to apply the analysis of ref. [3.47] to the case where the gluino (which plays the role of a massive quark) is now taken massless. While the analysis is not known to be quantitatively correct when the gluino is light, his conclusions are reasonable. Basically, Franco found that the supersymmetric hadron with mass $\tilde{M}$ decays with a total rate of a free massive gluino with effective mass $\tilde{M} B^{1 / 5}$ (where $B$ is estimated to be in the range corresponding to $0.7<B^{1 / 5}<0.8$ ). The photino energy spectrum differs from that of the free gluino as described above.

The remaining question is what is the value of $\tilde{M}$, the mass of supersymmetric hadrons which contain gluinos. A spectrum of gluino bound states has been worked out by Chanowitz and Sharpe [3.42] using the MIT bag model and by Mitra and Ono [3.48] using a Bethe-Saltpeter model (see also refs. $[3.109,3.110])$. Sample results are given in table 5. The notation we shall use is as follows. For q $\bar{q} \tilde{g}$ states, we shall write down the ordinary meson name (with an appended ) corresponding to the flavor structure of the $q \bar{q}$ pair with a subscript indicating the spin. For example, ud̃ $\tilde{g}$ can be either $\tilde{\pi}_{1 / 2}^{+}, \tilde{\rho}_{1 / 2}^{+}$, or $\tilde{\rho}_{3 / 2}^{+}$depending on whether the (color octet) q $\bar{q}$ pair has quantum numbers $J^{\mathrm{PC}}=0^{-+}$or $1^{--}$and how the spin $1 / 2$ of the gluino is added to give the final meiktino state. For g $\tilde{g}$ states we will write $\tilde{\Gamma}$ along with a subscript indicating its spin. Most of the states decay via strong interactions (e.g., $\tilde{\rho}_{1 / 2}^{+} \rightarrow \tilde{\Gamma}_{1 / 2}+\pi^{+}$and $\left.\tilde{\pi}^{+} \rightarrow \Gamma_{1 / 2}+\pi^{+}\right)$. However, one noteworthy feature of the spectra presented in table 5 is that the $\tilde{\mathrm{K}}_{1 / 2}^{* \pm}$ is strong-interaction stable, even for zero gluino mass. That is $\tilde{\mathrm{K}}_{1 / 2}^{*} \rightarrow \tilde{\Gamma}_{1 / 2}+\mathrm{K}^{+}$and $\tilde{\mathrm{K}}_{1 / 2}^{* \pm} \rightarrow \tilde{\pi}_{1 / 2}+\pi$ are kinematically forbidden (only weak decays such as $\mathrm{K}_{1 / 2}^{* \pm} \rightarrow \tilde{\gamma} \mathrm{K}$ are then permitted). Both groups of 
Table 5

Supersymmetric hadron masses. [We give some results from a number of calculations of supersymmetric hadron masses as a function of gluino masses. States with the lightest masses have been chosen for illustration. All masses below are given in units of $\mathrm{GeV}$. Model I is a bag model calculation [3.42] with the gluon self-energy parameter chosen to be (a) $C_{\mathrm{TE}}=1.00$ and (b) $C_{\mathrm{TE}}=2.36$ (the former value is a 'worst case' scenario whereas the latter is the preferred value). Model II uses a Bethe-Salpeter model of confinement [3.48]. In Model II, the $\tilde{\pi}_{1 / 2}, \tilde{\rho}_{1 / 2}, \tilde{\omega}_{1 / 2}$ and $\tilde{\eta}_{1 / 2}$ are degenerate in mass in the approximation used.]

\begin{tabular}{lllllllll}
\hline & & & \multicolumn{7}{c}{ Mass of gluino } \\
Supersymmetric hadron & Model & 0.0 & 0.5 & 1.0 & 1.5 & 2.0 & 5.0 & 10.0 \\
\hline $\bar{\Gamma}_{1 / 2}$ & Ia & & 0.80 & 1.45 & 2.02 & 2.56 & 5.66 & 10.70 \\
& Ib & & 1.45 & 2.04 & 2.59 & 3.12 & 6.18 & 11.21 \\
& II & 0.30 & 0.80 & 1.30 & 1.80 & 2.30 & & \\
$\tilde{\rho}_{1 / 2}$ & & & & & & & & \\
& I & & 1.02 & 1.54 & 2.06 & 2.60 & 5.66 & 10.68 \\
& II & 0.60 & 1.10 & 1.60 & 2.10 & 2.60 & & \\
$\overline{\mathrm{K}}_{1 / 2}$ & & & & & & & & \\
& I & & 1.22 & 1.71 & 2.24 & 2.75 & 5.79 & 10.81 \\
& II & 0.70 & 1.20 & 1.70 & 2.20 & 2.70 & & \\
$\tilde{\pi}_{1 / 2}$ & & & & & & & & \\
& I & & 1.36 & 1.78 & 2.24 & 2.73 & 5.73 & 10.74 \\
& II & 0.60 & 1.10 & 1.60 & 2.10 & 2.60 & & \\
\hline
\end{tabular}

authors $[3.42,3.48]$ conclude that there must be charged as well as neutral low lying supersymmetric hadrons which are strong interaction stable.

If true, this has important consequences for ruling out the possibility of (nearly) zero mass gluinos as discussed in the next section. Furthermore, the above analysis when combined with that of Franco [3.44] suggest that the lifetimes of the strong interaction stable supersymmetric hadrons of mass $\tilde{M}$ (even in the limit of $\tilde{M}_{\mathrm{g}}=0$ ) are unlikely to be much longer than $10^{-10} \mathrm{~s}$. This can be seen from eq. (3.22) by taking $\alpha_{\mathrm{s}}=1 / 3$ and summing over three quark flavors (u,d,s) which gives (assuming $\tilde{M}_{\gamma}=0$ and $\left.\tilde{M}_{\mathrm{L}}=\tilde{M}_{\mathrm{R}} \equiv \tilde{M}_{\mathrm{q}}\right)$

$$
\tau \sim\left[3 \times 10^{-12} \mathrm{~s} /\left(\tilde{M}_{\mathrm{g}} / 1 \mathrm{GeV}\right)^{5}\right]\left(\tilde{M}_{\mathrm{q}} / m_{\mathrm{w}}\right)^{4},
$$

where we should put, according to Franco, $\tilde{M}_{g}=\tilde{M} B^{1 / 5} \simeq 1 \mathrm{GeV}$. In principle, one can get around this result by either taking $\tilde{M}_{\gamma}$ large or by taking $\tilde{M}_{\mathrm{q}} \gg m_{\mathrm{w}}$. However, one must keep in mind that taking $\tilde{M}_{\mathrm{q}}$ too large would spoil supersymmetry as a solution to the various problems discussed in chapter 1 . That is, while one could treat $\tilde{M}_{\mathrm{q}}$ as a free parameter, in practice supersymmetry is of basic interest when the superpartner masses are on the order of $m_{\mathrm{w}}$. Also if $\tilde{M}_{\gamma} \geq \tilde{M}_{\mathrm{g}}$ then $\tilde{g}$ is (nearly) stable and can be detected by stable particle searches.

\section{(B) The search for light gluinos in present data}

If gluinos are light enough, they may already be present in low energy data. First, there may exist long-lived charged or neutral supersymmetric hadrons which leave visible tracks or gaps. Second, the gluino might be discovered by seeing the interaction of the resulting photino. This is the idea behind beam dump searches where the photino events would be interpreted as excess neutrino induced neutral current events.

Depending on the lifetime of the gluino, one of the two methods described above can probably rule 
out the possibility of light-mass gluinos. The situation is summarized in fig. 18. It is worth looking in detail to see what assumptions go into this result and to pose further experimental tests to tighten up the conclusion above.

Let us consider the significance of the beam dump experiments for the possible existence of light

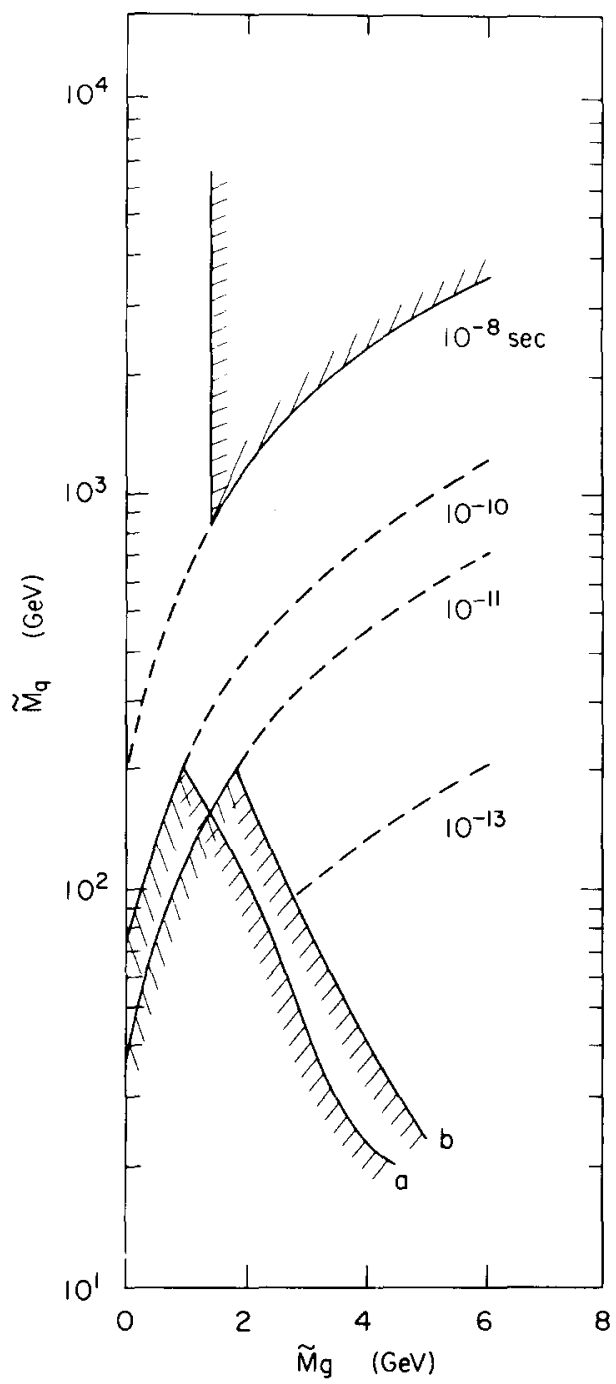

Fig. 18. This figure is to show the region of gluino masses which we believe is currently excluded by data. The result depends on assumptions. The figure is patterned after one from ref. [3.34] (we thank C. Quigg for making it available to us before publication); we have added the results from ref. [3.44] that even massless but confined gluinos will decay. To be specific for the graph, we have assumed that the mass of a gluino-gluon state is $\tilde{M}_{\mathrm{g}}+0.6 \mathrm{GeV}$, where $\tilde{M}_{\mathrm{g}}$ is the current algebra gluino mass, and that the parameter $B$ of ref. [3.44] is 0.2 . The shaded region is excluded by beam dump data where no gluino signal was detected [(a) is from ref. [3.49] and (b) from ref. [3.50]]. For a given $\tilde{M}_{\mathrm{q}}$ one can see what $\tilde{M}_{\mathrm{g}}$ is ruled out. Curves of constant lifetime for a $\overline{\mathrm{g}}$ decay are also shown. Constraints from stable particle searches rule out another region; they are limited by neutron background for $\tilde{M}_{\mathrm{g}}$ near $1 \mathrm{GeV}$, and by decreasing production cross section for $\tilde{M}_{\mathrm{g}}$ large. For $\bar{M}_{\mathrm{q}} \geqslant m_{\mathrm{w}}$ there is a region where light $\tilde{\mathrm{g}}$ could still exist, as emphasized in refs. [3.34,3.53]. However, nonobservation of $\psi \rightarrow \gamma \bar{\eta}_{\mathrm{g}}\left(\tilde{\eta}_{\mathrm{g}}=0^{-+} \tilde{\mathrm{g}} \tilde{\mathrm{g}}\right.$ states$)$ probably rules out $\tilde{\mathrm{g}}$ masses less than $1 \mathrm{GeV}$. For lifetimes longer than $10^{-10} \mathrm{~s}$ (CERN) or $10^{-11} \mathrm{~s}$ (FNAL) the beam dump experiments may not be sensitive because the $\tilde{g}$ will travel too far in the dump and may interact. Various assumptions are involved in interpreting the data to draw the conclusions shown, so each line should be considered to have some thickness. People with models having light gluinos should go back to the original equations to check their parameters in detail. In spite of the caveats, we think the main feature of the graph, that $\tilde{M}_{\mathfrak{g}} \geqslant 3 \mathrm{GeV}$ if $\tilde{M}_{\mathrm{q}} \leqslant m_{\mathrm{w}}$, is valid to a good approximation. 
gluinos. The main assumption made here is that the gluino, once produced, decays before it has a chance to interact again inside the target. This insures that the outgoing photino (one of the decay products) will be headed in the right direction to interact with the beam dump. In the two most recent beam dump experiments, the CHARM experiment [3.49] and the FERMILAB experiment [3.50], the experiments were sensitive to upper limits for the gluino lifetime of about $10^{-11}$ and $10^{-10} \mathrm{~s}$ respectively.

For gluinos heavier than 1-2 GeV, one can obtain approximate gluino cross sections using perturbative QCD (see next section). Accepting Franco's analysis, the same discussion applies to massless and light gluinos; although one cannot calculate the production cross section for light gluinos, it will be larger than that for heavier ones, so the limits obtained are general. Unfortunately, beam dump experiments are extremely sensitive to both experimental and theoretical considerations. On the theoretical side, two crucial points are - what is the subsequent interaction rate of those photinos and do the photinos ever reach the beam dump in the first place. We will discuss these issues in a moment, but first let us summarize the results of a FERMILAB beam dump experiment [3.50]. They see no events which can be interpreted as extra candidates for neutral current events. Their limits depicted in fig. 19 depend on $\tilde{M}_{\mathrm{q}}$ since the photino interaction rate varies as $\tilde{M}_{\mathrm{q}}^{-4}$. The CHARM experiment [3.48] has similar limits, slightly less stringent since their detector was further from the dump. Subject to the caveats of the next paragraph, one concludes that the gluino is heavier than $2-5 \mathrm{GeV}$.

To assign quantitative limits on $\tilde{M}_{\mathrm{g}}$ a careful analysis is needed. The results depend on photino and scalar-quark masses. It was assumed that the photino was massless. For a heavy enough photino (see eq. (3.22)) the decay modes of the gluino into photinos become negligible. Second, three-body decays of heavy objects typically induce a significant $p_{\mathrm{T}}$, the result being that the photino might miss the beam dump entirely; this has been included in the analyses by the experimental groups. Note that if the two-body decay of the gluino (into either $\mathrm{g} \tilde{\gamma}$ or $\mathrm{g} \tilde{\mathrm{G}}$ ) is significant, the sensitivity of the beam dump would increase since for two-body decays, almost all decay products emerge near the minimum opening angle given by $\cos \theta / 2=v$, where $v$ is the velocity of the decaying particle. Photinos may also decay into $\gamma \tilde{\mathrm{G}}$ or $\nu \tilde{\nu}$ or $\gamma \nu$; the signature of each of these pairs is different and has to be analyzed separately.

To interpret the absence of a signal in terms of a gluino mass, one must take account of the constituent mass of the gluino-gluon or $\tilde{g} q \bar{q}$ system. There are no definitive calculations; from glueball work we expect that one should subtract $0.8-1 \mathrm{GeV}$ from the limit to obtain the $\tilde{g}$ current algebra mass

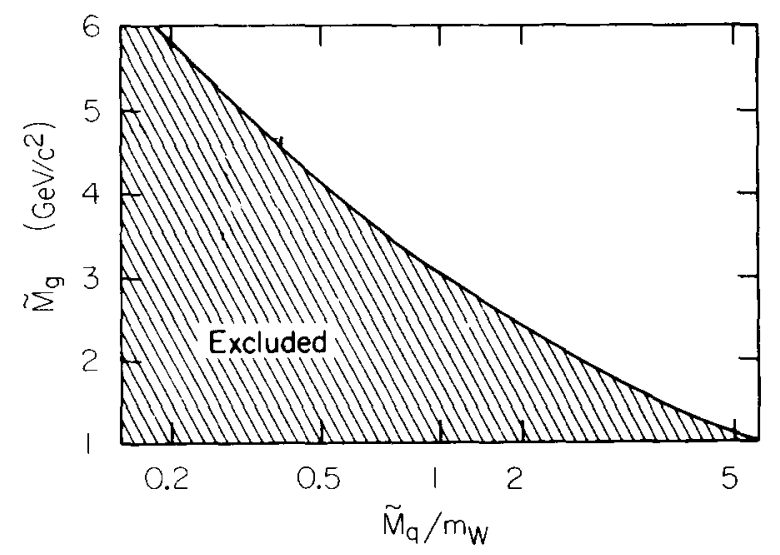

Fig. 19. This shows limits on gluino mass as a function of the scalar-quark mass (top axis) assuming the $\tilde{\mathrm{g}}$ decay $\tilde{\mathrm{g}} \rightarrow \mathrm{q} \overline{\mathrm{q}} \tilde{\gamma}$ is mediated by a scalarquark. The results, from ref. [3.50], also assume $\tilde{\gamma}$ lives long enough to get to the detector and interact, and that $\tilde{\mathrm{g}}$ does not live so long it interacts in the dump. The experimental numbers shown assume an $A$ dependence of $A^{1}$. These results are shown related to lifetimes in fig. 18. 
(but the possibility exists that for unknown dynamical reasons the nonperturbative constituent contribution is larger). Finally, one should note that logically it is possible that gluinos are stable or very long-lived-e.g. if photinos were heavier than gluinos. Then one would expect a new, stable or long-lived neutral (or charged) hadron, with strong interactions. It should be easy to detect at colliders, and the possibility of such a state should not be ignored.

Given the results of fig. 18 (and making standard assumptions about the gluino decay and the range of scalar-quark masses) one is able to rule out gluino masses less than about $2 \mathrm{GeV}$ if the lifetime of the lightest supersymmetric hadron is shorter than $10^{-10} \mathrm{~s}$. The analysis at the end of the previous section may then be used to rule out light gluino masses except for a small window if $\tilde{M}_{\mathrm{q}}>m_{\mathrm{w}}$.

The above argument is not ironclad, so we must investigate the sensitivity of experiments to light gluinos with lifetimes longer than $10^{-10} \mathrm{~s}$. Beam contamination searches rule out long-lived (i.e. lifetimes greater than $10^{-8} \mathrm{~s}$ ) charged and neutral supersymmetric hadrons with masses between about $1.5 \mathrm{GeV}$ and $10 \mathrm{GeV}[3.51,3.42,3.34]$. In addition, a negative hyperon beam at CERN, produced by $17 \mathrm{GeV}$ incident protons showed no evidence for additional well defined charged states [3.52]. The clean hyperon signals of that experiment suggest [3.43] that no charged supersymmetric hadrons with mass between about $1-2 \mathrm{GeV}$ with lifetimes $\geq 10^{-11} \mathrm{~s}$ can exist. This result should be considered in the context of the results of table 5 where it was argued that there must exist at least one charged supersymmetric hadron which is strong interaction stable. This observation taken together with the beam dump results would rule out gluino masses in the range of $1-2 \mathrm{GeV}$ and below.

Recently some authors $[3.53,3.34]$ have argued that there still may be a window for some $\tilde{M}_{\mathrm{q}}$ where light or massless gluinos could exist, contrary to our conclusion. There is no technical disagreement - if one assumes that only light neutral supersymmetric hadrons could have lifetimes longer than $10^{-10} \mathrm{~s}$ (whereas the charged ones decay strongly) then we agree that one cannot demonstrate just from data that such hadrons containing gluinos would already have been observed. However, we think one can extend the analysis in two ways. First, if we do suppose that light gluinos would not have decayed, they would have formed long-lived or stable neutral or charged hadrons of mass $1-1.5 \mathrm{GeV}$. While we cannot prove such hadrons do not exist, we think it likely that additional, strongly interacting, particles in this mass range would have been observed (could there be another neutron a few hundred $\mathrm{MeV}$ heavier than the normal one?). But we certainly agree with the authors of refs. [3.34, 3.53] that it would be good to explicitly exclude this window. Also, one can search for unusual decays occurring in neutral hyperon beams as this would be an ideal place to find long-lived gg or neutral $q \bar{q} \bar{g}$ states. Presumably, the decay of the lightest supersymmetric hadron would often lead to multi-body final states, by analogy with glueballs. But unusual events have never been reported in such a situation.

Secondly, as discussed above we think the argument of Franco is at least qualitatively correct in which case very long-lived or stable supersymmetric hadrons are ruled out (under the assumption that $\tilde{M}_{\mathrm{q}} \gg m_{\mathrm{w}}$ is unacceptable).

There is one more experimental test which we think unambiguously rules out zero mass gluinos and probably rules out gluinos lighter than about $1 \mathrm{GeV}$. Consider the gluinonium spectrum (i.e. g̃ g bound states) for light mass gluinos. As we shall show in section $D$, the lowest lying state has the quantum numbers of the $\eta\left(J^{\mathrm{PC}}=0^{-+}\right)$which we denote $\tilde{\eta}_{\mathrm{g}}$. The color octet nature of the gluino would substantially enhance $\psi \rightarrow \gamma \tilde{\eta}_{\mathrm{g}}$ relative to $\psi \rightarrow \gamma\left(\eta, \eta^{\prime}\right)[3.54,3.111,3.112]$. (Details are given at the end of section 5.2.) No such state is observed (with the possible exception of the $\iota(1440)$ ). This clearly rules out zero-mass gluinos; a more detailed analysis could set an interesting bound. In any case, the absence of $\tilde{\eta}_{\mathrm{g}}$ and of light charged supersymmetric hadrons closes the window for light gluinos even for $\tilde{M}_{\mathrm{q}} \gg m_{\mathrm{w}}$. 
Finally, there have been some purely theoretical works which argue [3.55] that a massless gluino would necessarily lead to an existence of a light pseudoscalar meson (presumably, the $\tilde{\eta}_{\mathfrak{g}}$ ) with a mass less than or equal to that of the pion mass. This again would rule out the possibility of a nearly massless gluino.

\section{(C) Production of gluinos in hadron colliders}

Because gluinos are color octet states, the cross section for gluino production is about an order of magnitude larger than for a heavy quark of the same mass [3.41]. For the signatures of gluinos, we shall use the fact that one of its decay products (the photino) escapes the apparatus carrying off unseen energy and momentum. The lack of an accompanying hard charged lepton in the event will be necessary to rule out the possibility of an escaping neutrino.

We shall assume for most of this section that the gluino decays via the three-body mode $\tilde{g} \rightarrow q \bar{q} \tilde{\gamma}$. Later on, we will briefly discuss the effects on the analysis of a two-body decay mode for the gluino.

Gluinos will be produced either in pairs or singly in association with scalar-quarks or photinos. The relevant diagrams are shown in figs. 14(d) and 20. Perturbative QCD gives us the ability to make fairly reliable computations of the cross sections $\mathrm{A}+\mathrm{B} \rightarrow \tilde{\mathrm{g}}+\tilde{\mathrm{q}}+\mathrm{X}, \mathrm{A}+\mathrm{B} \rightarrow \tilde{\mathrm{g}}+\tilde{\mathrm{g}}+\mathrm{X}$, and $\mathrm{A}+\mathrm{B} \rightarrow \tilde{\mathrm{g}}+\tilde{\gamma}+\mathrm{X}$ at large center-of-mass energy $\sqrt{s}$, where the outgoing gluino is emitted at large $p_{\mathrm{T}}$.

Let us first concentrate on the elementary processes involving two gluinos in the final state. Computations of the expected cross sections [3.34, 3.35] are shown in figs. 21 and 22. The following observations are noteworthy. First, the only supersymmetric particles in the diagrams of fig. 20(a) and the first diagram in fig. $20(\mathrm{~b})$ are the gluinos. Hence an experimental search for gluino production will be able to yield limits on the gluino mass independent of the mass of any other supersymmetric particle if one is sensitive to all the ways a gluino can behave (short lifetime with decay into light or heavy photinos, long lifetime, etc.). In addition, the strong (QCD) coupling constant appears at each vertex in fig. 20(a), (b). Hence, apart from phase space suppressions due to the gluino mass, we expect gluinos to be copiously produced in hadron machines.
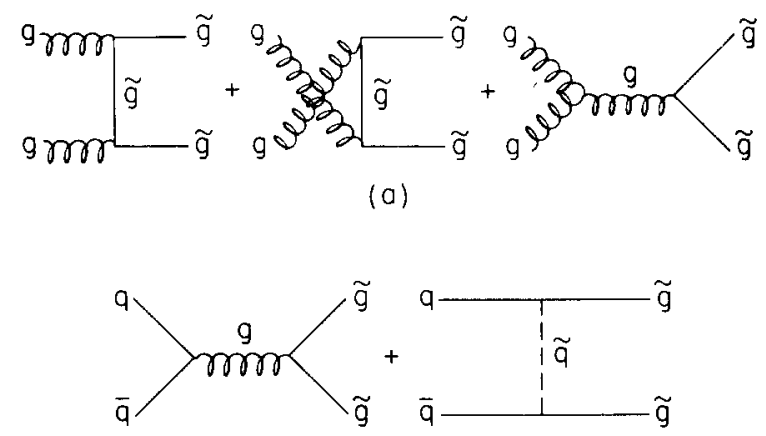

(b)

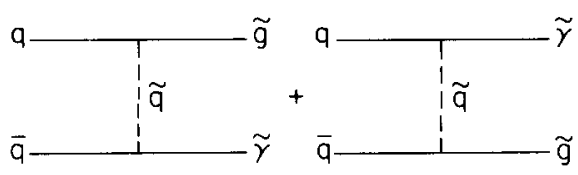

(c)

Fig. 20. Graphs for the production of gluinos in hadron-hadron colliders. Three subprocesses are considered: (a) gg $\rightarrow \tilde{\mathrm{g}} \tilde{\mathrm{g}}$, (b) $q \overline{\mathrm{q}} \rightarrow \mathrm{g} \tilde{\mathrm{g}}$, (c) $\mathrm{q} \overline{\mathrm{q}} \rightarrow \tilde{\mathrm{g}} \bar{\gamma}$. Graphs for $\tilde{g} \tilde{q}$ production are shown in fig. 14(d). 


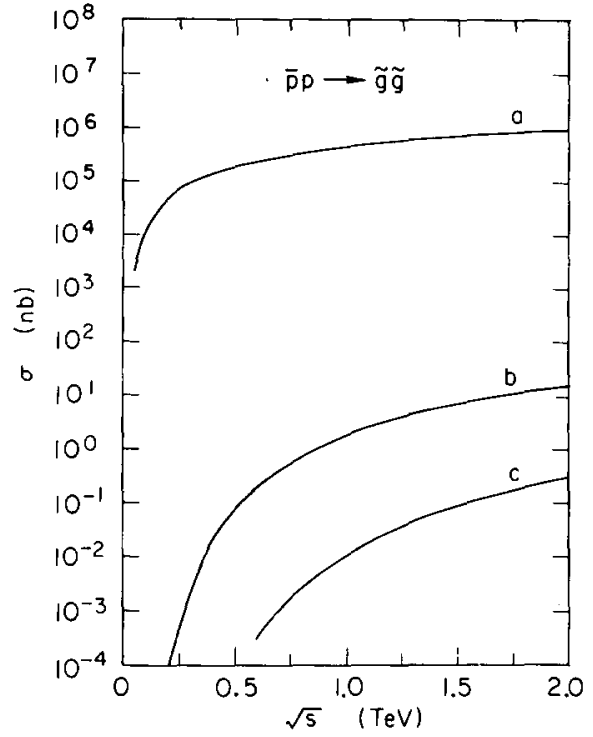

Fig. 21. Total cross section for $\mathrm{p} \overline{\mathrm{p}} \rightarrow \tilde{\mathrm{g}} \tilde{\mathrm{g}}+\mathrm{X}$ vs. $\sqrt{s}$. The three cases correspond to: (a) $\tilde{M}_{\mathrm{g}}=3 \mathrm{GeV}, \bar{M}_{y}=1 \mathrm{GeV}, \bar{M}_{\mathrm{q}}=\bar{M}_{\chi^{+}}=\bar{M}_{\chi^{0}}=$ $20 \mathrm{GeV}$; (b) All supersymmetric particles degenerate with mass of $50 \mathrm{GeV}$; (c) all supersymmetric particles degenerate with mass of $100 \mathrm{GeV}$. This figure has been taken from ref. [3.34] (we thank $\mathrm{S}$. Dawson for providing us with a copy before publication). The distribution functions of ref. [3.35] (with $A=0.29$ ) have been used.

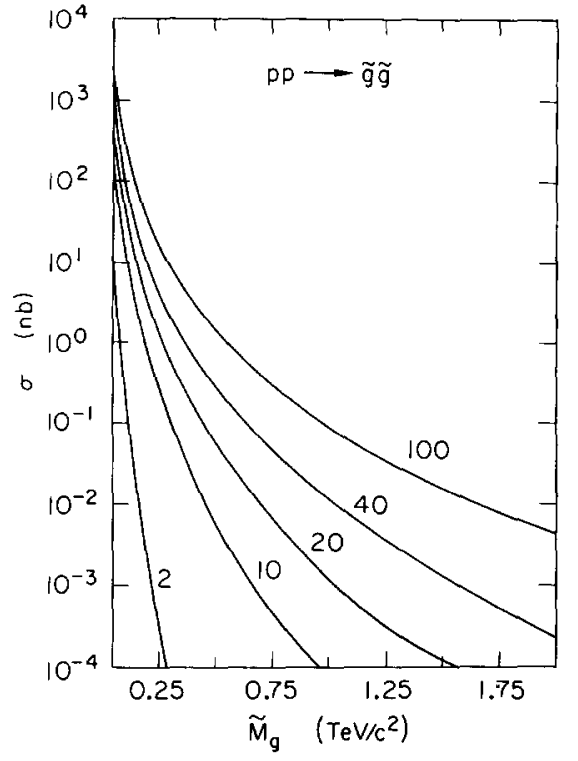

Fig. 22. Total cross section for $\mathrm{pp} \rightarrow \tilde{\mathrm{g}} \bar{\gamma}+\mathrm{X}$ vs. $\bar{M}_{\mathrm{g}}$ with $\sqrt{ } \bar{s}$ in $\mathrm{TeV}$ units shown on the curves, and computed for $\tilde{M}_{\mathrm{q}}=\tilde{M}_{\mathrm{g}}$. These curves are taken from ref. [3.35] (we thank E. Eichten and C. Quigg for providing us with copies before publication). For $\bar{p} p$ the rates are somewhat larger at large $\tilde{M}_{\mathrm{g}}$.

The main experimental task is to find a way to detect the gluinos. Here, one will have to use statistical arguments based on the fact that when gluinos decay, they emit a photino which escapes the detector. Therefore, on average, one should be able to detect a net $p_{\mathrm{T}}$ imbalance by studying a data sample with appropriate cuts. This requires a finely grained high resolution calorimeter with nearly $4 \pi$ angular coverage and no holes which would allow transverse energy to escape.

In refs. [3.29, 3.56], a Monte Carlo analysis was carried out using the program ISAJET [3.57]. The steps in the analysis can be briefly summarized as follows. First a reference axis (roughly, the jet axis in two jet events) is chosen for each event. In ref. [3.29], the sphericity tensor was calculated and the reference axis was associated with the eigenvector with the largest eigenvalue. The detected particles in the event are now divided into two groups corresponding to the two hemispheres defined with respect to the reference axis and beam axis (see fig. 23(a)). Add up all the momenta transverse to the beam in the hemisphere containing the reference axis and call the resultant $\boldsymbol{p}_{\mathrm{T}}$. The sum of the transverse momenta in the opposite hemisphere is denoted by $\boldsymbol{p}_{\mathbf{T}}^{\prime}$. Because of the way the reference axis was chosen, $\left|\boldsymbol{p}_{\mathrm{T}}\right|>\left|\boldsymbol{p}_{\mathrm{T}}^{\prime}\right|$. Two useful measures of missing $p_{\mathrm{T}}$ in the event are [3.29]:

$$
\begin{aligned}
& X_{\mathrm{E}}=\left(-\boldsymbol{p}_{\mathrm{T}} \cdot \boldsymbol{p}_{\mathrm{T}}^{\prime}\right) /\left|\boldsymbol{p}_{\mathrm{T}}\right|^{2}, \\
& \boldsymbol{p}_{\text {out }}=\boldsymbol{p}_{\mathrm{T}}^{\prime}+X_{\mathrm{E}} \boldsymbol{p}_{\mathrm{T}} .
\end{aligned}
$$

The geometrical significance of these quantities is shown in fig. 23(b). Note that $\left|p_{\text {out }}\right|=$ $\left(\left|p_{\mathrm{T}}^{\prime}\right|^{2}-X_{\mathrm{E}}^{2}\left|\boldsymbol{p}_{\mathrm{T}}\right|^{2}\right)^{1 / 2}$. For back-to-back jets, $\boldsymbol{p}_{\mathrm{T}}^{\prime}=-\boldsymbol{p}_{\mathrm{T}}$ which implies $X_{\mathrm{E}}=1$ and $\boldsymbol{p}_{\mathrm{out}}=0$; the QCD 


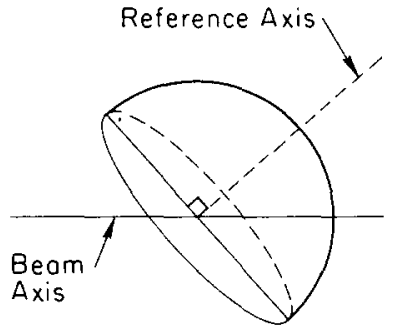

(a)

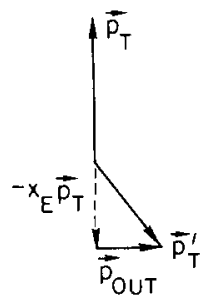

(b)

Fig. 23. A measure of transverse energy and momentum loss. Given an event at a hadron collider, a reference axis can be chosen via a jet-finding algorithm. This axis then defines two hemispheres as shown in (a). The vector sum of the momentum transverse to the beam axis of all particles in each hemisphere is denoted $p_{\mathrm{T}}$ and $p_{\mathrm{T}}$ respectively (where $p_{\mathrm{T}}$ corresponds to particles in the hemisphere containing the reference axis). The variables $X_{\mathrm{E}}$ and $p_{\text {out }}$ are defined as shown in (b).

background will look mainly that way. Thus, small values of $X_{\mathrm{E}}$ and large values of $\left|\boldsymbol{p}_{\text {out }}\right|$ will constitute our signal for missing $p_{\mathrm{T}}$ in the event. In fig. 24 we show $X_{\mathrm{E}}$ and $p_{\text {out }}$ distributions at $\sqrt{s}=800 \mathrm{GeV}$ for dominant background due to light constituents (gluons and light quarks) scattered to large $p_{\mathrm{T}}$ in hard collisions. The distributions due to gluinos of various mass are shown. This suggests that in order to enhance the gluino signal in the distributions shown, we must cut out all events with $X_{\mathrm{E}}>0.5$ and $p_{\text {out }}<5 \mathrm{GeV}$. We have yet to account for heavy (charm, etc.) quark production. In fact, a significant background would occur when a heavy quark decays semi-leptonically and a neutrino escapes with a significant amount of transverse energy. One can deal with this situation by cutting out events containing a visible lepton (for example, cut out events with $E_{\text {lepton }}>2 \mathrm{GeV}$ ). The effects of this cut is illustrated by the dashed lines in fig. 25 . One can see that the background is reduced by an order of magnitude with the cut on lepton energy. The end result of these cuts will be excess signal above

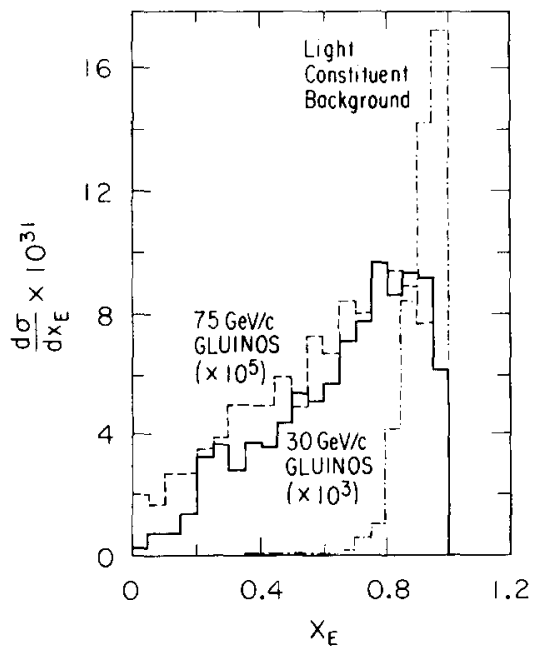

(a)

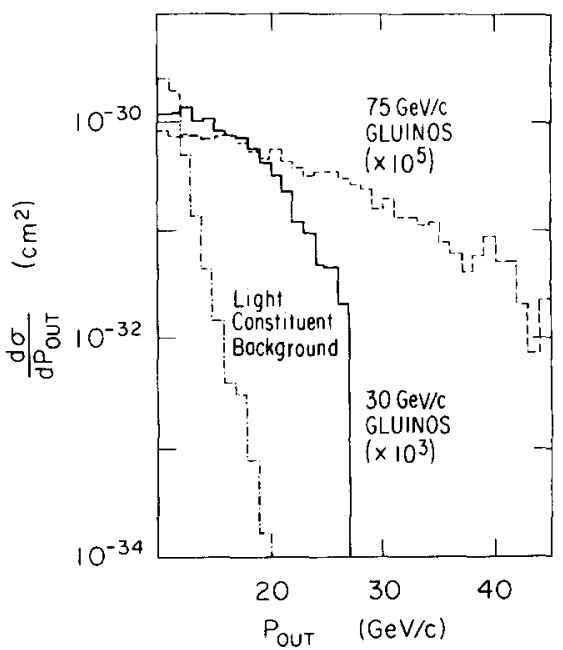

(b)

Fig. 24. Distributions for $X_{\mathrm{E}}$ and $p_{\text {out }}$ obtained by the ISAJET Monte Carlo program for light constituent background (dashed-dot line), $30 \mathrm{GeV}$ gluinos (solid line) and $75 \mathrm{GeV}$ gluinos (dashed line). Note that the gluino histograms are multipled by factors of $10^{3}$ and $10^{5}$ respectively. The center-of-mass energy for this run was $\sqrt{s}=800 \mathrm{GeV}$. This figure was taken from ref. [3.29]. 


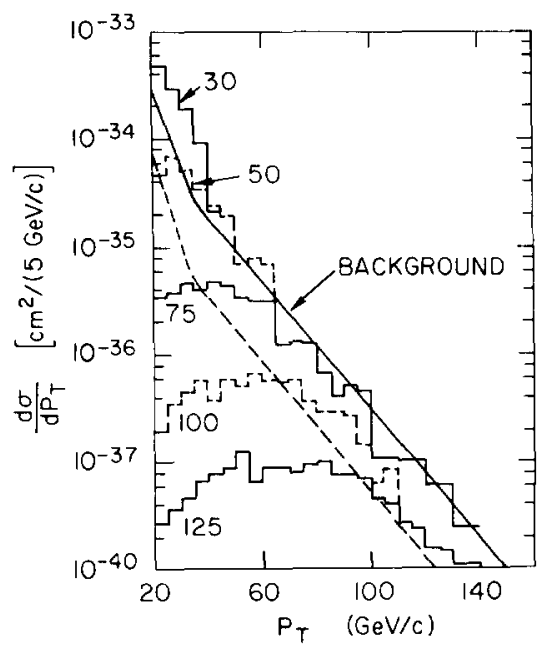

Fig. 25. Gluino cross section vs. $p_{\mathrm{T}}$ for $M_{\hat{\underline{z}}}=30,50,75,100$ and $125 \mathrm{GeV}$ at $\sqrt{s}=800 \mathrm{GeV}$ obtained by the ISAJET Monte Carlo program. The cuts $X_{\mathrm{E}}<0.5$ and $p_{\text {out }}>5 \mathrm{GeV} / c$ have been imposed. The background from Standard Model processes is denoted by the solid line. If in addition, all events with a hard lepton (with energy greater than $2 \mathrm{GeV}$ ) are omitted, the background decreases by about a factor of ten (as shown by the dashed line). This figure was taken from ref. [3.29].

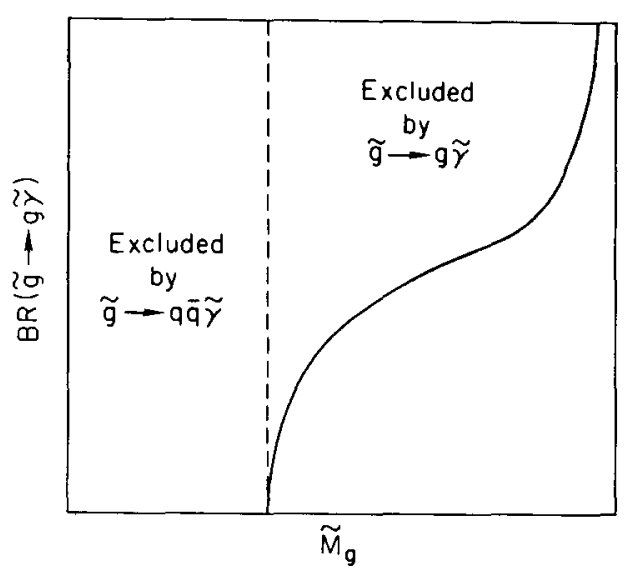

Fig. 26. Possible form for future graphs on gluino mass limits. If gluinos are not detected, the mass range excluded will be a function of the branching ratio for $\overline{\mathrm{g}} \rightarrow \mathrm{g} \tilde{\gamma}$ as compared to the dominant mode (assumed to be $\tilde{\mathrm{g}} \rightarrow \mathrm{q} \tilde{\mathrm{q}} \tilde{\gamma}$ ).

background assuming the gluino mass is not too heavy. Hence, this technique will either be able to discover the gluino* or set a limit on its mass as shown in fig. 26. Clearly, the gluino masses to which the experiment will be sensitive depends on the center of mass energy $\sqrt{s}$ and the luminosity of the accelerator.

The mode $\tilde{\mathrm{g}} \rightarrow \mathrm{g} \tilde{\gamma}$, discussed in section $\mathrm{A}$ above, is potentially very important for gluino detection, and for constraining the theory if gluinos are detected. Its importance for $\tilde{\mathrm{g}}$ detection arises because it is a two-body decay. The three-body mode $q \bar{q} \tilde{\gamma}$ must be searched for on a statistical basis, as described above, so large numbers of events are required (probably of order $10^{5}$ ) to allow a detectable signal to survive dramatic cuts designed to eliminate background. The two-body mode allows one to look for single event signatures such as acolinear central region jets plus missing $\boldsymbol{p}_{\mathrm{T}}$ and no hard charged lepton if both $\tilde{\mathrm{g}} \rightarrow \mathrm{g} \tilde{\gamma}$, or one hard jet opposite two hard jets plus missing $\boldsymbol{p}_{\mathrm{T}}$ and no hard charged lepton if one $\tilde{\mathrm{g}} \rightarrow \mathrm{q} \overline{\mathrm{q}} \tilde{\gamma}$ and the other $\tilde{\mathrm{g}} \rightarrow \mathrm{g} \tilde{\gamma}$. Then a signal could be based on as few as 10-50 events, which could more than compensate for the smaller branching ratio of the $\mathrm{g} \tilde{\gamma}$ mode. If gluinos are not discovered, eventually limits will be determined in the form of fig. 26. This will help constrain thinking about models - all models will predict a $\operatorname{BR}(\tilde{\mathrm{g}} \rightarrow \mathrm{g} \tilde{\gamma})$, and those with large $\tilde{\mathrm{g}} \rightarrow \mathrm{g} \tilde{\gamma}$ decays will be sensitive to larger mass $\tilde{\mathrm{g}}$. The quantitative effect depends on the characteristic of the collider and the detector; a $5-10 \% \tilde{g} \rightarrow \mathrm{g} \tilde{\gamma}$ mode could lead to an increase of a factor of two in what mass gluino might be detected.

Perhaps the best signal for gluinos at hadronic colliders is $p p \rightarrow \tilde{\mathrm{g}} \tilde{\gamma}+\mathrm{X}[3.113,3.46,3.34,3.35]$. Since the photino is assumed to escape, there is substantial missing transverse energy and such a reaction could undoubtedly be seen on an event-by-event basis. The only possible background that could be

\footnotetext{
* We have not included 'non-standard' backgrounds (e.g. other supersymmetric signals) in the above analysis. Hence discovery of a signal could suggest new physics other than gluinos.
} 


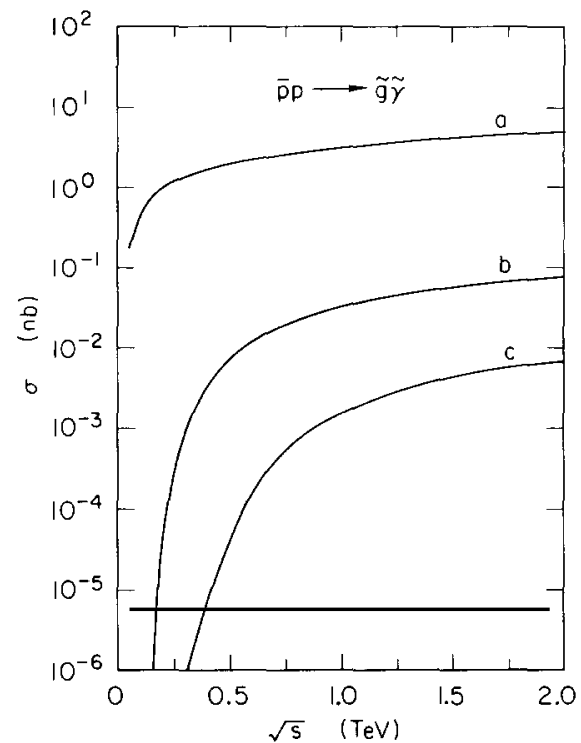

Fig. 27. Total cross section for $\mathrm{p} \overline{\mathrm{p}} \rightarrow \overline{\mathrm{g}} \tilde{y}+\mathrm{X}$ vs. $\sqrt{s}$. See caption to fig. 21 for notation. This figure has been taken from ref. [3.34].

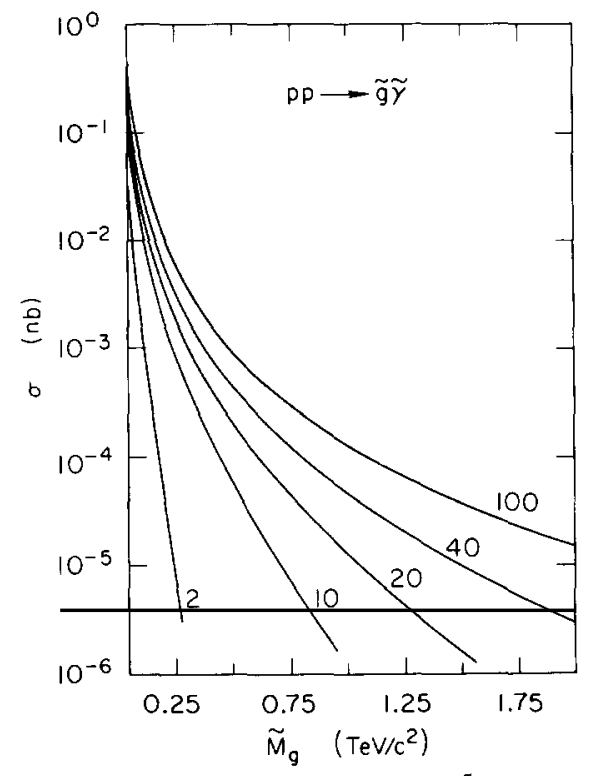

Fig. 28. Total cross section for $\mathrm{pp}, \mathrm{p} \overline{\mathrm{p}} \rightarrow \overline{\mathrm{g}} \tilde{\gamma}$ vs. $\tilde{M}_{\mathrm{g}}$. See caption to fig. 22 for notation. These graphs have been taken from ref. [3.35].

significant is $p p \rightarrow Z^{0}+g+X$ where the $Z^{0}$ decays into neutrinos and the emitted gluon is hard. However one could eventually subtract out such backgrounds on the basis of experimental measurements: by observing such events where $Z^{0} \rightarrow \mathrm{e}^{+} \mathrm{e}^{-}$, the $Z^{0}$ event rate where $Z^{0} \rightarrow \nu \bar{\nu}$ is then known precisely.

Cross sections for $\mathrm{pp}, \mathrm{p} \overline{\mathrm{p}} \rightarrow \tilde{\mathrm{g}} \tilde{\gamma}+\mathrm{X}$ are shown in figs. 27 and 28 . Note that unlike the case of $\mathrm{pp}, \mathrm{p} \overline{\mathrm{p}} \rightarrow \tilde{\mathrm{g}} \tilde{\mathrm{g}}+\mathrm{X}$, the $\tilde{\mathrm{g}} \tilde{\gamma}$ cross sections depends sensitively on the scalar-quark mass (due to scalar-quark exchange in fig. 20 (c)) and thus may be very small if the scalar-quark mass is too large. In addition, due to the production of the $\tilde{\gamma}$, one power of $\alpha_{\mathrm{s}}$ is replaced by $\alpha$ reducing further the $\tilde{\mathrm{g}} \tilde{\gamma}$ total cross section as compared to that of $\tilde{g} \tilde{g}$. However, the smaller cross sections may be compensated by the very dramatic signature of $\tilde{g} \tilde{\gamma}$ production at a higher luminosity machine.

The expected gluino mass sensitivity versus the expected turn-on date of future accelerators in the coming decade are summarized in chapter 11. In particular, gluinos of mass up to $25-50 \mathrm{GeV}$ should be detected at the CERN SPS collider in the next year or two if they exist!

\section{(D) Production of gluinos in ep and $e^{+} e^{-}$machines}

Gluinos can be produced in deep inelastic electroproduction in much the same way as gluons are produced $[3.58,3.5]$. Namely, one can ask what the probability is to find a constituent gluino inside the nucleon. This could be relevant if gluinos are light since gluons make up about $50 \%$ of the momentum of the nucleon and $\mathrm{g} \rightarrow \tilde{\mathrm{g}} \tilde{\mathrm{g}}$ occurs with strength $g_{\mathrm{s}}$. It is straightforward to compute the Altarelli-Parisi splitting functions $P_{i j}$ which govern the evolution equations of the various partonic constituents of the nucleon [3.45, 3.58-3.60]. This is discussed in chapter 7. The upshot of those results is that for large enough $Q^{2}$, one should be able to see evidence for the presence of supersymmetric constituents by careful evaluation of the deep-inelastic structure functions. It must be admitted that such an indirect

\footnotetext{
width.

* We assume that the number of light neutrinos is a known quantity. This will eventually be determined by precise measurements of the $Z^{0}$
} 


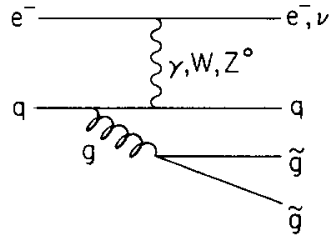

(o)

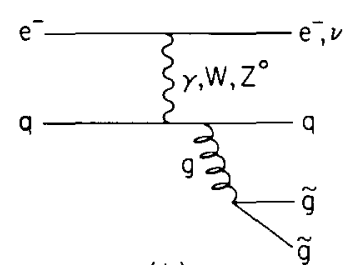

(b)
Fig. 29. Graphs for gluino pair production at ep machines.

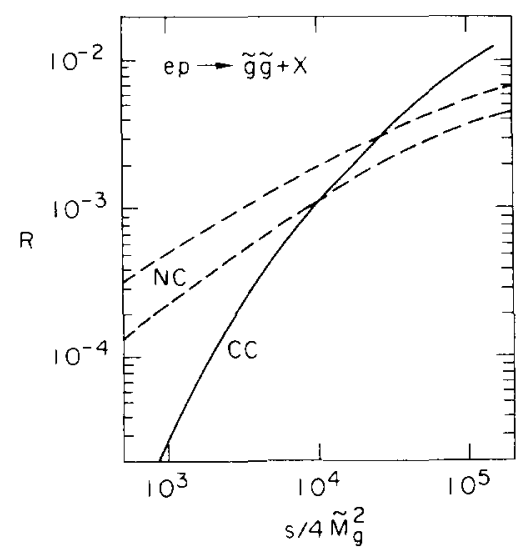

Fig. 30. Graphs of $R_{\mathrm{CC}}$ and $R_{\mathrm{NC}}$ defined by $R_{\mathrm{CC}} \equiv \sigma(\mathrm{ep} \rightarrow$ $\nu \tilde{\mathrm{g}} \tilde{\mathrm{gX}}) / \sigma(\mathrm{ep} \rightarrow \nu \mathrm{X}) \quad$ and $\quad R_{\mathrm{NC}} \equiv[\mathrm{d} \sigma(\mathrm{ep} \rightarrow \mathrm{e} \tilde{\mathrm{g}} \tilde{\mathrm{gX}}) / \mathrm{d} x \mathrm{~d} y] /[\mathrm{d} \sigma(\mathrm{ep} \rightarrow$ $\mathrm{eX}) / \mathrm{d} x \mathrm{~d} y$ ]. The solid line depicts $R_{\mathrm{CC}}$ and the dashed lines depict $R_{\mathrm{NC}}$ evaluated at $x=0.1, y=0.2$ (top line) and $x=0.05, y=0.1$ (bottom line). This figure was taken from refs. $[3.5,3.45]$.

measurement depends on very accurate experiments with little systematic error. Clearly, it would be better to observe directly the production of supersymmetric particles (in this case gluinos and scalar-quarks). Pair production of gluinos as shown in fig. 29 would provide the best method for producing gluinos; the expected rates are shown in fig. 30 taken from ref. [3.45]. Unfortunately, detecting the presence of gluinos (and separating them from heavy quark production) is a little trickier here compared with the techniques described in the previous section. One could construct kinematic variables sensitive to missing energy and momentum as before. However, there are new backgrounds to contend with. At large energies, weak processes such as $\ell \mathrm{q} \rightarrow \nu \mathrm{q}$ should come in which produce outgoing neutrinos which would carry off undetected energy. One would have to apply a cut on the data specifying exactly one lepton in the final state and be sure that this lepton originated from the electron vertex. As in the hadron collider, it will take a substantial statistical analysis to uncover evidence for gluinos. Similar comments also apply to associated production of gluinos and scalar-quarks.

In $\mathrm{e}^{+} \mathrm{e}^{-}$collisions, the production of gluons is a well-known experimental fact; hence, we may investigate the rate for gluino production. Here we have two choices: either in the continuum or on a quarkonium or $\mathrm{Z}^{0}$ resonance. The advantage of the latter is the increased luminosity. In addition, on a quarkonium resonance, almost the entire branching ratio is into three gluons. In the continuum, gluino production is much more difficult. Consider the process $\mathrm{e}^{+} \mathrm{e}^{-} \rightarrow \mathrm{q} \overline{\mathrm{q}} \tilde{\mathrm{g}} \tilde{\mathrm{g}}[3.45,3.61]$ which occurs when a bremsstrahlung gluon converts to a $\tilde{g} \tilde{g}$ pair. This process is of order $\alpha_{\mathrm{s}}^{2}$ and hence can only be discernible in a regime where four-jet events are visible. A very careful jet analysis along with ways of detecting small amounts of missing momentum will be required. In general, this seems difficult, especially if the gluinos are heavy. Once the $\mathrm{e}^{+} \mathrm{e}^{-}$center-of-mass energy is large enough, it will be possible to produce $\mathrm{e}^{+} \mathrm{e}^{-} \rightarrow \overline{\mathrm{q}} \tilde{\mathrm{q}} \tilde{\mathrm{g}}$ leading to supersymmetric three-jet events. Complicated jet analysis will be needed for picking these events out of background as discussed in refs. [3.62-3.63]. Such effects can also show up in QCD corrections to the total $\mathrm{e}^{+} \mathrm{e}^{-}$hadronic cross section. These effects are also quite small as illustrated in ref. [3.64].

In quarkonium physics, there are a number of possible signals. We concentrate on the ${ }^{3} S_{1}$ state $(\psi, \gamma, \ldots)$ as it is here where the luminosity is greatest. In fig. 31, we depict three possible ways of 

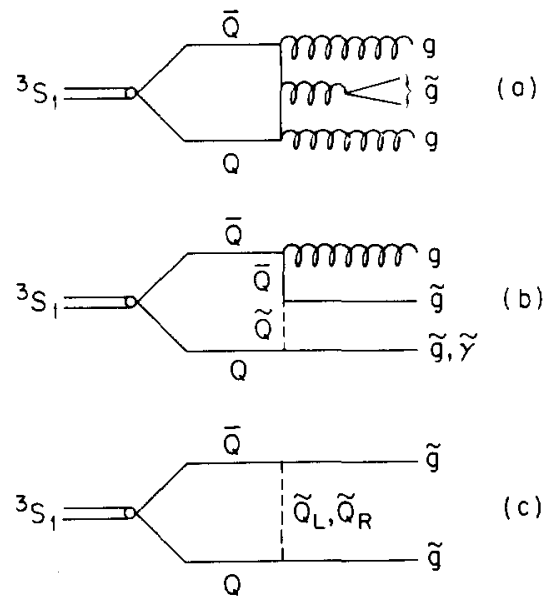

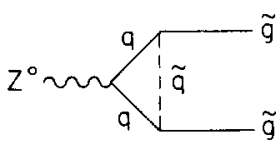

(0)

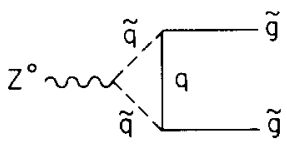

(b)

Fig. 31. The decay of the ${ }^{3} S_{1}$ quarkonium state (e.g., $\psi, Y, \ldots$ ) into gluinos and photinos.

Fig. 32. Graphs for $Z^{0} \rightarrow \tilde{g} \tilde{g}$.

producing gluinos. These will be discussed quantitatively in section 5.2. Here we briefly comment on the three mechanisms. (QD̄) $\rightarrow$ ggg̃ $\tilde{g}$ [3.45] has much the same problem as in the continuum case: It has a

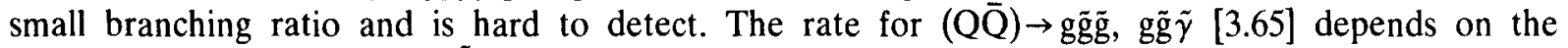
unknown scalar-quark mass $\tilde{M}_{\mathrm{Q}}$ and hence could be rare. However, the signal for gg $\tilde{\gamma}$ is likely to be good since the photino is assumed to escape the detector. Hence, nearly $50 \%$ of the energy of this event would be missing-it would appear as a three-jet event with one jet invisible. Finally, the reaction $(\overline{\mathrm{QQ}}) \rightarrow \tilde{\mathrm{g}} \tilde{\mathrm{g}}[3.66-3.68]$ with the $\overline{\mathrm{QQ}}$ in a ${ }^{3} \mathrm{~S}_{1}\left(1^{--}\right)$state violates $C$-invariance but can occur if $\tilde{M}_{\mathrm{QL}} \neq \tilde{M}_{\mathrm{QR}}$ (see the discussion in section A on the $C$-violating decay $\tilde{\mathrm{g}} \rightarrow \mathrm{g} \tilde{\gamma}$ ). Because there are only two strong interaction vertices as opposed to the usual three, it is not inconceivable that this could be a dominant decay of the ${ }^{3} S_{1}$ state (especially for $\vec{t} \vec{t}[3.68]$ ).

In $Z^{0}$ physics the decay $Z^{0} \rightarrow \tilde{g} \tilde{g}$ occurs at one loop, as in fig. 32 . The branching ratio depends on $m_{\mathrm{t}}$, $\tilde{M}_{\mathrm{q}}$ and $\tilde{M}_{\mathrm{g}}$. For typical values of these parameters one gets $\operatorname{BR}\left(Z^{0} \rightarrow \tilde{g} \tilde{g}\right) \geq 10^{-5}$ which is in principle detectable at a $Z^{0}$ factory $[3.69,3.70]$. One expects that gluinos will not be discovered this way, but this process could serve as a check on the theory once the gluino is found.

\section{(E) Gluinonium}

Gluinonium is a bound state of two (Majorana fermion) gluinos. Their properties are somewhat like quarkonium but there are a number of key differences [3.54,3.111,3.112]. First, because gluinos are color octets, we expect binding of $\tilde{g} \tilde{g}$ in other colored channels in addition to the color singlet channel. Consider the simple model where the color force is proportional to the product of two color generators $T^{(i)}, i=1,2$. The force is then attractive or repulsive depending on the sign of $T^{(1)} \cdot T^{(2)}$. The representation of the $\tilde{g} \tilde{g}$ determines the Casimir operator $\left(T^{(1)}+T^{(2)}\right)^{2}$, hence

$$
T^{(1)} \cdot T^{(2)}=\frac{1}{2}\left[\left(T^{(1)}+T^{(2)}\right)^{2}-\left(T^{(1)}\right)^{2}-\left(T^{(2)}\right)^{2}\right]
$$

Using the results of table 6 , it is easy to see that color singlet and color octet $\tilde{g} \tilde{g}$ states are expected to bind. Note that there are two possible color octet states depending on whether the two color octet gluinos are combined symmetrically or antisymmetrically. We shall denote these three gluinonium states by $\tilde{G}_{1}, \tilde{G}_{8}^{\mathrm{S}}$ and $\tilde{G}_{8}^{A}$. 
Table 6

Color coefficient for the gluino-gluino color interaction. [The potential between two gluinos in an overall color representation $R$ is attractive (repulsive) if the sign of $T_{1}^{\mathrm{a}} \cdot T_{2}^{\mathrm{a}}$ is negative (positive).]

\begin{tabular}{llll}
\hline$R$ & $\left(T_{1}^{\mathrm{a}}+T_{2}^{a}\right)^{2}$ & $T_{1}^{\mathrm{a}} \cdot T_{2}^{\mathrm{a}}$ & Comment \\
\hline 1 & 0 & -3 & bound \\
${ }^{8} \mathrm{~S}$ & 3 & $-3 / 2$ & bound \\
${ }^{8} \mathrm{~A}$ & 3 & $-3 / 2$ & bound \\
$\frac{10}{10}$ & 6 & 0 & threshold \\
27 & 6 & 0 & threshold \\
\hline
\end{tabular}

Table 7

The gluinonium spectrum ( $\tilde{g} \tilde{g}$ bound states). [We list the lowest lying states for $l \leq 2$. The spectrum of states depends whether the gluinonium state is in a symmetric $\left(\tilde{\mathrm{G}}_{1}, \tilde{\mathrm{G}}_{8}^{\mathrm{S}}\right)$ or an antisymmetric $\left(\tilde{\mathrm{G}}_{8}^{\mathrm{A}}\right)$ color representation.]

\begin{tabular}{|c|c|c|c|c|}
\hline$l$ & $S$ & $\tilde{\mathbf{G}}_{1}, \tilde{\mathrm{G}}_{\mathbf{8}}^{\mathrm{S}}$ & $\tilde{\mathrm{G}}_{8}^{\mathrm{A}}$ & Spectroscopic notation \\
\hline 0 & 0 & $0^{-+}$ & & ${ }^{1} \mathrm{~S}_{0}$ \\
\hline 0 & 1 & & $1^{-+}$ & ${ }^{3} S_{1}$ \\
\hline 1 & 0 & $\left(0^{++}\right.$ & $1^{++}$ & $\begin{array}{l}{ }^{1} \mathrm{P}_{1} \\
{ }^{3} \mathrm{P}_{0}\end{array}$ \\
\hline 1 & 1 & $\left\{\begin{array}{l}1^{++} \\
2^{++}\end{array}\right.$ & & $\begin{array}{l}{ }^{3} \mathrm{P}_{1} \\
{ }^{3} \mathrm{P}_{2}\end{array}$ \\
\hline 2 & 0 & $2^{-+}$ & $1^{-+}$ & $\begin{array}{l}{ }^{1} \mathrm{D}_{2} \\
{ }^{3} \mathrm{D}_{1}\end{array}$ \\
\hline 2 & 1 & & $\begin{array}{l}2^{-+} \\
3^{-+}\end{array}$ & $\begin{array}{l}{ }^{2} \mathrm{D}_{2} \\
{ }^{3} \mathrm{D}_{3}\end{array}$ \\
\hline
\end{tabular}

The quantum numbers of the color singlet gluinonium $\tilde{\mathrm{G}}_{1}$ are computed as follows. First, because gluinos are Majorana particles, the parity of each gluino is purely imaginary. Hence, the parity of an $l=0 \tilde{g} \tilde{g}$ state is -1 . (Note that the parity of an $l=0 q \bar{q}$ state is also -1 but for different reasons.) It follows that just as for $\mathrm{q} \overline{\mathrm{q}}$ the parity $(P)$ and charge conjugation $(C)$ of a $\tilde{g} \tilde{g}$ state with orbital angular momentum $l$ and total spin $s(s=0$ or 1$)$ are $P=(-1)^{l+1}$ and $C=(-1)^{l+s}$. However, gluinos are $C$-eigenstates (with $C=-1$ ) so that a $\tilde{g} \tilde{g}$ state must necessarily be $C=1$. Hence, $1=(-1)^{l+s}$ which requires $l+s$ to be even. ${ }^{*}$ A list of the low lying states is given in table 7 . Note in particular that there is no state analogous to the $\psi$ (i.e., $\left.{ }^{3} \mathrm{~S}_{1}\right)$. The most likely state which will be produced is the ${ }^{1} \mathrm{~S}_{0}\left(0^{-+}\right)$state analogous to the $\eta_{\mathrm{c}}$ which we denote by $\tilde{\eta}_{\mathrm{g}}$.

The above arguments about the quantum numbers are also valid for $\tilde{G}_{8}^{\mathrm{s}}$. However, for $\tilde{G}_{8}^{\mathrm{A}}$ there is one important change. Because the color wavefunction is anti-symmetric, using the Pauli principle for identical fermions now implies that $C=(-1)^{l+s+1}$ (the parity is unchanged). Hence, $l+s$ must now be odd and the quantum numbers of low lying states are changed. For massive gluinos, one expects both color octet and color singlet gluinonia to be produced in hadronic colliders. Although the color octet states cannot be asymptotic states, one may regard them as such for the purposes of a cross-section calculation based on perturbative QCD.

The properties of the color-singlet $\tilde{\eta}_{\mathrm{g}}$ can be obtained by standard charmonium techniques $[3.73,3.74]$. Essentially, only the color factors differ (due to the color octet gluinos). We simply quote the results of refs. [3.54,3.111]. The color factor for $\tilde{\eta}_{\mathrm{g}} \rightarrow \mathrm{gg}$ is a factor of $27 / 2$ larger than that for $\eta_{\mathrm{c}} \rightarrow \mathrm{gg}$; this leads to

$$
\Gamma\left(\tilde{\eta}_{\mathrm{g}} \rightarrow \mathrm{gg}\right)=\left(36 \alpha_{\mathrm{s}}^{2} / \tilde{M}^{2}\right) \frac{1}{2}|\tilde{R}(0)|^{2}
$$

where $\tilde{M}$ is the $\tilde{\eta}_{\mathrm{g}}$ mass $\left(\tilde{M}=2 \tilde{M}_{\mathrm{g}}\right)$ and extra factor of $1 / 2$ must be inserted to account for identical gluinos in the bound state. A similar calculation for the color octet $0^{-+}$state $\tilde{\eta}_{\mathbf{g}}^{(8)}$ yields

\footnotetext{
${ }^{*}$ We disagree with the results of Zuk et al. [3.71] who claim that $l+s$ is odd. A discussion of their error is given in refs. [3.54, 3.111, 3.112]. We note that the above results are consistent with those of Kayser [3.72] who showed that a Majorana particle can have only one electromagnetic form factor (corresponding here to the existence of only one spin 1 color singlet $\bar{g} \tilde{g}$ state; see table 7).
} 


$$
\Gamma_{8}\left(\tilde{\eta}_{\mathrm{g}}^{(8)} \rightarrow \mathrm{gg}\right)=\left(72 \alpha_{\mathrm{s}}^{2} / \tilde{M}^{2}\right) \frac{1}{2}\left|\tilde{R}_{\mathrm{s}}^{(8)}(0)\right|^{2}
$$

For large mass gluinos (e.g. $\tilde{M}_{\mathrm{g}}=\tilde{M} / 2 \approx 50 \mathrm{GeV}$ ), we may evaluate the wavefunctions in the Coulombic approximation: $\left|\tilde{R}_{\mathrm{s}}(0)\right|^{2}=27 \alpha_{\mathrm{s}}^{3} \tilde{M}^{3} / 16$ and $\left|\tilde{R}_{\mathrm{s}}^{(8)}(0)\right|^{2}=\frac{1}{8}\left|\tilde{R}_{\mathrm{s}}(0)\right|^{2}$. This gives $\Gamma\left(\tilde{\eta}_{\mathrm{g}} \rightarrow \mathrm{gg}\right)=\left(3 \alpha_{\mathrm{s}}\right)^{5} \tilde{M} / 8$ and $\Gamma_{8} / \Gamma=1 / 4$. Then

$$
\Gamma\left(\tilde{\eta}_{\mathrm{g}} \rightarrow \mathrm{gg}\right) / \tilde{M}=\left(3 \alpha_{\mathrm{s}}\right)^{5} / 8 \sim 4 \times 10^{-4} .
$$

The same color enhancement factor of $27 / 2$ as above leads us to predict that $\tilde{\eta}_{\mathrm{g}}$ will be produced an order of magnitude more copiously than an $\eta_{c}$-like object of the same mass in hadronic colliders. Unfortunately, it is extremely difficult to detect the $\tilde{\eta}_{\mathrm{g}}$ once it is produced.

In radiative decays of quarkonium, the production of $\tilde{\eta}_{\mathrm{g}}$ is again enhanced over $\eta_{\mathrm{c}}$-like objects due to color. Once again, the enhancement factor is $27 / 2$ [3.54,3.111,3.112]. Thus, the nonobservation of $\psi \rightarrow \gamma \tilde{\eta}_{\mathrm{g}}$ presumably rules out gluinos of masses below $1 \mathrm{GeV}$. In $Y$ decay, we expect BR $\left(Y \rightarrow \gamma \tilde{\eta}_{\mathrm{g}}\right) \sim$ $(1-3) \times 10^{-4}$ which could be observable in the coming years at the $Y$ factories. Unfortunately, a similar branching ratio rapidly decreases as the quarkonium mass increases so this technique is useless for gluino masses above $5 \mathrm{GeV}$.

One may also look for gluinonium production at hadron colliders [3.54]. The idea here is based on the fact that at very large $\sqrt{s}$, we expect large- $p_{\mathrm{T}}$ events to be dominated by gluon-gluon scattering. But the $\tilde{\eta}_{\mathrm{g}}$ (and nearly all the other gluinonium states) couple strongly to the two-gluon channel as shown by eq. (3.30). We can therefore search for resonant two-gluon scattering at large $p_{\mathrm{T}}$. Namely, compute the invariant mass distribution of a sample of two-jet events chosen with appropriate cuts [3.54] and search for a peak in the distribution. To succeed, one requires good resolution in the invariant mass (optimistically of order $10 \mathrm{GeV}$ ) and at least 1000 events per bin (after cuts). Goldman and Haber [3.54] find that one can expect a signal-to-background ratio of order $1-10 \%$, consistent with the requirements just stated. More detailed computations are required to check if this method is indeed practical under realistic conditions.

Gluinonium, if discovered, could provide the most accurate mass measurement of the gluino. It is worth pondering more on ways to uncover its presence.

\subsection{Charginos}

The $\mathrm{W}^{ \pm}$bosons, and the charged Higgs bosons $\mathrm{H}_{1}^{+}, \mathrm{H}_{2}^{-}$from the two weak doublets needed in a minimal supersymmetric theory, have supersymmetric partners $\tilde{\mathrm{W}}^{ \pm}, \tilde{\mathrm{H}}_{1}^{+}, \tilde{\mathrm{H}}_{2}^{-}$. These are weak eigenstates $-\tilde{\mathrm{W}}^{ \pm}$are in an $\mathrm{SU}(2)$ triplet, and $\tilde{\mathrm{H}}^{ \pm}$are in $\mathrm{SU}(2)$ doublets. A term $g_{2} \tilde{W}^{+} \tilde{H}^{-} H^{0}$ is allowed by $\mathrm{SU}(2) \times \mathrm{U}(1)$, and when $H^{0}$ gets a vacuum expectation value $v$ an off-diagonal mass term is generated in the $\tilde{W}^{+} \tilde{H}^{-}$mass matrix.

\section{(A) Spectrum}

Supersymmetry breaking could generate diagonal $\tilde{W}^{+} \tilde{W}^{-}$or $\tilde{H}^{+} \tilde{H}^{-}$masses as well, so the full mass matrix is, for the left-handed charginos,*

\footnotetext{
* The notation here is slightly different from that used in refs. [3.75-3.77]. A complete and consistent treatment of the mixing and detailed references are given in appendix C.2.
} 


$$
\left(\tilde{W}^{-} \tilde{H}_{1}^{-}\right)\left(\begin{array}{cc}
M & (1 / \sqrt{2}) g v_{2} \\
(1 / \sqrt{2}) g v_{1} & \mu
\end{array}\right)\left(\begin{array}{c}
\tilde{W}^{+} \\
\tilde{H}_{2}^{+}
\end{array}\right) .
$$

If $M=\mu=0$ then this matrix is diagonalized by adding and subtracting the weak eigenstates, so there are two Dirac spinors

$$
\begin{aligned}
& \tilde{\chi}_{1}^{+}=\left(\begin{array}{c}
\tilde{W}_{\mathrm{L}}^{+} \\
\tilde{H}_{\mathrm{IR}}^{*}
\end{array}\right), \\
& \tilde{\chi}_{2}^{+}=\left(\begin{array}{c}
\tilde{H}_{2 \mathrm{~L}}^{+} \\
\tilde{W}_{\mathrm{R}}^{-*}
\end{array}\right),
\end{aligned}
$$

with masses $g v_{1} / \sqrt{2}$ and $g v_{2} / \sqrt{2}$ respectively. If $M$ or $\mu$ is zero, or $M \mu \ll g^{2} v_{1} v_{2} / 2$, then the product of the eigenvalues $\tilde{M}_{1}, \tilde{M}_{2}$ is $g^{2} v_{1} v_{2} / 2$. Then

$$
m_{\mathrm{w}}^{2}-m_{1} m_{2}=g^{2}\left(v_{1}-v_{2}\right)^{2} / 4 \geq 0
$$

so one of the eigenstates $\tilde{M}_{1}$ or $\tilde{M}_{2}$ must have mass less than $m_{\mathrm{w}}$ [3.78-3.80,3.75]. However, it can happen that $M$ and $\mu$ are large, in which case there is no need for one chargino to be lighter than $m_{\mathrm{w}}$.

The situation with the chargino states is a little complicated, but it is somewhat familiar from the way ordinary fermions are treated in SU(2). The weak eigenstates for an electron are $\mathrm{e}_{\mathrm{L}}^{-}$(an $\mathrm{SU}(2)$ doublet) and $e_{R}^{-}$(an $S U(2)$ singlet). The mass eigenstate is a Dirac fermion,

$$
e^{-}=\left(\begin{array}{c}
e_{\mathrm{L}}^{-} \\
e_{\mathrm{R}}^{+*}
\end{array}\right)
$$

We speak of producing left-handed electrons by weak interactions. Similarly, we will produce the leftor right-handed parts of $\tilde{\chi}_{1,2}^{ \pm}$depending on the interactions.

For general $M, \mu, v_{2} / v_{1}$, the eigenstates are*

$$
\tilde{\chi}_{1}^{+}=\left(\begin{array}{c}
C_{11} \tilde{W}_{\mathrm{L}}^{+}+C_{12} \tilde{H}_{2 \mathrm{~L}}^{+} \\
C_{21} \tilde{W}_{\mathbf{R}}^{-*}+C_{22} \tilde{H}_{1 \mathbf{R}}^{-*}
\end{array}\right)
$$

with $\tilde{\chi}_{2}^{+}$given by the orthogonal combinations. By convention, we choose $\tilde{\chi}_{1}$ to be heavier than $\chi_{2}$ (see eqs. (C15), (C18) in Appendix C). When we look at charginos produced in various ways, different parts of the mass eigenstates are projected out. The photon couples to the charge $Q$, so it produces the mass eigenstates. The $Z^{0}$ couples to $T_{3}-Q \sin ^{2} \theta_{\mathrm{w}}$, so it treats $\tilde{\mathrm{W}}^{ \pm}$(with $T_{3}= \pm 1$ ) differently from $\tilde{\mathrm{H}}$ (with $T_{3}= \pm 1 / 2$ ). In $\mathrm{W}^{+} \rightarrow \tilde{\chi}^{+} \tilde{\chi}^{0}$, if $\tilde{\chi}^{0}$ is a weak isospin singlet, then the coupling is to the wino part of $\tilde{\chi}^{+}$since it has the same weak isospin as the $\mathrm{W}^{+}$; thus if the lightest chargino were largely made of $\tilde{\mathrm{H}}_{1}^{+}$, the decay $\mathrm{W}^{+} \rightarrow \tilde{\chi}^{+} \tilde{\chi}^{0}$ would be very suppressed. That would happen for $M \gg g v / \sqrt{2}$. The mixing is discussed in more detail in appendix C.2. Examples of chargino eigenvectors and mass values for a range of parameters are displayed in table 9 in section 3.6.

(B) Decays

Charginos have many allowed decay modes, as shown in fig. 33. The relative rates depend on the

* In terms of the notation used in appendix $\mathrm{C}$, we have $C_{1 j}=V_{1 j}$ and $C_{2 j}=U_{1 j}^{*}$ for $j=1,2$. 


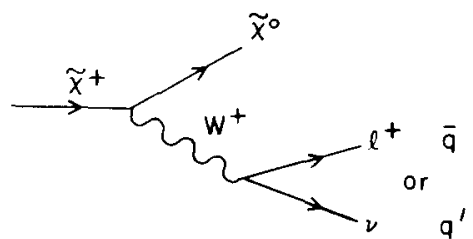

(0)

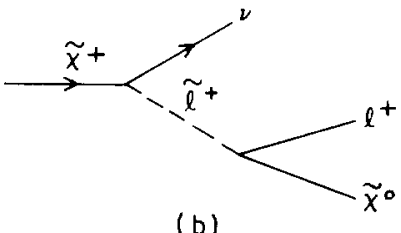

(b)

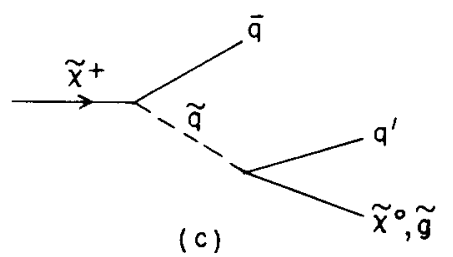

Fig. 33. Graphs for chargino $\left(\tilde{\chi}^{+}\right)$decay. The notation here is: $q$ and $\mathrm{q}^{\prime}$ are quarks of different charge and $\tilde{\chi}^{0}$ is any neutralino which is kinematically allowed.

superpartner masses, and on the mixing coefficients that determine the mass eigenstates (the mixing coefficients are themselves determined by the mass matrix). In the case of charginos, this uncertainty about which decays dominate considerably complicates the experimental search, and may effectively prevent setting general limits.

We note some possibilities. If $\tilde{\mathrm{q}}$ is not too heavy, the modes of fig. 33(c) may dominate since they are enhanced by a color factor. If $\tilde{M}_{\mathrm{g}}<\tilde{M}_{\chi^{+}}$, then $\tilde{\chi}^{+} \rightarrow \mathrm{q} \overline{\mathrm{q}} \tilde{\mathrm{g}} \rightarrow \mathrm{q} \overline{\mathrm{q}} \mathrm{q} \overline{\mathrm{q}} \tilde{\gamma}$, giving a difficult signature for $\tilde{\chi}^{+}$. If $\tilde{M}_{\mathrm{g}}>\tilde{M}_{\chi^{+}}$, only two jets and missing momentum appear, and that is easier to observe. Alternatively, if scalar-leptons are much lighter than scalar-quarks and W's, fig. 33(b) will dominate and the signature is a single charged lepton, with a typical three-body spectrum. Thus the possible final states are

$$
\begin{aligned}
\tilde{\chi}^{+} & \rightarrow \mathrm{q} \overline{\mathrm{q}} \mathrm{q} \overline{\mathrm{q}} \\
& \rightarrow \mathrm{q} \overline{\mathrm{q}} \\
& \rightarrow \ell^{+} \\
& \rightarrow \ell^{+} \ell^{+} \ell^{-} \\
& \rightarrow \ell^{+} \mathrm{q} \overline{\mathrm{q}}
\end{aligned}
$$

accompanied by missing momentum in each case. The last two arise from fig. 33(a) where $\tilde{\chi}^{0}=\tilde{\chi}_{2}^{0}$, and $\tilde{\chi}_{2}^{0}$ decays as discussed in section 3.6 (which discusses the neutralinos $\tilde{\chi}^{0}$ in detail).

The chargino decays are typical three-body weak decays, going as $\left(g^{2} / \tilde{M}^{2}\right)^{2} \tilde{M}_{\chi}^{5}$ or about as $G_{\mathrm{F}}^{2} \tilde{M}_{\chi}^{5}$, so the lifetimes are expected to be too short for any chargino to be directly observed.

In chapter 4 we specifically discuss the process $\mathrm{W}^{ \pm} \rightarrow \tilde{\chi}^{+} \tilde{\chi}^{0}$. 


\section{(C) Production}

Charginos can be produced in $\mathrm{e}^{+} \mathrm{e}^{-}$collisions $[3.75-3.77,3.81,3.91]$ as in fig. 34 , in hadron collisions $[3.75,3.77,3.82,3.83]$ as in fig. 35 and via $Z^{0}$ or $W^{ \pm}$decays $[3.78-3.80 ; 3.84-3.88]$. Of course the production in $\mathrm{Z}^{0}$ or $\mathrm{W}^{ \pm}$decays is a special case of lepton or hadron colliders, but it is convenient to arrange the discussion in those terms.

(a) $e^{+} e^{-}$colliders

A number of calculations have been made of $[3.75-3.77,3.81]$

$$
\mathrm{e}^{+} \mathrm{e}^{-} \rightarrow \tilde{\chi}_{i}^{+} \tilde{\chi}_{j}^{-}
$$

We will assume that only the lightest chargino is relevant for $\sqrt{s} \leq m_{\mathrm{z}}$.

At PETRA, PEP energies the dominant diagrams, from fig. 34, are the $\gamma$ and $\tilde{\nu}$ exchanges. The $\gamma$ contribution is precisely known apart from the phase space effects of $\tilde{M}_{\chi}$, but the $\tilde{\nu}$ exchange depends on the coefficients which determine the mass eigenstate and on the $\tilde{\nu}$ mass. If $\tilde{M}_{\nu}$ is small, this contribution can be quite large.

In any case, the $\gamma$ contribution gives a full unit of $R$, so at any $\sqrt{s}$ enough $\tilde{\chi}^{ \pm}$are produced to discover them if they are present. The problem in discovering them, or in putting limits on their mass if a signal is not found, is that the lack of knowledge of the masses prevents us from knowing the decay branching ratios, so searches must be conducted under a range of assumptions about decays (e.g., which contributions of fig. 33 dominate). Further, the final decay products $\left(\tilde{\chi}^{0}\right.$, e.g., the photino) can be heavy and carry off mass as well as momentum.

The various contributions can be separated by studies of the size of the cross section and the angular distribution. The $\gamma$ contribution gives a symmetric angular distribution, while the $\tilde{\nu}$ contribution has the $\tilde{\chi}^{+}$in the direction of $\mathrm{e}^{+}$if $\tilde{M}_{\nu}$ is not too large. When the $\mathrm{Z}^{0}$ contribution is significant there can be a large forward-backward asymmetry, depending on the form of the mass eigenstates. This has been

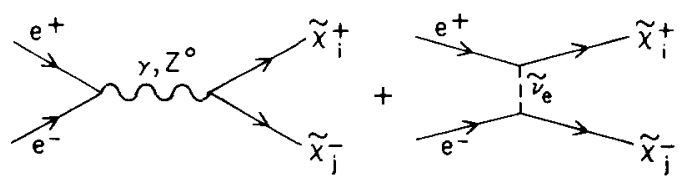

Fig. 34. Graphs for $\mathrm{c}^{+} \mathrm{e}^{-} \rightarrow \tilde{\chi}_{i}^{+} \tilde{\chi}_{j}^{-}(i, j=1,2)$.

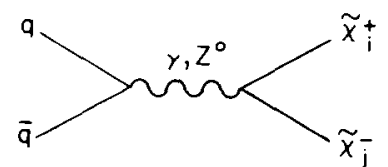

(a)

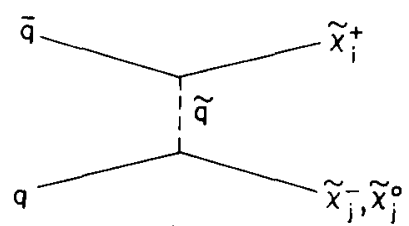

(b)

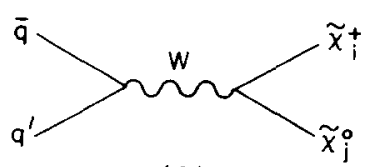

(c)

Fig. 35. Chargino production via the Drell-Yan mechanism. (See caption to fig. 33 for notation.) 
studied in refs. $[3.75,3.76]$. Figure 36 shows the asymmetry calculated for winos, higgsinos, wiggsinos, and normal leptons, including the effects of the final fermion mass - it is clear that if $\tilde{M}_{\chi^{ \pm}}$is not too large, one can nicely separate the various mixings and distinguish $\tilde{\chi}^{ \pm}$from a heavy lepton $\mathrm{L}^{ \pm}$.

The couplings to the $Z^{0}$ are quite large. Table 8 gives $g_{v}, g_{a}$ and $g_{v}^{2}+g_{a}^{2}$ for various mixings (and the contribution of a normal lepton for comparison). The corresponding asymmetries may then be computed. The differential cross section is given by

$$
\mathrm{d} \sigma / \mathrm{d} \Omega=\left(\alpha^{2} / 4 s\right)\left[A_{1}\left(1+\cos ^{2} \theta\right)+A_{2} \cos \theta\right] .
$$

Explicit expressions for $A_{1}$ and $A_{2}$ in terms of $g_{v}$ and $g_{a}$ are given in ref. [3.75]. The forward-backward asymmetry is then defined by

$$
A_{\mathrm{FB}}=\left[\frac{\mathrm{d} \sigma}{\mathrm{d} \Omega}(\theta)-\frac{\mathrm{d} \sigma}{\mathrm{d} \Omega}(\pi-\theta)\right] /\left[\frac{\mathrm{d} \sigma}{\mathrm{d} \Omega}(\theta)+\frac{\mathrm{d} \sigma}{\mathrm{d} \Omega}(\pi-\theta)\right]=\frac{\cos \theta}{1+\cos ^{2} \theta} \frac{A_{2}}{A_{1}} .
$$

We show the value of $A_{2} / A_{1}$ in fig. 36 for the various cases given in table 8 .

It is likely that $\tilde{\chi}^{+} \tilde{\chi}^{-}$production will show up in $R$, even if specific decays are hard to observe. Such a

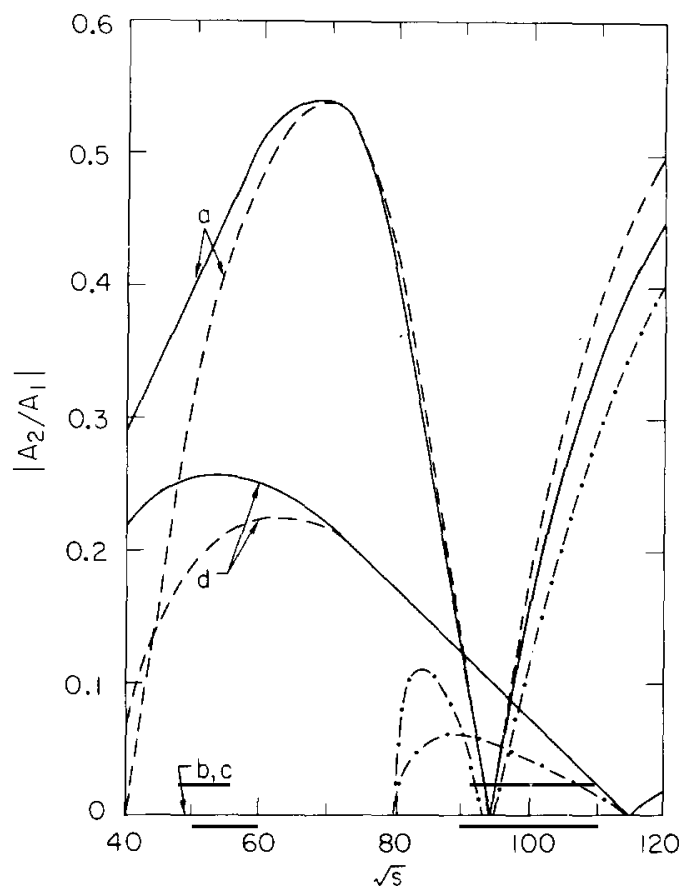

Table 8

The neutral current couplings of various mass eigenstates are given for purely diagonal $\left(\overline{\mathrm{h}}^{ \pm}\right.$and $\left.\tilde{\mathrm{w}}^{ \pm}\right)$and off-diagonal $\tilde{\omega}_{1}^{ \pm}$and $\tilde{\omega}_{2}^{ \pm}$) mass matrices and compared to those for a normal lepton. Cases (b) and (c) have no forward-backward asymmetry, case (a) has the asymmetry of the normal lepton and cases (d) and (e) have large forward-backward asymmetries of opposite sign. The forward-backward asymmetry in these five cases is shown in fig. 36

\begin{tabular}{llll}
\hline Mass eigenstate & $g_{\mathrm{v}}$ & $g_{\mathrm{a}}$ & $g_{\mathrm{v}}^{2}+g_{\mathrm{a}}^{2}$ \\
\hline (a) Normal lepton, $\ell^{-}$ & $-\frac{1}{2}+2 \sin ^{2} \theta_{\mathrm{w}}$ & $-\frac{1}{2}$ & 0.26 \\
(b) $\tilde{\mathrm{h}}^{ \pm}$ & $-1+2 \sin ^{2} \theta_{\mathrm{w}}$ & 0 & 0.31 \\
(c) $\tilde{\mathrm{w}}^{ \pm}$ & $-2+2 \sin ^{2} \theta_{\mathrm{w}}$ & 0 & 2.43 \\
(d) $\tilde{\omega}_{1}^{ \pm}$ & $-\frac{3}{2}+2 \sin ^{2} \theta_{\mathrm{w}}$ & $\frac{1}{2}$ & 1.37 \\
(e) $\tilde{\omega}_{2}^{ \pm}$ & $-\frac{3}{2}+2 \sin ^{2} \theta_{\mathrm{w}}$ & $-\frac{1}{2}$ & 1.37 \\
\hline
\end{tabular}

Fig. 36. Asymmetry in $\mathrm{e}^{+} \mathrm{e}^{-} \rightarrow \bar{\chi}^{+} \tilde{\chi}^{-}$due to $\mathrm{Z}^{0}$ exchange. $A_{\mathrm{FB}}$ from eq. (3.41) is shown, integrated over $-0.8 \leq \cos \theta \leq 0.8$, vs. $\sqrt{ } s$ for three values of the outgoing fermion mass: $\bar{M}=0$ (solid line), $\tilde{M}=20 \mathrm{GeV}$ (dashed line) and $\bar{M}=40 \mathrm{GeV}$ (dashed-dot line). The labels (a)-(d) are defined in table 8 . Note that cases (b) and (c) have no asymmetry. Case (e) is not drawn as it is the negative of case (d). For convenience, only the absolute value of the asymmetry is plotted; the curves, in fact, have continuous derivatives. This figure is due to J.-M. Frère. 
calculation has been presented for a specific model by Dicus et al. [3.81] (they compute a theoretical $R$, uncorrected for experimental cuts - some care is needed since considerable missing energy can be present for some decays). One concludes that $\mathrm{e}^{+} \mathrm{e}^{-}$colliders are very good places to study $\tilde{\chi}^{ \pm}$; if they are found it will be easy to determine the form of the mass eigenstates and the mass matrix.

\section{(b) Hadron colliders}

By fig. 35 one can produce $\tilde{\chi}^{+} \tilde{\chi}^{-}$at a hadron collider; since both decay, this channel does not have a good signature and we will not discuss it in detail. Figures 35(b), (c) provide significant cross sections for $\tilde{\chi}^{ \pm} \tilde{\chi}^{0}$ production, with the possibility of good signatures. A number of authors $[3.78-3.80 ; 3.83-3.88]$ have studied $\mathrm{W}^{ \pm} \rightarrow \tilde{\chi}^{ \pm} \tilde{\chi}^{0}$, and in ref. [3.77] the full calculation of figs. 35(b), (c) is done. The complete cross section will be even larger when gluon emission diagrams are added.

In principle, one can produce $\tilde{\chi}_{1,2}^{+}$and $\tilde{\chi}_{1,2,3,4}^{0}$, giving eight channels. In practice it is likely that $\tilde{\chi}_{2}^{+}$is significantly lighter than $\tilde{\chi}_{1}^{+}$, and that $\tilde{\chi}_{1,2}^{0}$ are considerably lighter than $\tilde{\chi}_{3,4}^{0}$, so that perhaps only two channels are probable, $\tilde{\chi}_{2}^{+} \tilde{\chi}_{1}^{0}$ and $\tilde{\chi}_{2}^{+} \tilde{\chi}_{2}^{0}$. Production will be mainly through the $\mathrm{W}^{ \pm}$channel when it is kinematically allowed.

\section{(D) Present limits}

Since a chargino is a spin $1 / 2$ charged particle it is like a charged lepton. Of course it can have different interactions so it can be produced differently from a charged lepton, and it can have different decays.

That a chargino is like a charged lepton originally led people to assume that the same limits would apply - i.e., that charginos of mass less than half the largest energy available at $\mathrm{e}^{+} \mathrm{e}^{-}$machines did not exist or they would have been seen (either in $\mathrm{R}$ or as new e $\mu$ events). However [3.75], the chargino decay modes could be sufficiently different from those of a standard heavy lepton to cause those limits to not exist. The differences are two-fold. First, if $\tilde{q}$ or $\tilde{\ell}$ is significantly lighter than the $\mathrm{W}^{ \pm}$, fig. 33(b) or (c) could dominate the decay. If fig. 33(c) dominates the decay giving a charged lepton is suppressed, and if fig. 33(b) dominates the decay giving $q \bar{q}$ is suppressed so there would be little contribution to $\mathrm{R}$. Thus to exclude a state one must look both at $\mathrm{e} \mu$ events (or excess $\mu \mu$ or ee events) and at R. Second, in all the decays except fig. 33(c) with gluinos lighter than charginos, the escaping neutralino $\tilde{\chi}^{0}-$ it is often called a photino, but need not be - can be rather massive, and thus carry off considerable energy. Since experiments have in the past generally not included in the definition of $\mathrm{R}$ events with more than $40-50 \%$ missing energy, chargino events might have been missed. Alternatively, since charged leptons with energies less than $2.5-3 \mathrm{GeV}$ have generally not been accepted, chargino pair events giving e $\mu$ pairs might not have been included in the data sample.

Thus the best one can do if no positive signal is obtained is to present limits as a function of the relevant unknown supersymmetric masses, and carefully state the branching ratio assumptions which went into the analysis.

For $\tilde{M}_{x^{0}} \approx 0$ the heavy lepton limits do apply (except possibly in the case where fig. 33(c) dominates and the missing energy is enough to lose some events in R). As $\tilde{M}_{x^{0}}$ increases, somewhere around $\tilde{M}_{\chi^{0}} \approx 3 \mathrm{GeV}$ the limits disappear, except that very recently the MARK-J group has presented an analysis [3.89] based on the assumption that the branching ratios implied by fig. 33(a) hold (either (a) dominates or (b), (c) occur in the same ratio as for (a)) and that $\tilde{M}_{\chi^{0}} \ll \tilde{M}_{x^{+}}$. They find no candidate chargino events for $\tilde{M}_{x^{+}} \leqslant 25 \mathrm{GeV}$. Hopefully, in the next months the search for charginos events will be carried out in its full generality. 


\subsection{Neutralinos}

The spin 1/2 partners of gauge bosons and Higgs bosons (apart from the gluino which we discuss separately in section 3.4) are perhaps the most promising of the supersymmetry partners for detection and study, because they may give the cleanest experimental signatures. They can be produced at any high luminosity collider, $\mathrm{e}^{+} \mathrm{e}^{-}$or hadron, and they tend to be light in models so that their absence could be a definitive constraint on supersymmetry models. To discuss their properties one must first examine the full set of such particles since the weak eigenstates mix with each other to produce the mass eigenstates that could be detected experimentally. We have given the nomenclature of these particles in chapter 2.

\section{(A) Spectrum}

The minimal set of such particles arises as partners of $\mathrm{W}^{3}, \mathrm{~B}^{0}, \mathrm{H}_{1}^{0}, \mathrm{H}_{2}^{0}$ giving supersymmetry partners $\tilde{\mathrm{W}}^{3}, \tilde{\mathrm{B}}^{0}, \tilde{\mathrm{H}}_{1}^{0}, \tilde{\mathrm{H}}_{2}^{0}$. These are weak (SU(2) $\left.\times \mathrm{U}(1)\right)$ eigenstates. $\left[\left(\mathrm{W}^{ \pm}, \mathrm{W}^{3}\right)\right.$ is in an $\mathrm{SU}(2)$ triplet, $\mathrm{B}^{0}$ is an $\mathrm{SU}(2)$ singlet, and $\mathrm{H}_{1}, \mathrm{H}_{2}$ are $\mathrm{SU}(2)$ doublets. One could equally well consider $\tilde{Z}^{0}, \tilde{\gamma}$, the partners of $\mathrm{Z}^{0}$, $\gamma$, instead of $\left.\tilde{\mathrm{W}}^{3}, \tilde{\mathrm{B}}^{0}\right]$. The partners are all spin $1 / 2$, uncolored and electrically neutral particles, differing only in their $\mathrm{SU}(2) \times \mathrm{U}(1)$ quantum numbers.

When $\mathrm{SU}(2) \times \mathrm{U}(1)$ is spontaneously broken by the Higgs mechanism, these states get off-diagonal contributions to their mass matrix. For example, a term in the Lagrangian $g \tilde{W}^{0} \tilde{H}_{1}^{0} H_{1}^{0}$ would give a $\tilde{W}^{0} \tilde{H}_{1}^{0}$ mass term $g v_{1}$ when $H_{1}^{0}$ gets a vacuum expectation value $v_{1}$. Other terms arise when $H_{2}^{0}$ gets a vacuum expectation value $v_{2}$. Additional mass terms may arise from SUSY breaking; if those terms do not also break $S U(2) \times U(1)$, the resulting mass matrix has the form*

$$
\left(\tilde{B}, \tilde{W}^{3}, \tilde{H}_{1}^{0}, \tilde{H}_{2}^{0}\right)\left(\begin{array}{cccc}
M^{\prime} & 0 & -g^{\prime} v_{1} / 2 & g^{\prime} v_{2} / 2 \\
0 & M & g v_{1} / 2 & -g v_{2} / 2 \\
-g^{\prime} v_{1} / 2 & g v_{1} / 2 & 0 & -\mu \\
g^{\prime} v_{2} / 2 & -g v_{2} / 2 & -\mu & 0
\end{array}\right)\left(\begin{array}{c}
\tilde{B} \\
\tilde{W}^{3} \\
\tilde{H}_{1}^{0} \\
\tilde{H}_{2}^{0}
\end{array}\right)
$$

where $M, M^{\prime}, \mu$ arise from supersymmetry breaking. The $\mathrm{SU}(2) \times \mathrm{U}(1)$ invariance requires that $M$ and $\mu$ above are the same quantities as in the chargino mass matrix, so only one new parameter $\left(M^{\prime}\right)$ is introduced above. In many models additional neutralino states will exist and the matrix will be larger, but this minimal set will always be present. Nothing definite is known about $M, M^{\prime}, \mu$. In grand unified models the wino mass $M$ and the bino mass $M^{\prime}$ will be related, typically by a ratio $\alpha_{2} / \alpha_{1}$ times a group-theoretic coefficient of order unity, while $\mu$ remains an independent parameter. We will generally assume $M^{\prime}=\left(5 \alpha_{1} / 3 \alpha_{2}\right) M$ as is usual in the literature. The vacuum expectation values $v_{1}$ and $v_{2}$ are constrained by $m_{\mathrm{w}}=g v / 2, v^{2}=v_{1}^{2}+v_{2}^{2}$, but $v_{2} / v_{1}$ is a free parameter. Thus there are basically three parameters needed to describe the neutralino mixing. The same parameters occur in the chargino mass matrix, so between the two they will quickly be measured if superpartners are ever found.

Since some mixing necessarily occurs, the mass eigenstates will be different from the weak eigenstates. In one sense this is unfortunate; for superpartners that are $\mathrm{SU}(3) \times \mathrm{SU}(2) \times \mathrm{U}(1)$ eigenstates, even though the masses are unknown, the couplings are known, so production rates, decay branching

\footnotetext{
* Technically speaking, the fermion fields which appear in eq. (3.42) are the left-handed (four-component) fields. For convenience of writing, we suppress the subscript $L$ which should appear. Note that our conventions here differ slightly from the one used in refs. [3.75-3.77]. A complete and consistent treatment of neutralino mixing and detailed references are given in appendix C. 3 .
} 
ratios, etc., can be reliably calculated. When mixing occurs, the amount of mixing is determined by the mass matrix, and so the couplings of the mass eigenstates are not fully known, although partial information still exists on the couplings.

As described in appendix C.3, we will denote the mass eigenstate neutralinos by $\tilde{\chi}_{i}^{0}$,

$$
\left(\begin{array}{c}
\tilde{\chi}_{1}^{0} \\
\tilde{\chi}_{2}^{0} \\
\tilde{\chi}_{3}^{0} \\
\tilde{\chi}_{4}^{0}
\end{array}\right)=\left(N_{i j}\right)\left(\begin{array}{c}
\tilde{B}^{0} \\
\tilde{W}^{3} \\
\tilde{H}_{1}^{0} \\
\tilde{H}_{2}^{0}
\end{array}\right)
$$

The weak eigenstates are (neutral) Majorana spinors. The mass eigenstates can arrange themselves as Majorana states also, or if two particles are degenerate in mass, the pair can combine to make a Dirac spinor (this depending on the values of $M, \mu, v_{2} / v_{1}$ ). In general the mass matrix eigenvalues will all be different, ranging from a fairly light eigenvalue to a heavy one. For special cases one can have a zero mass eigenvalue; for $M=M^{\prime}=0$ the lightest state is a massless photino,

$$
\tilde{\gamma}=\tilde{\chi}_{1}^{0}=\left(g^{\prime} \tilde{W}^{3}+g \tilde{B}\right) / \sqrt{g^{2}+g^{\prime 2}}=\sin \theta_{\mathrm{w}} \tilde{W}^{3}+\cos \theta_{\mathrm{w}} \tilde{B}
$$

(i.e., $N_{11}=\cos \theta_{\mathrm{w}}, N_{12}=\sin \theta_{\mathrm{w}}$ ) while for $\mu=0$ the lightest state is a massless higgsino,

$$
\tilde{h}^{0} \approx\left(v_{2} \tilde{H}_{1}^{0}+v_{1} \tilde{H}_{2}^{0}\right) / v
$$

for $v_{1} \simeq v_{2}$. One can quickly confirm that there is a massless eigenvalue for $M=M^{\prime}=0$ or $\mu=0$ by checking that the determinant of the mass matrix is zero. Note that whenever $\mu$ is small the lightest state is unlikely to be mainly a photino. Further discussion of the mixing is given in appendix C.3.

At present a variety of models exist with preferred values for $M, \mu, v_{2} / v_{1}$, but there is no consensus on what they should be. In table 9 we give for a range of interesting values for $M, \mu, v_{2} / v_{1}$, the lightest two neutralino eigenstates, the associated neutralino eigenvalues, and the chargino eigenvalues and eigenstates. Note that some sets are excluded either because the chargino eigenvalues are too light (see section 3.5) or because they give a photino in the mass range (below about half a $\mathrm{GeV}$ ) that is not consistent with the cosmological constraints. The mass spectrum has been studied in detail in refs. $[3.76,3.83]$. Note that a machine which can have $\sqrt{\hat{s}}$ available at good luminosity for constituent collisions up to about $300 \mathrm{GeV}$ can either find neutralinos or show that supersymmetry breaking does not produce phenomena on the scale of $1 \mathrm{TeV}$ or less.

\section{Table 9}

This table gives the neutralino and chargino mass eigenvalues and eigenvectors for a general mass matrix. Columns 1,2 give values for the entries in the mass matrices (all masses in $\mathrm{GeV}$ ). The next columns give the masses of the two lightest neutralino states (sometimes one of them is a photino), and then come their eigenvectors. The numbers shown give the amounts of $\left(\tilde{\mathrm{H}}_{1}^{0}, \tilde{\mathrm{H}}_{2}^{0}, \tilde{\mathrm{W}}^{3}, \tilde{\mathrm{B}}^{0}\right)$. Note that a photino is $\tilde{\gamma}=\sin \theta_{\mathrm{w}} \tilde{W}^{3}+\cos \theta_{\mathrm{w}} \tilde{B}=0.45 \tilde{W}^{3}+0.88 \tilde{B}$, so one can identify photino eigenvectors whenever they appear. For $M$ small and $\mu$ large there is often a light photino. For $\mu$ small and $M$ larger there is often a light higgsino. The next columns are the chargino mass eigenvalues, and then their eigenvectors. Chargino eigenvectors are labeled $(\tilde{H}, \tilde{W})$. If the eigenvectors of $M M^{+}$and $M^{+} M$ are different both are shown in that order; the eigenvectors of $M M^{+}$are what is shown otherwise, and the orthogonal combination is easily written. Finally the machines where these could be detected first are listed, just taking kinematical restrictions into account, but not production rates. For an $\mathrm{e}^{+} \mathrm{e}^{-}$collider with $\mathscr{L} \geq 5 \times 10^{31} / \mathrm{cm}^{2} \mathrm{~s}$ there will be no rates that are too small, but for smaller $\mathscr{L}$ some states may not be produced strongly enough to detect. For a hadron collider with $\mathscr{L}>10^{32} / \mathrm{cm}^{2} \mathrm{~s}$ such as a future supercollider (SC), similar remarks hold. Since we give a sampling of the full mass matrix, one can look up the consequences of any model here, at least approximately. We give separate tables for $v_{1} / v_{2}=4,1$ and for both relative signs of $\mu / M$. Note that in obtaining this table we have assumed that the bino mass parameter $M^{\prime}$ is related to $M$ as shown in eq. (C34). These tables were computed in collaboration with J.M. Frere and are shown on the following pages. 


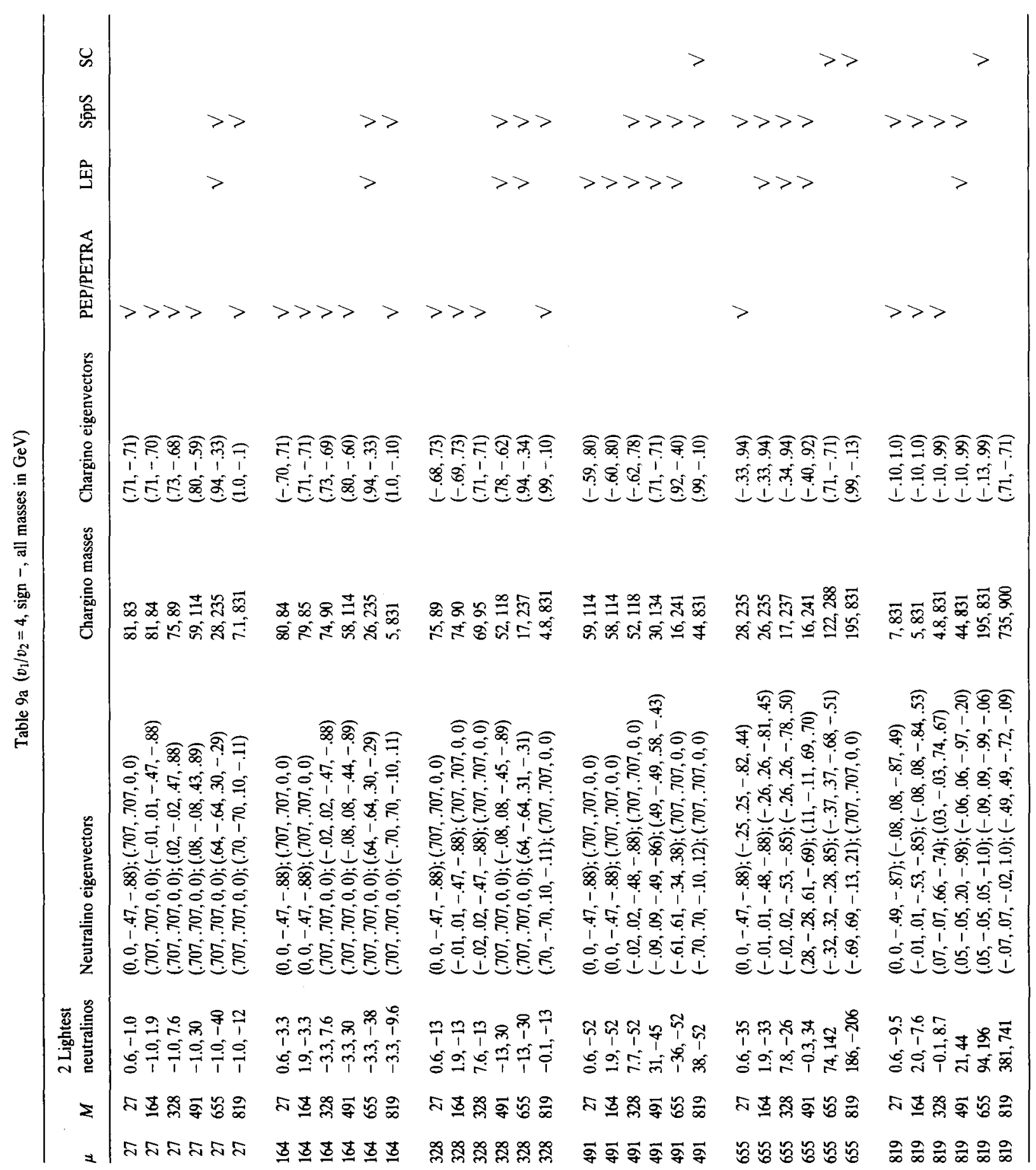




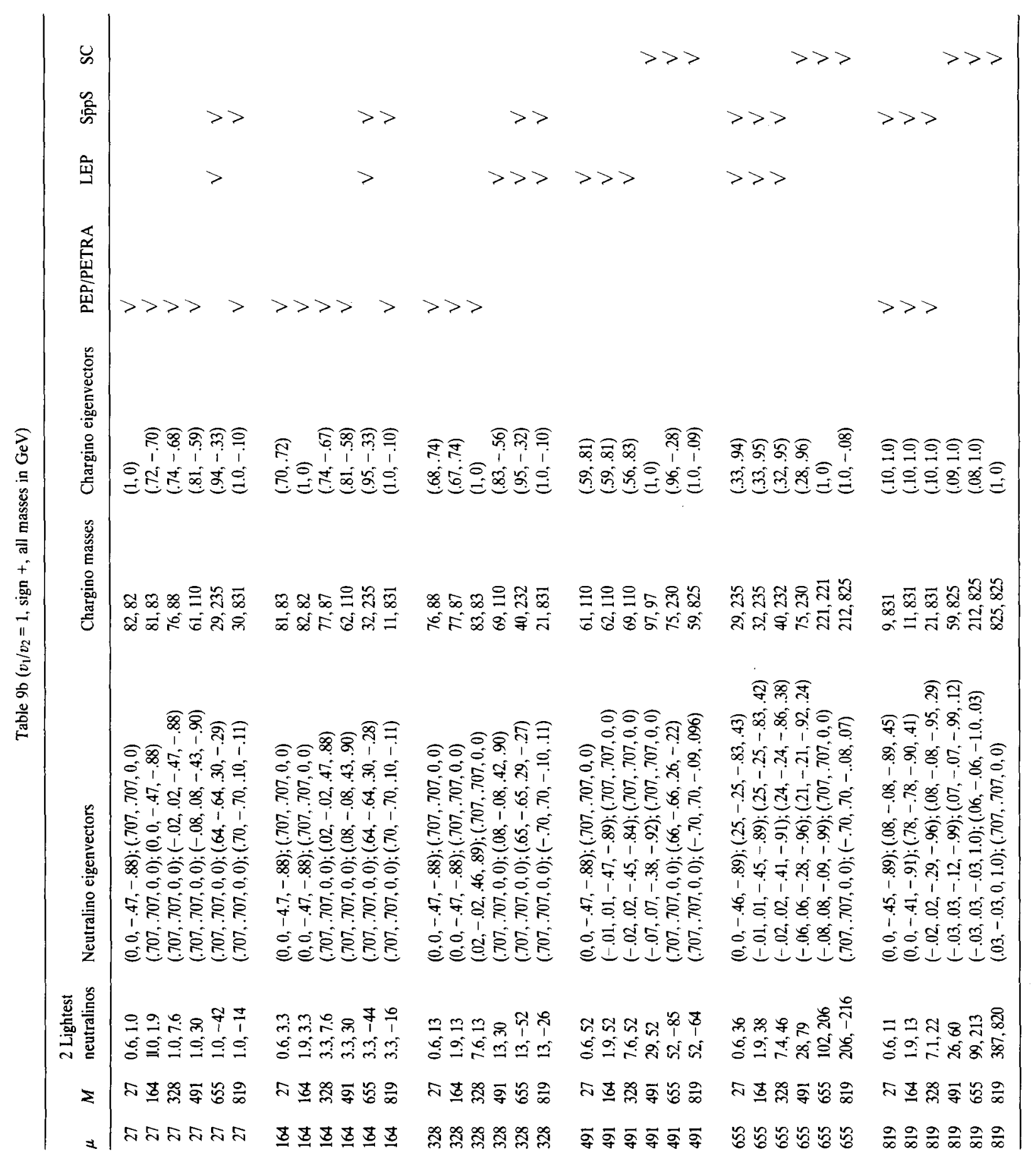




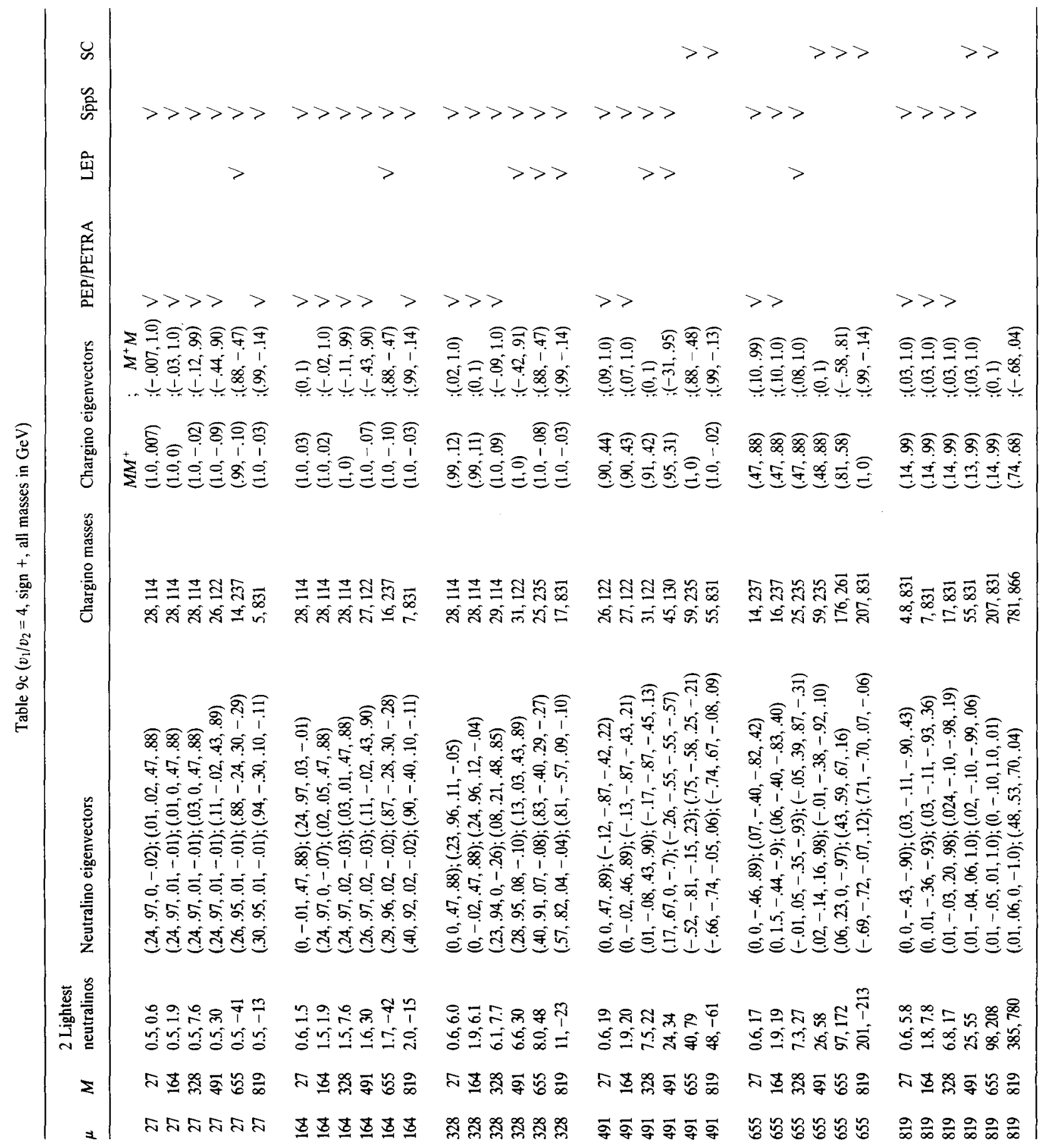




\section{(B) Decays}

Neutralino decays can proceed through a number of channels, as shown in fig. 37 . Which channel dominates depends on the masses, so no definite statement can be made. In many models the scalarquarks and scalar-leptons are lighter than $\mathrm{W}^{ \pm}, \mathrm{Z}^{0}$ so fig. 37(c) dominates. If the decay of fig. $37(\mathrm{~d})$ is allowed it will probably dominate, as it would be semi-weak and the $\mathrm{H}^{0}$ couplings to neutralinos can be large, via the wino component of one neutralino and the higgsino component of the other. (This is the supersymmetric version of $\mathrm{Z}^{0} \rightarrow \mathrm{H}_{1}^{0} \mathrm{H}_{2}^{0}$ which can occur with a fairly large branching ratio in multi-Higgs models [3.90].)

We will give detailed results for the case where fig. 37(c) dominates, as that often occurs in models. To evaluate the couplings, imagine each $\tilde{\chi}_{i}^{0}$ expanded into its weak eigenstate components. Then the fermion-higgsino components can be neglected because they are proportional to fermion masses, while for the $Z^{0}$ couplings only the higgsino components matter. The latter follows simply from the observation that the (tree-level) $\mathrm{Z}^{0}$ coupling to neutralinos is proportional to $T_{3}$ (since $Q=0$ ); but $T_{3}=0$ for neutral gauginos and $T_{3} \neq 0$ for higgsinos.

Expressing the vertices in terms of the mixing coefficients, we get [cf. eqs. (C77)]:

$$
\begin{array}{ll}
\sqrt{2}\left\langle\tilde{\chi}_{i}^{0} \mid \tilde{u}_{\mathrm{L}} u_{\mathrm{L}}\right\rangle=g N_{i 2}+\frac{1}{3} g^{\prime} N_{i 1}, & \sqrt{2}\left\langle\tilde{\chi}_{i}^{0} \mid \tilde{u}_{\mathrm{R}} u_{\mathrm{R}}\right\rangle=-\frac{4}{3} g^{\prime} N_{i 1}, \\
\sqrt{2}\left\langle\tilde{\chi}_{i}^{0} \mid \tilde{d}_{\mathrm{L}} d_{\mathrm{L}}\right\rangle=-g N_{i 2}+\frac{1}{3} g^{\prime} N_{i 1}, & \sqrt{2}\left\langle\tilde{\chi}_{i}^{0} \mid \tilde{d}_{\mathrm{R}} d_{\mathrm{R}}\right\rangle=\frac{2}{3} g^{\prime} N_{i 1}, \\
\sqrt{2}\left\langle\tilde{\chi}_{i}^{0} \mid \tilde{\nu}_{\ell} \nu_{\ell}\right\rangle=g N_{i 2}-g^{\prime} N_{i 1}, & \sqrt{2}\left\langle\tilde{\chi}_{i}^{0} \mid \tilde{\ell}_{\mathrm{L}}^{ \pm} \ell_{\mathrm{L}}^{\mp}\right\rangle=-g N_{i 2}-g^{\prime} N_{i 1}, \\
\sqrt{2}\left\langle\tilde{\chi}_{i}^{0} \mid \tilde{\ell}_{\mathrm{R}}^{ \pm} \ell_{\mathrm{R}}^{\mp}\right\rangle=2 g^{\prime} N_{i 1} . &
\end{array}
$$

The appropriate $\left(1 \pm \gamma_{5}\right)$ factors have been omitted, but can be restored by referring back to eq. (C77). Note that in the expression given above with a $\mathrm{q} \overline{\mathrm{q}}$ final pair we must in addition insert a color factor of 3. Using eqs. (C87a) and (C88), we find

$$
\left\langle Z^{0} \mid \tilde{\chi}_{i}^{0} \tilde{\chi}_{j}^{0}\right\rangle=(g / 2 \cos \theta) \operatorname{Re}\left(N_{i 3} N_{j 3}^{*}-N_{i 4} N_{j 4}^{*}\right)
$$

where a factor of $\gamma_{\mu} \gamma_{5}$ has been omitted. The Higgs vertex $\left\langle\tilde{\chi}_{i}^{0} \mid \tilde{\chi}_{j}^{0} H^{0}\right\rangle$ also depends on what linear combination of $\mathrm{H}_{1}^{0}, \mathrm{H}_{2}^{0}$ makes up the assumed light physical Higgs, so it is more complicated to write and depends on the Higgs sector of the theory. Presumably the couplings are flavor independent, so $\mathrm{c}$ and $t$ couplings are the same as $u$ couplings, etc.

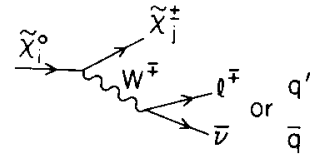

(a)

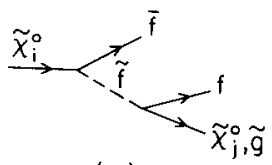

(c)

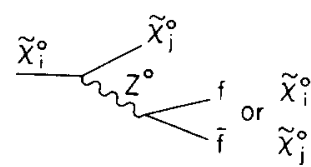

(b)

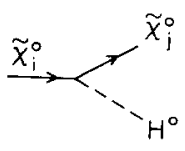

(d)

Fig. 37. Graphs for neutralino decays (see caption to fig. 33 for notation). The index $i$ (or $j$ ) labels different neutralino or chargino states. 
To estimate branching ratios one can substitute examples for $N_{i 1}, N_{i 2}$ from table 9 , and take $\tilde{M}_{\mathrm{q}}, \tilde{M}_{\ell}$ from models. Let us take two examples to see what patterns arise.

(I) Assume that $\tilde{\chi}_{2}^{0}$ is an equal mixture of all weak eigenstates, i.e. $N_{2 i}=1 / 2$, and $\tilde{\chi}_{1}^{0}$ is a photino $\tilde{\gamma}$, $N_{11}=\cos \theta_{\mathrm{w}}, N_{12}=\sin \theta_{\mathrm{w}}, N_{13}=N_{14}=0$. Also, assume $\tilde{\mathrm{g}}$ is too heavy to contribute, so the decays are $\tilde{\chi}_{2}^{0} \rightarrow \mathrm{ff} \tilde{\gamma}$. [If $\tilde{\mathrm{g}}$ is lighter than $\tilde{\chi}_{2}^{0}$ the decays are to f $\overline{\mathrm{f}} \tilde{\mathrm{g}}$ instead, with the same matrix elements for quarks and zero for leptons. The reader can redo the branching ratios for that case.] Then with $g=0.66$, $g^{\prime}=0.35$, one has matrix elements in the following ratios:

$$
\begin{gathered}
\tilde{\chi}_{2}^{0} \rightarrow \begin{cases}\bar{u}_{\mathrm{L}} u_{\mathrm{L}} \tilde{\gamma} & 0.39 / \tilde{M}_{\mathrm{uL}}^{2} \\
\bar{u}_{\mathrm{R}} u_{\mathrm{R}} \tilde{\gamma} & 0.17 / \tilde{M}_{\mathrm{uR}}^{2} \\
\bar{d}_{\mathrm{L}} d_{\mathrm{L}} \tilde{\gamma} & 0.14 / \tilde{M}_{\mathrm{dL}}^{2} \\
\bar{d}_{\mathrm{R}} d_{\mathrm{R}} \tilde{\gamma} & 0.06 / \tilde{M}_{\mathrm{dR}}^{2}\end{cases} \\
\tilde{\chi}_{2}^{0} \rightarrow \begin{cases}\bar{\nu} \nu \tilde{\gamma} & 0 \\
\ell_{\mathrm{L}} \ell_{\mathrm{L}} \tilde{\gamma} & 0.76 / \tilde{M}_{\ell \mathrm{L}}^{2} \\
\ell_{\mathrm{R}} \ell_{\mathrm{R}} \tilde{\gamma} & 0.53 / \tilde{M}_{\ell \mathrm{R}}^{2}\end{cases}
\end{gathered}
$$

Then consider two choices for the masses:

(i) All $\tilde{M}_{\mathrm{q}}$ are equal. This gives branching ratios (ignoring mass corrections and including color factors)

$$
\begin{array}{ll}
\operatorname{BR}\left(\tilde{\chi}_{2}^{0} \rightarrow \overline{\mathrm{u} u} \tilde{\gamma}\right) & 0.14 \\
\operatorname{BR}\left(\tilde{\chi}_{2}^{0} \rightarrow \overline{\mathrm{d} d} \tilde{\gamma}\right) & 0.015 \\
\operatorname{BR}\left(\tilde{\chi}_{2}^{0} \rightarrow \mathrm{e}^{+} \mathrm{e}^{-} \tilde{\gamma}\right) & 0.23 \\
\operatorname{BR}\left(\tilde{\chi}_{2}^{0} \rightarrow \overline{\mathrm{c}} \mathrm{\gamma}\right) & 0.14 \\
\operatorname{BR}\left(\tilde{\chi}_{2}^{0} \rightarrow \overline{\mathrm{ss}} \tilde{\gamma}\right) & 0.015 \\
\operatorname{BR}\left(\tilde{\chi}_{2}^{0} \rightarrow \mu^{+} \mu^{-} \tilde{\gamma}\right) & 0.23 \\
\operatorname{BR}\left(\tilde{\chi}_{2}^{0} \rightarrow \tau^{+} \tau^{-} \tilde{\gamma}\right) & 0.23 .
\end{array}
$$

Further, the $\mu$ and $\tau$ are polarized

$$
\left[\Gamma\left(\tilde{\chi}_{2}^{0} \rightarrow \mu_{\mathrm{L}}^{+} \mu_{\mathrm{L}}^{-} \tilde{\gamma}\right)-\Gamma\left(\tilde{\chi}_{2}^{0} \rightarrow \mu_{\mathrm{R}}^{+} \mu_{\mathrm{R}}^{-} \tilde{\gamma}\right)\right] / \Gamma\left(\tilde{\chi}_{2}^{0} \rightarrow \mu^{+} \mu^{-} \tilde{\gamma}\right)=0.35
$$

Certainly the $\mathrm{e}^{+} \mathrm{e}^{-} \tilde{\gamma}, \mu^{+} \mu^{-} \tilde{\gamma}$ modes have very good signatures at $\mathrm{e}^{+} \mathrm{e}^{-}$or hadron colliders, the cic modes do in some detectors, and the light modes give two jets which are probably also easy to identify.

(ii) Suppose $\tilde{M}_{1} \ll \tilde{M}_{\mathbf{q}}$. Then the $\mathrm{e}^{+} \mathrm{e}^{-} \tilde{\gamma}$ and $\mu^{+} \mu^{-} \tilde{\gamma}$ modes completely dominate and the signatures are excellent.

(II) Assume $\tilde{\chi}_{2}^{0}$ has a lot of $\tilde{\mathrm{B}}^{0}$ mixed in with higgsinos, but very little $\tilde{\mathrm{W}}^{3}$, i.e. put $N_{22}=0$. Then for equal $\tilde{M}$ the matrix elements occur in ratios $\frac{1}{3}:-\frac{4}{3}: \frac{1}{3}: \frac{2}{3}:-1:-1: 2$, so the relative rates (including color factors where appropriate) are in ratios $\frac{1}{3}: \frac{16}{3}: \frac{1}{3}: \frac{4}{3}: 1: 1: 4$. That gives

$$
\operatorname{BR}\left(\tilde{\chi}_{2}^{0} \rightarrow \mathrm{e}^{+} \mathrm{e}^{-} \tilde{\gamma}\right)=\operatorname{BR}\left(\tilde{\chi}_{2}^{0} \rightarrow \mu^{+} \mu^{-} \tilde{\gamma}\right)=0.15,
$$




$$
\begin{aligned}
& \operatorname{BR}\left(\tilde{\chi}_{2}^{0} \rightarrow \overline{\mathrm{c}} \mathrm{\gamma}\right)=0.17 \\
& \frac{\Gamma\left(\mu_{\mathrm{L}}^{+} \mu_{\mathrm{L}}^{-} \tilde{\gamma}\right)-\Gamma\left(\mu_{\mathrm{R}}^{+} \mu_{\mathrm{R}}^{-} \tilde{\gamma}\right)}{\Gamma\left(\mu^{+} \mu^{-} \tilde{\gamma}\right)}=-0.6 .
\end{aligned}
$$

Basically, as long as the scalar lepton masses are not larger than about $m_{\mathbf{w}}$, and a significant amount of either $\tilde{\mathrm{W}}^{3}$ or $\tilde{\mathrm{B}}^{0}$ is mixed into $\tilde{\chi}_{2}^{0}$, the branching ratios of $\tilde{\chi}_{2}^{0}$ to good signatures such as $\mathrm{e}^{+} \mathrm{e}^{-}$or $\mu^{+} \mu^{-}$are large and useful if $\tilde{M}_{\mathrm{g}}>\tilde{M}_{\chi_{2}}$. Similar analyses can be made for the decays of other neutralinos; we have concentrated on $\tilde{\chi}_{2}^{0}$ because it is (by definition) the second lightest neutralino, and its pair production or associated production with $\tilde{\chi}_{1}^{0}$ gives very good ways to detect superpartners. If gluinos are lighter than $\tilde{\chi}_{2}^{0}$, the modes $q \bar{q} \tilde{g}$ will dominate, so the signature is less good but still satisfactory.

\section{(C) Production}

Neutralinos can be produced in three ways, as shown in fig. 38(a), (b), (c), and the production can be accompanied by a hard radiated photon or gluon as in fig. 39; the accompanying $\gamma$ or g may aid in detection. The pairs (a) $\tilde{\chi}_{1}^{0} \tilde{\chi}_{1}^{0}$ (often called photino pair production), (b) $\tilde{\chi}_{1}^{0} \tilde{\chi}_{2}^{0}$ (associated production), and (c) $\tilde{\chi}_{2}^{0} \tilde{\chi}_{2}^{0}$ all give interesting signatures that may provide excellent ways to detect superpartners.

The most dramatic signal occurs for associated production of $\tilde{\chi}_{2}^{0} \tilde{\chi}_{1}^{0}$ where the $\tilde{\chi}_{1}^{0}$ is assumed to escape detection and the $\tilde{\chi}_{2}^{0}$ decays into $\tilde{\chi}_{1}^{0}$ (or a gluino, $\tilde{\mathrm{g}}$ ) plus either lepton pairs or quark jets. If the resulting decay products are energetic, this event will be quite striking; one hemisphere will be empty of large $p_{\mathbf{T}}$ particles. We call such events one-sided events. Such types of events have already been encountered in section 3.2 in $Z^{0} \rightarrow \tilde{\nu} \tilde{\nu}$ decays (see fig. 11) where one $\tilde{\nu}$ escapes and the other decays into four-body final

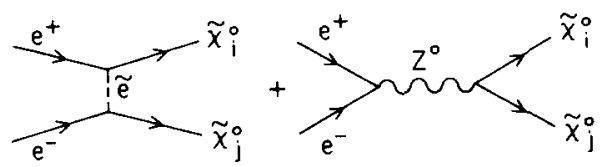

(a)

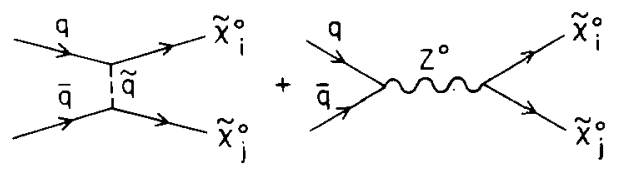

(b)
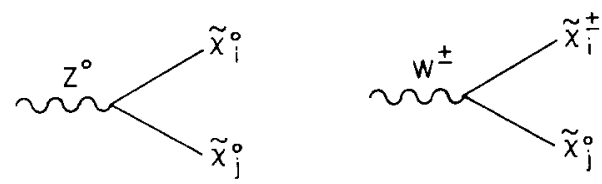

(c)

Fig. 38. Neutralino production in (a) $\mathrm{e}^{+} \mathrm{e}^{-}$annihilation, (b) Drell-Yan production and (c) $Z^{0}$ and $\mathrm{W}^{ \pm}$decay.

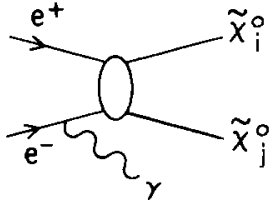

(a)

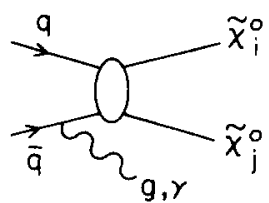

(b)
Fig. 39. Neutralino production accompanied by radiation of a single hard photon or gluon. The accompanying photon (or gluon) can signal the occurrence of an event if the neutralino energy escapes the detector. 
states. Hence, such events in general are characteristic of supersymmetry; as soon as some are found, further analysis will be required to determine which supersymmetric particle has been discovered.

Neutralinos were first discussed in general in ref. [3.91]. Associated production of $\tilde{\chi}_{1}^{0} \tilde{\chi}_{2}^{0}$ and one-sided events were first discussed in ref. [3.75]. Further study and calculations were presented in refs. [3.76-3.77]. Studies in a particular supergravity model were given in refs. [3.84-3.85]. See also refs. $[3.82,3.85,3.88,3.92$ and 3.93$]$.

\section{(a) $e^{+} e^{-}$colliders}

Perhaps the most hopeful way to find superpartners in the near future is by associated production of $\tilde{\chi}_{1}^{0} \tilde{\chi}_{2}^{0}[3.75-3.77,3.81,3.91,3.92]$. These are the two lightest neutralinos ( $\tilde{\chi}_{1}^{0}$ may be mainly a photino), and in many models the sum of their masses may be less than the top energies at PETRA or PEP. If they are not found it strongly constrains model building. If they are not found at PETRA or PEP, a new window opens at SLC or LEP, and eventually a high luminosity, high energy hadron collider will provide a definitive test of whether supersymmetry phenomena exist on the weak scale.

The signature for $\tilde{\chi}_{2}^{0} \tilde{\chi}_{1}^{0}$ associated production is very clear, and has no serious standard model background that could mask it. The $\tilde{\chi}_{1}^{0}$ may be the lightest superpartner and thus (probably) stable, or perhaps scalar neutrinos are lighter in which case $\tilde{\chi}_{1}^{0} \rightarrow \tilde{\nu} \nu$. In either case, $\tilde{\chi}_{1}^{0}$ escapes detection and nothing enters the detector in one hemisphere. As discussed in section B above, if gluinos are heavier than $\tilde{\chi}_{2}^{0}$, then it probably has fairly large branching ratios for decay to $\mathrm{e}^{+} \mathrm{e}^{-} \tilde{\chi}_{1}^{0}$ and $\mu^{+} \mu^{-} \tilde{\chi}_{1}^{0}$, so the full signature is a one-sided event with about $2 / 3$ of the total energy missing (since two $\tilde{\chi}_{1}^{0}$ escape, one with half the energy and one with $1 / 6$ of the energy on the average), and a single $\mathrm{e}^{+} \mathrm{e}^{-}$or $\mu^{+} \mu^{-}$pair. Other decay modes also give one-sided events with missing energy and $q+\bar{q}$ jets and may also be easily detectable. If gluinos are lighter than $\tilde{\chi}_{2}^{0}$, then $\tilde{\chi}_{2}^{0} \rightarrow \mathrm{ff} \tilde{g}$ giving two hard jets and $\tilde{\mathrm{g}} \rightarrow \mathrm{q} \overline{\mathrm{q}} \tilde{\gamma}$ opposite the missing $\tilde{\chi}_{1}^{0}$ on the other side.

There are two backgrounds for neutralino events from normal production of Standard Model particles. Probably the worst background is $\tau^{+} \tau^{-}$production, with both $\tau$ 's undergoing a semi-leptonic decay. Since $\tau^{+} \tau^{-}$gives e $\mu$ events as well as ee and $\mu \mu$, this background can be rather accurately subtracted; in addition, the $\mathrm{e}^{+} \mathrm{e}^{-}$or $\mu^{+} \mu^{-}$from $\tau^{+} \tau^{-}$are almost back-to-back at higher energies, while that is not so for neutralinos. Note that the $\tau^{+} \tau^{-}$background is present at $\mathrm{e}^{+} \mathrm{e}^{-}$colliders but not at hadron colliders. The other is two-photon events, which can be cut out because the missing momentum for $\tilde{\chi}_{2}^{0} \tilde{\chi}_{1}^{0}$ can point anywhere, while for two-photon events it will be in the beam direction.

An exact expression for the cross section for $\mathrm{e}^{+} \mathrm{e}^{-} \rightarrow \tilde{\chi}_{2}^{0} \tilde{\chi}_{1}^{0}$ is given in ref. [3.76]. Qualitatively, the cross section is a typical weak one if $\tilde{M}_{\mathrm{e}}$ is of order $m_{\mathrm{w}}$; it is suppressed by the mixing effects since only part of each neutralino will couple to eẽ. [Using eq. (3.46), the coupling of $\tilde{\chi}_{i}^{0}$ to $\mathrm{e}_{\mathrm{L}} \tilde{\mathrm{e}}_{\mathrm{L}}$ is $g N_{i 2}+g^{\prime} N_{i 1}$, and the coupling of $\tilde{\chi}_{i}^{0}$ to $\mathrm{e}_{\mathrm{R}} \tilde{\mathrm{e}}_{\mathrm{R}}$ is $2 g^{\prime} N_{i 1}$, where $g=e / \sin \theta_{\mathrm{w}}$ and $g^{\prime}=e / \cos \theta_{\mathrm{w}}$ are the SU(2) and U(1) couplings and $N_{i j}$ are the mixing coefficients, $\left|N_{i j}\right|<1$ ]. If $\tilde{M}_{\mathrm{e}}<m_{\mathrm{w}}$ the cross section is enhanced. Thus for kinematically allowed neutralino masses the cross section is $\sigma\left(\mathrm{e}^{+} \mathrm{e}^{-} \rightarrow \tilde{\chi}_{2}^{0} \tilde{\chi}_{1}^{0}\right) \geq \frac{1}{10} \mathrm{pb}$. Now that PETRA and PEP are collecting over one $\mathrm{pb}^{-1} /$ day integrated luminosity they could detect such a signal.

The cross section receives contributions from an s-channel $Z^{0}$. The branching ratio of $Z^{0}$ to $\tilde{\chi}_{2}^{0} \tilde{\chi}_{1}^{0}$ is (neglecting final state masses)

$$
\Gamma\left(Z^{0} \rightarrow \tilde{\chi}_{2}^{0} \tilde{\chi}_{1}^{0}\right) / \Gamma\left(Z^{0} \rightarrow \bar{\nu}_{\mathrm{e}} \nu_{\mathrm{e}}\right)=\left[\operatorname{Re}\left(N_{13} N_{23}^{*}-N_{14}^{*} N_{24}\right)\right]^{2},
$$

so it is large when the higgsino components of $\tilde{\chi}_{2}^{0}$ and $\tilde{\chi}_{1}^{0}$ are large. Even if the above branching ratio is of order $10^{-3}$ there would be several events/week at SLC and more at expected LEP luminosities, always with clear signatures. The ẽ exchange contribution to the cross section grows with energy until 
$s>\tilde{M}_{\mathrm{e}}^{2}$, and whatever the form of $\tilde{\chi}_{1,2}^{0}$ in terms of weak eigenstates, between the $Z^{0}$ and ex exchange contribution there is a good chance of producing one-sided $\tilde{\chi}_{2}^{0} \tilde{\chi}_{1}^{0}$ events. Almost all models with any superpartners at the weak scale or below will have $\tilde{M}_{\chi_{1}}+\tilde{M}_{\chi_{2}}<m_{z}$, so one-sided $\tilde{\chi}_{1}^{0} \tilde{\chi}_{2}^{0}$ associated production should be kinematically available at SLC, LEP.

(a) Pair production of $\tilde{\chi}_{2}^{0} \tilde{\chi}_{2}^{0}$ was emphasized in [3.91] as another good process to examine. Since $\tilde{M}_{\chi_{2}}>\tilde{M}_{\chi_{1}}$ it is kinematically suppressed compared to $\tilde{\chi}_{2}^{0} \tilde{\chi}_{1}^{0}$, but it could happen that the couplings to $\mathrm{Z}^{0}$ or to eẽ are larger. The signature is generally good, with a significant branching ratio for two $\ell^{+} \ell^{-}$pairs, plus missing energy from escaping $\tilde{\chi}_{1}^{0}$ (about $1 / 3$ of the energy is missing on the average) if the ff $\tilde{g}$ mode is not allowed. The exact cross section formula is given in [3.83]; again there are $Z^{0}$ and ẽ contributions and the basic size is typically weak, of order a pb.

(b) Pair production of $\tilde{\chi}_{1}^{0} \tilde{\chi}_{1}^{0}$ is also interesting. The cross section for production of any neutralino pair by $\tilde{\mathrm{e}}$ exchange (no $Z^{0}$ contribution) is given in ref. [3.94,3.12]. It is usually called photino pair production (see our discussion in section 3.1) although the lightest neutralino need not be a photino. Since both $\tilde{\chi}_{1}^{0}$ presumably escape the detector, at an $\mathrm{e}^{+} \mathrm{e}^{-}$collider some additional signature is necessary to establish an event occurred. As with the analogous situation for neutrinos, where $\mathrm{e}^{+} \mathrm{e}^{-} \rightarrow \nu \bar{\nu}$ is detectable via $\mathrm{e}^{+} \mathrm{e}^{-} \rightarrow \gamma \nu \bar{\nu}$ with a photon radiated off [3.13] an initial $\mathrm{e}^{+}$or $\mathrm{e}^{-}$, one can search for $\mathrm{e}^{+} \mathrm{e}^{-} \rightarrow \gamma \tilde{\chi}_{1}^{0} \tilde{\chi}_{1}^{0}$ (photino counting). This has been pointed out in refs. [3.11,3.95,3.106], and discussed extensively in ref. [3.12] where the cross section is estimated in the approximation of no $Z^{0}$ contribution. If the $\tilde{\mathrm{e}}$ mass is smaller than $m_{\mathrm{w}}$ the rate is larger than that for $\nu$ counting by $\left(m_{\mathrm{w}} / \tilde{M}_{\mathrm{e}}\right)^{4}$, so events could already be detected at PETRA or PEP. The MAC collaboration has used this to give the current strongest limit on $\tilde{M}_{\mathrm{e}}$ [3.14]; the limit depends on $\tilde{M}_{x_{1}}$ and is about $25 \mathrm{GeV}$ for $\tilde{M}_{x_{1}}=0$. As emphasized in refs. [3.11,3.12], the search for $\mathrm{e}^{+} \mathrm{e}^{-} \rightarrow \gamma \tilde{\gamma} \tilde{\gamma}$ is valuable at PETRA, PEP energies and is the most sensitive way to search for $\tilde{M}_{\mathrm{e}}$ up to $50-60 \mathrm{GeV}$. As $V_{s}$ increases at future machines the background from $\mathrm{e}^{+} \mathrm{e}^{-} \rightarrow \gamma \bar{\nu} \nu$ via an s-channel $\mathrm{Z}^{0}$ increases. In refs. [3.11,3.12] the $\mathrm{Z}^{0}$ contribution is neglected but is considered in ref. [3.95]. If the lightest neutralino is mainly a higgsino state its coupling to the $Z^{0}$ is large if $v_{1} \neq v_{2}$, and at any collider with reasonable luminosities there can be a large contribution to $\gamma \tilde{\chi}_{1}^{0} \tilde{\chi}_{1}^{0}$. The search is very useful at any higher energies.

\section{(b) Hadron colliders}

Pair production and associated production of neutralinos proceeds equally well through $\mathrm{q} \bar{q} \rightarrow \tilde{\chi}_{i}^{0} \tilde{\chi}_{j}^{0}$, by $\tilde{\mathrm{q}}$ or $Z^{0}$ exchange, as in fig. $38 \mathrm{~b}$. The signature for one-sided $\tilde{\chi}_{1}^{0} \tilde{\chi}_{2}^{0}$ events will be as good as at $\mathrm{e}^{+} \mathrm{e}^{-}$ colliders, with only an $\mathrm{e}^{+} \mathrm{e}^{-}$or $\mu^{+} \mu^{-}$or $\mathrm{q} \overline{\mathrm{q}}$ pair or $\mathrm{q} \overline{\mathrm{q}} \tilde{\mathrm{g}}$ in one hemisphere of the central region, and nothing in the other, and background questions may be less severe at hadron colliders.

Hadron colliders have the additional advantage that more center-of-mass energy is available for the constituent collision, so if $\tilde{M}_{\chi_{1}}+\tilde{M}_{\chi_{2}}$ is less than about $200 \mathrm{GeV}$ they are kinematically accessible at the SPS collider, and at the FNAL Tevatron Collider a mass of about $500 \mathrm{GeV}$ is kinematically accessible. Unfortunately, the cross sections for $\overline{\mathrm{p}} \mathrm{p} \rightarrow \tilde{\chi}_{1}^{0} \tilde{\chi}_{2}^{0}+\mathrm{X}$ are typically of order $0.1-1 \mathrm{pb}$, so integrated luminosities of order $10^{38} / \mathrm{cm}^{2}$ or more are needed to be confident of seeing neutralino production. At a future super-collider with $\int \mathscr{L} \mathrm{d} t \simeq 10^{40}$ and constituent center-of-mass energy greater than about $1 \mathrm{TeV}$, one-sided neutralino production events will certainly be observed if there are any supersymmetric partners with masses on the weak scale. In spite of the luminosity limitations, the signatures of one-sided events with a lepton or quark pair, or two-sided events with two lepton or quark pairs, should be watched for very carefully in case the cross section is fortuitously large. [The signatures are the same as for production by $\mathrm{e}^{+} \mathrm{e}^{-}$, and are described more fully above.] 
(c) $W^{ \pm}, Z^{0}$ decays

Neutralinos produced via a $Z^{0}$ have been discussed above. They also occur in the decay $\mathrm{W}^{ \pm} \rightarrow \tilde{\chi}_{i}^{ \pm} \tilde{\chi}_{j}^{0}$. We will discuss that way of detecting them more fully when we consider chargino detection in W decay in chapter 4.

\section{(D) Present limits}

At the time of writing there are no published limits on neutralino states. The combination of picobarn cross sections and large missing energy means that data studied up till now were not sensitive to one-sided neutralino pair events. Even if the events had occurred, they would have been missed because of the large missing energy. In the near future they could be found if they are kinematically accessible at PETRA, PEP energies, so if they are not detected useful limits will be available. ${ }^{*}$ Setting limits is not entirely straightforward, as the cross section depends on $\tilde{M}_{\mathrm{e}}, \tilde{M}_{\chi_{1}}, \tilde{M}_{\chi_{2}}$, and on the mixing coefficients for weak eigenstates into mass eigenstates; if a signal is not seen, approximate useful limits can be set from the cross section formula in ref. [3.76]. In setting limits, care should be taken to use correct cross sections. Since mixing of neutralinos necessarily occurs (see appendix C.3), calculations which assume production of $\tilde{z} \tilde{\gamma}$ will be misleading, and calculations which neglect the $Z^{0}$ contribution will be misleading. It is better to use formulas of ref. [3.76] and typical eigenstates of table 9.

\subsection{Goldstinos}

If supersymmetry were a spontaneously broken global symmetry (as opposed to an explicitly broken global symmetry or a broken local symmetry), a massless Goldstone fermion would appear [3.96], coupled to every particle and its superpartner. This gives rise to a number of interesting production and decay mechanisms, which could be relevant to experiment if the Goldstino coupling were large, which in turn (see below) would require the scale of supersymmetry breaking to be less than a $\mathrm{TeV}$.

We will briefly describe modifications that occur if massless Goldstinos are relevant. This alternative is not fashionable at present since in supergravity theories the Goldstino combines with the gravitino to make a massive spin-3/2 particle (via a supersymmetric analogue of the Higgs mechanism [3.97]); if there were a massless Goldstino around then the supersymmetry involved would not be related to gravity.

If there is a Goldstino, it couples every partner to its superpartner, with a coupling universal in form. The coupling is derived in a way analogous to the Goldberger-Treiman formula [3.98-3.100]. There is a supercurrent which would be conserved if the supersymmetry were not broken. A simple example is [3.101]

$$
S_{\mu}=\frac{1}{2}\left(F_{\rho \sigma}^{a} \gamma^{\rho} \gamma^{\sigma} \gamma_{\mu} \tilde{g}_{\mathrm{a}}\right)
$$

for the gluon-gluino current, where $F_{\rho \sigma}^{a}$ is the gluon field tensor and $a$ is a color index which is summed. If there is a broken supersymmetry, then just as one writes for a pion and the matrix element of the axial vector current an expression $\left\langle 0\left|A_{\mu}\right| \pi\right\rangle=f_{\pi} P_{\mu}$, one has here

$$
\left\langle 0\left|S_{\mu}\right| \tilde{G}\right\rangle=\gamma_{\mu} u_{\mathrm{G}} \Lambda_{\mathrm{SS}}^{2}
$$

where $\tilde{G}$ is the Goldstino, $u_{\mathrm{G}}$ the Goldstino spinor, and $A_{\mathrm{ss}}$ measures the scale of supersymmetry

\footnotetext{
${ }^{*}$ See note added in proof, p. 251.
} 
breaking. Then

$$
\left\langle g\left|S_{\mu}\right| \tilde{g}\right\rangle \simeq\left[\frac{1}{2} F_{\rho \sigma}^{a} \gamma^{\rho} \gamma^{\sigma} \gamma_{\mu}+A_{\mathrm{SS}}^{2} \lambda \gamma_{\mu}(1 / q) F_{\rho \sigma}^{a} \gamma^{\rho} \gamma^{\sigma}\right] \tilde{g}_{a}
$$

where the two contributions correspond to the supercurrent coupling to the $g \tilde{g}$ vertex, and the Goldstino pole contribution, as in fig. 40. The $G g \tilde{g}$ vertex must have the form shown by gauge invariance, and it has a strength $\lambda$ (to be determined). Then requiring $q_{\mu} S^{\mu}=0$ gives, after some spinor algebra,

$$
\lambda=\tilde{M}_{\mathrm{g}} / 2 \Lambda_{\mathrm{SS}}^{2} .
$$

This is the Goldstino coupling, and will hold for any particle and its superpartner. It increases as the mass splitting increases, so if $\Lambda_{\mathrm{ss}}$ is not too large it could get quite big. If $\Lambda_{\mathrm{ss}}$ were of order the weak scale, Goldstinos would be very important.

If Goldstinos are present, then all superpartners $\tilde{X}$ of some particle $X$ have a decay $\tilde{X} \rightarrow X \tilde{G}$ with lifetime [3.100]:

$$
\tau=8 \pi \Lambda_{\mathrm{SS}}^{4} / \tilde{M}_{\mathrm{X}}^{2}=1.65 \times 10^{-23} \mathrm{~s} \times\left(\Lambda_{\mathrm{SS}} / \tilde{M}_{\mathrm{X}}\right)^{4}\left(1 \mathrm{GeV} / \tilde{M}_{\mathrm{X}}\right)
$$

where the mass of $\mathrm{X}$ has been neglected. Then searches at colliders can take advantage of this two-body mode to look essentially on an event-by-event basis, as described in section 3.4 for $\tilde{g} \rightarrow \mathrm{g} \tilde{\gamma}$. There is no change for searches for $\tilde{\ell}$ or $\tilde{\mathrm{q}}$ since the modes $\tilde{\ell} \rightarrow \ell \tilde{\gamma}$ or $\tilde{\mathrm{q}} \rightarrow q \tilde{\gamma}$ play the same kinematical role as $\tilde{\ell} \rightarrow \ell \tilde{\mathrm{G}}$ or $\tilde{\mathrm{q}} \rightarrow \mathrm{qG}$ at a collider. For scalar-neutrinos the mode $\tilde{\nu} \rightarrow \nu \tilde{\mathrm{G}}$, if important, would mean that the branching ratio of observable four-body modes was smaller. Finally, a massive photino would be unstable: $\tilde{\gamma} \rightarrow \gamma \tilde{\mathrm{G}}$ which could lead to interesting signatures involving hard photons.

For beam dump experiments for gluinos the results from a possible $\tilde{\mathrm{G}}$ mode have already been included. The Goldstino interacts very much like a $\tilde{\gamma}$ in the detector [3.94], by exciting a $\tilde{\mathrm{q}}$, so the kinematical situation is similar. If $A_{\text {ss }}$ were as small as the weak scale, the Goldstino interaction cross section would be somewhat larger than a $\nu$ charged current interaction.

Goldstinos can be singly or pair produced in hadronic collisions [3.41], and an amusing situation arises; some diagrams are shown in fig. 41 . Since the couplings are proportional to $\tilde{M}_{\mathrm{g}}$ (see eq. (3.59)) the production cross sections increase as $\tilde{M}_{\mathrm{g}}$ gets larger. Thus the absence of a signal puts an upper bound on $\tilde{M}_{\mathfrak{g}}$, in any theory with $\Lambda_{\mathrm{ss}}$ not too large and with massless Goldstinos. Similar contributions occur with gluons replaced by quarks in hadrons, and $\tilde{\mathrm{g}}$ by $\tilde{\mathrm{q}}$, so $\tilde{M}_{\mathrm{q}}$ will also be bounded.

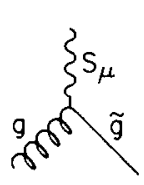

(a)

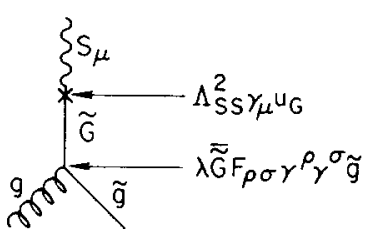

(b)

Fig. 40. The coupling of the supercurrent to gluon-gluino: (a) direct coupling; (b) coupling via the Goldstino pole.

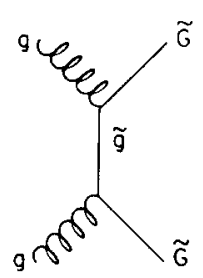

(o)

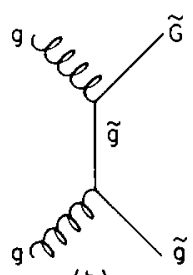

(b)
Fig. 41. Production of Goldstinos at hadronic colliders via gluongluon scattering: (a) pair production, and (b) single production in association with a gluino. Other possible diagrams not shown are possible when quark-gluon, quark-quark and quark-antiquark scattering processes are considered. 


\section{4. $W$ and $Z$ decay at the $\bar{p} p$ colliders}

Finding the $\mathrm{W}$ and $\mathrm{Z}$ intermediate vector bosons at the CERN $\bar{p} \mathrm{p}$ collider is one of the great particle physics discoveries [4.1,4.2]. Yet many hope that it represents only a beginning to the study of an entirely new energy scale. If current ideas about supersymmetry are correct, this new energy scale will be rich in new (supersymmetric) phenomena waiting to be discovered. Therefore, detailed study of the results of the CERN $\bar{p} p$ collider which is currently running at $\sqrt{s}=540 \mathrm{GeV}$ and the FERMILAB TEVATRON $I$ due to turn on at $\sqrt{s}=2000 \mathrm{GeV}$ in 1986 offer some of the best opportunities in the near future to uncover new physics.

In this section we will discuss the possibility that the $\mathrm{W}$ and $\mathrm{Z}$ bosons are decaying into new supersymmetric particles as well as into ordinary matter. Supersymmetry does not necessarily guarantee that this will occur because the new particles must have mass less than (roughly) $40 \mathrm{GeV}$. (See chapter 9 for a discussion of expectations of masses.) However, we emphasize our philosophy that the masses of the undiscovered particles are constrained only by experiment, and that independent of theoretical prejudice, one should search hard for new particles in unexplored regimes.

Consider the possible new decay modes for the $\mathrm{W}$ and $\mathrm{Z}$ in supersymmetric theories. The $\mathrm{W}$ and $\mathrm{Z}$ can decay either into a pair of scalar-leptons or scalar-quarks, or they can decay into a pair of supersymmetric fermions (charginos and/or neutralinos). The latter possibility is complicated by the mixing among charginos and neutralinos as discussed in sections 3.5 and 3.6. We will consider both of the above cases in turn. For the most part, we shall concentrate on the $\mathrm{W}$ decays for two reasons. First, W's are produced more prolifically than Z's in the p p colliders (by a factor of five). Second, the $Z^{0}$ decays will be far better studied at the $\left(\mathrm{e}^{+} \mathrm{e}^{-}\right) \mathrm{Z}^{0}$ factories SLC and LEP (see section 5.3).

\subsection{The decay of $W, Z$ into supersymmetric scalar-leptons}

The $\mathrm{W}$ and $\mathrm{Z}$ were discovered by their leptonic decay modes $\mathrm{W} \rightarrow \mathrm{e} \nu_{\mathrm{e}}$ and $\mathrm{Z}^{0} \rightarrow \mathrm{e}^{+} \mathrm{e}^{-}, \mu^{+} \mu^{-}$. The supersymmetric analogues of these decays are $\mathrm{W} \rightarrow \tilde{\mathrm{e}}_{\mathrm{L}} \tilde{\nu}_{\mathrm{e}}$ and $Z^{0} \rightarrow \tilde{\mathrm{e}}_{\mathrm{L}}^{+} \tilde{\mathrm{e}}_{\mathrm{L}}^{-}, \tilde{\mathrm{e}}_{\mathbf{R}}^{+} \tilde{\mathrm{e}}_{\mathbf{R}}^{-}$. For convenience we denote the scalar-leptons by their 'interaction eigenstates'. As discussed in section 3.1 and appendix C.1, the mass eigenstates could be mixtures of $\tilde{e}_{L}$ and $\tilde{e}_{R}$. The decay rate is easily computed as follows. To be totally general consider the following vertices:

$$
\begin{aligned}
& \mathrm{V}^{0} \mathrm{f}_{1} \overrightarrow{\mathrm{f}}_{2}: \quad \gamma_{\mu}\left(g_{\mathrm{L}} P_{\mathrm{L}}+g_{\mathrm{R}} P_{\mathrm{R}}\right), \\
& \mathrm{V}^{0} \mathrm{~A}_{1} \mathrm{~A}_{2}: \quad-i g_{\mathrm{S}}\left(p_{1}+p_{2}\right)_{\mu},
\end{aligned}
$$

where $P_{\mathrm{R}}, P_{\mathrm{L}}=\frac{1}{2}\left(1 \pm \gamma_{5}\right), \mathrm{V}=$ vector boson, $\mathrm{f}_{i}=$ fermion, $\mathrm{A}_{i}=$ complex scalar, and the incoming and outgoing momenta of the scalars are $p_{1}$ and $p_{2}$ respectively. If the fermions are taken to be massless and the scalars have mass $\tilde{M}_{i}$, then

$$
\Gamma\left(\mathrm{V}^{0} \rightarrow \mathrm{A}_{1} \overline{\mathrm{A}}_{2}\right) / \Gamma\left(\mathrm{V}^{0} \rightarrow \mathrm{f}_{1} \overline{\mathrm{f}}_{2}\right)=\left[g_{\mathrm{S}}^{2} / 2\left(g_{\mathrm{L}}^{2}+g_{\mathrm{R}}^{2}\right)\right] f\left(m_{\mathrm{v}}^{2}, \tilde{M}_{1}^{2}, \tilde{M}_{2}^{2}\right)
$$

The function $f\left(m_{\mathrm{v}}^{2}, \tilde{M}_{1}^{2}, \tilde{M}_{2}^{2}\right)$ is just the kinematical P-wave phase space suppression factor

$$
f\left(m_{\mathrm{v}}^{2}, \tilde{M}_{1}^{2}, \tilde{M}_{2}^{2}\right)=\left[\left(M_{\mathrm{v}}^{2}-\tilde{M}_{1}^{2}-\tilde{M}_{2}^{2}\right)^{2}-4 \tilde{M}_{1}^{2} \tilde{M}_{2}^{2}\right]^{3 / 2} / m_{\mathrm{v}}^{6} .
$$

For example, if we take $V^{0}$ to be a virtual photon, $g_{\mathrm{S}}=g_{\mathrm{L}}=g_{\mathrm{R}}=\mathrm{e}$, we get the familiar result that 
elementary scalars contribute $1 / 4$ of a unit of $R$ to the $\mathrm{e}^{+} \mathrm{e}^{-}$total cross section. For $\mathrm{W} \rightarrow \tilde{\mathrm{e}} \tilde{\nu}$ and $\mathrm{Z}^{0} \rightarrow \tilde{\nu} \tilde{\nu}$, $g_{\mathrm{L}}=g_{\mathrm{S}}$ and $g_{\mathrm{R}}=0$ which implies that neglecting the phase space suppression

$$
\Gamma(\mathrm{W} \rightarrow \tilde{\mathrm{e}} \tilde{\nu}) / \Gamma(\mathrm{W} \rightarrow \mathrm{e} \nu)=\Gamma\left(\mathrm{Z}^{0} \rightarrow \tilde{\nu} \bar{\nu}\right) / \Gamma\left(\mathrm{Z}^{0} \rightarrow \nu \bar{\nu}\right)=\frac{1}{2}
$$

This means that if the scalar-lepton masses are not too heavy, then there should be an observable scalar-lepton production at the p p collider. Even including mass effects, the scalar-lepton rate can be nonnegligible as shown in fig. 42.

Observation of such signals will require a detailed statistical analysis of the data. As an example, we look at $\mathrm{W} \rightarrow \tilde{e} \tilde{\nu}$ which has been discussed in refs. [4.3-4.6]. The signal depends on how the scalarneutrino decays (see section 3.2). If the scalar-neutrino decays into unobserved particles (such as $\nu \tilde{\gamma}$ ), then the signature for $\mathrm{W} \rightarrow \tilde{\mathrm{e}} \tilde{\nu}$ will be similar to $\mathrm{W} \rightarrow \mathrm{e} \nu$ events, i.e., it will consist of an isolated electron at large $p_{\mathrm{T}}$ with no associated large $p_{\mathrm{T}}$ hadronic jets. However, such events will have on average characteristic features which will distinguish them from the $\mathrm{W} \rightarrow \mathrm{e} \nu$ decays. This requires one to obtain distributions of various kinematical quantities. In the end an excess of events over the expected distributions coming from $\mathrm{W} \rightarrow \mathrm{e} \nu$ decays and other 'standard' background can be interpreted as new physics.

An example of such an analysis is given in ref. [4.6]. The strategy is to compare distributions of various kinematical quantities in the case of $\mathrm{W} \rightarrow \mathrm{e} \nu, \mathrm{W} \rightarrow \tilde{\mathrm{e}} \tilde{\nu}$ and background (for example, $\mathrm{W} \rightarrow \tau \nu, \tau \rightarrow$ e $\nu \nu$ will exhibit a similar signature). Then, experimental cuts can be selected which suppress $\mathrm{W} \rightarrow \mathrm{e} \nu$ events while affecting $\mathrm{W} \rightarrow \tilde{e} \tilde{v}$ events less. The experimentally measured quantities are the longitudinal and transverse momentum of the isolated electron $\left(p_{\mathrm{e}}^{\|}\right.$and $\left.p_{\mathrm{e}}^{\downarrow}\right)$ and the transverse momentum of the recoiling hadronic system $\left(p_{\text {had }}\right)$. If we postulate that the event we are examining is $\mathrm{W} \rightarrow \mathrm{e} \nu$, then we can determine the transverse momentum $\left(p_{\nu}^{\perp}=-p_{\mathrm{e}}^{\perp}-p_{\text {had }}^{\perp}\right)$ of the unobserved neutrino. We can also obtain the longitudinal momentum $\left(p_{\nu}^{\|}\right)$of the neutrino, but here one ends up solving a quadratic equation which leads to two solutions $p_{\nu}^{\|}( \pm)$, so that often there is an unresolved ambiguity. To be definite we choose $p_{\mathrm{m}}$ to be the solution $p_{\nu}^{\|}( \pm)$whose absolute value is minimum. One may also study the transverse mass $\left(m_{\mathrm{T}}\right)$ and the electron angle $\theta=\tan ^{-1}\left(p_{\mathrm{e}}^{\perp} / p_{\mathrm{e}}^{\|}\right)$in terms of variables already defined. Note that $p_{\nu}^{\perp}$ and $p_{\mathrm{m}}$ are well-defined variables although they lose their interpretation as neutrino momenta for processes other than $\mathrm{W} \rightarrow \mathrm{e} \nu$.

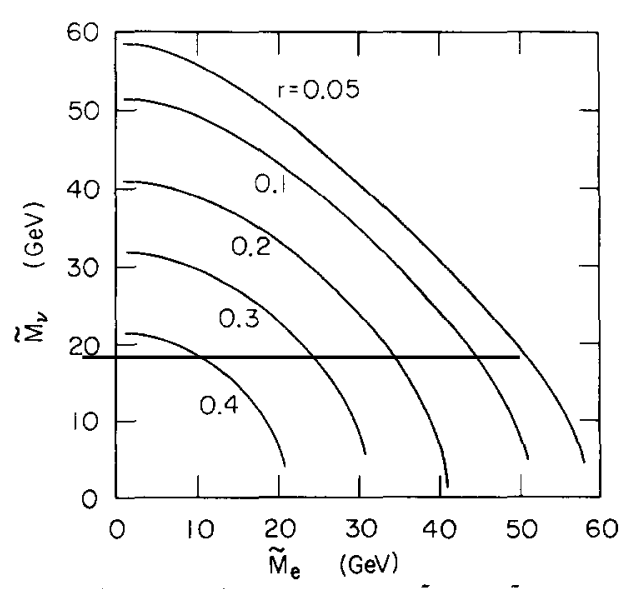

Fig. 42. Curves of constant $r$ are shown. $r \equiv \Gamma(\mathrm{W} \rightarrow \overline{\mathrm{e}} \bar{\nu}) / \Gamma(\mathrm{W} \rightarrow \mathrm{e} \nu)$ is a function of $\tilde{M}_{\nu}$ and $\bar{M}_{\mathrm{e}}$ (see eqs. (4.3)-(4.5)). There is a large range of mass parameters for which the decay of the $\mathrm{W}$ into scalar leptons would have a significant branching ratio. Since there is virtually no limit on $\tilde{M}_{\nu}$ and the limit on $\tilde{M}_{e}$ is about $22 \mathrm{GeV}$, even a value of $r=0.4$ is not yet excluded. 

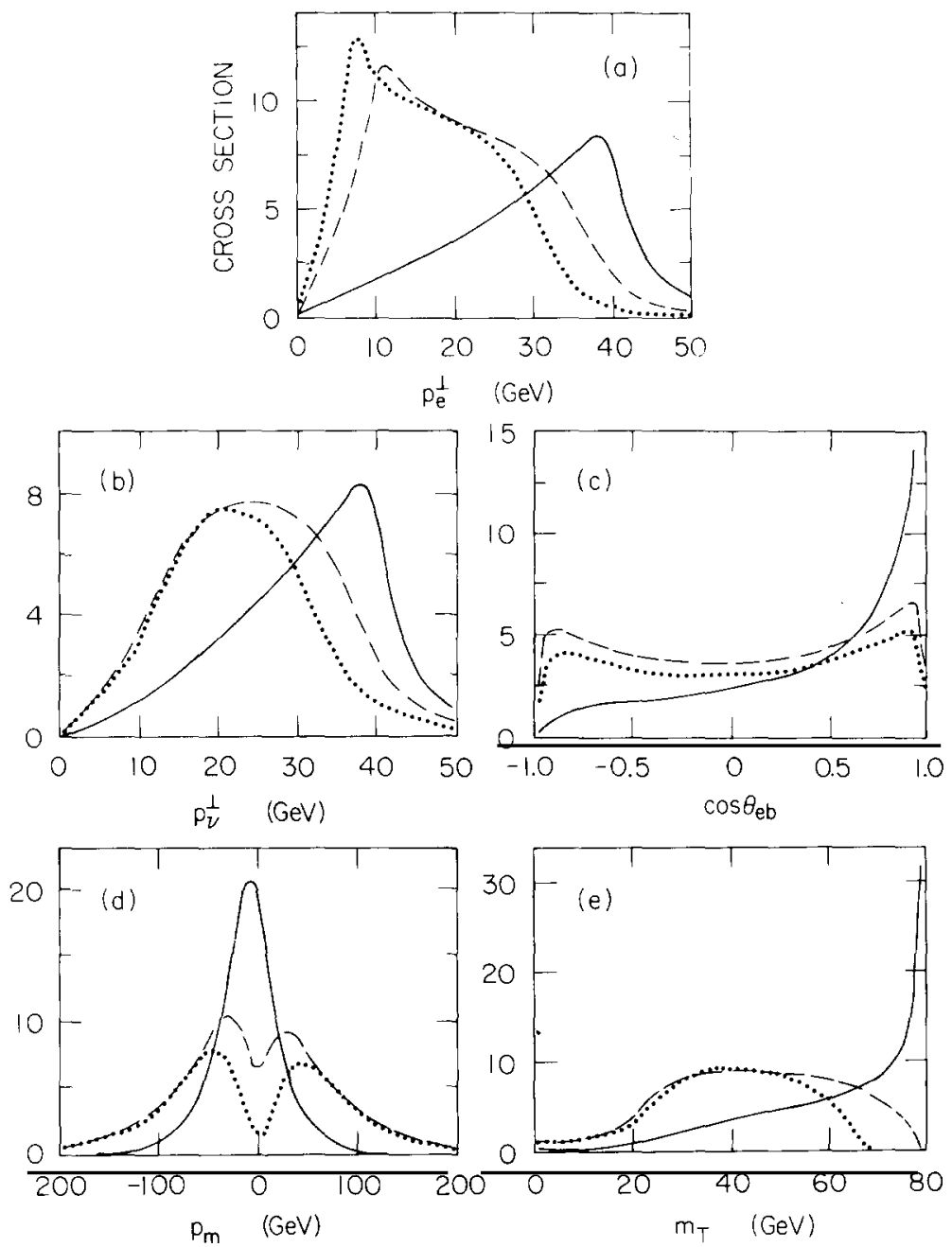

Fig. 43. The shapes of various distributions resulting from the decay of a $\mathrm{W}$ produced in $\mathrm{p} \overline{\mathrm{p}}$ collisions. We plot: (a) the transverse momentum $p_{\mathrm{e}} \frac{1}{\mathrm{e}}$ of the observed electron; (b) $p_{\nu}^{\perp}=-\left\{p_{\text {hadron }}^{\perp}+p_{\mathrm{e}}^{\perp} \mid\right.$; (c) the angle $\theta_{\mathrm{eb}}$ of the electron with respect to the proton beam axis; (d) $p_{\mathrm{m}}$ defined to be $p_{\nu}^{\|}$under the assumption that the observed decay is $\mathrm{W} \rightarrow \mathrm{e} \nu$; (e) the transverse mass, $m_{\mathrm{T}}$. The curves are normalized to equal area. In each case the solid curves refer to $\mathrm{W} \rightarrow \mathrm{e} \nu$; the two other curves refer to $\mathrm{W} \rightarrow \tilde{\mathrm{e}} \tilde{\nu}$ where $\tilde{\mathrm{e}} \rightarrow \mathrm{e} \tilde{\gamma}$. The dashed curve corresponds to $\bar{M}_{\mathrm{e}}=40 \mathrm{GeV}$ and $\tilde{M}_{\nu}=10 \mathrm{GeV}$. The dotted curve corresponds to $\tilde{M}_{\mathrm{e}}=\tilde{M}_{\nu}=30 \mathrm{GeV}$. This figure was taken from ref. [4.6].

The results of the analysis of ref. [4.6] are that the following cuts are useful. First, all events with $p_{\mathrm{e}}^{\perp}<12 \mathrm{GeV}$ are excluded. This is necessary because for low $p_{\mathrm{e}}^{\perp}$, the background (due to heavy quark semi-leptonic decays, for example) becomes significant. Second, eliminate all events which satisfy either $p_{\mathrm{e}}^{\perp}>33 \mathrm{GeV}$ or $p_{\nu}^{\perp}>35 \mathrm{GeV}$. This eliminates $\mathrm{W} \rightarrow \mathrm{e} \nu$ events in the Jacobian peak. Third, eliminate events with $\cos \theta>0.7$. This eliminates many $\mathrm{W} \rightarrow \mathrm{e} \nu$ and $\mathrm{W} \rightarrow \tau \nu$ events which are produced with $(1+\cos \theta)^{2}$ distributions* while not affecting as much $\mathrm{W} \rightarrow \tilde{\mathrm{e}} \tilde{\nu}$ events which are produced with $\sin ^{2} \theta$ distribution. Finally, the $p_{\mathrm{m}}$ distributions are quite different and can help further separate the signal from background. These results are summarized in figs. 43 and 44 . If we take $\tilde{M}_{\mathrm{e}}=\tilde{M}_{\nu}=30 \mathrm{GeV}$, we

\footnotetext{
* In making the $\cos \theta$ cut above, we assume that an electron has been detected. If a positron is being observed, simply replace $\cos \theta$ with $-\cos \theta$ in the analysis above.
} 

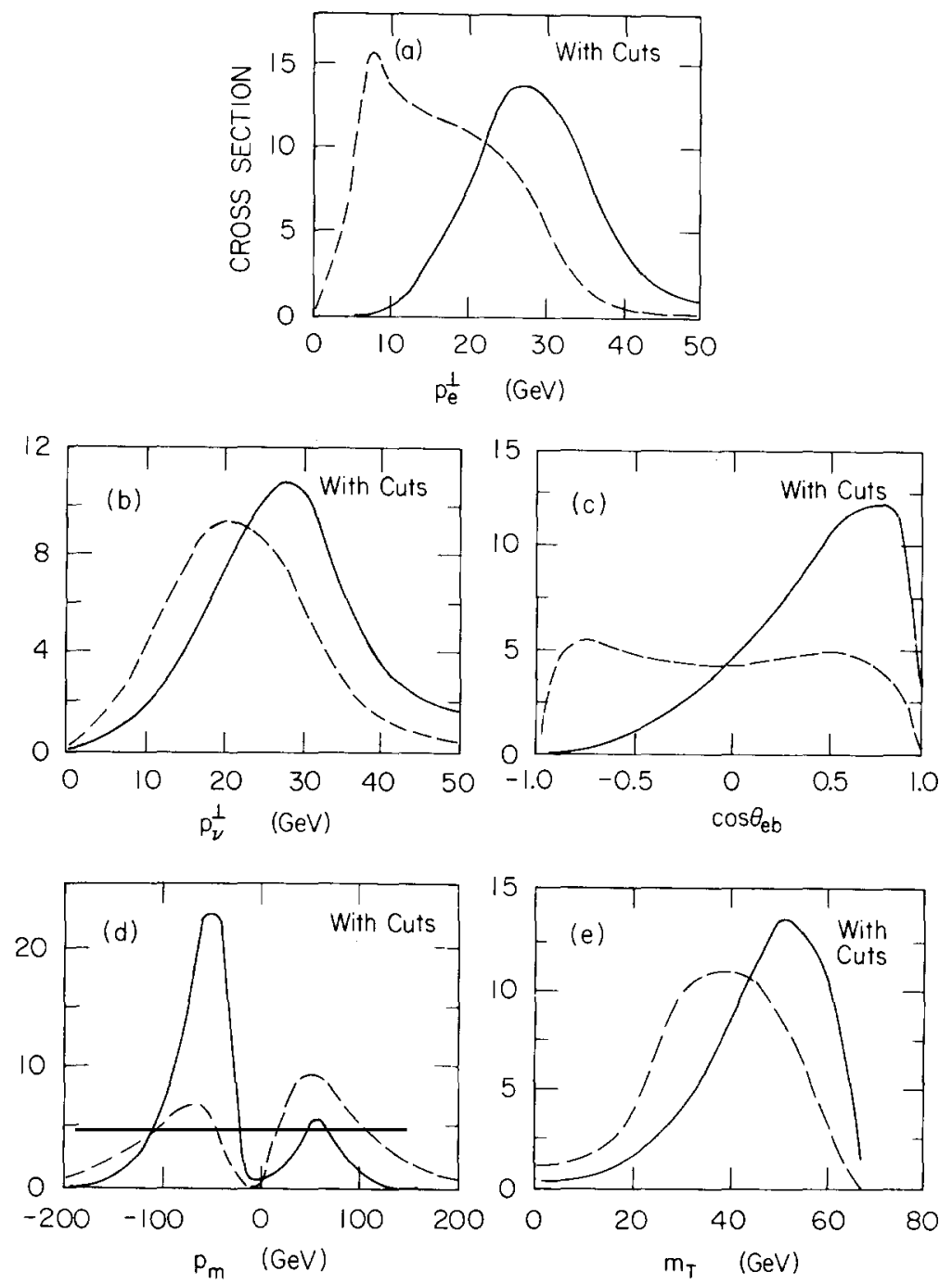

Fig. 44. The shapes of various distributions resulting from the decay of a $W$ produced in pp collisions. For notation see the caption to fig. 43 . We have eliminated all events with $p_{\mathrm{e}}^{\frac{1}{1}}>33 \mathrm{GeV}$, or $p_{\nu}^{\frac{1}{2}}>35 \mathrm{GeV}$, or $\cos \theta_{e \mathrm{~b}}>0.7$ or $-40<p_{\mathrm{m}}<20 \mathrm{GeV}$. (To be complete, we display the full range of $p_{e}^{\perp}$ despite the fact that events with $p_{e}^{\perp}>33 \mathrm{GeV}$ have been removed from the other graphs. Similar remarks hold for the other variables.) Although the overall normalization is arbitrary, the relative normalization of the two curves is fixed (this differs from the graphs of fig. 43). This figure was taken from ref. [4.6].

start with six times as many e $\nu$ events as compared to ẽ $\tilde{\nu}$ events (as shown in fig. 42). After the cuts, $90 \%$ of the e $\nu$ events are removed whereas only a third of the ẽ $\tilde{\nu}$ events are lost resulting in an equal number of events of both types surviving the cuts.

There still remain other sources of background. We have already remarked on the background from $\mathrm{W} \rightarrow \nu \tau, \tau \rightarrow \mathrm{e} \nu \nu$. (The nature of this background was first studied in refs. [4.4] and [4.7].) This background can in principle be removed since it is precisely calculable in the standard model (once the $\mathrm{W} \rightarrow \mathrm{e} \nu$ signal is measured). Furthermore, the fact that $\operatorname{BR}(\tau \rightarrow \mathrm{e} \nu \nu) \simeq 16 \%$ reduces the signal (compared to $\mathrm{W} \rightarrow \mathrm{e} \nu$ events) by a factor of six. Finally, the additional cuts, in particular the $\cos \theta$ cut, reduce the $\tau$ signal further. We conclude that the $\tau$ signal will not present difficulties in the search for $\mathrm{W} \rightarrow \tilde{\mathrm{e}} \tilde{\nu}$ 
events. One can also imagine the existence of a new heavy lepton $\mathrm{L}$ which like the $\tau$ events can mimic the single-electron events [4.7-4.9]. If $L$ behaves as a standard sequential lepton, then it could possibly be discovered in $\mathrm{W}$ decay and eventually subtracted out. However, there is always the possibility that it might have nonstandard couplings which would then mimic ẽ $\tilde{\nu}$ events. Still, it is hard to imagine a new heavy lepton $\mathrm{L}$ contributing in a substantial way to the single-electron events (due to phase space and semi-leptonic branching ratio suppressions). In any case it will be delightful to have to decide whether a new signal is a new heavy lepton or a supersymmetric particle. Thus, we conclude that despite contamination from $\tau$ 's and a possible new heavy lepton, respectable limits could be placed on $\tilde{\nu}$ and $\tilde{e}$ masses if they are not observed in W decay.

Another source of background is the production of a pair of heavy quarks both of which decay semi-leptonically. A small fraction of such events will have the energy of one quark's decay go primarily into an electron while the energy of the other quark's decay goes primarily into a neutrino. Monte Carlo studies have suggested that this background is not a problem [4.10]. In fact one can test this hypothesis experimentally by studying heavy quark pair production wherein both quarks decay semileptonically and the energy resides primarily in the charged leptons (say ee or e $\mu$ events). As long as one puts a cut on $p_{\mathrm{e}}^{\perp}$ (rejecting events with, say, $p_{\mathrm{e}}^{\perp}<12 \mathrm{GeV}$ ), we believe this source of background will be negligible.

In summary, by collecting events in $\mathrm{p} \overline{\mathrm{p}}$ collisions containing high $p_{\mathrm{T}}$ isolated electrons with no accompanying high $p_{\mathrm{T}}$ hadronic jet, one can eventually set limits on $\tilde{\mathrm{e}}$ and $\tilde{\nu}$ masses if no supersymmetric events are seen. For example, with a sample of $300 \mathrm{~W} \rightarrow \mathrm{e} \nu$ type events it should be possible to set limits on the region in fig. 42 where $r \geq \frac{1}{6}$.

We have concentrated in this section on ẽ $\tilde{\nu}$ decays where the $\tilde{\nu}$ decays invisibly. Clearly, similar conclusions are obtained for $\tilde{\mu} \tilde{\nu}_{\mu}$ events if the same assumptions are made (namely, $\tilde{\mu} \rightarrow \mu \tilde{\gamma}$ and the $\tilde{\nu}_{\mu}$ decays invisibly). On the other hand, as discussed in section 3.2 , it is possible that the $\tilde{\nu}$ will decay dominantly into charged jets (this will certainly be true if $\tilde{M}_{\mathrm{e}}<\tilde{M}_{\nu}$ ). Observing the supersymmetric signal is more problematical. In this case the e $\tilde{\nu}$ events would appear most often as an isolated electron recoiling against a hadronic jet, and occasionally recoiling against a spray of multi-leptons. In the former case it would be difficult to separate these events from heavy quark decays. The latter case should be more spectacular and perhaps immediately identifiable, but it will be suppressed by small semileptonic branching ratios. In both cases, the missing energies (due to neutrinos or photinos) is small due to the four-body decay of the scalar-neutrino. It should be noted that the scenario above requires a fairly light scalar-electron (we assume here that $\tilde{M}_{\nu}+\tilde{M}_{\mathrm{e}}<m_{\mathrm{w}}$, otherwise no ẽ $\tilde{\nu}$ events are possible).

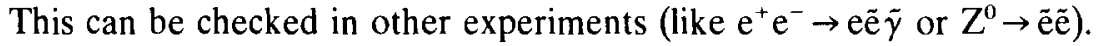

Finally, for completeness, we mention supersymmetric scalar production from $Z^{0}$ decay. The main problem here is that the $\mathrm{Z}^{0}$ production rate is about a factor of five less than the rate for $\mathrm{W}$ production. Hence, it is unlikely that any strong conclusions can be made before SLC and LEP turn on. (See section 5.3 for a discussion of $Z^{0}$ decay at $\mathrm{e}^{+} \mathrm{e}^{-}$machine.) In ref. [4.11], the possibility of observing $Z^{0} \rightarrow \tilde{e} e \tilde{~ i s ~}$ discussed. One looks for $\mathrm{p} \overline{\mathrm{p}} \rightarrow \mathrm{e}^{+} \mathrm{e}^{-} \mathrm{X}$ where substantial missing energy (due to missing photinos) occurs. It is argued that a priori, $Z^{0} \rightarrow$ ẽẽ events would be swamped by Drell-Yan events since $\tilde{\mathrm{e}} \rightarrow \mathrm{e} \tilde{\gamma}$ with the photino escaping leads to the appearance of $\mathrm{e}^{+} \mathrm{e}^{-}$pairs at invariant mass substantially below $m_{z}$. One can in principle make use of the missing (photino) transverse energy signal to eliminate most of the Drell-Yan events. Similar to the situation in W decay, one must still contend with backgrounds which in this case include $\tau^{+} \tau^{-}$Drell-Yan and double semi-leptonic decay of heavy quarks, both of which can result in substantial missing energy. The conclusion of ref. [4.11] is that a search for scalar-electrons with mass below $40 \mathrm{GeV}$ is feasible, but one will need dedicated running at the $p \bar{p}$ collider with more than $100 \mathrm{e}^{+} \mathrm{e}^{-}$events at the $\mathrm{Z}$ peak. 
Alternatively, one can look for $Z^{0} \rightarrow \tilde{\nu} \overline{\tilde{\nu}}$ events. Here, one needs the parameters of the model to be such that the $\tilde{\nu}$ decays fairly often into charged hadronic final states. Then it is possible that one can observe $\mathrm{Z}^{0} \rightarrow \tilde{\nu} \overline{\tilde{\nu}}, \overline{\tilde{\nu}} \rightarrow \bar{\nu} \tilde{\gamma}, \tilde{\nu} \rightarrow$ hadronic jets. This event would be quite spectacular as it would contain some large $p_{\mathrm{T}}$ jets close together and a large amount of missing energy (i.e. a one-sided event if the jets are energetic). The obvious background to this is the production of a $Z^{0}$ accompanied by a large $p_{\mathrm{T}}$ hadronic (gluon) jet followed by $Z^{0} \rightarrow \nu \bar{\nu}$. However, this background could be estimated precisely by observing similar events where $\mathrm{Z}^{0} \rightarrow \mathrm{e}^{+} \mathrm{e}^{-}$. On the other hand, if the $\tilde{\nu}$ decays entirely into four-body final states, separation from background (say $Z^{0} \rightarrow$ quark jets) will be extremely difficult. It is clear, however, that the high luminosity $Z^{0}$ factories will play a much larger role in uncovering nonstandard physics at the $Z^{0}$.

\subsection{The decay of $W, Z$ into supersymmetric fermions}

As discussed in sections 3.5 and 3.6, the $\mathrm{W}$ and $\mathrm{Z}$ can decay into pairs of charginos and neutralinos. This has also been discussed extensively in refs. [4.5, 4.7, 4.12-4.18, 4.24-4.25]. The main complication here is that the unknown mass matrices introduce too many presently unknown parameters to allow quantitative predictions. Nevertheless, it is worth surveying the various possibilities since these decay modes could be visible at the CERN pp collider.

We shall denote the two lightest neutralinos by $\tilde{\chi}_{1}^{0}$, and $\tilde{\chi}_{2}^{0}\left(\tilde{M}_{\chi_{1}}<\tilde{M}_{\chi_{2}}\right)$. Then, the possible decay modes of interest are:

$$
\mathrm{W}^{ \pm} \rightarrow \tilde{\chi}^{ \pm}+\tilde{\chi}_{1}^{0}, \quad \mathrm{~W}^{ \pm} \rightarrow \tilde{\chi}^{ \pm}+\tilde{\chi}_{2}^{0}, \quad \mathrm{Z}^{0} \rightarrow \tilde{\chi}_{1}^{0}+\tilde{\chi}_{2}^{0}, \quad \mathrm{Z}^{0} \rightarrow \tilde{\chi}^{+}+\tilde{\chi}^{-}
$$

The decay rates for each process above are dependent on the elements of the mass matrices discussed in appendix $\mathrm{C}$. To calculate the decay rates we shall write the following general vertices (using the notation of ref. [4.16]):

$$
\begin{array}{ll}
\mathrm{W}^{+} \chi_{i}^{-} \tilde{\chi}_{j}^{0}: & g \gamma_{\mu}\left(O_{i j}^{\mathrm{L}} P_{\mathrm{L}}+O_{i j}^{\mathrm{R}} P_{\mathrm{R}}\right) \\
\mathrm{Z}^{0} \tilde{\chi}_{i}^{+} \tilde{\chi}_{j}^{-}: & \left(g^{2}+g^{\prime 2}\right)^{1 / 2} \gamma_{\mu}\left(O_{i j}^{\prime L} P_{\mathrm{L}}+O_{i j}^{\prime \mathrm{R}} P_{\mathrm{R}}\right) \\
\mathrm{Z}^{0} \tilde{\chi}_{i}^{0} \tilde{\chi}_{j}^{0}: & \left(g^{2}+g^{\prime 2}\right)^{1 / 2} \gamma_{\mu}\left(O_{i j}^{\prime \prime} P_{\mathrm{L}}+O_{i j}^{\prime \prime} \mathrm{R} P_{\mathrm{R}}\right)
\end{array}
$$

where $P_{\mathrm{R}}, P_{\mathrm{L}}=\frac{1}{2}\left(1 \pm \gamma_{5}\right)$ and the various coefficient factors $\left(O^{\mathrm{L}}, O^{\mathrm{R}}\right.$, etc. $)$ are defined in terms of the mass matrices in appendix $C$. ${ }^{*}$ The decay rates have been computed in refs. [4.5, 4.7,4.12-4.18]. If we normalize to $\Gamma(\mathrm{W} \rightarrow \mathrm{e} \nu)=g^{2} m_{\mathrm{w}} / 48 \pi$ and $\Gamma\left(\mathrm{Z}^{0} \rightarrow \nu \bar{\nu}\right)=\left(g^{2}+g^{\prime 2}\right) m_{\mathrm{z}} / 96 \pi$, we find [4.16]:

$$
\frac{\Gamma\left(\mathrm{W}^{+} \rightarrow \tilde{\chi}_{i}^{+} \tilde{\chi}_{i}^{0}\right)}{\Gamma\left(\mathrm{W}^{+} \rightarrow \mathrm{e}^{+} \nu\right)}=\frac{\left(m_{\mathrm{w}}^{2}-\tilde{M}_{\chi^{+}}^{2}\right)\left(2 m_{\mathrm{w}}^{4}-m_{\mathrm{w}}^{2} \tilde{M}_{\chi^{+}}^{2}-\tilde{M}_{\chi^{+}}^{4}\right)}{m_{\mathrm{w}}^{6}} \times\left[\left(O_{i j}^{\mathrm{L}}\right)^{2}+\left(O_{i j}^{\mathrm{R}}\right)^{2}\right]
$$

where we have taken $\tilde{M}_{\chi^{0}}=0$ in eq. (4.10) for simplicity and

\footnotetext{
${ }^{*}$ The factor of $1 / 2$ which appears in the interaction Lagrangian (eq. (C.87)) does not appear in the Feynman rule (4.9) because the $\bar{\chi}_{i}^{0}$ are Majorana fermions.
} 


$$
\frac{\Gamma\left(\mathrm{Z}^{0} \rightarrow \tilde{\chi}_{i}^{+} \tilde{\chi}_{i}^{-}\right)}{\Gamma\left(\mathrm{Z}^{0} \rightarrow \nu \bar{\nu}\right)}=4\left(1-\frac{4 M_{x}^{2}}{m_{\mathrm{z}}^{2}}\right)^{1 / 2}\left[\left(1-\frac{\tilde{M}_{x}^{2}}{m_{\mathrm{z}}^{2}}\right)\left[\left(O_{i j}^{\prime \mathrm{L}}\right)^{2}+\left(O_{i j}^{\prime \mathrm{R}}\right)^{2}\right]+\frac{6 \tilde{M}_{x}^{2}}{m_{\mathrm{z}}^{2}} O_{i j}^{\prime \mathrm{L}} O_{i j}^{\prime \mathrm{R}}\right]
$$

(not summed over $i, j$ ). For $\Gamma\left(\mathrm{Z}^{0} \rightarrow \tilde{\chi}^{0} \tilde{\chi}^{0}\right)$, simply replace $O^{\prime}$ with $O^{\prime \prime}$ in eq. (4.11) and insert a factor of $1 / 2$ because the final state neutralinos are identical.

It is also useful to compute the angular distribution of the outgoing fermion in $\overline{\mathrm{q}}_{i} \mathrm{q}_{j} \rightarrow \tilde{\chi}_{i} \tilde{\chi}_{j}$ to be compared with the $(1 \pm \cos \theta)^{2}$ distribution for fermions with a pure V-A interactions and the $\sin ^{2} \theta$ distribution for scalars. Some of these distributions (and the resulting asymmetries) have been computed in refs. [4.7, 4.14, 4.17, 4.18].

The main conclusions we wish to draw are: (a) the decay rates of the supersymmetric channels can easily be comparable to that of the standard leptonic channels, and (b) the angular distributions in the $\mathrm{W}$ or $\mathrm{Z}$ rest frames need not resemble either massless fermions or scalars. One exception to this remark involves the decay $\mathrm{Z}^{0} \rightarrow \tilde{\chi}_{i}^{0}+\tilde{\chi}_{j}^{0}$. If one thinks in the $(\tilde{\gamma}, \tilde{\mathrm{H}})$ basis, then it is clear that $\Gamma\left(\mathrm{Z}^{0} \rightarrow \tilde{\gamma} \tilde{\gamma}\right) \ll$ $\Gamma\left(\mathrm{Z}^{0} \rightarrow \tilde{\gamma} \tilde{\mathrm{H}}\right) \ll \Gamma\left(\mathrm{Z}^{0} \rightarrow \tilde{\mathrm{H}} \tilde{\mathrm{H}}\right)$. Therefore, some of the rates for $\mathrm{Z}^{0}$ decay into neutralinos might be suppressed unless the lightest neutralinos had large higgsino components.

The cleanest signature of the reactions given by eq. (4.6) occurs when substantial energy is carried off by the lightest neutralino, $\tilde{\chi}_{1}^{0}$. As a result, let us focus on the two decays $W^{ \pm} \rightarrow \tilde{\chi}^{ \pm}+\tilde{\chi}_{1}^{0}$ and $Z^{0} \rightarrow \tilde{\chi}_{1}^{0}+\tilde{\chi}_{2}^{0}$. The signature of these decays will be determined by the branching ratios of the various $\tilde{\chi}^{ \pm}$and $\tilde{\chi}_{2}^{0}$ decays (discussed in sections 3.5 and 3.6 respectively). We shall assume here that the dominant decay of $\tilde{\chi}^{ \pm}$and $\tilde{\chi}_{2}^{0}$ consists of hadronic energy (in the form of jets if the masses are light enough) along with $\tilde{\chi}_{1}^{0}$ which escapes. Occasionally, one will find purely leptonic decays. These will be particularly useful as only a single lepton will be observable with the rest of the energy of the event deposited in unobserved photinos and neutrinos. Thus, we expect two classes of signals from these events, with both types of signals showing large energy-momentum imbalance. The first type of event will have an isolated electron and no visible large $p_{\mathrm{T}}$ hadronic matter. These events will resemble $\mathrm{W} \rightarrow \mathrm{e} \nu$ (and $\mathrm{W} \rightarrow \tilde{\mathrm{e}} \tilde{\nu}$ discussed in the previous section). Considerable analysis on the background coming from $\mathrm{W} \rightarrow \mathrm{e} \nu$ and $\mathrm{W} \rightarrow \tau \nu$ for this class of events has been done in ref. [4.7], but as yet we are not aware of any analysis of the major background from heavy quark semi-leptonic decay.

The second type of event will have no observable leptons but unbalanced hadronic matter. This is likely to be the dominant signal since the branching ratio of $\tilde{\chi}^{+}$and $\tilde{\chi}_{2}^{0}$ into quarks and gluinos is likely to be significant. In addition, this type of event is what we previously called the 'hallmark of supersymmetry' - namely substantial missing energy-momentum with no observable lepton. Perhaps the cleanest signal will be the channel where $\tilde{\chi}_{1}^{0}$ escapes and $\tilde{\chi}^{ \pm} \rightarrow q \bar{q} \tilde{\chi}_{1}^{0}$, so only a $q \bar{q}$ pair appears on one side of the central region (these are the 'zen' events of ref. [4.14]). Even in this case there will be serious Standard Model background which has not yet been estimated. Nevertheless, this is a major hope for finding a signal of supersymmetry in the near future.

The other decays listed in eq. (4.6) do not exhibit such a clear signal. In these events the amount of missing energy is far less and could only be detected by statistical averaging. In addition, these signatures are likely to be masked by large Standard Model backgrounds. If supersymmetric decays of the $\mathrm{W}$ and $\mathrm{Z}$ are discovered elsewhere, these types of events may eventually serve to check the theory and help measure parameters of the mass matrix.

\subsection{Comments on $W$ and $Z$ decay into supersymmetric particles}

It is interesting to note that the background for some of the supersymmetric signals consists of other 


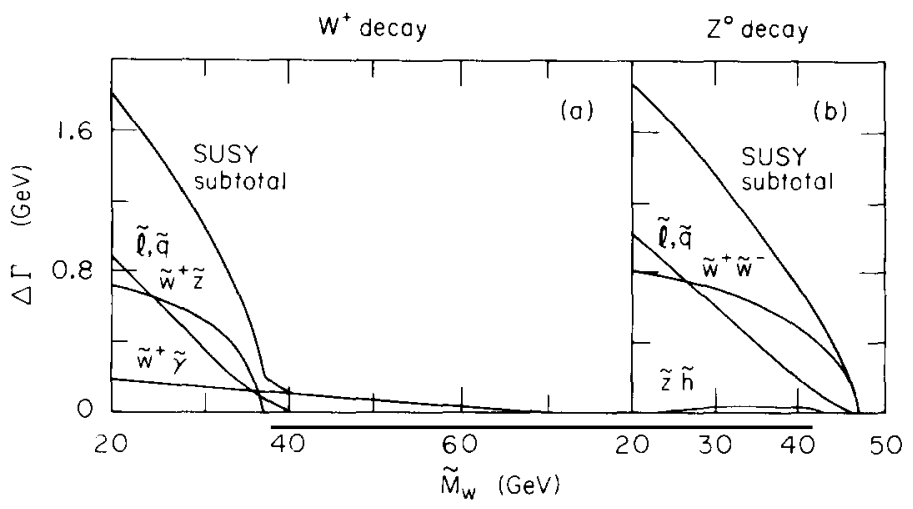

Fig. 45. Supersymmetric contributions $(\Delta \Gamma)$ to the $\mathrm{W}$ and $\mathrm{Z}$ decay widths are shown in (a) and (b) respectively. The partial contributions of various supersymmetric modes are shown. Both graphs are plotted as a function of the $\bar{w}$ mass (denoted by $\tilde{M}_{\mathrm{w}}$ ). The $\tilde{z}$ and $\tilde{h}$ masses were taken to be equal to the $\tilde{\mathrm{w}}$ mass and $\tilde{\ell}$ and $\overline{\mathrm{q}}$ masses were assumed to be given by $\tilde{M}_{\mathrm{f}}=\left(\tilde{M}_{\mathrm{w}}^{2}+m^{2}\right)^{1 / 2}, \mathrm{f}=\ell, \mathrm{q}$. The top quark mass was chosen to be $35 \mathrm{GeV} / c^{2}$. This figure was taken from ref. [4.17].

supersymmetric signals. For example, $\mathrm{W}^{ \pm} \rightarrow \tilde{\chi}^{ \pm}+\tilde{\chi}_{1}^{0}$ with $\tilde{\chi}^{ \pm}$decaying leptonically resembles $\mathrm{W}^{ \pm} \rightarrow \tilde{\mathrm{e}}^{ \pm} \tilde{\nu}$ [4.25] and $Z^{0} \rightarrow \tilde{\chi}_{1}^{0}+\tilde{\chi}_{2}^{0}$ with $\tilde{\chi}_{2}^{0}$ decaying into hadron jets resembles $Z^{0} \rightarrow \tilde{\nu} \bar{\nu}$ with one $\tilde{\nu}$ decaying invisibly and the other decaying into $\nu+$ hadronic jets. However, in principle, one could separate these types of events from each other if the statistical sample were large enough. One would need to make use of fairly detailed Monte Carlos and distinguish differences in various distributions. For example, the decay of supersymmetric fermions is not likely to occur with a $\sin ^{2} \theta$ distribution.

If supersymmetric particles are a major contribution to $\mathrm{W}$ and $\mathrm{Z}$ decays, then the total widths of the $\mathrm{W}$ and $\mathrm{Z}$ could be substantially larger than predicted by the standard model. This is demonstrated by fig. 45 taken from ref. [4.17] where a simple choice of chargino and neutralino mass matrix parameters has been made. It might be possible to detect this effect at the $p \bar{p}$ collider. The $Z^{0}$-width measurement at SLC or LEP will be an important constraint on supersymmetry.

One can also compute radiative corrections to the weak boson masses due to the virtual effects of supersymmetric particles. The mass shifts were computed by Grifols and Sola [4.26]; they find that the shift can be at most a few hundred $\mathrm{MeV}$. A measurement sensitive to such a shift is unlikely at a hadron machine but could be accomplished at a $Z^{0}$ factory.

One supersymmetric decay of the $Z^{0}$ which we briefly mentioned in section 3.4 is $Z^{0} \rightarrow \tilde{\mathrm{g}} \tilde{\mathrm{g}}$. This process occurs at one loop as shown in fig. 32, and its rate is expected to be small. Calculations $[4.19,4.20]$ show that for a reasonable value for unknown mass parameters (gluino and scalar-quark masses and the t-quark mass) one finds a branching ratio in the $10^{-5}$ to $10^{-4}$ range. Unfortunately, identifying the final state as $\tilde{g} \tilde{g}$ will be especially difficult, particularly in view of the rarity of the decay. Finally, a related process which may offer some additional possibilities is $Z^{0} \rightarrow q \bar{q} \tilde{g} \tilde{g}$ where the gluino pair is emitted from a bremsstrahlung gluon [4.21,4.22]. The decay rate into this mode is sensitive to the gluino mass as shown in fig. 46. The final state again is difficult to isolate but for light gluino masses the branching ratio could be on the order of $1 \%$ leading to many such events at the $Z^{0}$ factories.

It is important to realize that it is not guaranteed that any of the supersymmetric decays of the $\mathrm{W}$ and $\mathrm{Z}$ discussed above are kinematically allowed. Many models do suggest that at least some of the supersymmetric masses will be lighter than the $\mathrm{W}$ and $\mathrm{Z}$ so as to permit at least some of the decays. However, one can certainly choose parameters in such a way that the various mass matrices lead to only one light neutralino (the $\tilde{\gamma}$ ) or even no supersymmetric particles lighter than the $\mathrm{W}$ and $\mathrm{Z}$ [4.23]. This 


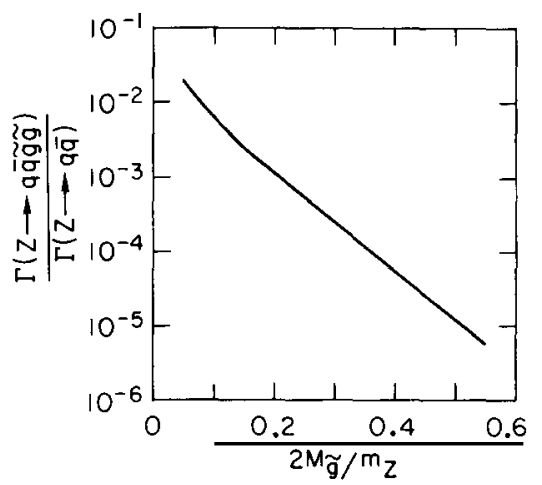

Fig. 46. The rate for $Z^{0} \rightarrow q \bar{q} \tilde{g} \tilde{g}$ relative to $Z^{0} \rightarrow q \bar{q}$ vs. gluino mass. (Figure taken from refs. $[4.21,4.22]$ ).

would be unfortunate, but there is no theorem which prevents it. The true tests to rule out supersymmetry at the weak scale will have to be carried out at a higher energy accelerator.

\section{Supersymmetry in $\mathrm{e}^{+} \mathrm{e}^{-}$(and related) physics}

There are three different areas in $\mathrm{e}^{+} \mathrm{e}^{-}$physics where one might find evidence for supersymmetry. First, in continuum $\mathrm{e}^{+} \mathrm{e}^{-}$scattering, one may produce new supersymmetric particles in pairs or observe their virtual effects. Second, one can study the decays of quarkonium systems $(\psi, Y, \ldots)$ for new phenomena. Third, one can study decays of the $Z^{0}$ at one of the future $Z^{0}$ factories (SLC or LEP). The advantage of the latter two is that the resonance enhances the production cross section for new physics. The disadvantage is that the resonance occurs at a fixed energy and precludes discovering phenomena which are accessible only at higher energy. On the other hand, the luminosity is of crucial importance. For example, for center-of-mass energies above the $\mathrm{Z}^{0}$ one will need $\mathrm{e}^{+} \mathrm{e}^{-}$machines with luminosity $>$ $10^{31} \mathrm{~cm}^{-2} \mathrm{~s}^{-1}$ in order to have any chance of seeing new physics (roughly 1000 events per year per unit of $R$ at $E_{\mathrm{cm}}=100 \mathrm{GeV}$ ).

\subsection{Continuum $e^{+} e^{-}$production of supersymmetry}

Many of the processes that follow have already been discussed in detail in other sections of this review, so we will just briefly summarize the relevant results here.

(a) $\mathrm{e}^{+} \mathrm{e}^{-} \rightarrow \tilde{\ell}^{+} \tilde{\ell}^{-}, \tilde{\ell} \rightarrow \ell \tilde{\gamma} \quad[5.1-5.4]$

These events are distinguished by the following properties. The total $\mathrm{e}^{+} \mathrm{e}^{-}$cross section increases by $1 / 4$ unit of $R$, but due to the P-wave threshold (i.e. a factor of $\beta^{3}$ ) the cross section increases slowly to its asymptotic value. A substantial amount of energy can be lost due to the escaping photinos. The

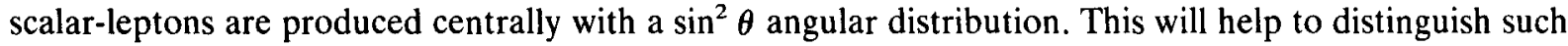
events from the production of a new heavy lepton.

The angular distribution can change somewhat in the particular case of $\mathrm{e}^{+} \mathrm{e}^{-} \rightarrow \tilde{\mathrm{e}}^{+} \tilde{\mathrm{e}}^{-}$where $\tilde{\gamma}$ exchange can also contribute (see eq. (3.4)). If the photino mass is nonzero, one finds an enhancement of the cross section near threshold (i.e. a term proportional to $\beta$ ) proportional to $\tilde{M}_{\gamma}^{2}$ which in principle 
could lead to an estimation of the photino mass [5.3,5.4]. Furthermore, as argued in ref. [5.3], with polarized beams one would have one further handle on the photino mass due to the contribution of the t-channel photino exchange to $\tilde{\mathrm{e}}_{\mathrm{L}}^{+} \tilde{\mathrm{e}}_{\mathrm{R}}^{-}$and $\tilde{\mathrm{e}}_{\mathrm{R}}^{+} \tilde{\mathrm{e}}_{\mathrm{L}}^{-}$scattering (which would vanish in the $\tilde{M}_{y}=0$ limit).

At center-of-mass energies approaching $m_{z}$, one must include $Z^{0}$ exchange and zino exchange (the latter only for $\mathrm{e}^{+} \mathrm{e}^{-} \rightarrow \tilde{\mathrm{e}}^{+} \tilde{\mathrm{e}}^{-}$) as remarked above eq. (3.2) [5.2].

(b) $\mathrm{e}^{+} \mathrm{e}^{-} \rightarrow \tilde{\mathrm{e}} \tilde{\gamma}, \tilde{\mathrm{e}} \rightarrow \mathrm{e} \tilde{\gamma} \quad[5.5-5.7]$

The final state of $\mathrm{e}^{+} \mathrm{e}^{-} \tilde{\gamma} \tilde{\gamma}$ is similar to case (a) above. (See eq. (3.5) and the discussion therein.) The advantage of this process is that it can be observed at energies below the $\tilde{\mathrm{e}}^{+} \tilde{\mathrm{e}}^{-}$threshold. See fig. 3 for the comparison of this process with process (a).

(c) $\mathrm{e}^{+} \mathrm{e}^{-} \rightarrow \mathrm{e}^{+} \mathrm{e}^{-} \tilde{\gamma} \tilde{\gamma}$

The direct production of the $\mathrm{e}^{+} \mathrm{e}^{-} \tilde{\gamma} \tilde{\gamma}$ state through two virtual scalar-electrons has a very small cross section but could be observable. (See eq. (3.6) and remarks therein.)

(d) $\mathrm{e}^{+} \mathrm{e}^{-} \rightarrow \tilde{\gamma} \tilde{\gamma} \gamma \quad[5.8-5.11,5.43,5.61], \mathrm{e}^{+} \mathrm{e}^{-} \rightarrow \tilde{\nu} \bar{\nu} \gamma$

For light photinos or scalar-neutrinos, the cross section could be observable even for scalar-electron masses much larger than the beam energy. This has been shown in fig. 3. The extra photon is used to tag the event since the photino or $\tilde{\nu}$ is assumed to be invisible. The Standard Model background $\mathrm{e}^{+} \mathrm{e}^{-} \rightarrow \nu \bar{\nu} \gamma$ will have a similar signature and a background subtraction will be required. Section 3.1 has an extensive discussion of these issues.

(e) $\mathrm{e}^{+} \mathrm{e}^{-} \rightarrow \tilde{\chi}_{i}^{0} \tilde{\chi}_{j}^{0} \quad[5.12-5.16]$

We consider here the more general case of neutralino production. We assume that at least one (or both) final state neutralinos decays. The decay branching ratios are highly model dependent. Two classes of decays will be $\tilde{\chi}_{i}^{0} \rightarrow \tilde{\chi}_{i}^{0} \ell^{+} \ell^{-}$involving only observable leptons and $\tilde{\chi}_{i}^{0} \rightarrow \tilde{\mathrm{g}} q \bar{q}$ which will be observed as hadronic jets. Such events all have substantial missing energy (with two stable neutralinos eventually escaping detection). In addition, one can have spectacular multi-lepton events if both neutralinos decay. If the hadronic mode dominates, the most observable signature will consist of events with one neutralino decay producing hadronic jets (with no observed leptons) in one hemisphere and an absence of particles in the opposite hemisphere. For more details see section 3.6.

(f) $\mathrm{e}^{+} \mathrm{e}^{-} \rightarrow \tilde{\chi}^{+} \tilde{\chi}^{-} \quad[5.12-5.16]$

Charginos can be produced in $\mathrm{e}^{+} \mathrm{e}^{-}$by directly coupling to the virtual photon. The charginos then decay into neutralinos in a one or two step process depending in part on how many neutralinos there are which are lighter than $\tilde{\chi}^{+}$. In general, the signature of $\tilde{\chi}^{+} \tilde{\chi}^{-}$events will resemble $\tilde{\chi}_{i}^{0} \tilde{\chi}_{j}^{0}$ events where both neutralinos decay. However, more complicated events involving long decay chains are possible. For more details see section 3.5.

(g) $\mathrm{e}^{+} \mathrm{e}^{-} \rightarrow \tilde{\nu} \overline{\tilde{\nu}} \quad[5.17]$

The signature of such events depends on the scalar-neutrino branching ratios. For such events to be detected, we need a substantial $\tilde{\nu}$ branching ratio into four-body modes involving charged particles. In that case $\tilde{\nu} \tilde{\nu}$ events could very well resemble $\tilde{\chi}_{i}^{0} \tilde{\chi}_{j}^{0}$ events discussed in case (e) above. However, although the $\tilde{\chi}_{i}^{0} \tilde{\chi}_{j}^{0}$ angular distributions will in general be model dependent, the $\tilde{\nu} \overline{\tilde{\nu}}$ events are predicted to occur with a (nearly) $\sin ^{2} \theta$ distribution. Careful Monte Carlos could distinguish between these events. 
(h) $\mathrm{e}^{+} \mathrm{e}^{-} \rightarrow \tilde{\mathrm{e} e} \tilde{\chi}_{i}^{0}, \tilde{\nu} \mathrm{e}^{+} \tilde{\chi}^{+} \quad[5.6]$

If the energies are sufficiently high, it should be possible to pair produce neutralinos and charginos in association with scalar-leptons. The various possible signatures are immense due to the many possible decay chains. The cross sections computed in ref. [5.6] are not very encouraging. Unless the masses of the supersymmetric particles are not too heavy (say, $\leqslant 40 \mathrm{GeV}$ ), it would be difficult to produce an observable number at LEP. On the other hand if the masses are small enough, they will probably be discovered in some of the previous processes considered here.

(i) $\mathrm{e}^{+} \mathrm{e}^{-} \rightarrow \tilde{\mathrm{q}} \overline{\tilde{\mathrm{q}}}, \tilde{\mathrm{q}} \rightarrow \mathrm{q} \tilde{\mathrm{g}}, \mathrm{q} \tilde{\gamma} \quad[5.2,5.18,5.58]$

Many of the remarks made in case (a) are relevant here. However, observation of $\overline{\mathrm{q}} \overline{\tilde{q}}$ (compared to $\tilde{\ell} \overline{\tilde{\ell}})$ is far more difficult. If the gluino is light, the $\tilde{\mathrm{q}} \rightarrow \tilde{\mathrm{q}} \tilde{\mathrm{g}}$ decay will dominate resulting in only a small fraction of missing energy when the gluino decays. The final state in that case will consist of $q \bar{q} \tilde{g} \tilde{g} \rightarrow(q \bar{q})(q \bar{q})(q \bar{q}) \tilde{\gamma} \tilde{\gamma}$. It might be possible to identify such events by studying a four-jet sample. Here one depends on being able to distinguish $q \bar{q} \tilde{g} \tilde{g}$ from $q \bar{q} g g$. The $\sin ^{2} \theta$ distribution of the $\tilde{q}$ might assist in the separation. One can also look for changes in the two-jet angular distribution and the energy-energy correlation due to the production of a pair of scalars [5.58]. Still, it is clear that very complicated Monte Carlo methods will be needed. For these events it will probably be easier to rule out scalar-quarks in a certain mass range than to detect their presence.

Scalar-quarks can bind together to form onium-type systems (called scalarium in ref. [5.18]). These states could show up as narrow resonances in $\mathrm{e}^{+} \mathrm{e}^{-}$annihilation. Various properties of these states have been worked out in ref. [5.18]. Unfortunately, they are likely to be difficult to observe. The reason is that in order to couple to a virtual photon, the scalarium state must be a p-wave bound state. Its coupling is suppressed due to the appearance of the derivative of the wavefunction [5.18]

$$
\Gamma\left(2 \mathrm{P} \rightarrow \mathrm{e}^{+} \mathrm{e}^{-}\right)=24 \alpha^{2} e_{\mathrm{q}}^{2}\left|R_{\mathrm{p}}^{\prime}(0)\right|^{2} / \tilde{M}^{4},
$$

where $\tilde{M}$ is the mass of the bound state. As a result, nonobservation of such states leads to weak limits on possible scalar quark masses as discussed in section 3.3. One interesting note concerns the possibility (suggested by eq. (8.8)) that the scalar-quark flavors could be very close in mass. In this circumstance, when the scalar-quark threshold is passed, one will find that multiple flavors of scalar-quarks are now being produced. This would also lead to multiple scalarium systems, some of them perhaps within the intrinsic resolution of the $\mathrm{e}^{+} \mathrm{e}^{-}$machine. This would lead to enhanced effects which could somewhat obviate the pessimistic conclusions above.

\section{(j) $\mathrm{e}^{+} \mathrm{e}^{-} \rightarrow \overline{\mathrm{q}} \tilde{\mathrm{q}} \tilde{\mathrm{g}} \quad[5.19-5.21]$}

These events are the supersymmetric analogs of three-jet ( $\mathrm{q} \overline{\mathrm{q} g}$ ) events. If the scalar-quark (and gluino) masses are light enough, it could be possible to detect them using three-jet Monte-Carlo studies. In refs. [5.19,5.20], various jet variables have been studied in order to detect the presence of a supersymmetric signal. It will also be important to demonstrate the loss of energy due to escaping photinos. The relative energy lost will be small, so that one will have to employ statistical tests similar to the $X_{\mathrm{E}}$ and $p_{\text {out }}$ variables discussed in section 3.4. One would then search for nonplanar events (the plane being defined by the beam axis and one of the outgoing jets) and look for statistical evidence of missing momentum transverse to the plane. In ref. [5.20], it is argued that the total three-jet cross section could be enhanced as much as $90 \%$ if all supersymmetric channels are open. In addition, it is recommended [5.20] that one studies the distribution of the Ellis-Karliner angle and the angular dis- 
tribution of the thrust axis. Finally, if the masses of $\tilde{q}_{L}$ and $\tilde{q}_{R}$ are sufficiently different, parity violating effects would show up which could perhaps be detected by measuring various angular asymmetries.

These analyses are only likely to be relevant at energies far above the scalar-quark threshold. Otherwise, the qã $\tilde{g}$ would lose its jet-like structure and render the jet analyses useless. Note also that virtual scalar-quark effects (below scalar-quark threshold) tend to be small. For example, in ref. [5.21], it is shown that first order QCD corrections due to virtual scalar-quark and gluino effects in the total hadronic $\mathrm{e}^{+} \mathrm{e}^{-}$cross section are negligible as compared to the standard correction due to one-gluon exchange. The conclusion here is the same as the one we reached in case (i) in studying $\mathrm{e}^{+} \mathrm{e}^{-} \rightarrow \tilde{\mathrm{q}} \overline{\tilde{q}}$. Namely, it will be difficult to observe a signal due to scalar-quarks unless one is sufficiently above the threshold for their production. The best one can hope to do is to obtain mass limits by showing that the Standard Model Monte Carlo reproduces well all features of the $\mathrm{e}^{+} \mathrm{e}^{-} \rightarrow$ jets data.

\section{(k) $\mathrm{e}^{+} \mathrm{e}^{-} \rightarrow \mathrm{q} \overline{\mathrm{q}} \tilde{g} \tilde{g} \quad[5.19,5.22]$}

Diagrams for this process are shown in fig. 47. The above final state matches that of cases (i) and (j), so many of the same remarks hold. However, there is one advantage of note in this particular case; namely, the cross section need not depend on the mass of the scalar-quark. If the scalar-quark masses are large compared to the beam energy, the diagram given in fig. 47(a) will dominate. The cross section can be related to the known four-quark ( $\left(\bar{q} \bar{q} q^{\prime} \bar{q}^{\prime}\right)$ cross section [5.23] by inserting the appropriate color factor and a factor of $1 / 2$ for identical gluinos in the final state [5.19]:

$$
\sum_{\mathbf{q}} \sigma\left(\mathrm{e}^{+} \mathrm{e}^{-} \rightarrow \mathrm{q} \overline{\mathrm{q}} \tilde{\mathrm{g}} \tilde{\mathrm{g}}\right) \sim \frac{1}{2} \frac{N_{\mathrm{c}}}{T_{\mathrm{f}} N_{\mathrm{f}}} \sum_{\mathbf{q q}} \sigma\left(\mathrm{e}^{+} \mathrm{e}^{-} \rightarrow \mathrm{q} \overline{\mathrm{q}} \mathrm{q}^{\prime} \overline{\mathrm{q}}^{\prime}\right)
$$

where $T_{\mathrm{f}}=1 / 2, N_{\mathrm{c}}=3$ and $N_{\mathrm{f}}$ is the number of quark flavors available to the beam energy. Note that the four-quark final state is substantially smaller than the qqugg state which dominates the process $\mathrm{e}^{+} \mathrm{e}^{-} \rightarrow$ four jets. Hence, observation of the gluino by this process will be extremely difficult.

\section{(l) $\mathrm{e}^{+} \mathrm{e}^{-} \rightarrow \tilde{\mathrm{g}} \tilde{\mathrm{g}} \quad[5.24]$}

Unlike the process discussed in case $(\mathrm{k})$ above, this process depends on the scalar-quark mass (the diagrams for this process are analogous to those given in fig. 32). In addition, in the regime where one-photon exchange dominates (via one loop), this process can only occur if charge conjugation is violated. This must be true simply because $\gamma^{*} \rightarrow \tilde{g} \tilde{g}$ involves a transition from a $C=-1$ state to a $C=+1$ state. In supersymmetric theories this requirement is not unreasonable; such a condition is met if $\tilde{\mathrm{q}}_{L}$ and $\tilde{\mathrm{q}}_{R}$ are split in mass. The cross section for $\mathrm{e}^{+} \mathrm{e}^{-} \rightarrow \tilde{\mathrm{g}} \tilde{\mathrm{g}}$ has been computed in ref. [5.24], with results consistent with the pessimistic view that scalar-quarks and gluinos are not likely to be discovered in $\mathrm{e}^{+} \mathrm{e}^{-}$physics.

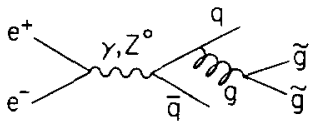

(a)

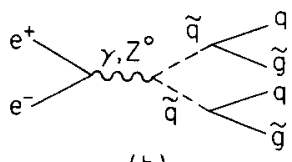

(b)

Fig. 47. Graphs for $\mathrm{e}^{+} \mathrm{e}^{-} \rightarrow \mathrm{q} \overline{\mathrm{q}} \tilde{\mathrm{g}} \tilde{\mathrm{g}}$. 


\subsection{Quarkonium decay into supersymmetric particles}

The advantage of studying quarkonium decay is that one can get higher luminosity over the $\mathrm{e}^{+} \mathrm{e}^{-}$ continuum. Rare decay modes can be a sensitive test for the presence of new physics. We will discuss here the most likely supersymmetric decay modes of the ${ }^{3} S_{1}$ state of quarkonium, and briefly mention possible decays of the ${ }^{1} \mathrm{~S}_{0}$ state and the P states. Most comments will, in principle, apply to the $\psi$ and $Y$ systems. However, because the scalar-quarks are heavy, it could very well turn out that it is necessary to wait for the toponium $(\mathrm{T})$ system in order to probe supersymmetry in quarkonium physics. Although a number of possibilities exist in this system to detect or limit the existence of superpartners, in practice they will be better studied by other data. Further, backgrounds are difficult; e.g. from $Y \rightarrow \tau^{+} \tau^{-}$one has an intrinsic, nonnegligible background which mimics the supersymmetric signal.

\section{(a) ${ }^{3} \mathrm{~S}_{1} \rightarrow \tilde{\mathrm{g}} \tilde{\mathrm{g}} \quad[5.25-5.27]$}

Because the gluino is an eigenstate of $C$, this reaction is $C$-violating. Its rate is therefore proprotional to $\Delta \tilde{M}_{\mathrm{q}}^{2} / \tilde{M}_{\mathrm{q}}^{2}$ where $\Delta \tilde{M}_{\mathrm{q}}^{2} \equiv \tilde{M}_{\mathrm{q}_{\mathrm{L}}}^{2}-\tilde{M}_{\mathrm{q}_{\mathrm{R}}}^{2}$ is a measure of $P$ (and $C$ ) violation introduced by supersymmetry. The decay rate is [5.25]

$$
\frac{\Gamma\left({ }^{3} \mathrm{~S}_{1} \rightarrow \tilde{\mathrm{g}} \tilde{\mathrm{g}}\right)}{\Gamma\left({ }^{3} \mathrm{~S}_{1} \rightarrow \mu^{+} \mu^{-}\right)}=\frac{4 C^{2}}{9 e_{\mathrm{Q}}^{2}}\left(\frac{\alpha_{\mathrm{s}}}{\alpha}\right)^{2}\left(1-\frac{\tilde{M}_{\mathrm{g}}^{2}}{m_{\mathrm{q}}^{2}}\right)^{3 / 2}
$$

where

$$
C=m_{\mathrm{q}}^{2} \Delta \tilde{M}_{\mathrm{q}}^{2} \cos 2 \theta /\left(m_{\mathrm{q}}^{2}-\tilde{M}_{\mathrm{g}}^{2}+\tilde{M}_{\mathrm{q}_{1}}^{2}\right)\left(m_{\mathrm{q}}^{2}-\tilde{M}_{\mathrm{g}}^{2}+\tilde{M}_{\mathrm{q}_{2}}^{2}\right) .
$$

Equation (5.3) is totally general and assumes that the scalar-quark mass eigenstates are rotated relative to $\tilde{\mathrm{q}}_{L}$ and $\tilde{\mathrm{q}}_{\mathrm{R}}$ by an angle $\theta$. It is normalized by the $\mu^{+} \mu^{-}$decay (via one-photon exchange) of the ${ }^{3} \mathrm{~S}_{1}$ state. One can imagine models with parameters leading to an overall branching ratio for ${ }^{3} \mathrm{~S}_{1} \rightarrow \tilde{\mathrm{g}} \tilde{\mathrm{g}}$ as large as $10 \%$. The signature for this process will take a statistical analysis which would uncover the missing energy of the escaping photinos (from gluino decay). Assuming the decay $\tilde{\mathrm{g}} \rightarrow \mathrm{q} \overline{\mathrm{q}} \tilde{\gamma}$ dominates, one would expect roughly one third of the energy missing.

The toponium system may be the best place to look for such a decay [5.25]. It could turn out that $\tilde{M}_{\text {t }}$ is not much larger than $m_{\mathrm{t}}$; furthermore, $\Delta \tilde{M}_{\mathrm{t}}^{2}$ is at present unconstrained (see section 8.2 ) and could be large.

\section{(b) ${ }^{3} \mathrm{~S}_{1} \rightarrow \gamma \tilde{\mathrm{g}} \tilde{\mathrm{g}}, \mathrm{g} \tilde{\mathrm{g}} \tilde{\mathrm{g}}, \mathrm{g} \tilde{\mathrm{g}} \tilde{\gamma} \quad[5.28-5.29]$}

These decays are parity conserving and therefore do not depend on the mass-splitting of scalar-quark eigenstates. However, these decays will be suppressed by the mass of the scalar-quark. The exact decay rate was computed in ref. [5.28] based on the diagrams shown in fig. 48. As a check, one can compute the decay rate in the limit of a massless gluino and infinitely heavy scalar-quarks $\tilde{Q}_{L}$ and $\tilde{Q}_{R}$ which are taken to be degenerate in mass (see fig. 88). This has been done in ref. [5.29] and some of the steps are provided in appendix E.3. Using the result of eq. (E22), we find that for $\tilde{M}_{\mathrm{QL}_{\mathrm{L}}}=\tilde{M}_{\mathrm{QR}} \equiv \tilde{M} \gg \sqrt{ } \bar{s}$,

$$
\Gamma\left({ }^{3} \mathrm{~S}_{1} \rightarrow \tilde{\gamma} \tilde{\gamma} \gamma\right)=8 \alpha^{3} e_{\mathrm{Q}}^{6} m_{\mathrm{V}}^{2}\left|R_{\mathrm{s}}(0)\right|^{2} / 9 \pi \tilde{M}^{4},
$$



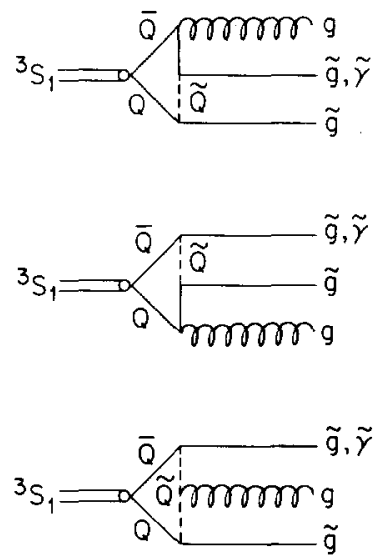

Fig. 48. Graphs for the decay of a $1^{--}$quarkonium state $\left({ }^{3} S_{1}\right)$ into supersymmetric particles. Two decays considered above are ${ }^{3} \mathrm{~S}_{1} \rightarrow g \tilde{g} \tilde{g}$ and ${ }^{3} S_{1} \rightarrow g \tilde{g} \tilde{y}$.
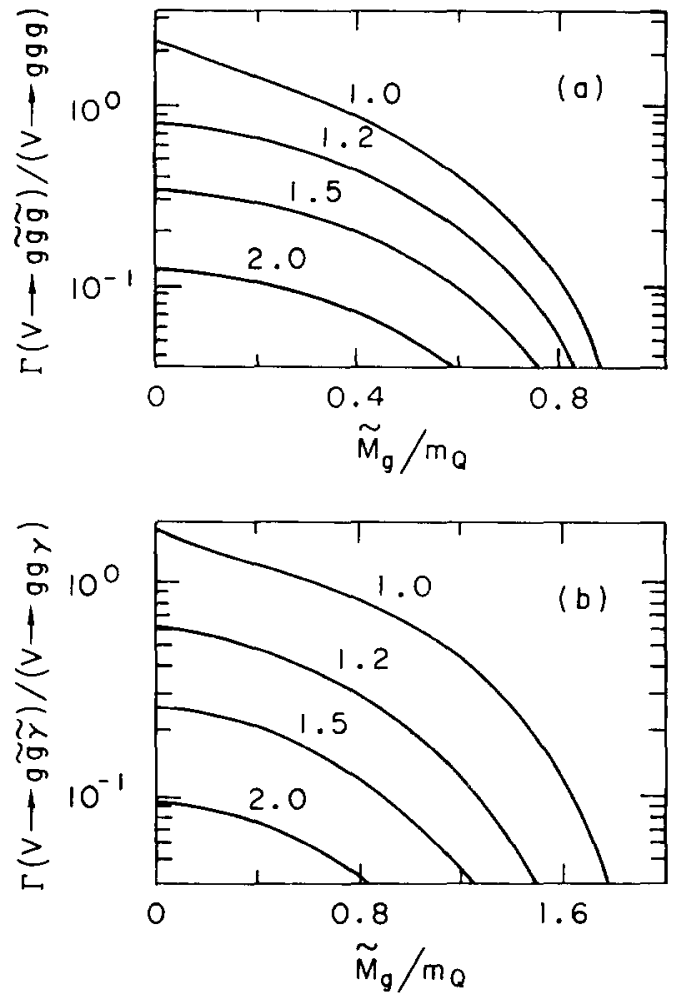

Fig. 49. The decay rate of a ${ }^{3} S_{1} Q \bar{Q}$ bound state (denoted by $V$ ) into (a) $\tilde{g} \tilde{g} g$ and (b) $\bar{g} \tilde{\gamma} \gamma$. The curves are labeled by the values of $\tilde{M}_{\mathrm{Q}} / m_{\mathrm{Q}}$, where $\tilde{M}_{\mathrm{Q}}$ is the mass of the scalar-quark partner of $\mathrm{Q}$. This figure has been obtained from Keung (private communication); it differs by an overall factor of four from what appears in ref. [5.28].

where $m_{\mathrm{V}}$ is the mass of the ${ }^{3} \mathrm{~S}_{1}$ state $\left(m_{\mathrm{V}} \approx 2 m_{\mathrm{O}}\right)$. To get other final states of interest, we must compute the relative color factors. Needed results are

$$
\Gamma(\gamma \tilde{\gamma} \tilde{\gamma}): \Gamma(\mathrm{g} \tilde{\mathrm{g}} \tilde{\gamma}): \Gamma(\mathrm{g} \tilde{\mathrm{g}} \tilde{\mathrm{g}})=\frac{3}{2}: \frac{2}{3} \alpha_{\mathrm{s}}^{2} / \alpha^{2} e_{\mathrm{Q}}^{4}: \frac{5}{36} \alpha_{\mathrm{s}}^{3} / \alpha^{3} e_{\mathrm{Q}}^{6},
$$

where a factor of $1 / 2$ was inserted in the first and third process to account for identical particles in the final state.

If we now normalize to $\Gamma\left({ }^{3} \mathrm{~S}_{1} \rightarrow \mathrm{ggg}\right)$ to get rid of the factor of $\left|R_{\mathrm{s}}(0)\right|^{2}$, we end up with

$$
\Gamma\left({ }^{3} \mathrm{~S}_{1} \rightarrow \mathrm{g} \tilde{\mathrm{g}} \tilde{\mathrm{g}}\right)=\frac{5}{24} \frac{\alpha_{\mathrm{s}}}{\alpha e_{\mathrm{Q}}^{2}} \Gamma\left({ }^{3} \mathrm{~S}_{1} \rightarrow \mathrm{g} \tilde{\mathrm{g}} \tilde{\gamma}\right)=\frac{1}{6\left(\pi^{2}-9\right)}\left(\frac{m_{\mathrm{V}}}{\tilde{M}_{\mathrm{Q}}}\right)^{4} \Gamma\left({ }^{3} \mathrm{~S}_{1} \rightarrow \mathrm{ggg}\right)
$$

The exact results from ref. [5.28] are very involved formulas, ${ }^{*}$ so we just present the graphical results in fig. 49. Note that the branching ratios are substantial only when $\tilde{M}_{\mathrm{Q}} \sim m_{\mathrm{Q}}$. For large scalar-quark

${ }^{*}$ In ref. [5.28] the formulas given there (with the correction given in an erratum) are in agreement with our eqs. (5.4) $-(5.6)$ in the limit of $\tilde{M} \rightarrow \infty$. 
masses, observation of a signal could be very difficult. One should note the inherent advantage of the

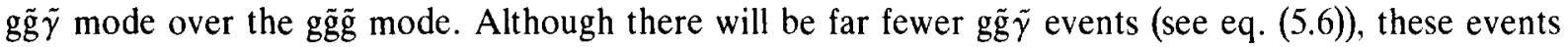
are noteworthy in that on average close to $50 \%$ of the energy escapes into photinos. Therefore, the gz $\tilde{\gamma}$ could be detected on an event-by-event basis. The signature would therefore be 'three-jet events' with one jet unobserved. One could check that the missing momentum did not point down the beam pipe or to a crack in the detector. One possible background would be $\operatorname{gg} \gamma$ events where the photon is missed. However, present detectors are very efficient in detecting high energy photons so this background is presumably not important at present luminosities.

(c) ${ }^{3} \mathrm{~S}_{1} \rightarrow$ ggg̃ $\tilde{g} \quad[5.22,5.30]$

This decay rate has the advantage that it does not depend on the mass of the scalar-quark since the lowest order diagram involves only the gg̃ g̃ vertex. The calculation done in ref. [5.22] for the $Y$ system leads to

$$
\Gamma(Y \rightarrow \operatorname{gg} \tilde{g} \tilde{g}) / \Gamma(Y \rightarrow \mathrm{ggg}) \leq 0.3
$$

(the equality being saturated for a zero-mass gluino [5.27]). A limit on the above branching ratio in principle can put a limit on the gluino mass. Experimentally, this could be a difficult process to measure at the $Y$ because the energy carried away by the photinos is small. Perhaps one could look for four-jet and/or nonplanar events at the $Y$ to enrich the gluino sample. Similar procedures could be repeated at toponium.

(d) ${ }^{3} \mathrm{~S}_{1} \rightarrow \tilde{\gamma} \tilde{\gamma}, \tilde{\gamma} \tilde{\gamma} \gamma$

The process ${ }^{3} \mathrm{~S}_{1} \rightarrow \tilde{\gamma} \tilde{\gamma}[5.25,5.26]$ is similar to ${ }^{3} \mathrm{~S}_{1} \rightarrow \tilde{\mathrm{g}} \tilde{\mathrm{g}}$ in that it needs parity violation to occur (i.e., $\tilde{M}_{\mathrm{q}_{\mathrm{L}}} \neq \dot{M}_{\mathrm{q}_{\mathrm{R}}}$. It is also suppressed in addition by the fact that $\alpha^{2}$ rather than $\alpha_{\mathrm{s}}^{2}$ occurs in the formula. Because the photinos escape. one would need to use an indirect method for observation. For example, in the $Y$ system, by looking at $Y^{\prime} \rightarrow Y \pi^{+} \pi^{-}$and identifying the $Y$ as the mass recoiling against the $\pi^{+} \pi^{-}$, one could interpret $Y^{\prime} \rightarrow \pi^{+} \pi^{-}+$'nothing' as $Y \rightarrow \tilde{\gamma} \tilde{\gamma}$. (This method was suggested to look for $\psi \rightarrow \nu \bar{\nu}$ [5.31] and $\psi \rightarrow$ gravitinos [5.32].) In the process ${ }^{3} \mathrm{~S}_{1} \rightarrow \tilde{\gamma} \tilde{\gamma} \gamma$, the $\gamma$ is observed and tags the event. The problem here is that for large $\tilde{M}_{\mathrm{Q}}$ the width (see eq. (5.4)) is proportional to $\alpha^{3}\left(m_{\mathrm{V}} / \tilde{M}_{\mathrm{Q}}^{2}\right)^{2}$ which leads to an unobservable rate.

(e) ${ }^{3} \mathrm{~S}_{1} \rightarrow \tilde{\chi}_{i}^{0} \tilde{\chi}_{j}^{0} \quad[5.26]$

This is the supersymmetric version of the Wilczek mechanism ${ }^{3} \mathrm{~S}_{1} \rightarrow \gamma \mathrm{H}^{0}[5.59]$. The rate depends on the parameters which make up the neutralino mass matrix. As an example, for ${ }^{3} \mathrm{~S}_{1} \rightarrow \tilde{\gamma} \tilde{\mathrm{H}}^{0}$ we get

$$
\frac{\Gamma\left({ }^{3} S_{1} \rightarrow \tilde{\gamma} \tilde{\mathrm{H}}^{0}\right)}{\Gamma\left({ }^{3} S_{1} \rightarrow g g g\right)}=\frac{81 \pi^{2} \alpha e_{O}^{2} G_{\mathrm{F}}}{20 \sqrt{2} \alpha_{\mathrm{s}}^{3}\left(\pi^{2}-9\right)} \frac{\left(m_{\mathrm{V}}^{2}-{\underline{M_{\mathrm{H}}^{2}}}^{2}\left(m_{\mathrm{V}}^{2}+2 \tilde{M}_{\mathrm{H}}^{2}\right)\right.}{\tilde{M}_{\mathrm{O}}^{4}},
$$

where we have assumed that the photino is massless and that there is no suppression due to the mixing of $\tilde{\mathrm{H}}^{0}$ with other neutralino mass eigenstates. (The formula for $\tilde{M}_{\gamma} \neq 0$ is given in ref. [5.26].) It is clear that only for heavy quarkonium states (close in mass to $M_{\mathrm{O}}$ ) can this branching ratio be appreciable. The signature for this decay is very dramatic (i.e. one-sided events). Assuming $\tilde{\chi}_{j}^{0} \rightarrow \tilde{\chi}_{i}^{0}+$ quark jets (or leptons), one would look for events with a hadronic jet or single lepton with over $50 \%$ of the energy missing and a substantial momentum imbalance. The kinematics of the event could easily separate it from two-photon physics or a beam-gas interaction. 


\section{(f) ${ }^{3} \mathrm{~S}_{1} \rightarrow \tilde{\mathrm{q}} \overline{\tilde{q}} \quad[5.25]$}

This decay can only be relevant for toponium (or heavier systems). It is not implausible that the $\mathrm{t}$-quark and scalar $\mathrm{t}$ may not be too different in mass. As shown in ref. [5.25], it is not implausible that ${ }^{3} \mathrm{~S}_{1} \rightarrow \tilde{\mathrm{tt}}$ could be the dominant decay of toponium. On the other hand, if $\tilde{M}_{\mathrm{t}}<m_{\mathrm{t}}$, then the t-quark will probably decay substantially into supersymmetric channels (such as $\tilde{\mathrm{t}} \tilde{\gamma}$ or $\tilde{\mathrm{t}} \tilde{\mathrm{g}}$ ).

\section{(g) ${ }^{3} \mathrm{~S}_{1} \rightarrow \gamma+\tilde{\eta}_{\mathrm{g}} \quad[5.54-5.56]$}

We discussed the spectrum of gluinonium - $\tilde{g} \tilde{g}$ bound states in section 3.4 , subsection $\mathrm{E}$. The lowest lying $0^{-+}$state was denoted by $\tilde{\eta}_{\mathrm{g}}$ in analogy with the $\eta_{\mathrm{c}}$. One can now study the decay ${ }^{3} \mathrm{~S}_{1} \rightarrow \gamma+\tilde{\eta}_{\mathrm{g}}$ which is analogous to the decay $Y \rightarrow \gamma+\eta_{\mathrm{c}}$ [5.57]. The results have been computed independently by three groups [5.54-5.56]. The results can be stated simply as follows. Due to color enhancement in $\tilde{\eta}_{\mathrm{g}}$ production, if we compare ${ }^{3} \mathrm{~S}_{1} \rightarrow \gamma+\tilde{\eta}_{\mathrm{g}}$ versus ${ }^{3} \mathrm{~S}_{1} \rightarrow \gamma+\eta_{\mathrm{Q}}$ where $\eta_{\mathrm{Q}}$ is the $L=0$ pseudoscalar QQ $\bar{Q}$ state, we find that for equal masses $\tilde{M}_{\eta_{\mathrm{g}}}=m_{\eta_{\mathrm{Q}}}$,

$$
\Gamma\left({ }^{3} \mathrm{~S}_{1} \rightarrow \gamma+\tilde{\eta}_{\mathrm{g}}\right) / \Gamma\left({ }^{3} \mathrm{~S}_{1} \rightarrow \gamma+\eta_{\mathrm{Q}}\right)=\frac{27}{4}\left|\tilde{R}_{\mathrm{s}}(0)\right|^{2} /\left|R_{\mathrm{s}}(0)\right|^{2}
$$

where $\left|R_{\mathrm{s}}(0)\right|^{2}$ is the appropriate wavefunction factor. Furthermore, assuming that the color singlet $\mathrm{q} \overline{\mathrm{q}}$ and $\tilde{\mathrm{g}} \tilde{\mathrm{g}}$ potentials are of the form $V_{\mathrm{q} \overline{\mathrm{q}}} \simeq C \alpha_{\mathrm{s}} r^{\mathrm{p}}$ and $V_{\tilde{\mathrm{g}} \tilde{\mathrm{g}}} \simeq \tilde{C} \alpha_{\mathrm{s}} r^{\mathrm{p}}$ (where the color factors are $C=4 / 3$ and $\tilde{C}=3$ ), then the ratio of wavefunctions in eq. (5.9) is expected to behave as $\left|\tilde{R}_{\mathrm{s}}(0)\right|^{2} /\left|R_{\mathrm{s}}(0)\right|^{2} \sim(9 / 4)^{3 /(2+p)}$ which further enhances $\tilde{\eta}_{\mathrm{g}}$ production. More complete formulas can be found in refs. [5.54-5.56]. The end result is for $1<\tilde{M}_{\tilde{\eta}_{\mathrm{g}}}<8 \mathrm{GeV}$, we find $\mathrm{BR}\left(Y \rightarrow \gamma+\tilde{\eta}_{\mathrm{g}}\right) \approx(1-3) \times 10^{-4}$. We expect this limit to be attainable in the near future. The signal would be detected by observing a monoenergetic photon at an unexpected energy. If no such signal were observed, one would rule out gluino masses lighter than $4 \mathrm{GeV} / \mathrm{c}^{2}$. Unfortunately, it is unlikely that such a technique could be useful at higher mass quarkonium factories (e.g., in toponium) because the radiative branching ratio into $\eta$-like states is extremely small.

One could consider other processes. For example ${ }^{3} \mathrm{~S}_{1} \rightarrow \tilde{\eta}_{\mathrm{g}}+$ gluon jet [5.54] would occur at a substantially larger rate (by a factor of $\alpha_{\mathrm{s}} / \alpha$ ) as compared to ${ }^{3} \mathrm{~S}_{1} \rightarrow \tilde{\eta}_{\mathrm{g}}+\gamma$. However, some sensitivity would be lost in attempting to measure the outgoing gluon jet. One could also study the production of higher gluinonium states such as the P-states $\left(0^{++}, 1^{++}, 2^{++}\right)$, although the rates are expected to be suppressed compared to the production of $\tilde{\eta}_{\mathrm{g}}[5.56]$.

\section{(h) ${ }^{1} \mathrm{~S}_{0},{ }^{3} \mathrm{P}_{0,1,2} \rightarrow \tilde{\mathrm{g}} \tilde{\mathrm{g}}, \tilde{\gamma} \tilde{\gamma} \quad[5.26,5.33,5.60]$}

These decays do not violate parity but their rates are suppressed by $\tilde{M}_{\mathrm{Q}}^{-4}$ for large scalar-quark masses. Unfortunately, considering the small production rate for the ${ }^{1} \mathrm{~S}_{0}$ state, it is unlikely that these decays could be seen in the near future. The P-states are produced more copiously in radiative decays of higher ${ }^{3} \mathrm{~S}_{1}$ states. Hence, under favorable circumstances, it may be possible to observe the decay of P-states into supersymmetric particles. Kuhn [5.60] argues that among the $t \mathrm{t} C$-even resonances, the $1^{++}$state can have an appreciable (perhaps dominant) branching ratio into $\tilde{\mathrm{g}} \tilde{\mathrm{g}}$ as compared to ordinary hadronic decays. However, severe backgrounds make the study of the P-states very difficult, and it will be hard to extract useful limits.

For pedagogical reasons we shall discuss the decay rate for ${ }^{1} \mathrm{~S}_{0} \rightarrow \tilde{\gamma} \tilde{\gamma}$. For details of the calculation see appendix E.2. We assume that $\tilde{\mathrm{Q}}_{\mathrm{L}}$ and $\tilde{\mathrm{Q}}_{\mathrm{R}}$ are the appropriate mass eigenstates and take their masses $\left(\tilde{M}_{\mathrm{L}}\right.$ and $\left.\tilde{M}_{\mathrm{R}}\right)$ to be unequal. To compute the desired decay rate, we must project the amplitude at threshold for $\mathrm{q} \overline{\mathrm{q}} \rightarrow \tilde{\gamma} \tilde{\gamma}$ onto a ${ }^{1} \mathrm{~S}_{0}$ state. The end result of the computation is 


$$
\Gamma\left({ }^{1} \mathrm{~S}_{0} \rightarrow \tilde{\gamma} \tilde{\gamma}\right)=\frac{3 \alpha^{2} e_{\mathrm{Q}}^{4} \tilde{M}_{\gamma}^{2}\left(m_{\mathrm{Q}}^{2}-\tilde{M}_{\gamma}^{2}\right)^{1 / 2}\left|R_{\mathrm{s}}(0)\right|^{2}}{m_{\mathrm{Q}}}\left[\frac{1}{\tilde{M}_{\mathrm{L}}^{2}+m_{\mathrm{Q}}^{2}-\tilde{M}_{\tilde{\gamma}}^{2}}+\frac{1}{\tilde{M}_{\mathrm{R}}^{2}+m_{\mathrm{Q}}^{2}-\tilde{M}_{\tilde{\gamma}}^{2}}\right]^{2},
$$

where the mass of the ${ }^{1} \mathrm{~S}_{0}$ state is $2 m_{\mathrm{Q}}$. In addition, note that $\Gamma(\tilde{\gamma} \tilde{\gamma}): \Gamma(\tilde{\mathrm{g}} \tilde{\mathrm{g}})=3: 2 \alpha_{\mathrm{S}}^{2} / 3 \alpha^{2} e_{\mathrm{Q}}^{4}$.

A number of features of eq. (5.10) are noteworthy. First, the decay rate is proportional to $\tilde{M}_{\gamma}^{2}$. This is reminiscent of the helicity suppression factor which occurs in leptonic $\pi$ decay. The reason it appears here is because the $Q \tilde{Q} \tilde{\gamma}$ vertices are chiral* (i.e., they contain factors of $1 \pm \gamma_{s}$; see eq. (C73)). As a result, the photinos would like to be emitted with opposite helicities; however, for a decaying spin zero state, they must be emitted with the same helicity. Second, if $\tilde{M}_{\mathrm{L}}=\tilde{M}_{\mathrm{R}}$, eq. (5.10) may be derived from eg. (E14) which implies that the decay rate is proportional to $\sigma(\mathrm{Q} \overline{\mathrm{Q}} \rightarrow \tilde{\gamma} \tilde{\gamma})$ at threshold. However, when $\tilde{M}_{\mathrm{L}} \neq M_{\mathrm{R}}$ eq. (E14) fails as can be seen by observing that at threshold, $\sigma(\mathrm{QQ} \rightarrow \tilde{\gamma} \tilde{\gamma})$ is not proportional to $M_{\bar{\gamma}}^{2}$. One reason for this is that when $M_{\mathrm{L}} \neq M_{\mathrm{R}}$ we have $C$-violation which implies that the $\tilde{\gamma} \tilde{\gamma}$ final state no longer automatically projects on to the $C$-even initial state. This observation was missed in ref. [5.33]; as a result an incorrect formula for $\Gamma\left({ }^{1} \mathrm{~S}_{0} \rightarrow \tilde{\gamma} \tilde{\gamma}\right)$ was obtained there. See appendix E.2 for further discussion on these points. In general, the spin 0 states $\left({ }^{1} \mathrm{~S}_{0},{ }^{3} \mathrm{P}_{0}\right)$ have $\tilde{\gamma} \tilde{\gamma}$ decay rates proportional to $\tilde{M}_{\gamma}^{2}$. No such factor (i.e. no helicity suppression) occurs for states of non-zero spin [5.60].

\subsection{Supersymmetry at the $Z^{0}$}

A good way to detect new supersymmetric particles is by studying decays of a $Z^{0}$ produced at an $\mathrm{e}^{+} \mathrm{e}^{-}$ collider on resonance. The expected $Z^{0}$ production cross section is roughly 5000 times that of $\mathrm{e}^{+} \mathrm{e}^{-} \rightarrow \gamma^{*} \rightarrow \mu^{+} \mu^{-}$. At SLC and LEP, it is expected that at least $10^{6} \mathrm{Z}^{0}$ events per year will be produced, perhaps allowing for the study of rare decay modes of the $Z^{0}$ which could provide hints of new physics beyond the Standard Model. If supersymmetric decays of the $Z^{0}$ are kinematically allowed, then the branching ratios should be substantial as emphasized in section 4.1 . If this is the case, $Z^{0}$ factories offer great promise for the discovery and/or study of supersymmetry.

We have already discussed in chapter 4 some of the consequences of $p \bar{p} \rightarrow Z^{0}+X$, where the $Z^{0}$ decays into supersymmetric particles. The experimental signature at the $Z^{0}$ factories will be cleaner. Supersymmetric decays of the $Z^{0}$ fall into three classes of events:

\section{(i) Neutrino counting [5.34]}

It is possible to have $\mathrm{Z}^{0} \rightarrow$ unobserved particles (analogous to $\mathrm{Z}^{0} \rightarrow \nu \bar{\nu}$ ). This would be the case for $\mathrm{Z}^{0} \rightarrow \tilde{\nu} \tilde{\tilde{\nu}}$ if the scalar neutrino were stable or its dominant decay were $\tilde{\nu} \rightarrow \nu \tilde{\gamma}$. In order to detect these events one must run the $Z^{0}$ factory at an energy slightly above the $Z^{0}$ and search for $\mathrm{e}^{+} \mathrm{e}^{-} \rightarrow \gamma \mathrm{Z}^{0} \rightarrow$ $\gamma+$ missing energy, where the bremsstrahlung photon tags the events. A precise measurement of this process would count the number of neutrino flavors. Any excess over the theoretically anticipated result could signal the presence of new physics.

(ii) One-sided events [5.10-5.14, 5.35]

These events are the hallmark of supersymmetric phenomenology. A typical example [5.12] consists of $Z^{0} \rightarrow \tilde{\chi}_{1}^{0} \tilde{\chi}_{2}^{0}$ where $\tilde{\chi}_{2}^{0} \rightarrow \tilde{\chi}_{1}^{0}+$ charged particles. The $\tilde{\chi}_{1}^{0}$ (the lightest neutralino) would escape detection thus leaving one hemisphere of the detector empty. Such a signal could also arise in $Z^{0} \rightarrow \tilde{\nu} \overline{\tilde{\nu}}$ if one scalar neutrino escapes undetected (say by decaying via $\tilde{\nu} \rightarrow \nu+\tilde{\gamma}$ ) and the other scalar neutrino decays

\footnotetext{
${ }^{*}$ If $\overline{\mathrm{Q}}_{\mathrm{L}}$ and $\tilde{\mathrm{Q}}_{\mathrm{R}}$ were not mass eigenstates (see appendix $\mathrm{C} .1$ ), it would no longer be true that $\Gamma\left({ }^{1} \mathrm{~S}_{0} \rightarrow \bar{\gamma} \tilde{\gamma}\right.$ ) is proportional to $\bar{M}_{\gamma}^{2}$. There would be an additional term proportional to the square of the $\tilde{Q}_{L}-\bar{Q}_{R}$ mixing parameter.
} 
into charged four body modes [5.17]. Backgrounds for such events would consist of beam-gas interactions, two-photon physics (where considerable energy can be lost down the beam pipe), and missed particle detection (due to a crack in the detector). These sources of background are easily dealt with, so that the one-sided event is by far the best signature for new physics.

\section{(iii) Two-sided events}

Events such as $Z^{0} \rightarrow \tilde{\chi}_{2}^{0} \tilde{\chi}_{2}^{0}, \tilde{\chi}^{+} \tilde{\chi}^{-}[5.10-5.13 ; 5.35-5.39]$ where both neutralinos or charginos decay into final state particles will in general show tracks in both hemispheres of the detector. Eventually, two neutralinos $\left(\tilde{\chi}_{1}^{0}\right)$ resulting from those decays will escape the detector unseen leading to events with missing energy and momentum transverse to the beam. Occasionally, the missing transverse energy will be substantial; however, statistical analysis of events will be required in order to prove that such a signal constitutes new physics.

For completeness we summarize the possible supersymmetric decays of the $Z^{0}$ which have been studied:

(a) $Z^{0} \rightarrow \tilde{\nu} \bar{\nu}[5.17,5.40]$. The branching ratio for this decay is obtained from eqs. (4.3) and (4.4) by putting $g_{R}=0$. Such a decay can fall into any one of the three classes of events described above depending on the branching ratio of the scalar-neutrino into charged four-body modes. This is illustrated by a model calculation [5.17]; the results are summarized in fig. 50 for a particular choice of supersymmetric masses.

(b) $Z^{0} \rightarrow \tilde{\ell}^{+} \tilde{\ell}^{-}$[5.40-5.42]. The branching ratio of this decay summing over $\tilde{\ell}_{L}$ and $\tilde{\ell}_{R}$ final states is given by eq. (3.7). These events are two-sided and could resemble $Z^{0} \rightarrow \tau^{+} \tau^{-}$or the production of a new sequential heavy lepton. The $\tilde{\ell}^{+} \tilde{\ell}^{-}$production could be differentiated by the (dominantly) $\sin ^{2} \theta$ angular distribution. Note further that $\tilde{\ell}^{+} \tilde{\ell}^{-}$production always leads to $\ell^{+} \ell^{-}+$missing energy and never results in $\mathrm{e}^{ \pm} \mu^{\mp}+$ missing energy as would be the case for heavy lepton production.

(c) $Z^{0} \rightarrow \tilde{\ell}+\ell^{-}+\tilde{\gamma}$ [5.42]. This process occurs as shown in fig. 51. In principle it allows one to be sensitive to scalar lepton masses larger than $m_{z} / 2$. The differential rate for the decay $Z^{0} \rightarrow \ell^{+} \tilde{\ell}^{-} \tilde{\gamma}$ has been computed by Johnson and Rudaz [5.42]. Let us define $x=\tilde{M}_{\ell}^{2} / m_{z}^{2}$. For $x \leqslant 1 / 4$, the decay occurs almost entirely with both scalar electrons on shell thus reducing the discussion to the previous case (b). For $x>1 / 4$, one scalar electron must be off-shell, and the branching ratio of $Z^{0} \rightarrow \ell^{+} \tilde{\ell}^{-} \tilde{\gamma}$ plummets as $x$ increases as

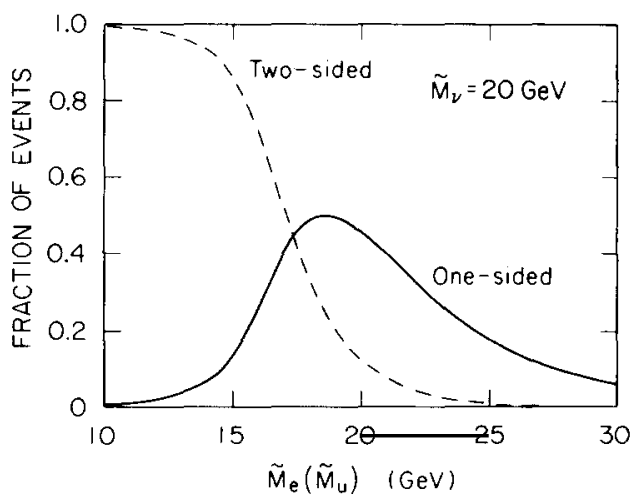

Fig. 50. The fraction of $Z^{0} \rightarrow \tilde{\nu} \bar{\nu}$ events which are two-sided (dashed line) and one-sided (solid line). Events not falling into either of these two categories require neutrino-counting techniques to be detected. See ref. [5.17] for assumptions on the model calculation.
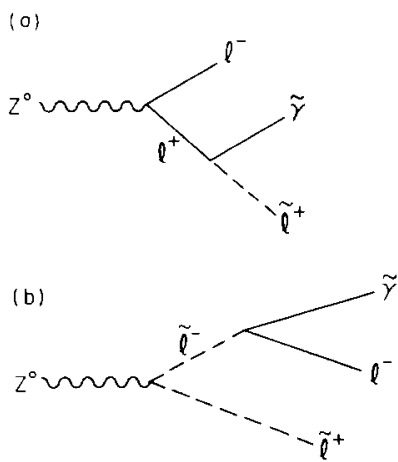

Fig. 51. Graphs for $Z^{0} \rightarrow \bar{\ell}^{+} \ell \dot{\gamma}$, where $\ell$ can be any charged lepton. 


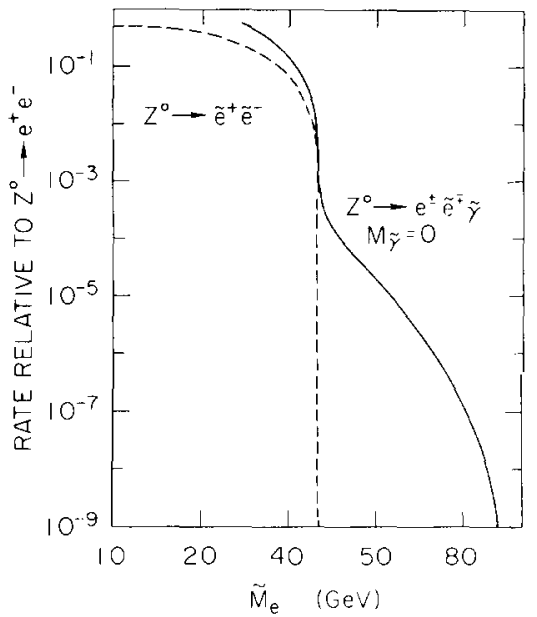

Fig. 52. Rates for $Z^{0} \rightarrow \mathrm{e}^{ \pm} \mathrm{e}^{\mp} \tilde{\gamma}$ (where we have summed over the decay rates for $\tilde{\mathrm{e}}_{\mathrm{L}}^{+}, \tilde{\mathrm{e}}_{\mathrm{L}}^{-}, \tilde{\mathrm{e}}_{\mathrm{R}}^{+}$ and $\tilde{\mathrm{e}}_{\mathbf{R}}$ ) relative to $Z^{0} \rightarrow \mathrm{e}^{+} \mathrm{e}^{-}$, as a function of scalar-electron mass. We have taken $\tilde{M}_{\gamma}=0$ and $\tilde{M}_{\mathrm{e}_{\mathrm{L}}}=\tilde{M}_{\mathrm{e}_{\mathrm{R}}} \equiv \tilde{M}_{\mathrm{e}}$. Also shown is the relative rate for $Z^{0} \rightarrow \tilde{\mathrm{e}}^{+} \tilde{\mathrm{e}}^{-}$(summed over $\tilde{\mathrm{e}}_{\mathrm{L}}$ and $\overline{\mathrm{e}}_{\mathrm{R}}$ ). This figure was taken from ref. [5.42].

shown in fig. 52. Thus in practice scalar leptons with masses much above $m_{z} / 2$ are unobservable, even at the high luminosity $\mathrm{Z}^{0}$ factories.

(d) $\mathrm{Z}^{0} \rightarrow \ell^{+} \ell^{-} \tilde{\gamma} \tilde{\gamma}$. One can repeat the analysis of case (c) where both scalar leptons are off-shell. The results are similar to the discussion in section 3.1 (see below eq. (3.5)); and one does not gain over the previous two cases.

(e) $Z^{0} \rightarrow \tilde{q} \overline{\tilde{q}}$. The decay rate of $Z^{0}$ into scalar quarks is enhanced over the rate into scalar leptons with $\Gamma(\tilde{\mathrm{d}} \overline{\overline{\tilde{d}}}): \Gamma(\tilde{u} \overline{\tilde{u}}): \Gamma(\tilde{\nu} \overline{\tilde{\nu}}): \Gamma(\tilde{\mathrm{e}} \overline{\tilde{e}})=14: 11: 6: 3$, assuming degenerate masses. Thus, the decay rate of $Z^{0}$ into scalar-quarks can be quite copious, unless it is suppressed by the (P-wave) phase space factor. $A$ careful measurement of the total $Z^{0}$ width at the $Z^{0}$ factory may reveal the potential for new physics. Life will be more difficult if one wants to identify the scalar-quarks directly.

The signal depends on how the scalar-quark decays. If the gluino is light, the decay $\tilde{q} \rightarrow q \tilde{g}$ will dominate leading to $q \bar{q} \tilde{g} \tilde{g}$ final states. As in the case of $\mathrm{e}^{+} \mathrm{e}^{-} \rightarrow \tilde{\mathrm{q}} \overline{\tilde{q}}$, this particular final state is very difficult to pick out of background as the undetected photino energy (via $\tilde{g} \rightarrow q \bar{q} \tilde{\gamma}$ ) will be small. Differentiating these decays from heavy quark decays of the $Z^{0}$ where the heavy quarks decay into nonleptonic final states will not be easy. If the gluino is heavy so that $\tilde{\mathrm{q}} \rightarrow \mathrm{q} \tilde{\gamma}$ dominates the scalar-quark decays, the final state from $Z^{0}$ decay is $q \bar{q} \tilde{\gamma} \tilde{\gamma}$ which could be identified by searching for events with significant energy loss and no observable leptons.

(f) $\mathrm{Z}^{0} \rightarrow \overline{\mathrm{q}} \tilde{\mathrm{q}} \tilde{\gamma}$ [5.42]. This process is the scalar-quark analog of case (c), and the results of fig. 52 apply by rescaling by a factor of the quark charge squared. The conclusions obtained in case (c) follow roughly unchanged.

(g) $Z^{0} \rightarrow q \bar{q} \tilde{g}[5.42]$. If the gluino is light (say $\tilde{M}_{\mathrm{g}} \leqslant 20 \mathrm{GeV}$ ), then this process could be important. The ratio $\Gamma\left(Z^{0} \rightarrow q \tilde{q} \tilde{g}\right) / \Gamma\left(Z^{0} \rightarrow q \bar{q}\right)$ can be obtained from fig. 52 by multiplying by a factor of $4 \alpha_{\mathrm{s}} / 3 \alpha \approx 27$. By this technique one could be sensitive to scalar-quark masses somewhat larger than $m_{z} / 2$, although once again the $q \bar{q} \tilde{g} \tilde{g}$ final state may be difficult to untangle from standard model backgrounds.

(h) $Z^{0} \rightarrow q \bar{q} \tilde{g} \tilde{g}[5.22,5.44]$. This process can occur via $Z^{0} \rightarrow \tilde{q} \overline{\tilde{q}}$ where each off-shell scalar quark decays $\tilde{\mathrm{q}} \rightarrow \mathrm{q} \tilde{\mathrm{g}}$. The rate due to this mechanism is expected to be small, as for our discussion of process (d). However, there is a far more likely mechanism to produce $q \bar{q} \tilde{g} \tilde{g}$. Namely, a quark bremsstrahlungs a gluon which converts into a gluino pair. We have already discussed this process in section 4.3; the decay rate is shown in fig. 46. Although the final state is difficult to identify, the total branching ratio could be as large as $1 \%$ for light gluino masses. With large samples of data from a $Z^{0}$ factory, perhaps such a final state could be detected by statistical means. 
(i) $Z^{0} \rightarrow \tilde{\chi}^{+} \tilde{\chi}^{-}, \tilde{\chi}_{i}^{0} \tilde{\chi}_{j}^{0}[5.12-5.16 ; 5.35-5.39]$. The decay of $Z^{0}$ into chargino and neutralino pairs given by eq. (4.11) (see also the comments below eq. (4.11)) are highly model dependent. But clearly the rates may be substantial as shown in fig. 45 for a sample choice of supersymmetric parameters. The signatures of these events can be of all three types discussed above. The most dramatic signal is the one-sided event signature which occurs when $Z^{0} \rightarrow \tilde{\chi}_{1}^{0} \tilde{\chi}_{2}^{0}, \tilde{\chi}_{2}^{0} \rightarrow \tilde{\chi}_{1}^{0}+$ charged particles.

(j) $Z^{0} \rightarrow \bar{g} \bar{g}[5.45,5.46]$. This decay occurs via the one-loop diagrams of fig. 32 and was discussed briefly in section 4.3 . The branching ratios are expected to be $\leqslant 10^{-4}$ and will be therefore difficult to detect.

\subsection{Supersymmetry in two-photon physics and miscellaneous processes}

Very high energy $\mathrm{e}^{+} \mathrm{e}^{-}$colliders such as LEP will also provide an arena for the study of two-photon physics [5.47]. Clearly, this is not the area where supersymmetry will first be observed. However, once the effects of new physics are observed elsewhere, the study of $\gamma \gamma$ physics can serve as a cross-check of our understanding of the new phenomena.

In addition linear $\mathrm{e}^{+} \mathrm{e}^{-}$colliders can be used to produce a high energy beam of real photons. This would allow for the study of $\gamma e$ collisions. Finally, one can imagine studying the physics of $\mathrm{e}^{-} \mathrm{e}^{-}$ collisions.

We briefly summarize here some of the studies made in the literature regarding the effects of supersymmetry in $\gamma \gamma$ collisions and miscellaneous processes at electron machines.

\section{(a) Structure function of the photon $[5.48,5.49]$}

The structure function of the photon as predicted by perturbative QCD in the standard model will change once the threshold for scalar quark pair production is surpassed. The relevant diagrams for $\gamma \gamma \rightarrow \tilde{\mathrm{q}} \tilde{\mathrm{q}}$ are shown in fig. 53. The supersymmetric corrections to the structure function $F_{2}^{\gamma}$ [5.48] and $F_{\mathrm{L}}^{\gamma}$ [5.49] have been computed. The analogous computations for the supersymmetric corrections to the structure functions of the proton obtained from deep inelastic ep scattering are discussed in section 7.1. The conclusions in both cases are the same: one needs to have sufficiently large energies (above the threshold for production of new supersymmetric particles) in order to obtain a discernible effect which can clearly be distinguished from an alternate explanation based on the Standard Model.

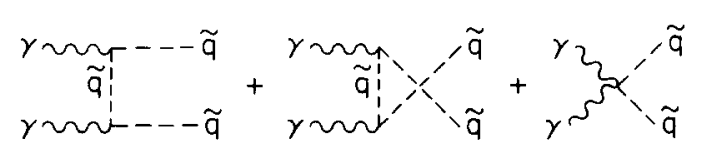

Fig. 53. Graphs for $\gamma \gamma \rightarrow \bar{q} \bar{q}$. The photons can be either on-shell or off-shell.

\section{(b) Production of scalar quarks and leptons [5.50]}

One can search directly for evidence for $\mathrm{e}^{+} \mathrm{e}^{-} \rightarrow \mathrm{e}^{+} \mathrm{e}^{-} \tilde{\ell}^{+} \tilde{\ell}^{-}$or $\mathrm{e}^{+} \mathrm{e}^{-} \rightarrow \mathrm{e}^{+} \mathrm{e}^{-} \tilde{\mathrm{q}} \tilde{\tilde{q}}$. The subprocesses responsible for these final states include $\gamma \gamma \rightarrow \tilde{\ell}^{+} \tilde{\ell}^{-}, \gamma \tilde{\gamma} \rightarrow \ell^{+} \tilde{\ell}^{-}$and $\tilde{\gamma} \tilde{\gamma} \rightarrow \ell^{+} \ell^{-}$. Computations performed in ref. [5.50] find for example that at $\sqrt{s}=200 \mathrm{GeV}$ and $\tilde{M}_{\ell} \simeq 15 \mathrm{GeV}$ leads to cross sections of order $10^{-36} \mathrm{~cm}^{2}$ which correspond to a few events per day at expected LEP luminosities. Backgrounds will be extremely severe and the viability of such a measurement is extremely doubtful. 


\section{(c) Photoproduction of supersymmetry [5.51, 5.52]}

At linear $\mathrm{e}^{+} \mathrm{e}^{-}$colliders or ep colliders, one can obtain an intense source of high energy real photons. One can then study $\gamma$ e collisions which have approximately the same energies and luminosities as the corresponding $\mathrm{e}^{+} \mathrm{e}^{-}$collisions. One can therefore investigate the production of supersymmetric particles in $\gamma \mathrm{e}$ collisions. The main advantage here would be that for (nearly) massless photinos, one can search for scalar-leptons with masses almost up to the beam energy by looking for $\gamma \mathrm{e} \rightarrow \tilde{\gamma} \tilde{\mathrm{e}}[5.51,5.52]$. The rates will be superior to $\mathrm{e}^{+} \mathrm{e}^{-} \rightarrow \mathrm{e}^{+} \tilde{\mathrm{e}}^{-} \tilde{\gamma}$ [5.5-5.8]; the signatures of such events will be similar. One can also look for $\gamma \mathrm{e} \rightarrow \tilde{\mathrm{w}} \tilde{\nu}$ [5.52] which will also have a distinctive signature. If polarized photons are available, it may be possible to discriminate between $\tilde{\ell}_{\mathrm{L}}$ and $\tilde{\ell}_{\mathrm{R}}$ production [5.52].

\section{(d) Supersymmetry in $e^{-} e^{-}$collisions [5.53]}

The study of $\mathrm{e}^{-} \mathrm{e}^{-} \rightarrow \tilde{\mathrm{e}}^{-} \tilde{\mathrm{e}}^{-}$via $\mathrm{t}$-channel photino exchange can reveal interesting features regarding the Majorana nature of the photino. This process apparently violates fermion number conservation, which is permissible in a theory containing Majorana particles. It is interesting to observe that such effects persist even when $\tilde{M}_{\gamma}=0$. In this limit we note that $\sigma\left(\mathrm{e}^{-} \mathrm{e}^{-} \rightarrow \tilde{\mathrm{e}}_{\mathrm{L}} \tilde{\mathrm{e}}_{\mathrm{L}}\right)=\sigma\left(\mathrm{e}^{-} \mathrm{e}^{-} \rightarrow \tilde{\mathrm{e}}_{\mathrm{R}} \tilde{\mathrm{e}}_{\mathrm{R}}\right)=0$ but $\sigma\left(\mathrm{e}^{-} \mathrm{e}^{-} \rightarrow\right.$ $\left.\tilde{\mathrm{e}}_{\mathrm{L}} \tilde{\mathrm{e}}_{\mathrm{R}}\right) \neq 0$. Of course, in an actual experiment, since $\tilde{\mathrm{e}} \rightarrow \mathrm{e} \tilde{\gamma}$, no fermion number violation would be apparent. Details of the process $\mathrm{e}^{-} \mathrm{e}^{--} \rightarrow \tilde{\mathrm{e}}^{--} \tilde{\mathrm{e}}^{-}$are worked out in ref. [5.54]. A pedagogical discussion of this calculation is given in appendix E.4.

\section{Supersymmetry at hadronic colliders}

Hadronic colliders may be copious sources of gluinos and scalar-quarks [6.1-6.7]. More important, if supersymmetric particle masses are larger than $m_{z} / 2$, very high energy hadronic colliders may be the only machines which can reveal the presence of new physics. Thus, it is very important to study seriously the production of new particles at the hadronic colliders.

The cross sections for the production of new supersymmetric particles, even if substantial, will be swamped by Standard Model processes. The extraction of new physics from Standard Model backgrounds will be a formidable task at the hadronic colliders. We have already discussed in detail some of the difficulties and the methods required to uncover gluino and scalar quark production in sections 3.3 and 3.4, and neutralinos in section 3.6. In this chapter we shall summarize the most likely signatures of supersymmetry in hadronic colliders. A comprehensive analysis of this subject has been given by Dawson, Eichten and Quigg [6.6].

Before we consider specific signatures due to the production of supersymmetric particles, we should note how general QCD predictions are modified if supersymmetry is taken into account. One can simply compute the one-jet inclusive cross section at large $p_{\mathrm{T}}$ and ask how the cross sections will change if supersymmetric particles are produced. Basically, one needs to have data at $p_{\mathrm{T}}$ substantially larger than the mass of the gluino and scalar quark. For example, in ref. [6.5], when the values $\tilde{M}_{\mathrm{g}}=2 \mathrm{GeV}$ and $\tilde{M}_{\mathrm{q}}=17 \mathrm{GeV}$ were chosen, for $\tilde{M}_{\mathrm{g}} \ll p_{\mathrm{T}} \ll \tilde{M}_{\mathrm{q}}$, the cross section was enhanced by a factor of about 1.3. If $p_{\mathrm{T}} \gg \tilde{M}_{\mathrm{q}}$, the cross section was enhanced significantly (e.g., by a factor of two at $p_{\mathrm{T}}=90 \mathrm{GeV}$ ).

Other tests can also be made which would differentiate supersymmetric QCD from ordinary QCD. In ref. [6.8] it is argued that by having polarized proton beams the measurement of helicity asymmetries could reveal departures from ordinary QCD. The helicity-dependent Born cross sections are computed for the various elementary processes into supersymmetric final states [6.8]. In principle, these results could be used to infer that gluino events were due to the production of a new heavy fermion. If, on the other hand, the gluino and scalar quark masses are much heavier (say $\mathrm{O}\left(m_{\mathrm{w}}\right)$ ), then such techniques will 
be of little value. The supersymmetric content of the observable cross sections will be small and careful analysis involving judicious cuts and Monte Carlos of Standard Model backgrounds [6.3] will be required to uncover the new physics. These latter techniques may be best in any case.

(a) Gluino production $[6.1-6.8]$

Gluinos can be produced via the diagrams shown in fig. 20. The cross sections of gluino production can be computed using the standard parton model techniques $[6.1,6.2,6.4-6.6]$. The relevant elementary cross sections have been collected in ref. [6.6]. The expected rates for gluino

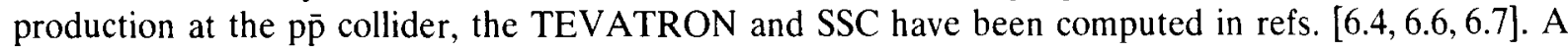
few of these results are displayed in figs. 21 and 22 . The $\tilde{g} \tilde{g}$ cross section is roughly a factor of ten larger than the production of heavy quarks of the same mass. This is due to larger color factors associated with the color octet gluinos.

The following features are noteworthy. First, the rates for $p p \rightarrow \tilde{g} \tilde{g}+X$ are only weakly dependent on the scalar-quark mass. The reason is that the dominant scattering mechanism $g \mathrm{~g} \rightarrow \tilde{\mathrm{g}} \tilde{\mathrm{g}}$ involves only the gg̃ $\tilde{g}$ vertex and hence depends only on the gluino mass. If $M_{\mathrm{q}} \gg M_{\mathrm{g}}$, then gluino pair production could be the only observable production of supersymmetric particles. The separation of $\tilde{g} \tilde{g}$ events from background is a nontrivial task as was described in section 3.4, subsection $C$. It will require detailed Monte Carlos of Standard Model backgrounds, and a judicious choice of cuts in variables such as $X_{\mathbf{E}}$ and $p_{\text {out }}$ (eqs. (3.27)-(3.28)) [6.3]. Second, the process $p p \rightarrow \tilde{\mathrm{g}} \tilde{\gamma}+\mathrm{X}$ [6.9] is the most dramatic signal of supersymmetry due to the large fraction of transverse energy lost in the outgoing photino. These rates are sensitive to the scalar-quark mass and thus will provide independent information on the supersymmetric parameters. However, there is a large background here from $\mathrm{pp} \rightarrow \nu \bar{\nu}+\mathrm{g}+\mathrm{X}$ which could result from associated production of a gluon and a $Z^{0}$ which decays into a neutrino pair (an $18 \%$ branching ratio). This background can be measured exactly by searching for $p p \rightarrow Z^{0}+g+X$ where $\mathrm{Z}^{0} \rightarrow \mathrm{e}^{+} \mathrm{e}^{-}$or $\mu^{+} \mu^{-}$. Thus, background subtraction should be fairly straightforward. Gluons can also be produced in association with scalar-quarks, charginos and neutralinos as discussed below.

\section{(b) Scalar-quark production [6.2-6.8,6.10,6.11]}

Scalar-quarks can be pair produced or singly produced in association with gluinos or photinos as shown in fig. 14. Elementary cross sections for the various processes depicted have been collected in ref. [6.6]. Computations of expected rates for scalar-quark production have been performed in refs. $[6.2,6.4-6.7]$. Roughly, one expects somewhat smaller rates for the production of a scalar-quark than for an ordinary heavy quark of the same mass. The exact results are model dependent and are sensitive to the gluino mass. Scalar-quarks can also be produced in association with charginos and neutralinos, although the rates tend to be smaller.

The signature of scalar-quark production is likely to be difficult to disentangle from the background, if the dominant decay of the scalar-quark is $\tilde{q} \rightarrow q+\tilde{g}$. On the other hand, if the dominant scalarquark decays are into other modes, the resulting signatures can be more favorable. For example, if $\tilde{M}_{\mathrm{g}}>\tilde{M}_{\mathrm{q}}$, then $\tilde{\mathrm{q}} \rightarrow \mathrm{q} \tilde{\gamma}$ is likely to dominate leading to substantial loss of transverse energy due to the escaping photino. Other decays, such as $\tilde{\mathrm{q}} \rightarrow \mathrm{qe} \tilde{\nu}[6.10]$ (if the scalar-neutrino were light) will probably be rarer and could be confused with ordinary heavy quark production. The most dramatic signal is $\mathrm{pp} \rightarrow \tilde{\mathrm{q}} \tilde{\gamma}+\mathrm{X}$ which can result in a significant loss of transverse energy even if the scalar-quark decays via $\tilde{\mathrm{q}} \rightarrow \mathrm{q} \tilde{\mathrm{g}}$. An additional advantage of this process is that the scalar-quark is singly produced so that sensitivity towards heavier scalar-quarks may be achieved. The disadvantage of this process is that it is suppressed by a factor of $e_{\mathrm{q}}^{2} \alpha / \alpha_{\mathrm{s}}$ over $\mathrm{pp} \rightarrow \tilde{\mathrm{q}} \tilde{\mathrm{g}}+\mathrm{X}$, which would be offset in rate only if the gluino were very massive. 
If the gluinos are light, then one can estimate the gluino content of the proton [6.12-6.15] (see section 7.1). The dominant production mechanism of scalar-quarks will simply consist of the elementary process $q+\tilde{g} \rightarrow \tilde{q}$ [6.11]. Although the scalar-quark will simply decay via the inverse process, occasionally the scalar-quark will decay via $\tilde{\mathrm{q}} \rightarrow \mathrm{q}+\tilde{\gamma}$. Such events will consist of one highly energetic unbalanced jet, i.e., a one-sided event.

Thus, it appears that signatures for scalar-quark production can be distinguished from background if one concentrates either on $\mathrm{pp} \rightarrow \tilde{\mathrm{q}} \tilde{\gamma}+\mathrm{X}$ or one looks for scalar-quarks which decay (albeit rarely if gluinos are light) into $\mathrm{q} \tilde{\gamma}$. These caveats suggest that high luminosity will be required to obtain sufficient data samples to uncover evidence for scalar-quarks.

\section{(c) Neutralino and chargino production [6.16-6.18]}

We have discussed in detail in chapter 4 how hadronic colliders can lead to supersymmetric particle production as final state decay products of the $\mathrm{W}$ and $\mathrm{Z}$ bosons, particularly with $\mathrm{W} \rightarrow \tilde{\mathrm{w}} \tilde{\gamma}, \tilde{\mathrm{w}} \rightarrow \overline{\mathrm{q}} \mathrm{q} \tilde{\gamma}$ giving a one-sided event with missing $p_{\mathrm{T}}$ and no charged lepton. Here, we simply emphasize that neutralinos and charginos can be produced via continuum quark-quark scattering processes as shown in figs. 35 and 38(b). The signatures for the various final states are the same as those discussed in section 4.2. The most dramatic signals will be the one-sided events which occur in $p p \rightarrow \tilde{\chi}_{1}^{0} \tilde{\chi}_{2}^{0}+X$ where $\tilde{\chi}_{2}^{0} \rightarrow \tilde{\chi}_{1}^{0}+$ charged particles. Eventually, at a high luminosity supercollider this process will provide the most definitive test of whether supersymmetry is present on the weak scale, because it has a clear signature and a cross section that is large enough to see even for quite heavy neutralinos. Neutralinos and charginos can also be produced in association with gluinos or scalar-quarks. Of these processes the $\tilde{w} \tilde{g}$ is likely to occur with the largest rate. However, precise predictions depend on the unknown chargino and neutralino mixing angles. Different choices for the mixing can lead to large variations in predicted cross sections.

\section{(d) Gluinonium production [6.19]}

In section (a) we noted that large color factors tended to enhance continuum gluino production over heavy quark production. The same reasoning suggests that hadronic production of gluinonium (a g̃ bound state) should be similarly enhanced over the production of quarkonium states. An additional feature, emphasized in section 3.4, subsection $\mathrm{E}$, is that the gluinonia can be produced in both color singlet and color octet states which will enhance the total signal. In order to detect the presence of gluinonium we concentrate on the production of the color singlet and color octet $\eta$-like states: $\tilde{\eta}_{\mathrm{g}}$ and $\tilde{\eta}_{\mathrm{g}}^{(8)}$. These will be produced as a resonance in gluon-gluon scattering: $\mathrm{gg} \rightarrow \tilde{\eta}_{\mathrm{g}} \rightarrow \mathrm{gg}$. To detect its presence one needs to search in a data sample of two-jet events for an enhancement in the two-jet invariant mass distribution. The background will be large, consisting of two-jet events arising from the hard 2-2 scattering of quarks and/or gluons. At sufficiently high energies the background will be dominated by gluon-gluon scattering and one can estimate the expected signal-to-nosie $(\mathrm{S} / \mathrm{N})$ due to the production of gluinonia of mass $\tilde{M}$. The calculation has been performed by Goldman and Haber in ref. [6.19]. The result they obtain (which depends on angular cuts applied to the data and the experimental resolution $\Gamma_{\mathrm{R}}$ of the two-jet mass measurement) is

$$
S / N \approx 5 \times 10^{-2} \times\left(1 / \alpha_{\mathrm{s}}^{2}\right)\left[\left(\tilde{\Gamma}+8 \tilde{\Gamma}_{8}\right) / \Gamma_{\mathrm{R}}\right],
$$

where $\tilde{\Gamma} \equiv \Gamma\left(\tilde{\eta}_{\mathrm{g}} \rightarrow \mathrm{gg}\right)$ is given by eq. (3.30) and $\tilde{\Gamma}_{8}=\frac{1}{4} \tilde{\Gamma}$ in the Coulombic approximation. Typically, $\tilde{\Gamma}+8 \tilde{\Gamma}_{\mathbf{8}} \sim \mathrm{O}(300 \mathrm{MeV})$. It is not unreasonable to expect future detectors to attain $\Gamma_{\mathbf{R}} \sim 10 \mathrm{GeV}$ which would lead to $S / N \sim 5 \%$. Such an experiment, if possible (as the estimates suggest), would provide an important means of identifying the gluino along with an accurate measurement of its mass. 


\section{Supersymmetry in ep physics}

In general, ep machines are not expected to be the place for the initial discoveries of supersymmetry. This is not because they cannot do it, but because other machines will cover the relevant mass ranges at an earlier date. Nevertheless, it is useful to consider the possible effects of the production of new supersymmetric particles. If supersymmetry is detected, some aspects can be well studied here. There are two facets to consider here. First, if gluinos and/or scalar-quarks are light, then the evolution of deep inelastic structure functions will be modified. Second, independent of the previous assumption, one may look for particular signatures which could signal the production of new supersymmetric particles. The ep machines may be particularly well suited to look for scalar-quarks and we shall discuss under what circumstances they could be first discovered here.

\subsection{Supersymmetric components of the nucleon}

Perturbative QCD allows one to predict the evolution of the distribution functions $f_{i}\left(x, Q^{2}\right)$ for the various partonic components of the nucleon. To leading order in $\alpha_{\mathrm{s}}$ and ignoring mass corrections $\mathrm{O}\left(m^{2} / Q^{2}\right)$ where $m$ is the mass of the parton in question, the $f_{i}\left(x, Q^{2}\right)$ satisfy

$$
\frac{\partial f_{i}\left(x, Q^{2}\right)}{\partial \ln Q^{2}}=\frac{\alpha_{\mathrm{s}}\left(Q^{2}\right)}{2 \pi} \sum_{j} \int_{x}^{1} \frac{\mathrm{d} y}{y} P_{i j}\left(\frac{x}{y}\right) f_{j}\left(y, Q^{2}\right)
$$

where $P_{i j}$ are the Altarelli-Parisi splitting functions [7.1] which specify the 'probability' of parton $j$ turning into parton $i$ in a deep inelastic scattering process. Note that the function $f_{i}\left(x, Q^{2}\right)$ is not determined by perturbative QCD; however, specifying the distribution functions at some initial point $Q^{2}=Q_{0}^{2}$ allows one to compute their evolution for $Q^{2}>Q_{0}^{2}$.

If scalar-quarks and gluinos are present, one would need to include their effects in the evolution equations given by eq. (7.1). Scalar-quarks have masses which are most likely greater than $15 \mathrm{GeV}$ so that their effects on the evolution of the structure functions at present energies are certainly negligible. On the other hand, light gluinos could exist (say, with masses below $5 \mathrm{GeV}$ ) as discussed in section 3.4. Hence, it seems plausible that light gluinos could have an observable effect on the behavior of structure functions observed at current facilities. This question has been studied in refs. [7.2-7.6]. The strategy is to set the gluino distribution function to zero at some $Q_{0}^{2}$ (chosen by ref. [7.4] to be $Q_{0}^{2}=2 \tilde{M}_{\mathrm{g}}^{2}$ ) and let it evolve as in eq. (7.1). ${ }^{*}$ Explicit forms for the splitting functions $P_{i j}$ taking gluinos into account can be found in refs. [7.2, 7.4,7.5] to lowest order and in ref. [7.3] including the next-to-leading order terms. Remarkably, the present data imposes virtually no new constraints on the gluino mass. For gluino masses above $1 \mathrm{GeV}$, the change in structure functions which might result from the effects of gluinos is entirely masked by the uncertainty in $\Lambda_{\mathrm{OCD}}$ in the regime $2<Q<15 \mathrm{GeV} / c$ where present data lies [7.3]. Therefore, we will need to go to higher energy ep machines (such as HERA) in order to have any chance of seeing new effects.

At larger $Q^{2}$, in addition to gluinos, one can be sensitive to the scalar-quarks. Furthermore, asymptotically (i.e., for $Q \gg \tilde{M}_{\mathrm{g}}, \tilde{M}_{\mathbf{q}}$ ), one expects that some nonzero fraction of the proton will reside

\footnotetext{
* This procedure certainly underestimates the supersymmetric content of the proton (as a similar procedure is known experimentally to underestimate the charm content of a proton). One could get large enhancements if the intrinsic charm model [7.13] could be applied to predict the intrinsic supersymmetry of the proton.
} 
Table 10

Asymptotic momentum fractions of constituents inside the hadron. [The following table, taken from ref. [7.5] gives the asymptotic momentum fractions of quarks (q), gluons $(\mathrm{g})$, scalar-quarks ( $\tilde{q})$ and gluinos $(\overrightarrow{\mathrm{g}})$ in four possible situations as labelled. (Supersymmetric QCD include both scalar-quarks and gluinos.) The numbers of flavors of quarks is denoted by $F$. Numerical values with $F=6$ are indicated in parentheses.]

\begin{tabular}{lllll}
\hline & Pure QCD & $\begin{array}{l}\text { Gluinos but no } \\
\text { scalar-quarks }\end{array}$ & $\begin{array}{l}\text { Scalar-quarks } \\
\text { but no gluinos }\end{array}$ & Supersymmetric QCD \\
\hline $\mathrm{q}$ & {$[3 F /(3 F+16)](0.53)$} & {$[3 F /(20+3 F)](0.47)$} & {$[3 F /(16+5 F)](0.39)$} & {$[3 F /(20+5 F)](0.36)$} \\
$\mathrm{g}$ & {$[16 /(3 F+16)](0.47)$} & {$[16 /(20+3 F)](0.42)$} & {$[16 /(16+5 F)](0.35)$} & {$[16 /(20+5 F)](0.32)$} \\
$\tilde{\mathrm{q}}$ & 0 & 0 & {$[2 F /(16+5 F)](0.26)$} & {$[2 F /(20+5 F)](0.24)$} \\
$\tilde{\mathrm{g}}$ & 0 & {$[4 /(20+3 F)](0.11)$} & 0 & {$[4 /(20+5 F)](0.08)$} \\
\hline
\end{tabular}

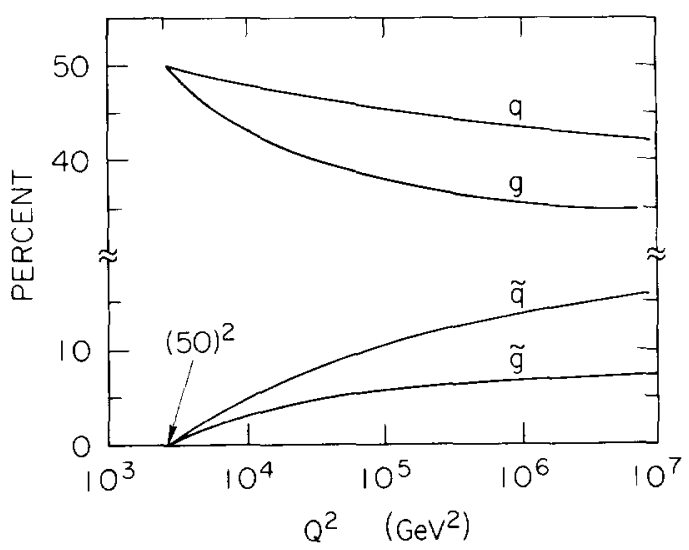

Fig. 54. Evolution of momentum fractions of the proton carried by various constituents as a function of $Q^{2}$ where the supersymmetric particles are switched on at $Q^{2}=(50 \mathrm{GeV})^{2}$. This figure was taken from ref. [7.5].

in supersymmetric particles, as shown in table 10, taken from ref. [7.5] (some of the entries were also computed in ref. [7.2,7.6]). Thus asymptotically, nearly one third of the content of the proton is supersymmetric. The approach to asymptotes is very slow however as indicated in fig. 54. Thus, one expects very weak limits for gluinos and scalar-quarks to be obtained from the HERA energy range $(Q \leqslant 300 \mathrm{GeV})$ by these methods. Still, an unexpected growth in the longitudinal structure function $\left(\sigma_{\mathrm{L}} / \sigma_{\mathrm{T}}\right)$ could be detected suggesting the presence of scalar-partons perhaps associated with supersymmetry [7.6]. However, evidence for associated production of new supersymmetric particles is likely to be a more fruitful area of study.

\subsection{Associated production of supersymmetric particles at ep machines}

The two main problems in extracting a supersymmetric signal in ep physics are low rates and significant backgrounds. Unlike the situation at hadron machines where one can trigger on missing energy and no accompanying lepton, here we have a lepton in the initial state. For example, the usual charge-current process ep $\rightarrow \nu \mathrm{X}$ leads to substantial missing energy with a rate significantly larger than the supersymmetric signals which are being looked for. We shall briefly survey the most likely supersymmetric reactions and their expected rates and signatures. Our conclusion is that the low rates 
and significant backgrounds expected at HERA $\left(\sqrt{s} \simeq 300 \mathrm{GeV}, \mathscr{L} \simeq 6 \times 10^{31} \mathrm{~cm}^{-2} \mathrm{~s}^{-1}\right)$ and other comparable or lower energy ep machines makes it highly unlikely that supersymmetry will first be discovered in ep physics. One possible exception to this forecast is the production of scalar-quarks in ep $\rightarrow \tilde{\nu} \tilde{\mathrm{g} X}$, as we shall discuss next.

\section{(a) $\mathrm{ep} \rightarrow \tilde{\mathrm{e}} \tilde{\mathrm{q} X}, \tilde{\nu} \tilde{\mathrm{q}} \mathrm{X}$}

This process occurs as shown in fig. 15. Its rate has been computed in refs. [7.5,7.7], although no complete analysis taking into account the possible mixing in the chargino and neutralino sector has been performed. We present two graphs from Jones and Llewellyn Smith in figs. 55 and 56 for the expected rates of these processes. For definiteness, they chose $\tilde{M}_{\gamma}=0, \tilde{M}_{\nu}=\tilde{M}_{\mathrm{e}}=\tilde{M}_{\mathrm{q}}=40 \mathrm{GeV}, \tilde{M}_{\mathrm{w}}=80 \mathrm{GeV}$ and $\tilde{M}_{\mathrm{z}}=90 \mathrm{GeV}$.

First, we consider the reaction ep $\rightarrow \tilde{\nu} \tilde{\mathrm{q} X}$. If the scalar-neutrino decays invisibly [e.g. $\tilde{\nu} \rightarrow \nu+\tilde{\gamma}$ as discussed in section 3.2(A)], then this process is difficult to distinguish from the Standard Model background ep $\rightarrow \nu \mathrm{X}$. One would have to somehow detect missing energy from the 'current jet' (i.e., the hadronic jet which arises from the parton which participated in the hard scattering). Jones and Llewellyn Smith [7.5] point out that if the scalar-quark loses a substantial amount of energy in the decay (as would be the case of $\tilde{\mathrm{q}} \rightarrow \mathrm{q} \tilde{\gamma}$ were the dominant decay), then the $x$ and $y$ variables measured in the decay (by measuring the outgoing hadronic system) are not their 'true' values. The end result is that one expects different results for the $x$ and $y$ distributions as measured in ep $\rightarrow \nu \mathrm{X}$ vs. ep $\rightarrow \tilde{\nu} \tilde{\mathrm{q} X}$.

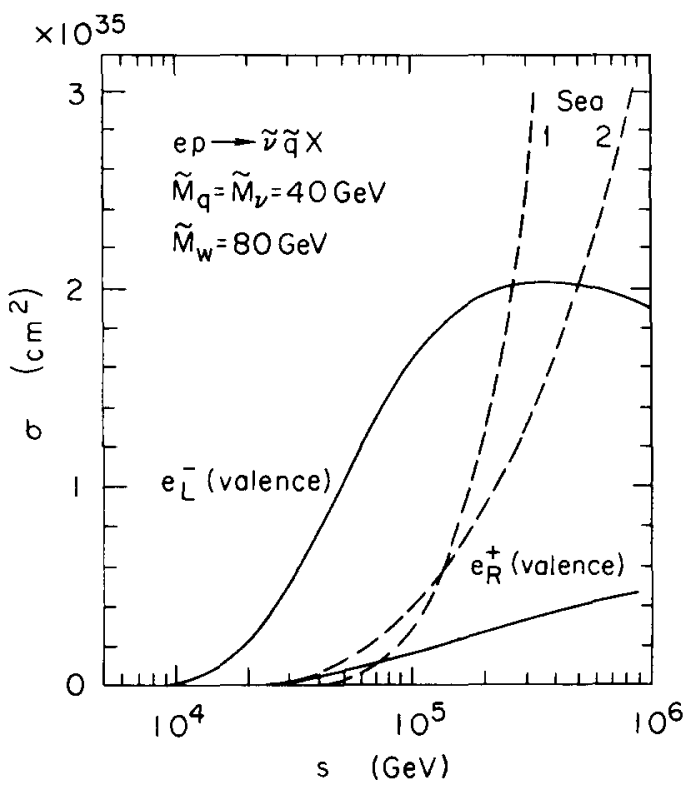

Fig. 55. Cross section for ep $\rightarrow \tilde{\nu} \tilde{q} X$ taken from ref. [7.5]. The valence (solid line) and sea (dashed line) contributions are shown separately; they must be added to get the full cross section. The sea contributions are very sensitive to the distribution functions used. Here 1 and 2 corresponds to the distributions of Owens and Reya [7.10] and Gluck, Hoffman and Reya [7.11] respectively.

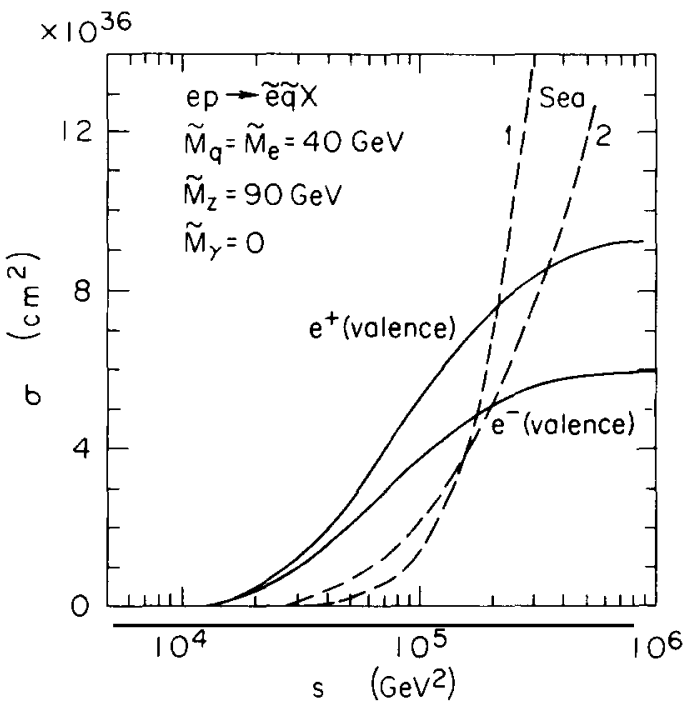

Fig. 56. Cross section for ep $\rightarrow \bar{e} \bar{q} X$ taken from ref. [7.5]. See caption to fig. 55 for details on notation. 
Appropriate cuts may be chosen to enhance the supersymmetric signal.* On the other hand, if the scalar-quarks decay via $\tilde{\mathrm{q}} \rightarrow \mathrm{q} \tilde{\mathrm{g}} \rightarrow \mathrm{q}(\mathrm{q} \tilde{\mathrm{q}} \tilde{\gamma})$, which would be the dominant decay if it is kinematically allowed, then the missing energy from the scalar-quark decay becomes harder to notice. However, $\sigma\left(\mathrm{e}^{-} \mathrm{p} \rightarrow \nu \mathrm{X}\right)=1.2 \times 10^{-34} \mathrm{~cm}^{2}$ at $s=10^{5} \mathrm{GeV}^{2}$ for left-handed electrons, nearly an order of magnitude larger in rate than the corresponding supersymmetric reaction. Under these circumstances, the search for a supersymmetric signal will be difficult.

Prospects are somewhat brighter if the scalar-neutrino decays into four-body charged modes (see section 3.2(A)). In this case there is a plethora of interesting signals which could be identified. The most likely decays of the scalar-neutrino will be into a lepton plus hadronic jets (e.g., $\tilde{\nu} \rightarrow$ eud $\tilde{\mathrm{d}}$ and $\tilde{\nu} \rightarrow \nu \mathrm{q} \overline{\mathrm{q}} \tilde{\mathrm{g}})$. These will lead to multi-jet events with relatively little missing energy if the $\tilde{\nu}$ decays into an electron. In addition, we would expect an occasional rare multi-lepton event in the event that the $\tilde{\nu}$ decays leptonically. The kinematics of such events would presumably allow them to be differentiated from the more copious multi-lepton events which occur when heavy quarks produced at the hadron vertex decay semi-leptonically. This is true because the scalar-neutrino decay occurs at the lepton vertex which is usually well separated kinematically from multi-body decays occurring at the hadron vertex. The observation of multi-jet decays originating from the lepton vertex would be an unambiguous signal of new physics. ${ }^{* *}$ This offers perhaps the best possible way to discover scalar-quarks. Unfortunately, if no such events were seen, it would not necessarily give (directly) new limits on scalar-quark masses. Rather, the limits one would obtain would be a function of the $\tilde{\nu}$ branching ratio into four-body states.

Second, we consider the reaction ep $\rightarrow \tilde{e} \tilde{q} X$. Because of the missing energy from $\mathrm{e} \rightarrow \tilde{\mathrm{e}} \tilde{\gamma}$ decays, $\mathrm{ep} \rightarrow \tilde{\mathrm{e}} \tilde{\mathrm{q} X}$ resembles ordinary ep $\rightarrow \mathrm{eX}$ at much lower $Q^{2}$ where the background is much higher in rate. However, because a substantial amount of energy is lost in the escaping photino, it should be possible to make the appropriate cuts in order to enhance the supersymmetric signal. In addition, in ep $\rightarrow \mathrm{eX}$, the measurement of the outgoing electron four-momentum allows one to predict (via the simple parton model) the direction and the energy of the 'current jet'. This correlation is clearly lost in ep $\rightarrow$ ẽ $\tilde{X}$ events, which could help distinguish a small signal from a large ep $\rightarrow \mathrm{eX}$ background. In addition, variables analogous to $X_{\mathrm{E}}$ and $\boldsymbol{p}_{\text {out }}$ (defined in the context of hadronic colliders; see eqs. (3.27), (3.28)) can be used to identify missing 'photino' energy. The major backgrounds which one must contend with are semi-leptonic decays of heavy quarks where the decay products consist of a highly energetic neutrino and a very low energy lepton which is not detected. Again, one can presumably separate out these kinds of events because the kinematics will differ from events where the missing energy is due to a particle emitted at the lepton vertex. Detailed Monte Carlos will be necessary to obtain quantitative estimates as to what scalar-quark and leptons masses HERA will be able to study.

\section{(b) ep $\rightarrow$ e g̃ $\tilde{g} X$}

This process is analogous to the similar process $e^{+} e^{-} \rightarrow q \bar{q} \tilde{g} \tilde{g}$ shown in fig. 47(a). It has the one advantage that its rate is independent of the mass of scalar-quarks and leptons. Unfortunately, even for light gluinos, the ratio $R \equiv \sigma(\mathrm{ep} \rightarrow \mathrm{e} \tilde{\mathrm{g}} \mathrm{gX}) / \sigma(\mathrm{ep} \rightarrow \mathrm{eX})$ is small as shown in refs. [7.2,7.7]. (As an example, for $s / 4 \tilde{M}_{\mathrm{g}}^{2}=10^{5}$ and $x=0.1, y=0.2$, Campbell, Ellis and Rudaz found $R \approx 5 \times 10^{-3}$ ). In addition to the small rate, the signature is extremely difficult to pick out of the background. Clearly, one

\footnotetext{
* Because of the large difference between the electron and proton beam energies at an ep collider (such as HERA), most events actually produce particles which are very near the beam direction and hence are unobservable. One must be very careful not to choose a set of kinematic cuts which eliminate a large fraction of observable events.

** New physics other than supersymmetry could explain such events. One such example would be the production of a new neutral heavy lepton which couples to electrons and can decay into multi-body final states.
} 
is far more likely to find evidence for $\tilde{\mathrm{g}} \tilde{\mathrm{g}}$ production in hadron machines where the rates are much larger.

(c) ep $\rightarrow \tilde{\mathrm{e}} \tilde{\chi}^{0}+\mathrm{X}, \tilde{\nu} \tilde{\chi}^{ \pm}+\mathrm{X}$

These processes involve the associated production of a scalar-lepton and a neutralino or chargino as shown in fig. 57. The rates depend on the unknown chargino and neutralino mass matrices. Salati and Wallet [7.8] compute a 'reduced' cross section $\sigma_{\mathrm{R}}=\sigma /\left(\alpha^{2}+\beta^{2}\right)$, where $\alpha$ and $\beta$ parameterize the eẽ $\tilde{\chi}^{0}$ and e $\tilde{\nu} \tilde{\chi}^{+}$vertices which are written as $-\mathrm{i} e\left(\alpha-\beta \gamma_{5}\right)$. They then plot $\sigma_{\mathrm{R}}$ as a function of the unknown masses and the center-of-mass energy. To get the actual cross section, one need only extract $\alpha$ and $\beta$ from the diagonalization of the chargino and neutralino mass matrices (see sections 3.5 and 3.6). The conclusion is that the rates are small, even at HERA. For example, at $\sqrt{s} \simeq 300 \mathrm{GeV}$ with luminosity $6 \times 10^{31} \mathrm{~cm}^{-2} \mathrm{~s}^{-1}$, and with $\tilde{M}_{x^{0}}=\tilde{M}_{\mathrm{e}}=20 \mathrm{GeV}$, we would expect about one e $\tilde{\chi}^{0}$ event per day. The reaction with the largest cross section is $\mathrm{e}^{-} \mathrm{p} \rightarrow \tilde{\mathrm{e}} \tilde{\gamma}+\mathrm{X}$. Neglecting the photino mass, Salati and Wallet estimate about $1 / 2$ to 20 e $\tilde{\gamma}$ events per day corresponding to $\tilde{M}_{\mathrm{e}}=20-60 \mathrm{GeV}$.

Even if the mass parameters are favorable, one still must be able to extract the ẽ $\tilde{\chi}$ events from Standard Model backgrounds. In addition, one must deal with non-supersymmetric versions of fig. 57 (such as $\mathrm{e}^{-} \mathrm{p} \rightarrow \mathrm{W}^{-}+\mathrm{X}$ ) which will lead to similar signatures. This background becomes comparable to the supersymmetric processes if the supersymmetric particle masses are larger than $60 \mathrm{GeV}$ [7.12].

(d) ep $\rightarrow$ eq̃̃ $X$, ep $\rightarrow$ eq̃̃ $X$

These processes may occur by the hard scattering processes $\gamma^{*} \mathrm{~g} \rightarrow \tilde{\mathrm{q}} \tilde{\mathrm{q}}$ and $\gamma^{*} \mathrm{q} \rightarrow \tilde{\mathrm{q}} \tilde{\mathrm{g}}$ as shown in fig. 58. Their rates were computed in refs. [7.5,7.7], and are very sensitive to scalar-quark masses. For $\tilde{M}_{\mathrm{q}}=40 \mathrm{GeV}$ (and ignoring the gluino mass), at $\sqrt{s} \simeq 300 \mathrm{GeV}$ with a luminosity of $6 \times 10^{31} \mathrm{~cm}^{-2} \mathrm{~s}^{-1}$, one expects roughly 10 events per day each of $\tilde{q} \tilde{q}$ and $\tilde{q} \tilde{g}$ type. Again, we expect the background to be severe and a statistical analysis using appropriate Monte Carlo techniques will be necessary. Once again, one is far more likely to find evidence for $\tilde{\mathrm{q}} \tilde{\mathrm{q}}$ and $\tilde{\mathrm{q}} \tilde{\mathrm{g}}$ production in hadron machines where the rates are higher.

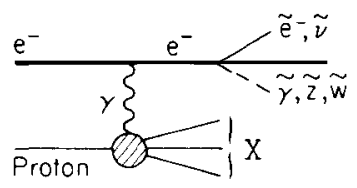

(a)

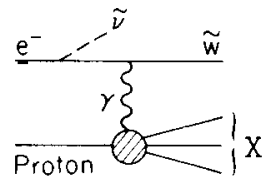

(b)

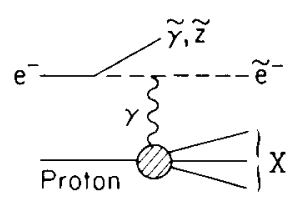

(c)

Fig. 57. Graphs for the associated production of a scalar-lepton and a neutralino or chargino. In these processes, it is the wino and zino components of the charginos and neutralinos which are important.

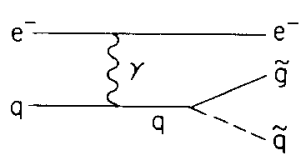

(o)

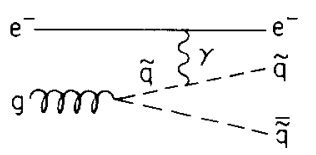

(c)

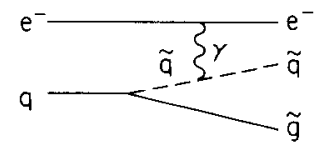

(b)

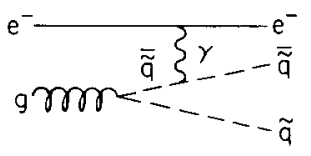

(d)

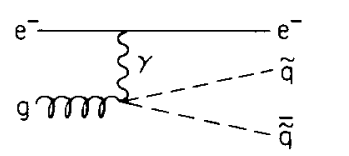

(e)

Fig. 58. Graphs for $\gamma \mathrm{g} \rightarrow \overline{\mathrm{q}} \overline{\tilde{q}}$ and $\gamma \mathrm{q} \rightarrow \tilde{\mathrm{q}} \bar{g}$ where the space-like photon is emitted from the incoming electron line. 


\section{Indirect constraints on supersymmetric parameters}

In the analysis presented so far in this review, various supersymmetric parameters (masses and mixing angles) were taken to be unknown quantities to be determined from experiment. Some direct constraints on the values of these parameters exist - these arise from the non-observation of supersymmetric particles at the present time. Those mass limits thus obtained were summarized in table 2 . In this section, we wish to summarize the indirect constraints which bear on the possible values of the supersymmetric parameters. These come from the effect of virtual supersymmetric particles, i.e., new particles which appear as intermediate states in Feynman diagrams for processes involving only ordinary particles as external states. In addition, we will consider briefly the implications of new supersymmetric particles on cosmology and the evolution of the early universe.

\section{1. $g-2$ of the electron and muon}

The anomalous magnetic moment of the electron (and muon) is one of the most accurately measured quantities on physics (with uncertainty appearing in the tenth significant figure for $\left.(g-2)_{e}\right)$. The theoretical prediction serves as a very accurate and at present successful test of QED. In fact, the experimental and theoretical numbers are such that one is nearly sensitive to virtual weak interaction effects. Thus, for any major change in the particle theory (such as supersymmetry) one must check that virtual effects due to the presence of new particles will not upset the previously described theoretical success.

We shall concentrate on the $g-2$ of the muon. Although the experimental measurement and theoretical prediction are not quite as accurate as in the electron case (here, the uncertainty appears in the eighth significant figure), it turns out that the contribution of new physics to $(g-2)_{\mu}$ is significantly larger than to $(g-2)$.

The limits allowed by experiment for $a_{\mu} \equiv(g-2)_{\mu} / 2$ are [8.1]

$$
-2 \times 10^{-8}<\Delta a_{\mu}<2.6 \times 10^{-8} \quad(95 \% \mathrm{CL}) .
$$

New graphs involving supersymmetric particles which contribute to $a_{\mu}$ are displayed in fig. 59 . Computations of $\Delta a_{\mu}$ which make various model assumptions about the supersymmetric spectrum have been performed in refs. [8.2-8.4, 8.95, 8.96]. One extracts $a_{\mu}$ by computing the graphs in fig. 59 and reading off the term which takes the form

$$
\left(\mathrm{i} e / 2 m_{\mu}\right) F\left(q^{2}\right) \bar{u} \sigma_{\mu \nu} q^{\nu} u
$$

and identifies $a_{\mu}=F(0)$. Note that because a factor of $m_{\mu}$ has been extracted (for dimensional reasons),

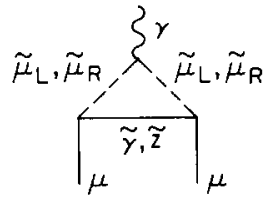

(a)

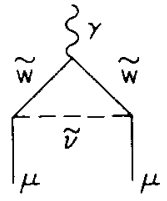

(b)

Fig. 59. Contributions to $g-2$ of the muon from supersymmetric particles. 
one immediately finds that $a_{\mu}$ must be proportional to $m_{\mu}$. In addition, because the operator given in (8.2) involves a L-R transition, a helicity flip must occur on one of the fermion lines in fig. 59. Thus, one typically expects on general principles either

$$
\Delta a_{\mu} \sim \alpha m_{\mu}^{2} / M_{\mathrm{x}}^{2}
$$

or

$$
\Delta a_{\mu} \sim \alpha m_{\mu} M_{\mathrm{F}} / M_{\mathbf{x}}^{2}
$$

to leading order in $m_{\mu}^{2} / M_{\mathrm{x}}^{2}$ where $M_{\mathrm{x}}$ is the mass of some heavy particle in the loop and $M_{\mathrm{F}}$ is the mass of one of the fermions in the loop. (Note that the appearance of $m_{\mu}$ explains why it is more fruitful to study $(g-2)_{\mu}$ as opposed to $(g-2)_{e}$.)

Clearly, if $M_{\mathrm{F}}$ is large, then a contribution such as eq. (8.3b) would dominate. However, in supersymmetry, one has chiral couplings appearing at the vertices (i.e., $V \pm A$ in the case of vectorfermion-fermion and $S \pm P$ in the case of scalar-fermion-fermion). Simple Dirac algebra or helicity arguments show that in the presence of chiral couplings, it is impossible to obtain a term in $a_{\mu}$ which is linear in $M_{\mathrm{F}}$ (more generally, $a_{\mu}$ must be an even function of $M_{\mathrm{F}}$ ). This would rule out eq. (8.3b) and would lead again to eq. (8.3a).

A possible method to circumvent the above argument goes as follows. Although it is true that one has chiral couplings in supersymmetry, these couplings are relevant for the 'interaction' eigenstates (e.g., $\tilde{\mu}_{\mathrm{L}}$ and $\tilde{\mu}_{\mathrm{R}}$ ). The mass eigenstates are mixtures of the interaction eigenstates and therefore may exhibit nonchiral coupling. In this case, eq. ( $8.3 b)$ is once again allowed; but in this case it will be multiplied by a mixing parameter which vanishes as one approaches mass degeneracy (or the pure 'chiral' eigenstates). This is not unlike the GIM cancellation. For example, consider the class of diagrams represented by fig. 59(a). The scalar muons which are exchanged are mixtures of $\tilde{\mu}_{\mathrm{L}}$ and $\tilde{\mu}_{\mathrm{R}}$ as described in appendix C.1. Using the notation of eqs. (C2)-(C5), one finds

$$
\Delta a_{\mu} \sim \cos \theta \sin \theta m_{\mu}\left(\tilde{M}_{\mu_{1}}^{2}-\tilde{M}_{\mu_{2}}^{2}\right) / \tilde{M}_{\chi}^{3}
$$

where here $\tilde{M}_{\chi}$ is the mass of the relevant neutralino (where it was assumed that $\left|\tilde{M}_{\mu_{1}}^{2}-\tilde{M}_{\mu_{2}}^{2}\right| \ll \tilde{M}_{\chi}^{2}$ ). As promised, this vanishes in the appropriate limits.

A similar discussion can be made regarding the effects of mixing in the chargino and neutralino sectors. Thus, naively, one would expect a formula similar to that of eq. (8.4) in this case as well. However, due to the mixing with higgsinos, one finds another factor of $m_{\mu}$ appearing from the $\mu \tilde{\mu} \tilde{\mathrm{H}}$ coupling. Thus, these contributions tend to be small.

Finally, it is interesting to note that in the exact supersymmetry limit, $a_{\mu}=0$ (not $\Delta a_{\mu}=0$ !) as originally shown in ref. [8.5]. This is true because there is no operator appearing in the effective potential which is a supersymmetric generalization of eq. (8.2). However, in realistic models of broken supersymmetry, this observation has no real practical implications.

We now turn to the limits implied by the above result on the supersymmetric parameters. The basic conclusion is that the constraints obtained are fairly weak [8.2-8.4, 8.95, 8.96]. If we ignore eq. (8.4) (say if $\tilde{M}_{\mu_{1}} \approx \tilde{M}_{\mu_{2}}$ ), then the constraints obtained are roughly $\tilde{M}_{\mu} \geqslant 15 \mathrm{GeV}$ and $\tilde{M}_{\mathrm{w}^{ \pm}} \geq 10 \mathrm{GeV}$ which are not as restrictive as the direct search limits. In addition, given $\tilde{M}_{\mathrm{w}^{ \pm}} \gtrsim 15 \mathrm{GeV}$, one obtains no restriction on the scalar neutrino mass. The only constraint of interest can come from eq. (8.4). Most models however predict 


$$
\tilde{M}_{\mu_{1}}^{2}-\tilde{M}_{\mu_{2}}^{2} \sim m_{\mu} M_{\mathbf{x}}
$$

which then implies the same weak constraints as eq. (8.3a). In principle, one can get a limit on the splitting of scalar muon eigenstates from eq. (8.4), but such limits do not appear so interesting when seen in the context of most models.

\subsection{Flavor changing neutral currents}

One of the major successes of the Standard Model is the natural explanation for the rarity of flavor changing neutral current processes. The GIM mechanism insures that there is no tree level $\bar{s} \mathrm{~d} Z^{0}$ coupling and leads to additional suppression of one-loop effects due to the appearance of $\Delta \mathrm{m}_{\mathrm{q}}^{2} / \mathrm{m}_{\mathrm{q}}^{2}$ factors ( $\Delta m_{\mathrm{q}_{12}}^{2} \equiv m_{\mathrm{q}_{1}}^{2}-m_{\mathrm{q}_{2}}^{2}$ where $\mathrm{q}_{1}$ and $\mathrm{q}_{2}$ are quarks of different flavor but the same electric charge). Such delicate cancellations could be upset by the introduction of new particles and interactions. Hence, one must carefully reconsider various neutral current processes in light of supersymmetry.

Various calculations appear in the literature which attempt to obtain constraints on supersymmetric models from the limits on flavor changing neutral current processes [8.6-8.15] (rare $\mathrm{K}$ and $\pi$ decays are discussed separately in section 8.6). As an example, consider the $\Delta S=2$ operator which contributes to $\mathrm{K}^{0}-\overline{\mathrm{K}}^{0}$ mixing. New box diagrams which are illustrated in fig. 60 can contribute. One interesting feature is the possible contributions of neutral gauge fermion exchange, in particular the gluino (which could be light). This would require the existence of off-diagonal $\mathrm{q}_{i} \tilde{\mathrm{q}}_{j} \tilde{\mathrm{g}}$ couplings $(i \neq j$ are flavor indices). Off-diagonal couplings would arise if the matrices which diagonalized the quark sector failed to diagonalize the scalar-quark sector. In fact, flavor changing neutral currents would be too large if the quark and scalar-quark diagonalizing matrices were not nearly equal. In most fashionable models (see chapter 9), these matrices are naturally exactly equal at tree level but do become unequal when radiative corrections are taken into account. In general, these corrections are not disastrous, but they do lead to interesting bounds on other parameters of the supersymmetry model.

A convenient table of results is presented by Campbell in ref. [8.10]. One typically finds appearance of $\Delta \tilde{M}_{\mathrm{q}_{i j}}^{2}$ and $\Delta \tilde{M}_{\ell_{i j}}^{2}$ factors which are a consequence of a super-GIM mechanism. The strongest constraints on the first two generations of scalar quarks and leptons come from the imaginary part of the $\mathrm{K}^{0}-\overline{\mathrm{K}}^{0}$ mass matrix and the non-observance of $\mu \rightarrow \mathrm{e} \gamma$. To get some feel for the numbers, consider the case where $\tilde{M}_{\mathrm{q}}, \tilde{M}_{\ell} \leqslant \tilde{M}_{\mathrm{w}} \approx m_{\mathrm{w}}$. Ignoring possible nondiagonal gluino couplings and setting ratios of mixing angles to unity, we find

$$
\begin{aligned}
& \Delta \tilde{M}_{\mathrm{q}_{i j}}^{2} / \tilde{M}_{\mathrm{q}}^{2} \lesssim \mathrm{O}\left(10^{-3}\right), \\
& \Delta \tilde{M}_{\ell_{i j}}^{2} / \tilde{M}_{\ell}^{2} \leqslant \mathrm{O}\left(10^{-3}\right) .
\end{aligned}
$$

These results are to be taken as a rough guide. Still, they have important implications. For example,

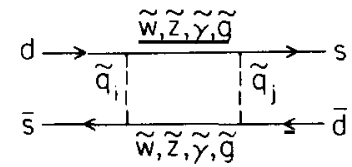

(a)

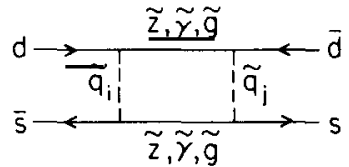

(b)

Fig. 60. Contributions to $\mathrm{K}^{0}-\overline{\mathrm{K}}^{0}$ mixing from box diagrams involving supersymmetry particles. 
successive scalar-quark flavors will be nearly degenerate in mass. This could have dramatic implications for $\mathrm{e}^{+} \mathrm{e}^{-}$physics should one be able to cross the scalar quark threshold [8.6]. One could find multiple flavors of scalar quarks all within the beam energy spread. In fact many supersymmetric models satisfy the mass formula (neglecting radiative corrections)

$$
\tilde{M}_{i}^{2}-\tilde{M}_{j}^{2}=m_{i}^{2}-m_{j}^{2}
$$

for quark and lepton flavors $i, j$ of the same electric charge. This formula is consistent with eq. (8.6) (recall that this formula is applicable only for the first two generations) as long as $\tilde{M}_{\mathrm{c}} \geqslant 50 \mathrm{GeV}$.

The analysis described above can also set limits on flavor changing gluino exchanges. Here the effect is potentially larger because gluinos may be light and interact with strong interaction coupling strength. The exact limits one can get depend in detail on the model considered. In a class of supergravity models, constraints were obtained in ref. [8.12] for the gravitino mass: $\tilde{M}_{\mathrm{g}_{3 / 2}} \geq 37 \mathrm{GeV}$. This is a fairly strong constraint with implications for other supersymmetric particles whose masses depend on $\tilde{M}_{\mathrm{g}_{3 / 2}}$.

\subsection{Parity violation in the strong interactions}

As discussed in appendix C.1, scalar-quark and scalar-lepton mass eigenstates are mixtures of the interaction eigenstates which we denote $\tilde{\mathrm{f}}_{\mathrm{L}}$ and $\tilde{\mathrm{f}}_{\mathrm{R}}$. If the masses of the physical states (denoted by $\tilde{\mathrm{f}}_{1}$ and $\tilde{\mathrm{f}}_{2}$ ) are different, then in general, the interactions of $\tilde{\mathrm{f}}_{1}$ and $\tilde{\mathrm{f}}_{2}$ will violate parity. ${ }^{*}$ It is possible that virtual exchange of $\tilde{f}_{1}$ and $\tilde{f}_{2}$ could lead to the introduction of parity violation into strong interaction processes [8.16,8.17]. (Examples of this feature have appeared previously in this review; see the discussion of the parity violating decay $\tilde{\mathrm{g}} \rightarrow \mathrm{g} \tilde{\gamma}$ in section 3.4(A)). The magnitude of parity violation is characterized by $\Delta \tilde{M}_{\mathrm{q}}^{2} / \tilde{M}_{\mathrm{q}}^{2}$ where $\Delta \tilde{M}_{\mathrm{q}}^{2} \equiv \tilde{M}_{\mathrm{q}_{1}}^{2}-\tilde{M}_{\mathrm{q}_{2}}^{2}, \tilde{\mathrm{q}}_{1}$ and $\tilde{\mathrm{q}}_{2}$ being the scalar-quark eigenstates corresponding to a given flavor. The most stringent limits come from the un and $\tilde{\mathrm{d}}$ scalar-quarks since it is virtual effects involving these particles which can result in apparent parity violating effects in the strong interactions. The Standard Model has been applied with some qualitative success in explaining nuclear parity violation in terms of nonleptonic weak interaction physics. We therefore conclude that parity violation from supersymmetric sources must be no bigger than that which is due to the Standard Model.

Suzuki and Duncan [8.16,8.17] have both analyzed the effect of supersymmetry on the parity violating component of the nucleon-nucleon interaction. One can study separately $\Delta I=0,1$, and 2 components of this interaction by computing effective four-fermion operators of definite isospin. The parity-violating pieces disappear when $\Delta \tilde{M}_{\mathrm{q}}^{2}=0$. In addition, the $\Delta I \neq 0$ pieces vanish if $\tilde{M}_{\mathrm{u}}=\tilde{M}_{\mathrm{d}}$. Thus, there are two mass-squared differences to constrain:

$$
\begin{aligned}
& d_{1}=\left|\left(\tilde{M}_{\mathrm{L}}^{2}-\tilde{M}_{\mathrm{R}}^{2}\right) / \tilde{M}_{0}^{2}\right| \\
& d_{2}=\left|\left(\delta \tilde{M}_{\mathrm{L}}^{2}-\delta \tilde{M}_{\mathrm{R}}^{2}\right) / \tilde{M}_{0}^{2}\right|
\end{aligned}
$$

where $\tilde{M}_{0}^{2} \approx \tilde{M}_{\mathrm{L}}^{2} \approx \tilde{M}_{\mathrm{R}}^{2}$ and $\delta \tilde{M}_{\mathrm{L}, \mathrm{R}}^{2} \equiv \tilde{M}_{\mathrm{d}, \mathrm{R}}^{2}-\tilde{M}_{\mathrm{uL}, \mathrm{R}}^{2}$. For simplicity, we have assumed that $\tilde{\mathrm{q}}_{\mathrm{L}}$ and $\tilde{\mathrm{q}}_{\mathrm{R}}$ are the appropriate mass eigenstates. The maximum splittings $d_{1}$ and $d_{2}$ allowed for a given gluino mass and scalar-quark mass $\tilde{M}_{0}$ are given in figs. 61 and 62 (taken from ref. [8.17]). Note that as the gluino and scalar-quark masses get larger, this constraint becomes less severe.

\footnotetext{
${ }^{*}$ The states $\left(\tilde{f}_{\mathrm{L}} \pm \overline{\mathrm{f}}_{\mathrm{R}}\right) / \sqrt{2}$ have definite parity quantum numbers. Hence, parity is violated if the physical states are mixtures of these two states.
} 


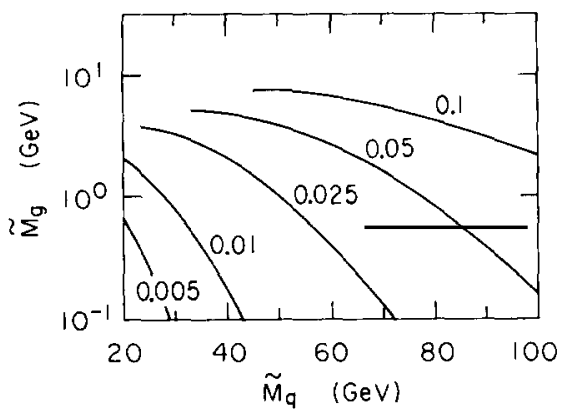

Fig. 61. Maximum helicity mass splittings for scalar-quarks $\left(d_{1}\right)$ allowed as a function of gluino and scalar-quark masses are displayed as numbers labelling the curves above. See eq. (8.9). This figure was taken from ref. [8.17].

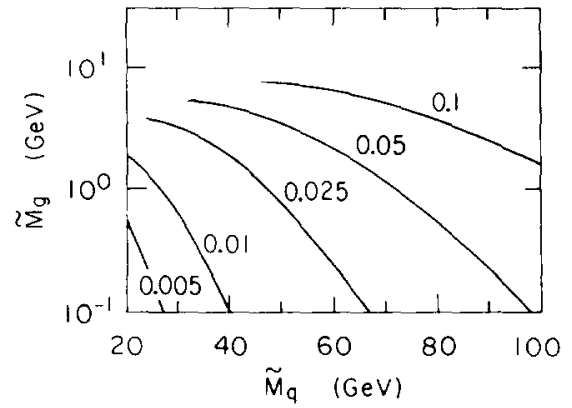

Fig. 62. Maximum isotopic mass splittings for scalar-quarks $\left(d_{2}\right)$ allowed as a function of gluino and scalar-quark masses are displayed as numbers labelling the curves above. See eq. (8.10). This figure was taken from ref. [8.17].

It should be pointed out that no such restrictions are known for second or third generation scalar quarks. This has some interesting consequences. For example, certain models naturally suggest small splittings of $\tilde{q}_{L}$ and $\tilde{q}_{R}$ masses except in the $\tilde{\mathrm{t}}$ scalar-quark sector. Thus, significant parity violation could occur in toponium decay, e.g. $\mathrm{V}(\mathrm{t} \overline{\mathrm{t}}) \rightarrow \tilde{\mathrm{g}} \tilde{\mathrm{g}}$ (see section 5.2(a)), or significant parity violation could appear in strange particle or charmed particle interactions or decays.

\subsection{The $\rho$-parameter}

The $\rho$-parameter is defined by

$$
\rho=M_{\mathrm{w}}^{2} / m_{\mathrm{z}}^{2} \cos ^{2} \theta_{\mathrm{w}}
$$

In the Standard Model of electroweak interactions, $\rho=1$ at tree level and thus is not an independent parameter. The symmetry point $\rho=1$ is the result of an approximate 'custodial' SU(2) symmetry which exists in the theory. Deviations from $\rho=1$ can occur in higher orders of perturbation theory. The most well-known example is the case where a quark doublet exists consisting of one heavy quark of mass $m_{\mathrm{q}}$ and one massless quark. Then the leading correction to $\rho=1$ is found to be [8.18]

$$
\delta \rho=3 \alpha m_{\mathrm{q}}^{2} / 16 \pi \sin ^{2} \theta_{\mathrm{w}} m_{\mathrm{w}}^{2}
$$

If we take the present experimental determination of $\rho$ to be $[8.103] \rho_{\exp }=1.002 \pm 0.015$, one obtains $m_{\mathrm{q}} \leq 300 \mathrm{GeV}$.

One can now ask whether the experimentally measured $\rho$-parameter puts any useful constraints on supersymmetric masses. This issue has been discussed in refs. [8.19-8.22]. Contributions to $\delta \rho$ can arise from mass splittings which may occur in weak doublets of supersymmetric particles. Thus, for example, $\delta \rho$ is sensitive to $\delta \tilde{M}_{\mathrm{L}}^{2}$ (using the notation of eq. (8.10)) since L-type scalar quarks occupy weak $\mathrm{SU}(2)$ doublets (whereas R-type scalar-quarks are singlets under weak SU(2)). For simplicity, we will assume that there is no mixing between L-type and R-type scalar-quarks. Then, for the $\tilde{t}_{L}$ and $\tilde{b}_{\mathrm{L}}$ scalar-quarks with masses $M_{1}$ and $M_{2}$ respectively, one finds approximately: 


$$
\delta \rho=\frac{3 \alpha}{16 \pi \sin ^{2} \theta_{\mathrm{w}} m_{\mathrm{w}}^{2}}\left[M_{1}^{2}+M_{2}^{2}-\frac{2 M_{1}^{2} M_{2}^{2}}{M_{1}^{2}-M_{2}^{2}} \log \left(\frac{M_{1}^{2}}{M_{2}^{2}}\right)\right]
$$

Note that for $M_{1}=M_{2}, \delta \rho=0$ as required. The strongest constraint is obtained by setting $M_{2}=0$. The resulting formula is identical to eq. (8.12) with $m_{\mathrm{q}}$ replaced by $M_{1}$. We conclude that the constraint imposed by the $\rho$-parameter simply prohibits very large $\mathrm{O}(100 \mathrm{GeV})$ mass splitting within scalar quark doublets. One can also examine contributions to $\delta \rho$ from the gaugino/higgsino sector and from the Higgs sector. Results for $\delta \rho$ on the whole tend to be small in realistic models (not much larger than a few parts in $10^{-3}$ ) and well within the experimental bounds. We conclude that the constraint due to $\delta \rho$ is not a severe limit on model builders.

\subsection{CP-violation}

There are three separate issues concerning $C P$-violation in supersymmetric models which have been discussed in the literature. The first is $C P$-violating parameters in the kaon system (the $\varepsilon$ and $\varepsilon^{\prime}$ parameters) [8.23-8.26, 8.14], the second is the neutron and electron electric dipole moments (denoted by $d_{\mathrm{n}}$ and $d_{\mathrm{e}}$ respectively) [8.26-8.30] and the third is the question of $\theta_{\mathrm{OCD}}$ and the strong $C P$ problem $[8.23,8.26,8.31-8.37]$.

Regarding the first two questions, supersymmetry considerations come into play because of new complex phases which can be introduced $[8.24-8.30,8.34]$. In the same way that a complex phase appears in the Kobayashi-Maskawa mass matrix, one also finds the possibility of complex phases in the chargino and neutralino mass matrices. (These phases were in fact ignored in our analysis of the mixing matrices in sections 3.5 and 3.6.) In addition, because there are multiple Higgs multiplets (at least two doublets) in the theories, relative phases may appear among the vacuum expectation values. This second point is of interest because it served as the basis for the Lee-Weinberg model of $C P$-violation [8.38] in which $C P$-violation was generated entirely through the Higgs couplings. Experimental limits on $\varepsilon^{\prime} / \varepsilon$ may have excluded this model as the sole explanation of $C P$-violation in the kaon sector [8.39] so it is of interest to see if a similar conclusion can be made for supersymmetric theories.

In a supersymmetric context, this issue is related to the presence of arbitrary phases which appear in the chargino, neutralino and gluino mass matrices. Supersymmetric theories also require at least two weak Higgs doublets so that we naturally have a structure similar to that of the Lee-Weinberg model. However, if the Higgs sector is again assumed to provide the sole explanation for the observed $C P$-violation, similar difficulties are encountered. Namely, the $\Delta S=1 C P$-violation parameter $(\xi)$ is larger than the $\Delta S=2$ piece and may lead to too large a value for $\varepsilon^{\prime} / \varepsilon$. Furthermore, the value for $d_{\mathrm{n}} / \xi$ is much larger than the experimental bound $[8.25,8.40]$, unless one performs an unnatural fine tuning of parameters (such as an adjustment of $\theta_{\mathrm{OCD}}$ which can provide additional contributions to $d_{\mathrm{n}}$ ). ${ }^{*}$

Finally, we come to the question of $\theta_{\mathrm{QCD}}$. This is known as the 'strong $C P$ problem' [8.42]. Although it is in some sense a theorist's problem, its solution can have powerful constraints on model building. Briefly stated, to account for the nontrivial topological structure of the QCD ground state, one uses an effective Lagrangian

$$
\mathscr{L}_{\text {eff }}=\mathscr{L}_{\text {QCD }}+\left(\theta g^{2} / 32 \pi^{2}\right) F_{\mu \nu} \tilde{F}^{\mu \nu}
$$

\footnotetext{
${ }^{*}$ Since $\theta_{\mathrm{OCD}}$ does not contribute to $d_{\mathrm{e}}$, a more accurate measurement of the dipole electric moment of the electron can add additional constraints to the model building [8.41].
} 
The second term in eq. (8.14) violates $P$ and $T$ invariance and thus contributes to the electric dipole moment of the neutron (though not the electron).

One cannot simply set $\theta=0$ since there is $C P$-violation in the weak interactions. A fermion mass matrix $M$ which can be made real and diagonal at the tree level will not remain so when radiative corrections are accounted for. Thus, we will have to rediagonalize $M$ with unitary transformations $U_{\mathbf{L}}$ and $U_{\mathrm{R}}$ :

$$
M_{\text {Diag }}=U_{\mathrm{R}}^{\dagger} M U_{\mathrm{L}}
$$

The result is that the physically relevant $C P$-violating parameter is given by

$$
\bar{\theta}=\theta+\arg \operatorname{det} U_{\mathrm{R}}^{\dagger} U_{\mathrm{L}} \text {. }
$$

It is this parameter which is constrained by the experimental upper bound on the electric dipole moment of the neutron: $\bar{\theta}_{\text {exp }} \lesssim \mathrm{O}\left(10^{-9}\right)$.

In the Standard Model, $\bar{\theta}$ is a parameter which must be renormalized. Still, it is hard to understand why such a parameter which was a priori arbitrary is so small. As we have mentioned, $\bar{\theta}=0$ is not a stable (natural) value as long as there are hard (i.e., dimension 4) operators which result in $C P$-violation elsewhere in the theory.

One solution proposed in the context of the Standard Model is the Peccei-Quinn mechanism [8.43]. This consists of expanding the Higgs sector to allow for a new U(1) chiral symmetry with which one could 'rotate' the $\theta$-parameter to zero. A consequence of this mechanism is the existence of the axion [8.44], a very light weakly interacting pseudoscalar. Because supersymmetry requires two Higgs doublets, supersymmetric models have been regarded as a natural home for the Peccei-Quinn mechanism. This is discussed in refs. [8.31-8.36, 8.26].

Supersymmetry, however, allows for a more novel solution to the strong $C P$ problem. Namely, the no-renormalization theorems of supersymmetry [8.45] guarantee that the fermion mass matrix $M$ (see eq. (8.15)) remains unchanged due to radiative corrections [8.23]. Thus, $\bar{\theta}$ is stable under radiative corrections and can be chosen to be zero (though, supersymmetry does not 'explain' this choice). When global supersymmetry is spontaneously broken, renormalization corrections to $\bar{\theta}$ are calculable and finite. Models can be constructed where the computed $\bar{\theta}$ is sufficiently small so as not to conflict with experiment. Supergravity theories are more subtle - in these theories, the effective low energy theory has supersymmetry broken by soft-supersymmetry breaking terms. This is not sufficient to guarantee that the renormalization corrections to $\bar{\theta}$ are finite. The reason is that the gluino mass is infinitely renormalized in a softly broken supersymmetric theory [8.46-8.48]. The imaginary part of the gluino mass parameter can contribute to $\bar{\theta}$ (similar to eq. (8.16)) and therefore $\bar{\theta}$ once again must have an infinity renormalized away. Presumably, in the context of supergravity, such renormalization corrections should be cut off at the Planck mass, $M_{\mathrm{p}}$. But, even so, calculations of $\bar{\theta}$ tend to lead to answers which are too large. Thus, it appears [8.37] that in supergravity models, one either has to accept the fine tuning of $\bar{\theta}$ to an experimentally acceptable value or else make use of the Peccei-Quinn mechanism which requires the axion.

\subsection{Rare decays of $K$ and $\pi$}

The experimental rarity (or absence) of flavor changing neutral currents imposes stringent constraints 
on any electroweak model. Here we would like to concentrate on rare decays of the $\mathrm{K}, \pi$ and $\mu$ into supersymmetric particles. The only possible supersymmetric particles which could result from the decay of such light particles are neutralinos or scalar-neutrinos. We shall usually take the neutralino to be a photino although it is also of interest to consider the higgsino case.

The decays of interest are of the following type: $\mathrm{K}^{ \pm} \rightarrow \pi^{ \pm} \tilde{\gamma} \tilde{\gamma}, \pi^{ \pm} \tilde{\nu} \tilde{\nu}$ and $\pi^{0} \rightarrow \tilde{\gamma} \tilde{\gamma}, \pi^{0} \rightarrow \tilde{\nu} \tilde{\nu}$. These are neutral current processes and are thus expected to be very small. Current and future experiments will typically be sensitive to branching ratios better than $10^{-10}$ so it is of interest to see whether such decays are potentially observable.

The decay $\mathrm{K}^{ \pm} \rightarrow \pi \tilde{\gamma} \tilde{\gamma}$ is an example of a general class of rare $\mathrm{K}$ decays of the form $\mathrm{K}^{ \pm} \rightarrow \pi^{ \pm}+$missing neutrals [8.49-8.51]. Thus the detection of such a process does not necessarily imply evidence for supersymmetry. In fact, such a decay is expected in the Standard Model, namely $\mathrm{K}^{ \pm} \rightarrow \pi^{ \pm} \nu \bar{\nu}$. The current experimental limit is $\mathrm{BR}\left(\mathrm{K}^{ \pm} \rightarrow \pi^{ \pm}+\right.$missing neutrals $) \leq 1.4 \times 10^{-7}(90 \% \mathrm{CL})$ although experiments now in progress at the AGS anticipate lowering this bound by over three orders of magnitude. The Standard Model prediction for $\mathrm{K}^{ \pm} \rightarrow \pi^{ \pm} \nu \bar{\nu}$ is obtained by calculating box diagrams which involve all possible quark generations. The result depends on the unknown top quark mass and unknown mixing angles involving the third generations. Various bounds can be obtained by using the $\mathrm{K}_{\mathrm{L}} \rightarrow \mu^{+} \mu^{-}$, the $\mathrm{K}_{\mathrm{L}}, \mathrm{K}_{\mathrm{S}}$ mass difference and the b-quark lifetime to constrain the various unknown parameters. As a result, Ellis and Hagelin [8.50] find $\mathrm{BR}\left(\mathrm{K}^{+} \rightarrow \pi^{+} \nu \bar{\nu}\right)=(1.4$ to 11$) \times 10^{-10}$ per neutrino. Observation of a branching ratio much larger than $3 \times 10^{-9}$ would signal the presence of new physics.

A number of groups have computed the branching ratio for $\mathrm{K}^{ \pm} \rightarrow \pi^{ \pm} \tilde{\gamma} \tilde{\gamma}$ [8.49-8.51]. A priori, one might think that this process could occur at tree level as shown in fig. 63(a). In that case, rough estimates would predict a branching ratio which could be as large as $10^{-7}$ which could be observable in the near future. However, as discussed in section 8.2, in most fashionable models one finds that the diagonalizing matrices which produce the quark mass eigenstates also produce the scalar-quark mass eigenstates (at tree level) which implies no $q_{i} \tilde{q}_{j} \tilde{\gamma}(i \neq j)$ tree-level couplings. Thus fig. 63(a) would not contribute and we must turn to fig. 63(b)-(d). The box diagrams have been computed in refs. [8.50, 8.51]; here we quote the result of ref. [8.51]:

$$
\mathrm{BR}\left(\mathrm{K}^{+} \rightarrow \pi^{+} \tilde{\gamma} \tilde{\gamma}\right)=7 \times 10^{-11}\left[20 \mathrm{GeV} / \tilde{M}_{\mathrm{c}}\right]^{4}\left[1+0.43 \log \left(\tilde{M}_{\mathrm{c}} / 20 \mathrm{GeV}\right)\right]
$$

Clearly, since it is almost certain that $\tilde{M}_{\mathrm{c}} \geq 20 \mathrm{GeV}$, the decay rate for $\mathrm{K}^{+} \rightarrow \pi^{+} \tilde{\gamma} \tilde{\gamma}$ due to the box diagrams is unobservable. Another mechanism to produce this decay comes in to play if $\pi^{0} \rightarrow \tilde{\gamma} \tilde{\gamma}$ occurs (see fig. 64). Then one can have the cascade process $\mathrm{K}^{+} \rightarrow \pi^{+} \pi^{0}, \pi^{0} \rightarrow \tilde{\gamma} \tilde{\gamma}$ producing the same final state. In fact, this is the experimental technique one would use to detect rare decays of the $\pi^{0}$. The decay rate for $\pi^{0} \rightarrow \tilde{\gamma} \tilde{\gamma}$ can be immediately obtained from eq. (5.10) by identifying [8.52] $f_{\pi}^{2}=3\left|R_{\mathrm{s}}(0)\right|^{2} /\left(2 \pi m_{\pi}\right), m_{\pi}=2 m_{\mathrm{Q}}$ and $e_{\mathrm{Q}}^{4}=\frac{1}{2}\left(e_{\mathrm{u}}^{2}-e_{\mathrm{d}}^{2}\right)^{2}=\frac{1}{18}$. (Here, $f_{\pi} \approx 93 \mathrm{MeV}$.) The result is

$$
\Gamma\left(\pi^{0} \rightarrow \tilde{\gamma} \tilde{\gamma}\right)=\left(4 \pi \alpha^{2} f_{\pi}^{2} \tilde{M}_{\tilde{\gamma}}^{2} m_{\pi} / \tilde{M}^{4}\right)\left(1-4 \tilde{M}_{\tilde{\gamma}}^{2} / m_{\pi}^{2}\right)^{1 / 2}
$$

where we have assumed that $\tilde{M}=\tilde{M}_{\mathrm{u}}=\tilde{M}_{\mathrm{d}}$ is large. Using $\mathrm{BR}\left(\mathrm{K}^{+} \rightarrow \pi^{+} \pi^{0}\right)=0.21$, one finds [8.51]

$$
\mathrm{BR}\left(\mathrm{K}^{+} \rightarrow \pi^{+} \tilde{\gamma} \tilde{\gamma}\right)_{\text {cascade }}=1.6 \times 10^{-11}\left(1-4 \tilde{M}_{\tilde{\gamma}}^{2} / m_{\pi}^{2}\right)^{1 / 2}[20 \mathrm{GeV} / \tilde{M}]^{4}\left[\tilde{M}_{\gamma} / 1 \mathrm{MeV}\right]^{2}
$$

For $2 \mathrm{MeV}<\tilde{M}_{y}<\frac{1}{2} m_{\pi}$, we see that eq. (8.19) dominates over eq. (8.17) although we will see in the next section that this mass range seems disfavored for reasons related to cosmological constraints. 


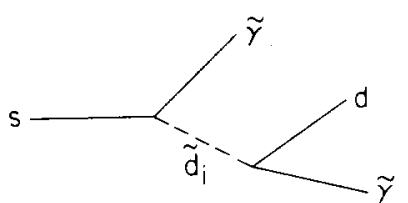

(a)

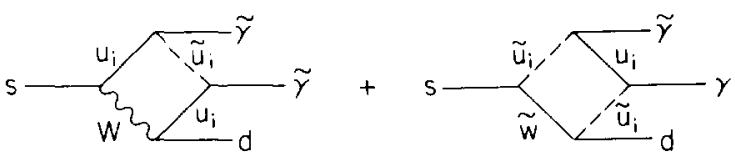

(b)

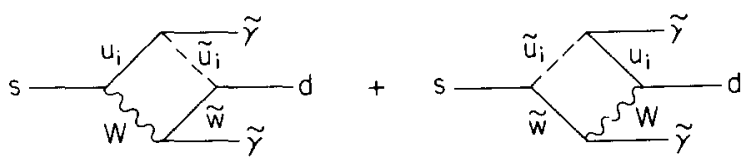

(c)

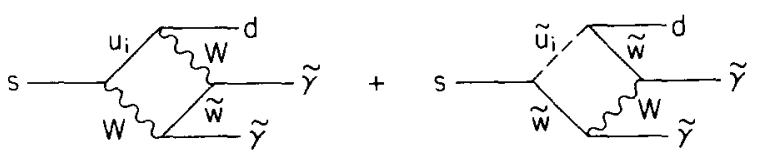

(d)

Fig. 63. Graphs contributing to $\mathrm{s} \rightarrow \mathrm{d} \tilde{\gamma} \tilde{\gamma}$ (which are relevant for $\mathrm{K}^{ \pm} \rightarrow$ $\pi^{ \pm} \bar{\gamma} \bar{\gamma}$ ). If the tree-level graph shown in (a) is forbidden (which will occur if the model does not allow flavor-changing photino interactions), then one must compute the box diagrams shown in (b), (c) and (d).

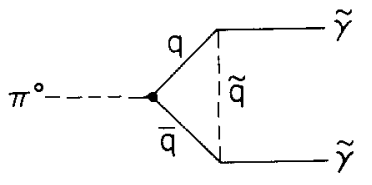

(a)

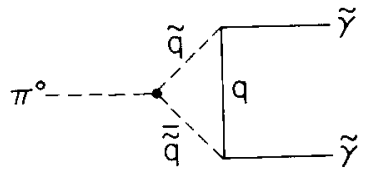

(b)

Fig. 64. The decay $\pi^{0} \rightarrow \tilde{\gamma} \tilde{\gamma}$ occurs via the graph shown in (a). The mechanism shown in (b) is highly suppressed because the large scalarquark masses imply that the scalar-quark content of the pion is negligible.

The conclusion to be drawn here is that it is unlikely that $\mathrm{K}^{+} \rightarrow \pi^{+} \tilde{\gamma} \tilde{\gamma}$ can be observed. If the lightest neutralino is a higgsino, then the situation is slightly improved. Ellis and Hagelin show [8.50] that $\mathrm{BR}\left(\mathrm{K}^{+} \rightarrow \pi^{+} \tilde{\mathrm{H}}^{0} \tilde{\mathrm{H}}^{0}\right) \approx 0.9 \mathrm{BR}\left(\mathrm{K}^{+} \rightarrow \pi^{+} \nu_{\mathrm{e}} \bar{\nu}_{\mathrm{e}}\right)$, if $\tilde{\mathrm{H}}^{0}$ is taken to be massless, which would imply that a light $\tilde{\mathrm{H}}^{0}$ would be counted as another flavor of neutrinos if experiments sensitive to $\mathrm{K}^{ \pm} \rightarrow \pi^{ \pm} \nu \bar{\nu}$ were performed. Other possible supersymmetric final states such as $\mathrm{K}^{ \pm} \rightarrow \pi^{ \pm} \tilde{\gamma} \tilde{\mathrm{G}}$ or $\pi^{ \pm} \tilde{\mathrm{G}} \tilde{\mathrm{G}}(\tilde{\mathrm{G}}=\mathrm{Goldstino}$ or a very light gravitino) have unobservably small branching ratios.

If the scalar neutrino is light enough, the decay $\mathrm{K}^{ \pm} \rightarrow \pi^{ \pm} \tilde{\nu} \overline{\bar{\nu}}$ may also contribute close to the level of $\pi^{ \pm} \nu \bar{\nu}$. The situation is briefly discussed in ref. [8.53].

Rare supersymmetric decays of the neutral pion would be searched for at a kaon factory via $\mathrm{K}^{+} \rightarrow \pi^{+} \pi^{0}, \pi^{0} \rightarrow$ missing neutrals as discussed above. The $\pi^{0} \rightarrow \tilde{\gamma} \tilde{\gamma}$ rate was given by eq. (8.18) (also, see discussion at the end of section 8.9). The decay $\pi^{0} \rightarrow \tilde{H}^{0} \tilde{H}^{0}$ (if the higgsino were sufficiently light) can also occur via the same mechanism (see fig. 64). However, the $\tilde{\mathrm{H}}^{0} \tilde{q} q$ coupling is suppressed by a factor of $m_{\mathrm{q}} / m_{\mathrm{w}}$ leading to a substantially smaller rate as compared with eq. (8.18). In addition, s-channel $\mathrm{Z}^{0}$-exchange diagrams also exist but these will be quite small.*

Finally, one could look for rare supersymmetric decays of the muon. It would be amusing to consider a method to detect the lepton-number violating process $\mu \rightarrow \mathrm{e} \tilde{\gamma} \tilde{\gamma}$. This process could occur in a theory

\footnotetext{
${ }^{*}$ In this regard consider the fact that the observed decay $\pi^{0} \rightarrow \mathrm{e}^{+} \mathrm{e}^{-}$occurs via a second-order electromagnetic process; the $\mathrm{Z}^{0}$-exchange contribution is too small to be observed.
} 
which permitted transitions between the scalar-leptons $\tilde{\mu}$ and ẽ. For example, in a model with unequal masses for scalar-neutrinos of different flavors, the scalar mass eigenstates would be Cabibbo rotated versions of the $\tilde{\mu}$ and $\tilde{e}$, so that the decay $\tilde{\mu} \rightarrow \mathrm{e} \tilde{\gamma}$ could occur (suppressed by appropriate mixing angles). The absence of $\tilde{\mu}-\tilde{e}$ transitions would impose further constraints on the supersymmetric theory in a manner similar to the constraints discussed in section 8.2 .

\subsection{Cosmological constraints}

There is at present a Standard Model of cosmology based on the assumption of an isotropic, homogeneous, adiabatically expanding universe [8.54]. In using this model, one can trace back the model in time to see how the early universe behaved. This cosmological model usually assumes the existence of a minimal spectrum of particles required by the Standard Model of particle physics. When one studies new models of particle physics which contain new particles, one must be careful not to upset some of the successful predictions of the Standard Model of cosmology.

As an example the Standard Model of cosmology predicts primordial abundances of helium and deuterium (relative to hydrogen) which depend in a sensitive way on the number of stable (nearly) massless neutrino-like particles [8.55]. If the number of such particles is greater than four, the predicted helium abundance would be inconsistent with observation. As there are at present three known flavors of neutrinos, the cosmological model can tolerate at most one new stable massless particle. Thus, one massless stable photino or higgsino is not inconsistent with the requirements of the cosmological model, although the existence of two such particles could be ruled out.

If new stable particles existed, it could have important consequences for cosmology. For example, at present, there is substantial evidence that $90 \%$ of the total mass density of the universe is made up of nonluminous nonbaryonic material. This 'dark matter' could be made up of massive neutrinos, or perhaps it consists of some new (presently undiscovered) stable particle [8.56]. Thus, new theories of particle physics could suggest new candidates for the dark matter, such as photinos [8.94] or scalarneutrinos [8.53]. However, one must be careful that the existence of such a new particle does not result in too much mass in the universe, as an upper limit for the total mass density of the universe (measured by its gravitational effects) is known. It is convenient to express this upper limit in terms of the critical density needed to close the universe: $\rho_{\mathrm{c}}=2 \times 10^{-29} h_{0}^{2} \mathrm{gm} / \mathrm{cm}^{3}$ (where $0.5 \leq h_{0} \leq 1$ depends on the possible range of the experimentally measured Hubble parameter). Then, we may conservatively say that the total mass density (baryonic and other) is $\rho \leq 2 \rho_{\mathrm{c}}$. (The baryonic mass density is certainly less than $0.1 \rho_{\mathrm{c}}$.)

As an example, consider the question of how massive could neutrinos be without contributing too much mass density to the universe. The crucial factor in the analysis is the neutrino decoupling temperature, $T_{\nu}^{\mathrm{d}}$. This is the temperature in the early universe above which the neutrinos are in thermal equilibrium with the rest of the elementary particles which make up the universe. Below $T_{\nu}^{\mathrm{d}}$, the expansion rate of the universe is much larger than the rate of neutrino interactions, and so the neutrinos decouple from the rest of the universe. As the universe expands, the number density of neutrinos at temperature $T_{\nu}$ simply decreases from its value at $T_{\nu}^{\mathrm{d}}$ by the red-shift factor $\left(T_{\nu} / T_{\nu}^{\mathrm{d}}\right)^{4}$. If the neutrino mass is less than $T_{\nu}^{\mathrm{d}}$, then it is straightforward to compute the total mass density that resides in neutrinos today. The result is $\rho_{\nu} / \rho_{\mathrm{c}}=\sum g_{\nu} m_{\nu} / 200 \mathrm{eV}$ (where $g_{\nu}=2,4$ for Majorana, Dirac neutrinos masses). Thus, the sum total of the three known neutrinos can be at most $100 \mathrm{eV}$. On the other hand, if neutrino masses are larger than $T_{\nu}^{\mathrm{d}}$, the resulting scenario changes. Once the temperature of the universe drops below the mass of a given particle, that particle can no longer be thermally pair 
produced and as a result, that particle drops out of thermal equilibrium. However, annihilation processes can still take place which depend on the strengths of the interactions and the remaining density of that particle. Thus, a dynamical calculation is required to determine how much mass density of the particle remains after annihilation processes stop. This calculation for heavy neutrinos was first performed by Lee and Weinberg [8.57], and Dicus, Kolb and Teplitz [8.58]. They found that for heavy neutrinos, the remaining mass density today would be $\rho_{\nu} / \rho_{\mathrm{c}}=\left(1.5 \mathrm{GeV} / m_{\nu}\right)^{2}$. The conclusion is that neutrino masses $100 \mathrm{eV} \leq m_{\nu} \leq 1.5 \mathrm{GeV}$ are cosmologically ruled out.

Such an analysis can be applied to any new stable particle, which may give a range of masses ruled out by cosmology. The exact range would depend on the decoupling temperature $T_{\mathrm{d}}$ to obtain the lower bound of the forbidden range and the efficiency of the annihilation to obtain the upper bound. Goldberg [8.59] was the first to apply this reasoning to obtain a Lee-Weinberg type bound for the photino. He noticed that photino annihilation into light fermions was suppressed due to a p-wave threshold for the annihilation (see eq. (E13) in appendix E and discussion following), which greatly affects the cosmological lower bound for heavy photinos. Because the photino annihilation into $\mathrm{f} \overline{\mathrm{f}}$ proceeds via $\mathrm{t}$-channel exchange of the scalar $\tilde{\mathrm{f}}$, the Lee-Weinberg bound for photinos will depend on the mass of the $\tilde{f}$. Recently, there have been a number of papers [8.60-8.63,8.53] which address the issue of cosmological constraints on new supersymmetric particles in more detail. We would like to comment briefly on the analysis performed in ref. [8.60] which included the effects of a complicated neutralino mass matrix. It is found that if the lightest neutralino is a photino, then either $\tilde{M}_{y} \lesssim$ $\mathrm{O}(100) \mathrm{eV}$; ${ }^{*}$ otherwise $\tilde{M}_{\gamma} \geq 0.5,1.8$, or $5 \mathrm{GeV}$ for scalar-quark and scalar-lepton masses equal to 20,40 , or $100 \mathrm{GeV}$. (The lower bound corresponds to $\rho_{\bar{\gamma}}=\rho_{\mathrm{c}}$.) On the other hand, if the lightest neutralino is a higgsino, the constraints are different. Although the lower restriction $\tilde{M}_{\mathrm{H}} \leqslant \mathrm{O}(100 \mathrm{eV}$ is unchanged, we note that the annihilation of higgsino pairs via t-channel exchange of a scalar-quark or scalar-lepton is much weaker than the corresponding annihilation of photino pairs because the $\tilde{H}^{0} \tilde{q} q$ vertex is suppressed by a factor $m_{\mathrm{q}} / m_{\mathrm{w}}$. However, one must also include the annihilation of higgsinos via s-channel $Z^{0}$ exchange. The end result is that the upper end of the forbidden mass region tends to be higher than was the case for the photino. Typical restrictions are $\tilde{M}_{\mathrm{H}} \geq 5-30 \mathrm{GeV}$ depending on the supersymmetric model.

In supersymmetry the lightest supersymmetric particle is almost certainly stable (see section 10.3 for comments on models where this is not the case). Above, we considered the possibility that this particle is a neutralino. One can imagine other possibilities. The most likely alternative candidate is the scalar-neutrino $[8.53,8.63]$. Here, no Lee-Weinberg type bound exists as the annihilation of scalarneutrinos can proceed through exchange of a Majorana neutralino, giving a cross section which does not depend on $\tilde{M}_{\nu}$. On the other hand, as there are three scalar-neutrino flavors (presumably nearly degenerate in mass), they cannot be too light without upsetting the nucleosynthesis calculation. The restriction of nucleosynthesis simply implies that the scalar-neutrinos had to be nonrelativistic by the time nucleosynthesis began. This would imply a lower bound $\tilde{M}_{\nu} \geq \mathrm{O}(10) \mathrm{MeV}$.

Other candidates for the lightest supersymmetric particle can be ruled out [8.60]. The gluino is probably not stable as discussed in section 3.4. Other candidates would be charged; but a new stable charged particle of mass less than $350 \mathrm{GeV}$ (with abundance of at least $10^{-21}$ times the baryon number of the universe) is ruled out by anomalous proton searches.

One supersymmetric particle which we have not mentioned in this section is the gravitino. First,

\footnotetext{
* A theoretical argument may be used to rule out photinos in this range $[8.60,8.93]$. Namely, grand unified theories lead to relations among gaugino masses. In particular, neglecting mixing with other neutralinos, $\tilde{M}_{\mathrm{g}} / \tilde{M}_{y}=\frac{3}{8} \alpha_{3} / \alpha_{2} \sin ^{2} \theta_{\mathrm{w}}$, where $\alpha_{3}$ and $\alpha_{2}$ are the SU(3) and SU(2) running coupling constraints evaluated at an appropriate scale. A photino mass less than $100 \mathrm{eV}$ would then imply an exceedingly light gluino which we have already argued against in section 3.4 .
} 
suppose that the gravitino is very light. Because the gravitino interacts extremely weakly, the gravitino decoupling temperature is much larger than the corresponding neutrino decoupling temperature $\left(T_{\nu}^{\mathrm{d}}\right)$ already mentioned. This implies $[8.97,8.99]$ that light gravitinos can be as heavy as one $\mathrm{keV}$ without exceeding the critical mass density of the universe $\rho_{\mathrm{c}}$. Although current fashionable models do not have the gravitino as being the lightest supersymmetric particle, its interactions are of gravitational strength so that its lifetime is exceedingly long. One can obtain Lee-Weinberg type bounds for a gravition which is heavier than $1 \mathrm{keV}[8.64,8.61]$. The upper bound corresponds to the requirement that the gravitino decay before nucleosynthesis begins. (More precisely, we require that if the gravitino decays after nucleosynthesis begins, the resulting reheating due to thermalization of the gravitino decay products raises the temperature above $0.2 \mathrm{MeV}$ so that nucleosynthesis can restart.) The resulting bound is [8.64]

$$
\tilde{M}_{\mathrm{g}_{3 / 2}} \lesssim 1 \mathrm{keV} \quad \text { or } \quad \tilde{M}_{\mathrm{g}_{3 / 2}} \geq 5.5 \times 10^{4} \mathrm{GeV}
$$

A more severe constraint arises when one considers the fact that the resulting gravitino must decay into particles which eventually include the lightest supersymmetric particle. These particles may be too cold to annihilate sufficiently resulting in a total mass density exceeding the observable limits. For example, if the photino is the lightest supersymmetric particle, one can get simultaneous limits on possible values of $\tilde{M}_{\gamma}$ and $\tilde{M}_{\mathbf{g}_{3 / 2}}$ [8.61].

The bounds given by eq. (8.20) are disturbing. In currently fashionable models based on supergravity it is the relation $\tilde{M}_{\mathrm{g}_{3 / 2}} \approx m_{\mathrm{w}}$ which is the basis for the hope that supersymmetry is relevant for explaining the magnitude of the weak scale.

The assumption behind the arguments above is that gravitinos are as abundant as all other species before they decouple. But because gravitinos interact gravitationally, they fall out of thermal equilibrium near $T \approx M_{\mathrm{p}}$. Can we trust the Standard Model of cosmology all the way back to this era? It is here where the model of the inflationary universe [8.65] may resolve the problem. Inflation alters the assumptions of the Standard Model of cosmology by postulating an epoch where a highly nonadiabatic phase transition occurred which pumped exponentially large amounts of entropy into the universe. After the phase transition is completed, the universe is reheated to a temperature somewhere between $10^{10}-10^{16} \mathrm{GeV}$ whereupon the Standard Model of cosmology takes over. If gravitinos decoupled before inflation, one only has to guarantee that after inflation the number density of gravitinos generated is not large enough to lead to the problems described above [8.66, 8.67]. The most recent calculations [8.61, 8.100] show that a consistent scenario emerges only if the universe reheats to a temperature not greater than about $10^{11} \mathrm{GeV}$ after inflation.

We have described above some of the cosmological implications of new particles which occur in supersymmetry. Cosmological constraints can always be avoided if one is willing to complicate sufficiently the cosmological model. Still the results described above are likely to be useful as particle physics theories continue to probe energy regimes accessible only to physics of the early universe.

\subsection{Proton decay and baryon number violation}

Let us review the current understanding as to why baryon number conservation is (nearly) an exact symmetry in the $S U(3) \times S U(2) \times U(1)$ Standard Model [8.68]. Consider starting with the fields of the Standard Model and construct the most general Lagrangian with no attempt to impose baryon number conservation. By imposing $\mathrm{SU}(3) \times \mathrm{SU}(2) \times \mathrm{U}(1)$ invariance (and Lorentz invariance) and allowing terms of dimension four or less (to preserve renormalizability), one finds that the theory automatically 
conserves baryon number and lepton number (separately). One can now construct (Lorentz scalar) operators of dimension greater than four. The result is that operators of dimension six or greater can be written down which obey $S U(3) \times S U(2) \times U(1)$ invariance and yet violate baryon number. Such operators can only arise from a more fundamental theory (which becomes relevant at some high energy scale $M$ ) which violated baryon number. Dimensional analysis implies that such dimension-six operators are proportional to $M^{-2}$. Although grand unified models have the above mentioned properties, the analysis just made is more general.

We may repeat the arguments for supersymmetric theories [8.69-8.76,8.79]. Already at step one, we run into a major difference. Because of the existence of colored scalar-quarks (with masses less than $1 \mathrm{TeV})$, we find that one can construct dimension-four operators which satisfy $\mathrm{SU}(3) \times \mathrm{SU}(2) \times \mathrm{U}(1)$ invariance and yet violate baryon number. An example of such an operator is: $\varepsilon_{i j k} \tilde{u}_{\mathrm{R}}^{i} d_{\mathrm{R}}^{j} s_{\mathrm{R}}^{k}$ where $i, j, k$ are color indices. This operator is a singlet under $\mathrm{SU}(3) \times \mathrm{SU}(2) \times \mathrm{U}(1)$ and yet carries net baryon number. Thus, in supersymmetric models, the previously natural explanation for the near absence of baryon number violation is lost.

To recover the desired results, such dimension-four operators must be banned. This is easy to do by imposing a discrete symmetry on the theory: Require the Lagrangian to be invariant under the transformations $\mathrm{q} \rightarrow-\mathrm{q}$ and $\tilde{\mathrm{q}} \rightarrow-\tilde{\mathrm{q}}$ for quark and scalar-quark fields. A similar requirement must be imposed on lepton and scalar-lepton fields, whereas the Higgs fields, gauge fields and their fermionic partners remain unchanged. The result then is that requiring $\mathrm{SU}(3) \times \mathrm{SU}(2) \times \mathrm{U}(1)$ invariance along with the discrete symmetry just described leads to automatic baryon number conservation for dimension-four (and less) operators in supersymmetric theories.

One can now proceed to analyze operators with dimensions greater than four. For example, the dimension-six operators which appeared in the non-supersymmetric model (resulting, say, from a grand unified theory) remain in the supersymmetric model also suppressed by a factor of the heavy mass squared. In supersymmetric grand unified models, the calculation of the grand unification mass (GUM) (defined as the point where the running coupling constants meet) via the renormalization group equations is modified because of the appearance of new particles (e.g., scalar-quarks) in the low energy theory which changes the evolution of the various running coupling constants [8.77-8.79]. The result is that $M_{\mathrm{SSGUM}}>M_{\mathrm{GUM}}$ (typically, $M_{\mathrm{GUM}} \approx 10^{14} \mathrm{GeV}$, whereas $M_{\mathrm{SSGUM}} \approx 10^{16}-10^{17} \mathrm{GeV}$ ). Thus, in supersymmetric grand unified models, the dimension-six operators (which mediate proton decay) are more suppressed than they are in the ordinary grand unified models. Since the proton lifetime is proportional to $M_{\mathrm{GUM}}^{4}$, it seems that the proton lifetime in supersymmetric models should be about $10^{8}$ times longer than in naive SU(5) which would explain the absence of experimentally observed proton decay.

However, supersymmetric $\mathrm{SU}(3) \times \mathrm{SU}(2) \times \mathrm{U}(1)$ (with the discrete symmetry described above) also possesses baryon number violating dimension-five operators (unlike the Standard Model which possesses no operators of this type). An example of such an operator would be: $\varepsilon_{i j k} \varepsilon_{a b} \varepsilon_{c d} \tilde{q}_{a}^{i} \tilde{q}_{b}^{j} q_{c}^{k} L_{d}$ (where $i, j, k$ are color $\mathrm{SU}(3)$ indices and $a, b, c, d$ are weak $\mathrm{SU}(2)$ indices so that $q_{a}=(u, d)$ and $\left.L_{d}=(\nu, \ell)\right)$. By dimensional analysis these operators are suppressed only by $M^{-1}$ so that naively one might fear that the proton would decay much too rapidly. A more detailed calculation shows that this conclusion is incorrect. If we consider how such operators would mediate proton decay in a supersymmetric grand unified model, we are led to diagrams such as the one in fig. 65. Proton decay via these dimension-five operators must occur via a loop diagram (fig. 65(a)) which leads to extra powers of the coupling constant compared to tree-level exchange of a superheavy baryon number violating gauge boson). Furthermore, one can estimate the short distance QCD corrections [8.79]. Whereas these corrections led to an enhancement of the dimension-six 


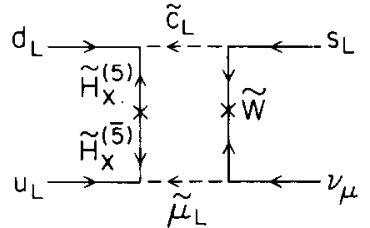

(a)

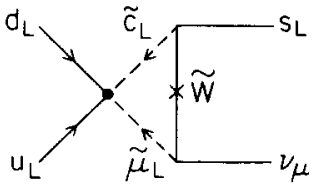

(b)

Fig. 65. Proton decay mediated by supersymmetric particles. The loop diagram (a) which is mediated by the exchange of one light and one superheavy supersymmetric fermion can be as important as the tree-level diagrams involving exchange of one superheavy grand unified gauge boson. The superheavy fermion line $\left(\tilde{\mathrm{H}}_{\mathrm{x}}\right)$ can be shrunk to a point as in diagram $(\mathrm{b})$ resulting in an effective dimension-five vertex.

operators in the ordinary grand unified theories, in the supersymmetric theory they lead to a suppression of the dimension-five operators. The end result is that the lifetime for the proton due to diagrams such as in fig. 65 remains roughly unchanged (although more uncertain due to model dependence). However, the decay final states are markedly different for reasons we now explain.

If one carefully writes out the dimension-five operator which is involved in proton decay, we find operators of the type (see, e.g., ref. [8.76]):

$$
\begin{aligned}
M_{\mathrm{ABCD}} & =\varepsilon_{i j k} \varepsilon_{a b} \varepsilon_{c c} \tilde{q}_{A a}^{i} \tilde{q}_{B b}^{j} q_{C c}^{k} L_{D d} \\
& =\varepsilon_{i j k}\left(\tilde{u}_{A}^{i} \tilde{d}_{B}^{j} u_{C}^{k} \ell_{D}-\tilde{d}_{A}^{i} \tilde{u}_{B}^{j} u_{C}^{k} \ell_{D}+\tilde{d}_{A}^{i} \tilde{u}_{B}^{j} d_{C}^{k} \nu_{D}-\tilde{u}_{A}^{i} \tilde{d}_{B}^{j} d_{C}^{k} \nu_{D}\right),
\end{aligned}
$$

where the notation used previously has been augmented with generation labels $A, B, C, D$. In supersymmetric theories, eq. (8.21) is related to a term where the two scalar-quark fields are replaced by their quark partners; the related term must occur in the theory with equal strength. If we set $A=B=C$, it is easy to see that this related term vanishes exactly. By supersymmetry it follows that $M_{A A A D}=0$ which means that the quarks and scalar-quarks cannot be all of the same generation in eq. (8.21). This explains why final state decay products containing a kaon are preferred. The dimension-five operator must contain members from at least two generations.

There is a second reason which favors the participation of higher generations in the context of SU(5) and similar models of grand unification [8.80]. In fig. 65 the cross marks the place where helicity flip must occur. ${ }^{*}$ One may ask whether a graph exists where the gauge fermion partners $\tilde{X}_{\mathrm{f}}$ of the baryon number violating superheavy $X$ gauge boson are exchanged. The answer is negative because the helicity-flip requirement implies that the $\tilde{\mathrm{W}}_{\mathrm{X}}$ mixes with $\tilde{\mathrm{H}}_{\mathrm{X}}$ - the fermionic partner of the Higgs boson which lives in the adjoint representation of the gauge group. (It is only such Higgs fermions which are relevant since it is this Higgs multiplet which breaks $S U(5)$ down to $S U(3) \times S U(2) \times U(1))$. But the adjoint Higgs field does not couple to fermions (for group-theoretic reasons). Hence, there can be no diagram analogous to fig. $65(\mathrm{a})$ where $\tilde{H}_{\mathrm{X}}^{(\mathrm{S})}$ and $\tilde{H}_{\mathrm{X}}^{(\overline{5})}$ are replaced with $\tilde{W}_{\mathrm{X}}$ and $\tilde{H}_{\mathrm{X}}$ respectively. Therefore, the only diagrams of the type shown in fig. 65(a) must involve the color-triplet Higgs fields $\tilde{H}_{\mathrm{X}}^{(5)}$ and $\tilde{H}_{\mathrm{X}}^{(\bar{S})}$ (these are the $\mathrm{SU}(5)$ partners of the two ordinary weak doublet Higgs fields required by supersymmetry). Because these fermionic Higgs couple to fermions and their scalar partners with strength proportional to the fermionic mass, it follows that members of the higher generations are enhanced. Thus, a diagram similar to fig. 65(a) with an intermediate state of $\tilde{t} \tilde{\tau}$ will also be important (depressed only by a mixing angle to convert $t$ to $s$ in the final state). The conclusion is that if dimension-five operators mediate proton decay, the dominant decay final states will be $\mathrm{K} \bar{\nu}_{\tau}, \mathrm{K} \bar{\nu}_{\mu}$, and

\footnotetext{
${ }^{*}$ In supersymmetric SU(5), $H^{(5)}$ and $H^{(\overline{5})}$ are Higgs multiplets which transform under SU(5) like a 5 and $\overline{5}$ respectively. A gauge invariant mass term $H_{\mathrm{F}}^{(5)} H_{\mathrm{f}}^{(5)}$ exists which occurs at the cross on fig. 65(a). To see that there must be a helicity flip, simply note that the couplings $u \tilde{c} \tilde{H}_{\mathrm{X}}^{(5)}$ and $d \tilde{\mu} \tilde{H}_{\mathrm{X}}^{(\overline{5})}$ are chiral (cf. the neutralino couplings to quarks given by eq. (C77)).
} 
$\mathrm{K} \mu^{+}[8.79,8.73,8.75]$. This differs drastically from ordinary $\mathrm{SU}(5)$ which favors $\pi^{0} \mathrm{e}^{+}$and has production of second generation fermions suppressed.

One may be concerned that the dimension-five operators may still result in a proton decay rate which contradicts the experimental limits. Current results from the proton decay experiments [8.81] quote $\tau_{\mathrm{p}} / \mathrm{BR}(\mathrm{p} \rightarrow \mathrm{K} \mu) \geq 2 \times 10^{31}$ years. For example, one would expect diagrams similar to the one shown in fig. 65 where the $\tilde{\mathrm{w}}$ exchange is replaced by neutral gaugino exchange. It seems plausible that gluino exchange could dominate. Because of the strong coupling constant which would appear, the result could be a proton decay rate larger than anticipated previously. In some models no such problem exists - for example it has been shown [8.82] that if one assumes that all scalar-quark and scalar-lepton masses are separately degenerate, then the sum of all Feynman diagrams involving the dimension-five operators and gluino exchange exactly vanishes. The reason is connected with the flavor independence of gluino interactions. Models which do not exhibit such mass degeneracies will have nonvanishing gluino exchanges [8.101]. Furthermore, note the appearance of the helicity flip which must occur on the gaugino line (in fig. 65). Thus, in principle, one could use the present limits on proton decay to constrain the values of the gaugino masses.

In conclusion, dimension-five operators in supersymmetric theories lead to new expectations for the proton decay branching ratios. One would expect final states involving kaons and either muons or neutrinos to be the dominant decay channels, whereas standard SU(5) modes such as $\pi^{0} \mathrm{e}^{+}$would be highly suppressed. At present there is still too much model dependence to determine whether the dimension-five operators lead to a proton decay rate which is inconsistent with present experimental limits. One always has the option of inventing a discrete symmetry to ban the baryon number violating dimension-five operators (in the same manner that we rid the theory of the baryon number violating dimension-four operators). If we were to do this, the proton decay would be mediated via dimension-six operators in the same way as it is in non-supersymmetric grand unified theories. Thus, we would now expect the branching ratio predictions to be similar to that of standard SU(5). The difference in the case of supersymmetric SU(5) would be a larger value of $M_{\mathrm{SSGUT}}$ so that the proton lifetime could be too long to be observable. Clearly, if proton decay is discovered, it will have significant implications for supersymmetric extensions of the Standard Model.

\subsection{Anomalies}

There are a number of interesting issues connected in one way or another with anomalies. There are two sorts of anomalies that we would like to consider. First, there are anomalies in gauged currents. If these exist, gauge invariance (and therefore renormalizability) would be destroyed, so we must demand that our supersymmetric model must have no anomalous gauge currents. Second, there are anomalies in global currents. These can have physical effects, the most famous of which is $\pi^{0} \rightarrow \gamma \gamma$ decay. We shall discuss some contraints on supersymmetric models which are related to both types of anomalies.

The Standard Model with gauge group $\mathrm{SU}(3) \times \mathrm{SU}(2) \times \mathrm{U}(1)$ has no anomalies in the gauge currents. This is somewhat of a miracle in that if one computes the anomalous triangle diagram involving three gauge currents, the result vanishes only when one sums over all fermions in the theory. The sum vanishes generation by generation so we say that the anomaly due to quark loops is cancelled out by the lepton loops. (In certain grand unification models such as $S O(10)$, the anomaly cancellation is a natural consequence of the group theory.)

In supersymmetric models, because new particles are introduced, one must check again that all anomalies cancel as above. If we simply take an anomaly-free theory and add supersymmetric partners, 
the cancellation is automatic. However, there have been many attempts in the past to go beyond the minimal extension of the Standard Model. We will discuss reasons for this in section 10.1. For now we shall summarize results for an $\mathrm{SU}(3) \times \mathrm{SU}(2) \times \mathrm{U}(1) \times \mathrm{U}(1)$ supersymmetric model. An extra $\mathrm{U}(1)$ has been considered by various authors $[8.83-8.86,8.69]$ as this allows one to construct a low-energy model of spontaneously broken supersymmetry where the gauge symmetry also breaks down to $\mathrm{SU}(3) \times \mathrm{U}(1)$. The problem many authors ran into is that such models were not anomaly-free: Here there is the extra complication of a new $\mathrm{U}(1)$ gauge current which also must be nonanomalous. When one tried to add appropriate new fields in order to cancel out the anomalies, other problems invariably arose (e.g., the theory no longer broke supersymmetry). Thus the requirement of anomaly cancellation imposed severe constraints on model building and appears to have essentially caused the end to investigations of supersymmetric models with an extra U(1) gauge symmetry.

We now turn to global currents in QCD. In the Standard Model with $N_{\mathrm{f}}$ quark flavors (with quark mass terms set to zero), there exists a global $\mathrm{SU}\left(N_{\mathrm{f}}\right)_{\mathrm{L}} \times \mathrm{SU}\left(N_{\mathrm{f}}\right)_{\mathrm{R}} \times \mathrm{U}(1)_{\mathrm{V}} \times \mathrm{U}(1)_{\mathrm{A}}$ symmetry. The $\mathrm{U}(1)_{\mathrm{V}}$ symmetry is global baryon number which we discussed in section 8.8 . The $\operatorname{SU}\left(N_{\mathrm{f}}\right)_{\mathrm{L}} \times \operatorname{SU}\left(N_{\mathrm{f}}\right)_{\mathrm{R}}$ is assumed to be broken dynamically in QCD down to the diagonal subgroup $\operatorname{SU}\left(N_{\mathrm{f}}\right)_{\mathrm{L}+\mathrm{R}}$. This picture seems well approximated for the two lightest quark flavors. The symmetry $\mathrm{SU}(2) \times \mathrm{SU}(2)$ is spontaneously broken down to diagonal SU(2) (strong isospin). The resulting three Goldstone bosons are identified as the pions which eventually gain a small mass when the up and down quark masses are turned on. Finally, there is a $\mathrm{U}(1)_{\mathrm{A}}$ symmetry which is in reality not a symmetry at all - it is anomalous.

When we consider a supersymmetric extension of the Standard Model, one has potentially new global currents to consider. If the masses of the (colored) supersymmetric particles are large, these new currents, corresponding to would-be symmetries, are explicitly broken by the mass terms and no new information is obtained. However, there is one colored supersymmetric particle which may be light - the gluino (see section 3.4). If the gluino were massless, there would correspondingly exist a new global current $\mathrm{U}(1)_{\mathrm{R}}$ called continuous $\mathrm{R}$-invariance $[8.87,8.88]$. This invariance simply corresponds to the chiral phase invariance of the gluino field. However, one quickly discovers that this $U(1)_{R}$ current is also anomalous* [8.90] (i.e., there is a triangle anomaly involving one $\mathrm{U}(1)_{\mathrm{R}}$ current and two gluon external lines). One can now consider linear combinations of this current and the anomalous $U(1)_{\mathrm{A}}$ current. There will exist one combination that will be anomaly free. Hence, in supersymmetric QCD with two zero mass flavors $(\mathrm{u}, \mathrm{d})$ and a massless gluino (but with explicit large mass terms for the scalar quarks ũ and $\tilde{\mathbf{d}}$ ), the appropriate exact global symmetry is $\mathrm{SU}(2) \times \mathrm{SU}(2) \times \mathrm{U}(1)$. QCD dynamics presumably breaks this symmetry and it has been argued that the remaining symmetry is again the SU(2) isospin group. If this is true, then one now expects four Goldstone bosons-the pions and a fourth $\tilde{\mathrm{g}} \tilde{\mathrm{g}}$ pseudoscalar which is just the $\tilde{\eta}_{\mathrm{g}}$ we discussed at the end of section 3.4. When the $\mathrm{u}$ and $\mathrm{d}$ quark masses are turned on, the pions and $\tilde{\eta}_{\mathrm{g}}$ gain mass which has been calculated in ref. [8.91] to be $M_{\tilde{\eta}_{\mathrm{g}}}^{2}=$ $m_{\pi}^{2} f_{\pi}^{2} /\left(f_{\pi}^{2}+\frac{2}{9} f_{\lambda}^{2}\right)$ where $f_{\lambda}$ is the $\tilde{\eta}_{\mathrm{g}}$ decay constant which was calculated to be $f_{\lambda}=\sqrt{3} f_{\pi}$ (in the large $N_{c}$ limit). Even if $f_{\lambda}$ is taken as unknown, we see that $\tilde{M}_{n_{\mathrm{g}}} \leq m_{\pi}$, a result clearly in conflict with experiment. These results were derived assuming a zero gluino mass so one concludes that the gluino mass cannot be zero. By giving the gluino a mass, the mass of the $\tilde{\eta}_{\mathrm{g}}$ is raised. As discussed in section $3.4 \mathrm{E}$, the fact that $\tilde{\eta}_{\mathrm{g}}$ is not seen in $\psi \rightarrow \gamma \tilde{\eta}_{\mathrm{g}}$ presumably restricts the gluino mass to be greater than $1 \mathrm{GeV}$.

One may ask if there are any consequences of the anomaly analogous to $\pi^{0} \rightarrow \gamma \gamma$ involving supersymmetric particles. In this regard, a calculation by Clark and Love [8.92] may be relevant. They

\footnotetext{
${ }^{*}$ The discrete R-parity $[8.83,8.89] R=(-1)^{3 B+L+2 j}$ which is a subgroup of the global $\mathrm{U}(1)_{\mathrm{R}}$ group is not affected by the anomaly.
} 
compute the supersymmetric generalization of $\pi^{0} \rightarrow \gamma \gamma$. If supersymmetry is exactly conserved, then one

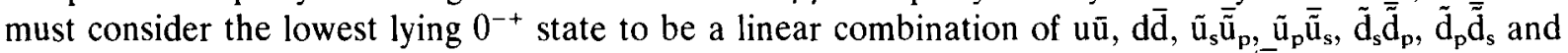
$\mathrm{g} \tilde{\mathrm{g}}$ (where we work with the pseudoscalar and scalar combinations $\left(\tilde{q}_{\mathrm{R}} \pm \tilde{q}_{\mathrm{L}}\right) / \sqrt{2}$, respectively - see appendix C.1.). Clark and Love found that the anomaly which gives rise to $\pi^{0} \rightarrow \gamma \gamma$ also yields $\pi^{0} \rightarrow \tilde{\gamma} \tilde{\gamma}$ via the effective Lagrangian (valid in the supersymmetric limit):

$$
\mathscr{L}_{\mathrm{eff}}=\frac{-e^{2} N_{\mathrm{c}}}{96 \pi^{2} f_{\pi}}\left[\varepsilon^{\mu \nu \lambda \rho} \pi^{0} F_{\mu \nu} F_{\lambda \rho}+2 \mathrm{i} m_{\pi} \pi^{0} \overline{\tilde{\gamma}} \gamma_{5} \tilde{\gamma}\right]
$$

where $F_{\mu \nu}$ is the electromagnetic field strength and $N_{\mathrm{c}}=3$ is the number of colors. The mechanism for $\pi^{0} \rightarrow \tilde{\gamma} \tilde{\gamma}$ here actually occurs via the $\tilde{q}_{\mathrm{s}} \overline{\tilde{q}}_{\mathrm{p}}$ and $\tilde{q}_{\mathrm{p}} \overline{\tilde{q}}_{\mathrm{s}}$ components of the pion as shown in fig. 64(b) (since the process $\pi^{0}((u \bar{u}-d \bar{d}) / \sqrt{2}) \rightarrow \tilde{\gamma} \tilde{\gamma}$ is proportional to $\tilde{M}_{y}^{2}$ as shown in eq. (8.18)). Thus, in the supersymmetric limit, $\pi^{0} \rightarrow \gamma \gamma$ and $\pi^{0} \rightarrow \tilde{\gamma} \tilde{\gamma}$ are of equal strength. (See appendix E.5 for another demonstration of this result.) However, it is clear that if the scalar-quark masses are large, the scalarquark component of the pion is negligible, so that the decay $\pi^{0} \rightarrow \tilde{\gamma} \tilde{\gamma}$ via the anomaly will be suppressed. An interesting consequence of the above analysis is that even in the limit of $M_{\tilde{\gamma}}=0$ where eq. (8.18) vanishes, we see that the decay $\pi^{0} \rightarrow \tilde{\gamma} \tilde{\gamma}$ is still allowed via the small mixing of scalar-quark components in the pion.

\section{Theoretical expectations for masses}

We have attempted throughout this review to analyze various supersymmetric processes in a fashion as model-independent as possible. Our basic assumption, described in chapter 1 , is that supersymmetry is responsible for the value of the weak scale. This implies that the masses of supersymmetric particles will be less than about $1 \mathrm{TeV}$ (some, of course, could be much lighter!). Other than this one assumption, we prefer to ignore model dependent biases whenever possible and let experiment provide us with input for future model building. Although this goal is appropriate, it is sometimes hard to avoid putting in some theoretical bias. The complete space of supersymmetric parameters is quite large and it is often judicious to restrict one's analysis to a smaller volume within that unknown space. The largest set of unknowns are the supersymmetric particle masses. In this chapter we shall briefly summarize the strategy behind the present fashionable supersymmetric model building and attempt to compile a reasonable theoretical expectation for the range of unknown supersymmetric masses.

To begin, let us sketch some of the basic theoretical ideas underlying supersymmetric model building. The fundamental property of such models is that supersymmetric partners of the ordinary particles normally do not have masses in excess of $1 \mathrm{TeV}$. To see how this property is insured, let us look briefly at global supersymmetry which is spontaneously broken. In such theories an analogous theorem to Goldstone's theorem requires the existence of a spin $1 / 2$ massless fermion called the Goldstino [9.1] $(\tilde{G})$. The Goldstino couples any ordinary particle $f$ to its supersymmetric partner $\tilde{\mathrm{f}}$. Current algebra techniques [9.1-9.4] then lead to a Goldberger-Treiman type relation for the mass squared splitting of $f$ (with mass $m_{\mathrm{f}}$ ) and $\tilde{\mathrm{f}}$ (with mass $\tilde{M}_{\mathrm{f}}$ ):

$$
\tilde{M}_{\mathrm{f}}^{2}-m_{\mathrm{f}}^{2}=f_{\overline{\mathrm{G}}} g_{\mathrm{f} \tilde{\mathrm{G}} \tilde{\mathrm{G}}}
$$

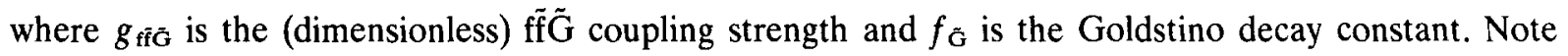


that $f_{\hat{\mathrm{G}}}^{2}$ measures the energy density of the vacuum relative to the unbroken supersymmetric case. For this reason, $\sqrt{f_{\overline{\mathrm{G}}}}$ is sometimes called the scale of supersymmetry breaking. Let us attempt to arrange the supersymmetric model such that $\tilde{M}_{\mathrm{f}}^{2}-m_{\mathrm{f}}^{2}$ is of order $m_{\mathrm{w}}^{2}$. The first serious attempt at model building was pursued by Fayet [9.5]. In his models and others which were constructed afterwards [9.6-9.11] $g_{\text {fŕ } \tilde{G}}$ was a typical tree-level coupling of order $e$ which implied that the scale of supersymmetry breaking, $\sqrt{f_{\tilde{\mathrm{G}}}}$, was less than $1 \mathrm{TeV}$. These models had severe problems both technically and phenomenologically. Further attempts at model building along these lines (with $\sqrt{f_{\overline{\mathrm{G}}}}=\mathrm{O}(1 \mathrm{TeV})$ ) arranged the Goldstino coupling to ordinary particles to be absent at tree level; the ff $\tilde{\mathrm{G}}$ coupling would then be generated radiatively [9.12-9.18]. The implication was that $g_{\text {ff } \tilde{G}}$ could be made much smaller than $e$ allowing for a larger scale of supersymmetry breaking $\sqrt{f_{\overline{\mathrm{G}}}}$ without upsetting the requirement that $\tilde{M}_{\mathrm{f}}^{2}-m_{\mathrm{f}}^{2}=\mathrm{O}\left(m_{\mathrm{w}}^{2}\right)$. Another approach originally introduced by Witten [9.19] led to the inverse hierarchy models [9.20-9.22]. In these models the scalar partner of the Goldstino (denoted by $\mathrm{Y}$ ) acquires a vacuum expectation value by radiative corrections which is much larger than the scale of supersymmetry breaking (to be identified with the grand unified mass, $M_{\mathrm{GUT}}$ ). Supersymmetry implies that if there is a f $\tilde{f} \tilde{G}$ vertex for some ordinary fermion $f$, then there must also be a ffY vertex with the same coupling strength $g_{\text {ff } \tilde{G}}$. Because $\langle Y\rangle=M_{\mathrm{GUT}}$ (by assumption), a fermion mass is generated [9.23]:

$$
m_{\mathrm{f}}=g_{\mathrm{f} \tilde{\mathrm{G}} \mathrm{G}} M_{\mathrm{GUT}} .
$$

Thus, $g_{\text {fr̃ } \tilde{G}}$ could be extremely small; using eq. (9.2), we find $\sqrt{f_{\tilde{\mathrm{G}}}} \geq 10^{10} \mathrm{GeV}$.

If $g_{\tilde{f} \tilde{f} \tilde{G}}$ were indeed as small in eq. (9.2), then gravitational corrections would begin to dominate [9.24-9.33]. It therefore seems inconsistent to consider such models without including the leading effects due to gravity. This led naturally to the study of supersymmetric models of gravity, i.e., supergravity. At present, there is no real understanding as to how one can consistently tie together a theory of gravity and renormalizable quantum field theory. Quantum gravity is notorious for being nonrenormalizable; in addition the infinities generated become far more severe when one attempts to couple gravity to matter. One hope for an eventual solution to these problems is supersymmetry. When the global supersymmetry is made a local symmetry, one naturally gets a theory of gravity embedded in a larger theory called supergravity. The infinities of supergravity seem less severe than those of gravity, although supergravity theories are also nonrenormalizable. One can enlarge supergravity to encompass interactions with matter. The most ambitious of such theories is $N=8$ supergravity which contains particles with spin 0 up to spin 2 all in one supergravity multiplet. This theory has many wonderful and mysterious properties which have led some authors to attempt to imagine how physics as we know it today might emerge from such a theory [9.34]. More recently, slightly less ambitious but still quite interesting work has been done by studying the coupling of spontaneously broken supergravity to ordinary matter $[9.29,9.30$, $9.33,9.35,9.36,9.39$ ] (spin $0,1 / 2$ fields and gauge fields). Because this theory is nonrenormalizable, it is taken to be only an effective theory at the Planck mass $M_{\mathrm{p}} \approx 10^{19} \mathrm{GeV}$; i.e., gravitational loop corrections are ignored. The limit $M_{\mathrm{p}} \rightarrow \infty$ is taken which results in the emergence of an effective low energy theory which is renormalizable and globally supersymmetric with the exception of some explicit supersymmetry breaking terms. The remarkable conclusion (first emphasized by Ovrut and Wess [9.24]) is that these explicit breaking terms which have direct and observable consequences on physics at $1 \mathrm{TeV}$ and below are a consequence of gravity!

Let us see what happens to eq. (9.1) in supergravity theories. First, the Goldstino is absorbed and becomes the helicity $\pm 1 / 2$ component of a massive spin $3 / 2$ gravitino $\left(\tilde{g}_{3 / 2}\right)$ [9.37]. It still makes sense,

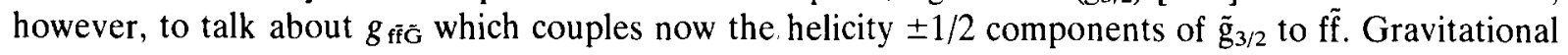


coupling of matter is related by supersymmetry to $g_{\mathrm{f} \tilde{\mathrm{f}} \overline{\mathrm{G}}}$ which is roughly*

$$
g_{\mathrm{f} \tilde{\mathrm{fO}}} \sim m_{\mathrm{w}} / M_{\mathrm{p}} \sim 10^{-17}
$$

which implies that $\sqrt{f_{\tilde{\mathrm{G}}}} \geqslant 10^{10} \mathrm{GeV}$. Thus, the picture in the most fashionable models at present is one where the scale of supersymmetry breaking is large but the model is arranged so that the mass squared splitting of the ordinary and supersymmetric particles is of order $m_{w}^{2}[9.27-9.33 ; 9.38-9.46]$. Such a picture is stable under radiative corrections due to matter loops, and one assumes that gravitational radiative corrections (which must be cut off at $M_{\mathrm{p}}$ ) are negligible. One further property of interest is that the gravitino mass turns out to be of order $m_{\mathrm{w}}$. This conclusion follows from some technical analysis of the supergravity model. It can be shown that when the parameters of the supergravity model are fine-tuned to insure a zero cosmological constant, ${ }^{* *}$ one obtains as a general feature a mass relation [9.37]

$$
m_{\mathbf{B}_{3 / 2}}=c f_{\bar{G}} / M_{\mathrm{p}}
$$

where $c$ is a model dependent constant of $\mathrm{O}(1)$.

The question which remains is how does $S U(2) \times U(1)$ spontaneously break to $U(1)$ in these approaches. There are two possible scenarios which can lead to electroweak symmetry breaking. We start with a model of spontaneously broken supergravity coupled to ordinary matter (and their supersymmetric partners). When the limit $M_{\mathrm{p}} \rightarrow \infty$ is taken, the result is an effective renormalizable Lagrangian which consists of two pieces. One piece has exact global supersymmetry and the second piece consists of terms which explicitly break the supersymmetry. $\dagger$ In one scenario the terms which explicitly break the supersymmetry (these terms are totally attributed to the effects of gravity) also turn out to break $\mathrm{SU}(2) \times \mathrm{U}(1)$. That is, one finds in these terms a negative mass squared term for a $\mathrm{SU}(2)$ weak doublet Higgs field. Such an approach has been considered in refs. [9.26, 9.30,9.32]. In the second scenario [9.27-9.29,9.31,9.38-9.46], the effective Lagrangian produced by the procedure described above does not break the $S U(3) \times S U(2) \times U(1)$ gauge symmetry at all. Let us focus on the scalar potential, i.e., the part of the Lagrangian which involves just the scalar fields of the model (the Higgs scalars and the supersymmetric scalars). To see whether $\mathrm{SU}(2) \times \mathrm{U}(1)$ is spontaneously broken at a scale of order $m_{\mathrm{w}}$, it is necessary to minimize the scalar potential and search for a minimum at field values of order $m_{\mathrm{w}}$. Is the scalar potential derived from the supergravity model trustworthy? To check, it is necessary to calculate the radiative corrections due to matter loops (ignoring further gravitational corrections). The renormalized effective potential will contain terms logarithmic in the scalar fields such as $g^{2} \phi^{4} \log \left(\phi^{2} / M_{\mathrm{p}}^{2}\right)$. For values of the scalar fields of order $M_{\mathrm{p}}$, these corrections are small; but for $\phi$ of order $m_{\mathrm{w}}$, these corrections can be large! Thus, the tree-level scalar potential is not trustworthy for values of the field of order $m_{\mathrm{w}}$. Therefore, we cannot determine whether $\mathrm{SU}(2) \times \mathrm{U}(1)$ is spontaneously broken by studying the tree-level potential. There is a simple procedure to avoid the problem of the large logarithms - we can attempt to sum up the leading logarithms to all orders in the coupling

\footnotetext{
* One class of models is arranged such that the Goldstino lives in a 'hidden sector' which has no communication with ordinary matter except through universal gravitational interactions.

** This is the most severe (unnatural) fine-tuning problem which remains totally unsolved in all presently known approaches.

†These explicit supersymmetry breaking terms are called 'soft' [9.47] because they do not reintroduce quadratic divergences into the unrenormalized theory (see section 1.2). The most general form of such terms is given by eq. (B.10) in Appendix B.
} 
constant. This procedure is equivalent to writing down a renormalization group equation for the effective potential and evolving it down from $M_{\mathrm{p}}$ to $m_{\mathrm{w}}$. As a result, one obtains a scalar potential which is valid when the fields take on values of order $m_{\mathrm{w}}$. The remarkable conclusion is that within a particular range of parameters, $\mathrm{SU}(2) \times \mathrm{U}(1)$ is found to be broken, as desired.

That such an outcome is possible is by no means trivial. Basically, one must calculate the effective scalar potential $V_{\text {eff }}(t)$ as a function of $t=\log \left(\phi_{i} / M_{\mathrm{p}}\right)$ where $\phi_{i}$ is any scalar field which appears in the low energy theory. Before radiative corrections are computed (i.e., at tree level, with no logarithmic terms present), $\mathrm{SU}(3) \times \mathrm{SU}(2) \times \mathrm{U}(1)$ is unbroken. That is, for any scalar field $\phi_{i}$, we find $\partial V /\left.\partial \phi_{i}\right|_{\phi_{i}=0}=0$ and $m_{\phi_{i}}^{2} \equiv \partial^{2} V /\left.\partial \phi_{i} \partial \phi_{i}^{*}\right|_{\phi_{i}=0}>0$. After renormalization group improvement, the origin $\phi_{i}=0$ is still a stationary point but we must check the sign of $m_{\phi_{i}}^{2}(t)=\partial^{2} V(t) /\left.\partial \phi_{i} \partial \phi_{i}^{*}\right|_{\phi_{i}=0}$ as $t \rightarrow-\infty$ (which corresponds to $\phi_{i} \rightarrow 0$ ). For example, it would be a disaster to have a model where the scalar-quark mass squared was negative. This would imply that both color and electric charge were spontaneously broken [9.48]. To find out which (if any) of the scalars have $m_{\phi_{i}}^{2}(t \rightarrow-\infty)<0$ requires solving a set of coupled renormalization group differential equations. The appropriate boundary condition states that $V(t=0)$ is the tree-level potential which means that $m_{\phi_{i}}^{2}(t=0)$ are all positive and of order $m_{3 / 2}^{2}$. The results for $m_{\phi_{i}}^{2}(t \rightarrow-\infty)$ depend on the range of values of various parameters which appear in the model.

Consider, for example, the class of models where the $S U(2) \times U(1)$ breaking occurs radiatively. In these models there is large range of parameters where one finds $m_{\phi_{i}}^{2}(t \rightarrow-\infty)>0$ for all scalar fields; that is the gauge symmetry remains unbroken. If on the other hand, if there is (at least) one large Higgs-fermion coupling constant (implying a large mass for the corresponding fermion, usually considered to be the t-quark), one finds that for one of the SU(2) weak doublet, color singlet Higgs, $\mathrm{H}$, the value of $m_{\mathrm{H}}^{2}(t)$ as $t \rightarrow-\infty$ is driven negative. At the same time, for all other scalar fields (i.e., the scalar-quarks, scalar-leptons, and other Higgs scalars), one finds $m_{\phi_{i}}^{2}(t \rightarrow-\infty)>0$. This is exactly the desired result $-\mathrm{SU}(2) \times \mathrm{U}(1)$ is broken to $\mathrm{U}(1)_{\mathrm{EM}}$, whereas color and electromagnetism remain as good symmetries. In addition, the value of $m_{\mathbf{H}}^{2}(t \rightarrow-\infty)$ is $-c m_{3 / 2}^{2}$ where $c$ is a constant of $\mathrm{O}(1)$. Thus, the weak scale is directly related to the gravitino mass. Models of this kind also exist where a large t-quark mass is not required in order to induce the $\mathrm{SU}(2) \times \mathrm{U}(1)$ breaking $[9.49,9.50]$. Once it is determined that spontaneous symmetry breaking occurs, one must investigate the new minima which appear away from $\phi_{i}=0$. Occasionally, one finds 'undesirable' minima (where the 'wrong' symmetries are broken) which have lower energy density than the desired ground state (where $\mathrm{SU}(2) \times \mathrm{U}(1)$ is broken to $\left.\mathrm{U}(1)_{\mathrm{EM}}\right)$. One can argue that as long as the desired ground state is at least metastable (with lifetime sufficiently greater than the age of the universe), such a model cannot be ruled out experimentally [9.42]. Alternatively, one can search the available space of parameters for a region where the desired ground state is indeed the one of lowest energy.

We shall not give the technical details on how the various scenarios described above occur, but we simply refer the reader to the references already mentioned where such a procedure has been carried out for various models. Good review articles on the low energy supergravity approach to constructing a viable low energy broken supersymmetric model can be found in refs. [9.4, 9.51-9.54].

One can now survey the various models for their predictions. Once the renormalization group improved effective potential has been derived, one can simply read off the values of the masses for the scalar-quarks and scalar-leptons and some of the Higgs bosons.*

\footnotetext{
* It is true that the mass of some of the Higgs particles is a free parameter as it is in the Standard Model. However, in the limit of exact supersymmetry the masses of three of the Higgs scalars (two charged and one neutral scalar) are equal to the $\mathrm{W}^{ \pm}$and $\mathrm{Z}^{0}$ boson masses as shown by eqs. (B29)-(B31). These Higgs scalar masses can be determined by the mechanism which breaks supersymmetry. See refs. [9.15, 9.57, 9.58].
} 
The gaugino masses are sensitive to the nature of the supergravity model. In the limit of exact supersymmetry (but where $\mathrm{SU}(2) \times \mathrm{U}(1)$ is broken to $\mathrm{U}(1)$ ), the gluino and photino would be massiess and the winos and zinos would be degenerate in mass with the $\mathrm{W}$ and $\mathrm{Z}$ bosons (see appendix $\mathrm{B}$ ). The question then arises: When supersymmetry is broken, are Majorana mass terms for the gauginos generated? In one class of models, no such terms are generated at tree level. But because the continuous R-invariance (the chiral invariance which protects gauginos from acquiring a Majorana mass) is usually broken, one finds that such mass terms are generated radiatively. One then expects Majorana mass terms of order $\alpha m_{3 / 2}$ where $\alpha$ is the coupling constant appropriate to the particular gaugino. For example, in refs. [9.54,9.55], the gluino mass is found to be fairly light (of order $1-2 \mathrm{GeV}$ ). In a second class of models (see, e.g., refs. [9.43,9.46,9.50]), there is a tree-level Majorana mass for the gaugino of order $m_{3 / 2}$. A word of caution regarding gaugino masses should be kept in mind. In both classes of models just described, one can show that there are radiative corrections to the gaugino mass which are logarithmically divergent (these usually occur at two loops) $[9.56,9.48,9.55]$. One can simply cut off these integrals at $M_{\mathrm{p}}$ in which case the above discussion is not altered. However, this divergence is telling us that the low energy effective theory still has some sensitivity to physics at the Planck scale. Perhaps a more sensible point of view would be to simply renormalize the gaugino mass (as one does for the mass parameter in a usual renormalizable field theory). In this view, the gaugino mass (at this stage) could not be predicted - it is simply a free parameter of the theory.

We end this chapter by presenting some 'typical' expectations for the masses of the supersymmetric particles in low energy supergravity models. We also include some of the Higgs particles and the top quark mass; the latter is often required to trigger the breaking of $\mathrm{SU}(2) \times \mathrm{U}(1)$ as described above. These results are shown in table 11. From our point of view, these results should be taken only as a very rough guide to where supersymmetric particles are likely to show up. Any clustering of mass values from different theorists should be taken as a random event with no particular physical significance at this time.

Table 11

Representative masses from low energy supergravity models. [We give model predictions of masses from four papers chosen at random: model I [9.42], model II [9.43], model III [9.45] and model IV [9.46]. We have picked only one given set of numbers per reference (although often more choices are given corresponding to different values of parameters). The models illustrate heavy scalar-quark and scalar-lepton masses (I), a light scalar-neutrino (II), a light gluino (III) and light scalar-lepton masses (IV). A heavy 'top'-quark is responsible for triggering $\mathrm{SU}(2) \times \mathrm{U}(1)$ breaking in models $I$ and II. It is possible that the 'top'-quark could be identified with a new fourth generation. All masses are in $\mathrm{GeV}$ units.]

\begin{tabular}{llllll}
\hline Particle & Symbol & I & II & III & IV \\
\hline Charged scalar-leptons & $\bar{\ell}$ & 154,151 & 71 & 73 & 32,22 \\
Scalar-neutrino & $\tilde{\nu}$ & 140 & 16 & 71 & 32,22 \\
$\tilde{u}, \bar{c}$ scalar-quarks & $\tilde{\mathrm{u}}, \tilde{\mathrm{c}}$ & 190,187 & 136,137 & $72-75$ & $60-66$ \\
$\tilde{\mathrm{d}}, \overline{\mathrm{s}}, \tilde{\mathrm{b}}$ scalar-quarks & $\tilde{\mathrm{d}}, \overline{\mathrm{s}}, \overline{\mathrm{b}}$ & 200,192 & 155,142 & $72-75$ & $60-66$ \\
$\hat{\text { t scalar-quarks }}$ & $\mathrm{i}$ & 84 & $120-150$ & 41,100 & 95,23 \\
Charginos & $\tilde{\chi}_{i}^{ \pm}$ & 33,128 & $20-40$ & 15,121 & $79-87$ \\
Neutralinos & $\tilde{\chi}_{i}^{0}$ & $4,55,101,102$ & $20-40$ & $3.2,61,66,132$ & $4.6,23,85,108$ \\
Gluino & $\tilde{\mathrm{g}}^{ \pm}$ & 140 & 147 & 15 & 47 \\
Charged Higgs & $\mathrm{H}^{ \pm}$ & 183 & 81 & 156 & 95 \\
Neutral Higgs & $\mathrm{H}^{0}$ & $58,162,182$ & $23-89$ & $4.6,134,163$ & $4.5,49,105$ \\
Gravitino & $\tilde{\mathrm{g}}_{3 / 2}$ & 145 & 50 & 72 & 15 \\
Top quark & $\mathrm{t}$ & 84 & 111 & 33 & 35 \\
\hline
\end{tabular}




\section{Alternative models and assumptions}

Throughout this review, we have discussed supersymmetric phenomena, making use of a framework which consisted of making a number of simplifying assumptions on the nature of the supersymmetric model. This entailed the following assumptions: (a) the effective low energy gauge group is $\operatorname{SU}(3) \times$ $\mathrm{SU}(2) \times \mathrm{U}(1)$; (b) the particle content consists of those particles of the minimal Glashow-WeinbergSalam model with two SU(2) weak doublets of Higgs scalars and their supersymmetric partners; ${ }^{*}$ (c) the low energy theory conserves lepton (L) and baryon (B) number global symmetries; (d) a discrete R-parity exists [10.1] implying that the lightest supersymmetric particle is stable; ${ }^{* *}$ (e) the Goldstino is either too weakly interacting to be of any consequence to the low energy theory or else it is absorbed in a gravitino with mass $\tilde{M}_{\mathrm{g}_{3 / 2}} \sim \mathrm{O}\left(m_{\mathrm{w}}\right)$. $\dagger$ In this chapter, we would like to relax each of the above assumptions in turn. We do this for the purposes of completeness; however, we shall just sketch briefly some comments regarding each case and direct the reader to the references for the details. Finally, we shall make some comments on attempts to embed supersymmetric models in the framework of grand unification.

\subsection{Enlarging the effective low energy gauge group}

At this time, there are no experimental indications which suggest the need to enlarge the effective low energy gauge group beyond $\mathrm{SU}(3) \times \mathrm{SU}(2) \times \mathrm{U}(1)$. However, there has been much discussion in the literature [10.1-10.6] about supersymmetric versions of $S U(3) \times S U(2) \times U(1) \times U(1)$. To understand why the extra U(1) factor has been added, one must go back to the 'early days' of supersymmetric model building. Originally, it was hoped that one could construct a globally supersymmetric gauge theory where the supersymmetry and local gauge symmetry were both spontaneously broken. There are basically two methods to spontaneously break global supersymmetry. If supersymmetry were unbroken, then the scalar potential $V$, evaluated by setting all scalar fields equal to their ground state values, would be zero [see eqs. (B3)-(B5)]. Since $V=\frac{1}{2} D^{a} D^{a}+F_{i}^{*} F_{i}^{*}$, in order that $\langle V\rangle \neq 0$, one must either have $\left\langle F_{i}\right\rangle \neq 0$ for some $i$ or $\left\langle D^{a}\right\rangle \neq 0$ for some $a$. These two types of supersymmetric breaking are called $F$-type and $D$-type breaking respectively. $F$-type breaking [10.7] (often called $O$ 'Raifeartaigh models) is arranged by constructing a model where the set of equations $F_{i}=0$ cannot be consistently solved. However, it was found that such models necessarily obeyed sum rules, involving the tree-level masses of the particles appearing in each supermultiplet of the theory, of the form [10.8-10.10]

$$
\sum_{J=0,1 / 2,1}(-1)^{2 J}(2 J+1) m_{J}^{2}=0
$$

This would imply for example that one of the scalar-electrons would be lighter than the electron contrary to experimental observation.

One way around this problem was investigated in detail by Fayet $[10.1,10.2]$. He investigated $D$-type supersymmetry breaking which requires the gauge group to have an explicit $U(1)$ factor, and corresponding to it the presence of a Fayet-Iliopolos term $\xi[10.11]$ [see eqs. (B8), (B9)]. When $\xi \neq 0$, one can construct a model such that $\left\langle D^{a}\right\rangle \neq 0$ for some $a$. Supersymmetry is spontaneously broken, but there still

\footnotetext{
* Furthermore, the masses of the supersymmetric partners are expected to be of order of the $\mathrm{W}$ boson mass (to within an order of magnitude).

${ }^{* *}$ Assumptions (c) and (d) are related since $R=(-1)^{2 j+3 B+L}$ for a particle of spin $j$.

† We briefly discussed the implications of a Goldstino which can significantly interact with other particles in section 3.7 .
} 
remains a sum rule involving the tree-level masses of a given supermultiplet. The new sum rule is $[10.9,10.10]$

$$
\sum_{J=0,1 / 2,1}(-1)^{2 J}(2 J+1) m_{J}^{2}=2 \sum_{a}\left\langle D^{a}\right\rangle \operatorname{Tr} T^{a} .
$$

We thus have the possibility of altering the sum rule in a phenomenologically acceptable way. For non-Abelian generators, $\operatorname{Tr} T^{a}=0$ so that eq. (10.2) reduces to eq. (10.1). Furthermore, for the Standard Model which contains a U(1) factor-the hypercharge $Y$, the particle content is such that $\operatorname{Tr} Y=0$. Thus, in order to obtain a nontrivial result for the right-hand side of eq. (10.2), we need to introduce an additional $U(1)$ to the theory (i.e., a new hypercharge which we call $Y^{\prime}$ ) such that $\operatorname{Tr} Y^{\prime} \neq 0$. Fayet showed $[10.1,10.2]$ that it was then possible to construct an $\mathrm{SU}(3) \times \mathrm{SU}(2) \times \mathrm{U}(1) \times$ U(1) model which contained spontaneous $D$-type supersymmetry breaking and had a phenomenologically acceptable mass spectrum for the supersymmetric scalars.

Two main problems remained with such models. The first was a theoretical one-the model constructed by Fayet had an anomaly in the new gauged U(1) current (see section 8.9). Attempts to construct an anomaly free low energy $\mathrm{SU}(3) \times \mathrm{SU}(2) \times \mathrm{U}(1) \times \mathrm{U}(1)$ supersymmetric gauge model were pursued by a number of authors $[10.3,10.4,10.6]$. Those efforts required adding many more multiplets of particles to the low energy theory to cancel anomalies, resulting in very complicated models which often had other severe problems (such as an unbroken supersymmetric ground state). The second problem of such models involved the new neutral current it predicted (due to an extra Z-boson corresponding to the new hypercharge $Y^{\prime}$ ). The model predicted effects in various neutral current asymmetries which so far have not been observed. This work has been well reviewed $[10.2,10.12]$ and we will not pursue it further here.

As an aside, it is worth commenting here about how other model building approaches avoided the dire consequences of eq. (10.1). Recall that the mass sum rules eq. (10.1) and eq. (10.2) are valid for the tree-level masses. One can construct $F$-type supersymmetry breaking models where the supersymmetry breaking occurs in a sector of the theory separate from the ordinary particles. Supersymmetry breaking is communicated to the ordinary low energy sector via radiative corrections. These radiative corrections also induce $\mathrm{SU}(2) \times \mathrm{U}(1)$ gauge symmetry breaking [10.13]. Thus, eq. (10.1) is trivially satisfied because at tree level, the fermion masses and their scalar partners are all zero. When supersymmetry breaking occurs, the scalars obtain masses radiatively - these masses need not satisfy eq. (10.1). The most important observation is that the scalars can gain $S U(2) \times U(1)$ invariant masses; whereas the fermion masses arise due to $\mathrm{SU}(2) \times \mathrm{U}(1)$ breaking. The end result is that the scalar partner masses can all be larger than the corresponding fermion masses.

In the supergravity approach, where $n$ chiral supermultiplets are minimally coupled to the supergravity, one finds that eq. (10.1) is modified to [10.14]

$$
\sum_{J=0,1 / 2,1,3 / 2}(-1)^{2 J}(2 J+1) m_{J}^{2}=2(n-1) \tilde{M}_{\mathbf{g}_{3 / 2}}^{2}
$$

where $\tilde{M}_{8_{3 / 2}}$ is the gravitino mass. We have assumed for simplicity that $\left\langle D^{a}\right\rangle=0$ (for the more general case, see ref. [10.15]). Thus, one sees that acceptable tree-level mass formulas are possible without resorting to $D$-type supersymmetry breaking and a Fayet-Iliopoulos term [10.11]. Indeed, one finds in these models (see chapter 9), that the scalar partners of the ordinary fermions have masses of order $\tilde{M}_{g_{3 / 2}}$. 


\subsection{Enlarging the particle content of the low energy theory}

One can imagine increasing the particle content of the low energy theory. We know of three separate reasons for contemplating such an extension.

\section{(a) Increasing the Higgs sector}

There are two basic extensions of the Higgs sector which have been discussed in the literature. The first is the addition of an $\mathrm{SU}(3) \times \mathrm{SU}(2) \times \mathrm{U}(1)$ Higgs singlet field. The reason for this addition is that the minimal supersymmetric extension of the Standard Model which uniquely breaks the $\mathrm{SU}(2) \times \mathrm{U}(1)$ gauge symmetry to $\mathrm{U}(1)_{\mathrm{EM}}$ requires two Higgs doublets and one Higgs singlet* $[10.16,10.17]$. However, in supersymmetric grand unified theories or supergravity theories, it has been argued that for technical reasons $[10.18,10.19]$, a light singlet scalar field (with mass of order $m_{w}$ ) destabilizes the hierarchy of scales (i.e., $m_{\mathrm{w}} / M_{\mathrm{p}} \sim 10^{-17}$ ). There have been efforts to alleviate this problem as discussed in refs. $[10.20,10.21]$. Recently these arguments have been disputed in ref. [10.54]. Still, the simplicity of such a model as a low energy effective theory make it quite attractive. The second extension of the Higgs sector involves adding further Higgs doublets. The reasons for this addition is usually connected with attempts to embed the low energy model in a grand unified theory. For example, in SU(5), one often chooses to have two 5 's and two $\overline{5}$ 's of Higgs multiplets (corresponding to four weak doublets) in order to generate the baryon asymmetry in the early universe [10.22].

\section{(b) Additional matter multiplets}

Adding totally new matter multiplets to the low energy theory often occurs as a result of grand unification where extra multiplets have been added for various theoretical reasons [10.23, 10.24] which we shall discuss at the end of this chapter. Typically, one finds new supersymmetric multiplets of color octets and weak triplets with masses of order $m_{\mathrm{w}}$. The phenomenology of such new particles has yet to be considered in detail.

The possibility of new color octet fermions has been previously considered by Fayet [10.25] as a way to make gluinos heavy. Basically, these fermions can mix with the gluinos leading to Dirac mass terms for the resulting color octet fermions. Such a mechanism was considered by Fayet as a possible way to give gluinos a mass without breaking the continuous $R$-invariance.

Another set of matter multiplets sometimes considered are so-called 'mirror fermions'. These particles consist of repeating the Standard Model spectrum with opposite chirality so that the entire low energy sector is vector-like. The masses of the mirror fermions (and their supersymmetric partners) are assumed to be of order $m_{\mathrm{w}}$. These particles are needed if one wants to construct models based on $N=2$ (or higher) supersymmetry [10.26], where $N$ counts the number of supersymmetric fermionic generators. Models arising from these theories must be vector-like since for every helicity $+1 / 2$ state, two supersymmetric generators can be applied to convert it into a helicity $-1 / 2$ state. As of yet, there has been little work to investigate the phenomenological properties of such models.

\subsection{Theories which violate $R$-parity}

We may define the $R$-parity [10.1] of a state in the low energy theory with spin $j$, baryon number $B$ and lepton number $L$ to be $R=(-1)^{2 j+3 B+L}$. All ordinary particles have even $R$-parity; all supersym-

\footnotetext{
* This minimal model which we describe in appendix B requires the addition of (soft) explicit terms to break the supersymmetry. One could also drop the singlet Higgs field and break the $\mathrm{SU}(2) \times \mathrm{U}(1)$ gauge symmetry using explicit supersymmetry breaking terms.
} 
metric partners have odd $R$-parity. If $R$-parity is exactly conserved (as it must if baryon and lepton numbers are conserved), then the lightest $R$-odd particle is exactly stable. One can imagine models where $R$-parity is broken in the effective low energy theory; however, since this must be accompanied by either $B$ or $L$ violation, one must be careful to construct a model which does not immediately contradict experimental observations. An example of such a model would be one where the scalar neutrino gains a vacuum expectation value. If $\langle\tilde{\nu}\rangle \neq 0$, then both lepton number and $R$-parity would be broken. One can construct models with such a property in which the rates for $\mu \rightarrow \mathrm{e} \gamma$ or neutrinoless double beta decay do not contradict present experimental limits. One consequence of such a model is that the lightest $R$-odd particle would no longer be stable. For example, if the lightest $R$-odd particle were the photino, it could decay via $\tilde{\gamma} \rightarrow \gamma+\nu$. These issues have been discussed in detail in refs. $[10.53,10.27]$.

\subsection{A phenomenologically important Goldstino (or gravitino)}

In section 3.7, we discuss some of the implications of a phenomenologically important Goldstino (or gravitino). The main consequence of such an assumption would be that the Goldstino would be the lightest $R$-odd particle; all other $R$-odd particles would decay into the Goldstino with a lifetime given by eq. (3.60). In addition, one can look for 'invisible' decays of certain particles. One example [10.28] of such a decay would be the decay of a $2^{++}$meson $X$ into $\tilde{G} \tilde{G}$ (e.g., $X$ could be the $f(1270)$ or the $\chi_{\mathrm{c}}(3555)$ ). This could be searched for in radiative quarkonium decay $\mathrm{V} \rightarrow \gamma \mathrm{X}, \mathrm{X} \rightarrow$ nothing seen. Observation of such a process could also be a signal for the direct decay process $V \rightarrow \gamma \tilde{G} \tilde{G}$ [10.29]. The same phenomenological consequences could be applied to a very light gravitino. If both the gravitino and photino were light, Fayet [10.30] has argued that an interesting limit could be obtained by the nonobservation of $\psi \rightarrow \tilde{\gamma} \tilde{g}_{3 / 2}$. This is achieved experimentally by studying the decay $\psi^{\prime} \rightarrow \pi^{+} \pi^{-} \psi$, $\psi \rightarrow$ nothing seen, thereby obtaining an upper limit for BR $(\psi \rightarrow$ nothing seen). The present experimental limit is not very constraining and Fayet obtains a limit on the gravitino mass of $\tilde{M}_{\mathrm{g}_{3 / 2}}>1.5 \times 10^{-8} \mathrm{eV}$. Gravitinos with masses on the order of $1 \mathrm{keV}$ have interesting cosmological implications which have been discussed by Pagels and Primack [10.31]. They could account for the dark matter of the universe. In addition, the Jeans mass of collisionless gravitinos of this mass would be roughly equal to the mass of a typical galaxy - a result which could be used to explain the origin of galaxies.

\subsection{Grand unification and supersymmetry}

Our final topic is a discussion of how supersymmetric models might fit in with the grand unification picture. A successful supersymmetric grand unified model would hopefully have the following properties: (a) the successful features of minimal SU(5), i.e., predictions for $\sin ^{2} \theta_{\mathrm{w}}$ and $m_{\mathrm{b}} / m_{\tau}$ would be preserved; (b) the grand unification mass $M_{\mathrm{X}}$ should be less than the Planck mass $M_{\mathrm{p}}$; (c) the model should be consistent with the present nonobservation of proton decay. In addition, three further features of such models would be highly desirable. First, one would like a natural explanation for the vastly different mass scales of the weak Higgs doublet and color Higgs triplet which usually lie in the same grand unification Higgs multiplet. Supersymmetry guarantees that such a difference of mass scales, once put into the theory at tree level, is stable under further radiative corrections [10.32-10.35]. Still, a far more satisfying solution would be to find some 'natural' group theoretical explanation for this feature. Second, it would be desirable to construct a model where the unique ground state is $\mathrm{SU}(3) \times \mathrm{U}(1)$. Often, one finds that supersymmetric models have multiple degenerate ground states 
[10.33-10.35]. Third, one would like to find a natural explanation for the ratio $M_{\mathrm{X}} / M_{\mathrm{p}}$ rather than put in both scales into the theory separately by hand.

First, we briefly review the predictions for $\sin ^{2} \theta_{\mathrm{w}}, M_{\mathrm{X}}, \alpha_{\mathrm{GUM}}$ and $m_{\mathrm{b}} / m_{\tau}$ in supersymmetric grand unified theories. ( $M_{\mathrm{X}}$ is the grand unification mass and $\alpha_{\mathrm{GUM}}$ is the value of the unified coupling constant at that mass.) We compare the results for the standard and supersymmetric versions of the model in the case of $N_{\mathrm{g}}$ generations of quarks, leptons and their scalar partners and $N_{\mathrm{H}}$ doublets of Higgs scalars and their fermionic partners. These results have been computed using the two-loop $\beta$-function in refs. [10.36-10.38]. In the calculation, it was assumed that the masses of all supersymmetric partners lie below a mass scale $A$ (which by the arguments of section 1.2 should be less than $1 \mathrm{TeV}$ ). We summarize the results which are noteworthy: In the supersymmetric model with $N_{\mathbf{H}}=2$ or 4 (as compared with the Standard Model), $\alpha_{\mathrm{GUM}}$ is larger by about a factor of two, $M_{\mathrm{X}}$ is larger by one to two orders of magnitude* and $\sin ^{2} \theta_{\mathrm{w}}$ is slightly larger. The reason for this behavior is connected with the addition of new light fermions to the low energy effective theory. This causes $\alpha_{3}$ to run more slowly and therefore delays unification. Unification therefore takes place at a larger mass $M_{\mathrm{X}}$ and at a larger value for $\alpha_{\mathrm{GUM}}$. If too many new light scalars are introduced, this behavior can be substantially modified.

The effects of new light fields not normally assumed to exist in the Standard Model can alter the above results. It is nontrivial to add a set of new light fields to the model while maintaining acceptable values for $M_{\mathrm{X}}, \sin ^{2} \theta_{\mathrm{w}}$ and $\alpha_{\mathrm{GUM}}$. It is interesting to note that there is an (almost) unique set of low energy supermultiplets which leads to acceptable results $[10.23,10.24]$. These particles transform as $(8,1,0)+(1,3,0)+2(1,1,1)+2(1,1,-2)$ under $\mathrm{SU}(3) \times \mathrm{SU}(2) \times \mathrm{U}(1)$. In such models, $M_{\mathrm{X}}$ is roughly equal to the Planck scale; this has the advantage that no independent grand unification scale need be introduced. One reason for adding an extra set of light fields will be discussed below.

One relation we have yet to talk about is the prediction of $m_{\mathrm{b}} / m_{\tau}$. This is a successful relation in SU(5) only for the third generation. In supersymmetric grand unified models, this relations is only very slightly altered [10.36-10.38], so the conclusions regarding this relation are the same as in standard SU(5). One may also be able to utilize gravitational corrections [10.39] to explain why this relation fails so badly for the first generation fermions.

In the SU(5) grand unification model, the Standard Model weak Higgs doublet is part of an SU(5) representation: $H=\mathbf{5}=(3,1,-2 / 3)+(1,2,1)$. On the other hand, the color triplet Higgs must be very heavy (with mass $\geq 10^{10} \mathrm{GeV}$ ) in order to avoid too rapid a rate for proton decay. Splitting these masses is one aspect of the gauge hierarchy problem. The Higgs scalars will tend to have masses of order $M_{\mathrm{X}}$ unless the parameters of the model are fine-tuned to extreme accuracy. In supersymmetric theories, such a gauge hierarchy will be stable against radiative corrections; however, it still must be arranged by hand in the tree-level potential.

Various methods to explain the tree-level hierarchy in a natural way have been proposed. We refer the reader to the references for a description of the 'sliding-singlet' mechanism [10.40-10.42, 10.21] and the 'missing partner' mechanism $[10.43,10.44]$. The major implication of these mechanisms is that new multiplets beyond the Standard Model must be introduced. In particular, new light fields will often appear which do not occur in the Standard Model. As we discussed above, one must be very careful to check that these new fields do not upset the successful predictions of $\sin ^{2} \theta_{\mathrm{w}}, M_{\mathrm{X}}$ and $\alpha_{\mathrm{GUM}}$.

There are numerous examples of supersymmetric grand unified models in the literature. One can build such models based on SU(5) or generalize to larger grand unified gauge group such as $\mathrm{SO}(10)$ and

\footnotetext{
* The effect of such a large $M_{\mathrm{X}}$ is a much longer lived proton unless dimension-five baryon number violating operators exist. See section 8.8 .
} 
beyond. $\mathrm{SO}(10)$ grand unified models are interesting in that they offer novel ways to naturally split the light weak Higgs doublets from the superheavy color Higgs triplets [10.45-10.50]. Most recently, much effort has been given for building supersymmetric grand unified models using the low-energy supergravity approach. (For a review and further references, see [10.51, 10.52].) Many of these models imply that (if the dimension-five operators are absent) the proton lifetime is too long to be observable (because $M_{\mathrm{X}}$ is too large). On the other hand, these same models invariably predict that new light fields must exist including new color octets and weak triplets [10.24]. These fields could have dramatic implications for supersymmetric phenomenology in the future.

\section{Future prospects and conclusions}

In this section we summarize some estimates of what mass superpartners might be detectable at various present and future facilities. Some estimates are based on a detailed analysis and others are just estimates. Probably relative sensitivities are more meaningful than absolute ones. Table 12 indicates what various machines are most sensitive to, and figs. 66 and 67 indicate what might be in store for finding partners of gauge bosons as time goes on.

In the next few years there are three main ways we can hope to find evidence for supersymmetry: (1) pair production or associated production of gluinos at hadron colliders, detected by the signature of missing $p_{\mathrm{T}}$ and no hard charged lepton, (2) associated production of the two lightest neutralinos at $\mathrm{e}^{+} \mathrm{e}^{-}$ or hadron colliders, giving one-sided events with fermion pairs and missing $p_{\mathrm{T}}$, and (3) pair production of $\tilde{w} \tilde{\gamma}$ (directly or through $\mathrm{W}$ decays) giving leptons or jets and missing $p_{\mathrm{T}}$. All of these are discussed in detail in the appropriate sections (see contents). We have given systematic techniques for looking; in addition, one can hope for a lucky situation where masses and rates allow some events with

Table 12

Maximum mass superpartner that could be detected by systematic techniques, apart from occasional individual events in fortunate circumstances; see separate chapters for all subtleties. Other machine configurations can be extrapolated from these. [All masses are in GeV.]

\begin{tabular}{lllllll}
\hline & $\tilde{\ell}^{ \pm}$ & $\tilde{\nu}$ & $\tilde{\mathrm{q}}$ & $\tilde{\mathrm{g}}$ & $\tilde{\chi}^{ \pm}$ & $\tilde{\chi}^{0}(\bar{\gamma} \ldots)$ \\
\hline PETRA/PEP & 25 & 20 & 20 & - & 20 & 40 \\
Sp̃pS & (a) & (a) & $20-40$ & $25-50$ & (b) & $(\mathrm{b})$ \\
FNAL $\bar{p} p$ & (a) & (a) & $50-75$ & $75-125$ & (b) & $($ b) \\
SLC/LEP & 50 & 45 & 45 & 35 & 45 & 90 \\
HERA & 60 & (c) & (c, d) & - & $15(\mathrm{e})$ & $15(\mathrm{e})$ \\
$10 \mathrm{TeV}$ pp & 100 & (a) & 800 & 1500 & 300 & 500 \\
$40 \mathrm{TeV}$ pp & 200 & (a) & 1600 & 3000 & 400 & 600 \\
\hline
\end{tabular}

(a) $\tilde{M}_{\nu}+\tilde{M}_{\ell}<m_{\mathrm{w}}$. (b) $\tilde{M}_{x^{+}}+\tilde{M}_{x^{0}}<m_{\mathrm{w}}$. (c) $\tilde{M}_{\nu}+\tilde{M}_{\mathrm{q}} \leqslant 150 \mathrm{GeV}$ if $\tilde{\nu}$ decays appreciably into four-body final states. (d) $\tilde{M}_{\mathrm{e}}+\tilde{M}_{\mathrm{q}} \lesssim$ $130 \mathrm{GeV}$ including cuts. (e) Only if scalar-leptons are light.
Table 13

This table lists some probable signatures of supersymmetric pair production and decays. For a jet (quark or gluon) we write $\mathrm{j}$, and for a charged lepton $\ell$. Interpret $\nu$ and $\bar{\gamma}$ as objects which escape any collider detector, and $\bar{v}$ as probably escaping any collider detector. Note all examples give missing $P_{\mathrm{T}}$, due to two or more particles which escape detection. See separate sections of the review for details of the modes. [Part (a) lists typical decays; those in parentheses would require special circumstances to dominate. Part (b) gives examples of pairs that might be produced and the simplest signatures in favorable circumstances.] No account is taken of jets that might not be counted in a real detector or overlapping jets.

(a)

\begin{tabular}{|c|c|}
\hline 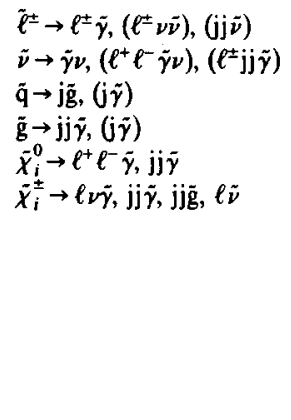 & 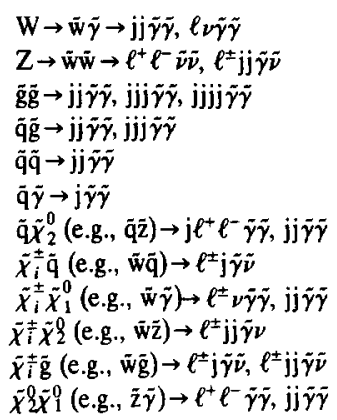 \\
\hline
\end{tabular}




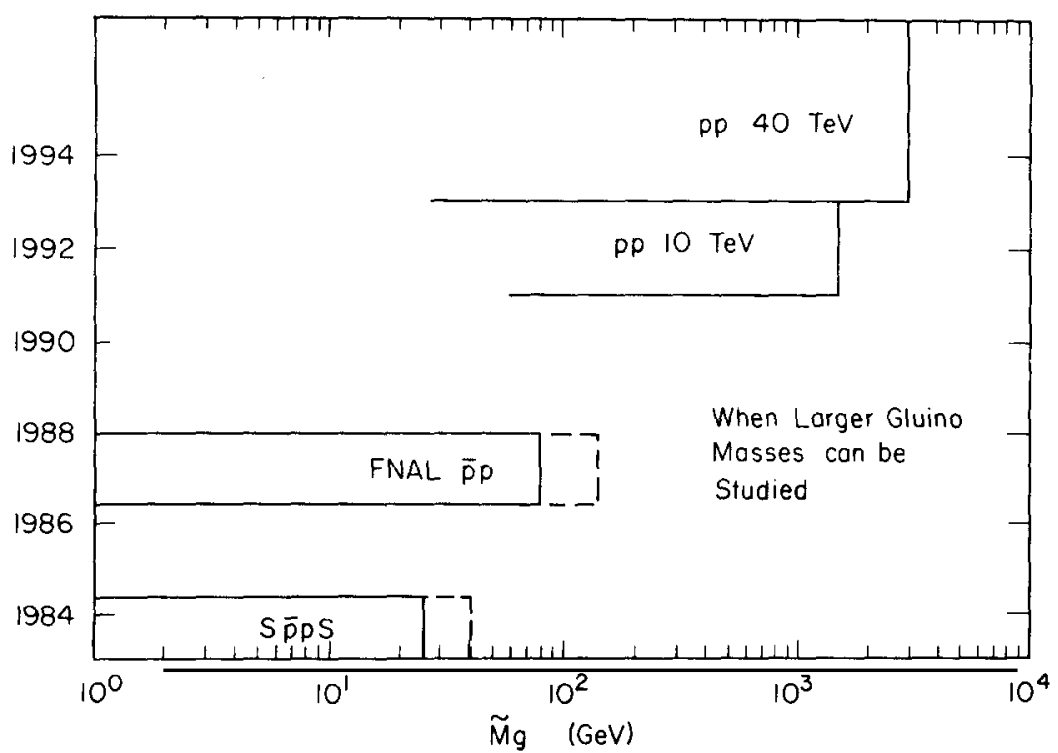

Fig. 66. This figure indicates when there will be further opportunity to find gluinos or to learn more about limits on gluino masses. Estimates on both scales are crude, since careful analyses of what $\tilde{M}_{\varepsilon}$ can be observed at a given machine and detector have not been carried out, and obviously statements about future machines are speculative. The dashed extensions on some curves suggest the increase in sensitivity that might be possible if the mode $\tilde{\mathrm{g}} \rightarrow \mathrm{g} \tilde{\gamma}$ (or $\tilde{\mathrm{g}} \rightarrow \mathrm{g} \tilde{\mathrm{G}}$ ) has at least a few percent branching ratio. See the section on gluinos to understand $\tilde{\mathrm{g}}$ production and signatures.

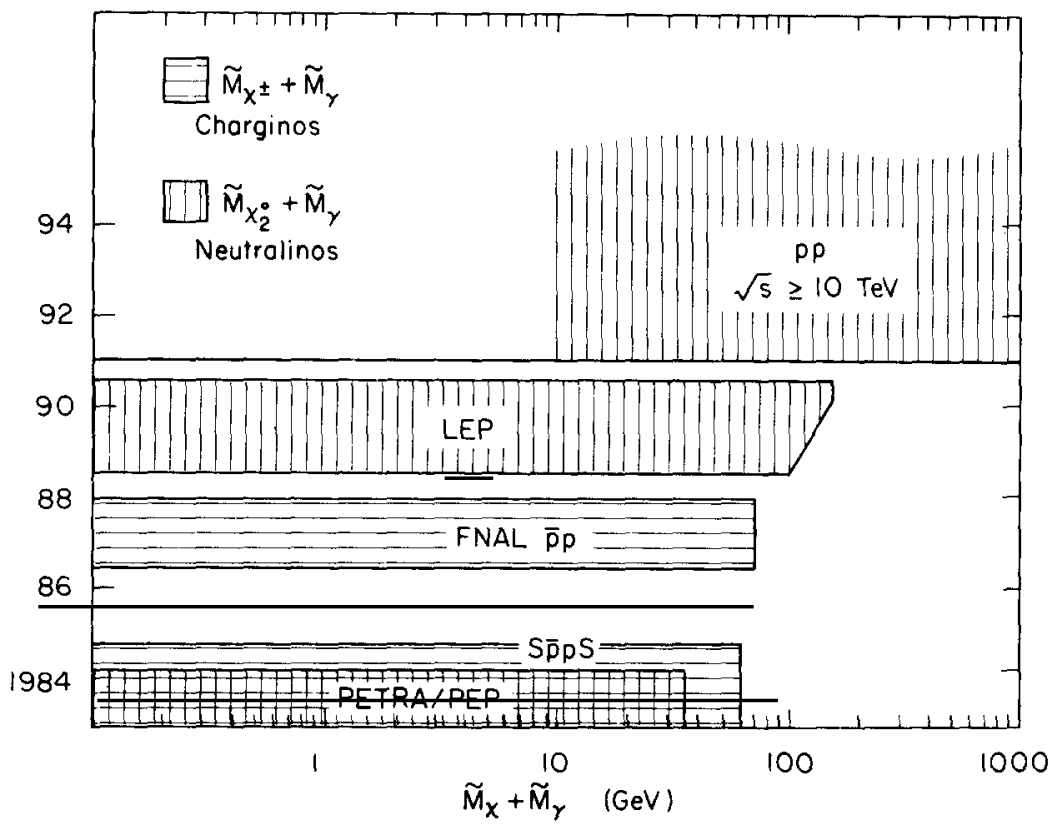

Fig. 67. This figure indicates when there will be further opportunity to learn about chargino and neutralino masses. Estimates on both scales are crude, since careful analyses of what can be detected at a given machine and detector have not been carried out, and obviously statements about future machines are speculative. Since chargino and neutralino cross sections are typically weak, luminosity is crucial to observe them. Probably $\int \mathscr{L} \mathrm{d} t \geqslant 3 \times 10^{38} / \mathrm{cm}^{2}$ is needed for neutralino detection. We assume charginos are mainly detected via $\mathrm{W}^{ \pm} \rightarrow \bar{\chi}^{ \pm} \tilde{\gamma}$, although at higher $\mathscr{L}$ machines one can have direct production of $\tilde{\chi}^{ \pm}$at an observable rate, so higher mass $\bar{\chi}^{ \pm}$can be looked for. 
supersymmetric partners to be observed. We have summarized these and other possibilities in a table of supersymmetric signatures (see table 13).

We have given our reasons for writing this review in the introduction. We only want to conclude that (1) the physics of supersymmetry is nice enough so that experimenters should take it very seriously and really search for evidence of supersymmetry, (2) theorists should take supersymmetry seriously enough to help think of better ways to search, and (3) fortunately, if nature is not supersymmetric on the weak scale, it will be possible to know this definitively with the accelerators and detectors that should be available within about the next decade and the kinds of analysis we have discussed.

\section{Acknowledgements}

We would like to thank our colleagues R.M. Barnett, J. Ellis, J.-M. Frère, T. Goldman, J. Gunion, J. Hagelin, K. Lackner, J. Leveille, S. Petkov, S. Raby and W. Rolnick for collaborations during which we have learned a great deal that appears in this review. We are grateful to F. Paige for many useful discussions, and we appreciate helpful interactions with J. Bagger, M. Chanowitz, M. Chen, S. Dawson, M. Demarteau, M. Duncan, E. Eichten, M. Einhorn, I. Hinchliffe, D.R.T. Jones, W.-Y. Keung, L. Littenberg, B. Ovrut, M. Peskin, C. Quigg, W. Repko and A. Savoy-Navarro. We thank P. Langacker and S. Rudaz very much for carefully examining the manuscript. Finally, we are very grateful to Connie Barker and Ibi Ward for an excellent job of turning many versions of a handwritten paper into a properly typed manuscript.

\section{Appendix A. Spinor notation and conventions}

It is convenient to use two-component notation for spinors when deriving supersymmetric Lagrangians. However, due to the way most particle physicists have been brought up, it is more convenient to state Feynman rules using four-component notation. This will lead to some complications when (four-component) Majorana fermions are involved. A recipe for dealing with Majorana fermions is given in appendix $\mathrm{D}$.

We begin by briefly reviewing the two-component formalism. We warn the reader that there are almost as many variations in the conventions used as there are papers on supersymmetry. If sufficient care is not applied, it is very easy to make sign errors. We shall, for the most part, use the conventions described by Wess and Bagger [A.1]. However, we shall use a different metric convention which will lead to slight variations in the definitions as given below. If the rules below are followed, all Lagrangians given in ref. [A.1] can immediately be translated in agreement with our rules.

We take our metric to be

$$
g_{\mu \nu}=\operatorname{diag}(1,-1,-1,-1)
$$

The momentum four vector is $p^{\mu}=(E ; p)$. We introduce the Pauli matrices:

$$
\sigma^{\mu}=(1, \boldsymbol{\sigma}), \quad \bar{\sigma}^{\mu}=(1,-\boldsymbol{\sigma})
$$

The two-component spinor $\xi_{\alpha}$ transforms under a matrix $M$ of $\operatorname{SL}(2, \mathrm{C})$. Similarly, the spinors $\bar{\xi}_{\dot{\alpha}}, \xi^{\alpha}$, and $\bar{\xi}^{\dot{\alpha}}$ transform under $M^{*}, M^{-1}$ and $\left(M^{-1}\right)^{*}$ respectively. In fact, we may define $\bar{\xi}_{\dot{\alpha}} \equiv \xi_{\alpha}^{*}$, etc. The 
Dirac equation in two-component notation is

$$
\left(\bar{\sigma}_{\mu} p^{\mu}\right)^{\dot{\alpha} \beta} \xi_{\beta}=m \bar{\eta}^{\dot{\alpha}}, \quad\left(\sigma_{\mu} p^{\mu}\right)_{\alpha \dot{\beta}} \bar{\eta}^{\dot{\beta}}=m \xi_{\alpha}
$$

This allows us to introduce four-component notation. Ordinarily, one introduces a four-component spinor which satisfies

$$
\left(\gamma_{\mu} p^{\mu}-m\right) \psi=0
$$

It follows that

$$
\begin{aligned}
& \psi=\left(\begin{array}{c}
\xi_{\alpha} \\
\bar{\eta}^{\dot{\alpha}}
\end{array}\right), \quad \gamma_{\mu}=\left(\begin{array}{cc}
0 & \sigma_{\mu \alpha \dot{\beta}} \\
\bar{\sigma}_{\mu}^{\alpha \beta} & 0
\end{array}\right) \\
& \gamma_{5}=\mathrm{i} \gamma^{0} \gamma^{1} \gamma^{2} \gamma^{3}=\left(\begin{array}{cc}
-1 & 0 \\
0 & 1
\end{array}\right) \\
& \sigma^{\mu \nu}=\frac{\mathrm{i}}{2}\left[\gamma^{\mu}, \gamma^{\nu}\right]=2 \mathrm{i}\left(\begin{array}{cc}
\sigma_{\alpha}^{\mu \nu \beta} & 0 \\
0 & \bar{\sigma}_{\dot{\beta}}^{\mu \nu \dot{\alpha}}
\end{array}\right),
\end{aligned}
$$

where

$$
\begin{aligned}
& \sigma_{\alpha}^{\mu \nu \beta}=\frac{1}{4}\left(\sigma_{\alpha \dot{\alpha}}^{\mu} \bar{\sigma}^{\nu \alpha \beta}-\sigma_{\alpha \dot{\alpha}}^{\nu} \bar{\sigma}^{\mu \dot{\alpha} \beta}\right), \\
& \tilde{\sigma}_{\dot{\beta}}^{\mu \nu \dot{\alpha}}=\frac{1}{4}\left(\bar{\sigma}^{\mu \dot{\alpha} \alpha} \sigma_{\alpha \dot{\beta}}^{\nu}-\bar{\sigma}^{\nu \dot{\alpha} \alpha} \sigma_{\alpha \dot{\beta}}^{\mu}\right)
\end{aligned}
$$

This is called the chiral representation of the $\gamma$-matrices. We define the left- and right-handed projection operators by

$$
P_{\mathrm{L}} \equiv \frac{1}{2}\left(1-\gamma_{5}\right), \quad P_{\mathrm{R}} \equiv \frac{1}{2}\left(1+\gamma_{5}\right)
$$

Then, using the notation $\psi_{\mathrm{L}, \mathrm{R}} \equiv P_{\mathrm{L}, \mathrm{R}} \psi$, we see that ${ }^{*}$

$$
\psi \equiv\left(\begin{array}{l}
\psi_{\mathrm{L}} \\
\psi_{\mathrm{R}}
\end{array}\right)
$$

As usual, one defines $\bar{\psi}=\psi^{+} \gamma^{0}$. The charge conjugation operator $C$ allows us to define the charge conjugated spinor:

$$
\psi^{\mathrm{c}}=C \bar{\psi}^{\mathrm{T}}
$$

In the chiral representation, $C=-\mathrm{i} \gamma^{2} \gamma^{0}$. In two-component notation, one defines an antisymmetric

${ }^{*}$ A word of caution is in order here. Most textbooks define $\sigma_{\mu}=(1, \sigma)$ and $\bar{\sigma}_{\mu}=(1,-\sigma)$ (as opposed to eq. (A2)). This would then lead to an interchange of $\psi_{\mathrm{L}}$ and $\psi_{\mathrm{R}}$ in eq. (A11). 
tensor $\left(\varepsilon^{\alpha \beta}=-\varepsilon^{\beta \alpha}\right)$ :

$$
\varepsilon^{\alpha \beta}=-\varepsilon_{\alpha \beta}=\mathrm{i} \sigma^{2}=\left(\begin{array}{rr}
0 & 1 \\
-1 & 0
\end{array}\right)
$$

The $\varepsilon_{\alpha \beta}$ tensor can raise and lower spinor indices

$$
\xi^{\alpha}=\varepsilon^{\alpha \beta} \xi_{\beta}, \quad \xi_{\beta}=\varepsilon_{\beta \alpha} \xi^{\alpha}
$$

Identical relations hold by replacing undotted with dotted spinor indices in eqs. (A13) and (A14).* Then, in the chiral representation

$$
C=-\mathrm{i} \gamma^{2} \gamma^{0}=\left(\begin{array}{cc}
\varepsilon_{\beta \alpha} & 0 \\
0 & \varepsilon^{\dot{\beta} \dot{\alpha}}
\end{array}\right)
$$

and

$$
\bar{\Psi}^{\mathbf{T}}=\left(\frac{\eta^{\alpha}}{\bar{\xi}_{\dot{\alpha}}}\right)
$$

It follows that

$$
\Psi^{\mathfrak{c}}=\left(\begin{array}{l}
\eta_{\beta} \\
\bar{\xi}^{\beta}
\end{array}\right)
$$

A four-component Majorana spinor has the property that $\eta=\xi$ which implies that $\Psi^{\mathrm{c}}=\Psi$. That is,

$$
\Psi_{\mathrm{M}}=\left(\begin{array}{c}
\xi_{\alpha} \\
\bar{\xi}^{\dot{\alpha}}
\end{array}\right)=\left(\begin{array}{c}
\psi_{\mathrm{L}} \\
\mathrm{i} \sigma^{2} \psi_{\mathrm{L}}^{*}
\end{array}\right)
$$

We complete our translation of two-component formalism into four-component formalism by the following set of equations:

$$
\begin{aligned}
& \bar{\Psi}_{1} \Psi_{2}=\eta_{1} \xi_{2}+\bar{\eta}_{2} \bar{\xi}_{1}, \\
& \bar{\Psi}_{1} \gamma_{5} \Psi_{2}=-\eta_{1} \xi_{2}+\bar{\eta}_{2} \bar{\xi}_{1}, \\
& \bar{\Psi}_{1} \gamma^{\mu} \Psi_{2}=\bar{\xi}_{1} \bar{\sigma}^{\mu} \xi_{2}-\bar{\eta}_{2} \bar{\sigma}^{\mu} \eta_{1}, \\
& \bar{\Psi}_{1} \gamma^{\mu} \gamma_{5} \Psi_{2}=-\bar{\xi}_{1} \bar{\sigma}^{\mu} \xi_{2}-\bar{\eta}_{2} \bar{\sigma}^{\mu} \eta_{1}, \\
& -\frac{1}{2} \mathrm{i} \bar{\Psi}_{1} \sigma^{\mu \nu} \Psi_{2}=\eta_{1} \sigma^{\mu \nu} \xi_{2}-\bar{\eta}_{2} \bar{\sigma}^{\mu \nu} \bar{\xi}_{1},
\end{aligned}
$$

where the subscripts 1 and 2 label two different four-component spinors and their associated twocomponent spinors. In eqs. (A19)-(A23), we have used:

* We warn the reader that in many papers, the convention $\varepsilon_{\alpha \beta}=\varepsilon^{\alpha \beta}$ is used and eq. (A14) is modified accordingly. 


$$
\begin{aligned}
& \eta \xi=\eta^{\alpha} \xi_{\alpha}=\xi \eta, \\
& \bar{\eta} \bar{\xi}=\bar{\eta}_{\dot{\alpha}} \bar{\xi}^{\dot{\alpha}}=\bar{\xi} \bar{\eta}, \\
& \bar{\eta}_{2} \bar{\sigma}^{\mu} \eta_{1}=\bar{\eta}_{2 \dot{\alpha}} \bar{\sigma}^{\mu \dot{\alpha} \beta} \eta_{1_{\beta}}=-\eta_{1} \sigma^{\mu} \bar{\eta}_{2}, \\
& \bar{\eta} \bar{\sigma}^{\mu \nu} \bar{\xi}=\bar{\eta}_{\dot{\alpha}} \bar{\sigma}_{\dot{\beta}}^{\mu \nu \dot{\alpha}} \bar{\xi}^{\dot{\beta}}=-\bar{\xi} \bar{\sigma}^{\mu \nu} \bar{\eta}, \\
& \eta \sigma^{\mu \nu} \xi=\eta^{\alpha} \sigma_{\alpha}^{\mu \nu \beta} \xi_{\beta}=-\xi \sigma^{\mu \nu} \eta .
\end{aligned}
$$

In eqs. (A24)-(A27), the first equality is one of definition. The second equality follows from eqs. (A13), (A14) and the fact that the spinors anticommute. Manipulations of the two component spinors is illustrated in detail in appendix A of ref. [A.1]. The definition of $\bar{\eta} \bar{\xi}$ has been chosen so that $(\eta \xi)^{\dagger}=\bar{\eta} \bar{\xi}$. Using eqs. (A.19)-(A23), one can convert all Lagrangians written in two-component form into four-component notation satisfying all our conventions. In this regard, it is particularly useful to rewrite eqs. (A19)-(A22) as follows:

$$
\bar{\Psi}_{1} P_{\mathrm{L}} \Psi_{2}=\eta_{1} \xi_{2}, \quad \bar{\Psi}_{1} P_{\mathrm{R}} \Psi_{2}=\bar{\eta}_{2} \bar{\xi}_{1}, \quad \bar{\Psi}_{1} \gamma^{\mu} P_{\mathrm{L}} \Psi_{2}=\bar{\xi}_{1} \bar{\sigma}^{\mu} \xi_{2}, \quad \bar{\Psi}_{1} \gamma^{\mu} P_{\mathrm{R}} \Psi_{2}=-\bar{\eta}_{2} \bar{\sigma}^{\mu} \eta_{1},
$$

where $P_{\mathrm{L}, \mathrm{R}}$ are the projection operators defined in eq. (A10). From this, we see how one can build up four-component Dirac and Majorana spinors and interactions from a Lagrangian expressed in twocomponent notation.

Finally, we note some useful identities which follow from eqs. (A18)-(A23). If $\Psi_{1}, \Psi_{2}$ are anticommuting four-component Majorana spinors, then:

$$
\begin{aligned}
& \bar{\Psi}_{1} \Psi_{2}=\bar{\Psi}_{2} \Psi_{1}, \\
& \bar{\Psi}_{1} \gamma_{5} \Psi_{2}=\bar{\Psi}_{2} \gamma_{5} \Psi_{1}, \\
& \bar{\Psi}_{1} \gamma_{\mu} \Psi_{2}=-\bar{\Psi}_{2} \gamma_{\mu} \Psi_{1}, \\
& \bar{\Psi}_{1} \gamma_{\mu} \gamma_{5} \Psi_{2}=\bar{\Psi}_{2} \gamma_{\mu} \gamma_{5} \Psi_{1}, \\
& \bar{\Psi}_{1} \sigma_{\mu \nu} \Psi^{2}=-\bar{\Psi}_{2} \sigma_{\mu \nu} \Psi_{1},
\end{aligned}
$$

One useful consequence of the above equations is:

$$
\bar{\Psi}_{1} \gamma_{\mu} P_{\mathrm{L}} \Psi_{2}=-\bar{\Psi}_{2} \gamma_{\mu} P_{\mathrm{R}} \Psi_{1}
$$

\section{Appendix B. How to construct Lagrangians for an $\mathrm{SU}(2) \times \mathrm{U}(1)$ model of broken supersymmetry}

\section{Recipe for constructing Lagrangians}

We begin by providing a recipe for constructing interaction Lagrangians for supersymmetric gauge theories. These theories consist of gauge bosons $V_{\mu}^{a}$ and their (two-component) gaugino fermionic 
partners $\lambda^{\alpha}$ in the adjoint representation of the gauge group $\mathrm{G}$, and matter fields consisting of complex scalar fields $A_{i}$ and two-component fermions $\psi_{i}$ which transform under some representation $\mathrm{R}$ of $\mathrm{G}$.

We begin by writing down the interaction Lagrangian (omitting kinetic energy pieces) employing two-component notation for the fermions. The summation convention is employed throughout this appendix (unless otherwise noted). The interaction Lagrangian consists of three pieces:

(a) Self-interaction of the gauge multiplet

This piece contains the usual 'three-gluon' and 'four-gluon' vertices which we do not write down explicitly. These are given for example in ref. [A.41]; we follow their convention where the covariant derivative is given by $D_{\mu}=\partial_{\mu}+\mathrm{i} g A_{\mu}^{a} T^{a}$. In addition, the gauginos interact with the gauge field via the following term:

$$
\mathrm{ig} f_{a b c} \lambda^{a} \sigma^{\mu} \bar{\lambda}^{b} V_{\mu}^{c}
$$

where $f_{a b c}$ are the structure constants of $\mathrm{G}$.

(b) Interactions of the gauge and matter multiplets

The following interaction terms arise:

$$
-g T_{i j}^{a} V_{\mu}^{a}\left(\bar{\psi}_{i} \bar{\sigma}^{\mu} \psi_{j}+\mathrm{i} A_{i}^{*} \stackrel{\leftrightarrow}{\partial}_{\mu} A_{j}\right)+\mathrm{i} g \sqrt{2} T_{i j}^{a}\left(\lambda^{a} \psi_{j} A_{i}^{*}-\bar{\lambda}^{a} \bar{\psi}_{i} A_{j}\right)+g^{2}\left(T^{a} T^{b}\right)_{i j} V_{\mu}^{a} V^{\mu b} A_{i}^{*} A_{j}
$$

where $T^{a}$ is the (Hermitian) group generator in representation $\mathrm{R}$.

\section{(c) Self-interactions of the matter multiplet}

Here we must introduce some notation. The superpotential $W$ is some cubic gauge-invariant function of the scalar matter fields $A_{i}$ (and does not depend on $A_{i}^{*}$ ). Define the auxiliary functions:

$$
\begin{aligned}
& F_{i}=\partial W / \partial A_{i}, \\
& D^{a}=g A_{i}^{*} T_{i j}^{a} A_{j} .
\end{aligned}
$$

Then, the ordinary scalar potential $V$ consists of

$$
V=\frac{1}{2} D^{a} D^{a}+F_{i}^{*} F_{i}
$$

(note that it is $-V$ which appears in the interaction Lagrangian), and the Yukawa interactions and fermion mass terms are contained in

$$
-\frac{1}{2}\left[\left(\partial^{2} W / \partial A_{i} \partial A_{j}\right) \psi_{i} \psi_{j}+\left(\partial^{2} W / \partial A_{i} \partial A_{j}\right)^{*} \overline{\psi_{i}} \bar{\psi}_{j}\right]
$$

as we shall illustrate later.

It is easy to generalize these formulas to groups $\mathrm{G}$ which contain a $\mathrm{U}(1)$ factor. The following rules should be noted corresponding to the three cases above:

(i) There is no interaction between the $U(1)$ gaugino $\lambda^{\prime}$ and the $U(1)$ gauge field $V_{\mu}^{\prime}$ (i.e. set $f_{a b c}=0$ ).

(ii) For a $U(1)$ gauge multiplet, replace $g T_{i j}^{a} V_{\mu}^{a}$ in eq. (B2) with $\frac{1}{2} g^{\prime} y_{i} \delta_{i j} V_{\mu}^{\prime}$ (no sum over $i$ ) where $y_{i}$ is the $\mathrm{U}(1)$ quantum number of the matter field $\psi_{i}$ (which is the same as for $A_{i}$ ). A similar comment holds for the 
$\lambda-\psi-A$ term in eq. (B2). Note that in a $\mathrm{G} \times \mathrm{U}(1)$ gauge theory, the last term in eq. (B2) would be written:

$$
A_{i}^{*} A_{j}\left(g T_{i k}^{a} V_{\mu}^{a}+\frac{1}{2} g^{\prime} y_{i} \delta_{i k} V_{\mu}^{\prime}\right)\left(g T_{k j}^{b} V^{\mu b}+\frac{1}{2} g^{\prime} y_{j} \delta_{k j} V^{\prime \mu}\right)
$$

This leads to two-scalar boson-two gauge boson vertices where each gauge boson can either be from $\mathrm{G}$ or $U(1)$. This can lead to surprising interaction terms such as a $g-\gamma-\tilde{q}-\tilde{q}$ vertex.

(iii) In order to include a U(1) gauge multiplet, eq. (B5) must be modified. The scalar potential is now

$$
V=\frac{1}{2}\left(D^{a} D^{a}+\left(D^{\prime}\right)^{2}\right)+F_{i}^{*} F_{i}
$$

where the new term above is defined by

$$
D^{\prime}=\frac{1}{2} g^{\prime} y_{i} A_{i}^{*} A_{i}+\xi
$$

(the index $i$ is summed over). The constant $\xi$, called the Fayet-Iliopoulos term [A.2], is in principle a new constant of the theory. In the context of many models (see e.g. ref. [A.3]), it can be argued that $\xi$ must be very small and so we will henceforth set $\xi=0$.

This completes the discussion of the recipe for constructing supersymmetric Lagrangians. Nature, however, does not exhibit supersymmetry in the low energy particle spectrum. Therefore, supersymmetry must be broken. For reasons discussed in chapters 1 and 9 , it is sufficient to consider the addition of all possible explicit soft-supersymmetry breaking terms to the interaction Lagrangian described by eqs. (B1)-(B9). In this context, soft-supersymmetry breaking terms are terms which do not introduce quadratic divergences to the unrenormalized theory (which would reintroduce a new hierarchy problem in the renormalized theory). Girardello and Grisaru [A.4] have categorized all possible explicit soft-supersymmetry breaking terms. They are displayed below:

$$
\tilde{M}_{1} \operatorname{Re} A^{2}+\tilde{M}_{2} \operatorname{Im} A^{2}+c\left(A^{3}+\text { h.c. }\right)+\tilde{M}_{3}\left(\lambda^{a} \lambda^{a}+\bar{\lambda}^{a} \bar{\lambda}^{a}\right)+\tilde{M}_{4}\left(\lambda^{\prime} \lambda^{\prime}+\overline{\lambda^{\prime}} \overline{\lambda^{\prime}}\right),
$$

where $A^{2}$ and $A^{3}$ are group invariant combinations of the scalar fields $A_{i}$ (e.g., $A^{3} \equiv d_{i j k} A_{i} A_{j} A_{k}$, etc.). The effect of these terms are as follows. The parameter $\tilde{M}_{1}$ splits the mass of the complex scalar $A_{i}$ from its fermionic partner $\psi_{i}$. If we express $A_{i}$ in terms of two real spin-zero fields, then $\tilde{M}_{2}$ splits the masses of these two fields. The coupling constant $c$ corresponds to a new (non-supersymmetric) scalar interaction term. Finally, $\tilde{M}_{3}$ and $\vec{M}_{4}$ are Majorana mass terms for the gauginos corresponding to the groups $\mathrm{G}$ and $\mathrm{U}(1)$. Note that the interaction in eq. (B10) must be gauge invariant.

It is interesting to note the effect of coupling spontaneously broken supergravity to matter and examine the effective low energy theory which results. One finds [A.5, A.6] that the low energy theory consists of a globally supersymmetric theory (with interactions described by eqs. (B1)-(B9)) modified by soft-supersymmetric terms of the form displayed in eq. (B10)." Thus it is possible to propose a theory which determines the parameters $M_{i}$ and $c$ of eq. (B10); these lead to the predictions reviewed in chapter 9.

For phenomenological purposes, it is appropriate to take the parameters of eq. (B10) as arbitrary and to be determined by experiment or a future theory.

\footnotetext{
${ }^{*}$ One also finds that the superpotential has been modified somewhat in a way consistent with supersymmetry. This just adds new supersymmetric interaction terms.
} 


\section{A model of supersymmetric $S U(2) \times U(1)$ in which the gauge symmetry breaks to $U(1)$}

For pedagogical reasons it is valuable to illustrate a supersymmetric model where the gauge symmetry $\mathrm{SU}(2)_{\mathrm{w}} \times \mathrm{U}(1)_{\mathrm{y}}$ breaks to $\mathrm{U}(1)_{\mathrm{EM}}$. We will describe the model in detail and write down the interaction Lagrangian and Feynman rules. (Later on, we will explicitly add soft-supersymmetry breaking terms.) Although we will not be completely general, most of the results we obtain will survive future generalizations.

The simplest supersymmetric generalization of the Glashow-Weinberg-Salam model consists of the fields listed in table 14. (An additional Higgs scalar and its supersymmetric fermionic partner has been added for reasons to be discussed below.) The model is specified completely once we provide the superpotential $W$ [see eqs. (B3)-(B6)]. We choose [A.7]*

$$
W=h \varepsilon_{i j} H_{1}^{i} H_{2}^{j} N+s N+W_{\mathrm{F}},
$$

where $i, j$ are $\mathrm{SU}(2)_{\mathrm{w}}$ indices, $H_{1}$ and $H_{2}$ are $\mathrm{SU}(2)_{\mathrm{w}}$ Higgs doublets, $N$ is an $\mathrm{SU}(2)_{\mathrm{w}} \times \mathrm{U}(1)_{\mathrm{y}}$ Higgs singlet and $W_{\mathrm{F}}$ contain terms which are responsible for quark and lepton masses:

$$
W_{\mathrm{F}}=\varepsilon_{i j}\left[f_{a b} H_{1}^{i} \tilde{L}_{a}^{j} \tilde{R}_{b}+h_{a b} H_{1}^{i} \tilde{Q}_{a}^{j} \tilde{D}_{b}+h_{a b}^{\prime} H_{2}^{i} \tilde{Q}_{a}^{j} \tilde{U}_{b}\right]
$$

where $a, b$ are generation (flavor) labels. $\tilde{Q}$ and $\tilde{L}$ are SU(2) doublets of scalar-quarks and scalarleptons, and $\tilde{U}, \tilde{D}$, and $\tilde{R}$ are $\mathrm{SU}(2)_{\mathrm{w}}$ singlets of scalar-quarks and scalar-leptons i.e. for one generation,

Table 14

We list the gauge and matter multiplets of the supersymmetric $\mathrm{SU}(2) \times \mathrm{U}(1)$ model. The charge $Q$ is obtained via $Q=T_{3}+y / 2$. The labels are as follows: $a=1,2,3$ labels the $\mathrm{SU}(2)$ triplet of gauge bosons and $i, j=1,2$ are $\mathrm{SU}(2)$ indices. Labels referring to multiple generations of quarks, leptons and their scalar

\begin{tabular}{|c|c|c|c|c|}
\hline & Boson fields & Fermionic partners & $\mathrm{SU}(2)_{\mathrm{w}}$ & $y$ \\
\hline \multicolumn{5}{|l|}{ Gauge multiplets } \\
\hline & $V^{a}$ & $\lambda^{a}$ & triplet & 0 \\
\hline & $V^{\prime}$ & $\lambda^{\prime}$ & singlet & 0 \\
\hline \multicolumn{5}{|l|}{ Matter multiplets } \\
\hline \multirow[t]{3}{*}{ Scalar-leptons } & $\tilde{L}^{j}=\left(\tilde{\nu}, \tilde{e}_{\mathrm{L}}\right)$ & $\left(\nu, e^{-}\right)_{\mathbf{L}}$ & doublet & -1 \\
\hline & $\tilde{R}=\tilde{\ell}_{\mathrm{R}}^{+}$ & & singlet & 2 \\
\hline & $\tilde{Q}^{i}=\left(\tilde{\mathrm{u}}_{\mathbf{L}}, \tilde{\mathrm{d}}_{\mathrm{L}}\right)$ & $(u, d)_{\mathrm{L}}$ & doublet & $1 / 3$ \\
\hline \multirow[t]{3}{*}{ Scalar-quarks } & $\tilde{U}=\tilde{u}_{\mathbf{R}}^{*}$ & $u_{\mathrm{L}}^{\mathrm{c}}$ & singlet & $-4 / 3$ \\
\hline & $\tilde{D}=\tilde{d_{\mathbf{R}}^{*}}$ & $d_{\mathrm{L}}^{c}$ & singlet & $2 / 3$ \\
\hline & $H_{1}^{j}$ & $\left(\tilde{H}_{\mathrm{l}}^{0}, \tilde{H}_{1}^{-}\right)_{\mathrm{L}}$ & doublet & -1 \\
\hline \multirow[t]{2}{*}{ Higgs bosons } & $H \dot{i}$ & $\left(\tilde{H}_{2}^{+}, \tilde{H}_{2}^{0} \mathrm{\iota}\right.$ & doublet & 1 \\
\hline & $N$ & & singlet & 0 \\
\hline
\end{tabular}
partners are suppressed

A version of this model consisting only of $H_{1}, H_{2}, N$, its fermionic partners, and the gauge bosons and gauginos was first constructed by Fayet [A.8] and subsequently investigated in detail in ref. [A.9]. 


$$
\tilde{Q}=\left(\begin{array}{c}
\tilde{u}_{\mathbf{L}} \\
\tilde{d}_{\mathrm{L}}
\end{array}\right), \quad \tilde{L}=\left(\begin{array}{c}
\tilde{\nu} \\
\tilde{e}_{\mathrm{L}}^{-}
\end{array}\right), \quad \tilde{U}=\tilde{u}_{\mathrm{R}}^{*}, \quad \tilde{D}=\tilde{d}_{\mathrm{R}}^{*} \quad \text { and } \quad \tilde{R}=\tilde{e}_{\mathrm{R}}^{+}
$$

The Yukawa couplings $f_{a b}, h_{a b}$ and $h_{a b}^{\prime}$ are related to the quark and lepton mass matrices as can be seen by using eq. (B6). Note that one needs two Higgs doublets $H_{1}$ and $H_{2}$ to provide the necessary fermion masses. The reason is that supersymmetry does not allow the use of complex conjugates $H_{1}^{*}$ and $H_{2}^{*}$ in eq. (B12). Because quantum numbers (i.e. gauge invariance) prohibit a $H_{1} \tilde{Q} \tilde{U}$ coupling, if $H_{2}$ were to be omitted then no up-quark mass could be generated. (The up-quark mass arises from the $H_{2} \tilde{Q} \tilde{U}$ coupling via eq. (B6) when $\mathrm{H}_{2}$ gains a vacuum expectation value.) With the possible exception of the t-quark, these Yukawa couplings are small (proportional to fermion masses) and we shall henceforth neglect them (except for the discussion at the end of appendix $\mathrm{C}$ ).

It is worthwhile to comment on the appearance of the $\mathrm{SU}(2) \times \mathrm{U}(1)$ singlet $N$. In principle, it is not required for a sensible low energy softly-broken supersymmetric theory. "However, it is required if one wants to construct an unbroken supersymmetric model which at tree level exhibits a unique ground state which breaks $\mathrm{SU}(2) \times \mathrm{U}(1)$ to $\mathrm{U}(1)$. Thus, it is useful to introduce this new singlet field for the purposes of illustrating this toy model although it is not required for phenomenology.

It is now straightforward to show that $S U(2) \times U(1)$ is broken to $U(1)$. The scalar potential is obtained using the superpotential given by eq. (B11) (setting $W_{\mathrm{F}}=0$ for convenience) and eqs. (B3), (B4), (B8), and (B9) (with $\xi=0$ ). In order to keep the number of terms from getting out of hand, we shall work out our results assuming only one generation of quarks and leptons. First, compute $F_{i}(i$ runs over all scalar fields) and $D_{a}, D^{\prime}$. When $W_{\mathrm{F}}=0$, the only nonzero $F$ terms (eq. (B3)) correspond to the Higgs scalars $H_{1}, H_{2}, N$. One finds

$$
\begin{aligned}
& F_{N}=h \varepsilon_{i j} H_{1}^{i} H_{2}^{j}+s, \\
& F_{H_{1}}=h \varepsilon_{i j} H_{2}^{j} N, \\
& F_{H_{2}}=h \varepsilon_{i j} H_{1}^{j} N .
\end{aligned}
$$

The $D$ terms (eqs. (B4), (B9)) are given by

$$
\begin{aligned}
& D^{a}=\frac{1}{2} g\left[H_{1}^{i *} \tau_{i j}^{a} H_{1}^{j}+H_{2}^{i *} \tau_{i j}^{a} H_{2}^{j}+\tilde{Q}^{i *} \tau_{i j}^{a} \tilde{Q}^{j}+\tilde{L}^{i *} \tau_{i j}^{a} \tilde{L^{j}}\right] \\
& D^{\prime}=\frac{1}{2} g^{\prime}\left[H_{2}^{i *} H_{2}^{i}-H_{1}^{i *} H_{1}^{i}+y_{Q} \tilde{Q}^{i *} \tilde{Q}^{i}+y_{\mathrm{u}} \tilde{U}^{*} \tilde{U}+y_{\mathrm{d}} \tilde{D}^{*} \tilde{D}-\tilde{L}^{i *} \tilde{L}^{i}+2 \tilde{R}^{*} \tilde{R}\right]
\end{aligned}
$$

For $\operatorname{SU}(2), T^{a}=\frac{1}{2} \tau^{a}$; the values for the hypercharges $y_{\mathrm{Q}}, y_{\mathrm{u}}$, and $y_{\mathrm{d}}$ are $1 / 3,-4 / 3$ and $2 / 3$ respectively. The scalar potential is computed using eq. (B8) by using the identity

$$
\tau_{i j}^{a} \tau_{k l}^{a}=2 \delta_{i l} \delta_{j k}-\delta_{i j} \delta_{k l}
$$

The result for $V$ is

\footnotetext{
* In fact, there are some technical arguments in the literature [A.10] which suggest that a light $\mathrm{SU}(2) \times \mathrm{U}(1)$ singlet field is incompatible if one wants to solve the hierarchy problem. However, these arguments have been recently disputed [A.40].
} 


$$
\begin{aligned}
V= & h^{2}\left(H_{1}^{i *} H_{1}^{i}+H_{2}^{i *} H_{2}^{i}\right) N^{*} N+\mid h \varepsilon_{i j} H_{1}^{i} H_{2}^{j}+s^{2}+\frac{1}{8} g^{2}\left[4\left|H_{1}^{i *} H_{2}^{i}\right|^{2}-2\left(H_{1}^{i *} H_{1}^{i}\right)\left(H_{2}^{i *} H_{2}^{i}\right)\right. \\
& +4\left|H_{1}^{i *} \tilde{Q}^{i}\right|^{2}-2\left(H_{1}^{i *} H_{1}^{i}\right)\left(\tilde{Q}^{i *} \tilde{Q}^{i}\right)+4\left|H_{1}^{i *} \tilde{L}^{i}\right|^{2}-2\left(H_{1}^{i *} H_{1}^{i}\right)\left(\tilde{L}^{i *} \tilde{L}^{i}\right) \\
& +4\left|H_{2}^{i *} \tilde{Q}^{i}\right|^{2}-2\left(H_{2}^{i *} H_{2}^{i}\right)\left(\tilde{Q}^{i *} \tilde{Q}^{i}\right)+4\left|H_{2}^{i *} \tilde{L}^{i}\right|^{2}-2\left(H_{2}^{i *} H_{2}^{i}\right)\left(\tilde{L}^{i *} \tilde{L}^{i}\right) \\
& \left.+4\left|\tilde{Q}^{i *} \tilde{L}^{i}\right|^{2}-2\left(\tilde{Q}^{i *} \tilde{Q}_{i}\right)\left(\tilde{L}^{i *} \tilde{L}^{i}\right)+\left(H_{1}^{i *} H_{1}^{i}\right)^{2}+\left(H_{2}^{i *} H_{2}^{i}\right)^{2}+\left(\tilde{Q}^{i *} \tilde{Q}^{i}\right)^{2}+\left(\tilde{L}^{i *} \tilde{L}^{i}\right)^{2}\right] \\
& +\frac{1}{8} g^{\prime 2}\left[H_{2}^{i *} H_{2}^{i}-H_{1}^{i *} H_{1}^{i}+y_{\mathrm{Q}} \tilde{Q}^{i *} \tilde{Q}^{i}+y_{\mathrm{u}} \tilde{U}^{*} \tilde{U}+y_{\mathrm{d}} \tilde{D}^{*} \tilde{D}-\tilde{L}^{i *} \tilde{L}^{i}+2 \tilde{R}^{i *} \tilde{R}^{i}\right]^{2}
\end{aligned}
$$

From eq. (B19), the vacuum state is easily obtained: The only scalar fields which acquire nonzero vacuum expectation values are

$$
\left\langle H_{1}\right\rangle=\frac{v}{\sqrt{2}}\left(\begin{array}{l}
1 \\
0
\end{array}\right), \quad\left\langle H_{2}\right\rangle=\frac{v}{\sqrt{2}}\left(\begin{array}{l}
0 \\
1
\end{array}\right),
$$

which breaks $\mathrm{SU}(2) \times \mathrm{U}(1)$ down to $\mathrm{U}(1)_{\mathrm{EM}}$. The constant $v$ is related to $h$ and $s$ of eq. (B11) by $\frac{1}{2} v^{2} h+s=0$. By inserting this solution back into eq. (B19), we see that at this minimum, $V=0$ thus implying that the theory remains supersymmetric [A.11].

The interaction Lagrangian for the $\mathrm{SU}(2) \times \mathrm{U}(1)$ gauge theory broken down to $\mathrm{U}(1)_{\mathrm{EM}}$ can now be obtained in a straightforward manner. We shall work in the unitary (physical) gauge which is obtained by replacing $H_{i}, i=1,2$ by $H_{i}+\left\langle H_{i}\right\rangle$. Some of the supersymmetric multiplets must get arranged (in a supersymmetric way) to reflect the fact that the $\mathrm{W}^{ \pm}$and $\mathrm{Z}^{0}$ gauge bosons get mass via the Higgs mechanism. The simplest way to see the results of this rearrangement is to first compute the fermion mass matrix which couples higgsinos and gauginos. This mass matrix arises because of the coupling $\lambda \psi A$ which occurs in eq. (B2); when $A$ gains a vacuum expectation value, this results in a fermion mass term. We illustrate this step with one part of the calculation. Consider the following piece of the $\lambda \psi A$ terms:

$$
\frac{\mathrm{i} g}{\sqrt{2}} \tau_{i j}^{a} \lambda^{a} \psi_{H_{1}}^{j} H_{1}^{i^{*}}-\frac{\mathrm{i} g^{\prime}}{\sqrt{2}} \lambda^{\prime} \delta_{i j} \psi_{H_{1}}^{j} H_{1}^{i^{*}}+\text { h.c. }
$$

Inserting eq. (B20) in for $H_{1}^{j}$ (i.e. $H_{1}^{i}=(v / \sqrt{2}) \delta_{1 j}$ ), we concentrate on the $a=1,2$ part of eq. (B21). Redefining $\lambda^{ \pm}=(1 / \sqrt{2})\left(\lambda^{1} \mp i \lambda^{2}\right)$, we find

$$
\mathscr{L}_{\text {mass }}=(\mathrm{i} v g / \sqrt{2}) \lambda^{+} \psi_{H_{1}}^{2}+\text { h.c. }
$$

To see that this is indeed a mass term, let us translate this into four-component language. Using eq. (A19), it follows that eq. (B22) is equal to

$$
\mathscr{L}_{\text {mass }}=-m_{\mathrm{w}} \overline{\bar{\omega}}_{1} \tilde{\omega}_{1}, \quad \tilde{\omega}_{1}=\left(\begin{array}{l}
\psi_{H_{1}}^{2} \\
\mathbf{i} \bar{\lambda}^{+}
\end{array}\right)
$$

where $m_{\mathrm{w}}=g v / \sqrt{2}$ is the W-boson mass, which is recognized by computing the vector boson mass matrix. We have thus identified the physical fermion mass eigenstate - it is the fermionic partner of the $\mathrm{W}^{-}$and degenerate in mass as required by supersymmetry (which at this point remains unbroken). Continuing in this fashion for the rest of the terms, we find the following fermion mass terms*;

\footnotetext{
${ }^{*}$ Our conventions listed in table 4 imply that all supersymmetric partners have a ( $)$. We apply this convention to four-component fermions only. In appendix B, as a general rule, two-component fermions will be denoted by either $\psi$ or $\lambda$ with no ( )
} 


$$
\begin{aligned}
\mathscr{L}_{\text {mass }}= & -\mathrm{i} v\left[\left(g^{2}+g^{\prime 2}\right) / 2\right]^{1 / 2} \lambda_{\mathrm{z}}\left(\psi_{H_{2}}^{2}-\psi_{H_{1}}^{1}\right) / \sqrt{2}+v h \psi_{N}\left(\psi_{H_{1}}^{1}+\psi_{H_{2}}^{2}\right) / \sqrt{2} \\
& +(\mathrm{i} v g / \sqrt{2})\left(\lambda^{-} \psi_{H_{2}}^{1}+\lambda^{+} \psi_{H_{1}}^{2}\right)+\text { h.c. }
\end{aligned}
$$

where $\lambda^{ \pm}=(1 / \sqrt{2})\left(\lambda^{1} \mp \mathrm{i} \lambda^{2}\right), \lambda_{\mathrm{z}}=\left(g \lambda^{3}-g^{\prime} \lambda^{\prime}\right) / \sqrt{g^{2}+g^{\prime 2}}$ and the superscripts 1,2 are $\mathrm{SU}(2)_{\mathrm{W}}$ indices. By computing the vector-boson mass matrix, one learns that $m_{\mathrm{w}}^{2}=\frac{1}{2} g^{2} v^{2}$ and $m_{\mathrm{z}}^{2}=\frac{1}{2}\left(g^{2}+g^{\prime 2}\right) v^{2}$. We may now convert the two-component notation of eq. (B24) into four-component notation by using eq. (A19). This allows us to identify the following four-component Dirac fermions which exist in the spectrum:

$$
\begin{array}{ll}
\tilde{\omega}_{1}^{-}=\left(\begin{array}{l}
\psi_{H_{1}}^{2} \\
\overline{\mathrm{i}} \bar{\lambda}^{+}
\end{array}\right), \quad \tilde{\omega}_{2}^{+}=\left(\begin{array}{l}
\psi_{H_{2}}^{1} \\
\mathrm{i} \bar{\lambda}^{-}
\end{array}\right), & \text {with mass } m_{\mathrm{w}}, \\
\tilde{\zeta}=\left(\begin{array}{c}
\left(\psi_{H_{1}}^{1}-\psi_{H_{2}}^{2}\right) / \sqrt{2} \\
\mathrm{i} \bar{\lambda}_{\mathrm{z}}
\end{array}\right), & \text { with mass } m_{\mathrm{z}}, \\
\tilde{h}=\left(\begin{array}{c}
\left(\psi_{H_{1}}^{1}+\psi_{H_{2}}^{2}\right) / \sqrt{2} \\
\bar{\psi}_{N}
\end{array}\right), & \text { with mass } m_{\mathrm{h}} \equiv h v .
\end{array}
$$

Finally, there is a photino $\lambda_{\gamma}=\left(g^{\prime} \lambda^{3}+g \lambda^{\prime}\right) / \sqrt{g^{2}+g^{\prime 2}}$ which is the orthogonal state to $\lambda_{z}$. No term of the form $\lambda_{\gamma} \lambda_{\gamma}$ appears in eq. (B24) implying that the photino remains massless. In four-component notation, it is expressed as a Majorana fermion:*

$$
\tilde{\gamma}=\left(\begin{array}{c}
-\mathrm{i} \lambda_{\gamma} \\
\mathrm{i} \bar{\lambda}_{\gamma}
\end{array}\right)
$$

The final step is to compute the scalar masses. By shifting $H_{i}(i=1,2)$ by their vacuum expectation values in eq. (B19) and identifying the quadratic pieces, we may compute the masses of the physical Higgs bosons. First consider the terms quadratic in the charged fields, $H_{1}^{2}$ and $H_{2}^{1}$. Letting $H_{1}^{1} \rightarrow$ $H_{1}^{1}+\dot{v} / \sqrt{2}$ and $H_{2}^{2} \rightarrow H_{2}^{2}+v / \sqrt{2}$ in eq. (B19), we find that the terms in $V$ quadratic in $H_{1}^{2}$ and $H_{2}^{1}$ are

$$
-\mathscr{L}_{\text {mass }}=\frac{1}{4} g^{2} v^{2}\left[\left|H_{1}^{2}\right|^{2}+\left|H_{2}^{1}\right|^{2}+2 \operatorname{Re} H_{1}^{2} H_{2}^{1 *}\right]
$$

If we define $H^{+}=\left(H_{2}^{1}+H_{1}^{2 *}\right) \sqrt{2}, G^{+}=\left(H_{2}^{1}-H_{1}^{2 *}\right) / \sqrt{2}, H^{-}=\left(H^{+}\right)^{*}$ and $G^{-}=\left(G^{+}\right)^{*}$, then eq. (B29) can be written as

$$
-\mathscr{L}_{\text {mass }}=m_{\mathrm{w}}^{2}\left|H^{+}\right|^{2}
$$

Equation (B30) reflects the existence of a complex charged Higgs scalar $\mathrm{H}^{+}$(and its antiparticle $\mathrm{H}^{-}$) with mass $m_{\mathrm{w}}$. That is, the Higgs mechanism plus supersymmetry has generated a multiplet of particles $\left(H^{ \pm}, \tilde{\omega}_{1}, \tilde{\omega}_{2}, W^{ \pm}\right)$which are degenerate in mass. Note that no mass term appeared for $G^{ \pm}$, the states orthogonal to $H^{ \pm}$. These states are simply the charged Goldstone bosons which are eaten by the $W^{ \pm}$ gauge bosons thereby giving mass to the $W^{ \pm}$. The remaining pieces in $V$ which are quadratic in the

\footnotetext{
* The factors of $\mathrm{i}$ which occur in eqs. (B25)-(B28) are artifacts of the convention used by Wess and Bagger [A.1] to define the two-component gaugino field. Note that if $\eta=-\mathrm{i} \lambda^{+}$, then $\bar{\eta}=+\mathrm{i} \bar{\lambda}^{+}$, etc.
} 
remaining scalar fields may be analyzed in a similar fashion. We simply quote the results. The complete list of physical massive Higgs scalars is as follows:

$$
\begin{aligned}
& H^{+}=(1 / \sqrt{2})\left(H_{2}^{1}+H_{1}^{2 *}\right) \quad \text { with mass } m_{\mathrm{w}}, \quad H^{0}=\operatorname{Re}\left(H_{2}^{2}-H_{1}^{1}\right) \quad \text { with mass } m_{\mathrm{z}}, \\
& h_{1}^{0}=\operatorname{Re}\left(H_{1}^{1}+H_{2}^{2}\right), \quad h_{2}^{0}=\operatorname{Im}\left(H_{2}^{2}+H_{1}^{1}\right), \quad h_{3}^{0}=\sqrt{2} \operatorname{Re} N, \quad h_{4}^{0}=\sqrt{2} \operatorname{Im} N, \quad \text { with mass } m_{\mathrm{h}},
\end{aligned}
$$

where $m_{\mathrm{h}}=h v$ (the parameter $h$ appears in the potential, see. eq. (B19)). Of the massless Higgs states, we identify $G^{0}=\operatorname{Im}\left(H_{2}^{2}-H_{1}^{1}\right)$ as the neutral Goldstone boson which gives mass to the $Z^{0}$. The remaining massless scalars are the scalar-quarks and scalar-leptons. Because we set $W_{\mathrm{F}}=0$ in eq. (B11), the quarks and leptons remain massless; the supersymmetry insures that their scalar partners are also massless.

We summarize the new supersymmetric multiplets as follows. One massless gauge multiplet $(\tilde{\gamma}, \gamma)$ consisting of a Majorana fermion (the photino) and the photon remains unchanged. Some matter and gauge multiplets have combined leading to new massive multiplets $\left(H^{-}, \tilde{\omega}_{1}^{-}, W^{-}\right),\left(H^{+}, \tilde{\omega}_{2}^{+}, W^{+}\right)$and $\left(H^{\mathrm{o}}, \tilde{\zeta}^{0}, Z^{0}\right)$. Each multiplet consists of one real scalar field, one Dirac fermion and one gauge field all with the same mass and charge. Finally, there is a massive multiplet $\left(h_{i}, \tilde{h}\right)(i=1, \ldots, 4)$ consisting of two scalars, two pseudoscalars and one Dirac fermion. Note that each multiplet has equal numbers of fermionic and bosonic degrees of freedom.

The fermionic spectrum described above consists of some Majorana fermions $\tilde{\gamma}$ which have two degrees of freedom and some Dirac fermions $\tilde{\omega}_{1}^{-}, \tilde{\omega}_{2}^{+}$and $\tilde{\zeta}^{0}$ each of which have four degrees of freedom. It is important to note that Majorana fermions cannot carry any conserved additive quantum number. This is why the charged fermions had to combine into four-component Dirac spinors, $\tilde{\omega}_{1}^{-}$and $\tilde{\omega}_{2}^{+}$. On the other hand, the existence of the four component Dirac spinor $\tilde{\zeta}^{0}$ is special to the model considered so far. Hence, it will be useful to rewrite $\tilde{\zeta}^{0}$ in terms of the two degenerate Majorana fermions. We will find later that the masses of these two fermions will be different in more general circumstances, for example, when an explicit Majorana gaugino mass term is added.

Let us define (in terms of two-component fields):

$$
\begin{aligned}
& \psi_{1}=\left(-\mathrm{i} \lambda_{\mathrm{z}}+\psi\right) / \sqrt{2} \\
& \mathrm{i} \psi_{2}=\left(-\mathrm{i} \lambda_{\mathrm{z}}-\psi\right) / \sqrt{2}
\end{aligned}
$$

where

$$
\psi \equiv\left(\psi_{H_{1}}^{1}-\psi_{H_{2}}^{2}\right) / \sqrt{2}
$$

It then follows from eq. (B24) that the terms which depend on $\lambda_{z}$ may be written as

$$
\mathscr{L}_{\text {mass }}=m_{\mathrm{z}}\left(\mathrm{i} \lambda_{\mathbf{z}} \psi-\mathrm{i} \bar{\lambda}_{\mathbf{z}} \bar{\psi}\right)=-\frac{1}{2} m_{\mathbf{z}}\left(\psi_{1} \psi_{1}+\bar{\psi}_{1} \bar{\psi}_{1}+\psi_{2} \psi_{2}+\bar{\psi}_{2} \bar{\psi}_{2}\right)
$$

Note the factor of $i$ on the left-hand side of eq. (B32); this has been chosen to insure that the fermion mass eigenvalue is positive. If we define two Majorana spinors $\tilde{\zeta}_{1}$ and $\tilde{\zeta}_{2}$ : 


$$
\tilde{\zeta}_{1}=\left(\begin{array}{l}
\psi_{1} \\
\bar{\psi}_{1}
\end{array}\right), \quad \tilde{\zeta}_{2}=\left(\begin{array}{l}
\psi_{2} \\
\bar{\psi}_{2}
\end{array}\right),
$$

then, by using eq. (A19), we find that eq. (B34) in four-component language is equal to

$$
\mathscr{L}_{\text {mass }}=-\frac{1}{2} m_{z}\left(\tilde{\tilde{\zeta}}_{1} \tilde{\zeta}_{1}+\overline{\tilde{\zeta}}_{2} \tilde{\zeta}_{2}\right)
$$

In order to obtain the Feynman rules for the interactions of this model, one needs to proceed with the following steps.

(a) Start with all interactions listed in eqs. (B1)-(B9).

(b) Shift the scalar fields $H_{i} \rightarrow H_{i}+\left\langle H_{i}\right\rangle, i=1,2$.

(c) Insert the correct definitions of the physical particles (i.e. the mass eigenstates) given in part by eqs. (B25)-(B28), (B31) and (B35).

The last step will also involve switching from two-component to four-component notation. In this regard, eqs. (A19)-(A28) will be extremely useful.

To illustrate the above procedure, we shall work out the interactions of photinos with scalar leptons and leptons. Equation (B2) contains the relevant interaction term in two-component notation. The one we are interested in is:

$$
(\mathrm{i} / \sqrt{2})\left(g \tau_{i j}^{a} \lambda^{a}-g^{\prime} \delta_{i j} \lambda^{\prime}\right) \psi_{L}^{j} L_{i}^{*}+(2 i / \sqrt{2}) g^{\prime} \lambda^{\prime} \psi_{\mathrm{R}} R^{*}+\text { h.c. },
$$

where $\psi_{\mathrm{L}}^{j}$ is the weak $\left(\begin{array}{c}v \\ e\end{array}\right)$ doublet of two-component fields, $\psi_{\mathrm{R}}$ is the two-component $e^{+}$field, $L_{i}$ is the scalar-lepton doublet $\left(\tilde{\tilde{e}}_{\tilde{\mathrm{L}}}^{\tilde{\mathrm{L}}}\right)$ and $R$ is $\tilde{e}_{\mathrm{R}}^{+}$. For the present discussion we need only consider the $a=3$ piece of $\tau_{i j}^{a} \lambda^{a}$. If we express $\lambda^{3}$ and $\lambda^{\prime}$ in terms of $\lambda_{z}$ and $\lambda_{y}$ we find

$$
\begin{aligned}
& g \tau_{i j}^{3} \lambda^{3}-g^{\prime} \delta_{i j} \lambda^{\prime}=g\left(\begin{array}{cc}
\lambda_{z} / \cos \theta_{w} & 0 \\
0 & \left(-\cos 2 \theta_{w} / \cos \theta_{w}\right) \lambda_{z}-2 \sin \theta_{w} \lambda_{y}
\end{array}\right) \\
& \lambda^{\prime}=-\sin \theta_{w} \lambda_{z}+\cos \theta_{w} \lambda_{\gamma} .
\end{aligned}
$$

Thus, using $g \sin \theta_{\mathrm{w}}=g^{\prime} \cos \theta_{\mathrm{w}}$, the photino interaction terms become

$$
\sqrt{2} g \sin \theta_{\mathrm{w}}\left(-\mathrm{i} \lambda_{\gamma}\right)\left[\psi_{\mathrm{L}}^{2} L_{2}^{*}-\psi_{\mathrm{R}} R^{*}\right]+\text { h.c. }
$$

We now convert to four-component notation. The photino spinor is given by eq. (B28) and the electron spinor is denoted by

$$
e=\left(\begin{array}{l}
\psi_{\mathrm{L}}^{2} \\
\overline{\psi_{\mathrm{R}}}
\end{array}\right)
$$

Using eq. (A28), we see that eq. (B40) can be written as

$$
\sqrt{2} g \sin \theta_{\mathrm{w}}\left[\overline{\tilde{\gamma}} P_{\mathrm{L}} e L_{2}^{*}-\bar{e} P_{\mathrm{L}} \tilde{\gamma} R^{*}\right]+\text { h.c. }
$$


Because $e=g \sin \theta_{\mathrm{w}}>0$ is the electric charge, we note that the minus sign between the two terms of eq. (B42) arises from the fact that $L_{2}^{*}$ creates a negatively charged $\tilde{e}_{\mathrm{L}}^{-}$whereas $R^{*}$ creates a positively charged $\tilde{e}_{\mathrm{R}}^{+}$. This is somewhat inconvenient so we shall henceforth define:

$$
\tilde{e}_{\mathrm{L}}^{*}=L_{2}^{*}, \quad \tilde{e}_{\mathrm{R}}^{* *}=R,
$$

where now $\tilde{e}_{\mathrm{L}}^{*}$ and $\tilde{e}_{\mathrm{R}}^{*}$ both create negative charge. The interaction eq. (B42) written out in full becomes

$$
\sqrt{2} g \sin \theta_{\mathrm{w}}\left[\overline{\bar{\gamma}} P_{\mathrm{L}} e \tilde{e}_{\mathrm{L}}^{*}+\bar{e} P_{\mathrm{R}} \tilde{\gamma} \tilde{e}_{\mathrm{L}}-\overline{\tilde{\gamma}} P_{\mathrm{R}} e \tilde{e}_{\mathrm{R}}^{*}-\bar{e} P_{\mathrm{L}} \tilde{\gamma} \tilde{e}_{\mathrm{R}}\right]
$$

In appendix $\mathrm{C}$, we explicitly write out the Lagrangian and derive the Feynman rules.

\section{Appendix C. The Lagrangian and Feynman rules}

In appendix B, we outlined the steps needed in building an interaction Lagrangian for an SU(2) $\times U(1)$ model of broken supersymmetry. We then provided as an example a supersymmetric $S U(2) \times U(1)$ model where the supersymmetry remained unbroken but the $S U(2) \times U(1)$ gauge symmetry was broken to $\mathrm{U}(1)_{\mathrm{EM}}$.

We now generalize those results so that they may be applied to a larger class of realistic models. We do this in a number of ways:

(a) We shall not require that the superpotential have the form specified in eq. (B11). In particular, we will omit the $\mathrm{SU}(2) \times \mathrm{U}(1)$ singlet fields $\left(N, \psi_{\mathrm{N}}\right)$. This will allow us to focus on the minimal set of particles required for the supersymmetric extension of $\mathrm{SU}(2) \times \mathrm{U}(1)$. In addition, it will allow a more general set of vacuum expectation values than that of eq. (B20). We shall allow for

$$
\left\langle H_{1}\right\rangle=\frac{v_{1}}{\sqrt{2}}\left(\begin{array}{l}
1 \\
0
\end{array}\right), \quad\left\langle H_{2}\right\rangle=\frac{v_{2}}{\sqrt{2}}\left(\begin{array}{l}
0 \\
1
\end{array}\right),
$$

where $v_{1} \neq 2$. (We may choose the phases of $H_{1}$ and $H_{2}$ so that the $V_{i}$ are real.)

(b) The supersymmetry will be broken explicitly by terms of the form of eq. (B10). This will require us to recompute the physical fields (mass eigenstates) of the model. The results of eqs. (B25)-(B28) and eq. (B31) will therefore change for this reason (as well as changing because we are omitting $\psi_{\mathrm{N}}$ from the model).

The analysis in this section will not be completely general in that we only consider the minimal number of Higgs multiplets. In addition, because we do not specify the superpotential of the model, we shall have to omit discussing some of the interaction terms which would arise from it.

The plan of this appendix is as follows. We first discuss how various states of the theory mix and how the mass eigenstates are obtained. We illustrate both the mixing of scalar-leptons (or quarks) and the mixing of gauginos and higgsinos. Then, we write down a general interaction Lagrangian for the class of models considered. From it, we derive a set of Feynman rules. (One complication involving Feynman rules of Majorana fermions is discussed in appendix D.)

\section{Mixing of scalar-leptons (or quarks)}

There are two (complex) scalar-leptons (denoted $\tilde{\ell}_{\mathrm{L}}$ and $\tilde{\ell}_{\mathrm{R}}$ ) which are the supersymmetric partners of 
the left and right helicity parts of the four component fermion $\ell$. Before supersymmetry breaking, they are degenerate in mass with $\ell$.

If we allow for the soft symmetry breaking terms proportional to $m_{1}$ and $m_{2}$ in eq. (B10), then we have two effects: the generation of diagonal mass terms for $\tilde{\ell}_{\mathrm{L}}$ and $\tilde{\ell}_{\mathrm{R}}$ different from $m_{\ell}$ (as well as different from each other), and the mixing of $\tilde{\ell}_{\mathrm{L}}$ and $\tilde{\ell}_{\mathrm{R}}$ through nondiagonal mass terms. It is convenient to parameterize the scalar-lepton mass matrix as follows [A.12]:

$$
\left(\tilde{\ell}_{\mathrm{L}}, \tilde{\ell}_{\mathrm{R}}\right)\left(\begin{array}{cc}
L^{2} \tilde{m}^{2}+m_{\ell}^{2} & A \tilde{m} m_{\ell} \\
A \tilde{m} m_{\ell} & R^{2} \tilde{m}^{2}+m_{\ell}^{2}
\end{array}\right)\left(\begin{array}{c}
\tilde{\ell}_{\mathrm{L}} \\
\tilde{\ell}_{\mathrm{R}}
\end{array}\right)
$$

where $\tilde{m}$ is some mass parameter and $L, R$, and $A$ are dimensionless constants. This form is suggested by low-energy supergravity models, but it is completely general (if $m_{\ell}=0$, simply write $A m_{\ell}=A^{\prime}$ where $A^{\prime}$ is a new constant). The mass eigenstates are easily obtained by diagonalization. The resulting states are

$$
\tilde{\ell}_{1}=\tilde{\ell}_{\mathrm{L}} \cos \theta+\tilde{\ell}_{\mathrm{R}} \sin \theta, \quad \tilde{\ell}_{2}=-\tilde{\ell}_{\mathrm{L}} \sin \theta+\tilde{\ell}_{\mathrm{R}} \cos \theta,
$$

where

$$
\tan 2 \theta=2 A m_{\ell} /\left(L^{2}-R^{2}\right) \tilde{m},
$$

with respective masses

$$
\tilde{M}_{\ell_{1,2}}^{2}=m_{\ell}^{2}+\frac{1}{2}\left[\left(L^{2}+R^{2}\right) \tilde{m}^{2} \pm\left[\left(L^{2}-R^{2}\right)^{2} \tilde{m}^{4}+4 A^{2} m_{\ell}^{2} \tilde{m}^{2}\right]^{1 / 2}\right] .
$$

The effects of the mixing on interactions can be illustrated in the following simple model. Consider supersymmetric QED where the supersymmetry is broken solely due to the scalar-lepton mass matrix given by eq. (C2). Thus, the photon and photino remain massless. From eq. (B44), we may write the $\tilde{\gamma}-\tilde{\ell}-\ell$ interaction as:

$$
\sqrt{2} e\left[\bar{\ell} P_{\mathbf{R}} \tilde{\gamma} \tilde{\ell}_{\mathbf{L}}-\bar{\ell} P_{\mathbf{L}} \tilde{\gamma} \tilde{\ell}_{\mathbf{R}}\right]+\text { h.c. }
$$

in terms of four-component spinors $\ell$ and $\tilde{\gamma}$ and the left- and right-handed projection operators $P_{\mathrm{R}, \mathrm{L}}=\frac{1}{2}\left(1 \pm \gamma_{5}\right)$. Substituting in eq. (C3) gives the interaction in terms of mass eigenstates. Note from eq. (C5) that, in general, parity is violated because $\tilde{\ell}_{1}$ and $\tilde{\ell}_{2}$ (which usually have different masses) have both scalar and pseudoscalar couplings to leptons and photinos. However, parity is violated if and only if $L \neq R$. If $L=R$, it follows from eq. (C3b) that $\theta=45^{\circ}$ and the two mass eigenstates [eq. (C3)] are $\left(\tilde{\ell}_{\mathrm{R}} \pm \tilde{\ell}_{\mathrm{L}}\right) / \sqrt{2}$. Using eq. (C5), these combinations are pseudoscalar and scalar bosons, respectively.

The above discussion holds for all flavors of leptons and quarks. Note that a priori the mass matrix parameters $L, R, A$ and $\tilde{m}$ are different for each quark or lepton flavor. In addition, the lack of observed parity violation in the strong and electromagnetic interactions imposes certain restrictions on the values of $\tilde{M}_{\ell_{1}}^{2}, \tilde{M}_{\ell_{2}}^{2}, L$ and $R$. These restrictions are discussed in section 8.3 .

\section{Mixing of charged gauginos and higgsinos*}

We saw in appendix $B$ that in the supersymmetric $S U(2) \times U(1)$ model (where the gauge symmetry

\footnotetext{
${ }^{*}$ Chargino mixing has been discussed in refs. [A.13-A.21]. Here, we follow closely the techniques discussed in ref. [A.19].
} 
breaks to $\mathrm{U}(1)_{\mathrm{EM}}$ ), there are two four-component fermions degenerate with the $\mathrm{W}$ bosons [see eq. (B25)]. These fermions are made up of gauginos and higgsinos which mix as in eq. (B24). The mixing matrix becomes more complicated when the supersymmetry is broken [say, via soft supersymmetry breaking terms given in eq. (B10)]. The possible mass terms in two-component notation are as follows:

$$
(\mathrm{ig} / \sqrt{2})\left[v_{1} \lambda^{+} \psi_{H_{1}}^{2}+v_{2} \lambda^{-} \psi_{H_{2}}^{1}\right]+M \lambda^{+} \lambda^{-}-\mu \psi_{H_{1}}^{2} \psi_{H_{2}}^{1}+\text { h.c. }
$$

Comparing with eq. (B22), the following differences are noteworthy. First for superpotentials more general than given in eq. (B.11), we can have the term in eq. (C6) proportional to $\mu$. This would arise from applying eq. (B6) to an additional supersymmetric term of the form $W=\mu \varepsilon_{i j} H_{1}^{i} H_{2}^{j}$. Second, due to a more general superpotential and additional soft-supersymmetry breaking terms, the minimum of the scalar potential is assumed to be of the form specified by eq. (C1) with $v_{1} \neq v_{2}$. Finally, among the possible soft-supersymmetry breaking terms is a Majorana mass term for the gaugino [eq. (B10) with $\tilde{M}_{3} \equiv M$; note that $\left.2 \lambda^{+} \lambda^{-}=\lambda^{1} \lambda^{1}+\lambda^{2} \lambda^{2}\right]$.

We rewrite eq. (C6) as follows. Let

$$
\psi_{j}^{+}=\left(-\mathrm{i} \lambda^{+}, \psi_{H_{2}}^{1}\right), \quad \psi_{j}^{-}=\left(-\mathrm{i} \lambda^{-}, \psi_{H_{1}}^{2}\right), \quad j=1,2
$$

Equation (C6) then takes the form

$$
-\frac{1}{2}\left(\psi^{+} \psi^{-}\right)\left(\begin{array}{cc}
0 & X^{\mathrm{T}} \\
X & 0
\end{array}\right)\left(\begin{array}{l}
\psi^{+} \\
\psi^{-}
\end{array}\right)+\text {h.c. }
$$

where

$$
X=\left(\begin{array}{cc}
M & m_{\mathrm{w}} \sqrt{2} \cos \theta_{\mathrm{v}} \\
m_{\mathrm{w}} \sqrt{2} \sin \theta_{\mathrm{v}} & \mu
\end{array}\right),
$$

and

$$
\begin{aligned}
& m_{\mathrm{w}}^{2} \equiv \frac{1}{4} g^{2}\left(v_{1}^{2}+v_{2}^{2}\right) \\
& \tan \theta_{\mathrm{v}} \equiv v_{1} / v_{2}
\end{aligned}
$$

Let the mass eigenstates be defined as

$$
\chi_{i}^{+}=V_{i j} \psi_{j}^{+}, \quad \chi_{i}^{-}=U_{i j} \psi_{j}^{-}, \quad i=1,2,
$$

where $V$ and $U$ are unitary matrices chosen such that

$$
U^{*} X V^{-1}=M_{\mathrm{D}}
$$

where $M_{\mathrm{D}}$ is a diagonal matrix with nonnegative entries. Equation (C8) can then be written as [note eq. (A24)]

$$
-\left(\chi_{i}\left(M_{\mathrm{D}}\right)_{i j} \chi_{j}^{+}+\text {h.c. }\right) \text {. }
$$


Using eq. (A19), we may write eq. (C14) in four-component notation as

$$
-\left(\tilde{M}_{+} \overline{\bar{\chi}}_{1} \tilde{\chi}_{1}+\tilde{M}_{-} \overline{\tilde{\chi}}_{2} \tilde{X}_{2}\right)
$$

where $\tilde{\chi}_{1}$ and $\tilde{\chi}_{2}$ are charged four-component Dirac spinors:

$$
\tilde{\chi}_{1}=\left(\begin{array}{c}
\chi_{1}^{+} \\
\bar{\chi}_{1}^{-}
\end{array}\right), \quad \tilde{\chi}_{2}=\left(\begin{array}{c}
\chi_{2}^{+} \\
\bar{\chi}_{2}^{\frac{1}{2}}
\end{array}\right) \text {. }
$$

By convention, we choose $\tilde{x}_{1}$ to be heavier than $\tilde{x}_{2}$, i.e. $\tilde{M}_{+}>\tilde{M}_{-}$. It remains to work out expressions for the masses $\tilde{M}_{+}$and $\tilde{M}_{-}$and the matrices $U$ and $V$.

Note that the eigenvalues of $X$ can be either positive or negative whereas we require $M_{\mathrm{D}}$ to contain only nonnegative entries. Therefore, it is useful to consider the eigenvalue problem for $X^{+} X$. The positive square roots of the eigenvalues of $X^{\dagger} X$ will be the diagonal entries of $M_{\mathrm{D}}$. From eq. (C14), we see that

$$
M_{\mathrm{D}}^{2}=V X^{+} X V^{-1}=U^{*} X X^{\dagger}\left(U^{*}\right)^{-1}
$$

Thus, the diagonalizing matrices $U^{*}$ and $V$ can easily be obtained by computing the eigenvectors corresponding to the eigenvalues of $X^{\dagger} X$ and $X X^{\dagger}$ respectively. Note that at this stage, $U$ and $V^{*}$ are not unique. This just reflects the fact that certain arbitrary phases can be absorbed into the definition of the physical fields. In addition, it is useful to choose $U^{*}$ and $V$ [using eq. (C13)] such that $M_{\mathrm{D}}$ has no nonnegative entries.

Because we are dealing with $2 \times 2$ matrices here, exact expressions can be obtained for the masses and mixing matrices. For simplicity we will assume that the parameters $M$ and $\mu$ are real. We find

$$
\begin{aligned}
& \tilde{M}_{ \pm}^{2}=\frac{1}{2}\left\{M^{2}+\mu^{2}+2 m_{\mathrm{w}}^{2} \pm\left[\left(M^{2}-\mu^{2}\right)^{2}+4 m_{\mathrm{w}}^{4} \cos ^{2} 2 \theta_{\mathrm{v}}+4 m_{\mathrm{w}}^{2}\left(M^{2}+\mu^{2}+2 M \mu \sin 2 \theta_{\mathrm{v}}\right)\right]^{1 / 2}\right\}, \\
& U=O_{-}, \quad V=\left\{\begin{array}{ll}
O_{+}, & \operatorname{det} X \geq 0 \\
\sigma_{3} O_{+}, & \operatorname{det} X<0
\end{array}, \quad O_{ \pm}=\left(\begin{array}{cc}
\cos \phi_{ \pm} & \sin \phi_{ \pm} \\
-\sin \phi_{ \pm} & \cos \phi_{ \pm}
\end{array}\right),\right.
\end{aligned}
$$

where the Pauli matrix $\sigma_{3}$ is inserted when det $X<0$, so that the masses $M_{ \pm}$which appear as diagonal elements of $M_{\mathrm{D}}$ (eq. (C.13)) are both positive. The angles $\phi_{ \pm}$are given by

$$
\begin{aligned}
& \tan 2 \phi_{-}=2 \sqrt{2} m_{\mathrm{w}}\left(\mu \cos \theta_{\mathrm{v}}+M \sin \theta_{\mathrm{v}}\right) /\left(M^{2}-\mu^{2}+2 m_{\mathrm{w}}^{2} \cos 2 \theta_{\mathrm{v}}\right), \\
& \tan 2 \phi_{+}=2 \sqrt{2} m_{\mathrm{w}}\left(\mu \sin \theta_{\mathrm{v}}+M \cos \theta_{\mathrm{v}}\right) /\left(M^{2}-\mu^{2}-2 m_{\mathrm{w}}^{2} \cos 2 \theta_{\mathrm{v}}\right) .
\end{aligned}
$$

Further formulas can be found in ref. [A.44].

To get a feeling for these results we consider a simple case where $\mu=0$ and $v_{1}=v_{2}$ (i.e., $\theta_{\mathrm{v}}=45^{\circ}$ ). Then $\phi_{+}=\phi_{-} \equiv \phi$ and we obtain

$$
\begin{aligned}
& \tilde{M}_{ \pm}=\left[m_{\mathrm{w}}^{2}+\frac{1}{4} M^{2}\right]^{1 / 2} \pm \frac{1}{2} M, \\
& \cos \phi=\left(\frac{\tilde{M}_{+}}{\tilde{M}_{+}+\tilde{M}_{-}}\right)^{1 / 2}
\end{aligned}
$$


The physical eigenstates are the Dirac spinors

$$
\begin{aligned}
& \tilde{\chi}_{1}=\left(\begin{array}{c}
-\mathrm{i} \lambda^{+} \cos \phi+\psi_{H_{2}}^{1} \sin \phi \\
\mathrm{i} \bar{\lambda}^{-} \cos \phi+\bar{\psi}_{H_{1}}^{2} \sin \phi
\end{array}\right), \quad \text { mass }=\tilde{M}_{+}, \\
& \tilde{\chi}_{2}=\left(\begin{array}{c}
-\mathrm{i} \lambda^{+} \sin \phi-\psi_{H_{2}}^{1} \cos \phi \\
-\mathrm{i} \bar{\lambda}^{-} \sin \phi+\psi_{H_{1}}^{2} \cos \phi
\end{array}\right), \quad \text { mass }=\tilde{M}_{-} .
\end{aligned}
$$

The question now arises: How is the supersymmetric limit reached as $M \rightarrow 0$ (recall that $\mu=0$ and $v_{1}=v_{2}$ in this example so that $M \rightarrow 0$ should bring us back to eq. (B25)). From eqs. (C22) and (C23) $M=0$ implies two degenerate states with mass $m_{\mathrm{w}}$ and $\phi=45^{\circ}$. Thus, in this limit, the connection with eq. (B25) is

$$
\tilde{\omega}_{1}^{-}=(1 / \sqrt{2})\left(\tilde{\chi}_{2}^{\mathrm{c}}+\tilde{\chi}_{1}^{\mathrm{c}}\right), \quad \tilde{\omega}_{2}^{+}=(1 / \sqrt{2})\left(\tilde{\chi}_{1}-\tilde{\chi}_{2}\right)
$$

[the $\mathrm{c}$ denotes charge conjugation as in eq. (A12)].

Let us now investigate how the Feynman rules are computed for the physical states. Consider the interaction terms $\tilde{\chi}^{ \pm} e \tilde{\nu}$ and $\tilde{\chi}^{ \pm} \tilde{e}_{\mathrm{L}} \nu$ [which correspond to the $\lambda \psi A$ term of eq. (B2)]. These are the supersymmetric versions of the usual $\mathrm{W}^{ \pm} \mathrm{e} \nu$ interaction. Note that in the limit where the fermion masses are neglected, the $\mathrm{H}^{ \pm} \mathrm{e} \nu$ interaction is absent, as are the supersymmetric versions, $\psi_{\mathrm{H}} \mathrm{e} \nu$ and $\psi_{\mathrm{H}} \mathrm{e} \tilde{\nu}$. Thus, we need only focus on the term (in two-component notation)

$$
\operatorname{ig}\left[\lambda^{-} \nu_{\mathrm{L}} \tilde{e}_{\mathrm{L}}^{*}-\bar{\lambda}^{-} \bar{\nu}_{\mathrm{L}} \tilde{e}_{\mathrm{L}}+\lambda^{+} e_{\mathrm{L}} \tilde{\nu}^{*}-\bar{\lambda}^{+} \bar{e}_{\mathrm{L}} \tilde{\nu}\right]
$$

This has been obtained from eq. (B2) where we have put $\lambda^{ \pm}=(1 / \sqrt{2})\left(\lambda^{1} \mp i \lambda^{2}\right)$. The notation of eq. (A11) is used for the electron and neutrino spinors. We shall write eq. (C26) in four-component notation as follows. Define the weak interaction eigenstates

$$
\tilde{W}=\left(\begin{array}{c}
-\mathrm{i} \lambda^{+} \\
\mathrm{i} \bar{\lambda}^{-}
\end{array}\right), \quad \tilde{H}=\left(\begin{array}{c}
\psi_{H_{2}}^{1} \\
\bar{\psi}_{H_{1}}^{2}
\end{array}\right)
$$

In addition, $\nu_{\mathrm{L}}=P_{\mathrm{L}} \nu$ and $e_{\mathrm{L}}=P_{\mathrm{L}} e$ where $\nu$ and $e$ are four-component spinors. Using eq. (A28), we find that the interaction specified in eq. (C26) can be written as

$$
-g\left[\overline{\tilde{W}} P_{\mathrm{L}} \nu \tilde{e}_{\mathrm{L}}^{*}+\bar{\nu} P_{\mathrm{R}} \tilde{W} \tilde{e}_{\mathrm{L}}+\overline{\tilde{W}}^{\mathrm{c}} P_{\mathrm{L}} e \tilde{\nu}^{*}+\bar{e} P_{\mathrm{R}} \tilde{W}^{\mathrm{c}} \tilde{\nu}\right]
$$

We shall comment in a moment on the appearance of $\tilde{W}^{c}$ in the interaction. At this point, eq. (C28) does not involve physical particles. We must express the mass eigenstates $\tilde{\chi}_{1}$ and $\tilde{\chi}_{2}$ [defined in eq. (C16)] in terms of $\tilde{W}$ and $\tilde{H}$. This is easily done using eqs. (C7) and (C12) from which we obtain

$$
\begin{aligned}
& P_{\mathrm{R}} \tilde{W}=P_{\mathrm{R}}\left(U_{11} \tilde{\chi}_{1}+U_{21} \tilde{\chi}_{2}\right) \\
& P_{\mathrm{R}} \tilde{W}^{\mathrm{c}}=P_{\mathrm{R}}\left(V_{11} \tilde{\chi}_{1}^{\mathrm{c}}+V_{21} \tilde{\chi}_{2}^{\mathrm{c}}\right)
\end{aligned}
$$

Inserting into eq. (C28) yields the desired form for the interaction 


$$
\begin{aligned}
& -g\left\{\left(U_{11}^{*} \overline{\tilde{\chi}}_{1}+U_{21}^{*} \overline{\tilde{\chi}}_{2}\right) P_{\mathrm{L}} \nu \tilde{e}_{\mathrm{L}}^{*}+\bar{\nu} P_{\mathrm{R}}\left(U_{11} \tilde{\chi}_{1}+U_{21} \tilde{\chi}_{2}\right) \tilde{e}_{\mathrm{L}}\right. \\
& \left.+\left(V_{11}^{*} \overline{\tilde{\chi}}_{1}^{\mathrm{c}}+V_{21}^{*} \overline{\tilde{\chi}}_{2}^{\mathrm{c}}\right) P_{\mathrm{L}} e \tilde{\nu}^{*}+\bar{e} P_{\mathrm{R}}\left(V_{11} \tilde{\chi}_{1}^{\mathrm{c}}+V_{21} \tilde{\chi}_{2}^{\mathrm{c}}\right) \tilde{\nu}\right\}
\end{aligned}
$$

The following convention will now be established. All fermions will be represented diagrammatically by a solid line accompanied by an arrow. In the case of leptons, it is conventional to take the arrow to indicate the flow of lepton number. For quarks, the arrow indicates the direction of flow of the flavor quantum number. In theories with fermion number conservation, all Feynman graphs will contain a continuous flow of fermion number as indicated by the direction of the arrows. In supersymmetric theories, fermion number (as understood in the conventional sense) is violated. This is usually seen most easily by noting the presence of massive neutral Majorana fermions in the spectrum. However, we can already see the effect of fermion number nonconservation in the charged sector here. Let us by convention represent the fermion $\tilde{\chi}_{1}^{+}$and $\tilde{\chi}_{2}^{+}$by solid lines accompanied by an arrow indicating the flow of positive electric charge. The appearance of the charge conjugate states $\tilde{\chi}_{1}^{c}$ and $\tilde{\chi}_{2}^{c}$ in eq. (C30) along with $\tilde{\chi}_{1}$ and $\tilde{\chi}_{2}$ signals the breakdown of fermion number. Diagrammatically, the interactions given in eq. (C30) are depicted in fig. 68. The Feynman rules for the diagrams shown in fig. 68(a) and (b) are easily read off from the interaction given in eq. (C30). On the other hand, note that the arrows clash in fig. 68(c) and (d) (caused by the presence of $\tilde{\chi}_{1}^{c}$ and $\tilde{\chi}_{2}^{c}$ in the interaction). This results in the presence of the charge conjugation matrix $C$ in the rules for graphs (c) and (d) which occurs because of eq. (A.12). These issues will be discussed in detail in appendix $D$.

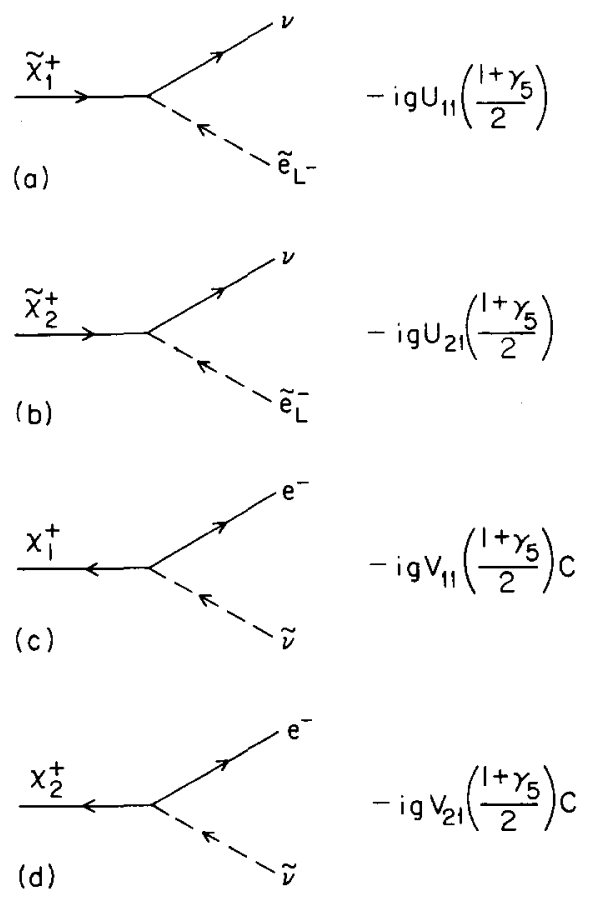

Fig. 68. Feynman rules for chargino interactions. The rules may be read off from eq. (C30). The arrow on the chargino line indicates the flow of positive electric charge. The appearance of the charge conjugation matrix $C$ in (c) and (d) is a consequence of the appearance of charge conjugated states in the interaction (eq. C30) and is accompanied diagrammatically by 'clashing' arrows. See appendix D for a more complete set of rules. 


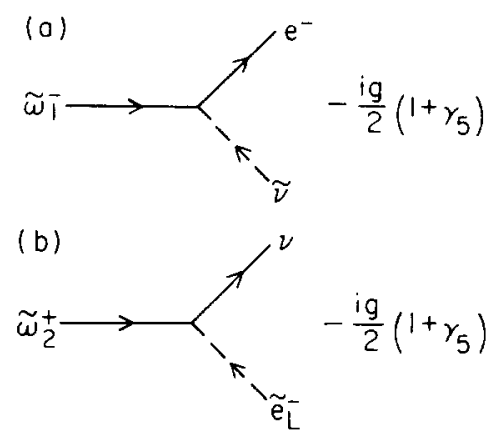

Fig. 69. Feynman rules in the supersymmetry limit. In this limit the wino and higgsino combine to form four-component (Dirac) wiggsino states $\bar{\omega}_{1}^{-}$ and $\tilde{\omega}_{2}^{+}$.

It is useful to examine eq. (C30) in the special case considered previously; namely $\mu=0$ and $v_{1}=v_{2}$. Then, $U$ and $V$ are given by eq. (C19) with $\phi_{+}=\phi_{-}=\phi$ given by eqs. (C22) and (C23). In this limit, eq. (C30) reduces to

$$
\begin{aligned}
& -g\left\{\left(\overline{\tilde{\chi}}_{1} \cos \phi-\overline{\tilde{\chi}}_{2} \sin \phi\right) P_{\mathrm{L}} \nu \tilde{e}_{\mathrm{L}}^{*}+\bar{\nu} P_{\mathrm{R}}\left(\tilde{\chi}_{1} \cos \phi-\tilde{\chi}_{2} \sin \phi\right) \tilde{e}_{\mathrm{L}}\right. \\
& \left.+\left(\overline{\tilde{\chi}}_{1}^{\mathrm{c}} \cos \phi+\overline{\tilde{\chi}}_{2}^{\mathrm{c}} \sin \phi\right) P_{\mathrm{L}} e \tilde{\nu}^{*}+\bar{e} P_{\mathrm{R}}\left(\tilde{\chi}_{1}^{\mathrm{c}} \cos \phi+\tilde{\chi}_{2}^{\mathrm{c}} \sin \phi\right) \tilde{\nu}\right\}
\end{aligned}
$$

In the supersymmetric limit $(M=0), \phi=45^{\circ}$ we may use eq. (C25) to obtain the following interaction terms:

$$
-g\left[\overline{\tilde{\omega}}_{1} P_{\mathrm{L}} e \tilde{\nu}^{*}+\bar{e} P_{\mathrm{R}} \tilde{\omega}_{1} \tilde{\nu}+\overline{\tilde{\omega}}_{2} P_{\mathrm{L}} \nu \tilde{e}_{\mathrm{L}}^{*}+\bar{\nu} P_{\mathrm{R}} \tilde{\omega}_{2} \tilde{e}_{\mathrm{L}}\right]
$$

The corresponding Feynman rules are shown in fig. 69. The supersymmetric limit has two features of note. First, no charge conjugated fields are present in eq. (C32) implying that there is no longer any fermion number violation in the charged sector. Second, the $\tilde{\omega}_{1}$ couples only to $e \tilde{\nu}$ but not $\tilde{e}_{\mathrm{L}} \nu$ whereas the $\tilde{\omega}_{2}$ couples in the opposite fashion. This simply reflects the fact that the $W^{+}$couples only to $e^{+} \nu$ whereas the $W^{-}$couples only to $e^{-} \bar{\nu}$.

It is sometimes convenient to allow some of the entries of the diagonal mass matrix $M_{\mathrm{D}}$ to be negative. For example, previously when $\mu=0$ and $v_{1}=v_{2}$ we could have chosen

$$
U=V=\left(\begin{array}{cc}
\cos \phi & \sin \phi \\
-\sin \phi & \cos \phi
\end{array}\right), \quad \cos \phi=\left(\frac{\tilde{M}_{+}}{\tilde{M}_{+}-\tilde{M}_{-}}\right)^{1 / 2}, \quad \tilde{M}_{ \pm}=\frac{1}{2} M \pm\left[m_{\mathrm{w}}^{2}+\frac{1}{4} M^{2}\right]^{1 / 2}
$$

which is to be compared with eqs. (C19)-(C23). Consistency is then regained by replacing $\tilde{\chi}_{2}$ by $\gamma_{5} \tilde{\chi}_{2}$. One can easily check that the interaction given by eq. (C30) is invariant under $\tilde{\chi}_{2} \rightarrow \gamma_{5} \tilde{\chi}_{2}$ and $V_{2 i} \rightarrow-V_{2 i}$ $(i=1,2)$. To summarize, if a given mass eigenvalue is negative, the corresponding eigenspinor must be multiplied by $\gamma_{5}$.

\section{Mixing of neutral gauginos and higgsinos*}

The discussion of the neutral mass eigenstates is similar to the previous discussion of the charged

${ }^{*}$ Neutralino mixing has been discussed in refs. [A.13-A.21]. Here we follow closely the techniques discussed in ref. [A.19]. 
mass eigenstates. The main complication here is that there are at least four neutral states mixing: $\lambda_{\gamma}$, $\lambda_{\mathrm{Z}}, \psi_{H_{1}}^{1}$ and $\psi_{H_{2}}^{2}$. (In models which contain an $\mathrm{SU}(2) \times \mathrm{U}(1)$ singlet field $N$, a fifth neutral state $\psi_{\mathrm{N}}$ must be included.) The supersymmetric limit (including the fifth state $\psi_{\mathrm{N}}$ ) was discussed in appendix B. The resulting mass eigenstates were displayed in eqs. (B26)-(B28) [see also eqs. (B32)-(B36)]. In this section, we will consider the general case of mixing of the four states $\lambda_{\gamma}, \lambda_{\mathrm{Z}}, \psi_{H_{1}}^{1}$ and $\psi_{H_{2}}^{2}$. The possible mass terms in two-component notation are as follows:

$$
\begin{aligned}
& \frac{1}{2} i\left(g^{2}+g^{\prime 2}\right)^{1 / 2} \lambda_{\mathrm{Z}}\left(v_{1} \psi_{H_{1}}^{1}-v_{2} \psi_{H_{2}}^{2}\right)+\frac{1}{2}\left(M \cos ^{2} \theta_{\mathrm{w}}+M^{\prime} \sin ^{2} \theta_{\mathrm{w}}\right) \lambda_{\mathrm{Z}} \lambda_{\mathrm{Z}} \\
& \quad+\left(M-M^{\prime}\right) \sin \theta_{\mathrm{w}} \cos \theta_{\mathrm{w}} \lambda_{\mathrm{Z}} \lambda_{\gamma}+\frac{1}{2}\left(M^{\prime} \cos ^{2} \theta_{\mathrm{w}}+M \sin ^{2} \theta_{\mathrm{w}}\right) \lambda_{\gamma} \lambda_{\gamma}+\mu \psi_{H_{1}}^{1} \psi_{H_{2}}^{2}+\text { h.c. }
\end{aligned}
$$

For comments on the origin of these terms, see the discussion below eq. (C6). Note that the parameters $\mu$ and $M$ are the same as those in eq. (C6). Also, $\lambda_{z}$ and $\lambda_{\gamma}$ are defined in terms of $\lambda^{3}$ and $\lambda^{\prime}$ as described below eq. (B24). In the context of the $\mathrm{SU}(2) \times \mathrm{U}(1)$ theory, $M$ and $M^{\prime}$ are independent parameters. However, if $\mathrm{SU}(2) \times \mathrm{U}(1)$ is embedded in a grand unified theory, these parameters become related (in much the same way as $\sin ^{2} \theta_{w}$ becomes determined). One finds that:

$$
M^{\prime}=\frac{5}{3}\left(g^{\prime 2} / g^{2}\right) M
$$

It is often more convenient to express the mass matrix in terms of $\lambda^{3}$ and $\lambda^{\prime}$ rather than $\lambda_{\mathrm{z}}$ and $\lambda_{\gamma}$. Equation (C33) then has the form

$$
\frac{1}{2} i g \lambda^{3}\left(v_{1} \psi_{H_{1}}^{1}-v_{2} \psi_{H_{2}}^{2}\right)-\frac{1}{2} \mathrm{i} g^{\prime} \lambda^{\prime}\left(v_{1} \psi_{H_{1}}^{1}-v_{2} \psi_{H_{2}}^{2}\right)+\frac{1}{2} M \lambda^{3} \lambda^{3}+\frac{1}{2} M^{\prime} \lambda^{\prime} \lambda^{\prime}+\mu \psi_{H_{1}}^{1} \psi_{H_{2}}^{2}+\text { h.c. }
$$

Defining

$$
\psi_{j}^{0}=\left(-i \lambda^{\prime},-i \lambda^{3}, \psi_{H_{1}}^{1}, \psi_{H_{2}}^{2}\right),
$$

eq. (C35) can be written as

$$
-\frac{1}{2}\left(\psi^{0}\right)^{\mathrm{T}} Y \psi^{0}+\text { h.c. }
$$

where

$$
Y=\left(\begin{array}{cccc}
M^{\prime} & 0 & -m_{\mathrm{z}} \sin \theta_{\mathrm{v}} \sin \theta_{\mathrm{w}} & m_{\mathrm{z}} \cos \theta_{\mathrm{v}} \sin \theta_{\mathrm{w}} \\
0 & M & m_{\mathrm{z}} \sin \theta_{\mathrm{v}} \cos \theta_{\mathrm{w}} & -m_{\mathrm{z}} \cos \theta_{\mathrm{v}} \cos \theta_{\mathrm{w}} \\
-m_{\mathrm{z}} \sin \theta_{\mathrm{v}} \sin \theta_{\mathrm{w}} & m_{\mathrm{z}} \sin \theta_{\mathrm{v}} \cos \theta_{\mathrm{w}} & 0 & -\mu \\
m_{\mathrm{z}} \cos \theta_{\mathrm{v}} \sin \theta_{\mathrm{w}} & -m_{\mathrm{z}} \cos \theta_{\mathrm{v}} \cos \theta_{\mathrm{w}} & -\mu & 0
\end{array}\right)
$$

where $\theta_{v}$ was defined in eq. (C11). The mass eigenstates are defined by

$$
\chi_{i}^{0}=N_{i j} \psi_{j}^{0}, \quad i=1, \ldots, 4,
$$

where $N$ is a unitary matrix satisfying

$$
N^{*} Y N^{-1}=N_{\mathrm{D}}
$$


where $N_{\mathrm{D}}$ is a diagonal matrix with nonnegative entries. To determine $N$, it is easiest to square eq. (C40) obtaining

$$
N_{\mathrm{D}}^{2}=N Y^{+} Y N^{-1}
$$

which is analogous to eq. (C17). Thus, $N_{\mathrm{D}}$ can be chosen to have nonnegative entries by appropriate choice of $N$. Using eqs. (A19) and (C39) we may write eq. (C37) in terms of four-component neutral Majorana spinors. Defining

$$
\tilde{\chi}_{i}^{0}=\left(\begin{array}{c}
\chi_{i}^{0} \\
\bar{\chi}_{i}^{0}
\end{array}\right)
$$

the mass term becomes

$$
-\frac{1}{2} \sum_{i} \tilde{M}_{i} \overline{\tilde{\chi}}_{i}^{0} \tilde{\chi}_{i}^{0}
$$

where $\tilde{M}_{i}$ are the diagonal elements of $N_{\mathrm{D}}$. In principle, it is simple to solve for the matrix $N$ and masses $\tilde{M}_{i}$ numerically.

An easily worked out example is the case where $\mu=0$ and $M=M^{\prime}$. It is clear from eq. (C33) that this corresponds to a massive photino with mass $\tilde{M}$ which decouples from the other fermions. In addition, there is a massless fermion* proportional to $v_{2} \psi_{H_{1}}^{1}+v_{1} \psi_{H_{2}}^{2}$. Thus, in this case, the convenient basis to work in is

$$
\psi_{j}^{0 \prime}=\left(-\mathrm{i} \lambda_{\gamma},-\mathrm{i} \lambda_{\mathrm{z}}, \frac{v_{1} \psi_{H_{1}}^{1}-v_{2} \psi_{H_{2}}^{2}}{\left(v_{1}^{2}+v_{2}^{2}\right)^{1 / 2}}, \frac{v_{2} \psi_{H_{1}}^{1}+v_{1} \psi_{H_{2}}^{2}}{=}\right.
$$

To distinguish between the two bases, we shall replace eqs. (C39) and (C40) with

$$
\chi_{i}^{0}=N_{i j}^{\prime} \psi_{j}^{0 \prime}, \quad N^{\prime *} Y^{\prime} N^{\prime-1}=N_{\mathrm{D}}
$$

where $Y^{\prime}$ is the mass matrix in the basis specified by eq. (C43) [analogous to $Y$ defined by eq. (C37)]. In the example where $M=M^{\prime}$ and $\mu=0, Y^{\prime}$ takes the simple form

$$
Y^{\prime}=\left(\begin{array}{cccc}
M & 0 & 0 & 0 \\
0 & M & m_{\mathrm{z}} & 0 \\
0 & m_{\mathrm{z}} & 0 & 0 \\
0 & 0 & 0 & 0
\end{array}\right)
$$

All we need to analyze is the nontrivial $2 \times 2$ submatrix of $Y$. The $2 \times 2$ matrix $N^{\prime}$ which diagonalizes this submatrix is

$$
N^{\prime}=\left(\begin{array}{cc}
\cos \phi & \sin \phi \\
-\mathrm{i} \sin \phi & \mathrm{i} \cos \phi
\end{array}\right)
$$

* One motivation for introducing an extra guage singlet field $N$ is that $\psi_{\mathrm{N}}$ combines with this massless fermion to produce a massive fermion [cf. eq. (B27)]. 
with $\cos \phi$ given by

$$
\begin{aligned}
& \cos \phi=\left[\tilde{M}_{+} /\left(\tilde{M}_{+}+\tilde{M}_{-}\right)\right]^{1 / 2}, \\
& \tilde{M}_{ \pm}=\left[m_{\mathrm{z}}^{2}+\frac{1}{4} M^{2}\right]^{1 / 2} \pm \frac{1}{2} M .
\end{aligned}
$$

Note that the factors of $\mathrm{i}$ in eq. (C46) have been inserted so that the resulting mass eigenvalues $\tilde{M}_{ \pm}$are nonnegative. Using eq. (C44), the two mass eigenstates with masses $\tilde{M}_{ \pm}$are

$$
\chi_{2}^{0}=-\mathrm{i} \lambda_{\mathrm{z}} \cos \phi+\left(\frac{v_{1} \psi_{H_{1}}^{1}-v_{2} \psi_{H_{2}}^{2}}{\left(v_{1}^{2}+v_{2}^{2}\right)^{1 / 2}}\right) \sin \phi, \quad-\mathrm{i} \chi_{3}^{0}=\mathrm{i} \lambda_{\mathrm{z}} \sin \phi+\left(\frac{v_{1} \psi_{H_{1}}^{1}-v_{2} \psi_{H_{2}}^{2}}{\left(v_{1}^{2}+v_{2}^{2}\right)^{1 / 2}}\right) \cos \phi
$$

Note that in the supersymmetric limit where $v_{1}=v_{2}$ and $M=0$ (i.e. $\phi=45^{\circ}$ ), we regain eqs. (B32)(B36).

Returning to the general case, let us write the interaction terms for $\tilde{\chi}_{i}^{0} e \tilde{e}$ and $\tilde{\chi}_{i}^{0} \nu \tilde{\nu}$. As in the previous section, we neglect the fermion masses so that the interaction terms of interest originate from the $\lambda \psi A$ term of eq. (B2). We focus on the following terms (using two-component notation):

$$
\text { (i } / \sqrt{2})\left\{\left(g \lambda^{3}-g^{\prime} \lambda^{\prime}\right) \nu_{\mathrm{L}} \tilde{\nu}^{*}-\left(g \lambda^{3}+g^{\prime} \lambda^{\prime}\right) e_{\mathrm{L}} \tilde{e}_{\mathrm{L}}^{*}-2 g^{\prime} \lambda^{\prime} e_{\mathrm{R}} \tilde{e}_{\mathrm{R}}\right\}+\text { h.c. }
$$

To rewrite this equation in four-component notation, define four-component Majorana spinors:

$$
\tilde{B}=\left(\begin{array}{c}
-\mathrm{i} \lambda^{\prime} \\
\mathrm{i} \bar{\lambda}^{\prime}
\end{array}\right), \quad \tilde{W}_{3}=\left(\begin{array}{c}
-\mathrm{i} \lambda^{3} \\
\mathrm{i} \bar{\lambda}^{3}
\end{array}\right), \quad \tilde{H}_{1}=\left(\begin{array}{c}
\psi_{H_{1}}^{1} \\
\bar{\psi}_{H_{1}}^{1}
\end{array}\right), \quad \tilde{H}_{2}=\left(\begin{array}{c}
\psi_{H_{2}}^{2} \\
\bar{\psi}_{H_{2}}^{2}
\end{array}\right)
$$

Using eq. (A28), we find that eq. (C50) becomes

$$
(-1 / \sqrt{2})\left\{\left(g \overline{\tilde{W}}_{3}-g^{\prime} \overline{\tilde{B}}\right) P_{\mathrm{L}} \nu \tilde{\nu}^{*}-\left(g \tilde{W}_{3}+g^{\prime} \overline{\tilde{B}}\right) P_{\mathrm{L}} e \tilde{e}_{\mathrm{L}}^{*}-2 g^{\prime} \bar{e} P_{\mathrm{L}} \tilde{B} \tilde{e}_{\mathrm{R}}\right\}+\text { h.c. }
$$

Finally, one may express this interaction in terms of mass eigenstates $\tilde{\chi}_{i}^{0}$ defined in eq. (C41) by using the inverse of eq. (C39).

One may also wish to make use of the basis given by eq. (C43). Then, defining

$$
\tilde{\gamma}=\left(\begin{array}{c}
-\mathrm{i} \lambda_{y} \\
\mathrm{i} \overline{\lambda_{\gamma}}
\end{array}\right), \quad \tilde{Z}=\left(\begin{array}{c}
-\mathrm{i} \lambda_{z} \\
\mathrm{i} \bar{\lambda}_{z}
\end{array}\right)
$$

the equation analogous to eq. (C52) is

$$
\begin{aligned}
& \frac{1}{\sqrt{2}}\left\{\frac{\bar{g}}{\cos \theta_{\mathrm{w}}} \overline{\tilde{Z}} P_{\mathrm{L}} \nu \tilde{\nu}^{*}+\left[\left(g^{\prime} \sin \theta_{\mathrm{w}}-g \cos \theta_{\mathrm{w}}\right) \overline{\tilde{Z}}-2 g \sin \theta_{\mathrm{w}} \overline{\tilde{\gamma}}\right] P_{\mathrm{L}} e \tilde{e}_{\mathrm{L}}^{*}\right. \\
& \left.\quad+2 g^{\prime} \bar{e} P_{\mathrm{L}}\left(-\sin \theta_{\mathrm{w}} \tilde{Z}+\cos \theta_{\mathrm{w}} \tilde{\gamma}\right) \tilde{e}_{\mathrm{R}}\right\}+ \text { h.c. }
\end{aligned}
$$

As an example, consider again the case where $M=M^{\prime}$ and $\mu=0$. We found that the physical neutralinos consisted of a massive photino $\tilde{\gamma}$, two Majorana fermions: 


$$
\tilde{\chi}_{i}^{0}=\left(\begin{array}{c}
\chi_{i}^{0} \\
\bar{\chi}_{i}^{0}
\end{array}\right), \quad i=2,3
$$

where $\chi_{2}^{0}, \chi_{3}^{0}$ are given in eq. (C49), and one massless state. Here, one must be careful in going from two-component to four-component notation because of the extra factor of $i$ in the definition of $\chi_{3}^{0}$. One finds from eq. (C49):

$$
P_{\mathrm{L}} \tilde{Z}=P_{\mathrm{L}}\left(\tilde{\chi}_{2}^{0} \cos \phi+\mathrm{i} \tilde{\chi}_{3}^{0} \sin \phi\right), \quad \overline{\tilde{Z}} P_{\mathrm{L}}=\left(\overline{\bar{\chi}}_{2}^{0} \cos \phi+\mathrm{i} \overline{\tilde{\chi}}_{3}^{0} \sin \phi\right) P_{\mathrm{L}},
$$

which can be inserted into eq. (C54) to obtain the interaction of physical neutralinos in this example.

It is interesting to consider the supersymmetric limit $\left(\phi=45^{\circ}\right.$ and $\left.M=0\right)$ of the above example. Then, $\chi_{2}^{0}$ and $\chi_{3}^{0}$ become degenerate in mass with the $Z^{0}$; thus confirming our previous results given in eqs. (B32)-(B36). Suppose we wish to express these two degenerate Majorana fermions as one Dirac fermion $\tilde{\zeta}$ [given by eq. (B26)]. Then, the interaction terms involving $\tilde{\zeta}$ [from eq. (C54)] becomes

$$
\frac{-1}{\sqrt{2}}\left\{\frac{g}{\cos \theta_{\mathrm{w}}} \overline{\tilde{\zeta}} P_{\mathrm{L}} \nu \tilde{\nu}^{*}+\left(g^{\prime} \sin \theta_{\mathrm{w}}-g \cos \theta_{\mathrm{w}}\right) \overline{\tilde{\zeta}} P_{\mathrm{L}} e e_{\mathrm{L}}^{*}-2 g^{\prime} \sin \theta_{\mathrm{w}} \bar{e} P_{\mathrm{L}} \tilde{\zeta}^{\mathrm{c}} \tilde{e}_{\mathrm{R}}\right\}+\text { h.c. }
$$

The corresponding Feynman rules are shown in fig. 70 . Note the appearance of $\tilde{\zeta}^{\mathrm{c}}$ above. Unlike the case of the chargino interactions [eq. (C32)], we see here that fermion number violating interactions remain even in the supersymmetric limit.

The factor of $i$ inserted into the definition of $\chi_{3}^{0}$ (see eq. (C49)) is awkward. Without the factor of $i$, the corresponding mass eigenvalue would have been negative. We have discussed the effects of a negative mass eigenvalue at the end of appendix C.2. A similar analysis can be applied in this case, namely one must use $\gamma_{5} \tilde{\chi}_{3}^{0}$ as the eigenspinor corresponding to the negative mass eigenvalue. The
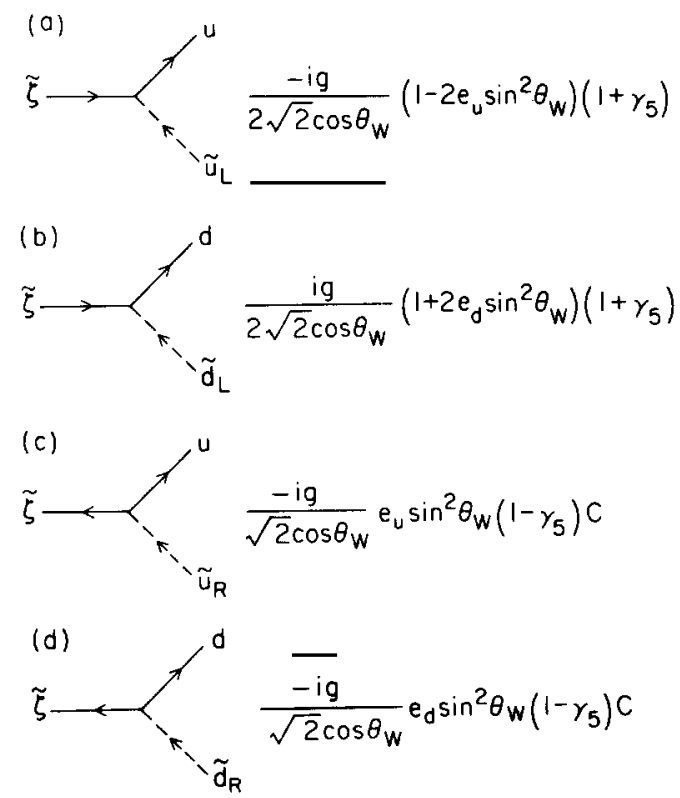

Fig. 70. Feynman rules in the supersymmetric limit. In this limit the zino and higgsino combine to form one four-component (Dirac) ziggsino state, $\tilde{\zeta}$. The quark charges are $e_{\mathrm{u}}=2 / 3, e_{\mathrm{d}}=-1 / 3$. 
resulting Feynman rules will be somewhat different as to the appearance of factors of $i$. The physical results are unchanged.

\section{The Feynman rules}

We are now ready to write down the interaction Lagrangian for the broken supersymmetric $S U(2) \times U(1)$ theory. We shall focus on the pieces of most interest to the phenomenological study of supersymmetry. The main complication in what follows arises from the complicated pattern of mixing described in the previous three sections. To make the rules as transparent as possible, we shall often rewrite a given interaction term in a number of different ways. For simplicity, we treat only one generation of quarks and leptons, thereby avoiding complications of quark (and scalar-quark) generational mixing.

(a) Quark-quark-gauge boson

In two-component notation,

$$
\mathscr{L}_{\mathrm{qqV}}=-\frac{1}{2}\left(g \tau_{i j}^{a} V_{\mu}^{a}+y_{\mathrm{Q}} g^{\prime} V_{\mu}^{\prime} \delta_{i j}\right) \bar{\psi}_{\mathrm{Q}}^{i} \bar{\sigma}^{\mu} \psi_{\mathrm{O}}^{j}-\frac{1}{2} g^{\prime} y_{\mathrm{u}} V_{\mu}^{\prime} \bar{\psi}_{\mathrm{u}} \bar{\sigma}^{\mu} \psi_{\mathrm{u}}-\frac{1}{2} g^{\prime} y_{\mathrm{d}} V_{\mu}^{\prime} \bar{\psi}_{\mathrm{d}} \bar{\sigma}^{\mu} \psi_{\mathrm{d}}
$$

In the above equation, $\psi_{\mathrm{O}}^{i}(i=1,2)$ denotes the weak doublet $\left(u_{\mathrm{L}}, d_{\mathrm{L}}\right)$ of two-component fermions, and $\psi_{\mathrm{u}}, \psi_{\mathrm{d}}$ denote the two-component fermion fields $u_{\mathrm{L}}^{\mathrm{c}}, d_{\mathrm{L}}^{\mathrm{c}}$ respectively.

The quark hypercharges $y_{i}$ are related to each other and the quark charges $e_{i}\left(e_{\mathrm{u}}=2 / 3, e_{\mathrm{d}}=-1 / 3\right)$ by

$$
y_{\mathrm{u}}=-1-y_{\mathrm{Q}}=-2 e_{\mathrm{u}}, \quad y_{\mathrm{d}}=1-y_{\mathrm{Q}}=-2 e_{\mathrm{d}} \text {. }
$$

Note that by writing the expression in this form, eq. (C58) can be used for the leptons as well, with appropriate choice of $e_{\mathrm{u}}$ and $e_{\mathrm{d}}$.

As usual we define:

$$
\begin{aligned}
& W_{\mu}^{ \pm}=(1 / \sqrt{2})\left(V_{\mu}^{\mathrm{1}} \mp \mathrm{i} V_{\mu}^{2}\right), \quad A_{\mu}=\sin \theta_{\mathrm{w}} V_{\mu}^{3}+\cos \theta_{\mathrm{w}} V_{\mu}^{\prime}, \\
& Z_{\mu}=\cos \theta_{\mathrm{w}} V_{\mu}^{3}-\sin \theta_{\mathrm{w}} V_{\mu}^{\prime}, \quad \tan \theta=g^{\prime} / g, \quad e=g \sin \theta_{\mathrm{w}}>0 .
\end{aligned}
$$

Then, in four-component notation, we find

$$
\begin{aligned}
\mathscr{L}_{\mathrm{qqV}}= & \frac{-g}{\sqrt{2}}\left[W_{\mu}^{+} \bar{u} \gamma^{\mu} P_{\mathrm{L}} d+W_{\mu}^{-} \bar{d} \gamma^{\mu} P_{\mathrm{L}} u\right]-\frac{g}{\cos \theta_{\mathrm{w}}} Z_{\mu}\left\{\bar{u} \gamma_{\mu}\left[\left(\frac{1}{2}-e_{\mathrm{u}} \sin ^{2} \theta_{\mathrm{w}}\right) P_{\mathrm{L}}-e_{\mathrm{u}} \sin ^{2} \theta_{\mathrm{w}} P_{\mathrm{R}}\right] u\right. \\
& \left.-\bar{d} \gamma_{\mu}\left[\left(\frac{1}{2}+e_{\mathrm{d}} \sin ^{2} \theta_{\mathrm{w}}\right) P_{\mathrm{L}}+e_{\mathrm{d}} \sin ^{2} \theta_{\mathrm{w}} P_{\mathrm{R}}\right] d\right\}-e A_{\mu}\left(e_{\mathrm{u}} \bar{u} \gamma^{\mu} u+e_{\mathrm{d}} \bar{d} \gamma^{\mu} d\right),
\end{aligned}
$$

where $P_{\mathbf{R}, \mathbf{L}}=\left(1 \pm \gamma_{5}\right) / 2$ as before. The Feynman rules as shown in fig. 71 . For future reference, it is worth noting that the Zqq coupling above is a special case of

$$
\mathscr{L}_{\mathrm{ffZ}}=\frac{-g}{\theta_{\mathrm{w}}} Z_{\mu} \bar{f} \gamma^{\mu}\left[\left(T_{3}^{\mathrm{L}}-e_{\mathrm{f}} \sin ^{2} \theta_{\mathrm{w}}\right) P_{\mathrm{L}}+\left(T_{3}^{\mathrm{R}}-e_{\mathrm{f}} \sin ^{2} \theta_{\mathrm{w}}\right) P_{\mathrm{R}}\right] f,
$$

where

$$
f \equiv\left(\begin{array}{l}
f_{\mathrm{L}} \\
f_{\mathrm{R}}
\end{array}\right)
$$



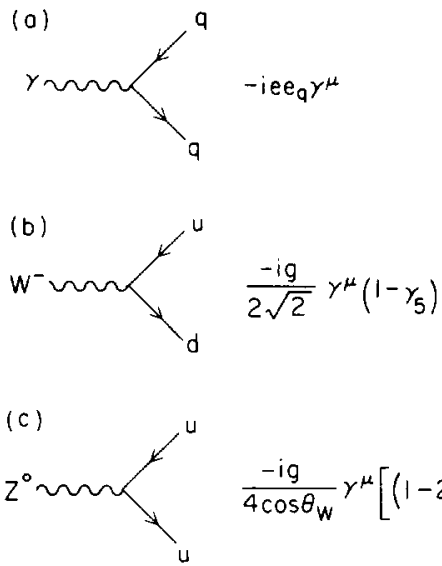

$$
\frac{-i g}{4 \cos \theta_{w}} \gamma^{\mu}\left[\left(1-2 e_{u} \sin ^{2} \theta_{w}\right)\left(1-y_{5}\right)-2 e_{u} \sin ^{2} \theta_{w}\left(1+y_{5}\right)\right]
$$

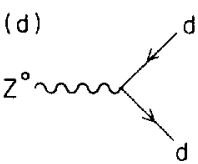

$$
\frac{\frac{19}{4 \cos \theta_{W}}}{\mu}\left[\left(1+2 e_{d} \sin ^{2} \theta_{W}\right)\left(1-\gamma_{5}\right)+2 e_{d} \sin ^{2} \theta_{W}\left(1+\gamma_{5}\right)\right]
$$

Fig. 71. Feynman rules for the interaction of a gauge boson with a pair of quarks. The quark charges are $e_{\mathrm{u}}=2 / 3, e_{\mathrm{d}}=-1 / 3$.

is a four-component spinor and $T_{3}^{\mathrm{L}, \mathrm{R}}$ are the $T_{3}$ quantum numbers of $\mathrm{f}_{\mathrm{L}}$ and $\mathrm{f}_{\mathrm{R}}$ respectively, and $e_{\mathrm{f}}$ is the charge of $\mathrm{f}_{\mathrm{L}}$ in units of $e$.

The gluon interaction may similarly be obtained. One subtlety here is that whereas $\psi_{\mathrm{Q}}^{i}(i=1,2)$ are color triplets, $\psi_{\mathrm{u}}$ and $\psi_{\mathrm{d}}$ are color antitriplets. If $T_{j \mathrm{k}}^{a}$ are the color triplet generators, then one must use $-T_{j k}^{* a}=-T_{k j}^{a}$ for the color antitriplet generators. Hence in two-component notation,

$$
\mathscr{L}_{\mathrm{QQ}_{\mathrm{g}}}=-g_{\mathrm{s}} A_{\mu}^{a} T_{j k}^{a}\left(\bar{\psi}_{\mathrm{Q}_{j}}^{i} \bar{\sigma}^{\mu} \psi_{\mathrm{Q}_{k}}^{i}-\bar{\psi}_{\mathrm{u}_{k}} \bar{\sigma}^{\mu} \psi_{\mathrm{u}_{j}}-\bar{\psi}_{\mathrm{d}_{k}} \bar{\sigma}^{\mu} \psi_{\mathrm{d}_{j}}\right)
$$

where $j$ and $k$ are color indices. Defining the four-component spinor

$$
u=\left(\begin{array}{c}
\psi_{\mathrm{Q}}^{1} \\
\bar{\psi}_{\mathrm{u}}
\end{array}\right), \quad d=\left(\begin{array}{c}
\psi_{\mathrm{O}}^{2} \\
\bar{\psi}_{\mathrm{d}}
\end{array}\right)
$$

we may use eq. (A21) to obtain

$$
\mathscr{L}_{\mathrm{QQ}_{\mathrm{g}}}=-g_{\mathrm{s}} A_{\mu}^{a} \bar{q}_{j} \gamma^{\mu} T_{j k}^{a} q_{k},
$$

where $q=\left(\begin{array}{l}u \\ d\end{array}\right)$ is a doublet of four component fermions.

\section{(b) Scalar-quark-scalar-quark-gauge boson}

We shall write the interaction in terms of the $q_{\mathrm{L}}-q_{\mathrm{R}}$ basis. See appendix C.1 for the effects of mixing. We use the following notation: $\tilde{\mathrm{q}}^{*}(\tilde{\mathrm{q}})$ creates (destroys) the scalar-quark $\tilde{\mathrm{q}}$; where $\tilde{\mathrm{q}}_{\mathrm{L}}^{*}$ and $\tilde{\mathrm{q}}_{\mathrm{R}}^{*}$ create the same value of electric and color charge. Then, 


$$
\begin{aligned}
\mathscr{L}_{\tilde{\mathbf{q}} \tilde{\mathbf{q}} \mathrm{V}}= & \frac{-\mathrm{i} g}{\sqrt{2}}\left[W_{\mu}^{+}\left(\tilde{u}_{\mathrm{L}}^{*} \stackrel{\leftrightarrow}{\partial}^{\mu} \tilde{d}_{\mathrm{L}}\right)+W_{\mu}^{-}\left(\tilde{d}_{\mathrm{L}}^{*} \stackrel{\leftrightarrow}{\partial}^{\mu} \tilde{u}_{\mathrm{L}}\right)\right]-\frac{\mathrm{i} g}{\cos \theta_{\mathrm{w}}} Z_{\mu} \sum_{i}\left(T_{3 i}-e_{i} \sin ^{2} \theta_{\mathrm{w}}\right) \tilde{q}_{i}^{*} \stackrel{\leftrightarrow}{\partial}^{\mu} \tilde{q}_{i} \\
& -\mathrm{i} e A_{\mu} \sum_{i} e_{i} \tilde{q}_{i}^{*} \stackrel{\leftrightarrow}{\partial}^{\mu} \tilde{q}_{i},
\end{aligned}
$$

where the sums run over $\tilde{u}_{\mathrm{L}}, \tilde{u}_{\mathrm{R}}, \tilde{d}_{\mathrm{L}}$ and $\tilde{d}_{\mathrm{R}}$. (Note that $e_{\tilde{\mathrm{u}}_{\mathrm{L}}}=e_{\tilde{\mathrm{u}}_{\mathrm{R}}}=e_{\mathrm{u}}$, etc.) The Feyman rules are shown in fig. 72 .

The gluon interactions may be similarly obtained:

$$
\mathscr{L}_{\tilde{\mathbf{q}} \tilde{\mathbf{q} g}}=-\mathrm{i} g_{\mathrm{s}} A_{\mu}^{a} \sum_{i} \tilde{q}_{i}^{\sharp j} T_{j k}^{a} \stackrel{\leftrightarrow}{\partial}^{\mu} \tilde{q}_{i}^{k}
$$

where $j, k$ are color indices, and the sum over $i$ is as in eq. (C66).
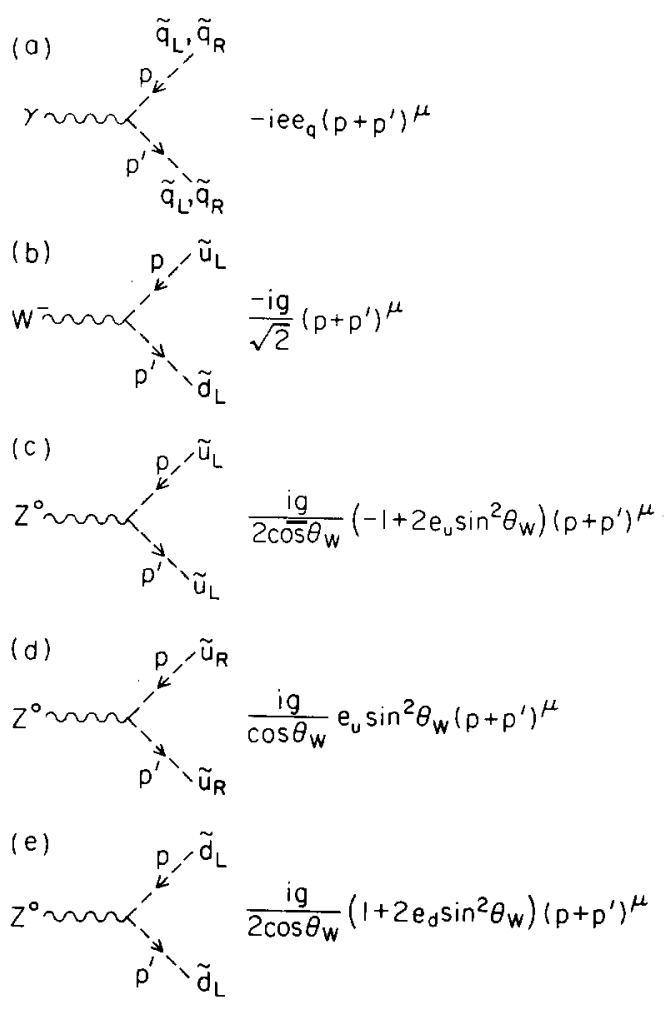

(f) $z^{\circ} \sim \sim_{p^{\prime}, \tilde{d}_{R}}^{p,} \frac{i g}{\cos \theta_{w}} e_{d^{\prime}} \sin ^{2} \theta_{w}\left(p+p^{\prime}\right)^{\mu}$

Fig. 72. Feynman rules for the interaction of a gauge boson with a pair of scalar-quarks. The quark charges are $e_{\mathrm{u}}=2 / 3, e_{\mathrm{d}}=-1 / 3$. 
(c) Quark-scalar-quark-chargino

We have discussed the lepton-scalar-lepton-chargino interactions in eq. (C27)-(C30). For the quarks, the interaction is obtained by simple replacement of $(\nu, e) \rightarrow(\mathrm{u}, \mathrm{d})$. We summarize the results here. In two component notation,

$$
\mathscr{L}_{\mathbf{q} \tilde{q}^{+}}=\mathrm{ig}\left[\lambda^{-} u_{\mathrm{L}} \tilde{d}_{\mathrm{L}}^{*}-\bar{\lambda}^{-} \bar{u}_{\mathrm{L}} \tilde{d}_{\mathrm{L}}+\lambda^{+} d_{\mathrm{L}} \tilde{u}_{\mathrm{L}}^{*}-\bar{\lambda}^{+} \bar{d}_{\mathrm{L}} \tilde{u}_{\mathrm{L}}\right]
$$

To rewrite this in four-component notation, we need to choose a basis consisting of the mass eigenstates for the four-component chargino states. However, it is often more convenient to use a different set of basis states. If we use $\tilde{\omega}_{1}$ and $\tilde{\omega}_{2}$ as basis states (see eq. (B25)), then we find

$$
\mathscr{L}_{\mathrm{q} \overline{\mathrm{q}} \tilde{x}^{+}}=-g\left[\overline{\tilde{\omega}}_{2} P_{\mathrm{L}} u \tilde{d}_{\mathrm{L}}^{*}+\tilde{u} P_{\mathrm{R}} \tilde{\omega}_{2} \tilde{d}_{\mathrm{L}}+\overline{\tilde{\omega}}_{1} P_{\mathrm{L}} d \tilde{u}_{\mathrm{L}}^{*}+\bar{d} P_{\mathrm{R}} \tilde{\omega}_{1} \tilde{u}_{\mathrm{L}}\right]
$$

The physical states can be expressed in terms of $\tilde{\omega}_{1}$ and $\tilde{\omega}_{2}$ using

$$
\begin{aligned}
& P_{\mathrm{R}} \tilde{\omega}_{1}=P_{\mathrm{R}}\left(V_{11} \tilde{\chi}_{1}^{\mathrm{c}}+V_{21} \tilde{\chi}_{2}^{\mathrm{c}}\right), \\
& P_{\mathrm{R}} \tilde{\omega}_{2}=P_{\mathrm{R}}\left(U_{11} \tilde{\chi}_{1}+U_{21} \tilde{\chi}_{2}\right) .
\end{aligned}
$$

We end up with

$$
\begin{aligned}
\mathscr{L}_{\mathbf{q} \bar{q} \bar{\chi}^{+}}= & -g\left[\left(U_{11}^{*} \overline{\tilde{\chi}}_{1}+U_{21}^{*} \overline{\tilde{\chi}}_{2}\right) P_{\mathrm{L}} u \tilde{d}_{\mathrm{L}}^{*}+\bar{u} P_{\mathrm{R}}\left(U_{11} \tilde{\chi}_{1}+U_{21} \tilde{\chi}_{2}\right) \tilde{d}_{\mathrm{L}}\right. \\
& \left.+\left(V_{11}^{*} \overline{\tilde{\chi}}_{1}^{\mathrm{c}}+V_{21}^{*} \overline{\tilde{\chi}}_{2}^{\mathrm{c}}\right) P_{\mathrm{L}} d \tilde{u}_{\mathrm{L}}^{*}+\bar{d} P_{\mathrm{R}}\left(V_{11} \tilde{\chi}_{1}^{\mathrm{c}}+V_{21} \tilde{\chi}_{2}^{\mathrm{c}}\right) \tilde{u}_{\mathrm{L}}\right] .
\end{aligned}
$$

The Feynman rules depend on the elements of the mixing matrices. The rules given in fig. 68 are appropriate here if we replace $\mathrm{e}, \nu, \tilde{\mathrm{e}}_{\mathrm{L}}, \tilde{\nu}$ with $\mathrm{d}, \mathrm{u}, \tilde{\mathrm{d}}_{\mathrm{L}}, \tilde{\mathrm{u}}_{\mathrm{L}}$ respectively. Note that we are working in the limit of massless quarks so that there is no quark-scalar-quark-higgsino interaction terms. For $m_{\mathrm{q}} \neq 0$ the Feynman rules are given in ref. [A.37].

\section{(d) Quark-scalar-quark-neutralino}

In two component notation,

$$
\mathscr{L}_{\mathrm{q} \bar{q} \bar{x}^{0}}=\frac{\mathrm{i}}{\sqrt{2}}\left(g \tau_{i j}^{3} \lambda^{3}+y_{\mathrm{Q}} g^{\prime} \delta_{i j} \lambda^{\prime}\right) \psi_{\mathrm{Q}}^{i} \tilde{Q}_{j}^{*}+\frac{\mathrm{i}}{\sqrt{2}} g^{\prime}\left(y_{\mathrm{u}} \lambda^{\prime} \psi_{\mathrm{u}} \tilde{U}^{*}+y_{\mathrm{d}} \lambda^{\prime} \psi_{\mathrm{d}} \tilde{D}^{*}\right)+\text { h.c. }
$$

where $\tilde{Q}_{j}, \tilde{U}$, and $\tilde{D}$ are the scalars partners of $\psi_{\mathrm{Q}}, \psi_{\mathrm{u}}$ and $\psi_{\mathrm{d}}$ respectively (see table 14 ).

In four-component notation, let us choose as a basis $\tilde{\gamma}, \tilde{Z}, \tilde{H}_{1}$ and $\tilde{H}_{2}$ (see eqs. (C51) and (C53)). Then, eq. (C72) becomes

$$
\begin{aligned}
\mathscr{L}_{\mathrm{q} \tilde{q} \tilde{x}^{(0)}=} & \frac{-\sqrt{2} g}{\cos \theta_{\mathrm{w}}}\left\{\sum_{i=u, d}\left(T_{3 i}-e_{i} \sin ^{2} \theta_{\mathrm{w}}\right)\left(\overline{\tilde{Z}} P_{\mathrm{L}} q_{i} \tilde{q}_{i \mathrm{~L}}^{*}+\bar{q}_{i} P_{\mathrm{R}} \tilde{Z} \tilde{q}_{i \mathrm{~L}}\right)+\sum_{i=u, d} e_{i} \sin ^{2} \theta_{\mathrm{w}}\left(\overline{\tilde{Z}} P_{\mathrm{R}} q_{i} \tilde{q}_{i \mathrm{R}}^{*}+\bar{q}_{i} P_{\mathrm{L}} \tilde{Z} \tilde{q}_{i \mathrm{R}}\right)\right\} \\
& -\sqrt{2} e\left\{\sum_{i=u, d} e_{i}\left(\overline{\tilde{\gamma}} P_{\mathrm{L}} q_{i} \tilde{q}_{i \mathrm{~L}}^{*}+\bar{q}_{i} P_{\mathrm{R}} \tilde{\gamma} \tilde{q}_{i \mathrm{~L}}\right)-e_{i}\left(\overline{\tilde{\gamma}} P_{\mathrm{R}} q_{i} \tilde{q}_{i \mathrm{R}}^{*}+\bar{q}_{i} P_{\mathrm{L}} \tilde{\gamma} \tilde{q}_{i \mathrm{R}}\right)\right\}
\end{aligned}
$$


If the photino $\tilde{\gamma}$ is a mass eigenstate, then we may immediately obtain the Feynman rules for photino interactions shown in fig. 73. Note the change in sign which occurs, for example, between the two terms involving the photino. The origin of this sign change comes from the fact that the scalar partners of the antiquarks are opposite in sign to the scalar partners of the quarks (see table 14). We have defined $\tilde{u}_{\mathrm{R}}^{*}=U$ and $\tilde{d}_{\mathrm{R}}^{*}=\tilde{D}$ above so that $\tilde{q}_{\mathrm{L}}$ and $\tilde{q}_{\mathrm{R}}$ have the same sign as the corresponding quark charge (cf. discussion below eq. (B42)), but remnants of this sign change remain.

In terms of the physical states, we shall use eq. (C44) which defines the matrix $N^{\prime}$. Then, we find

$$
\begin{aligned}
& P_{\mathrm{L}} \tilde{\gamma}=P_{\mathrm{L}} \sum_{j=1}^{4} N_{j 1}^{\prime *} \tilde{\chi}_{j}, \\
& P_{\mathrm{R}} \tilde{\gamma}=P_{\mathrm{R}} \sum_{j=1}^{4} N_{j 1}^{\prime} \tilde{\chi}_{j},
\end{aligned}
$$

and two similar equations for $P_{\mathrm{L}, \mathrm{R}} \tilde{Z}$ which involve $N_{j 2}^{\prime}$. Inserting these equations into eq. (C73) leads to the desired interaction terms involving physical states:

$$
\begin{aligned}
\mathscr{L}_{\mathrm{q} \overline{\mathrm{q}} \tilde{\chi}^{0}}= & -\sqrt{2} \sum_{i j} \bar{q}_{i} P_{\mathrm{R}} \tilde{\chi}_{j}^{0} \tilde{q}_{i \mathrm{~L}}\left[\left(g / \cos \theta_{\mathrm{w}}\right)\left(T_{3 i}-e_{i} \sin ^{2} \theta_{\mathrm{w}}\right) N_{j 2}^{\prime}+e e_{i} N_{j_{1}}^{\prime}\right] \\
& -\sqrt{2} \sum_{i j} \bar{q}_{i} P_{\mathrm{L}} \tilde{\chi}_{j}^{0} \tilde{q}_{i \mathrm{R}}\left[\left(\mathrm{g} / \cos \theta_{\mathrm{w}}\right) e_{i} \sin ^{2} \theta_{\mathrm{w}} N_{j 2}^{\prime *}-e e_{i} N_{j 1}^{*}\right]+\text { h.c. }
\end{aligned}
$$

Let us now repeat the computation using the $\tilde{W}_{3}, \tilde{B}, \tilde{H}_{1}, \tilde{H}_{2}$ basis. In this basis, eq. (C73) now becomes

$$
\mathscr{L}_{\mathrm{q} \tilde{\mathfrak{q}} \tilde{x}^{0}}=-\sqrt{2} g \sum_{i} \bar{q}_{i} P_{\mathrm{R}}\left[T_{3 i} \tilde{W}_{3}-\tan \theta_{\mathrm{w}}\left(T_{3 i}-e_{i}\right) \tilde{B}\right] \tilde{q}_{i \mathrm{~L}}+\sqrt{2} g \tan \theta_{\mathrm{w}} \sum_{i} e_{i} \bar{q}_{i} P_{\mathrm{L}} \tilde{B} \tilde{q}_{i \mathrm{R}}+\text { h.c. }
$$

We may write this in terms of mass eigenstates by using the matrix $N$ defined in eq. (C39). The end result is an equation analogous to eq. (C75):
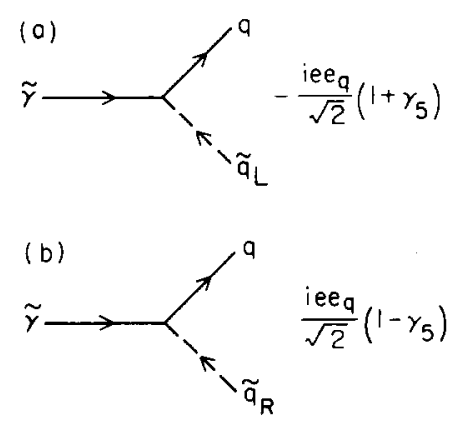

Fig. 73. Feynman rules for the interaction of photinos with a quark-scalar-quark pair. It is assumed that the photino is one of the neutralino mass eigenstates. 


$$
\mathscr{L}_{\mathrm{q} \tilde{\bar{q}} \bar{\chi}^{0}}=-\sqrt{2} g \sum_{i j} \bar{q}_{i} P_{\mathrm{R}} \tilde{\chi}_{j}^{0} \tilde{q}_{i \mathrm{~L}}\left[T_{3 i} N_{j 2}-\tan \theta_{\mathrm{w}}\left(T_{3 i}-e_{i}\right) N_{j 1}\right]+\sqrt{2} g \tan \theta_{\mathrm{w}} \sum_{i j} \bar{q}_{i} P_{\mathrm{L}} \tilde{\chi}_{j}^{0} \tilde{q}_{i \mathrm{R}}\left[e_{i} N_{j 2}^{*}\right]+\text { h.c. }
$$

Of course, eqs. (C75) and (C77) are identical because $N$ and $N^{\prime}$ are related in the appropriate way as one may easily verify.

The corresponding Feynman rules depend on the neutralino mixing matrix. As an example, in the supersymmetric limit, the appropriate rules are given in figs. 70 and 73. Note that the higgsinos decouple in the approximation of massless quarks. For $m_{\mathrm{q}} \neq 0$, the Feynman rules are given in ref. [A.37].

(e) Lepton and scalar-lepton interactions

These may be obtained from the quark and scalar-quark interactions by the replacement $(u, d) \rightarrow$ $\left(\nu_{\mathrm{e}}, \ell\right), e_{\nu}=0$, and $e_{\ell}=-1$.

\section{(f) Chargino-neutralino- $W^{ \pm}$}

This interaction has two sources: eqs. (B1) and (B2), because the chargino and neutralinos are made up of gaugino pieces and higgsino pieces. First, we look at the interaction in two component form:

$$
\begin{aligned}
& g\left\{W_{\mu}^{+}\left(\bar{\lambda}^{+} \bar{\sigma}^{\mu} \lambda^{3}-\bar{\lambda}^{3} \bar{\sigma}^{\mu} \lambda^{-}\right)+W_{\mu}^{-}\left(\bar{\lambda}^{3} \bar{\sigma}^{\mu} \lambda^{+}-\bar{\lambda}^{-} \bar{\sigma}_{\mu} \lambda^{3}\right)\right\} \\
& \quad-(g / \sqrt{2})\left\{W_{\mu}^{+}\left(\bar{\psi}_{H_{2}}^{1} \bar{\sigma}^{\mu} \psi_{H_{2}}^{2}+\bar{\psi}_{H_{1}}^{1} \bar{\sigma}^{\mu} \psi_{H_{1}}^{2}\right)+W_{\mu}^{-}\left(\bar{\psi}_{H_{2}}^{2} \bar{\sigma}^{\mu} \psi_{H_{2}}^{1}+\bar{\psi}_{H_{1}}^{2} \bar{\sigma}^{\mu} \psi_{H_{1}}^{1}\right)\right\} .
\end{aligned}
$$

Using eq. (A21), (A22), (C27) and (C51), we may immediately write this in four-component form:

$$
g W_{\mu}^{-}\left[\overline{\tilde{W}}_{3} \gamma^{\mu} \tilde{W}-(1 / \sqrt{2})\left(\overline{\tilde{H}}_{2} \gamma^{\mu} P_{\mathrm{L}} \tilde{H}-\overline{\tilde{H}}_{1} \gamma^{\mu} P_{\mathrm{R}} \tilde{H}\right)\right]+\text { h.c. }
$$

If the photino is a mass eigenstate, we may obtain the Feynman rules for the $W^{-} \tilde{\chi}^{+} \tilde{\gamma}$ vertex from eq. (C79). First, we recall that $\tilde{W}_{3}=\cos \theta_{\mathrm{w}} \tilde{Z}+\sin \theta_{\mathrm{w}} \tilde{\gamma}$. Second, we must replace $\tilde{W}$ with physical chargino eigenstates. This is done by inserting $P_{\mathbf{L}}+P_{\mathbf{R}}=1$ into the $W^{-} \tilde{W}_{3} \tilde{W}^{+}$interaction term in eq. (C79) and making use of eq. (C29a) and the following equation:

$$
P_{\mathrm{L}} \tilde{W}=P_{\mathrm{L}}\left[V_{11}^{*} \tilde{\chi}_{1}+V_{21}^{*} \tilde{\chi}_{2}\right]
$$

The resulting interaction is

$$
\mathscr{L}_{\mathrm{W}^{-} \tilde{\chi}^{+} \tilde{\gamma}}=e W_{\mu}^{-} \overline{\tilde{\gamma}} \gamma^{\mu}\left(P_{\mathrm{R}} U_{11}+P_{\mathrm{L}} V_{11}^{*}\right) \tilde{\chi}_{1}^{+}+e W_{\mu}^{-} \overline{\tilde{\gamma}} \gamma^{\mu}\left(P_{\mathrm{R}} U_{21}+P_{\mathrm{L}} V_{21}^{*}\right) \tilde{\chi}_{2}^{+}+\text {h.c. }
$$

The corresponding Feynman rules are shown in fig. 74.

The general $W^{-} \tilde{\chi}^{+} \tilde{\chi}^{0}$ interaction requires the use of the neutralino mixing matrix. In terms of the physical states we obtain

$$
\mathscr{L}_{\mathrm{W}^{-} \bar{\chi}^{+} \tilde{\chi}^{0}}=g W_{\mu}^{-} \overline{\tilde{\chi}}_{i}^{0} \gamma^{\mu}\left[O_{i j}^{\mathrm{L}} P_{\mathrm{L}}+O_{i j}^{\mathrm{R}} P_{\mathrm{R}}\right] \tilde{\chi}_{j}^{+},
$$

where

$$
O_{i j}^{\mathrm{L}}=-(1 / \sqrt{2}) N_{i 4} V_{j 2}^{*}+N_{i 2} V_{j 1}^{*}, \quad O_{i j}^{\mathrm{R}}=+(1 / \sqrt{2}) N_{i 3}^{*} U_{j 2}+N_{i 2}^{*} U_{j 1} .
$$

The Feynman rules are shown in fig. 75. 

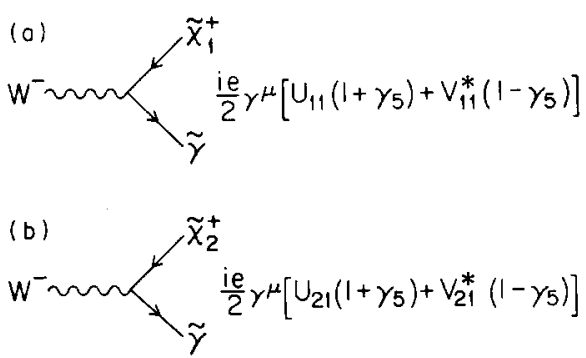

Fig. 74. Feynman rules for the interaction of the $\mathrm{W}^{+}$with a photino and chargino (assuming that the photino is one of the neutralino mass eigenstates). $U$ and $V$ are chargino mixing matrices (see appendix C.2).
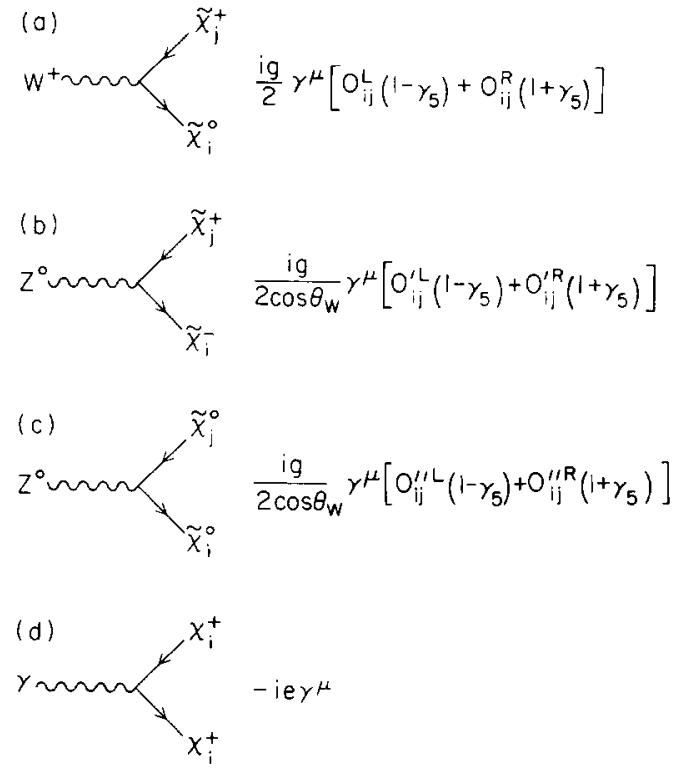

Fig. 75. Feynman rules for the interaction of a gauge boson with charginos and neutralinos. The matrices $O, O^{\prime}$ and $O^{\prime \prime}$ are defined by eqs. (C83) and (C88).

(g) Chargino-chargino- $\left(Z^{0}, \gamma\right)$; neutralino-neutralino- $\left(Z^{0}, \gamma\right)$

In two-component form, the relevant interaction is given by

$$
-g V_{\mu}^{3}\left(\bar{\lambda}^{+} \bar{\sigma}^{\mu} \lambda^{+}-\bar{\lambda}^{-} \bar{\sigma}^{\mu} \lambda^{-}\right)-\frac{1}{2}\left(g \tau_{i j}^{3} V_{\mu}^{3}+g^{\prime} \delta_{i j} V_{\mu}^{\prime}\right) \bar{\psi}_{H_{2}}^{i} \bar{\sigma}^{\mu} \psi_{H_{2}}^{j}-\frac{1}{2}\left(g \tau_{i j}^{3} V_{\mu}^{3}-g^{\prime} \delta_{i j} V_{\mu}^{\prime}\right) \bar{\psi}_{H_{1}}^{i} \bar{\sigma}^{\mu} \psi_{H_{1}}^{j} .
$$

Following our previous steps, we find that eq. (C84) in four-component form is given by

$$
-g V_{\mu}^{3} \tilde{\tilde{W}} \gamma^{\mu} \tilde{W}-\frac{1}{2}\left(g V_{\mu}^{3}+g^{\prime} V_{\mu}^{\prime}\right) \tilde{\tilde{H}} \gamma^{\mu} \tilde{H}+\frac{1}{4}\left(g V_{\mu}^{3}-g^{\prime} V_{\mu}^{\prime}\right)\left(\overline{\tilde{H}}_{1} \gamma^{\mu} \gamma_{5} \tilde{H}_{1}-\overline{\tilde{H}}_{2} \gamma^{\mu} \gamma_{5} \tilde{H}_{2}\right)
$$

In terms of the photon field $\left(A_{\mu}\right)$ and the $Z^{0}$ field, we find

$$
\begin{aligned}
& \left(g / \cos \theta_{\mathrm{w}}\right) Z_{\mu}\left[-\cos ^{2} \theta_{\mathrm{w}} \overline{\tilde{W}} \gamma^{\mu} \tilde{W}-\frac{1}{2}\left(\cos ^{2} \theta_{\mathrm{w}}-\sin ^{2} \theta_{\mathrm{w}}\right) \overline{\tilde{H}} \gamma^{\mu} \tilde{H}\right. \\
& \left.\quad+\frac{1}{4}\left(\tilde{H}_{1} \gamma^{\mu} \gamma_{5} \tilde{H}_{1}-\tilde{\tilde{H}}_{2} \gamma^{\mu} \gamma_{5} \tilde{H}_{2}\right)\right]-e A_{\mu}\left[\tilde{\tilde{W}} \gamma^{\mu} \tilde{W}+\tilde{\tilde{H}} \gamma^{\mu} \tilde{H}\right]
\end{aligned}
$$

Note that because $\tilde{H}_{1}$ and $\tilde{H}_{2}$ are neutral Majorana fermions, $\overline{\tilde{H}}_{i} \gamma^{\mu} \tilde{H}_{i}=0(i=1,2)$. Finally, we express eq. (C86) in terms of physical states:

$$
\begin{aligned}
& \mathscr{L}_{Z^{0} \tilde{\chi} \tilde{\chi}}=\left(g / \cos \theta_{\mathrm{w}}\right) Z_{\mu}\left[\overline{\tilde{\chi}}_{i}^{+} \gamma^{\mu}\left(O_{i j}^{\prime \mathrm{L}} P_{\mathrm{L}}+O_{i j}^{\prime \mathrm{R}} P_{\mathrm{R}}\right) \tilde{\chi}_{j}^{+}+\frac{1}{2} \overline{\tilde{\chi}}_{i}^{0} \gamma^{\mu}\left(O_{i j}^{\prime \mathrm{L}} P_{\mathrm{L}}+O_{i j}^{\prime \mathrm{R}} P_{\mathrm{R}}\right) \tilde{\chi}_{j}^{0}\right], \\
& \mathscr{L}_{\gamma \tilde{\chi}^{+} \tilde{\chi}^{-}}=-e A_{\mu} \overline{\tilde{\chi}}_{i}^{+} \gamma^{\mu} \tilde{\chi}_{i}^{+},
\end{aligned}
$$

where 


$$
\begin{aligned}
& O_{i j}^{\prime \mathrm{L}}=-V_{i 1} V_{j 1}^{*}-\frac{1}{2} V_{i 2} V_{j 2}^{*}+\delta_{i j} \sin ^{2} \theta_{\mathrm{w}}, \quad O_{i j}^{\prime \mathrm{R}}=-U_{i 1}^{*} U_{j 1}-\frac{1}{2} U_{i 2}^{*} U_{j 2}+\delta_{i j} \sin ^{2} \theta_{\mathrm{w}}, \\
& O_{i j}^{\prime \mathrm{L}}=-\frac{1}{2} N_{i 3} N_{j 3}^{*}+\frac{1}{2} N_{i 4} N_{j 4}^{*}, \quad O_{i j}^{\prime \mathrm{R}}=-O_{i j}^{\prime \mathrm{L} *} .
\end{aligned}
$$

In deriving these equations, we have used the unitarity properties of the matrices $U$ and $V$.

One subtlety should be pointed out in obtaining the Feynman rules for the $Z^{0} \tilde{\chi}_{i}^{0} \tilde{\chi}_{j}^{0}$ vertex. Because the Majorana field $\tilde{\chi}_{i}^{0}$ contains both creation and annihilation operators, the Feynman rule for the $Z^{0} \tilde{\chi}_{i}^{0} \tilde{\chi}_{i}^{0}$ vertex is obtained by taking the appropriate term of the interaction Lagrangian and multiplying by 2 as shown in fig. 75c. For $i \neq j$, the same result is obtained by using eq. (A34).

\section{(h) Quark-scalar-quark-gluino}

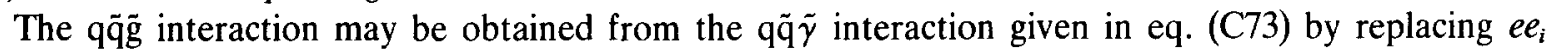
with $g_{\mathrm{s}} T^{a}$. Thus,

$$
\mathscr{L}_{\mathbf{q} \tilde{q} \tilde{\mathrm{g}}}=-\sqrt{2} g_{\mathrm{s}} T_{j k}^{a} \sum_{i=\mathrm{u}, \mathrm{d}}\left(\overline{\tilde{g}}_{a} P_{\mathrm{L}} q_{i}^{k} \tilde{q}_{i \mathrm{~L}}^{j *}+\bar{q}_{i}^{j} P_{\mathrm{R}} \tilde{g}_{a} \tilde{q}_{i \mathrm{~L}}^{k}-\overline{\tilde{g}}_{a} P_{\mathrm{R}} q_{i}^{k} \tilde{q}_{i \mathrm{R}}^{i *}-\bar{q}_{i}^{j} P_{\mathrm{L}} \tilde{g}_{a} \tilde{q}_{i \mathrm{R}}^{k}\right)
$$

where $j, k$ are color indices. The origin of the relative minus sign here is due to the fact that $\tilde{U}=u_{\mathrm{R}}^{*}$ and $\tilde{D}=d_{\mathrm{R}}^{*}$ are color antitriplets; the color generator is $-T^{*}$.

(i) Gluino-gluino-gluon

In two-component notation, we have from eq. (B1)

$$
\mathscr{L}_{\mathrm{g} \tilde{\mathrm{g}} \tilde{\mathrm{g}}}=\mathrm{i} g_{\mathrm{s}} f_{a b c} \lambda_{\mathrm{g}}^{a} \sigma^{\mu} \bar{\lambda}_{\mathrm{g}}^{b} A_{\mu}^{c} .
$$

The four-component Majorana spinor for the gluino is

$$
\tilde{g}_{a}=\left(\begin{array}{c}
-\mathrm{i} \lambda_{\mathrm{g}}^{a} \\
\mathrm{i} \bar{\lambda}_{\mathrm{g}}^{a}
\end{array}\right)
$$

In four-component notation, using eqs. (A18) and (A21), we find

$$
\mathscr{L}_{\mathrm{g} \overline{\mathrm{g} \tilde{\mathrm{E}}}}=\frac{1}{2} \mathrm{i} g_{\mathrm{s}} f_{a b c} \overline{\tilde{g}}_{a} \gamma_{\mu} \tilde{g}_{b} A_{c}^{\mu}
$$

Note that because $f_{a b c}$ is totally antisymmetric, $f_{a b c} \overline{\tilde{g}}_{a} \gamma_{\mu} \gamma_{s} \tilde{g}_{b}=0$ follows from the Majorana nature of the gluino. As before, one must multiply by 2 to obtain the Feynman rule shown in fig. 76 .

(j) Gauge boson-gauge boson-scalar-quark-scalar-quark

This interaction arises from the last term in eq. (B2):

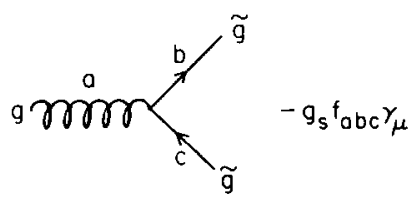

Fig. 76. Feynman rule for the interaction of a gluon with a pair of gluinos 


$$
\mathscr{L}_{\tilde{\mathbf{q} \tilde{q} \mathrm{qV}}}=g_{a} g_{b}\left(T^{a} T^{b}\right)_{i j} V_{\mu}^{a} V^{\mu b} A_{i}^{*} A_{j}
$$

The interesting feature of this interaction is that it can connect both gluons and weak gauge bosons to the same vertex. The matrices $T^{a}$ should be viewed as $\mathrm{SU}(3) \times \mathrm{SU}(2) \times \mathrm{U}(1)$ gauge group generators in block diagonal form (e.g., see eq. (B7)). It is then straightforward to derive the various interaction terms. First,

$$
\begin{aligned}
\mathscr{L}_{\mathrm{VV} \tilde{\mathrm{q}} \overline{\mathrm{q}}}= & \frac{1}{2} g^{2} W_{\mu}^{+} W^{\mu-}\left(\tilde{u}_{\mathrm{L}}^{*} \tilde{u}_{\mathrm{L}}+\tilde{d}_{\mathrm{L}}^{*} \tilde{d}_{\mathrm{L}}\right)+\frac{g}{\sqrt{2}} y_{\mathrm{Q}}\left[e A^{\mu}-\frac{g \sin ^{2} \theta_{\mathrm{w}} Z^{\mu}}{-\cos ^{-} \theta_{\mathrm{w}}^{*}}\right]\left[\tilde{u}_{\mathrm{L}}^{*} \tilde{d}_{\mathrm{L}} W_{\mu}^{+}+\tilde{d}_{\mathrm{L}}^{*} \tilde{u}_{\mathrm{L}} W_{\mu}^{-}\right] \\
& +e^{2} A_{\mu} A^{\mu} \sum_{i} e_{i}^{2} \tilde{q}_{i}^{*} \tilde{q}_{i}+\frac{g^{2}}{\cos ^{2} \theta_{\mathrm{w}}} Z_{\mu} Z^{\mu} \sum_{i}\left(T_{3 i}-e_{i} \sin ^{2} \theta_{\mathrm{w}}\right)^{2} \tilde{q}_{i}^{*} \tilde{q}_{i} \\
& +\frac{2 g e}{\cos \theta_{\mathrm{w}}} A_{\mu} Z^{\mu} \sum_{i} e_{i}\left(T_{3 i}-e_{i} \sin ^{2} \theta_{\mathrm{w}}\right) \tilde{q}_{i}^{*} \tilde{q}_{i},
\end{aligned}
$$

where the sum over $i$ goes over L-type and R-type scalar-quarks of all flavors and $y_{\mathrm{Q}}=-1+2 e_{\mathrm{u}}=$ $1+2 e_{\mathrm{d}}$. The Feynman rules are shown in fig. 77. The quantum numbers $y_{\mathrm{Q}}, e_{i}$, and $T_{3 i}$ may be obtained from table 14 . Note that because $\tilde{q}_{\mathrm{R}}$ has $T_{3}=0$, the corresponding electric charges only appears squared in eq. (C94) so the sign convention for $\tilde{q}_{R}$ does not matter. Note that the model also has four-point interactions involving two gauge bosons and two scalar-leptons. These are easily obtained from eq. (C.94) by using the appropriate values for $y_{i}, e_{i}$ and $T_{3 i}$ given in table 14 .

Second, for the term involving gluinos, we need the following formula valid for SU(3) generators:

$$
\left(T^{a} T^{b}\right)_{i j}=\frac{1}{6} \delta_{a b} \delta_{i j}+\frac{1}{2}\left(d_{a b c}+i f_{a b c}\right) T_{i j}^{c}
$$

The totally antisymmetric piece $\left(f_{a b c}\right)$ does not contribute to eq. (C93) and we are left with

$$
\mathscr{L}_{\tilde{\mathbf{q}} \overline{q g g}}=\frac{1}{6} g_{\mathrm{s}}^{2} A_{\mu}^{a} A^{\mu a} \sum_{i} \tilde{q}_{i}^{*} \tilde{q}_{i}+\frac{1}{2} g_{\mathrm{s}}^{2} d_{a b c} A_{\mu}^{a} A^{\mu b} \sum_{i}\left(\tilde{q}_{i \mathrm{~L}}^{*} T_{i j}^{c} \tilde{q}_{j \mathrm{~L}}+\tilde{q}_{i \mathrm{R}}^{*} T_{i j}^{c} \tilde{q}_{j \mathrm{R}}\right)
$$

Third, we have the mixed terms

$$
\begin{aligned}
\mathscr{L}_{\tilde{\mathbf{q}} \tilde{\mathrm{q} g} \mathrm{~g}}= & \left(\sqrt{2} g g_{\mathrm{s}}\right) A_{a}^{\mu}\left(W_{\mu}^{-} \tilde{d}_{\mathrm{L} i}^{*} T_{i j}^{a} \tilde{u}_{\mathrm{L} j}+W_{\mu}^{+} \tilde{u}_{\mathrm{L} i}^{*} T_{i j}^{a} d_{\mathrm{L} j}\right) \\
& +2 g_{\mathrm{s}} e A_{\mu} A_{a}^{\mu} \sum_{i} e_{i} \tilde{q}_{i}^{*} T_{i j}^{a} \tilde{q}_{j}+2 g_{\mathrm{s}}(g / \cos \theta) Z_{\mu} A_{a}^{\mu} \sum_{i}\left(T_{3 i}-e_{i} \sin ^{2} \theta_{\mathrm{w}}\right) \tilde{q}_{i}^{*} T_{i j}^{a} \tilde{q}_{j} .
\end{aligned}
$$

As before $e_{\tilde{\mathrm{u}} \mathrm{L}}=e_{\tilde{\mathrm{u}}_{\mathrm{R}}}=e_{\mathrm{u}}$, etc. The corresponding Feynman rules are shown in fig. 78 .

(k) Interaction of Higgs bosons

Interactions involving the Higgs bosons are model dependent for two reasons. First, the correct Higgs boson mass eigenstates depend on the details of the Higgs potential which depends on the choice of the superpotential $W$ (see eqs. (B3)-(B5)) and possible soft-supersymmetry-breaking terms (see eq. (B10)). Second, the interaction of the Higgs bosons with higgsinos depends explicitly on the super- 


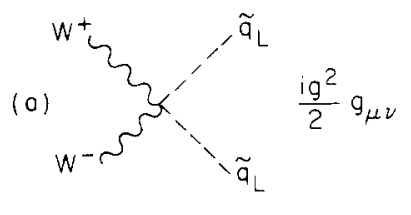

(b)

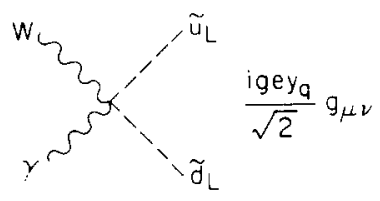

(c)

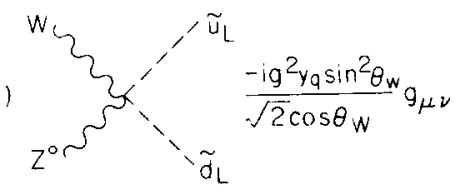

(d)<smiles>[Y][As]([Y])([Y])[Hg][3H]</smiles>

(e)

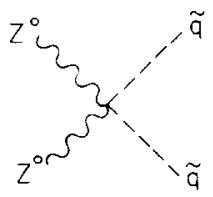

$$
\frac{2 i g^{2}}{\cos ^{2} \theta_{W}}\left(T_{3 q}-e_{q} \sin ^{2} \theta_{W}\right)^{2} g_{\mu \nu}
$$

(f)<smiles>[Y][As]([Z20])([Hg])[Hg]</smiles>

$$
\frac{2 i g e e_{q}}{\cos \theta_{w}}\left(T_{3 q}-e_{q} \sin ^{2} \theta_{w}\right) g_{\mu \nu}
$$

Fig. 77. Feynman rules for the four-point vertices involving two gauge bosons and two scalar-quarks. The quantum numbers $e_{q}, y_{q}$, and $T_{3 q}$ can be obtained from table 14. In particular, note that $y_{\mathrm{q}}=e_{\mathrm{u}}+e_{\mathrm{d}}$. Rules involving scalar-leptons are obtained by using the appropriate quantum numbers.

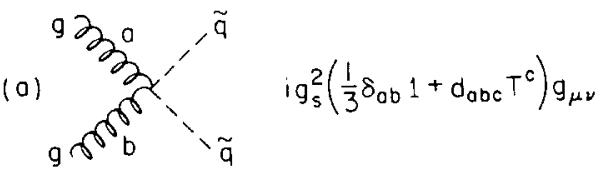

(b)

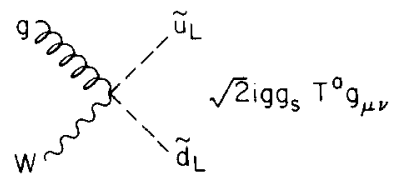

(c)

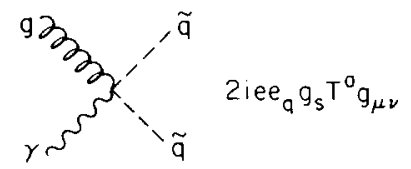

(d)

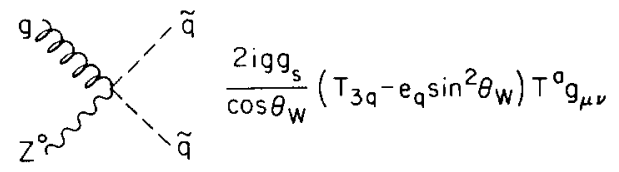

Fig. 78. Feynman rules for the four-point vertices involving two gauge bosons (one or two of which is a gluon) and two scalar-quarks. See caption to fig. 77 .

potential $W$ as shown in eq. (B6). Only the interactions of the Higgs bosons with gauginos and gauge bosons (eq. (B2)) are fixed by the supersymmetry.

We shall illustrate the calculation of Higgs interaction terms using the supersymmetric $S U(2) \times U(1)$ model introduced in appendix B.2. The superpotential is given by eq. (B11); we shall set $W_{\mathrm{F}}=0$ which is appropriate in the approximation that fermion masses are negligible as compared with gauge boson masses. We have already written out the spectrum of physical Higgs bosons in eq. (B31). Recall that in this model, the vacuum expectation values of the two Higgs fields are equal: $v_{1}=v_{2}$. We therefore make the shift: $H_{i} \rightarrow H_{i}+\left\langle H_{i}\right\rangle$ (see eq. (B20)) in the Higgs potential (eq. (B19)) and in the Higgs interaction terms eqs. (B2) and (B6). In the unitary gauge, we may simply set the Goldstone boson field $G^{+}=\left(H_{2}^{1}-H_{1}^{2 *}\right) / \sqrt{2}, G^{-}=\left(G^{+}\right)^{*}$ and $G^{0}=\operatorname{Im}\left(H_{2}^{2}-H_{1}^{1}\right)$ to zero. We then find five classes of interaction terms. 
First, there are three-point and four-point interaction terms involving gauge bosons and the SU(2) weak doublet Higgs bosons which arise from the first and third terms of eq. (B2). These may in fact be obtained from eqs. (C66) and (C94) with appropriate values of $T_{3 i}, y_{\mathrm{q}}$ and $e_{i}$ inserted. The result is

$$
\begin{aligned}
\mathscr{L}_{\mathrm{HHV}}= & \frac{-\mathrm{i} g}{\sqrt{2}}\left[W_{\mu}^{+}\left(H_{1}^{1 *} \stackrel{\leftrightarrow}{\partial}^{\mu} H_{1}^{2}+H_{2}^{1 *} \stackrel{\leftrightarrow}{\partial}^{\mu} H_{2}^{2}\right)+\text { h.c. }\right]-\frac{\mathrm{i} g}{2 \cos \theta_{\mathrm{w}}} Z_{\mu}\left\{\left(H_{1}^{1 *} \stackrel{\leftrightarrow}{\partial}^{\mu} H_{1}^{1}-H_{2}^{2 *} \stackrel{\leftrightarrow}{\partial^{\mu}} H_{2}^{2}\right)\right. \\
& \left.+\left(-1+2 \sin ^{2} \theta_{\mathrm{w}}\right)\left(H_{1}^{2 *} \stackrel{\leftrightarrow}{\partial}^{\mu} H_{1}^{2}-H_{2}^{1 *} \stackrel{\partial}{\partial}^{\mu} H_{2}^{1}\right)\right\}+\mathrm{i} e A_{\mu}\left(H_{1}^{2 *} \overleftrightarrow{\partial}^{\mu} H_{1}^{2}-H_{2}^{1 *} \stackrel{\leftrightarrow}{\partial}^{\mu} H_{2}^{1}\right), \\
\mathscr{L}_{\mathrm{HHVV}}= & \frac{1}{2} g^{2} W_{\mu}^{+} W^{\mu-}\left(\left|H_{1}^{1}\right|^{2}+\left|H_{1}^{2}\right|^{2}+\left|H_{2}^{1}\right|^{2}+\left|H_{2}^{2}\right|^{2}\right) \\
& -\frac{g}{\sqrt{2}}\left(e A^{\mu}-\frac{g \sin ^{2} \theta_{\mathrm{w}}}{-\cos ^{-} \theta_{\mathrm{w}}^{-*}} Z^{\mu}\right)\left(H_{1}^{1 *} H_{1}^{2} W_{\mu}^{+}+H_{1}^{2 *} H_{1}^{1} W_{\mu}^{-}\right. \\
& \left.-H_{2}^{1 *} H_{2}^{2} W_{\mu}^{+}-H_{2}^{2 *} H_{2}^{1} W_{\mu}^{-}\right)+e^{2} A_{\mu} A^{\mu}\left(\left|H_{1}^{2}\right|^{2}+\left|H_{2}^{1}\right|^{2}\right) \\
& +\frac{g^{2}}{4 \cos ^{2} \theta_{\mathrm{w}}} Z_{\mu} Z^{\mu}\left\{\left|H_{1}^{1}\right|^{2}+\left|H_{2}^{2}\right|^{2}+\left(1-2 \sin ^{2} \theta_{\mathrm{w}}\right)^{2}\left(\left|H_{1}^{2}\right|^{2}+\left|H_{2}^{1}\right|^{2}\right)\right\} \\
& +\frac{e g}{\cos \theta_{\mathrm{w}}} A_{\mu} Z^{\mu}\left(1-2 \sin ^{2} \theta_{\mathrm{w}}\right)\left(\left|H_{1}^{2}\right|^{2}+\left|H_{2}^{1}\right|^{2}\right) .
\end{aligned}
$$

The above expressions are not yet written in terms of physical fields. It is here where the model dependence creeps in. We shall use the physical fields given in eq. (B31) and at the same time we shall set the Goldstone boson fields to zero. This procedure implies that

$$
\begin{aligned}
& H_{1}^{1}=\frac{1}{2}\left(h_{1}^{0}+\mathrm{i} h_{2}^{0}-H^{0}\right)+v / \sqrt{2}, \\
& H_{2}^{2}=\frac{1}{2}\left(h_{1}^{0}+\mathrm{i} h_{2}^{0}+H^{0}\right)+v / \sqrt{2}, \\
& H_{2}^{1}=H_{1}^{2 *}=(1 / \sqrt{2}) H^{+} \\
& H_{1}^{2}=H_{2}^{1 *}=(1 / \sqrt{2}) H^{-}
\end{aligned}
$$

Inserting eqs. (C100)-(C103) into eqs. (C98)-(C99), we end up with the following set of interactions:

$$
\begin{aligned}
\mathscr{L}_{\mathrm{HHV}}= & -\frac{1}{2} \mathrm{i} g\left[W_{\mu}^{+} H^{-} \stackrel{\leftrightarrow}{\partial}^{\mu}\left(H^{0}+\mathrm{i} h_{2}^{0}\right)+\text { h.c. }\right] \\
& -\frac{g Z_{\mu}}{2 \cos \theta_{\mathrm{w}}}\left[H^{0} \stackrel{\leftrightarrow}{\partial}^{\mu} h_{2}^{0}-\left(2 \sin ^{2} \theta_{\mathrm{w}}-1\right) \mathrm{i} H^{-} \vec{\partial}^{\mu} H^{+}\right]-\mathrm{i} e A_{\mu} H^{-} \stackrel{\leftrightarrow}{\partial}^{\mu} H^{+}, \\
\mathscr{L}_{\mathrm{HHVV}}= & \frac{1}{4} g^{2} W_{\mu}^{+} W^{\mu-}\left(\left[H^{0}\right]^{2}+\left[h_{1}^{0}\right]^{2}+\left[h_{2}^{0}\right]^{2}+2 H^{+} H^{-}\right) \\
& +\frac{g}{2}\left[e A^{\mu}-\frac{g \sin ^{2} \theta_{\mathrm{w}}}{\cos \theta_{\mathrm{w}}} Z^{\mu}\right]\left[W_{\mu}^{+} H^{-}\left(H^{0}+\mathrm{i} h_{2}^{0}\right)+\text { h.c. }\right]+e^{2} A_{\mu} A^{\mu} H^{+} H^{-} \\
& +\frac{g^{2}}{8 \cos ^{2} \theta_{\mathrm{w}}} Z_{\mu} Z^{\mu}\left\{\left[H^{0}\right]^{2}+\left[h_{1}^{0}\right]^{2}+\left[h_{2}^{0}\right]^{2}+2\left(1-2 \sin ^{2} \theta_{\mathrm{w}}\right)^{2} H^{+} H^{-}\right\} \\
& +\frac{g e}{\cos ^{-} \theta_{\mathrm{w}}}\left(1-2 \sin ^{2} \theta_{\mathrm{w}}\right) A_{\mu} Z^{\mu} H^{+} H^{-},
\end{aligned}
$$




$$
\mathscr{L}_{\mathrm{HVV}}=g m_{\mathrm{w}} W_{\mu}^{+} W^{\mu-} h_{1}^{0}+\frac{g m_{\mathrm{z}}}{2 \cos \theta_{\mathrm{w}}} Z_{\mu} Z^{\mu} h_{1}^{0}
$$

Note that we have used $m_{\mathrm{w}}=g v / \sqrt{2}$ and $m_{\mathrm{z}}=m_{\mathrm{w}} / \cos \theta_{\mathrm{w}}$ in obtaining eq. (C106). The above interaction terms have been obtained in more general (non-supersymmetric) two-Higgs doublet models in ref. [A.36]. By comparing with the results of the next section, one sees that the neutral Higgs bosons have definite quantum numbers under parity (P) and charge conjugation (C), namely $H^{0}$ and $h_{1}^{0}$ are $0^{++}$ scalars and $h_{2}^{0}$ is a $0^{-+}$pseudoscalar.

The second class of interactions are the Higgs boson couplings to a pair of scalar-quarks or scalar-leptons. In general, the Hã $\tilde{q}$ coupling can arise from two sources - either the $F$ terms or the $D$ terms (see eqs. (B3)-(B5)). The Hã q̃ couplings which arise from the $F$-terms are proportional to the quark mass, $m_{\mathbf{q}}$ in the same way that the Hqq couplings in the standard model are proportional to $m_{\mathbf{q}}$. We shall ignore all such couplings here, but we will consider their effects in the next section. The Hã $\tilde{q}$ couplings which arise from the $D$ terms survive in the $m_{\mathrm{q}} \rightarrow 0$. We may compute these couplings by making the shift $H_{i} \rightarrow H_{i}+\left\langle H_{i}\right\rangle$ in the scalar potential (eq. (B19)) and using the expressions (eqs. (C100)-(C103)) for the physical Higgs fields. The calculation is straightforward, and we find

$$
\begin{aligned}
\mathscr{L}_{\mathrm{H} \bar{q} \tilde{q}}= & -\frac{g m_{\mathrm{w}}}{\sqrt{2}}\left[H^{+} \tilde{u}_{\mathrm{L}}^{*} \tilde{d}_{\mathrm{L}}+H^{-} \tilde{d}_{\mathrm{L}}^{*} \tilde{u}_{\mathrm{L}}\right] \\
& +\frac{g m_{\mathrm{z}}}{\cos \theta_{\mathrm{w}}} H^{0}\left(\sum_{i}\left[\left(T_{3 i}-e_{i} \sin ^{2} \theta_{\mathrm{w}}\right) \tilde{q}_{i \mathrm{~L}}^{*} \tilde{q}_{i \mathrm{~L}}+e_{i} \sin ^{2} \theta_{\mathrm{w}} \tilde{q}_{i \mathrm{R}}^{*} \tilde{q}_{i \mathrm{R}}\right]\right),
\end{aligned}
$$

where $e_{\mathrm{u} \mathrm{L}}=e_{\mathrm{u} \mathrm{L}}=e_{\mathrm{u}}$, etc. Note the sign change above; see the discussion below eq. (C73). The corresponding Feynman rules are shown in fig. 79. It should be emphasized that this is a Higgs coupling which is not proportional to the light fermion masses. It may give rise to large Higgs cross sections in some situations. Similar terms arise for scalar-leptons.

The third class of interactions are the Higgs boson couplings to a pair of charginos and/or neutralinos. There are two sources for these couplings: the gaugino-Higgsino-Higgs boson vertex (eq. (B2)) and the higgsino-higgsino-Higgs boson vertex (eq. (B6)) which depends on the form of the superpotential $W$.

For the specific choice for $W$ given by eq. (B11), we see that the resulting interaction in two-component notation is

$$
\begin{aligned}
\mathscr{L}_{\mathbf{H} \tilde{x} \tilde{x}}= & -h \varepsilon_{i j} N \psi_{H_{1}}^{i} \psi_{H_{2}}^{j}+\mathrm{i} g\left(H_{1}^{1 *} \lambda^{+} \psi_{H_{1}}^{2}+H_{1}^{2 *} \lambda^{-} \psi_{H_{1}}^{1}+H_{2}^{1 *} \lambda^{+} \psi_{H_{2}}^{2}+H_{2}^{2 *} \lambda^{-} \psi_{H_{2}}^{1}\right) \\
& +(\mathrm{i} / \sqrt{2})\left[\left(g \tau_{i j}^{3} \lambda^{3}-g^{\prime} \delta_{i j} \lambda^{\prime}\right) \psi_{H_{1}}^{j} H_{1}^{i *}+\left(g \tau_{i j}^{3} \lambda^{3}+g^{\prime} \delta_{i j} \lambda^{\prime}\right) \psi_{H_{2}}^{j} H_{2}^{i *}\right]
\end{aligned}
$$

Recall that in the notation of eq. (B31), $N=\left(h_{3}^{0}+\mathrm{i} h_{4}^{0}\right) / \sqrt{2}$, and $h=g m_{\mathrm{h}} /\left(\sqrt{2} m_{\mathrm{w}}\right)$. Furthermore, there are five two-component neutralino states, so we must generalize the formalism of section C.2. We do this by defining (analogous to eq. (C43), where $v_{1}=v_{2} \equiv v$, and eq. (C44)):

$$
\psi_{j}^{0 \prime}=\left(-\mathrm{i} \lambda_{\gamma},-\mathrm{i} \lambda_{\mathrm{z}},\left(\psi_{H_{1}}^{1}-\psi_{H_{2}}^{2}\right) / \sqrt{2},\left(\psi_{H_{1}}^{1}+\psi_{H_{2}}^{2}\right) / \sqrt{2}, \psi_{\mathrm{N}}\right), \quad \chi_{i}^{0}=N_{i j}^{\prime} \psi_{j}^{0 \prime},
$$

where the indices $i, j$ can run from 1 to 5 . 


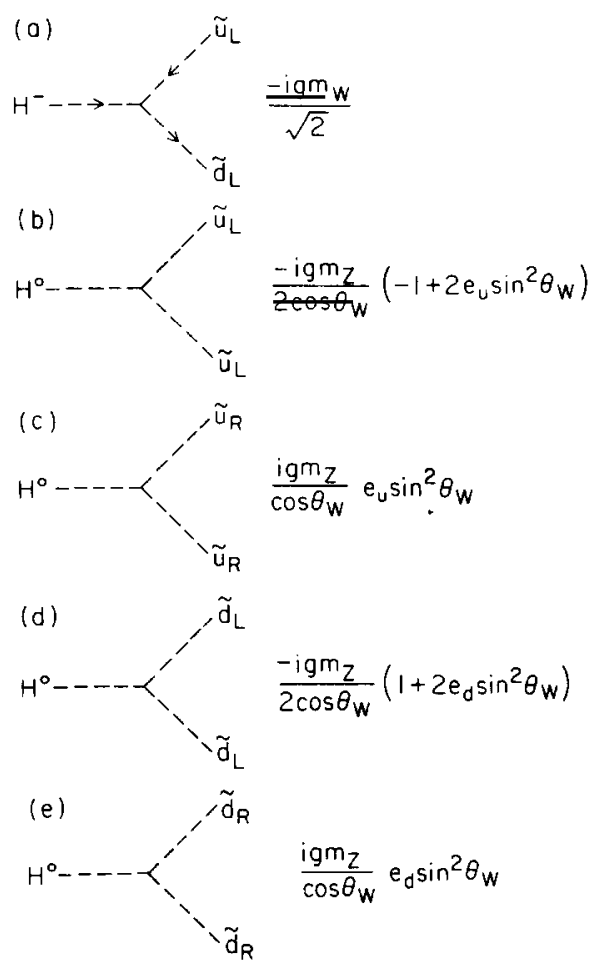

Fig. 79. Feynman rules for the interaction of the Higgs boson and a pair of scalar-quarks in the supersymmetric limit. In this limit, the $\mathrm{H}^{-}$is degenerate in mass with the $\mathrm{W}^{-}$and the $\mathrm{H}^{0}$ is degenerate in mass with the $\mathrm{Z}^{0}$. The coupling of the other physical neutral Higgs bosons to the scalar-quarks is proportional to the (fermionic) quark mass which we have neglected here. The quark charges are $e_{\mathrm{u}}=2 / 3, e_{\mathrm{d}}=-1 / 3$.

The remainder of the analysis is straightforward and involves expressing eq. (C108) in terms of mass eigenstates and converting to four-component notation. As these interactions are more model-dependent than the others considered in this appendix, we shall omit the final resulting expression, which can be found in ref. [A.37].

The fourth class of interactions consists of four-point vertices involving two Higgs bosons and two scalar-quarks (or scalar-leptons). These may be trivially read off from the scalar potential given in eq. (B19).

The fifth class of interactions are the self-couplings of the Higgs bosons. The various couplings can be obtained from the scalar potential (eq. (B19)) after shifting $H_{i} \rightarrow H_{i}+\left\langle H_{i}\right\rangle$. The algebra is straightforward, and we shall again omit the final expression.

\section{(l) Effects of nonzero fermion masses [A.37]}

Throughout these appendices, we have assumed that the quark and lepton masses were zero. This was implemented by a particular choice of the superpotential, e.g., by setting $W_{F}=0$ (see eq. (B12)). In this section, we wish to summarize briefly the effects of nonzero fermion masses. We shall designate the generic up-type and down-type quark (or lepton) masses by $m_{\mathrm{u}}$ and $m_{\mathrm{d}}$ respectively.

Consider the effects of $m_{\mathrm{u}} \neq 0, m_{\mathrm{d}} \neq 0$ in the $\mathrm{SU}(2) \times \mathrm{U}(1)$ model which result from the superpotential given by eq. (B11) and a nonzero $W_{\mathrm{F}}$ given by eq. (B12). The only change is that the $F$ terms (eqs. (B13)-(B15)) are modified, resulting in a more complicated scalar potential $V$ (which replaces (B19)). The calculation is straightforward and we shall omit all the intermediate steps. If we focus on 
just one generation of quarks (with Yukawa couplings $\lambda_{\mathbf{d}}$ and $\lambda_{\mathbf{u}}$, cf. eq. (B12)), then we find a relation between the Higgs boson-quark couplings and the quark masses

$$
\begin{aligned}
& \lambda_{\mathrm{d}}=g m_{\mathrm{d}} / m_{\mathrm{w}}, \\
& \lambda_{\mathrm{u}}=g m_{\mathrm{u}} / m_{\mathrm{w}} .
\end{aligned}
$$

In the present calculation, because supersymmetry is unbroken, we find that the up- and down-type scalar-quarks are degenerate in mass with their quark partners. This degeneracy would be broken if supersymmetry-breaking terms such as those given by eq. (B10) were introduced.

The coupling of the quarks to the Higgs boson is obtained by using eq. (B6). By working in the unitary gauge, we may use eqs. $(\mathrm{C} 100)-(\mathrm{C} 103)$ which express the components of $H_{i}^{j}$ in terms of the physical Higgs states. The resulting interaction is

$$
\begin{aligned}
\mathscr{L}_{\mathrm{Hq} \overline{\mathrm{q}}}= & -\frac{g m_{\mathrm{d}}}{2 m_{\mathrm{w}}}\left[\bar{d} d\left(h_{1}^{0}-H^{0}\right)-\mathrm{i} \bar{d} \gamma_{5} d h_{2}^{0}\right]-\frac{g m_{\mathrm{u}}}{2 m_{\mathrm{w}}}\left[\bar{u} u\left(h_{1}^{0}+H^{0}\right)-\mathrm{i} \bar{u} \gamma_{5} u h_{2}^{0}\right] \\
& +\frac{g}{\sqrt{\bar{\Sigma}} m_{\mathrm{w}}}\left[\bar{u}\left(m_{\mathrm{d}} P_{\mathrm{R}}+m_{\mathrm{u}} P_{\mathrm{L}}\right) d H^{+}+\text {h.c. }\right],
\end{aligned}
$$

where we have used eq. (A28). Equation (C112) allows us to identify $h_{1}^{0}$ and $H^{0}$ as scalars and $h_{2}^{0}$ as a pseudoscalar. The charged Higgs bosons exhibit both scalar and pseudoscalar couplings.

One also finds trilinear couplings of the scalar-quarks to the Higgs bosons which are proportional to the fermionic quark masses.* These terms arise directly from the scalar potential $V$ (which is now more complicated than eq. (B19) due to the additional $F$ terms) when the shift $H_{i} \rightarrow H_{i}+\left\langle H_{i}\right\rangle$ is made (see eq. (B20)). The computation is straightforward, and the result is

$$
\begin{aligned}
\mathscr{L}_{\mathrm{H} \tilde{q} \tilde{\bar{q}}}= & -\frac{g m_{\mathrm{u}} m_{\mathrm{h}}}{\sqrt{2} m_{\mathrm{w}}}\left[\tilde{u}_{\mathrm{L}}^{*} \tilde{u}_{\mathrm{R}} N+\text { h.c. }\right]-\frac{g m_{\mathrm{d}} m_{\mathrm{h}}}{\sqrt{2} m_{\mathrm{w}}}\left[\tilde{d}_{\mathrm{L}}^{*} \tilde{d}_{\mathrm{R}} N+\text { h.c. }\right] \\
& -\frac{g m_{\mathrm{u}}^{2}}{m_{\mathrm{w}}}\left(h_{\mathrm{L}}^{0}+H^{0}\right)\left(\tilde{u}_{\mathrm{R}}^{*} \tilde{u}_{\mathrm{R}}+\tilde{u}_{\mathrm{L}}^{*} \tilde{u}_{\mathrm{L}}\right)-\frac{g m_{\mathrm{d}}^{2}}{m_{\mathrm{w}}}\left(h_{1}^{0}-H^{0}\right)\left(\tilde{d}_{\mathrm{R}}^{*} \tilde{d}_{\mathrm{R}}+\tilde{d}_{\mathrm{L}}^{*} \tilde{d}_{\mathrm{L}}\right) \\
& +\frac{\sqrt{2} g m_{\mathrm{u}} m_{\mathrm{d}}}{m_{\mathrm{w}}}\left[\tilde{u}_{\mathrm{R}}^{*} \tilde{d}_{\mathrm{R}} H^{+}+\text {h.c. }\right]+\frac{g\left(m_{\mathrm{u}}^{2}+m_{\mathrm{d}}^{2}\right)}{\sqrt{2} m_{\mathrm{w}}}\left[\tilde{u}_{\mathrm{I}}^{*} \tilde{d}_{\mathrm{L}} H^{+}+\text {h.c. }\right]
\end{aligned}
$$

where the complex Higgs boson $\mathrm{N}$ (see eq. (B31)) has mass $m_{\mathrm{h}}=h v$. Note that the Hã $\tilde{q}$ interaction obtained above must be added to the interaction found in eq. (C107). The sources for the two interactions are different. In the latter case, the interaction is a result of the $D$ terms (eqs. (B16), (B17)) and arises because $\mathrm{H}^{ \pm}, \mathrm{H}^{0}$ are the supersymmetric partners of the $\mathrm{W}^{ \pm}, \mathrm{Z}^{0}$. In the case described above

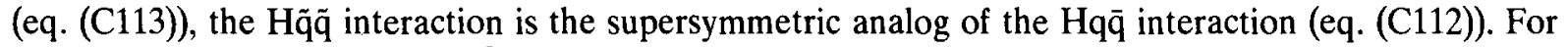
Higgs bosons other than $\mathrm{H}^{ \pm}, \mathrm{H}^{\mathbf{0}}$, the entire interaction with the scalar-quarks must be proportional to the (fermionic) quark mass.

\footnotetext{
${ }^{*}$ That is, even if the scalar-quarks get mass through supersymmetry breaking effects, the Hã $\tilde{q}$ couplings are proportional to the corresponding quark mass and not the scalar-quark mass.
} 


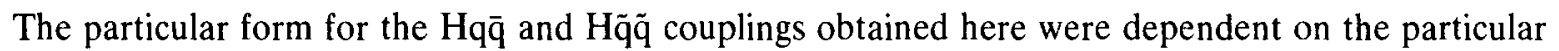
choice of the superpotential (eq. (B11)). In a more general two Higgs doublet model, eq. (C112) would be somewhat more complicated and would depend, for example, on the ratio of vacuum expectation values, $v_{1} / v_{2}[\mathrm{~A} .38$, A.39]. Likewise, eq. (C113) would also be changed. One would not in general expect $\mathrm{h}_{2}^{0}$ to decouple from the scalar-quarks as it does here in our simple model. A catalogue of the Feynman rules involving Higgs bosons in the more general supersymmetric model may be found in ref. [A.37].

\section{Appendix D. Feynman rules for Majorana particles}

We first introduce the charge conjugation matrix which satisfies the following properties:*

$$
\begin{aligned}
& \text { (i) } C^{\dagger}=C^{-1}, \\
& \text { (ii) } C^{\mathbf{T}}=-C, \\
& \text { (iii) For } \Gamma_{i}=1, \mathrm{i} \gamma_{5}, \gamma_{\mu} \gamma_{5}, \gamma_{\mu}, \sigma_{\mu \nu}=\frac{1}{2} \mathrm{i}\left[\gamma_{\mu}, \gamma_{\nu}\right], C^{-1} \Gamma_{i} C=\eta_{i} \Gamma_{i}^{\mathbf{T}} \text {, }
\end{aligned}
$$

where $\eta_{i}=+1$ for the first six $\Gamma_{i}$ and $\eta_{i}=-1$ for the last ten $\Gamma_{i}$. The $\Gamma_{i}$ have been chosen such that $\Gamma_{i}^{\dagger}=\gamma^{0} \Gamma_{i} \gamma^{0}$.

A Majorana field $\psi_{\mathrm{M}}$ satisfies:**

$$
\psi_{\mathrm{M}}=\psi_{\mathrm{M}}^{\mathrm{c}} \equiv C \bar{\psi}_{\mathrm{M}}^{\mathrm{T}}
$$

where $\bar{\psi} \equiv \psi^{\dagger} \gamma^{0}$. In general, the $u$ and $v$ spinors for either Dirac of Majorana fermions are related via

$$
u(k, s)=C \bar{v}^{\mathbf{T}}(k, s), \quad v(k, s)=C \bar{u}^{\mathbf{T}}(k, s),
$$

where $s= \pm 1 / 2$ labels spin.

Feynman rules for Majorana particles have been given in a convenient form by Jones and Llewellyn Smith [A.22]. They choose the Majorana representation $\left(\gamma_{\mu}^{*}=-\gamma_{\mu}, C=-\gamma^{0}\right.$, and $\left.\psi_{M}=\psi_{M}^{*}\right)$ in which to state their rules. Here, we shall state the rules in a representation independent fashion.

We use the following notation: $\lambda, \psi$, and $\phi$ stand for Majorana fermion, Dirac fermion and bosonic (spin 0 or 1) fields respectively. The interaction Lagrangian is assumed to have the form:

$$
\begin{aligned}
\mathscr{L}= & \frac{1}{2} \mathrm{i} \bar{\lambda}_{a}\left(\mathrm{i} \not \partial-M_{a}\right) \lambda_{a}+\bar{\psi}_{a}\left(\mathrm{i} \not \partial-m_{a}\right) \psi_{a}+\frac{1}{2} g_{a b c}^{i} \bar{\lambda}_{a} \Gamma_{i} \lambda_{b} \phi_{c}+\frac{1}{2} g_{a b c}^{i *} \bar{\lambda}_{b} \Gamma_{i} \lambda_{a} \phi_{c}^{*} \\
& +k_{a b c}^{i} \bar{\lambda}_{a} \Gamma_{i} \psi_{b} \phi_{c}^{*}+k_{a b c}^{i *} \bar{\psi}_{b} \Gamma_{i} \lambda_{a} \phi_{c} .
\end{aligned}
$$

Using eqs. (D3) and (D4), and remembering that fermion fields anticommute, we find that the following constraint must be satisfied:

$$
g_{a b c}^{i}=\eta_{i} g_{b a c}^{i} .
$$

${ }^{*}$ Note that $C^{\mathrm{T}}$ means the transpose of $C$.

${ }^{* *}$ An arbitrary phase in $C$ has been chosen so that $\left(\psi^{c}\right)^{c}=\psi$. 
We shall present the rules in a diagrammatic fashion. First, all external fermions are denoted by a straight solid line and an arrow. For Dirac fermions, the direction of the arrow is set by convention and indicates the flow of some quantum number. For Majorana fermions, the direction of the arrow is arbitrary (as Majorana fermions do not carry a conserved additive quantum number). One is free to choose the direction of arrow as a matter of convenience; the particular choice made dictates which Feynman rules are used.

Corresponding to each external fermion line in some Feynman diagram, one attaches a $u$ or $v$ spinor. For Dirac fermions, one uses $u(p)[(\bar{u}(p)]$ for an incoming [outgoing] particle state and $\bar{v}(p)[v(p)]$ for an incoming [outgoing] antiparticle state (as shown in fig. 80). For Majorana spinors, the above rule appears ambiguous as there is no distinction between particle and antiparticle. The correct procedure is to first choose a direction for the arrow on a given Majorana fermion line (the choice is arbitrary). Then, an incoming Majorana fermion line will be called a particle line if the arrow points in an incoming direction (i.e. into the Feynman diagram); if the arrow points in an outgoing direction, the line will be called an antiparticle line. Similarly, an outgoing Majorana fermion line will be called a particle line if the arrow points in an outgoing direction; if the arrow points in an incoming direction, the line will be called an antiparticle line. At this point, one may use unambiguously the rules shown in fig. 80 for external Majorana fermion lines with the additional constraint that the $u$ and $v$ spinors satisfy eq. (D5). This constraint is important when one deals with spin sums. In diagrams involving Dirac fermions, one makes use of

$$
\begin{aligned}
& \sum_{s} u^{(s)}(p) \bar{u}^{(s)}(p)=\not p+M, \\
& \sum_{s} v^{(s)}(p) \bar{v}^{(s)}(p)=\not p-M .
\end{aligned}
$$

In diagrams involving Majorana fermions the above formulas remain valid, however other combinations of the $u$ and $v$ spinors also arise. Using eq. (D5), the required relations are

$$
\begin{aligned}
& \sum_{s} u^{(s)}(p) v^{(s) \mathrm{T}}(p)=(\not p+M) C^{\mathbf{T}}, \\
& \sum_{s} \bar{u}^{(s) \mathrm{T}}(p) \bar{v}^{(s)}(p)=C^{-1}(\not p-M),
\end{aligned}
$$

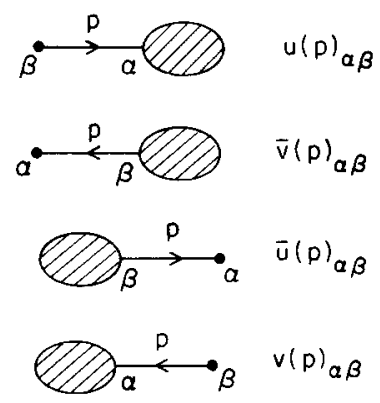

Fig. 80. Feynman rules for an external (Dirac or Majorana) fermion line. The labels $\alpha, \beta$ are spinor indices. The arrows denote flow of fermion number and do not indicate direction of momentum flow. In the graphs above momentum flow (denoted by $p$ ) is from left to right. 


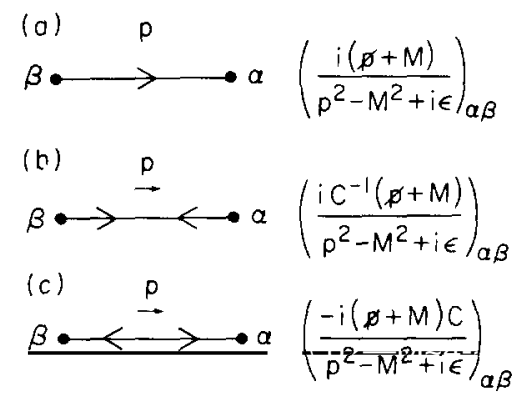

Fig. 81. Feynman rules for the Majorana fermion propagator. The matrix $C$ is the charge conjugation matrix which appears in the 'fermion-number violating' propagators which have clashing arrows (b) and (c)). The momentum flow is from left to right.

$$
\begin{aligned}
& \sum_{s} \bar{v}^{(s) \mathrm{T}}(p) \bar{u}^{(s)}(p)=C^{-1}(\not p+M), \\
& \sum_{s} v^{(s)}(p) u^{(s) \mathrm{T}}(p)=(\not p-M) C^{\mathbf{T}},
\end{aligned}
$$

where $C$ is the charge conjugation matrix which satisfies eqs. (D1)-(D3). The derivation of projection operators for specific helicity states is also straightforward.

Second, for internal fermion lines (i.e. propagators), the Dirac fermions are again denoted by a line and an arrow. However, for the Majorana fermion, there are three possibilities as shown in fig. 81 . These three cases correspond to $\lambda \bar{\lambda}, \lambda \lambda$, and $\bar{\lambda} \bar{\lambda}$; the latter two are 'fermion-number violating' propagators. Note that the numerators of the propagators (deleting the factor of $i$ ) simply correspond to the projection operators given in eqs. (D8), (D10) and (D12).

Third, the rules for the fermion-fermion-boson vertices can be read off from eq. (D6) as follows. The rules for (a) and (d) given in fig. 82 are the usual ones which we would write down for Dirac fermions. To get the rules (b) and (f), simply note that (by using eq. (D4)) $\bar{\lambda}_{a} \Gamma_{i} \lambda_{b}=-\lambda_{a}^{\mathrm{T}} C^{-1} \Gamma_{i} \lambda_{b}$. Finally, rules (c) and (e) are obtained by using $\bar{\lambda}_{a} \Gamma_{i} \lambda_{b}=\bar{\lambda}_{a} \Gamma_{i} C \bar{\lambda}_{b}^{\mathrm{T}}$. A similar analysis leads to the rules given in fig. 83 .

Finally, a factor of $1 / 2$ must be associated with each closed Majorana fermion loop due to the exchange symmetry between the internal lines.

A few additional remarks concerning the rules stated in this appendix will be helpful. First, note that the factor of $1 / 2$ which appears in eq. (D6) does not appear in the rules. To understand this point, we must consider the expansion of the Majorana fermion field in plane waves

$$
\lambda(x)=\int \frac{\mathrm{d}^{3} k}{(z \pi)^{3} 2 k_{0}} \sum_{s= \pm 1 / 2}\left[b_{s}(k) u^{(s)}(k) \exp (-\mathrm{i} k \cdot x)+b_{s}^{\dagger}(k) v^{(s)}(k) \exp (+\mathrm{i} k \cdot x)\right]
$$

where $b^{\dagger}$ and $b$ are creation and annihilation operators which satisfy the usual anticommutation relations. In order to compute the transition $\phi+\lambda_{b} \rightarrow \lambda_{a}$, we need to evaluate the matrix element

$$
\left\langle 0\left|b_{a, s_{i}}\left(k_{f}\right) \bar{\lambda}_{a}^{-} \frac{1}{2} g_{a b c}^{j} \Gamma_{j} \lambda_{b} b_{b, s_{i}}^{+}\left(k_{\mathrm{i}}\right)\right| 0\right\rangle .
$$

Inserting eq. (D14), and using appropriate anticommutation relations, we obtain

$$
\frac{1}{2}\left[g_{a b c}^{j} \bar{u}_{a}\left(k_{f}\right) \Gamma_{j} u_{b}\left(k_{i}\right)-g_{b a c}^{j} \bar{v}_{b}\left(k_{i}\right) \Gamma_{j} v_{a}\left(k_{f}\right)\right]
$$


(a)<smiles>CC(C)C(C)C(=O)OC(=O)O</smiles>

(b)

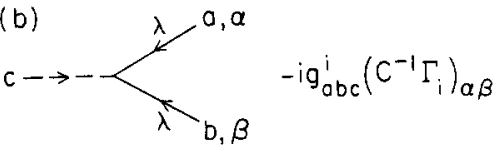<smiles>CC1C(C)C(C)(C)C1C(=O)O</smiles>

(d)

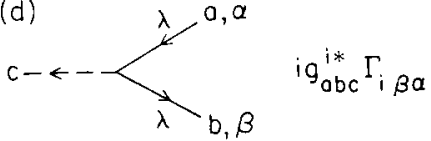

(e)<smiles>CC1C(C)C(C)(O)C1[18O]</smiles>
$i g_{o b c}^{i *}\left(\Gamma_{i} C\right)_{\beta a}$

(f)

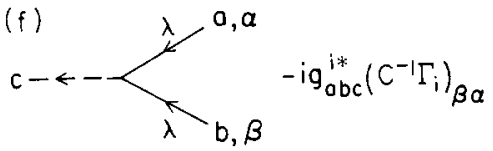

Fig. 82. Feynman rules for the interaction of a scalar (or vector) field with a pair of Majorana fermions $\lambda$. The labels $\alpha, \beta$ are spinor indices and $a, b, c$ are flavor indices. Given the interaction specified by graph (a), all the remaining rules follow. The coupling coefficients $g_{a b c}^{i}$ must satisfy $g_{a b c}^{i}=\eta_{i} g_{b a c}^{i}$, where $\eta_{i}$ depends on which combination of gamma matrices $\Gamma_{i}$ appears in rule (a) (see eq. (D3)). (o)<smiles>CCC1CC1C(C)C(=O)O</smiles>

(b)

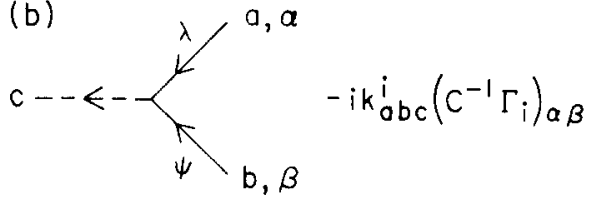

(c) $i k_{a b c}^{i *} \Gamma_{i \beta a}$<smiles>CCC1CCCCC1C(C)C</smiles><smiles>CCC1C(C(=O)O)C1(C)C</smiles><smiles>[TeH4]</smiles>

Fig. 83. Feynman rules for the interaction of a scalar (or vector) field with one Majorana $(\lambda)$ and one Dirac $(\psi)$ fermion. See caption to fig. 82. Note that there is no constraint on the coupling coefficients $k_{a b c}^{i}$.

Finally, using eqs. (D2), (D3), (D5) and (D7), one sees that the two terms above are equal and we obtain

$$
g_{a b c}^{j} u_{a}\left(k_{f}\right) \Gamma_{j} u_{b}\left(k_{\mathrm{i}}\right)
$$

which is the result one would obtain by using rule (a) of fig. 82 .

Second, it appears that the rules of fig. 82 are ambiguous. For example, why not write rule (c) as $\mathrm{i} g_{b a c}^{i}\left(\Gamma_{i} C\right)_{\beta \alpha}$ ? In fact, using eqs. (D2), (D3) and (D7), we find

$$
\mathrm{i} g_{a b c}^{i}\left(\Gamma_{i} C\right)_{\alpha \beta}=\mathrm{i} g_{a b c}^{i}\left(\Gamma_{i} C\right)_{\beta \alpha}^{\mathrm{T}}=-\mathrm{i} \eta_{i} g_{a b c}^{i}\left(\Gamma_{i} C\right)_{\beta \alpha}=-\mathrm{i} g_{b a c}^{i}\left(\Gamma_{i} C\right)_{\beta \alpha},
$$

which appears to be evidence for an ambiguity in sign. To see what this means, consider the decay process $\phi_{c} \rightarrow \lambda_{1}+\lambda_{2}$, using rule (c). If we regard 1 as $a$ and 2 as $b$, then

$$
-\mathrm{i} M=\mathrm{i} g_{12 c}^{i} \bar{u}_{1} \Gamma_{i} C \bar{u}_{2}^{\mathrm{T}}=\mathrm{i} g_{12 c}^{i} \bar{u}_{1} \Gamma_{i} v_{2}
$$


where we have used eq. (D5). On the other hand, if we regard 1 as $b$ and 2 as $a$, then

$$
-\mathrm{i} M=\mathrm{i} g_{21 c}^{i} \bar{u}_{2} \Gamma_{i} C \bar{u}_{1}^{\mathrm{T}} \text {. }
$$

Since the matrix element $M$ is a scalar, we may take the transpose of this equation: making use of eqs. (D2), (D5) and (D7), we end up with

$$
-\mathrm{i} M=-\mathrm{i} g_{12 c}^{i} \bar{u}_{1} \Gamma_{i} v_{2}
$$

which is the negative of eq. (D19). The source of the problem can be seen by returning to first principles. For $\phi_{c} \rightarrow \lambda_{1}+\lambda_{2}$, the matrix element analogous to eq. (D15) is

$$
\begin{aligned}
\left\langle 0\left|b_{2, s_{2}}\left(k_{2}\right) b_{1, s_{1}}\left(k_{1}\right) \bar{\lambda}_{a} \frac{1}{2} g_{a b c}^{j} \Gamma_{j} \lambda_{b}\right| 0\right\rangle & =\frac{1}{2}\left[g_{12 c}^{j} \bar{u}_{1}\left(k_{1}\right) \Gamma_{j} v_{2}\left(k_{2}\right)-g_{21 c}^{j} \bar{u}_{2}\left(k_{2}\right) \Gamma_{j} v_{1}\left(k_{1}\right)\right] \\
& =g_{12 c}^{j} \bar{u}_{1}\left(k_{1}\right) \Gamma_{j} v_{2}\left(k_{2}\right)=-g_{21 c}^{j} \bar{u}_{2}\left(k_{2}\right) \Gamma_{j} v_{1}\left(k_{1}\right) .
\end{aligned}
$$

This seems to imply the eq. (D19) is correct in sign whereas eq. (D20) is incorrect by an overall factor of -1 . However, we could have just as well written $\langle 0| b_{1, s_{1}}\left(k_{1}\right) b_{2, s_{2}}\left(k_{2}\right)$ in the matrix element above thereby reversing all signs in the final expressions of eq. (D22). The lesson to be learned here is that the overall sign of the Feynman rules given in fig. 82 is not well-defined in an absolute sense. One may use them to calculate any specific process by fixing ones conventions from the start (i.e., which Majorana fermion is particle $a$ and which one is particle $b$ ?). The overall sign of the matrix element will be ambiguous (but irrelevant), but the relative sign between interfering diagrams will be well defined.

One final comment about relative signs of matrix elements. The rules here are the same as they are for Dirac fermions. For example, there are two types of diagrams for $\mathrm{e}^{+} \mathrm{e}^{-} \rightarrow \tilde{\gamma} \tilde{\gamma}$ depending on whether the outgoing photinos are crossed or uncrossed. One must insert a relative minus sign between these two diagrams. (This is analogous to the relative sign between the crossed and uncrossed graphs in Moller scattering).

We will give examples of how to use these rules in appendix $\mathrm{E}$ by computing $\mathrm{e}^{+} \mathrm{e}^{-} \rightarrow \tilde{\gamma} \tilde{\gamma}, \mathrm{e}^{-} \mathrm{e}^{-} \rightarrow \tilde{\mathrm{e}}^{-} \tilde{\mathrm{e}}^{-}$ via $\tilde{\gamma}$-exchange and $\tilde{q} \overline{\tilde{q}} \rightarrow \tilde{\gamma} \tilde{\gamma}$. The only real subtlety which arises involves determining the sign of the interference term in the squared matrix element of a given process.

It is useful to have a further check on such troublesome signs. We shall illustrate one such procedure applied to the case $\mathrm{e}^{+} \mathrm{e}^{-} \rightarrow \tilde{\gamma} \tilde{\gamma}$ which has been used by Fayet [A.23]. The scattering $\mathrm{e}^{+} \mathrm{e}^{-} \rightarrow \tilde{\gamma} \tilde{\gamma}$ occurs via $\mathrm{t}$-channel exchange of $\tilde{e}_{\mathrm{L}}$ and $\tilde{\mathrm{e}}_{\mathrm{R}}$ scalar-electrons. Furthermore, one must consider two cases where the outgoing fermion lines are uncrossed and crossed. The relevant Feynman rules are given by eq. (B44). Let us ignore for the moment the $\tilde{e}_{\mathrm{L}}$ exchange diagrams. In the limit of large $\tilde{M}_{\mathrm{e}_{\mathrm{R}}}$, the scalar electron propagator is simply $-\mathrm{i} / \tilde{M}_{\mathrm{e}_{\mathrm{R}}}^{2}$ which leads to a four-fermion effective Lagrangian

$$
\mathscr{L}_{\mathrm{eff}}^{(\mathrm{R})}=\left(-2 e^{2} / \tilde{M}_{\mathrm{e}}^{2}\right) \overline{\tilde{\gamma}} P_{\mathrm{R}} e \bar{e} P_{\mathrm{L}} \tilde{\gamma}
$$

where $e=g \sin \theta_{\mathrm{w}}$. One next uses the Fierz rearrangement formula

$$
\bar{\lambda}_{1} P_{\mathrm{R}} \psi_{1} \bar{\psi}_{2} P_{\mathrm{L}} \lambda_{2}=\frac{1}{2} \bar{\lambda}_{1} P_{\mathrm{R}} \gamma^{\mu} \lambda_{2} \bar{\psi}_{2} P_{\mathrm{L}} \gamma_{\mu} \psi_{1}
$$

However, for a Majorana spinor, $\bar{\lambda} \gamma^{\mu} \lambda=0$ (see eq. (A31)). Therefore, 


$$
\mathscr{L}_{\mathrm{eff}}^{(\mathrm{R})}=\left(e^{2} / 4 \tilde{M}_{\mathrm{e}}^{2}\right) \overline{\tilde{\gamma}} \gamma^{\mu} \gamma_{5} \tilde{\gamma} \bar{e} \gamma_{\mu}\left(1+\gamma_{5}\right) e .
$$

On the other hand, if we have considered only $\tilde{\mathrm{e}}_{\mathrm{L}}$ exchange, we would interchange $L \leftrightarrow R$ in eq. (D23), leading to

$$
\mathscr{L}_{\mathrm{eff}}^{(\mathrm{L})}=\left(e^{2} / 4 \tilde{M}_{\mathrm{eR}}^{2}\right) \bar{\gamma} \gamma^{\mu} \gamma_{5} \tilde{\gamma} \bar{e} \gamma_{\mu}\left(1-\gamma_{5}\right) e .
$$

Note that if $\tilde{M}_{\mathrm{e}_{\mathrm{L}}}=\tilde{M}_{\mathrm{er}} \equiv \tilde{M}$, we would get

$$
\mathscr{L}_{\mathrm{eff}}^{(\mathrm{R})}+\mathscr{L}_{\mathrm{eff}}^{(\mathrm{L})}=\left(e^{2} / 2 \tilde{M}^{2}\right) \overline{\tilde{\gamma}} \gamma^{\mu} \gamma_{5} \tilde{\gamma} \bar{e} \gamma_{\mu} \gamma_{5} e
$$

which is manifestly parity invariant as expected. In order to convert eq. (D27) into an effective Feynman rule, we must multiply by 2 since the photinos are self-conjugate particles. The resulting rule is shown in fig. 84(a). Using this rule, one needs not cross the final state photinos. That is, this rule embodies the interference between the original crossed and uncrossed graphs.

If the photinos are massless, the chiral properties of the above vertices allows us to describe the photino as a two-component Weyl fermion (analogous to the neutrino). To see how to arrange this, we must first write the effective Lagrangian entirely in terms of a left-handed photino field. Since $\overline{\tilde{\gamma}} \gamma_{\mu} \tilde{\gamma}=0$, it is clear that eq. (D27) is equivalent to

$$
\mathscr{L}_{\mathrm{eff}}^{(\mathrm{L})}+\mathscr{L}_{\mathrm{eff}}^{(\mathrm{R})}=\left(e^{2} / \tilde{M}^{2}\right) \overline{\tilde{\gamma}} \gamma^{\mu} P_{\mathrm{L}} \tilde{\gamma} \bar{e} \gamma_{\mu} \gamma_{5} e .
$$

Using this effective Lagrangian, we may simply regard the photino as being a left-handed Weyl fermion, whereas the right-handed photino does not exist. We then distinguish between $\tilde{\gamma}$ and $\overline{\tilde{\gamma}}$ (which are now not to be considered as identical) which is completely analogous to the way one treats ordinary neutrinos. We would then write an effective Feynman rule as shown in fig. 84(b). The advantage of doing this is that there are no longer any Majorana fermions involved and the ordinary Feynman rule techniques may be employed. This method serves as one more additional check that our calculations are correct. We shall illustrate each method described above in appendix E.

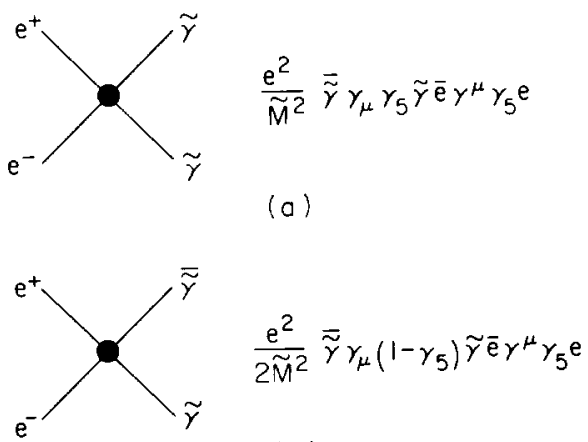

(b)

Fig. 84. Effective four-fermion vertices which arise from the exchange of a very heavy scalar-electron. We have assumed that $\tilde{M}_{\mathrm{e}_{\mathrm{L}}}=\tilde{M}_{\mathrm{e}_{\mathrm{R}}} \equiv \bar{M}$. Graph (a) is the usual rule where the photino is a Majorana fermion. Graph (b) can be used if $\bar{M}_{\gamma}=0$, in which case the $\tilde{\gamma}$ may be treated as Weyl fermion which differs from its antiparticle $\overline{\bar{\gamma}}$. 


\section{Appendix E. Sample calculations}

In this appendix, we provide details on a number of explicit calculations which should help clarify the rules given in appendices $\mathrm{C}$ and $\mathrm{D}$.

1. $e^{+} e^{-} \rightarrow \tilde{\gamma} \tilde{\gamma}$

The Feynman diagrams for this process are given in fig. 85. (For simplicity, we assume that $\tilde{e}_{\mathrm{L}}$ and $\tilde{e}_{\mathrm{R}}$ are the appropriate mass eigenstates.) The Feynman rules are given by eq. (B44) and by the rules stated in fig. 83. The rules we need are summarized in fig. 86, (the rules for $\tilde{e}_{\mathrm{L}}$ are obtained simply by replacing $\gamma_{5}$ by $-\gamma_{5}$ and multiplying the overall rule by -1$)$. Consider first $\tilde{e}_{\mathrm{R}}$ exchange. The respective amplitudes for $\mathrm{e}^{-}\left(p_{1}\right)+\mathrm{e}^{+}\left(p_{2}\right) \rightarrow \tilde{\gamma}\left(k_{1}\right)+\tilde{\gamma}\left(k_{2}\right)$ (where the four-momenta are specified in parentheses) are:

$$
\begin{aligned}
& M_{b}=\left[e^{2} / 2\left(\tilde{M}_{\mathrm{e}}^{2}-t\right)\right] \bar{u}\left(k_{1}\right)\left(1+\gamma_{5}\right) u\left(p_{1}\right) \bar{v}\left(p_{2}\right)\left(1-\gamma_{5}\right) v\left(k_{2}\right), \\
& M_{d}=\left[-e^{2} / 2\left(\tilde{M}_{\mathrm{e} \mathbf{R}}^{2}-u\right)\right] v\left(k_{2}\right)^{\mathrm{T}} C^{-1}\left(1+\gamma_{5}\right) u\left(p_{1}\right) \bar{v}\left(p_{2}\right)\left(1-\gamma_{5}\right) C \bar{u}\left(k_{1}\right)^{\mathrm{T}},
\end{aligned}
$$

where $t=\left(p_{1}-k_{1}\right)^{2}$. Using eq. (D5), we may rewrite $M_{d}$ as

$$
M_{d}=\left[e^{2} / 2\left(\tilde{M}_{\mathrm{eR}}^{2}-u\right)\right] \bar{u}\left(k_{2}\right)\left(1+\gamma_{5}\right) u\left(p_{1}\right) \bar{v}\left(p_{2}\right)\left(1-\gamma_{5}\right) v\left(k_{1}\right),
$$

where $u=\left(p_{1}-k_{2}\right)^{2}$. Note that we could have obtained $M_{d}$ directly by redrawing the arrows on fig. $85(\mathrm{~d})$ as shown in fig. 87. Thus, we see that in this case, the choice of direction for the arrows is not consequential. The next step is to compute $\left|M_{b}-M_{d}\right|^{2}$, where the relative minus sign arises due to Pauli

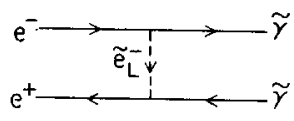

(a)

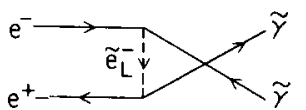

(c)

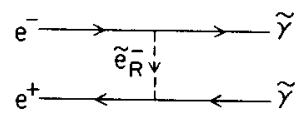

(b)

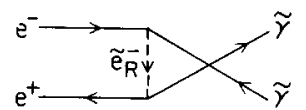

(d)
Fig. 85. Graphs for $\mathrm{e}^{+} \mathrm{e}^{-} \rightarrow \tilde{\gamma} \tilde{\gamma}$.
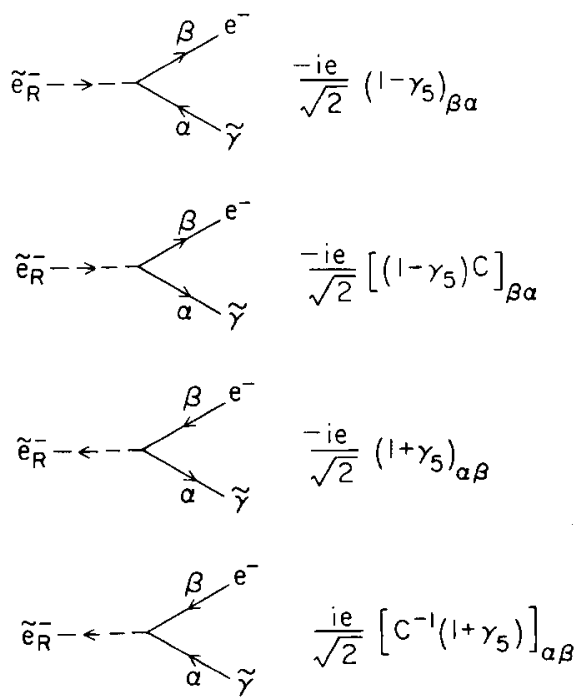

Fig. 86. Feynman rules for the $\tilde{\mathrm{e}}_{\mathrm{R}} \rightarrow \mathrm{e}^{+} \tilde{\gamma}$ vertex. Rules for the $\tilde{\mathrm{e}}_{\mathrm{L}} \rightarrow \mathrm{e}^{+} \tilde{\gamma}$ vertex can be obtained from the rules above simply by changing the sign of $\gamma_{5}$ in each case and multiplying by an overall -1 . 


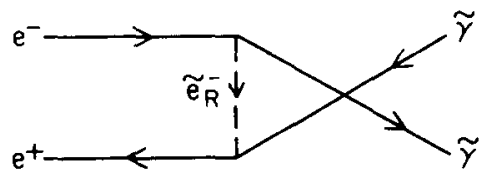

Fig. 87. Graph for $\mathrm{e}^{+} \mathrm{e}^{-} \rightarrow \tilde{\gamma} \bar{\gamma}$. A different convention for the direction of arrows on the Majorana fermion photino lines has been chosen as compared with fig. 85(d).

statistics. ${ }^{*}$ Summing over initial and final spins,

$$
\begin{aligned}
& \sum_{\text {spins }}\left|M_{b}\right|^{2}=\left[4 e^{4}\left(t-\tilde{M}_{\gamma}^{2}-m_{\mathrm{e}}^{2}\right)^{2}\right] /\left(\tilde{M}_{\mathrm{e}}^{2}-t\right)^{2} \\
& \sum_{\text {spins }}\left|M_{d}\right|^{2}=\left[4 e^{4}\left(u-\tilde{M}_{\gamma}^{2}-m_{\mathrm{e}}^{2}\right)^{2}\right] /\left(\tilde{M}_{\mathrm{e}_{\mathrm{R}}}^{2}-u\right)^{2}
\end{aligned}
$$

We work out the interference term in detail. Using eqs. (E1) and (E3),

$$
\begin{aligned}
-2 \sum_{\text {spins }} M_{b} M_{d}^{*}= & \frac{-e^{4}}{2\left(\tilde{M}_{\mathrm{eR}}^{2}-t\right)\left(\tilde{M}_{\mathrm{eR}}^{2}-u\right)} \operatorname{Tr}\left[\left(1+\gamma_{5}\right)\left(\not p_{1}+m_{\mathrm{e}}\right)\left(1-\gamma_{5}\right)\right. \\
& \left.\times\left(\mathcal{K}_{2}+\tilde{M}_{\gamma}\right) C^{\mathrm{T}}\left(1-\gamma_{5}\right)^{\mathrm{T}}\left(\not p_{2}-m_{\mathrm{e}}\right)^{\mathrm{T}}\left(1+\gamma_{5}\right)^{\mathrm{T}} C^{-1}\left(K_{1}+\tilde{M}_{\gamma}\right)\right],
\end{aligned}
$$

where we have used the projection operators given by eqs. (D8), (D9), (D10) and (D12). Using eqs. (D2) and (D3),

$$
C^{\mathrm{T}}\left(1-\gamma_{5}\right)^{\mathrm{T}}\left(\not p_{2}+m_{\mathbf{e}}\right)^{\mathrm{T}}\left(1-\gamma_{5}\right)^{\mathrm{T}} C^{-1}=\left(1-\gamma_{5}\right)\left(\not p_{2}+m_{\mathrm{e}}\right)\left(1+\gamma_{5}\right) .
$$

Therefore,

$$
\begin{aligned}
-2 \sum_{\text {spins }} M_{b} M_{d}^{*} & =\frac{-4 e^{4} \tilde{M}_{\gamma}^{2}}{\left(\tilde{M}_{\mathrm{e}_{\mathrm{R}}}^{2}-t\right)\left(\tilde{M}_{\mathrm{e}}^{2}-u\right)} \operatorname{Tr}\left[\left(1-\gamma_{5}\right) \not p_{1} \not p_{2}\right] \\
& =\left[-8 e^{4} \tilde{M}_{\tilde{\gamma}}^{2}\left(s-2 m_{\mathrm{e}}^{2}\right)\right] /\left(\tilde{M}_{\mathrm{e}}^{2}-t\right)\left(\tilde{M}_{\mathrm{e}_{\mathrm{R}}}^{2}-u\right) .
\end{aligned}
$$

The complete answer may now be obtained using similar manipulations. We quote the final result for the spin-averaged differential cross section (in the limit of $\tilde{M}_{\mathrm{er}}=\tilde{M}_{\mathrm{e}}$ ):

$$
\frac{\mathrm{d} \sigma}{\mathrm{d} \Omega}=\frac{\alpha^{2}}{4 s}\left(\frac{s-4 \tilde{M}_{\gamma}^{2}}{s-4 m_{\mathrm{e}}^{2}}\right)^{1 / 2}\left[\left(\frac{t-\tilde{M}_{\gamma}^{2}-m_{\mathrm{e}}^{2}}{\tilde{M}_{\mathrm{e}}^{2}-t}\right)^{2}+\left(\frac{u-\tilde{M}_{\gamma}^{2}-m_{\mathrm{e}}^{2}}{\tilde{M}_{\mathrm{e}}^{2}-u}\right)^{2}+\frac{\left.16 m_{\mathrm{e}}^{2} \tilde{M}_{\gamma}^{2}-2 s\left(\tilde{M}_{\gamma}^{2}+m_{\mathrm{e}}^{2}\right)\right]}{\left(\tilde{M}_{\mathrm{e}}^{2}-t\right)\left(\tilde{M}_{\mathrm{e}}^{2}-u\right)}\right]
$$

To check the sign of the interference term, let us take $\tilde{M}_{\mathrm{e}}$ large and use the effective vertex given by fig. 84(a). Instead of four diagrams, all we need to compute is the one diagram given by the effective

\footnotetext{
* The relative sign arising here is analogous to the sign which occurs between the t-channel and u-channel diagrams in $\mathrm{e}^{-} \mathrm{e}^{-} \rightarrow \mathrm{e}^{-} \mathrm{e}^{-}$. The overall sign of
} the amplitude is undefined, but irrelevant. 
vertex. The relevant trace computation is

$$
\begin{aligned}
& \left(e^{2} / \tilde{M}_{\mathrm{e}}^{2}\right)^{2} \operatorname{Tr}\left[\gamma_{\mu} \gamma_{s}\left(k_{1}+\tilde{M}_{\gamma}\right) \gamma_{\nu} \gamma_{5}\left(k_{2}-\tilde{M}_{\gamma}\right)\right] \operatorname{Tr}\left[\gamma^{\nu} \gamma_{s}\left(\not p_{1}+m_{\mathrm{e}}\right) \gamma^{\mu} \gamma_{s}\left(\not \not_{2}-m_{\mathrm{e}}\right)\right] \\
& \quad=\left(4 e^{4} / \tilde{M}_{\mathrm{e}}^{4}\right)\left[\left(t-m_{\mathrm{e}}^{2}-\tilde{M}_{\gamma}^{2}\right)^{2}+\left(u-m_{\mathrm{e}}^{2}-\tilde{M}_{\gamma}^{2}\right)^{2}+16 m_{\mathrm{e}}^{2} \tilde{M}_{\gamma}^{2}-2 s\left(\tilde{M}_{\gamma}^{2}+m_{\mathrm{e}}^{2}\right)\right]
\end{aligned}
$$

which clearly reproduces eq. (E9) in the limit of large $\tilde{M}_{\mathrm{e}}$.

Finally, it is instructive to see how one may treat photinos as left-handed Dirac particles when $\tilde{M}_{\gamma}=0$. In that case, we may use the effective vertex given by fig. $84(\mathrm{~b})$. The relevant trace computation is

$$
\begin{aligned}
\left(e^{2} / 2 \tilde{M}_{\mathrm{e}}^{2}\right)^{2} \operatorname{Tr}\left[\gamma_{\mu} \gamma_{5}\left(\not p_{1}+m_{\mathrm{e}}\right) \gamma_{\nu} \gamma_{5}\left(\not \not p_{2}-m_{\mathrm{e}}\right)\right] \operatorname{Tr}\left[\gamma^{\nu}\left(1-\gamma_{5}\right) k_{1} \gamma^{\mu}\left(1-\gamma_{5}\right) k_{2}\right] & \\
& =\left(2 e^{4} / \tilde{M}_{\mathrm{e}}^{4}\right)\left[\left(t-m_{\mathrm{e}}^{2}\right)^{2}+\left(u-m_{\mathrm{e}}^{2}\right)^{2}-2 s m_{\mathrm{e}}^{2}\right]
\end{aligned}
$$

This result is exactly one half of the expression given by eq. (E10) when $\tilde{M}_{\gamma}$ is set to zero. The reason for this factor of $\frac{1}{2}$ difference is that the cross section computed with eq. (E11) is for $\mathrm{e}^{+} \mathrm{e}^{-} \rightarrow \tilde{\gamma} \overline{\tilde{\gamma}}$ where the final states particles are not identical. On the other hand, the results of eqs. (E9) and (E10) are for $\mathrm{e}^{+} \mathrm{e}^{-} \rightarrow \tilde{\gamma} \tilde{\gamma}$ so that when the integrated total cross section* is computed, a factor of $\frac{1}{2}$ must be inserted in order that one avoid double counting of identical photinos in the final state.

The limit $\tilde{M}_{\mathrm{eL}} \rightarrow \infty$ is also an interesting limit to consider. That is, we simply ignore graphs (a) and (c) in fig. 85. The resulting cross section which replaces eq. (E9) is

$$
\frac{\mathrm{d} \sigma}{\mathrm{d} \Omega}=\frac{\alpha^{2}}{8 s}\left(\frac{s-4 \tilde{M}_{\gamma}^{2}}{s-4 m_{e}^{2}}\right)^{1 / 2}\left[\left(\frac{t-\tilde{M}_{\gamma}^{2}-m_{\mathrm{e}}^{2}}{\tilde{M}_{\mathrm{e}}^{2}-t}\right)+\left(\frac{u-\tilde{M}_{\gamma}^{2}-m_{\mathrm{e}}^{2}}{\tilde{M}_{\mathrm{e}}^{2}-u}\right)^{2}+\frac{2 \tilde{M}_{\gamma}^{2}\left(2 m_{\mathrm{e}}^{2}-s\right)}{\left(\tilde{M}_{\mathrm{e}}^{2}-t\right)\left(\tilde{M}_{\mathrm{e}}^{2}-u\right)}\right]
$$

The same expression (with $\tilde{M}_{\mathrm{e}}$ replaced with $\tilde{M}_{\mathrm{eL}}$ ) would result if we instead took $\tilde{M}_{\mathrm{e}} \rightarrow \infty$. Note that if $m_{\mathrm{e}}=0$, then eq. (E12) is exactly one-half of eq. (E9). The reason for this is that for $m_{\mathrm{e}}=0$, there is no interference between $\tilde{\mathrm{e}}_{\mathrm{L}}$ and $\tilde{\mathrm{e}}_{\mathrm{R}}$ exchanges so that the cross section is an incoherent sum of the $\tilde{\mathrm{e}}_{\mathrm{L}}$ and $\tilde{\mathrm{e}}_{\mathrm{R}}$ exchange diagrams (the crossed and uncrossed graphs still interfer except when $M_{\tilde{\gamma}}=0$ ).

For practical applications, the appropriate approximation to make is $m_{\mathrm{e}}=0$. For convenience, we shall take $\tilde{M}_{\mathrm{e}}^{2} \gg s$ which allows us to rewrite eq. (E9) as

$$
\frac{\mathrm{d} \sigma}{\mathrm{d} \Omega}=\frac{\alpha^{2} s}{8 \tilde{M}_{\mathrm{e}}^{4}}\left(1-\frac{4 \tilde{M}_{\gamma}^{2}}{s}\right)^{3 / 2}\left(1+\cos ^{2} \theta\right)
$$

Note the factor of $\left(1-4 \tilde{M}_{\gamma}^{2} / s\right)^{3 / 2}$ in eq. (E13); this signifies a P-wave annihilation. This is most easily understood using the effective Lagrangian of eq. (D27). The photinos couple to the $\mathrm{e}^{+} \mathrm{e}^{-}$current $j_{\mu}=\bar{e} \gamma_{\mu} \gamma_{s} e$. For massless electrons, this current is conserved which implies that the $\mathrm{e}^{+} \mathrm{e}^{-}$annihilation must occur in a spin 1 state. Using Fermi statistics, a spin $1 \tilde{\gamma} \tilde{\gamma}$ state must be in a P-wave. This fact can be simply read off from table 7 which is also applicable here. (If $m_{\mathrm{e}} \neq 0$, then the $\mathrm{e}^{+} \mathrm{e}^{-}$annihilation can occur in a spin 0 zero state. This would lead to a $\left(1-4 \tilde{M}_{\gamma}^{2} / s\right)^{1 / 2}$ term in eq. (E13) proportion to the electron mass.) These arguments were used by Goldberg [A.24] in discussing the cosmological constraints on the photino mass.

\footnotetext{
* The total cross section is computed by integrating over the full $4 \pi$ steradians.
} 


\section{2. ${ }^{1} S_{0} \rightarrow \tilde{\gamma} \tilde{\gamma}$}

The decay of the quarkonium state ${ }^{1} \mathrm{~S}_{0}$ into $\tilde{\gamma} \tilde{\gamma}$ may be trivially obtained from eq. (E9) (assuming that $\tilde{M}_{\mathrm{eL}}=\tilde{M}_{\mathrm{eR}}$ ). We may use the standard formula [A.25]

$$
\Gamma\left(\mathrm{S}_{0} \rightarrow \tilde{\gamma} \tilde{\gamma}\right)=4 \frac{\left|R_{\mathrm{s}}(0)\right|^{2}}{4 \pi} \lim _{s \rightarrow 4 m_{\mathrm{O}}^{2}} \frac{\left(s-4 m_{\mathrm{Q}}^{2}\right)^{1 / 2}}{m_{\mathrm{Q}}} \sigma(\mathrm{QQ} \overrightarrow{\mathrm{Q}} \rightarrow \tilde{\gamma} \tilde{\gamma})
$$

where $m_{\mathrm{Q}}$ is the mass of the quark, $v_{\text {rel }} \equiv\left(s-4 m_{\mathrm{Q}}^{2}\right)^{1 / 2} m_{\mathrm{Q}}^{-1}$ is the relative velocity of the q $\bar{q}$ pair, $\sigma(\mathrm{QQ} \rightarrow \tilde{\gamma} \tilde{\gamma})$ is the spin averaged cross section and the factor of 4 in front corrects for the fact that the ${ }^{1} \mathrm{~S}_{0}$ is pure singlet (and not a statistical mixture). $R_{\mathrm{s}}(0)$ is the radial wavefunction evaluated at the origin. Integrating eq. (E9) over angles, and inserting a factor of $1 / 2$ for identical photinos and a factor of 3 for color, we achieve

$$
\Gamma\left({ }^{1} \mathrm{~S}_{0} \rightarrow \tilde{\gamma} \tilde{\gamma}\right)=\frac{12 \alpha^{2} e_{\mathrm{Q}}^{4} \tilde{M}_{\tilde{\gamma}}^{2}\left(m_{\mathrm{Q}}^{2}-\tilde{M}_{\gamma}^{2}\right)^{1 / 2}\left|R_{\mathrm{s}}(0)\right|^{2}}{m_{\mathrm{Q}}\left(\tilde{M}_{\mathrm{Q}}^{2}+m_{\mathrm{Q}}^{2}-\tilde{M}_{\gamma}^{2}\right)^{2}}
$$

which agrees with the result stated in eq. (5.10).

If we take $\tilde{M}_{\mathrm{Q}_{\mathrm{L}}} \neq \tilde{M}_{\mathrm{Q}_{\mathrm{R}}}$, it turns out that it is incorrect to use eq. (E14). The reason is somewhat subtle. If $\tilde{M}_{\mathrm{Q}_{\mathrm{L}}} \neq \tilde{M}_{\mathrm{Q}_{\mathrm{R}}}$, the theory is not $P$ or $C$ invariant (although $C P$ is still conserved). Thus, the process $\mathrm{QQ} \rightarrow \tilde{\gamma} \tilde{\gamma}$ no longer guarantees that the initial $\mathrm{Q} \bar{Q}$ state is in a $C$-even state. Hence, we must compute $\Gamma\left({ }^{1} \mathrm{~S}_{0} \rightarrow \tilde{\gamma} \tilde{\gamma}\right)$ by correctly projecting out the ${ }^{1} \mathrm{~S}_{0}$ state from the $\mathrm{Q} \overline{\mathrm{Q}} \rightarrow \tilde{\gamma} \tilde{\gamma}$ amplitude. This is clearly illustrated by taking the limit where $\tilde{M}_{\mathrm{Q}_{\mathrm{L}}} \rightarrow \infty$, so that diagrams involving $\tilde{Q}_{\mathrm{L}}$ exchange are omitted. The correct expression for $\Gamma\left({ }^{1} \mathrm{~S}_{0} \rightarrow \tilde{\gamma} \tilde{\gamma}\right)$ is obtained by taking $\frac{1}{4}$ of eq. (E15) (with $\tilde{M}_{\mathrm{Q}}$ replaced by $\tilde{M}_{\mathrm{Q}_{\mathrm{R}}}$ ). Had we used eq. (E12) in eq. (E14), we would have gotten the wrong answer.

The reason that the factor of $\frac{1}{4}$ is correct can be seen by using the effective Lagrangians given by eqs. (D25)-(D27). If

$$
M(\overline{\mathrm{QQ}} \rightarrow \tilde{\gamma} \tilde{\gamma})=\left(e^{2} e_{\mathrm{O}}^{2} / 2 \tilde{M}^{2}\right) \bar{u}\left(k_{1}\right) \gamma_{\mu} \gamma_{5} v\left(k_{2}\right) \bar{v}\left(p_{2}\right) \gamma^{\mu}\left(g_{\mathrm{V}}+g_{\mathrm{A}} \gamma_{5}\right) u\left(p_{1}\right)
$$

then, we find in the nonrelativistic approximation (i.e., $s \rightarrow m_{\mathrm{Q}}^{2}$ ):

$$
M\left({ }^{1} \mathrm{~S}_{0} \rightarrow \tilde{\gamma} \tilde{\gamma}\right)=\left(g_{\mathrm{A}} e^{2} e_{\mathrm{Q}}^{2} / \tilde{M}^{2}\right) \sqrt{2 m_{\mathrm{O}}}\left(R_{\mathrm{s}}(0) / \sqrt{4 \pi}\right) \bar{u}\left(k_{1}\right) \gamma_{0} \gamma_{5} v\left(k_{2}\right)
$$

Thus, the width $\Gamma\left({ }^{1} \mathrm{~S}_{0} \rightarrow \tilde{\gamma} \tilde{\gamma}\right)$ is proportional to $g_{\mathrm{A}}^{2}$. Since $g_{\mathrm{A}}=1$ if only $\tilde{\mathrm{Q}}_{\mathrm{L}}$ contributes but $g_{\mathrm{A}}=2$ if both $\tilde{Q}_{L}$ and $\tilde{Q}_{R}$ of equal mass contribute, we arrive at the conclusion stated above. The exact formula for unequal scalar-quark masses is given in eq. (5.10).

\section{3. $e^{+} e^{-} \rightarrow \gamma \tilde{\gamma} \tilde{\gamma},{ }^{3} S_{1} \rightarrow \gamma \tilde{\gamma} \tilde{\gamma}$}

The process $\mathrm{e}^{+} \mathrm{e}^{-} \rightarrow \gamma \tilde{\gamma} \tilde{\gamma}$ has been computed in refs. [A.26-A.29, A.42, A.43]. We may calculate the rate in the limit of large $\tilde{M}_{\mathrm{e}_{\mathrm{L}}}=\tilde{M}_{\mathrm{e}} \equiv \tilde{M}$. This allows us to use the effective vertices given in fig. 84. For example, for ${ }^{3} \mathrm{~S}_{1} \rightarrow \gamma \tilde{\gamma} \tilde{\gamma}$, we would compute the diagrams similar to the ones given in fig. 88 . There are two diagrams 


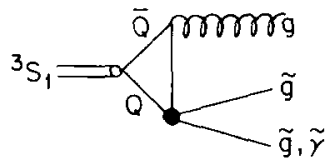

(a)

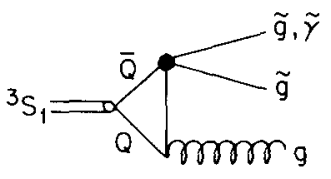

(b)

Fig. 88. Graphs for the decay of the quarkonium state ${ }^{3} \mathrm{~S}_{1}$ into supersymmetric particles using the effective vertex given in fig. 84 .

since the photon can be emitted from two possible places. ${ }^{*}$ We define the variables $x=2 k_{\gamma} / \sqrt{s}$ and $y=\cos \theta$ where $\sqrt{s}$ is the $\mathrm{e}^{+} \mathrm{e}^{-}$center-of-mass energy, $k_{\gamma}$ is the outgoing photon momentum in the $\mathrm{e}^{+} \mathrm{e}^{-}$ center-of-mass frame and $\theta$ is the photon scattering angle (with respect to the electron) in this frame. The kinematical limits are $0 \leq x \leq 1,-1 \leq y \leq 1$. The result of the calculation is

$$
\mathrm{d} \sigma / \mathrm{d} x \mathrm{~d} y=\left(x / 2^{11} \pi^{4}\right)\left(1-4 m_{\mathrm{e}}^{2} / s\right)^{-1 / 2} F\left(x, y, s, m_{e}^{2}, \tilde{M}_{\tilde{\gamma}}^{2}\right),
$$

where the exact formula for $F$ is complicated. For $m_{\mathrm{e}}=\tilde{M}_{\gamma}=0$, we find

$$
F(x, y, s, 0,0)=32 \pi e^{6} s(1-x)\left[x^{2} y^{2}+(2-x)^{2}\right] / 3 \tilde{M}^{4} x^{2}\left(1-y^{2}\right) .
$$

Inserting this into eq. (E18) we obtain an answer which is twice the result obtained by Ellis and Hagelin [A.28]. This is consistent because ref. [A.28] takes $\tilde{M}_{\mathrm{eL}} \rightarrow \infty$ (i.e., omits $\tilde{e}_{\mathrm{L}}$ exchange) whereas we have set $\tilde{M}_{\mathrm{el}}=\tilde{M}_{\mathrm{e}}$. When $m_{\mathrm{e}}=0$, there is no interference between $\tilde{\mathrm{e}}_{\mathrm{L}}$ and $\tilde{\mathrm{e}}_{\mathrm{R}}$ exchanges so that our answer should be twice as large as theirs.

We may also use eq. (E18) to obtain $\Gamma\left({ }^{3} \mathrm{~S}_{1} \rightarrow \gamma \tilde{\gamma} \tilde{\gamma}\right)$. If $\tilde{M}_{\mathrm{Q}_{\mathrm{L}}}=\tilde{M}_{\mathrm{Q}_{\mathrm{R}}} \equiv \tilde{M}$, we may use the standard formula [A.25]

$$
\Gamma\left({ }^{3} \mathrm{~S}_{1} \rightarrow \tilde{\gamma} \tilde{\gamma} \gamma\right)=\frac{4}{3} \frac{\left|R_{s}(0)\right|^{2}}{4 \pi} \lim _{s \rightarrow 4 m_{\mathrm{Q}}^{2}} \frac{\left(s-4 m_{\mathrm{Q}}^{2}\right)^{1 / 2}}{m_{\mathrm{Q}}} \sigma(\mathrm{Q} \overline{\mathrm{Q}} \rightarrow \tilde{\gamma} \tilde{\gamma} \gamma)
$$

We may use eq. (E18); the relevant expression for $F$ is

$$
\lim _{s \rightarrow 4 m_{\mathrm{O}}^{2}} F\left(x, y, s, m_{\mathrm{Q}}^{2}, 0\right)=128 \pi e^{6} m_{\mathrm{Q}}^{2}(2-x) / 3 \tilde{M}^{4}
$$

for large $\tilde{M}$ and zero photino mass. The final result (remembering to include a factor of $\frac{1}{2}$ for identical final state photinos) is

$$
\Gamma\left({ }^{3} \mathrm{~S}_{1} \rightarrow \tilde{\gamma} \tilde{\gamma} \gamma\right)=32 \alpha^{3} e_{\mathrm{Q}}^{6} m_{\mathrm{Q}}^{2}\left|R_{s}(0)\right|^{2} / 9 \pi \tilde{M}^{4}
$$

To compute the decay rate for the processes shown in fig. 88 one needs to insert the appropriate color factors (see eq. (5.5)).

\footnotetext{
* In principle, the photon can be emitted from the effective vertex itself (i.e., off the scalar-electron line), but for large scalar-electron mass, this is negligible.
} 


\section{4. $e^{-} e^{-} \rightarrow \tilde{e}^{-} \tilde{e}^{-}$}

The calculation of this process was performed by Keung and Littenberg [A.30]. It is instructive to see how the rules of appendix $D$ (regarding the t-channel photino exchange) are used. These calculations are also relevant for $q q \rightarrow \tilde{q} \tilde{q}$ which is one way to make scalar-quarks in hadronic colliders.

We shall consider separately the processes $\mathrm{e}^{-} \mathrm{e}^{-} \rightarrow \tilde{\mathrm{e}}_{L}^{-} \tilde{\mathrm{e}}_{R}^{-}, \mathrm{e}^{-} \mathrm{e}^{-} \rightarrow \tilde{\mathrm{e}}_{L}^{-} \tilde{\mathrm{e}}_{L}^{-}$and $\mathrm{e}^{-} \mathrm{e}^{-} \rightarrow \tilde{\mathrm{e}}_{R}^{-} \tilde{\mathrm{e}}_{R}^{-}$. In each case there are two graphs shown in fig. 89.

We find (taking the electron as massless)

$$
\begin{aligned}
& M_{a}\left(\mathrm{e}^{-} \mathrm{e}^{-} \rightarrow \tilde{\mathrm{e}}_{\mathrm{L}}^{-} \tilde{\mathrm{e}}_{\mathrm{R}}^{-}\right)=\frac{-e^{2}}{2 t} u^{\mathrm{T}}\left(p_{2}\right)\left[C^{-1}\left(1+\gamma_{5}\right)\right]^{\mathrm{T}}\left(p_{1}-k_{1}+\tilde{M}_{\gamma}\right)\left(1-\gamma_{5}\right) u\left(p_{1}\right), \\
& M_{b}\left(\mathrm{e}^{-} \mathrm{e}^{-} \rightarrow \tilde{\mathrm{e}}_{L}^{-} \tilde{\mathrm{e}}_{\mathrm{R}}^{-}\right)=\frac{-e^{2}}{2 u} u^{\mathrm{T}}\left(p_{2}\right)\left[C^{-1}\left(1-\gamma_{5}\right)\right]^{\mathrm{T}}\left(\not p_{1}-k_{2}+\tilde{M}_{\gamma}\right)\left(1+\gamma_{5}\right) u\left(p_{1}\right),
\end{aligned}
$$

where we have used the rules of figs. 80, 81, and 86. From eqs. (D2), (D3) and (D5),

$$
u^{\mathrm{T}}\left(p_{2}\right)\left[C^{-1}\left(1+\gamma_{5}\right)\right]^{\mathrm{T}}=\bar{v}\left(p_{2}\right)\left(1+\gamma_{5}\right)
$$

The rest of the computation is straightforward. We compute $\left|M_{a}+M_{b}\right|^{2}$ averaged over initial spins as usual. Note that there is a relative plus sign between $M_{a}$ and $M_{b}$ in spite of identical fermions in the initial state. ${ }^{*}$ The interference term in this case is actually zero and the end result is

$$
\frac{\mathrm{d} \sigma}{\mathrm{d} t}\left(\mathrm{e}^{-} \mathrm{e}^{-} \rightarrow \tilde{\mathrm{e}}_{\mathrm{L}}^{-} \tilde{\mathrm{e}}_{\mathrm{R}}^{-}\right)=\frac{\pi \alpha^{2}\left(t u-\tilde{M}_{\mathrm{eL}}^{2} \tilde{M}_{\mathrm{e}}^{2}\right)}{s^{2}}\left[\frac{1}{\left(t-\tilde{M}_{y}^{2}\right)^{2}}+\frac{1}{\left(u-\tilde{M}_{y}^{2}\right)^{2}}\right] .
$$

Note that when $\tilde{M}_{\tilde{y}}=0$, this process still occurs. This illustrates the fact that fermion-number violation (which occurs in this process) persists even in the limit of a zero-mass photino! The exchange of the Majorana photino is the source of this fermion-number violation. In practice, the scalar-electrons would

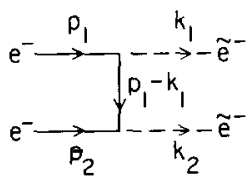

(a)

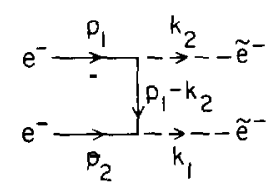

(b)

Fig. 89. Graphs for $\mathrm{e}^{-} \mathrm{e}^{-} \rightarrow \tilde{\mathrm{e}}^{-} \tilde{\mathrm{e}}^{-}$. Graph (b) is the crossed version of graph (a). The final state can be either $\tilde{e}_{\mathbf{L}}^{-} \bar{e}_{\bar{L}}, \bar{e}_{\bar{R}} \bar{e}_{\bar{R}}^{-}$or $\mathrm{e}_{\bar{L}}^{-} e_{\bar{R}}$. The four-momenta of all the lines are indicated.

* To check this claim, note that we may rewrite $M_{b}$ as follows. First take the transpose of $M_{b}$ (which does not affect it since it is a scalar). Using eq. (D2) and (D3) and putting $p_{1}-k_{2}=k_{1}-p_{2}$, we end up with

$$
M_{b}\left(\mathrm{e}^{-} \mathrm{e}^{--} \rightarrow \tilde{\mathrm{e}}_{\mathrm{L}}^{-} \overline{\mathrm{e}}_{\mathrm{R}}^{-}\right)=\frac{e^{2}}{2 u} u^{\mathrm{T}}\left(p_{1}\right)\left[C^{-1}\left(1+\gamma_{5}\right)\right]^{\mathrm{T}}\left(p_{2}-k_{1}+\tilde{M}_{y}\right)\left(1-\gamma_{5}\right) u\left(p_{2}\right)
$$

Comparing with eq. (E23), we see that $M_{b}$ can be obtained from $M_{a}$ by interchanging $p_{1}$ and $p_{2}$ and multiplying by -1 . This is consistent with the Pauli principle applied to the incoming electrons. No additional minus sign is needed. 
immediately decay $\tilde{\mathrm{e}}^{-} \rightarrow \mathrm{e}^{-}+\tilde{\gamma}{ }^{*}$ so that one could not directly observe this fermion-number violation. We now turn to $\mathrm{e}^{-} \mathrm{e}^{-} \rightarrow \tilde{\mathrm{e}}_{\mathrm{L}}^{-} \tilde{\mathrm{e}}_{\mathrm{L}}^{-}$. The amplitudes are similar to those given by eq. (E23) and (E24) except that all factors of $1-\gamma_{5}$ which appear there now become $1+\gamma_{5}$ and vice versa. Simple Dirac algebra then shows that the amplitudes are proportional to $\tilde{M}_{\tilde{\gamma}}$. The interference term does not vanish in this case, but it is constructive. The final result is

$$
\frac{\mathrm{d} \sigma}{\mathrm{d} t}\left(\mathrm{e}^{-} \mathrm{e}^{-} \rightarrow \tilde{\mathrm{e}}_{\mathrm{L}}^{-} \tilde{\mathrm{e}}_{\mathrm{L}}^{-}\right)=\frac{1}{2} \frac{\pi \alpha^{2} \tilde{M}_{\gamma}^{2}}{s}\left(\frac{1}{t-\tilde{M}_{\gamma}^{2}}+\frac{1}{u-\tilde{M}_{\gamma}^{2}}\right)^{2} .
$$

The factor of $\frac{1}{2}$ in front is inserted when integrating over $4 \pi$ steradians to get the total cross section in order to account for identical particles in the final state. The cross section for $\mathrm{e}^{-} \mathrm{e}^{-} \rightarrow \tilde{\mathrm{e}}_{\mathrm{R}}^{-} \tilde{\mathrm{e}}_{\mathrm{R}}^{-}$is identical to the one given in eq. (E27).

It is instructive to see what would happen to the computation of the matrix element for $\mathrm{e}^{-} \mathrm{e}^{-} \rightarrow \tilde{\mathrm{e}}_{\mathrm{L}}^{-} \tilde{\mathrm{e}}_{\mathrm{R}}^{-}$ if we switched the direction of the arrow on the photino line in fig. 89 and used the conventions shown in fig. 90 . We now obtain

$$
M_{a}=\frac{-e^{2}}{2 t} u^{\mathrm{T}}\left(p_{2}\right)\left(1+\gamma_{5}\right)^{\mathrm{T}}\left(k_{1}-\not p_{1}+\tilde{M}_{\gamma}\right)^{\mathrm{T}} C^{-1}\left(1-\gamma_{5}\right) u\left(p_{1}\right)
$$

where we have again used the rules of figs. 80, 81, 83, and 86. From eqs. (D3) and (D5),

$$
u^{\mathrm{T}}\left(p_{2}\right)\left(1+\gamma_{5}\right)^{\mathrm{T}}\left(k_{1}-\not p_{1}+\tilde{M}_{\gamma}\right)^{\mathrm{T}} C^{-1}=-\bar{v}\left(p_{2}\right)\left(1+\gamma_{5}\right)\left(\not p_{1}-k_{1}+\tilde{M}\right)
$$

Comparing these results with those of eqs. (E23) and (E25), we see that the two matrix elements (obtained by the different arrow conventions on the photino line) differ by an overall minus sign. Thus, the matrix element appears ambiguous in sign. This ambiguity is exactly analogous to the ambiguous sign on the $\phi \lambda \lambda$ rule discussed below eq. (D17) in appendix D. Namely in this case, one can always redefine the initial state as to which electron comes first (the two choices differ in sign). The conclusion is the same as discussed in appendix D. One must fix the convention from the start. The overall sign of the matrix element is undefined but irrelevant. The relative sign between graphs (a) and (b) of fig. 89 is physical. Thus, one must choose the direction of the photino arrow in graphs (a) and (b) in the same manner. This will insure the correct positive sign for the interference term as obtained in eq. (E27).

Finally, we may use the results of eqs. (E26) and (E27) to get the cross section for qq $\rightarrow \tilde{q} \tilde{q}$. Let us define the cross section to be

$$
\begin{aligned}
& \frac{\mathrm{d} \sigma}{\mathrm{d} t}(\mathrm{qq} \rightarrow \tilde{\mathrm{q}} \tilde{\mathrm{q}}) \equiv \frac{\mathrm{d} \sigma}{\mathrm{d} t}\left(\mathrm{qq} \rightarrow \tilde{\mathrm{q}}_{\mathrm{L}} \tilde{\mathrm{q}}_{\mathrm{L}}\right)+\frac{\mathrm{d} \sigma}{\mathrm{d} t}\left(\mathrm{qq} \rightarrow \tilde{\mathrm{q}}_{\mathrm{R}} \tilde{\mathrm{q}}_{\mathrm{R}}\right)+\frac{\mathrm{d} \sigma}{\mathrm{d} t}\left(\mathrm{qq} \rightarrow \tilde{\mathrm{q}}_{\mathrm{L}} \tilde{\mathrm{q}}_{\mathrm{R}}\right) .
\end{aligned}
$$

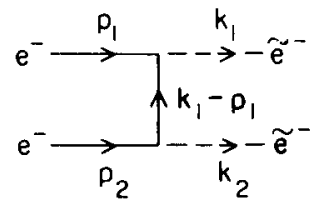

Fig. 90. Graph for $\mathrm{e}^{-} \mathrm{e}^{-} \rightarrow \tilde{\mathrm{e}}^{-} \tilde{\mathrm{e}}^{-}$. The arrow convention on the exchanged photino is different from the one chosen in fig. 89(a).

${ }^{*}$ Note that $\mathrm{e}^{-}$and $\tilde{\mathrm{e}}^{-}$carry one unit of lepton number, so that the lepton number is still conserved. 
The color factors [A.31] for $\left|M_{\mathrm{A}}\right|^{2}$ and $\left|M_{\mathrm{B}}\right|^{2}$ are $2 / 9$ and for $2 \operatorname{Re} M_{\mathrm{A}} M_{\mathrm{B}}^{*}$ is $-2 / 27$. Thus, the end result (where we take $\tilde{M}_{\mathrm{q}_{\mathrm{L}}}=\tilde{M}_{\mathrm{q}_{\mathrm{R}}} \equiv \tilde{M}_{\mathrm{q}}$ for simplicity) is

$$
\frac{\mathrm{d} \sigma}{\mathrm{d} t}=\frac{2 \pi \alpha_{\mathrm{s}}^{2}}{9 s^{2}}\left[\left(s \tilde{M}_{\gamma}^{2}+u t-\tilde{M}_{\mathrm{q}}^{4}\right)\left(\frac{1}{\left(\tilde{M}_{\gamma}^{2}-t\right)^{2}}+\frac{1}{\left(\tilde{M}_{\gamma}^{2}+u\right)^{2}}\right)-\frac{2}{3\left(\tilde{M}_{\gamma}^{2}-t\right)\left(\tilde{M}_{\gamma}^{2}-u\right)}\right] .
$$

A similar expression has been obtained in ref. [A.32]. In order to compute the total cross section, a factor of $1 / 2$ might be inserted into the expression of ref. [A.32]. The resulting answer then agrees with our result given by eq. (E31).

\section{5. ${ }^{1} S_{0} \rightarrow \tilde{\gamma} \tilde{\gamma}$ revisited}

In this section, we note that one can have scalar-quarkonium states with $0^{-+}$quantum numbers which can decay into two photinos. For simplicity, take the masses of $\tilde{Q}_{L}$ and $\tilde{Q}_{R}$ to be equal. Then, by using the results of appendix C.1, we define scalar and pseudoscalar states:

$$
\tilde{Q}_{\mathrm{p}} \equiv(1 / \sqrt{2})\left(\tilde{q}_{\mathrm{R}}+\tilde{q}_{\mathrm{L}}\right), \quad \tilde{Q}_{\mathrm{s}} \equiv(1 / \sqrt{2})\left(\tilde{q}_{\mathrm{R}}-\tilde{q}_{\mathrm{L}}\right)
$$

In terms of these states, the interaction given by eq. (B44) in appendix B becomes:

$$
-e\left[\bar{Q} \tilde{\gamma} \tilde{Q}_{\mathrm{s}}+\overline{\tilde{\gamma}} Q \tilde{Q}_{\mathrm{s}}^{*}+\bar{Q} \gamma_{5} \tilde{\gamma} \tilde{Q}_{\mathrm{p}}-\overline{\tilde{\gamma}} \gamma_{\mathrm{s}} Q \tilde{Q}_{\mathrm{p}}^{*}\right]
$$

The ${ }^{1} \mathrm{~S}_{0}$ state arises from a bound state of $\tilde{\mathrm{Q}}_{s} \overline{\tilde{Q}}_{\mathrm{p}}$ and $\tilde{\mathrm{Q}}_{\mathrm{p}} \overline{\tilde{Q}}_{\mathrm{s}}$. The relevant Feynman graphs are given by fig. 91 ; note the convention we have chosen for the photino arrows. We compute the continuum scattering (at threshold) for $\tilde{\mathrm{Q}}_{\mathrm{s}}\left(p_{1}\right)+\tilde{\mathrm{Q}}_{\mathrm{p}}\left(p_{2}\right) \rightarrow \tilde{\gamma}\left(k_{1}\right)+\tilde{\gamma}\left(k_{2}\right)$. The matrix elements are

$$
\begin{aligned}
M_{a} & =\frac{e^{2} e_{\mathrm{Q}}^{2}}{t=m_{\mathrm{O}}^{2}} \bar{u}\left(k_{1}\right)\left(k_{1}-\not p_{1}+m_{\mathrm{Q}}\right) \gamma_{5} v\left(k_{2}\right), \\
M_{b} & =\frac{\overline{e^{2} e_{\mathrm{Q}}^{2}}}{u-m_{\mathrm{Q}}^{2}} v^{\mathrm{T}}\left(k_{2}\right)\left(-C^{-1}\right)\left(k_{2}-\not p_{1}+m_{\mathrm{O}}\right) \gamma_{5} C \bar{u}\left(k_{1}\right)^{\mathrm{T}} .
\end{aligned}
$$

We rewrite $M_{b}$ by noting that it is a scalar so that we can take its transpose without changing its value:

$$
M_{b}=\left(-e^{2} e_{\mathrm{O}}^{2} / u-m_{\mathrm{O}}^{2}\right) \bar{u}\left(k_{1}\right) C \gamma_{\mathrm{S}}^{\mathrm{T}}\left(k_{2}-\not p_{1}+m_{\mathrm{Q}}\right)^{\mathrm{T}} C^{-1} v\left(k_{2}\right) \text {. }
$$

Using eq. (D3), we end up with

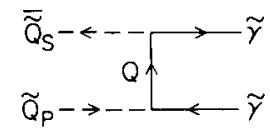

(0)

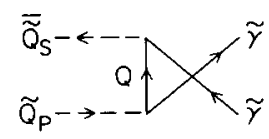

(b)

Fig. 91. Graphs $\overline{\mathrm{Q}}_{\mathrm{s}}+\overline{\tilde{\mathrm{Q}}}_{\mathrm{p}} \rightarrow \bar{\gamma}+\tilde{\gamma}$, where $\tilde{\mathrm{Q}}_{\mathrm{s}}$ and $\overline{\mathrm{Q}}_{\mathrm{p}}$ are the scalar and pseudoscalar combinations of the scalar-quarks $\tilde{\mathrm{Q}}_{\mathrm{L}}, \overline{\mathrm{Q}}_{\mathrm{R}}$ (assumed to be degenerate in mass). These scalar-quarks would be constituents of a $0^{-+}$scalar-quarkonium state which could decay into a pair of photinos. 


$$
M_{b}=\frac{-e^{2} e_{\mathrm{Q}}^{2}}{u=m_{\mathrm{Q}}^{2}} \bar{u}\left(k_{1}\right)\left(k_{2}-\not p_{1}+m_{\mathrm{Q}}\right) \gamma_{5} v\left(k_{2}\right)
$$

In order to satisfy the Pauli principle, we must supply an extra minus sign. To see why, we rewrite eq. (E37) using eq. (D5):

$$
M_{b}=\frac{+e^{2} e_{\mathrm{Q}}^{2}}{u-m_{\mathrm{Q}}^{2}} v^{\mathrm{T}}\left(k_{1}\right) C^{-1}\left(k_{2}-\not p_{1}+m_{\mathrm{Q}}\right) \gamma_{5} C \bar{u}\left(k_{2}\right)^{\mathrm{T}} .
$$

Taking the transpose and using eq. (D3), we end up with

$$
M_{b}=\frac{e^{2} e_{\mathrm{Q}}^{2}}{u-m_{\mathrm{O}}^{2}} \bar{u}\left(k_{2}\right)\left(k_{2}-\not p_{1}+m_{\mathrm{Q}}\right) \gamma_{5} v\left(k_{1}\right)
$$

Comparing with eq. (E34), it is clear that an additional minus sign is required. The full matrix element, using eqs. (E34) and (E37) with an additional sign inserted is

$$
M=e^{2} e_{\mathrm{Q}}^{2} \bar{u}\left(k_{1}\right)\left(\frac{k_{1}-\not p_{1}+m_{\mathrm{Q}}}{t-m_{\mathrm{Q}}^{2}}+\frac{k_{2}-\not p_{1}+m_{\mathrm{Q}}}{u-m_{\mathrm{Q}}^{2}}\right) \gamma_{\mathrm{s}} v\left(k_{2}\right)
$$

The resulting cross section is given by

$$
\frac{\mathrm{d} \sigma}{\mathrm{d} \Omega}=\frac{3 \alpha^{2} e_{\mathrm{Q}}^{4} \beta^{\prime}}{\underline{16 s \beta}}\left(\frac{1}{m_{\mathrm{Q}}^{2}-t}+\frac{1}{\underline{m}_{\mathrm{Q}}^{2}-u}\right)^{2}\left[s^{2}-\beta^{2} \beta^{\prime 2} \cos ^{2} \theta-4 s\left(\tilde{M}_{\mathrm{Q}}^{2}+\tilde{M}_{\gamma}^{2}-m_{\mathrm{Q}}^{2}\right)+16 \tilde{M}_{\mathrm{Q}}^{2} \tilde{M}_{\gamma}^{2}\right]
$$

where

$$
\begin{aligned}
& \beta \equiv\left(s-4 \tilde{M}_{\mathrm{Q}}^{2}\right)^{1 / 2}, \quad \beta^{\prime} \equiv\left(s-4 \tilde{M}_{\gamma}^{2}\right)^{1 / 2}, \\
& t=\tilde{M}_{\mathrm{Q}}^{2}+\tilde{M}_{\gamma}^{2}-\frac{1}{2}\left(s-\beta \beta^{\prime} \cos \theta\right), \quad u=\tilde{M}_{\mathrm{Q}}^{2}+\tilde{M}_{\gamma}^{2}-\frac{1}{2}\left(s+\beta \beta^{\prime} \cos \theta\right)
\end{aligned}
$$

Included in eq. (E41) is a factor of 3 for color and a factor of $1 / 2$ to account for identical photinos in the final state. Finally,

$$
\begin{aligned}
\Gamma\left({ }^{1} \mathrm{~S}_{0} \rightarrow \tilde{\gamma} \tilde{\gamma}\right) & =\left(\left|R_{\mathrm{s}}(0)\right|^{2} / 4 \pi \tilde{M}_{\mathrm{Q}}\right) \lim _{\beta \rightarrow 0} \beta \sigma\left(\overline{\tilde{Q}}_{\mathrm{s}} \tilde{\mathrm{Q}}_{\mathrm{p}} \rightarrow \tilde{\gamma} \tilde{\gamma}\right) \\
& =\left[6 \alpha^{2} e_{\mathrm{Q}}^{4} m_{\mathrm{Q}}^{2}\left(\tilde{M}_{\mathrm{Q}}^{2}-\tilde{M}_{\gamma}^{2}\right)^{1 / 2} / \tilde{M}_{\mathrm{Q}}\left(\tilde{M}_{\mathrm{Q}}^{2}-\tilde{M}_{\gamma}^{2}+m_{\mathrm{Q}}^{2}\right)^{2}\right]\left|R_{\mathrm{s}}(0)\right|^{2}
\end{aligned}
$$

One can also consider the bound state of $\overline{\mathrm{Q}}_{\mathrm{p}} \tilde{\mathrm{Q}}_{\mathrm{s}}$ decaying into $\tilde{\gamma} \tilde{\gamma}$; the width is identical to the result just obtained [eq. (E43)]. It is instructive to compare the result obtained to eq. (E15) and to the decay rate of a $\mathrm{q} \bar{q} 0^{-+}$bound state (such as the $\eta_{\mathrm{c}}$ ) into $\gamma \gamma$ :

$$
\Gamma\left({ }^{1} \mathrm{~S}_{0}(\mathrm{q} \overline{\mathrm{q}}) \rightarrow \gamma \gamma\right)=3 \alpha^{2} e_{\mathrm{Q}}^{4}\left|R_{\mathrm{s}}(0)\right|^{2} / m_{\mathrm{O}}^{2} .
$$

In the supersymmetric limit where $\tilde{M}_{y}=0$ and $\tilde{M}_{\mathrm{Q}}=m_{\mathrm{Q}}$, 


$$
\Gamma\left({ }^{1} S_{0}\left(\tilde{\mathrm{Q}}_{\mathrm{s}} \overline{\mathrm{Q}}_{\mathrm{p}}\right) \rightarrow \tilde{\gamma} \tilde{\gamma}\right)+\Gamma\left({ }^{1} \mathrm{~S}_{0}\left(\tilde{\mathrm{Q}}_{\mathrm{p}} \overline{\mathrm{Q}}_{\mathrm{s}}\right) \rightarrow \tilde{\gamma} \tilde{\gamma}\right)=\Gamma\left({ }^{1} \mathrm{~S}_{0}(\mathrm{q} \overline{\mathrm{q}}) \rightarrow \gamma \gamma\right)
$$

Of course, in this limit, all the ${ }^{1} \mathrm{~S}_{0}$ states above would mix.

6. $e^{+} e^{-} \rightarrow \tilde{\nu} \bar{\nu}$

The process $\mathrm{e}^{+} \mathrm{e}^{-} \rightarrow \tilde{\nu} \tilde{\tilde{\nu}}$ proceeds via s-channel $\mathrm{Z}^{0}$ exchange and t-channel wino exchange as shown in fig. 92. We neglect higgsino exchange since the coupling is proportional to the electron mass. The matrix element for wino exchange will depend on the chargino mixing parameters. We shall illustrate the calculation in two steps. First, we shall consider the limit where the charginos are degenerate in mass with the $\mathrm{W}$. Second, we will show how the calculation changes when the chargino mass matrix is altered.

In the limit where the charginos are degenerate in mass with the $\mathrm{W}$, the two eigenstates are denoted by $\tilde{\omega}_{1}$ and $\tilde{\omega}_{2}$; each is $50 \%$ wino and $50 \%$ higgsino as shown in eq. (B25). The relevant interactions are specified by eq. (C32). It is apparent that only $\tilde{\omega}_{1}$-exchange contributes in fig. 92(a). The calculation is straightforward and we summarize the results [A.33]:

$$
\begin{aligned}
& \sum_{\text {spins }}\left|M_{a}\right|^{2}=-g^{4}\left[s t+\left(\tilde{M}_{\nu}^{2}-t\right)^{2}\right] /\left(m_{\mathrm{w}}^{2}-t\right)^{2}, \\
& \sum_{\mathrm{spins}}\left|M_{b}\right|^{2}=-g^{4}\left[s t+\left(\tilde{M}_{\nu}^{2}-t\right)^{2}\right]\left[1+\left(4 \sin ^{2} \theta_{\mathrm{w}}-1\right)^{2}\right] / 8\left[\left(m_{\mathrm{z}}^{2}-s\right)^{2}+\Gamma_{\mathrm{z}}^{2} m_{\mathrm{z}}^{2}\right] \cos ^{4} \theta_{\mathrm{w}}, \\
& 2 \operatorname{Re} \sum_{\text {spins }} M_{a} M_{b}^{*}=-g^{4}\left[s t+\left(\tilde{M}_{\nu}^{2}-t\right)^{2}\right]\left(m_{\mathrm{z}}^{2}-s\right)\left(2 \sin ^{2} \theta_{\mathrm{w}}-1\right) /\left(m_{\mathrm{w}}^{2}-t\right)\left[\left(m_{\mathrm{z}}^{2}-s\right)^{2}+\Gamma_{\mathrm{z}}^{2} m_{\mathrm{z}}^{2}\right] .
\end{aligned}
$$

We have set the $\tilde{\omega}_{1}$ mass to $m_{\mathrm{w}}$ and allowed for a nonzero width $\Gamma_{\mathrm{z}}$ for the $\mathrm{Z}^{0}$.

In calculating the total cross section, it is convenient to normalize to $\sigma_{\mu \mu} \equiv 4 \pi \alpha^{2} / 3 \mathrm{~s}$. Defining $R \equiv \sigma / \sigma_{\mu \mu}$, the final result is the sum of three pieces corresponding to eqs. (E46)-(E48):

$$
\begin{aligned}
& R=R_{a}+R_{b}+R_{a b}, \\
& R_{a}=\frac{3}{16 \sin ^{4} \theta_{\mathrm{w}}}\left[-2 \beta+(1-2 \gamma) \underline{\left.\log \left(\frac{m_{\mathrm{w}}^{2}-t^{-}}{m_{\mathrm{w}}^{2}-t^{+}}\right)\right]}\right. \\
& R_{b}=s^{2} \beta^{3}\left[\left(4 \sin ^{2} \theta_{\mathrm{w}}-1\right)^{2}+1\right] / 256 \sin ^{4} \theta_{\mathrm{w}} \cos ^{4} \theta_{\mathrm{w}}\left[\left(m_{\mathrm{z}}^{2}-s\right)^{2}+\Gamma^{2} m_{\mathrm{z}}^{2}\right], \\
& R_{a b}=\frac{3\left(2 \sin ^{2} \theta_{\mathrm{w}}-1\right)\left(m_{\mathrm{z}}^{2}-s\right) s}{\left.16 \sin ^{4} \theta_{\mathrm{w}} \cos ^{2} \theta_{\mathrm{w}}\left(m_{\mathrm{z}}^{2}-\mathrm{J}\right)^{2}+\Gamma^{2} m_{\mathrm{z}}^{2}\right]}\left[\frac{1}{2} \beta(1-2 \gamma)-\left(\gamma^{2}+\frac{m_{\mathrm{w}}^{2}}{s}\right) \log \left(\frac{m_{\mathrm{w}}^{2}-t^{-}}{m_{\mathrm{w}}^{2}-t^{+}}\right)\right], \\
& \mathrm{e}^{+} \longrightarrow \mathrm{e}_{\tilde{\mathrm{w}}^{-}}^{---\tau}, \overline{\widetilde{v}}
\end{aligned}
$$
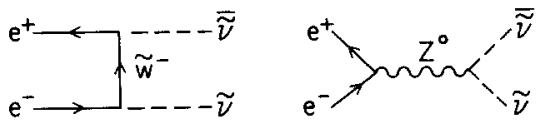

(a) (b)

Fig. 92. Graphs for $\mathrm{e}^{+} \mathrm{e}^{-} \rightarrow \tilde{\tilde{\nu}} \tilde{\nu}:$ (a) t-channel exchange of the wino (in general, this exchange involves the wino component of the chargino); (b) s-channel $Z^{0}$ exchange. 
where,

$$
\begin{aligned}
& \beta \equiv\left(1-4 \tilde{M}_{\nu}^{2} / s\right)^{1 / 2}, \\
& \gamma \equiv\left(\tilde{M}_{\nu}^{2}-m_{\mathrm{w}}^{2}\right) / s, \\
& t^{ \pm}=\tilde{M}_{\nu}^{2}-\frac{1}{2} s(1 \mp \beta) .
\end{aligned}
$$

Note that in the limit of $\beta \rightarrow 0$, if one expands out the logarithms in eqs. (E50) and (E52), one finds that $R \sim \beta^{3}$ as expected for the pair production of scalar particles.

Let us now investigate the changes that occur if the chargino mass matrix is modified. We shall choose a simple example described by eqs. (C22)-(C24). The relevant chargino interactions are given in eq. (C31). We see now that both $\tilde{\chi}_{1}$ and $\tilde{\chi}_{2}$ exchanges are possible. Let us define $R_{A}$ as the contribution of $\tilde{\chi}_{1}$ and $\tilde{\chi}_{2}$ exchanges plus their interference, $R_{B}$ is the $Z^{0}$-exchange contribution and $R_{A B}$ as the interference of the $Z^{0}$ exchange with $\tilde{\chi}_{1}$ and $\tilde{\chi}_{2}$ exchanges. $R_{B}=R_{b}$ which is given by eq. (E51), whereas $R_{A}$ and $R_{A B}$ are given below:

$$
R_{A}=R_{a}\left(\tilde{M}_{\chi_{1}}^{2}\right) \cos ^{4} \phi+R_{a}\left(\tilde{M}_{\chi_{2}}^{2}\right) \sin ^{4} \phi+\frac{3 s \sin ^{2} \phi \cos ^{2} \phi}{8 \sin ^{4} \theta_{\mathrm{w}}\left(\tilde{M}_{\chi_{2}}^{2}-\tilde{M}_{\chi_{1}}^{2}\right)}\left[f\left(\tilde{M}_{\chi_{1}}^{2}\right)-f\left(\tilde{M}_{\chi_{2}}^{2}\right)\right],
$$

where

$$
\begin{aligned}
& f\left(\tilde{M}_{\chi}^{2}\right) \equiv \frac{1}{2} \beta(1-2 \delta)-\left(\delta^{2}+\frac{\tilde{M}_{x}^{2}}{s}\right) \log \left(\frac{\tilde{M}_{\chi}^{2}-t^{-}}{\tilde{M}_{\chi}^{2}-t^{+}}\right), \\
& \delta \equiv\left(\tilde{M}_{\nu}^{2}-\tilde{M}_{\chi}^{2}\right) / s,
\end{aligned}
$$

and $R_{a}\left(\tilde{M}_{\chi}^{2}\right)$ is obtained by replacing $\gamma$ and $m_{\mathrm{w}}^{2}$ in $R_{a}$ (given by eq. (E50)) with $\delta$ and $\tilde{M}_{\chi}^{2}$, respectively. Finally,

$$
R_{A B}=R_{a b}\left(\tilde{M}_{\chi_{1}}^{2}\right) \cos ^{2} \phi+R_{a b}\left(\tilde{M}_{\chi_{2}}^{2}\right) \sin ^{2} \phi,
$$

using similar notation as above. The angle $\phi$ is defined by eq. (C23):

$$
\sin \phi=\left[\tilde{M}_{\chi_{2}} /\left(\tilde{M}_{\chi_{1}}+\tilde{M}_{\chi_{2}}\right)\right]^{1 / 2} .
$$

In the model of chargino mixing we have used, the masses of $\tilde{\chi}_{1}$ and $\tilde{\chi}_{2}$ are split above and below the $M$ mass respectively. One can ask whether a light chargino mass for $\tilde{\chi}_{2}$ could enhance the $\mathrm{e}^{+} \mathrm{e}^{-} \rightarrow \tilde{\nu} \overline{\tilde{\nu}}$ cross section substantially. From our equations (E56)-(E60), we see that in fact the cross section is not enhanced. Although indeed $R_{a}\left(\tilde{M}_{x_{2}}^{2}\right)>R_{a}\left(m_{\mathrm{w}}^{2}\right)$, we see that the presence of the factor $\sin ^{4} \phi$ in eq. (E56) offsets any benefit of the enhanced propagator. The physical reason behind this effect is that in the mixing model we have chosen, the lighter chargino has a larger higgsino component (whose coupling to light fermions is negligible) as compared with its wino component. In the heavier chargino, the wino component is larger. The end result is that the total $\mathrm{e}^{+} \mathrm{e}^{-} \rightarrow \tilde{\nu} \overline{\tilde{\nu}}$ cross section is not changed much by varying the parameter $M$ which governs the mixing model we have studied here (see eq. (C9); we have set $\mu=0$ above). 
It is important to remark that the observation above is not a general property of all mixing models. The other extreme case would be to set $M=0$ and vary $\mu$ [see eq. (C9)]. The result would be exactly opposite to the one above. Namely, the lighter chargino would have the larger gaugino component and the heavier chargino would have the larger higgsino component. In such models, the rate for $\mathrm{e}^{+} \mathrm{e}^{-} \rightarrow \tilde{\nu} \bar{\nu}$ could be significantly enhanced from the result given by eq. (E49)-(E52).

\section{Cancellation of quadratic divergences}

One of the key properties of supersymmetric theories (where the supersymmetry is either exact or broken spontaneously or by explicit 'soft' terms), is that the unrenormalized theory contains no quadratic divergences to all orders in perturbation theory. The mechanism by which the quadratic divergences disappear is the exact cancellation of graphs involving fermion and boson loops. The former come with an extra factor of -1 which allows for the cancellation. We can demonstrate this (to one loop) in the simplest supersymmetric model: the Wess-Zumino model [A.34]. The Lagrangian is

$$
\begin{aligned}
\mathscr{L}= & \frac{1}{2}\left(\partial_{\mu} A\right)^{2}+\frac{1}{2}\left(\partial_{\mu} B\right)^{2}+\frac{1}{2} \mathrm{i} \bar{\psi} \not \partial \psi-\frac{1}{2} m^{2}\left(A^{2}+B^{2}\right)-\frac{1}{2} m \bar{\psi} \psi-g m A\left(A^{2}+B^{2}\right) \\
& -\frac{1}{2} g^{2}\left(A^{2}+B^{2}\right)^{2}-g \bar{\psi}\left(A-\mathrm{i} B \gamma_{5}\right) \psi,
\end{aligned}
$$

where $A$ and $B$ are real scalar fields and $\psi$ is a (four-component) Majorana spinor.

At one-loop, the only potential quadratic divergence can occur in the self-energies of the scalars. We shall illustrate the cancellation of quadratic divergences in the one-loop graphs of the $A$ self-energy. All possible one-loop graphs are given in fig. 93. Only graphs (c), (d), and (e) have quadratic divergences, so we concentrate on those. The necessary Feynman rules are given in fig. 94 . It is also important to remember the symmetry factors of $1 / 2$ which appear with the loops of graphs (c), (d) and (e). The sum of the two boson-loop graphs (c) and (d) is the quadratically divergent integral

$$
8 g^{2} \int \frac{d^{4} k}{k^{2}-m^{2}}
$$

The fermion-loop graph (e) is given by

$$
-2 g^{2} \operatorname{Tr} \int \mathrm{d}^{4} k \frac{(k+m)(k-\not p+m)}{\left(k^{2}-m^{2}\right)\left[(k-p)^{2}-m^{2}\right]}
$$

The trace is easily evaluated. We write it in the following convenient way:

$$
\begin{aligned}
\operatorname{Tr}(k+m)(k-\not p+m) & =4\left(k^{2}-k \cdot p+m^{2}\right) \\
& =2\left[\left(k^{2}-m^{2}\right)+\left((k-p)^{2}-m^{2}\right)-p^{2}+4 m^{2}\right] .
\end{aligned}
$$

Inserting this expression into eq. (E63), we obtain:

$$
-4 g^{2} \int \frac{\mathrm{d}^{4} k}{k^{2}-m^{2}}-4 g^{2} \int \frac{\mathrm{d}^{4} k}{(k-p)^{2}-m^{2}}+I(p, m),
$$


(a)

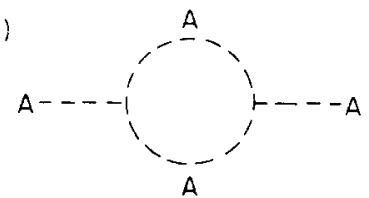

(b)

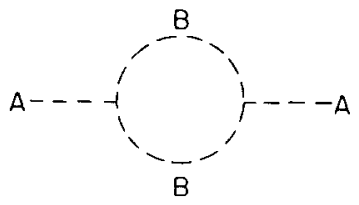

(c)

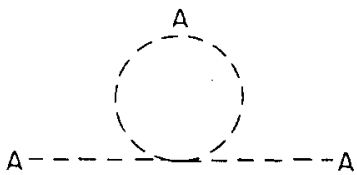

(d)
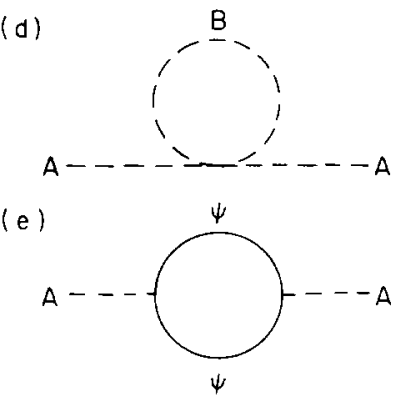

Fig. 93. One-loop corrections to the mass of the scalar $A$. Graphs (b) (c) and (d) contain quadratic divergences which exactly cancel with (e) in the supersymmetric limit.<smiles>[3H]C([3H])([3H])[3H]</smiles><smiles>[Te][Te]</smiles>

(a)<smiles>[B]C([B])([B])[B]</smiles>

(b)<smiles>[3H]C([B])([B])[B]</smiles>

$-4 i g^{2}$

(c)

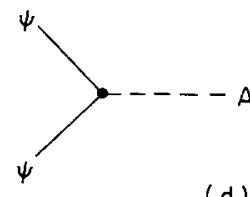

$-2 i g$

(d)

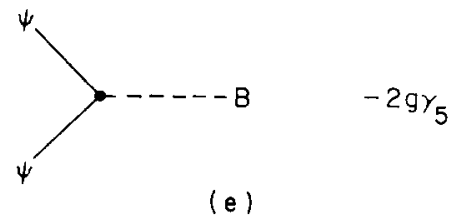

Fig. 94. Feynman rules for the Wess-Zumino model.

where the integral $I(p, m)$ is only logarithmically divergent. If we shift variables $k \rightarrow k+p$ in the second integral in eq. (E65), it then becomes clear that the first two terms of eq. (E65) exactly cancel the result given in eq. (E62). Thus, the quadratic divergence has indeed cancelled.

More complicated examples of the cancellation of quadratic divergences in models containing gauge fields have been studied in ref. [A.35, A.9].

Note added in proof.

The first published limits have appeared as this review was going to press. The JADE collaboration [W. Bartel et al., Phys. Lett. 146B (1984) 126] and experiments using MARK-J [3.89] have presented limits on neutralino masses which depend on various assumptions regarding supersymmetric masses and branching ratios.

\section{References}

[1.1] S.L. Glashow, Nucl. Phys. 22 (1961) 579;

S. Weinberg, Phys. Rev. Lett. 19 (1967) 1264;

A. Salam, Proc. 8th Nobel Symposium Stockholm 1968, ed., N. Svartholm (Almqvist and Wiksells, Stockholm 1968) p. 367.

[1.2] For reviews and references to the original literature, see H.D. Politzer, Phys. Rep. 14 (1974) 129;

W. Marciano and H. Pagels, Phys. Rep. 36 (1978) 137. 
[1.3] UA1 Collaboration: G. Arnison et al., Phys. Lett. 122B (1983) 103; 126B (1983) 398; 129B (1983) 273;

UA2 Collaboration: G. Banner et al., Phys. Lett. 122B (1983) 476;

P. Bagnaia et al., Phys. Lett. 129B (1983) 130.

[1.4] M. Kobayashi and T. Maskawa, Prog. Theor. Phys. 49 (1973) 652.

[1.5] For a discussion and review, see M. Veltman, Acta Phys. Pol. B8 (1977) 475; in: Proc. of the Int. Europhysics Conf. High Energy Phys., Brighton, eds., J. Guy and C. Costain (Rutherford Appleton Laboratory, 1983) p. 880.

[1.6] For a review and references to the original literature, see Michael E. Peskin, in: Proc. 1981 Int. Symp. Lepton and Photon Interactions at High Energies, Bonn, ed., W. Pfeil (Universitat Bonn, 1981) p. 880

[1.7] S. Weinberg, Phys. Rev. D13 (1976) 974 and D19 (1979) 1277;

L. Susskind, Phys. Rev. D20 (1979) 2619;

E. Farhi and L. Susskind, Phys. Rep. 74 (1981) 277.

[1.8] For a recent review and references to the original literature, see K. Lane, in: Proc. 1982 DPF Summer Study of Elementary Particle Physics and Future Facilities, eds., R. Donaldson, R. Gustafson and F. Paige (American Physical Society, New York, 1982) p. 222.

[1.9] Recent results for mass limits on new particles are summarized by S. Yamada, in: Proc. 1983 Int. Symp. Lepton and Photon Interactions at High Energies, Cornell, eds., D.G. Cassel and D.L. Kreinick (Cornell University, 1983) p.525.

[1.10] G.L. Kane, in: Gauge Theories in High Energy Physics, Proc. 1981 Les Houches Summer School of Theoretical Physics, eds., M.K. Gaillard and R. Stora (North-Holland, Amsterdam, 1983) p. 415.

[1.11] For a review and references to the original literature, see P. Langacker, Phys. Rep. 72C (1981) 185.

[1.12] C.H. Llewellyn Smith and G.G. Ross, Phys. Lett. 105B (1981) 38.

[1.13] K. Wilson, Phys. Rev. D3 (1971) 1818;

H. Georgi and A. Pais, Phys. Rev. D10 (1974) 539;

G. 't Hooft, in: Recent Developments in Gauge Theories, Proc. NATO Advanced Summer Institute, Cargese, 1979, eds., G. 't Hooft, et al. (Plenum, New York, 1980) p. 135.

[1.14] E. Witten, Nucl. Phys. B185 (1981) 513.

[1.15] S. Dimopoulos and H. Georgi, Nucl. Phys. B193 (1981) 150; N. Sakai, Z. Phys. C11 (1981) 153.

[1.16] H. Georgi, H.R. Quinn and S. Weinberg, Phys. Rev. Lett. 33 (1974) 451;

E. Gildener, Phys. Rev. D14 (1976) 1667;

A.J. Buras, J. Ellis, M.K. Gaillard and D.V. Nanopoulos, Nucl. Phys. B135 (1978) 66;

C. Sachrajda, Phys. Lett. $98 B$ (1981) 74;

Y. Kazama and Y.-P. Yao, Phys. Rev. D25 (1982) 1650.

[1.17] S. Weinberg, Phys. Lett. 82B (1979) 387;

M. Veltman, Acta Phys. Pol. B12 (1981) 437;

R.K. Kaul and P. Majumdar, Nucl. Phys. B199 (1982) 36.

[1.18] T. Inami, H. Nishino and S. Watamura, Phys. Lett. 117B (1982) 197; N.G. Deshpande, R.J. Johnson and E. Ma, Phys. Lett. 130B (1983) 61.

[1.19] For arguments along these lines, see J. Ellis, in: Proc. 1983 Int. Symp. Lepton and Photon Interactions at High Energies, Cornell, eds., D.G. Cassel and D.L. Kreinick (Cornell University, 1983) p. 439.

[1.20] For reviews of finite theories, see K.S. Stelle, in: Quantum Theory of Gravity: Essays in honor of the 60th birthday of Bryce S. DeWitt, ed., S.M. Christensen (Hilger, Bristol, 1984) p. 345.

P. West, in: Problems in Unification and Supergravity (AIP Conf. Proc. No. 116) eds., G. Farrar and F. Henyey (Am. Inst. Phys., New York, 1984) p. 167.

[1.21] For a review and references to the original literature, see P. Van Nieuwenhuizen, Phys. Rep. 68 (1981) 189.

[1.22] P. Ramond, Field Theory: A Modern Primer (Benjamin, Reading, Mass., 1981) p. 55.

[1.23] J. Wess and B. Zumino, Phys. Lett. 49B (1974) 52.

[1.24] For recent reviews and further references, see J. Polchinski, in: Proc. Fourth Workshop on Grand Unification, eds., H.A. Weldon, P. Langacker and P.J. Steinhardt (Birkhauser, Boston, 1983) p. 325;

R. Barbieri and S. Ferrara, Surveys in High-Energy Physics 4 (1983) 33;

P. Nath, R. Arnowitt and A.H. Chamseddine, Applied $N=1$ Supergravity; the ICTP Series in Theoretical Physics, Vol. I (World Scientific, Singapore, 1984);

H.P. Nilles, Phys. Rep. 110 (1984) 1.

[1.25] P. Fayet, Nucl. Phys. B90 (1975) 104.

[1.26] L.J. Hall and M. Suzuki, Nucl. Phys. B231 (1984) 419.

[1.27] J. Ellis and D.V. Nanopoulos, Phys. Lett. 110B (1982) 44;

R. Barbieri and R. Gatto, Phys. Lett. 110B (1982) 211;

T. Inami and C.S. Lim, Nucl. Phys. B207 (1982) 533;

B.A. Campbell, Phys. Rev. D28 (1983) 203;

M.J. Duncan, Nucl. Phys. B221 (1983) 285;

J.F. Donoghue, H.P. Nilles and D. Wyler, Phys. Lett. 128B (1983) 55. 
[1.28] A. Salam and J. Strathdee, Nucl. Phys. B87 (1975) 85; G.R. Farrar and S. Weinberg, Phys. Rev. D27 (1983) 2732.

[1.29] P. Fayet, Phys. Lett. 69B (1977) 489; G. Farrar and P. Fayet, Phys. Lett. 76B (1978) 575.

[1.30] P. Fayet and J. Iliopoulos, Phys. Lett. 51B (1974) 461.

[1.31] S. Deser and B. Zumino, Phys. Rev. Lett. 38 (1977) 1433.

[1.32] L. Gladney et al., Phys. Rev. Lett. 51 (1983) 2253; E. Fernandez et al., Phys. Lett. 52 (1984) 22.

[1.33] H.J. Behrend et al., Phys. Lett. 114B (1982) 287; R. Brandelik et al., Phys. Lett. 117B (1982) 365; Y. Ducros, Acta Phys. Pol. B14 (1983) 589.

[1.34] F. Bergsma et al., Phys. Lett. 121B (1983) 429; R.C. Ball et al., Phys. Rev. Lett. 53 (1984) 1314.

[1.35] G.L. Kane and W.B. Rolnick, Nucl. Phys. B232 (1984) 21.

[1.36] P. Fayet, Phys. Lett. 86B (1979) 272.

[1.37] R.P. Feynman, Photon-Hadron Interactions (Benjamin, Reading, Mass., 1972).

[1.38] G.L. Kane and J.P. Leveille, Phys. Lett. 112B (1982) 227.

[1.39] P. Fayet and S. Ferrara, Phys. Rep. 32 (1977) 249.

[1.40] A. Salam and J. Strathdee, Fortschr. Phys. 26 (1978) 57.

[1.41] P. Fayet, in: New Frontiers in High Energy Physics, Proc. 1978 Orbis Scientiae, Coral Gables (Plenum, New York, 1978) p. 413; in: Proc. 1980 Europhysics Study Conference on Unification of Fundamental Interactions, Erice, eds., J. Ellis, et al. (Plenum, New York, 1980) p. 587; in: QCD and Lepton Physics, Proc. 16th Rencontre de Moriond, ed., J. Tran Thanh Van (Editions Frontieres, France, 1981) p. 347; in: Quarks, Leptons and Supersymmetry, Proc. 17th Rencontre de Moriond, ed., J. Tran Thanh Van (Editions Frontieres, France, 1982) p. 483; in: Proc. 21st Int. Conf. on High Energy Physics, Paris, eds., P. Petiau and M. Porneuf (Les Editions de Physique, France, 1982) p. C3-673; in: Proc. 14th Summer School on Particle Physics, Gif-sur-Yvette, France, 1982, p. 43.

[1.42] E. Witten, lectures at the 1981 Summer Workshop on Particle Physics at Trieste, Princeton preprint.

[1.43] I. Hinchliffe and L. Littenberg, in: Proc. 1982 DPF Summer Study of Elementary Particle Physics and Future Facilities, eds., R. Donaldson, R. Gustafson and F. Paige (American Physical Society, New York, 1982) p. 242.

[1.44] Proc. Supersymmetry Confronting Experiment Workshop, eds. D.V. Nanopoulos and Savoy-Navarro, Phys. Rep. 105 (1984) 1.

[1.45] J. Wess and J. Bagger, Supersymmetry and Supergravity (Princeton University Press, Princeton, NJ, 1983).

[1.46] J. Gates, M. Grisaru, M. Rocek and W. Siegel, Superspace or 1001 Lectures in Supersymmetry (Benjamin, Reading, Mass., 1983).

[1.47] F. Legovini, Trieste preprint SISSA-24/83/EP (1983).

[1.48] C.A. Savoy, in: Beyond the Standard Model, Proc. 18th Rencontre de Moriond, 1983, ed., J. Tran Thanh Van (Editions Frontieres, France, 1984) p. 299.

[1.49] A. Savoy-Navarro, Phys. Rep. 105 (1984) 91.

[1.50] P. Nath, R. Amowitt and A.H. Chamseddine, Harvard preprint HUTP-83/A077 (1983).

[1.51] J.S. Hagelin, G.L. Kane and S. Raby, Nucl. Phys. B 241 (1984) 638.

[1.52] B. Adeva et al., Phys. Rev. Lett. 53 (1984) 1806.

[3.1] G. Farrar and P. Fayet, Phys. Lett. 89B (1980) 191.

[3.2] B. Adeva et al., MARK J Collaboration, Phys. Rev. Lett. 48 (1982) 967

[3.3] S. Wolfram, Phys. Lett. 82B (1979) 65.

[3.4] CELLO: H. Behrend et al., Phys. Lett. 114B (1982) 287;

JADE: W. Bartel et al., Phys. Lett. 114B (1982), 211;

MARK J: D. Barber et al., Phys. Rev. Lett. 45 (1981) 1904;

TASSO: R. Brandelik et al., Phys. Lett. 117B (1982) 365;

MARK II: C.A. Blocker et al., Phys. Rev. Lett. 49 (1982) 517.

For a summary of all the present data, see S. Yamada, in: Proc. 1983 Int. Symp. Lepton and Photon Interactions at High Energies, Cornell, eds., D.G. Cassel and D.L. Kreinick (Cornell University; 1983) p. 525.

[3.5] I. Hinchliffe and L. Littenberg, Proc. 1982 DPF Summer Study on Elementary Particle Physics and Future Facilities, eds., R. Donaldson, R. Gustafson and R. Paige (American Physical Society, New York, 1982) p. 242.

[3.6] M.K. Gaillard, L. Hall and I. Hinchliffe, Phys. Lett. 116B (1982) 279.

[3.7] G.L. Kane and M.L. Perl, in: Proc. 1982 DPF Summer Study on Elementary Particle Physics and Future Facilities, eds., R. Donaldson, R. Gustafson and R. Paige (American Physical Society, New York, 1982) p. 18.

[3.8] L. Gladney et al., Phys. Rev. Lett. 51 (1983) 2253; E. Fernandez et al., Phys. Rev. Lett. 52 (1984) 22.

[3.9] C.-C. Chen, H.B. Wald and R. Weinstein, Northeastern preprint NUB \#2581 (1983).

[3.10] J. Ellis and J. Hagelin, private note (1983).

[3.11] P. Fayet, Phys. Lett. 117B (1982) 460.

[3.12] J. Ellis and J. Hagelin, Phys. Lett. 122B (1982) 303.

[3.13] E. Ma and J. Okada, Phys. Rev. Lett. 41 (1978) 287 and Phys. Rev. D18 (1978) 4219; 
K.J.F. Gaemers, R. Gastmans and F.M. Renard, Phys. Rev. D19 (1979) 1605;

G. Barbiellini, B. Richter and J.L. Siegrist, Phys. Lett. 106B (1981) 414.

[3.14] MAC Collaboration, SLAC experiment in progress, reported by H.R. Band, SLAC-PUB-3335 (1984), invited talk presented at the 1984 Rencontre de Moriond: Electroweak Interactions and Unified Theories, La Plagne, France, Feb. 26-March 4, 1984.

[3.15] D. Burke et al., PEP-21 Experimental Proposal (April, 1983).

[3.16] N. Cabibbo, L. Maiani and S. Petrarca, Phys. Lett. 132B (1983) 195.

[3.17] C.N. Yang, Phys. Rev. 77 (1950) 242.

[3.18] C.S. Aulakh and R.N. Mohapatra, Phys. Lett. 121B (1983) 147.

[3.19] L.J. Hall and M. Suzuki, Nucl. Phys. B231 (1984) 419.

[3.20] J.S. Hagelin, G.L. Kane and S. Raby, Nucl. Phys. B241 (1984) 638.

[3.21] L.E. Ibanez, Phys. Lett. 137B (1984) 160.

[3.22] D.A. Dicus, S. Nandi and X. Tata, Phys. Lett. 129B (1983) 451.

[3.23] R.M. Barnett, H.E. Haber and K.S. Lackner, Phys. Lett. 126B (1983) 64.

[3.24] H.E. Haber, R.M. Barnett and K.S. Lackner, Phys. Rev. D29 (1984) 1990.

[3.25] R.M. Barnett, H.E. Haber and K.S. Lackner, Phys. Rev. Lett. 51 (1983) 176.

[3.26] R. Barbieri, N. Cabibbo, L. Maiani and S. Petrarca, Phys. Lett. 129B (1983) 458.

[3.27] R.M. Barnett, H.E. Haber and K.S. Lackner, Phys. Rev. D29 (1984) 1381.

[3.28] G.L. Kane and W. Rolnick, Nucl. Phys. B232 (1984) 21.

[3.29] S.H. Aronson et al., Proc. 1982 DPF Summer Study on Elementary Particle Physics and Future Facilities, eds., R. Donaldson, R. Gustafson and R. Paige (American Physical Society, New York, 1982) p. 505.

[3.30] P.R. Harrison and C.H. Llewellyn Smith, Nucl. Phys. B213 (1983) 223 (E: B223 (1983) 542).

[3.31] M. Gluck and E. Reya, Phys. Rev. Lett. 51 (1983) 867 (E: 51 (1983) 1507).

[3.32] I. Antoniadis, L. Baulieu and F. Delduc, Z. Phys. C23 (1984) 119.

[3.33] M.J. Herrero, L.E. Ibanez, C. Lopez and F.J. Yndurain, Phys. Lett. 132B (1983) 199 (E: 142B (1984) 455).

[3.34] S. Dawson, E. Eichten and C. Quigg, Fermilab-PUB-83/82-THY, LBL-16540 (1984).

[3.35] E. Eichten, I. Hinchliffe, K. Lane and C. Quigg, Rev. Mod. Phys. 56 (1984) 579.

[3.36] C. Nappi, Phys. Rev. D25 (1982) 84.

[3.37] S. Yamada, in: Proc. 1983 Int. Symp. Lepton and Photon Interactions at High Energies, Cornell, eds., D.G. Cassel and D.L., Kreinick (Cornell University, 1983) p. 525.

[3.38] M.J. Duncan, Nucl. Phys. B214 (1983) 21.

[3.39] P. Fayet, Phys. Lett. 78B (1978) 417.

[3.40] G.R. Farrar and P. Fayet, Phys. Lett. 76B (1978) 575; 79B (1978) 442.

[3.41] G.L. Kane and J.P. Leveille, Phys. Lett. 112B (1982) 227.

[3.42] M. Chanowitz and S. Sharpe, Phys. Lett. 126B (1983) 225.

[3.43] T. Goldman, Phys. Lett. 78B (1978) 110.

[3.44] E. Franco, Phys. Lett. 124B (1983) 271.

[3.45] B.A. Campbell, J. Ellis and S. Rudaz, Nucl. Phys. B198 (1982) 1.

[3.46] H.E. Haber and G.L. Kane, Nucl. Phys. B232 (1984) 333.

[3.47] G. Altarelli, N. Cabibbo, G. Corbo, L. Maiani and G. Martinelli, Nucl. Phys. B208 (1982) 365.

[3.48] A. Mitra and S. Ono, Z. Phys. C25 (1984) 245.

[3.49] F. Bergsma et al., Phys. Lett. 121B (1983) 429.

[3.50] R.C. Ball et al., Phys. Rev. Lett. 53 (1984) 1314.

[3.51] B. Alper et al., Phys. Lett. 46B (1973) 265;

J.A. Appel et al., Phys. Rev, Lett. 32 (1974) 428;

R. Gustafson et al., Phys. Rev. Lett. 37 (1976) 474;

D. Cutts et al., Phys. Rev. Lett. 41 (1978) 363.

[3.52] J. Badier et al., Phys. Lett. 39B (1972) 414.

[3.53] G.R. Farrar, Phys. Rev. Lett. 53 (1984) 1029; Physica 124A (1984) 293.

[3.54] T. Goldman and H.E. Haber, Los Alamos preprint LA-UR-83-2323 (1984), to appear in: Proc. Los Alamos Workshop on Supersymmetry in Pliysics, to be published in Physica D.

[3.55] M.I. Eides and M.I. Vysotsky, Phys. Lett. 124B (1983) 83; A.V. Smilga and M.I. Vysotsky, Phys. Lett. 125B (1983) 227.

[3.56] L.S. Littenberg, F.E. Paige and D.T. Weygand, Update to the CBA Study on Supersymmetry, Brookhaven preprint (1983).

[3.57] F.E. Paige and S.D. Protopopescu, ISAJET: A Monte Carlo Event Generator for pp and pō Interactions - Version 3, in: Proc. 1982 DPF Summer Study on Elementary Particle Physics and Future Facilities, eds., R. Donaldson, R. Gustafson and F.E. Paige (American Physical Society, New York, 1982) p.471.

[3.58] S.K. Jones and C.H. Llewellyn Smith, Nucl. Phys, B217 (1983) 145.

[3.59] I. Antoniadis, C. Kounnas and R. Lacaze, Nucl. Phys. B211 (1983) 216.

[3.60] C. Kounnas and D.A. Ross, Nucl. Phys. B214 (1983) 317.

[3.61] P. Nelson and P. Osland, Phys. Lett. 115B (1982) 407. 
[3.62] M.J. Herrero, C. Lopez and F.J. Yndurain, Phys. Lett. 127B (1983) 463.

[3.63] H.D. Dahmen, D.H. Schiller and D. Wahner, Nucl. Phys. B227 (1983) 291.

[3.64] G. Altarelli, B. Mele and R. Petronzio, Phys. Lett. 129B (1983) 456.

[3.65] W.-Y. Keung, Phys. Rev. D28 (1983) 1129 (E: D29 (1984) 1544).

[3.66] G. Eilam and A. Khare, Phys. Lett. 134B (1984) 269.

[3.67] B.A. Campbell, J.A. Scott and M.K. Sundaresan, Phys. Lett. 131B (1983) 213.

[3.68] J. Ellis and S. Rudaz, Phys. Lett. 128B (1983) 248.

[3.69] G.L. Kane and W. Rolnick, Nucl. Phys. B217 (1983) 117.

[3.70] B.A. Campbell, J.A. Scott and M.K. Sundaresan, Phys. Lett. 126B (1983) 376.

[3.71] J. Zuk, G.C. Joshi and J.W.G. Wignall, Phys. Rev. D28 (1983) 1706.

[3.72] B. Kayser, Phys. Rev. D26 (1982) 1662.

[3.73] J.D. Jackson, Proc. 1976 SLAC Summer Institute in Particle Physics, SLAC-198 (1976).

[3.74] V.A. Novikov et al., Phys. Rep. 41 (1978) 1.

[3.75] J.-M. Frere and G.L. Kane, Nucl. Phys. B223 (1983) 331.

[3.76] J. Ellis, J.-M. Frere, J.S. Hagelin, G.L. Kane and S.T. Petcov, Phys. Lett. 132B (1983) 436.

[3.77] V. Barger, R.W. Robinett, W.Y. Keung and R.J.N. Phillips, Phys. Lett. 131B (1983) 372.

[3.78] S. Weinberg, Phys. Rev. Lett. 50 (1983) 387.

[3.79] A.H. Chamseddine, R. Arnowitt and P. Nath, Phys. Rev. Lett. 49 (1982) 970

[3.80] P. Fayet, Phys. Lett. 125B (1983) 178.

[3.81] D.A. Dicus, S. Nandi, W.W. Repko and X. Tata, Phys. Rev. Lett. 51 (1983) 1030.

[3.82] D.A. Dicus, S. Nandi, W.W. Repko and X. Tata, Phys. Rev. D29 (1984) 67.

[3.83] J. Ellis, J.S. Hagelin, D.V. Nanopoulos and M. Srednicki, Phys. Lett. 127B (1983) 233

[3.84] A.H. Chamseddine, P. Nath and R. Arnowitt, Phys. Lett. 129B (1983) 445 (E: 132B (1983) 467).

[3.85] D.A. Dicus, S. Nandi and X. Tata, Phys. Lett. 129B (1983) 451.

[3.86] B. Grinstein, J. Polchinski and M.B. Wise, Phys. Lett. 130B (1983) 285

[3.87] V. Barger, R.W. Robinett, W.Y. Keung and R.J.N. Phillips, Phys. Rev. D28 (1983) 2912.

[3.88] P. Fayet, Phys. Lett. 133B (1983) 363.

[3.89] B. Adeva et al., Phys. Rev. Lett. 53 (1984) 1806

[3.90] H. Huffel and G. Pocsik, Z. Phys. C8 (1981) 13;

G. Pocsik and G. Zsigmond, Z. Phys. C10 (1981) 367.

[3.91] J. Ellis and Graham G. Ross, Phys. Lett. 117B (1982) 397.

[3.92] D.A. Dicus, S. Nandi, W.W. Repko and X. Tata, Phys. Rev. D29 (1984) 1317.

[3.93] J.A. Grifols, Phys. Lett. 120B (1983) 217.

[3.94] P. Fayet, Phys. Lett. 86B (1979) 272.

[3.95] J.A. Grifols, X. Mor-Mur and J. Sola, Phys. Lett. 114B (1982) 35.

[3.96] P. Fayet and J. Iliopoulos, Phys. Lett. 51B (1974) 461.

[3.97] S. Deser and B. Zumino, Phys. Rev. Lett. 38 (1977) 1433.

[3.98] B. deWit and D.Z. Freedman, Phys. Rev. Lett. 35 (1975) 827.

[3.99] P. Fayet, Phys. Lett. 84B (1979) 421.

[3.100] N. Cabibbo, G.R. Farrar and L. Maiani, Phys. Lett. 105B (1981) 155.

[3.101] B. deWit and D.Z. Freedman, Phys. Rev. D12 (1975) 2286.

[3.102] M. Gluck and E. Reya, Phys. Lett. B130 (1983) 423.

[3.103] T. Kobayashi and M. Kuroda, Phys. Lett. B134 (1984) 271.

[3.104] P. Salati and J.C. Wallet, Phys. Lett. 122B (1983) 397.

[3.105] M. Kuroda, K. Ishikawa, T. Kobayashi and S. Yamada, Phys. Lett. 127B (1983) 467.

[3.106] K. Grassie and P.N. Pandita, Phys. Rev. D30 (1984) 22.

[3.107] J.E. Johnson and S. Rudaz, Phys. Rev. D30 (1984) 1590.

[3.108] R.J. Cashmore, in: Proc. Workshop 'Experimentation at HERA', NIKHEF Amsterdam, 1983, DESY HERA 83/20 (1983).

[3.109] S. Ono, preprint LPTHE Orsay 83/35 (1983).

[3.110] D.V. Nanopoulos, S. Ono and T. Yanagida, Phys. Lett. 137B (1984) 363.

[3.111] W.-Y. Keung and A. Khare, Phys. Rev. D29 (1984) 2657.

[3.112] J.H. Kuhn and S. Ono, Phys. Lett. 142B (1984) 436.

[3.113] A. Savoy-Navarro, Phys. Rep. 105 (1984) 91.

[3.114] T. Kobayashi and M. Kuroda, Phys. Lett. 139B (1984) 208.

[3.115] J.D. Ware and M.E. Machacek, Phys. Lett. 142B (1984) 300.

[4.1] UA1 Collaboration, G. Arnison et al., Phys. Lett. 122B (1983) 103; 129B (1983) 273; UA2 Collaboration, G. Banner et al., Phys. Lett. 122B (1983) 476.

[4.2] UA1 Collaboration, G. Arnison et al., Phys. Lett. 126B (1983) 398; UA2 Collaboration, P. Bagnaia et al., Phys. Lett. 129B (1983) 130.

[4.3] R.M. Barnett, K.S. Lackner and H.E. Haber, Phys. Rev. Lett. 51 (1983) 176. 
[4.4] R. Barbieri, N. Cabibbo, L. Maiani and S. Petrarca, Phys. Lett. 127B (1983) 458.

[4.5] D.A. Dicus, S. Nandi and X. Tata, Phys. Lett. 129B (1983) 451

[4.6] R.M. Barnett, H.E. Haber and K.S. Lackner, Phys. Rev. D29 (1984) 1381.

[4.7] D.A. Dicus, S. Nandi, W.W. Repko and X. Tata, Phys. Rev, D29 (1984) 67.

[4.8] D. B. Cline and C. Rubbia, Phys. Lett. 127B (1983) 277.

[4.9] S. Gottlieb and T. Weiler, Phys. Rev. D29 (1984) 2005.

[4.10] F. Paige, in: Proton-Antiproton Collider Physics, eds., V. Barger, D. Cline and F. Halzen (American Institute of Physics, New York, 1982) p. 168.

[4.11] N. Cabibbo, L. Maiani and S. Petrarca, Phys. Lett. 132B (1983) 195.

[4.12] R. Amowitt, A.H. Chamseddine and P. Nath, Phys. Rev. Lett. 50 (1983) 232; Phys. Lett. 129B (1983) 445 (E: 132B (1983) 467).

[4.13] S. Weinberg, Phys. Rev. Lett. 50 (1983) 387.

[4.14] J. Ellis, J.S. Hagelin, D.V. Nanopoulos and M. Srednicki, Phys. Lett. 127B (1983) 233.

[4.15] P. Fayet, Phys. Lett. 133B (1983) 363.

[4.16] B. Grinstein, J. Polchinski and M.B. Wise, Phys. Lett. 130B (1983) 285.

[4.17] V. Barger, R.W. Robinett, W.Y. Keung and R.J.N. Phillips, Phys. Rev. D28 (1983) 2912.

[4.18] G.L. Kane and J.-M. Frere, Nucl. Phys. B223 (1983) 331.

[4.19] G.L. Kane and W.B. Rolnick, Nucl. Phys. B217 (1983) 117.

[4.20] B.A. Campbell, J.A. Scott and M.K. Sundaresan, Phys. Lett. 126B (1983) 376.

[4.21] B.A. Campbell, J. Ellis and S. Rudaz, Nucl. Phys. B198 (1982) 1.

[4.22] I. Hinchliffe and L. Littenberg, in: Proc. 1982 DPF Summer Study on Elementary Particle Physics and Future Facilities, eds., R. Donaldson, R. Gustafson and F. Paige (American Physical Society Society, New York, 1983) p. 242.

[4.23] Mark Claudson, L.J. Hall and Ian Hinchliffe, Nucl. Phys. B228 (1983) 501.

[4.24] M. Mangano, CERN preprint, ref. TH.3717 (1983).

[4.25] R.M. Barnett and H.E. Haber, Santa Barbara preprint, NSF-ITP-84-78 (1984), to be published in Phys. Rev. D.

[4.26] J.A. Grifols and J. Sola, Phys. Lett. 137B (1984) 257.

[4.27] H.E. Haber, R.M. Barnett and K.S. Lackner, Phys. Rev. D29 (1984) 1990.

[5.1] G.R. Farrar and P. Fayet, Phys. Lett. 89B (1980) 191.

[5.2] I. Hinchliffe and L. Littenberg, in: Proc. 1982 DPF Summer Study on Elementary Particle Physics and Future Facilities, eds., R. Donaldson, R. Gustafson and F. Paige (American Physical Society Society, New York, 1983) p. 242.

[5.3] M. Gluck and E. Reya, Phys. Lett. 130B (1983) 423; Dortmund preprint DO-TH 83/24 (1983).

[5.4] T. Kobayashi and M. Kuroda, Phys. Lett. 134B (1984) 271.

[5.5] M.K. Gaillard, L. Hall and I. Hinchliffe, Phys. Lett. 116B (1982) 279.

[5.6] P. Salati and J.C. Wallet, Phys. Lett. 122B (1983) 397.

[5.7] M. Kuroda, K. Ishikawa, T. Kobayashi and S. Yamada, Phys. Lett. 127B (1983) 467.

[5.8] J.A. Grifols, X. Mor-Mur and J. Sola, Phys. Lett. 114B (1982) 35.

[5.9] P. Fayet, Phys. Lett. 117B (1982) 460.

[5.10] J. Ellis and J.S. Hagelin, Phys. Lett. 122B (1983) 303.

[5.11] K. Grassie and P.N. Pandita, Phys. Rev. D30 (1984) 22.

[5.12] J. Ellis and G.G. Ross, Phys. Lett. 117B (1982) 397.

[5.13] J.-M. Frere and G.L. Kane, Nucl. Phys. B223 (1983) 331.

[5.14] D.A. Dicus, S. Nandi, W.W. Repko and X. Tata, Phys. Rev. Lett. 51 (1983) 1030; Phys. Rev. D29 (1984) 1317.

[5.15] J. Ellis, J.-M. Frere, J.S. Hagelin, G.L. Kane and S.T. Petcov, Phys. Lett. 132B (1983) 436.

[5.16] V. Barger, R.W. Robinett, W.Y. Keung and R.J.N. Phillips. Phys. Lett. 131B (1983) 372.

[5.17] R.M. Barnett, H.E. Haber and K.S. Lackner, Phys. Rev. Lett. 51 (1983) 176; Phys. Rev. D29 (1984) 1381.

[5.18] C.R. Nappi, Phys. Rev. D25 (1982) 84.

[5.19] M.J. Herrero, C. Lopez and F.J. Yndurain, Phys. Lett. 127B (1983) 463.

[5.20] H.D. Dahmen, D.H. Schiller and D. Wahner, Nucl. Phys. B227 (1983) 291.

[5.21] G. Altarelli, B. Mele and R. Petronzio, Phys. Lett. 129B (1983) 456.

[5.22] B.A. Campbell, J. Ellis and S. Rudaz, Nucl. Phys. B198 (1982) 1.

[5.23] D. Danckaert, P. deCausmaecker, R. Gastmans, W. Troost and T.T. Wu, Phys. Lett. 114B (1982) 203.

[5.24] P. Nelson and P. Osland, Phys. Lett. 115B (1982) 407.

[5.25] J. Ellis and S. Rudaz, Phys. Lett. 128B (1983) 248.

[5.26] B.A. Campbell, J.A. Scott and M.K. Sundaresan, Phys. Lett. 131B (1983) 213.

[5.27] G. Eilam and A. Khare, Phys. Lett. 134B (1984) 269.

[5.28] W.-Y. Keung, Phys. Rev. D28 (1983) 1129 (E: D29 (1984) 1544).

[5.29] H.E. Haber, unpublished.

[5.30] G. Eilam and M. Gluck, Phys. Rev. D 30 (1984) 99.

[5.31] J. Rich and D.R. Winn, Phys. Rev. D14 (1976) 1283.

[5.32] P. Fayet, Phys. Lett. 84B (1979) 421; 86B (1979) 272.

[5.33] M. Gluck, Phys. Lett. 129B (1983) 257. 
[5.34] E. Ma and J. Okada, Phys. Rev. D18 (1978) 4219;

K. Gaemers, R. Gastmans and F. Renard, Phys. Rev. D19 (1979) 1605;

D. Dicus, Phys. Rev. D21 (1980) 1767;

G. Barbiellini, B. Richter and J.L. Siegrist, Phys. Lett. 106B (1981) 414.

[5.35] J. Ellis, J.S. Hagelin, D.V. Nanopoulos and M. Srednicki, Phys. Lett. 127B (1983) 233.

[5.36] R. Arnowitt, A.H. Chamseddine and P. Nath, Phys. Rev. Lett. 50 (1983) 232; Phys. Lett. 129B (1983) 445 (E: 132B (1983) 467).

[5.37] P. Fayet, Phys. Lett. 125B (1983) 178; Phys. Lett. 133B (1983) 363.

[5.38] B. G. instein, J. Polchinski and M.B. Wise, Phys. Lett. 130B (1983) 285.

[5.39] V. Barger, R.W. Robinett, W.Y. Keung and R.J.N. Phillips, Phys. Rev. D28 (1983) 2912.

[5.40] D.A. Dicus, S. Nandi and X. Tata, Phys. Lett. 129B (1983) 451.

[5.41] N. Cabibbo, L. Maiani and S. Petrarca, Phys. Lett. 132B (1983) 195.

[5.42] J.E. Johnson and S. Rudaz, Phys. Rev. D30 (1984) 1590.

[5.43] J.D. Ware and M.E. Machacek, Phys. Lett. 142B (1984) 300.

[5.44] I. Hinchliffe and L. Littenberg, in: Proc. 1982 DPF Summer Study on Elementary Particle Physics and Future Facilities, eds., R. Donaldson, R. Gustafson and F. Paige (American Physical Society, New York, 1983) p. 242.

[5.45] G.L. Kane and W.B. Rolnick, Nucl. Phys. B217 (1983) 117.

[5.46] B.A. Campbell, J.A. Scott and M.K. Sundaresan, Phys. Lett. 126B (1983) 376.

[5.47] For a review, see D.L. Burke, in: Proc. 21st Int. Conf. High Energy Physics, Paris, eds., P. Petiau and M. Porneuf (Les Editions de Physique, France, 1982) p. C3-513.

[5.48] E. Reya, Phys. Lett. 124B (1983) 424

[5.49] D.M. Scott and W.J. Stirling, Phys. Rev. D29 (1984) 157.

[5.50] J.A. Grifols and J. Sola, Z. Phys. C18 (1983) 185.

[5.51] M. Gluck, Phys. Lett. 129B (1983) 255.

[5.52] J.A. Grifols and R. Pascual, Phys. Lett. 135B (1984) 319 (E: 142B (1984) 455).

[5.53] W.Y. Keung and L. Littenberg, Phys. Rev. D28 (1983) 1067.

[5.54] T. Goldman and H.E. Haber, Los Alamos preprint LA-UR-83-2323 (1984), to appear in: Proc. Los Alamos Workshop on Supersymmetry in Physics, to be published in Physica D.

[5.55] W.-Y. Keung and A. Khare, Phys. Rev. D29 (1984) 2657.

[5.56] J.H. Kuhn and S. Ono, Phys. Lett. 142B (1984) 436.

[5.57] B. Guberina and J. Kuhn, Nuov. Cim. Lett. 32 (1981) 295;

A. Devoto and W.W. Repko, Phys. Lett. 106B (1981) 501;

T. Fujiwara and M. Igarashi, Prog. Theor. Phys. 66 (1981) 617.

[5.58] F. Almeida and D.H. Schiller, Z. fur Phys. C21 (1983) 103.

[5.59] F. Wilczek, Phys. Rev. Lett. 39 (1977) 1304.

[5.60] J.H. Kühn, Phys. Lett. 141B (1984) 433.

[5.61] T. Kobayashi and M. Kuroda, Phys. Lett. 139B (1984) 208.

[6.1] G.L. Kane and J.P. Leveille, Phys. Lett. 112B (1983) 227.

[6.2] I. Hinchliffe and L. Littenberg, in: Proc. 1982 DPF Summer Study on Elementary Particle Physics and Future Facilities, eds., R. Donaldson, R. Gustafson and F. Paige (American Physical Society, New York, 1983) p. 242.

[6.3] S. Aronson et al., in: Proc. 1982 DPF Summer Study on Elementary Particle Physics and Future Facilities, eds., R. Donaldson, R. Gustafson and F. Paige (American Physical Society, New York, 1983) p. 505.

[6.4] P.R. Harrison and C.H. Llewellyn Smith, Nucl. Phys. B213 (1983) 223 (E: B223 (1983) 542).

[6.5] I. Antoniadis, L. Baulieu and F. Delduc, Z. Phys. C23 (1984) 119.

[6.6] S. Dawson, E. Eichten and C. Quigg, Fermilab-PUB-83/82-THY, LBL-16540 (1984).

[6.7] E. Eichten, I. Hinchliffe, K. Lane and C. Quigg, Rev. Mod. Phys. 56 (1984) 579.

[6.8] N.S. Craigie, K. Hidaka and P. Ratcliffe, Phys. Lett. 129B (1983) 310; N.S. Craigie, K. Hidaka, M. Jacob and F.M. Renard, Phys. Rep. 99 (1983) 69.

[6.9] A. Savoy-Navarro, Phys. Rep. 105 (1984) 91.

[6.10] M. Gluck and E. Reya, Phys. Rev. Lett. 51 (1983) 867.

[6.11] M.J. Herrero, L.E. Ibanez, C. Lopez and F.J. Yndurain, Phys. Lett. 132B (1983) 199 (E: 142B (1984) 455).

[6.12] B.A. Campbell, J. Ellis and S. Rudaz, Nucl. Phys. B198 (1982) 1.

[6.13] I. Antoniadis, C. Kounnas and R. Lacaze, Nucl. Phys. B211 (1983) 216.

[6.14] C. Kounnas and D.A. Ross, Nucl. Phys. B214 (1983) 317.

[6.15] S.K. Jones and C.H. Llewellyn Smith, Nucl. Phys. B217 (1983) 145.

[6.16] J.M. Frere and G.L. Kane, Nucl. Phys. B223 (1983) 331.

[6.17] J. Ellis, J.S. Hagelin, D.V. Nanopoulos and M. Srednicki, Phys. Lett. 127B (1983) 233.

[6.18] V. Barger, R.W. Robinett, W.Y. Keung and R.J.N. Phillips. Phys. Lett. 131B (1983) 372.

[6.19] T. Goldman and H.E. Haber, Los Alamos preprint LA-UR-83-2323 (1984), to appear in: Proc. Los Alamos Workshop on Supersymmetry in Physics, to be published in Physica D.

[6.20] R. Cutler and D. Sivers, Phys. Rev. D17 (1978) 196. 
[7.1] G. Altarelli and G. Parisi, Nucl. Phys. B126 (1977) 298.

[7.2] B.A. Campbell, J. Ellis and S. Rudaz, Nucl. Phys. B198 (1982) 1.

[7.3] I. Antoniadis, C. Kounnas and R. Lacaze, Nucl. Phys. B211 (1983) 216.

[7.4] C. Kounnas and D.A. Ross, Nucl. Phys. B214 (1983) 317.

[7.5] S.K. Jones and C.H. Llewellyn Smith, Nucl. Phys. B217 (1983) 145.

[7.6] F.J. Yndurain, Phys. Lett. 130B (1983) 313.

[7.7] I. Hinchliffe and L. Littenberg, in: Proc. 1982 DPF Summer Study of Elementary Particle Physics and Future Facilities, eds., R. Donaldson, R. Gustafson and F. Paige (American Physical Society, New York, 1982) p. 242.

[7.8] P. Salati and J.C. Wallet, Phys. Lett. 122B (1983) 397.

[7.9] R.J. Cashmore, in: Proc. Workshop: 'Experimentation at HERA', NIKHEF Amsterdam, 1983, p. 381, DESY HERA 83/20 (1983).

[7.10] J.F. Owens and E. Reya, Phys. Rev. D17 (1978) 3003.

[7.11] M. Gluck, E. Hoffman and E. Reya, Z. Phys. C13 (1982) 119.

[7.12] L. Maiani, in: Proc. Workshop: 'Experimentation at HERA', NIKHEF Amsterdam, 1983, p. 3, DESY HERA 83/20 (1983).

[7.13] S. Brodsky, C. Peterson and N. Sakai, Phys. Rev. D23 (1981) 2745.

[8.1] J. Bailey et al., Nucl. Phys. B150 (1979) 1.

[8.2] P. Fayet, in: Unification of the Fundamental Particle Interactions, eds., S. Ferrara, J. Ellis and P. van Nieuwenhuizen (Plenum, New York, 1980) p. 587.

[8.3] J. Ellis, J. Hagelin and D.V. Nanopoulos, Phys. Lett. 116B (1982) 283.

[8.4] R. Barbieri and L. Maiani, Phys. Lett. 117B (1982) 203.

[8.5] S. Ferrara and E. Remiddi, Phys. Lett. 53B (1974) 3471.

[8.6] J. Ellis and D.V. Nanopoulos, Phys. Lett. 110B (1982) 44.

[8.7] R. Barbieri and R. Gatto, Phys. Lett. 110B (1982) 211.

[8.8] M. Suzuki, Berkeley preprint UCB-PTH-82/8 (1982).

[8.9] T. Inami and C.S. Lim, Nucl. Phys. B207 (1982) 533.

[8.10] B.A. Campbell, Phys. Rev. D28 (1983) 209.

[8.11] M.J. Duncan, Nucl. Phys. B221 (1983) 285.

[8.12] J.F. Donoghue, H.P. Nilles and D. Wyler, Phys. Lett. 128B (1983) 55.

[8.13] A.B. Lahanas and D.V. Nanopoulos, Phys. Lett. 129B (1983) 461.

[8.14] M.J. Duncan and J. Trampetic, Phys. Lett. 134B (1984) 439.

[8.15] E. Franco and M. Mangano, Phys. Lett. 135B (1984) 445.

[8.16] M. Suzuki, Phys. Lett. 115B (1982) 40.

[8.17] M.J. Duncan, Nucl. Phys. B214 (1983) 21.

[8.18] D. Ross and M. Veltman, Nucl. Phys. B95 (1975) 135;

M. Veltman, Nucl. Phys. B123 (1977) 89.

[8.19] T.K. Kuo and N. Nakagawa, Nuovo Cim. Lett. 36 (1983) 560.

[8.20] L. Alvarez-Gaume, J. Polchinski and M.B. Wise, Nucl. Phys. B221 (1983) 495.

[8.21] R. Barbieri and L. Maiani, Nucl. Phys. B224 (1983) 32.

[8.22] C.S. Lim, T. Inami and N. Sakai, Phys. Rev. D29 (1984) 1488.

[8.23] J. Ellis, S. Ferrara and D.V. Nanopoulos, Phys. Lett. 114B (1982) 231

[8.24] F. del Aguila, J.A. Grifols, A. Mendez, D.V. Nanopoulos and M. Srednicki, Phys. Lett. 129B (1983) 77.

[8.25] J.-M. Frere and M.B. Gavela, Phys. Lett. 132B (1983) 107.

[8.26] I.I. Bigi, invited talk given at the VI Warsaw Symposium on Elementary Particle Physics, Aachen preprint PITHA 83/14.

[8.27] J. Polchinski and M.B. Wise, Phys. Lett. 125B (1983) 393.

[8.28] F. del Aguila, M.B. Gavela, J.A. Grifols and A. Mendez, Phys. Lett. 126B (1983) 71 (E: 129B (1983) 473).

[8.29] M.J. Duncan, Nucl. Phys. B224 (1983) 289.

[8.30] D.V. Nanopoulos and M. Srednicki, Phys. Lett. 128B (1983) 61.

[8.31] H.P. Nilles and S. Raby, Nucl. Phys. B198 (1982) 102.

[8.32] W. Buchmuller and D. Wyler, Phys. Lett. 121B (1983) 321.

[8.33] R.N. Mohapatra, S. Ouvry and G. Senjanovic, Phys. Lett. 126B (1983) 329.

[8.34] J.-M. Frere and J.-M. Gerard, Nuovo Cim. Lett. 37 (1983) 135.

[8.35] G. Senjanovic, Acta Phys. Pol. B15 (1984) 511.

[8.36] R. Akhoury and I.I. Bigi, Nucl. Phys. B234 (1984) 549.

[8.37] R. Akhoury, I.I. Bigi and H.E. Haber, Phys. Lett. 135B (1984) 113.

[8.38] T.D. Lee, Phys. Rev. D8 (1973) 1226;

S. Weinberg, Phys. Rev. Lett. 31 (1976) 657.

[8.39] For a recent review, see N.G. Deshpande, in: Proc. Theoretical Symposium on Intense Medium Energy Sources of Strangeness, Santa Cruz, 1983, eds., T. Goldman, H.E. Haber and H.F.-W. Sadrozinski (American Institute of Physics, New York, 1983) p. 107.

[8.40] J.-M. Frere, private communication.

[8.41] M. Gavela and H. Georgi, Phys. Lett. 119B (1982) 141. 
[8.42] G. 't Hooft, Phys. Rev. Lett. 37 (1976) 8; Phys. Rev. D14 (1976) 3432;

R. Jackiw and C. Rebbi, Phys. Rev. Lett. 37 (1976) 172;

C.G. Callan, R.F. Dashen and D.J. Gross, Phys. Lett. 63B (1978) 334.

[8.43] R.D. Peccei and H.R. Quinn, Phys. Rev. Lett. 38 (1977) 1440; Phys. Rev. D16 (1977) 1791.

[8.44] S. Weinberg, Phys. Rev. Lett. 40 (1978) 223;

F. Wilczek, Phys. Rev. Lett. 40 (1978) 279.

[8.45] M.T. Grisaru, M. Rocek and W. Siegel, Nucl. Phys. B159 (1979) 429.

[8.46] C.S. Aulakh and R.N. Mohapatra, Phys. Lett. 121B (1983) 147.

[8.47] J.-M. Frere, D.R.T. Jones and S. Raby, Nucl. Phys. B222 (1983) 11.

[8.48] R. Barbieri, L. Girardello and A. Masiero, Phys. Lett. 127B (1983) 429.

[8.49] R. Shrock, in: Proc. 1982 DPF Summer Study on Elementary Particle Physics and Future Facilities, eds., R. Donaldson, R. Gustafson and F. Paige (American Physical Society, New York, 1982) p. 291.

[8.50] J. Ellis and J.S. Halgein, Nucl. Phys. B217 (1983) 189.

[8.51] M.K. Gaillard, Y.-C. Kao, I.-H. Lee and M. Suzuki, Phys. Lett. 123B (1983) 241.

[8.52] H.E. Haber, G.L. Kane and T. Sterling, Nucl. Phys. B 161 (1979) 493. (Note that the value of $f_{\pi}$ in this reference is $\sqrt{2}$ larger than what we use in this review.)

[8.53] J.S. Hagelin, G.L. Kane and S. Raby, Nucl. Phys. B241 (1984) 638.

[8.54] S. Weinberg, Gravitation and Cosmology (Wiley, New York, 1972).

[8.55] For a pedagogical review see G. Steigman, in: Proc. 1982 SLAC Summer Institute on Particle Physics, p. 651, ed., A. Mosher, SLAC-259 (1983). The most recent analysis is given by: J. Yang, M.S. Turner, G. Steigman, D. Schramm and K. Olive, Astrophys. J. 281 (1984) 493.

[8.56] For a recent review, see J. Primack, in: Proc. Fourth Workshop on Grand Unification (Birkhauser, Boston, 1983) p. 256.

[8.57] B.W. Lee and S. Weinberg, Phys. Rev. Lett. 39 (1977) 165.

[8.58] D. Dicus, E.W. Kolb and V. Teplitz, Phys. Rev. Lett. 39 (1977) 168.

[8.59] H. Goldberg, Phys. Rev. Lett. 50 (1983) 1419.

[8.60] J. Ellis, J.S. Hagelin, D.V. Nanopoulos, K. Olive and M. Srednicki, Nucl. Phys. B238 (1984) 453.

[8.61] L.M. Krauss, Nucl. Phys. B227 (1983) 556.

[8.62] K. Freese and D.B. Schramm, Nucl. Phys. B233 (1984) 167.

[8.63] L.E. Ibanez, Phys. Lett. 137B (1984) 160.

[8.64] S. Weinberg, Phys. Rev. Lett. 48 (1982) 1303.

[8.65] A. Guth, Phys. Rev. D23 (1981) 347;

A.D. Linde, Phys. Lett. 108B (1982) 389;

A. Albrecht and P.J. Steinhardt, Phys. Rev. Lett. 48 (1982) 1220;

S.W. Hawking and I.G. Moss, Phys. Lett. 110B (1982) 35.

[8.66] J. Ellis, A.D. Linde and D.V. Nanopoulos, Phys. Lett. 118B (1982) 59.

[8.67] D.V. Nanopoulos, K.A. Olive and M. Srednicki, Phys. Lett. 127B (1983) 30.

[8.68] S. Weinberg, Phys. Rev. Lett. 43 (1979) 1566;

F. Wilczek and A. Zee, Phys. Rev. Lett. 43 (1979) 1571.

[8.69] S. Weinberg, Phys. Rev. D26 (1982) 287.

[8.70] N. Sakai and T. Yanagida, Nucl. Phys. B197 (1982) 533.

[8.71] S. Dimopoulos, S. Raby and F. Wilczek, Phys. Lett. 112B (1982) 133.

[8.72] G. Costa, F. Feruglio and F. Zwirner, Nuovo Cim. 70A (1982) 201.

[8.73] P. Salati and J.C. Wallet, Nucl. Phys. B209 (1982) 389.

[8.74] N. Sakai, Phys. Lett. 121B (1983) 130; Nucl. Phys. B238 (1984) 317.

[8.75] W. Lucha, Nucl. Phys. B221 (1983) 300.

[8.76] S. Chadha and M. Daniel, Nucl. Phys. B229 (1983) 105; Phys. Lett. 137B (1984) 374.

[8.77] S. Dimopoulos and H. Georgi, Nucl. Phys. B193 (1981) 150; N. Sakai, Z. Phys. C11 (1981) 153; S. Dimopoulos, S. Raby and F. Wilczek, Phys. Rev. D24 (1981) 1681.

[8.78] M.B. Einhorn and D.R.T. Jones, Nucl. Phys. B196 (1982) 475; W.J. Marciano and G. Senjanovic, Phys. Rev. D25 (1982) 3092.

[8.79] J. Ellis, D.V. Nanopoulos and S. Rudaz, Nucl. Phys. B202 (1982) 43.

[8.80] H. Georgi, in: Grand Unified Theories and Related Topics, Proc. 1981 Kyoto Summer School, eds., M. Konuma and T. Maskawa (World Scientific, Singapore, 1981) p. 109.

[8.81] E. Fiorini, in: Proc. 1983 Int. Symp. on Lepton and Photon Interactions at High Energies, Cornell, eds., D.G. Cassel and D.L. Kreiknick (Cornell Univeristy, 1983) p. 405.

[8.82] V.M. Belyaev and M.I. Vysotsky, Phys. Lett. 127B (1983) 215.

[8.83] P. Fayet, Phys. Lett. 69B (1977) 489.

[8.84] L.J. Hall and I. Hinchliffe, Phys. Lett. 112B (1982) 351.

[8.85] R. Barbieri, S. Ferrara and D.V. Nanopoulos, Phys. Lett. 116B (1982) 16. 
[8.86] A.B. Lahanas and T.R. Taylor, Phys. Lett. 114B (1982) 132.

[8.87] A. Salam and J. Strathdee, Nucl. Phys. B87 (1975) 85.

[8.88] P. Fayet, Nucl. Phys. B90 (1975) 104.

[8.89] G.R. Farrar and P. Fayet, Phys. Lett. B76 (1978) 575.

[8.90] G.R. Farrar and S. Weinberg, Phys. Rev. D27 (1983) 2732.

[8.91] M.I. Eides and M.I. Vysotsky, Phys. Lett. 124B (1983) 83;

A.V. Smilga and M.I. Vysotsky, Phys. Lett. 125B (1983) 227.

[8.92] T.E. Clark and S.T. Love, Phys. Lett. 138B (1984) 1289.

[8.93] J. Ellis and S. Rudaz, Phys. Lett. 128B (1983) 248.

[8.94] N. Cabibbo, G. Farrar and L. Maiani, Phys. Lett. 105B (1981) 155.

[8.95] J.A. Grifols and A. Mendez, Phys. Rev. D26 (1982) 1809.

[8.96] D.A. Kosower, L.M. Kruass and N. Sakai, Phys. Lett. 133B (1983) 305.

[8.97] H. Pagels and J. Primack, Phys. Rev. Lett. 48 (1982) 223.

[8.98] M. Fukugita and N. Sakai, Phys. Lett. 114B (1982) 23.

[8.99] A. Bouquet and C.E. Vayonakis, Phys. Lett. 116B (1982) 219.

[8.100] S. Weinberg, to appear in: Intersection Between Elementary Particle Physics and Cosmology, Proc. Jerusalem Winter School for Theoretical Physics, Dec., 1983-Jan., 1984

[8.101] J. Milutinovic, P.B. Pal and G. Senjanovic, Phys. Lett. 140B (1984) 324.

[8.102] Y. Asano et al., Phys. Lett. 107B (1981) 159.

[8.103] M. Davier, in: Proc. 21st Int. Conf. on High Energy Physics, Paris, eds., P. Petiau and M. Porneuf (Les Editions de Physique, France, 1982) p. C3-471.

[9.1] P. Fayet and J. Iliopoulos, Phys. Lett. 51B (1974) 461.

[9.2] B. deWit and D.Z. Freedman, Phys. Rev. Lett. 35 (1975) 827.

[9.3] P. Fayet, Phys. Lett. 70B (1977) 461.

[9.4] J. Polchinski, in: Proc. Fourth Workshop on Grand Unification, eds., H.A. Weldon, P. Langacker and P.J. Steinhardt (Birkhäuser, Boston, 1983) p. 325.

[9.5] P. Fayet, Nucl. Phys. B78 (1974) 14; Nucl. Phys. B90 (1975) 104; Phys. Lett. 64B (1976) 159; 69B (1977) 489; 84B (1979) 416.

[9.6] E. Witten, Nucl. Phys. B185 (1981) 513.

[9.7] S. Dimopoulos and H. Georgi, Nucl. Phys. B193 (1981) 150.

[9.8] N. Sakai, Z. Phys. C11 (1981) 153.

[9.9] S. Weinberg, Phys. Rev. D25 (1982) 287.

[9.10] L.J. Hall and I. Hinchliffe, Phys. Lett. 112B (1982) 351.

[9.11] A.B. Lahanas and T.R. Taylor, Phys. Lett. 114B (1982) 132.

[9.12] M. Dine and W. Fischler, Phys. Lett. 110B (1982) 227.

[9.13] C.R. Nappi and B.A. Ovrut, Phys. Lett. 113B (1982) 1751.

[9.14] L. Alvarez-Gaume, M. Claudson and M.B. Wise, Nucl. Phys. B207 (1982) 96.

[9.15] K. Inoue, A. Kakuto, H. Komatsu and S. Takeshita, Prog. Theor. Phys. 67 (1982) 1889; 68 (1982) 927 (E: 70 (1983) 330$) ; 71$ (1984) 413.

[9.16] J. Ellis, L.E. Ibanez and G.G. Ross, Phys. Lett. 113B (1982) 283; Nucl. Phys. B221 (1983) 29.

[9.17] M. Dine and W. Fischler, Nucl. Phys. B204 (1982) 346; B227 (1983) 477.

[9.18] R. Barbieri, S. Ferrara and D.V. Nanopoulos, Z. Phys. C13 (1982) 267; Phys. Lett. 116B (1982) 16.

[9.19] E. Witten, Phys. Lett. 105B (1982) 267.

[9.20] T. Banks and V. Kaplonovsky, Nucl. Phys. B206 (1982) 45

[9.21] S. Dimopoulos and S. Raby, Nucl. Phys. B219 (1983) 479

[9.22] V. Kaplunovsky, Nucl. Phys. B233 (1984) 336.

[9.23] V. Kaplunovsky, Ph.D. Dissertation, Tel-Aviv University (1983).

[9.24] B. Ovrut and J. Wess, Phys. Lett. 112B (1982) 347; 119B (1982) 105.

[9.25] R. Barbieri, S. Ferrara, D.V. Nanopoulos and K. Stelle, Phys. Lett. 113B (1982) 219

[9.26] A. Chamseddine, R. Arnowitt and P. Nath, Phys. Rev. Lett. 49 (1982) 970; Phys, Lett. 120B (1983) 145; 121B (1983) 33; Nucl, Phys. B227 (1983) 121.

[9.27] H.P. Nilles, Phys. Lett. 115B (1982) 193; Nucl. Phys. B217 (1983) 366.

[9.28] J. Ellis and D.V. Nanopoulos, Phys. Lett. 116B (1982) 133.

[9.29] L. Ibanez, Phys. Lett. 118B (1982) 73; Nucl. Phys. B218 (1983) 514.

[9.30] R. Barbieri, S. Ferrara and C.A. Savoy, Phys. Lett. 119B (1982) 343.

[9.31] L. Alvarez-Gaume, J. Polchinski and M.B. Wise, Nucl. Phys. B221 (1983) 495.

[9.32] E. Cremmer, P. Fayet and L. Girardello. Phys. Lett. 120B (1983) 41.

[9.33] L. Hall, J. Lykken and S. Weinberg, Phys. Rev. D27 (1983) 2359.

[9.34] For a review and further references, see J. Ellis, in: Proc. 1983 Int. Symp. Lepton and Photon Interactions at High Energies, Cornell, eds., D.G. Cassel and D.L. Kreinick (Cornell University, 1983) p. 439.

[9.35] E. Cremmer, S. Ferrara, L. Girardello and A. Van Proeyen, Phys. Lett. 116B (1982) 231; Nucl. Phys. B212 (1983) 413. 
[9.36] S.K. Soni and H.A. Weldon, Phys. Lett. 126B (1983) 215.

[9.37] S. Deser and B. Zumino, Phys. Rev. Lett. 38 (1977) 1433.

[9.38] J. Ellis, D.V. Nanopoulos and K. Tamvakis, Phys. Lett. 121B (1983) 123.

[9.39] H. Nilles, M. Srednicki and D. Wyler, Phys. Lett. 124B (1983) 337.

[9.40] B.A. Ovrut and S. Raby, Phys. Lett. 125B (1983) 270; 130B (1983) 277.

[9.41] J. Ellis, J.S. Hagelin, D.V. Nanopoulos and K. Tamvakis, Phys. Lett. 125B (1983) 275.

[9.42] M. Claudson, L.J. Hall and I. Hinchliffe, Nucl. Phys. B228 (1983) 501.

[9.43] L.E. Ibanez and C. Lopez, Phys. Lett. 126B (1983) 54; Nucl. Phys. B233 (1984) 511.

[9.44] L.E. Ibanez and G.G. Ross, Phys. Lett. 131B (1983) 335.

[9.45] J. Ellis, A.B. Lahanas, D.V. Nanopoulos and K. Tamvakis, Phys. Lett. 134B (1984) 429.

[9.46] C. Kounnas, A.B. Lahanas, D.V. Nanopoulos and M. Quiros, Phys. Lett. 132B (1983) 95; Nucl. Phys. B236 (1984) 438.

[9.47] L. Girardello and M.T. Grisaru, Nucl. Phys. B194 (1982) 65.

[9.48] J.-M. Frère, D.R.T. Jones and S. Raby, Nucl. Phys, B222 (1983) 11.

[9.49] S.K. Jones and G.G. Ross, Phys. Lett. 135B (1984) 69.

[9.50] J.-P. Derendinger and C.A. Savoy, Nucl. Phys. B237 (1984) 307.

[9.51] R. Barbieri and S. Ferrara, Surveys in High Energy Physics 4 (1983) 33.

[9.52] S. Ferrara, CERN preprint Ref. TH.3570 (1983), Lectures given at the 4th Silarg Symposium on Gravitation, Caracas, Venezuela, 1982.

[9.53] C.A. Savoy, in: Beyond the Standard Model, Proc. 18th Rencontre de Moriond, 1983, ed., J. Tran Thanh Van (Editions Frontieres, France, 1984) p. 299.

[9.54] P. Nath, R. Arnowitt and A.H. Chamseddine, Applied $N=1$ Supergravity, The ICTP Series in Theoretical Physics, Vol. I (World Scientific, Singapore, 1984)

[9.55] R. Barbieri, L. Girardello and A. Masiero, Phys. Lett. 127B (1983) 429.

[9.56] C.S. Aulakh and R.N. Mohapatra, Phys. Lett. 121B (1983) 147.

[9.57] R. Flores and M. Sher, Ann. Phys. (NY) 148 (1983) 95.

[9.58] J. Gunion and H.E. Haber, SLAC-PUB-3404 (1984).

[10.1] P. Fayet, Phys. Lett. 69B (1977) 489;

G. Farrar and P. Fayet, Phys. Lett. 76B (1978) 575.

[10.2] P. Fayet, in: New Frontiers in High Energy Physics, Proc. 1978 Orbis Scientiae, Coral Gables (Plenum, New York, 1980) p. 413; in: Proc. 1980 Europhysics Study Conference on Unification of Fundamental Interactions, Erice, eds., J. Ellis et al. (Plenum, New York, 1980) p. 587; Phys. Lett. 95B (1980) 285; Phys. Lett. 96B, (1980) 83;

P. Fayet and M. Mezard, Phys. Lett. 104B (1981) 226.

[10.3] S. Weinberg, Phys. Rev. D26 (1982) 287.

[10.4] L.J. Hall and I. Hinchliffe, Phys. Lett. 112B (1982) 351.

[10.5] R. Barbieri, S. Ferrara and D.V. Nanopoulos, Z. Phys. C13 (1982) 267; Phys. Lett. 116B (1982) 16.

[10.6] A.B. Lahanas and T.R. Taylor, Phys. Lett. 114B (1982) 132.

[10.7] L. O'Raifeartaigh, Nucl. Phys. B96 (1975) 331.

[10.8] P. Fayet, Phys. Lett. 84B (1979) 421.

[10.9] S. Ferrara, L. Girardello and F. Palumbo, Phys. Rev. D20 (1979) 403.

[10.10] J. Polchinski and M.B. Wise, Nucl. Phys. B218 (1983) 297.

[10.11] P. Fayet and J. Iliopoulos, Phys. Lett. 51B (1974) 461.

[10.12] P. Fayet, in: Quarks, Leptons and Supersymmetry, Proc. 17th Rencontre de Moriond, ed., J. Tran Thanh Van (Editions Frontiere, France, 1982) p. 483; in: Proc. 21st Int. Conf. High Energy Physics, Paris, 1982, eds., P. Petiau and M. Porneuf (Les Editions de Physique, France, 1982) p. C3-673.

[10.13] J. Polchinski and L. Susskind, Phys. Rev. D26 (1982) 3661.

[10.14] E. Cremmer, S. Ferrara, L. Girardello and A. Van Proeyen, Phys. Lett. 116B (1982) 231.

[10.15] E. Cremmer, S. Ferrara, L. Girardello and A. Van Proeyen, Nucl. Phys. B212 (1983) 413.

[10.16] P. Fayet, Nucl. Phys. B90 (1975) 104.

[10.17] E. Cremmer, P. Fayet and L. Girardello. Phys. Lett. 122B (1983) 41.

[10.18] H.P. Nilles, M. Srednicki and D. Wyler, Phys. Lett. 124B (1983) 337.

[10.19] A. Lahanas, Phys. Lett. 124B (1983) 341.

[10.20] S. Ferrara, D.V. Nanopoulos and C.A. Savoy, Phys. Lett. 123B (1983) 214.

[10.21] D. Nemeschansky, Nucl. Phys. B234 (1984) 379.

[10.22] H.E. Haber, Phys. Rev. D26 (1982) 1317.

[10.23] L.E. Ibanez, Phys. Lett. 126B (1983) 196.

[10.24] B.A. Ovrut and S. Raby, Phys. Lett. 125B (1983) 270; 130B (1983) 277; 138 B (1984) 72.

[10.25] P. Fayet, Phys. Lett. 78B (1978) 417.

[10.26] F. del Aguila et al., Harvard preprint HUTP-84/A001, 1984; J.-M. Frere, L. Mezincescu and Y.-P. Yao, Phys. Rev. D29 (1984) 1196; D30 (1984) 2238.

[10.27] L.J. Hall and M. Suzuki, Nucl. Phys. B231 (1984) 419. 
[10.28] O. Nachtmann and M. Wirbel, Z. Phys. C23 (1984) 85.

[10.29] O. Nachtmann, A. Reiter and M. Wirbel, Z. Phys. C23 (1984) 199.

[10.30] P. Fayet, Phys. Lett. 84B (1979) 421.

[10.31] H. Pagels and J.R. Primack, Phys. Rev. 48 (1982) 223.

[10.32] M.T. Grisaru, W. Siegel and M. Rocek, Nucl. Phys. B159 (1979) 429.

[10.33] E. Witten, Nucl. Phys. B185 (1981) 513.

[10.34] S. Dimopoulos and H. Georgi, Nucl. Phys. B193 (1981) 150.

[10.35] N. Sakai, Z. Phys. C11 (1981) 153.

[10.36] M.B. Einhorn and D.R.T. Jones, Nucl. Phys. B196 (1982) 475.

[10.37] W.J. Marciano and G. Senjanovic, Phys. Rev. D25 (1982) 3092.

[10.38] J. Ellis, D.V. Nanopoulos and S. Rudaz, Nucl. Phys. B202 (1982) 43.

[10.39] D.V. Nanopoulos and M. Srednicki, Phys. Lett. 124B (1983) 37.

[10.40] L.E. Ibanez and G.G. Ross, Phys. Lett. 110B (1982) 215.

[10.41] E. Witten, Phys. Lett. 105B (1981) 267.

[10.42] S. Dimopoulos and S. Raby, Nucl. Phys. B219 (1983) 479.

[10.43] A. Masiero, D.V. Nanopoulos, K. Tamvakis and T. Yanagida, Phys. Lett. 115B (1982) 380

[10.44] C. Kounnas, D.V. Nanopoulos, M. Quiros and M. Srednicki, Phys. Lett. 127B (1983) 82.

[10.45] R.N. Cahn, I. Hinchliffe and L.J. Hall, Phys. Lett. 109B (1982) 426.

[10.46] L.E. Ibanez, Phys. Lett. 114B (1982) 243.

[10.47] T.E. Clark, T.K. Kuo and N. Nakagawa, Phys. Lett. 115B (1982) 26.

[10.48] C.S. Aulakh and R.N. Mohapatra. Phys. Rev. D28 (1982) 217.

[10.49] B. Sathiapalan, Phys. Lett. 122B (1983) 49.

[10.50] J. Leon, M. Quiros and M. Ramon Medrano, Nucl. Phys. B222 (1983) 104.

[10.51] D.V. Nanopoulos, CERN preprint ref. TH.3699, invited lecture at the 1983 Europhysics Study Conference on 'Electroweak Effects at High Energies', Erice.

[10.52] P. Nath, R. Amowitt and A.H. Chamseddine, Applied $N=1$ supergravity, The ICTP Series in Theoretical Physics, Vol. I (World Scientific, Singapore, 1984).

[10.53] C.S. Aulakh and R.N. Mohapatra, Phys. Lett. 121B (1983) 147.

[10.54] J. Kubo and S. Sakakibara, Phys. Lett. 140B (1984) 223.

[A.1] J. Wess and J. Bagger, Supersymmetry and Supergravity (Princeton University Press, Princeton, NJ, 1983).

[A.2] P. Fayet and J. Iliopoulos, Phys. Lett. 51B (1974) 461.

[A.3] L. Alvarez-Gaume, J. Polchinski and M.B. Wise, Nucl. Phys. B221 (1983) 495.

[A.4] L. Girardello and M.T. Grisaru, Nucl. Phys. B194 (1982) 65.

[A.5] E. Cremmer, S. Ferrara, L. Girardello and A. van Proeyer, Phys. Lett. 116B (1982) 231; Nucl. Phys. B212 (1983) 413.

[A.6] L. Hall, J. Lykken and S. Weinberg, Phys. Rev. D27 (1983) 2359.

[A.7] H.E. Haber, R.M. Barnett and K.S. Lackner, Phys. Rev. D29 (1984) 1990.

[A.8] P. Fayet, Nucl. Phys. B90 (1975) 104.

[A.9] R.K. Kaul and P. Majumdar, Nucl. Phys. B199 (1982) 36.

[A.10] H.P. Nilles, M. Srednicki and D. Wyler, Phys. Lett. 124B (1983) 327; A. Lahanas, Phys. Lett. 124B (1983) 341.

[A.11] E. Witten, Nucl. Phys. B185 (1981) 513.

[A.12] J. Ellis and S. Rudaz, Phys. Lett. 128B (1983) 248.

[A.13] J. Ellis and G.G. Ross, Phys. Lett. 117B (1982) 397.

[A.14] J. Ellis, L.E. Ibanez and G.G. Ross, Nucl. Phys. B221 (1983) 29.

[A.15] J.-M. Frere and G.L. Kane, Nucl. Phys. B223 (1983) 331

[A.16] J. Ellis, J.S. Hagelin, D.V. Nanopoulos and M. Srednicki, Phys. Lett. 127B (1983) 233.

[A.17] A.H. Chamseddine, P. Nath and R. Arnowitt, Phys. Lett. 129B (1983) 445.

[A.18] D.A. Dicus, S. Nandi and X. Tata, Phys. Lett. 129B (1983) 451.

[A.19] B. Grinstein, J. Polchinski and M.B. Wise, Phys. Lett. 130B (1983) 285.

[A.20] V. Barger, R.W. Robinett, W.Y. Keung and R.J.N. Phillips, Phys. Lett. 131B (1983) 372.

[A.21] J. Ellis, J.-M. Frere, J.S. Hagelin, G.L. Kane and S.T. Petcov, Phys. Lett. 132B (1983) 436.

[A.22] S.K. Jones and C.H. Llewellyn Smith, Nucl. Phys. B217 (1983) 145.

[A.23] P. Fayet, Phys. Lett. 86B (1979) 272.

[A.24] H. Goldberg, Phys. Rev. Lett. 50 (1983) 1419.

[A.25] For a review, see J.D. Jackson, in: Proc. 1976 SLAC Summer Institute in Particle Physics, SLAC-198 (1976).

[A.26] J.A. Grifols, X. Mor-Mur and J. Sola, Phys. Lett. 114B (1982) 35.

[A.27] P. Fayet, Phys. Lett. 117B (1982) 303.

[A.28] J. Ellis and J. Hagelin, Phys. Lett. 122B (1982) 303.

[A.29] K. Grassie and P.N. Pandita, Phys. Rev. D30 (1984) 22. 
[A.30] W.-Y. Keung and L. Littenberg, Phys. Rev. D28 (1983) 1067.

[A.31] R. Cutler and D. Sivers, Phys. Rev. D17 (1978) 196.

[A.32] P.R. Harrison and C.H. Llewellyn Smith, Nucl. Phys. B213 (1983) 223 (E: B223 (1983) 542).

[A.33] R.M. Barnett, H.E. Haber and K.S. Lackner, Phys. Rev. Lett. 51 (1983) 176; Phys. Rev. D29 (1984) 1381

[A.34] J. Wess and B. Zumino, Phys. Lett. 49B (1974) 52.

[A.35] R.K. Kaul, Phys. Lett. 109B (198?) 19.

[A.36] H. Huffel and G. Pocsik, Z. Phys. C8 (1981) 13;

G. Pocsik and G. Zsigmond, Z. Phys. C10 (1981) 367.

[A.37] J.F. Gunion and H.E. Haber, SLAC-PUB-3404 (1984).

[A.38] H.E. Haber, G.L. Kane and T. Sterling, Nucl. Phys. B161 (1979) 493.

[A.39] J.F. Donoghue and L.F. Li, Phys. Rev. D19 (1979) 945.

[A.40] J. Kubo and S. Sakakibara, Phys. Lett. 140B (1984) 223.

[A.41] I.J.R. Aitchison and A.J.G. Hey, Gauge Theories in Particle Physics (Adam Hilger, Bristol, 1982).

[A.42] T. Kobayashi and M. Kuroda.

[A.43] J.D. Ware and M.E. Machacek, Phys. Lett. 142B (1984) 300.

[A.44] R.M. Barnett and H.E. Haber, Santa Barbara preprint, NSF-ITP-84-78 (1984), to be published in Phys. Rev. D. 